

\section{Re 118.801}

\section{Library}

of the

University of Toronto 




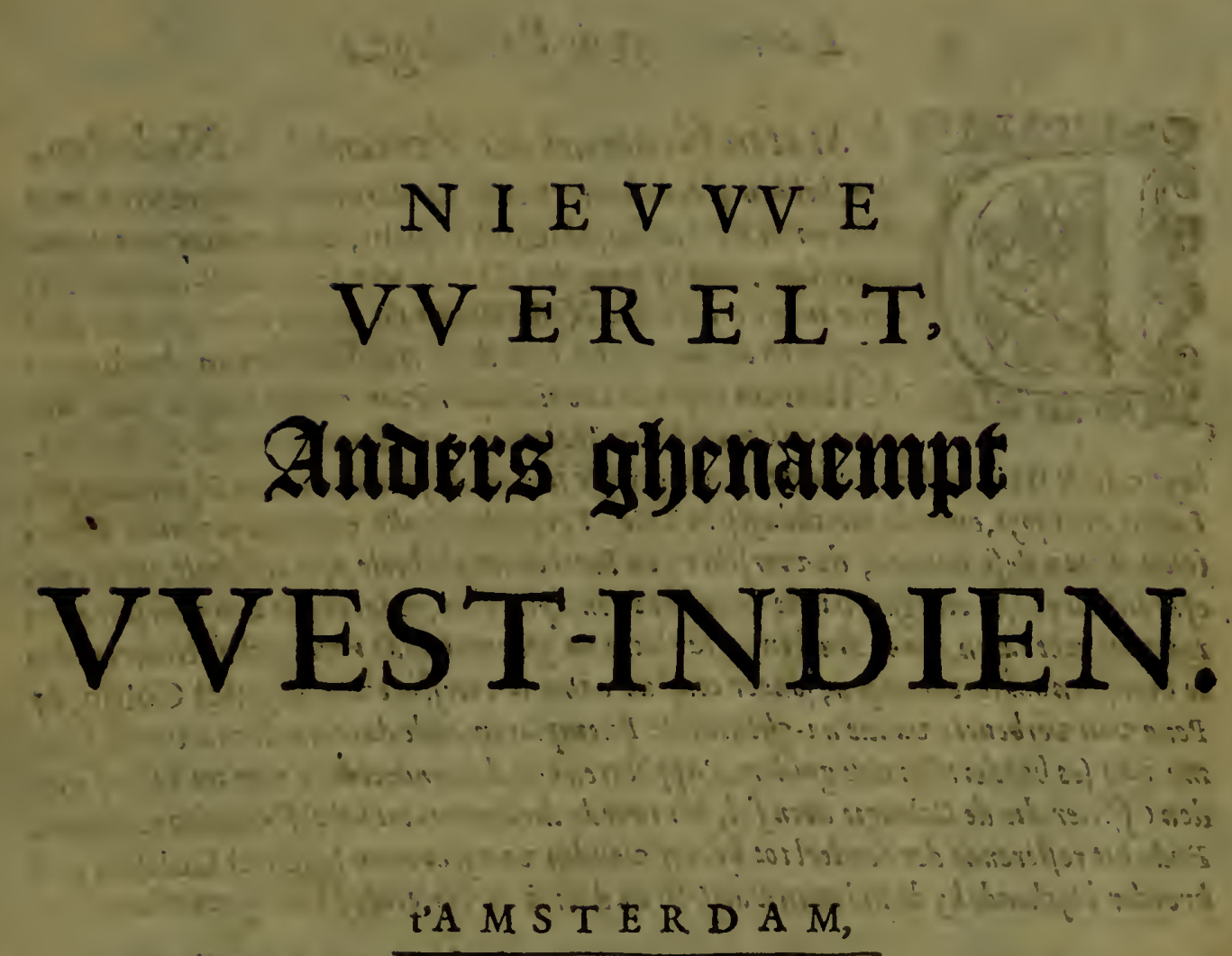

25p Micbiel Colijn, 25oeck-bercooper op't mater by de gDude 23zugh / in't tapus-21boeck.

Zinna 622

(. ' ONes Privilege. 


\section{Extract uyt de Privileges.}

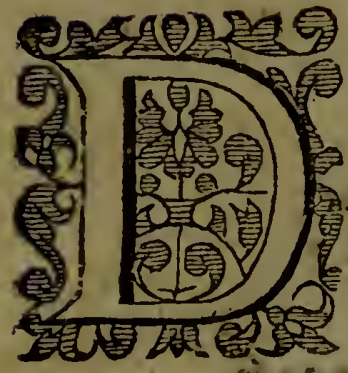

E Staten Generael der Vereenichde Nederlan. den, bebben ghecon entecrit, ende gheodtroyeert, Consentecren ende octroyeren mits de fen, Michiel Colijn, Boeck-vercooper tot Am. ftelicdam, dat by voor dentÿt wan acht.Iaren naeft-comende, al. lecne ivide Vercenichde 2 ederlanden fil mogen doen drucken ende uyegheven de Bocken by bem doen tranfrateren wan Anthonio de Herrera opperfte Cronÿck-Schrijver vande Indicn van He. in ghen den Coninck vian Spaignien, sracterende sande glseleghent-

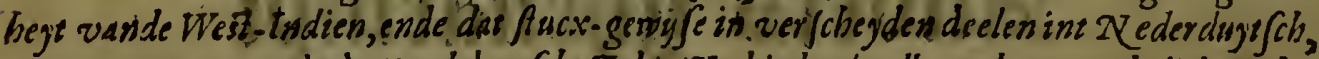
Latÿn,Françoys, exde de Hoochdugt Cche Tale. Verbiedende alle ende een yeghelick Inghe. Jetenen ruan defe Landen, de woor Jchreven boecken int ghebeele, of te ten decle, int groote, of ie ine cleyne, In ecnighe vande voor folbrëwen Spraecken, geduerende den voor cobreven tyt van acht haren, na te drucken, ofie elders na gedrucke, ivide vercenclide Nedertanden te brengheriomie vercoupen, fonder con fent vanaen voor fetreven Michiel Colijn, by Pene van verbeurte vande na-ghedrucke Exemplaren, ende daer-en-boven vande fomme van fes hondert Carolus guldens, t'appliceren een derdendeel dacr van zot beboef wan den officier die de Calengie doen fal, bet tweede derdendepl tot behoef vanden urmen, Ende bet refterende derdendeel tot behoef wanden voor fcbreven Michiel Coli)n, alles breeder blyckende by de Brieven ruan Octroy daer van werlecnt, fijnde gbereeckent

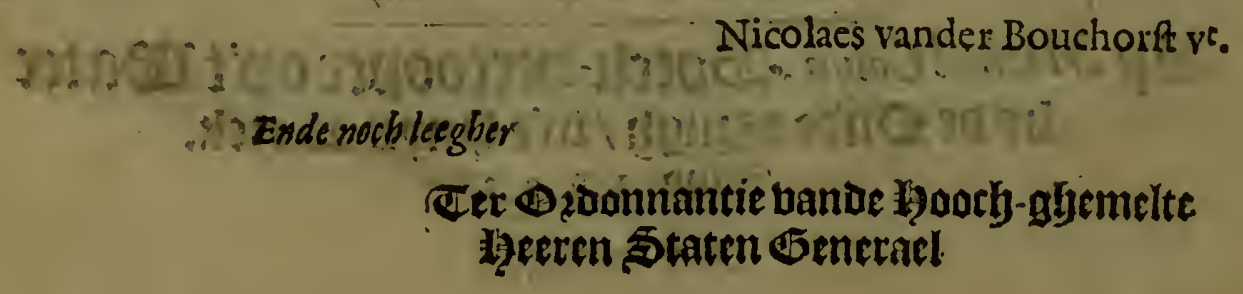

C. Acrffens. 


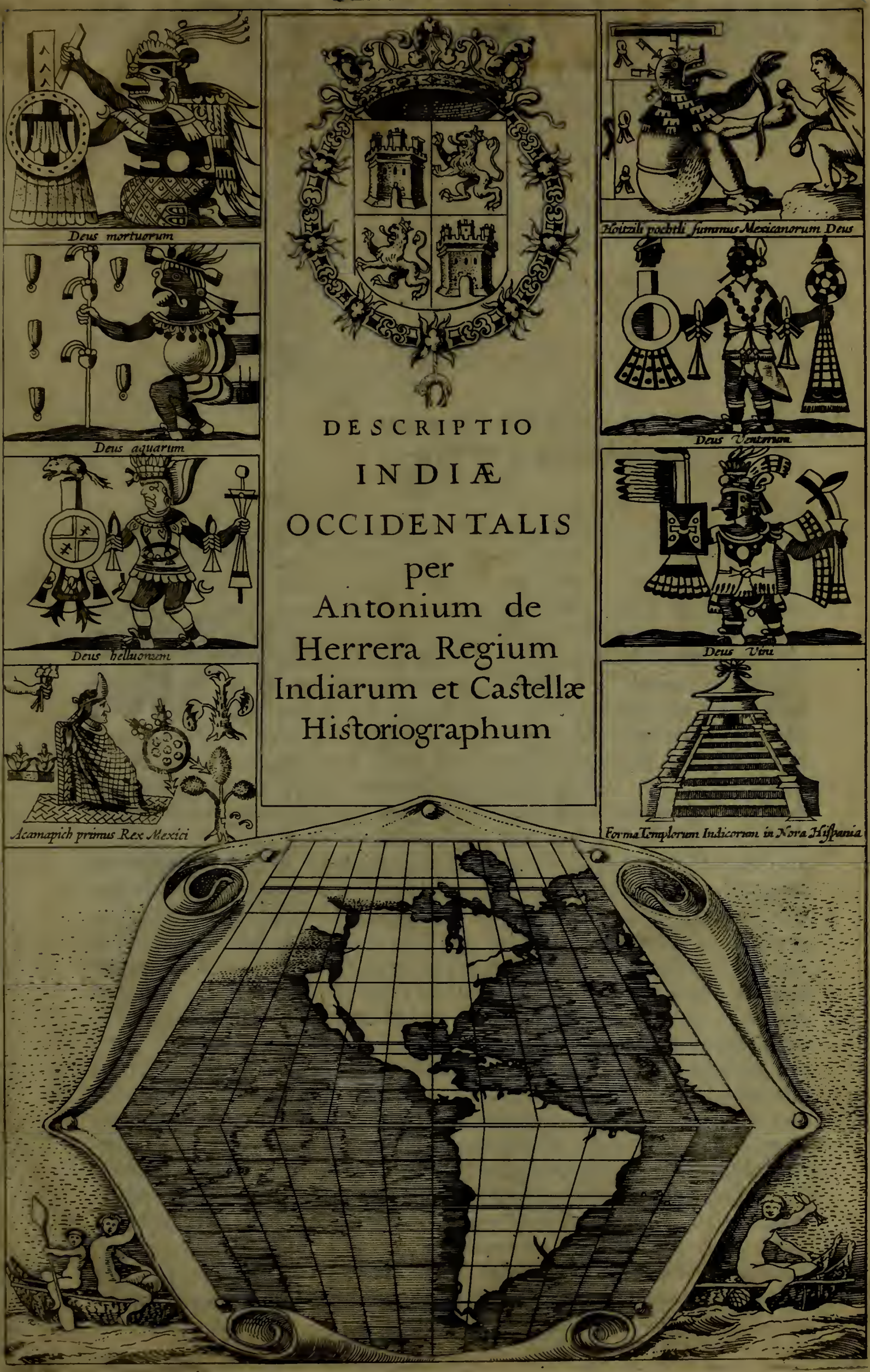




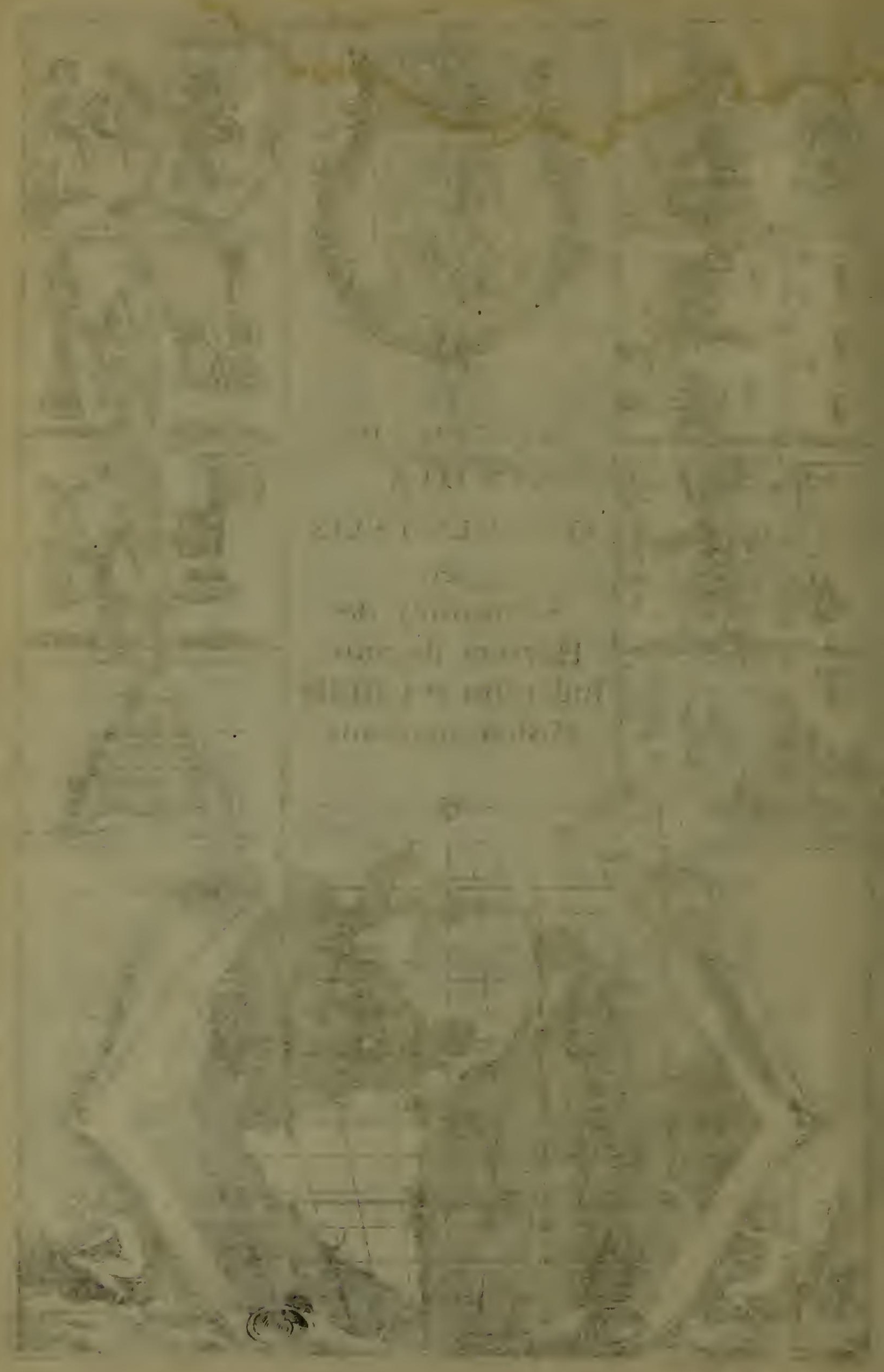




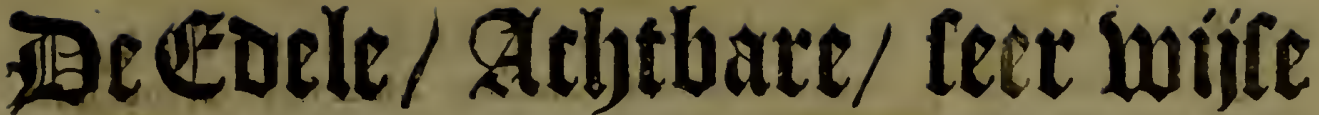

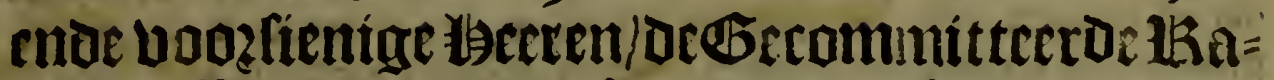 Den Det zldmitalitept/ Lefiderende tot ZImetcroam.
}

\author{
Ioncker Fredcrick van Baer, Heere tot slanghenberch, $Q c$. \\ Aclbrecht Heere was Schaghen, Burchorn, $O c$. \\ Nicolaes VVouter 2 . Burgermeester sot Haerlem. \\ $V$ Villem VVarmont, out Schepen en Vroet Schap tot Leyden. \\ Adriaen Pieter $\sqrt{z}$. Raep, out Schepen ende Vroet $\int$ chap lot Amfterdam. \\ lacob de Vry, out Schopen en Vroes fchap binnen der Goude. \\ Theunis I an $\sqrt{z}$. Burgermeester tot Edam. \\ Ian Adriaen $\sqrt{2}$. Zoggaert, out Schepen ende Raedt der siadi Middelburch. \\ Iacob Lieftinck, Vroetschap tot VV trecht. \\ Ioof Iongbeflal. \\ Dirck Nykerck, Burgermeefer tot Znol. \\ D. Hendrick Storm, Raedt ende Advocaet Fifcael, \\ lacob Lauren $\sqrt{z}$. Secretarius.
}

\section{Smenfogt Micfrief Cofiiy Mafr soorgatnde

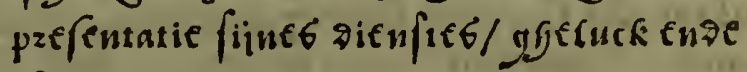 Eaficfrequt.}

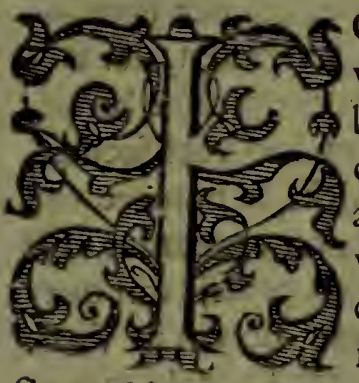

Ck prefenteere V. E. met hoochfter eerbiedicheyt, het vierde deel van den Aerdt-Cloot, niet foo wijdt ende' brecdt, ghelijck het in fich felve is, maer als het in een cleyn begrijp van Caerten, ende corte bcfchrijvinge van, alle ende yeghelijcke plactfen t'famen ghetoghen, ende vervatet is: op dat V.E. haer ghe moet, 'twelck met veele' ende ghewichtige faecken befich is, altemet verluftigen? met een Reyfe door die Laniden, waerin de materie van $\boldsymbol{r}$ menfchen giericheyt ende begecrlijcheyt is begraven en befloten. Daer zijn voorwaer veele dingen, die V.E.tot een naerder onderfoeck van't Americaen: fibe Ryck, ende'tlefen defer Hiftorie connen noodighen. VVant beneffens, datter nicten is, 'twelck Regenten ende voornementlijcke perfoonen beter. betaemt, dan de kenniffe van de werelt, waer in fy in eere verheven fijń, Heerfchappse voeren, na Rijckdom trachten, Oorloghen, ende 'tgantfche menfchclijcke geflacht woelt, ende niet onbetaemlijcker can zijn, dan geen wetenfchap te hebben van die Landen, over welcke te ghcbieden niet min profijtclijck als heerlijck foude zijn: roo durve ick dacr-en boven fegghen, datter defe Republijeque, in de welcke V. E. foo notable dicinften doen, niet weynich, aen gheleghen is, dat V. E.' fecckere ende volle kenniffe van defe Nicuwe VVerelt, mïtfgaders van de Stcden, Sterckten, Havens, Rivieren, ende Volckeren , dacr in zijnde, becomen. Nac dat foo veele treffelijcke feyreñ binnen ende búyten Lants, te Lande ende te Warer, geluckelijek fijn. uytgherecht, na dat de Spángjaerts bý nac uyt de Moluck fchc Eylanden zijn, ghédreven, nac dat de weghen vánt Noorden ende de Tartarifche Zee zijin befocht', hebt ghy oock ten lactften beraedtnacelit, een voct inde Nicuwe. Werelt te fetten. Men heeft tot allen tijden gcoordeclt, dat liet de befte wijfe van Oorloghen was, den Vyandt nict alleen op onfen cyglien boden ende ontreht onfe cuften te verwach ten: macr hen oock in fijne Rijeken, daci hy fonder forghe of vrecfe is, nae 'texempel der Romeynen, te beftoocken. Het is kenlijck, dat Hannibal door defen trecck uyt Italien weder in Africa is ghedreven, Ende dit weten oock de Medecijns, dic, wannecr 'trechter been 
eenich letfel heeft, het andere altemet wijffelijck fnijden, om den Vyandt der ghefontheyt van de crancke plaetfe af te locken. Maer fulcx fal met minder voortganck ende voorfpoet ghefchieden, ten fy faecke V.E. wel ende grondclijck verftacn, welcke den wech zy, die daer toe leydt, waermen onfe Livius $x x \bar{y}$. inghefeiene beft planten fal, wat profijt daer uyt voor den handel can ontftaen, ende hoe alles feeckerlijck can worden int werck gheftelt. Livius prijft dit in Hannibal, dat bem alle de zhelegentbeden vande Vyanden, niet min als yyne eyghene bekent waren, waerom hy oock beveelt dat men der vyanden heyr, de fotuatie der plaet fen, ende den aerdi van't Lande verfpiede ende door fie, Macr dit alles canmen niet beter comen te weten, dan door de Geographifche befchrij vinghe, dewelcke ons voor ooghen ftelt, felfs die plaetfen, die wy noyt hebben gefien, ende den lefer,al blyft hy fchoon te huys, door de geheele werelt,ende de alderveerfte oorden van Landt ende Zee, met cen gheluckighe ende feer ghencuch lijcke feylagie fijns ghemoets om voert ende leydt. Tot dien eynde heb ick beftaen V. E. toe te brenghen, den aldernetften fchrijver van West-Indien, Ansonio de Herrera Spangjaert, den welcke ick andere talen heb doen fpreecken. Een Autheur, die, zijnde vereert geweeft met den Tijtul van des Conincx Hiftory-fchrijver, ende tot in't binnenfte der fecreten van den Raedt van Indien toegelaten, defe Hiftoric met groote trouwe ende waetheyt, maer weynich tot voordeel van fijne Natie, fchijnt uytghegeven te hebben. Dit gaet feccker, dat niemant de palen ende fcheydingen der Provincien, ende de groote van de felve, de Zee-cuften, Capen, Eylanden, der Revieren vloeden, cromten, gaten ende uytgangen, de gelegentheyt ende breed. te vande Meeren, ende ftaende wateren, de ghedaente van foo veel Steden, Schanfen, Vlecken, Dorpen, Havens, Reeden, en eyndelijck, de natuere van de Landen in't befonder, watfe voortbrenghen, ofte niet, met meerder vlijt ende duydelijcker befchreven heeft. In dien hier uyt eenich profijt can ontftaen voor onfe Republijcque, indien dit werck V. E. can dienen in V. E. treffelijck voor nemen, fo fal het my niet berouwen, dat icker fo veele moeyten ende coften om heb gedaen. Ghy fult hier claerlijck fien, Mijn Heeren, hoe wijdt ende breedt de macht van uwen Vyandt fich uyt breydt, die niet te vreden zijnde met een deel van Europa, Afia ende Africa, defe gheheele Nieuwe Werelt by fijne Croonen heeft ghevoecht : Ende hoe groot fijne fchatten ende macht ten Oorloghe van daer gheworden zijn, heeft de Chriftenheyt bevonden ende geproeff, dewelcke fchier aen alle hoecken bevochten is door fijne wapenen, ende nu (Godt betert) in veele plaetfen buckt onder'tAmericacnfche gout. Ick en verhale dit niet om V. E. herten te ontrocren, maer op dat $\mathrm{V}$. E. der voorgaende dapperheyt indachtich zijnde, met een volftandighe cloeckmoedicheyt, onder 'tbeleydt van den gheluckighen Prince van Oraengien,'tgewelt van dien feer machtigen Vyandt tegen ftaen, ftuyten ende breccken, want by aldien ghy hem overwint, fo fal den lof van de viktorie foo veel te heerlijcker zijn, hoe veel te mach tigher den vyandt is, waer teghens ghy hebt te ftrijden gchadt. De Almogende Godt, de Behouder van onfe Vaderlandt, wille V.E. de victoric verleenen:Macr waer de vietoric behoort ende mach vercreghen worden, fal dit Boeck aenwijfen, het welcke ick V. E. bidde foo gunftelijck te ontfang hen, als het comt van fulck eene, welckers wenfch ende bede is, dat de Heere ende Regierder van al, mildelijck ende rijckelijck geve, alles, wat tor V. E, cnde defer Landen welvaren ftrcckens san. 


\title{
Voor-rededes Autheurs,
}

\author{
Aen $P$ aulum de Laguna, Licentiact, Prefident van \\ den Opperften Raedt vian Indien.
}

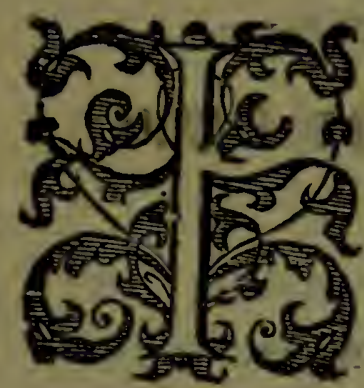

Ohan de Ovasdo Licentiact, Yoorfaet van uiwe E. de welc: ke Prefident fijnde, het Ampt van Hiftori-Schrijver van d'Indiens eerft hecft opgherecht met groote nzerficheyt ende arbeyt by-een-ghebracht, alle de fucken, acten ende monumenten, die to tdefe faecke mochten dienen, ende tot ontdeckinghe van Landen, Steden, ende Plaítinge van volcke ftreckten; nict alleen uyt Spaengien; maer oack, nyt Weft-Indien felfs; op dat alfoo de feyten, die by de Spaengjaerden in de nieuwe Werelt uytgherecht zijn, met dies te meerder waerheyt ende lof berchreven mochten worden:Maer na fijn orerlijden is' t ghebeurt, dat door verfuym vail andere, de befchrijvinghe van die Hiftorie eenighe Iaren is blijven fteecken. Maer als ghy tot het Ampt, ende de Weerdicheyt van Prefident verheven zijt, hebt ghy gheoordeclt, datter niet onbeh oorlicker was, dan dat fulcke treffelicke daden door ftilfwijgen fouden begraven worden; mits dien doch de Hiftorie de Schilderijen fou verre overtreft, hoevieel de ziele weerdigher is, als 'tlichaem: ende hebt dacrom alle moeyte', vernuft ende vlift daer henen ghewender, dat dees Hifturie moche voltrocken worden: Daer-en-bovén, foo is't oock door V. E.ghefchiet, dat een goede gagie ghcordineért is gewrorden voor, den Hiftori-Schrijver, op dat de felve aldus door den prickel van fulck tractement ende lof opgheweckt fijnde, dat heerlick ende gewichtich Ampt met meerder vlije mocht uytvoeren. Soo comt dan V.E. voornemclick toe de eere van defe Hiftorie, als oock den danck, die V.E. fehuldich fijn, alle de ghene, welckers voor-Ouderen lof ende daden met uytghedruckte namen in defe Hiftorie worden uytghecondighet. Want d'opinie van lobannes Baptifia Ramufius is barbarifch ende gant fch, onrechtveerdich; dic hem niet en heeft ghefchaemt te fchrijven in't derde Boeck van fyne Zee-vaerten; dat het ydel ende befpottelick is, dat de Schrijvers vali d'Indifche faecken foo befich fijn, om de namen ende'tVaderlandt, van de ghene; die door loffelicke daden in die quatrieren beroèmt zijn gheweelt, aen te teeckenen ende te verhalen. Voorwaer die menfch ontdeckt hier mede de fieckte van fijne nijdicheyt, ende fijne quaetwillicheyt teghens de Coninghen ende Natie van Spaengien. - Want het yoorhaemfte ooghemerck der Hiftorie is, den ghenen, die wel hebben ghedaen, met prijs ende lof te vereeren, ende daerenteghen fulcke, die het qualick acnftellen, met die fchande, die zy verdienen; te brantmercken; ende fulcx den. Nacomelinghen tot cen exempel : het wclcke nochtans niet can ghefchieden, by al dien de meeninghe-van Baprifla plactfo comt te houden. Maer V.E.oordeelt anders ende béter, ende fal dertralven de Spaen: Iche Natie, die om defe oorfaecke rondelick bekent; $V, E$. eeưi ighe danckbbaêrheyz fchuldich fe fijn, gheenfins lijden, dat uwer $E$. ghedachteniffe ende prijs t'eenighein tijde.come te vervallen. De almoghende Godt wille V. E.behoeden. Ghegheven tö Valladolit, den Is: OAtober, Anno i 601 .

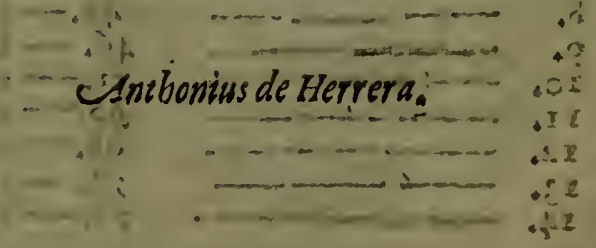




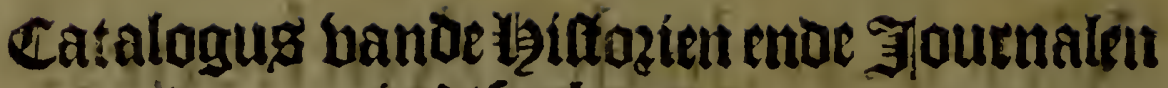 in oeren begrepen.}

$V$ Vest.Indien, befchreven van Antoni de Herrera, ende Pedro de ordonnez de Cevallos.

Voyage van Iacoble CMaire, door de Strate le Maire.

Vàn Capiteyn Don Ioan de Moré, door de Strate le Maire.

\section{Acrtchepden 1kelatien ban die geene die de Strate} gagellanes beblen gyepactert.

I. Ferdinando Magellanes, Anno I 520.

2. Don Iutieres Carvaial,

3. Langhs de Zuydt Zee, door Capiteyn Ladrillero.

4. Don Fray Garcia, lofre de Loayfa. Is2s.

s. Sr. Francifco. Draeck. I 577.

6. Ianghs de Zuyt Zee Pedro Sarmiento, afghevaren van $\mathrm{Li}$. ma. I 579.

7. Thomas Candifch. I 587 .

8. De tweede van Thomas Candifch. I 591 .

9. Van Iaques Mahu ende Symon de Cordes. I 598.

10. Van Olivier van Noort. I 598 .

¿\$De Udotabulaer vande Tale vande Eeplanden.

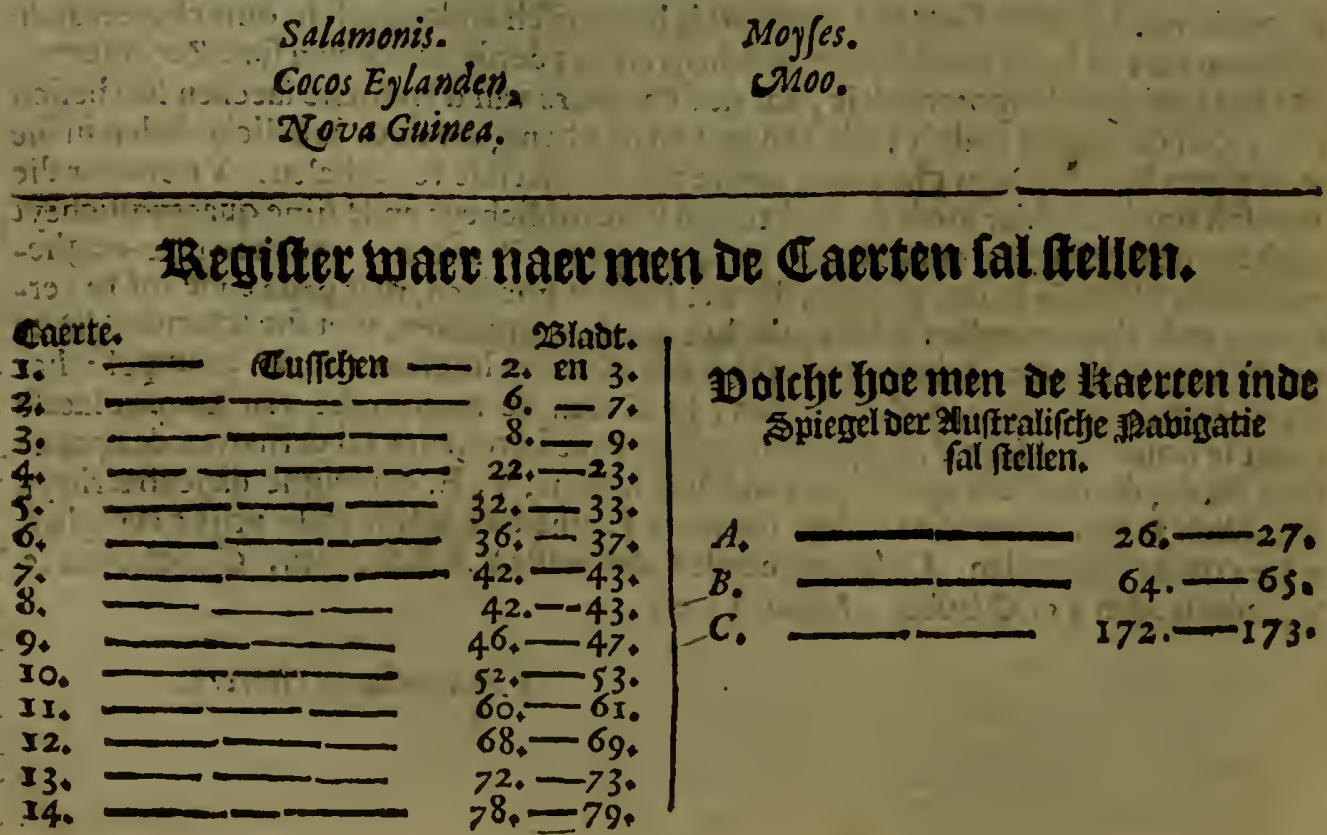




\section{Refchálúntge bande efplanden/

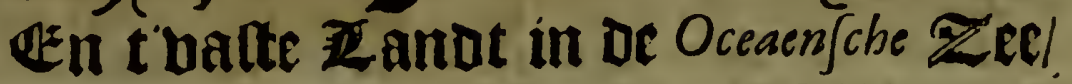 Diemen noemt W ES T-I N D I N N.}

\section{boot \\ A NTONIO ban HERRERA;

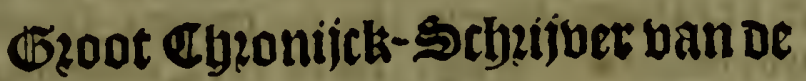 \\ Indiens, $\mathfrak{m}$ Caftilien.}

Het I. Capittel.

$V$ an de toedeylinghe, en deelinghe var de Wert-Indiens.

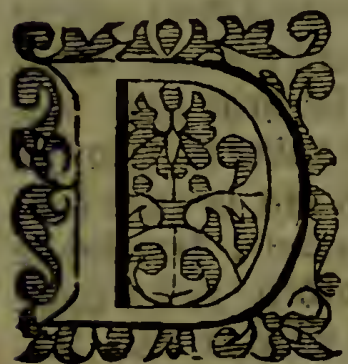

Ge omgang Degalertbodents beltaet in Daie hondert en tie = eticly graden/ Die gljebzacbt ziin= de tot Caftiliaenfche $\mathfrak{A B}$ iilen $/$, ulackiender fes Duplent en dxie handert : lup beu Dmgangh oes. Zlerdetrijex / bet= atactmen oock teffend of zee / De= fe twee Gilementen maecken een Cloot/ wiens aen weder-fijden ne= Derdalentae ulackec / ten beele ig De Zlerde / en ten deele. De zee. De Ouden bebuen ghedeplt bet ztert= riick in dic acelen/en bebben ghe $=$

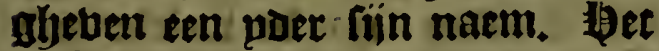
eertte noemden $3 \mathrm{p}$ Europam, 'tmee = At boven andere gljepzelen. Het. tweede luas Afia, lyet grootite ban D'andere / begriipende in ljem bet groote Riich ban China:- bet ber = be beel is Africa. De grentrlyen siinde in meeninglye dat de toe rele niet meer en biel dan be obe= noentoe beelen / met de felfoe niet: te becoen siinde / begaluen faer tot. oe contele ban nauigeren/. en tot be Linoiltalje ban groote Sctjepen/Dic maet fentoe alloo dat $3 \mu$ condố uer $=$ Dengen de cracht van de Golbé des̆
Zeeg: : en in die contte bebluen be ae spaens Spaengiaerts alle andere ßatien giarts bette

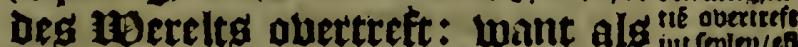
in Caftilien en Leon regeerden of tman greocen Doozlurbtige Catbolijeke Conin = \$atyeyem. glyen Ferdinandus oc bijfae/ en fiin Bzoutue Íabelle, De alderbifife en vailiantfte Coningunne. En in Portegael D. Iohan de twetue/ ghe = naent de Pellicaen, die d'efulwighe glyelyeugenife altoos fal weeroirf 3iin. Don Chriftoffei Colon, D'eer= Ite zlomirael ban Weft-Indien, ghe= leeft lyebluende beel jaren $\%$ en ghe = trout in bifpanien/ met oe raed ban Martijn uan Bohemen, Joatu= gijg/ghebooztich bant jet Éplanot Fayal, bermaett Aftrologus, en een bpfonder oozdelaer/ en andere daet bu mede comnumiceerde / Jeeft bet beginne ghegheben ban dontoes= Kinglye deg: Xandts/ Datmen feden reeckent het biermbed oes $10 E=$ reltg /'tgrootette ban alten. Én ne= menoe lijn couts Derbaerts nacr be Son onver gaet/ varende af ban Palos, een Stabt ban de Graef uan palos rom

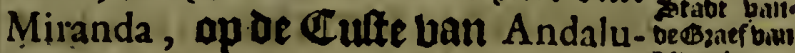
fien, fenloe foo langh booz d'O. ceaen, dat fy bont bit groote Zlant/ let welcke Dooz bet mioosn gljefne= Den wozt bande Equinoctiacl : $\mathbf{m}^{\text {i }}$ tgaet foo leet Zupo-waerts / oat Ijet comt tot op twecnbiiftich gea ben en een balf : ende foo uerde Roozt-waere bat het ous fefunlt onder den Polo: Aretico, conder te weten Ijet rinoe. 
De grootyeut ban dit bierde deel Der hoocljten jïn die/ dicntê neeme lyeeft oc sgenfeljen ghebzacljt in en telt ban de Polo af die reeclier

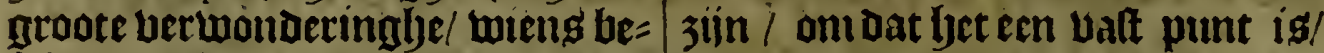

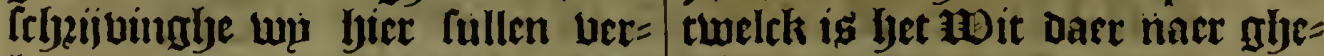
handelen / onoer De naem ban Ëy= frboten wert/ met de welelie men landen/ en lyet Lalte ILant/ ban den Oceaen, oul te beren oluringt baul die $\mathbb{Z} \mathfrak{c} / \mathrm{en}$ gljeleghen int $\mathrm{mBelten:}$ en die ziin gheneenlick ghenoeme Weft-Indien, en de niculwe merelt/ cul begrepen in de demarcatic of toe = Deplinglye ban de Coningyen ban Caftilien, en van Leon, 'twelck is een Hemifphxrium, en de belft ban de Derelt ban 180 . graden/ begolt

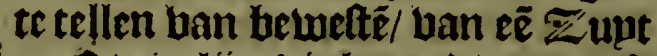
seaer $000 ;$ Dat be Zumia en Roozt-lijn/Die loopt Do02 39. oft bull be beps ungbe loopt. 40.graden Der lengte/betwelten den Meridiaen ban Toledo, weltke gaet over den mont ban de Hibier Maranjon, en booz fyet Bofterdeel ober de Stadt Malacca, alloo dat bu we= gljen uan 2o.legtuas op een graet/ Defe demarcatie oberobeers bau D'eene tot D'ander fijoe dzie duufent ell negen bondert Caftiliaenfche $l e=$ guag/elch gbareeckent op daie bup= fent Haften/ ban tuif Caftiliaenfche koe-boeten: t'welckmen rept Dat fouden ziin feg handert 3taliaen= frlje ghiilen/Lan't Bolten / tot bet Helten / 'twelck het Zze-Bolck noemt (boft en 1 bat / en die tellin= glye van twintich lequas booz em graet / ig conform met die ban P tolomzo, en oock bet ghetuolen ban vele curieule lupoê. alndere hebduen gledicht datter trebentich lioom= filje oft 5 taliaenfelye mijlen in een graet fijn / en dat 3 niet meer en maecken Dan feluenthié en een balf Caftiliaenfche leguien; twaick gebou= Den loct of luefte tellinghe.

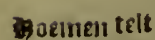
Degradé bait De laugte.
De graden ber lenghten/ tweick

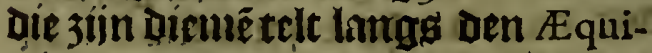
noet iael, gaende van ljet Boften na 'tibeften/aber bet nuiboen ban fet rout / en de Cloot Der Zterden / en beeftuen loo wel niet connen waer nemen / on Datmen in de lgentel gljeen balt teecken mbert: graden allier aenwiffinge fal doen in defe befelyzijuinglje.

Dace is ont oeckt en befcult wan maater dulse

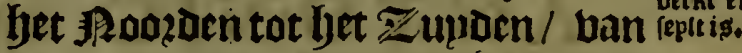

De 60. graden \$Roozdeliclte breete/ tot de 53 . Zupdelicke/ twacleli zijn Duprent / negljen ljondert en leven= en trebentich leguen lants / twelels Dit bafte lanot bout in lengte. $\mathbb{E} \mathfrak{n}$ al wacrt op fijn beetlte is lyout lict ouplent dzie bondert / en op fijn ininfte tot achfbien / twelck is lyet fmalte van Nombre de Dios, of Porto belo, tot Panama, Doot welclie natuplick dit 1 ant gljedeult wort/ latende bp naelt de helft baer ban int \$oozoen/en d'ander belft in lyet Zupden / twelck zijn de twee dees Ien ban die demarcarie. Het Derde Decl zijn be Eplanoen / en let valte Zlant twelck bp Boften ban Malacca is / waer ober de linie van de Deplingtye is gaendo / tuffeben de Croonen van Caftilien, en Portugael, die een Deel maecken uan OoftIndien, ten relpecte ban Caftilien, werden $3 \mu$ uljenoemt Indien Lan't Weiten, ghelijckmen fien call in be alghemeene Caette die bier nace bolght.

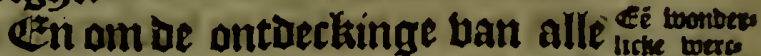
Dere Tamien / uut oe welcke beré kinge ban oe

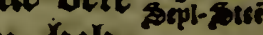
Coninckrijeken ghetracken hyeb= ben foo aroote riicksoommen / nien moet dien toetefyijuen den SeplSteen / wiens Londerlicke cracht in die tijoen is ontoeckt booz Don Anthonio Oforio kiduer ban Valladolid enoe was togene dat de Sepl-5teen lyet Hor mede-deplde een heftigher amtreckende cracht/

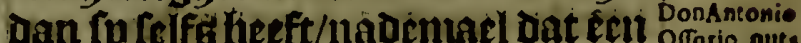
Gifer gfjeboectjt acn de tant ban be bersere groos

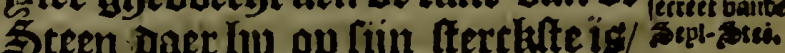

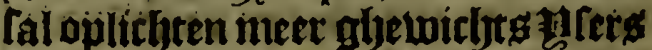
ald bo felfg beeclit. . Op alfulcke wijle 


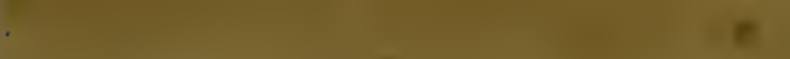

$$
\text { as }
$$

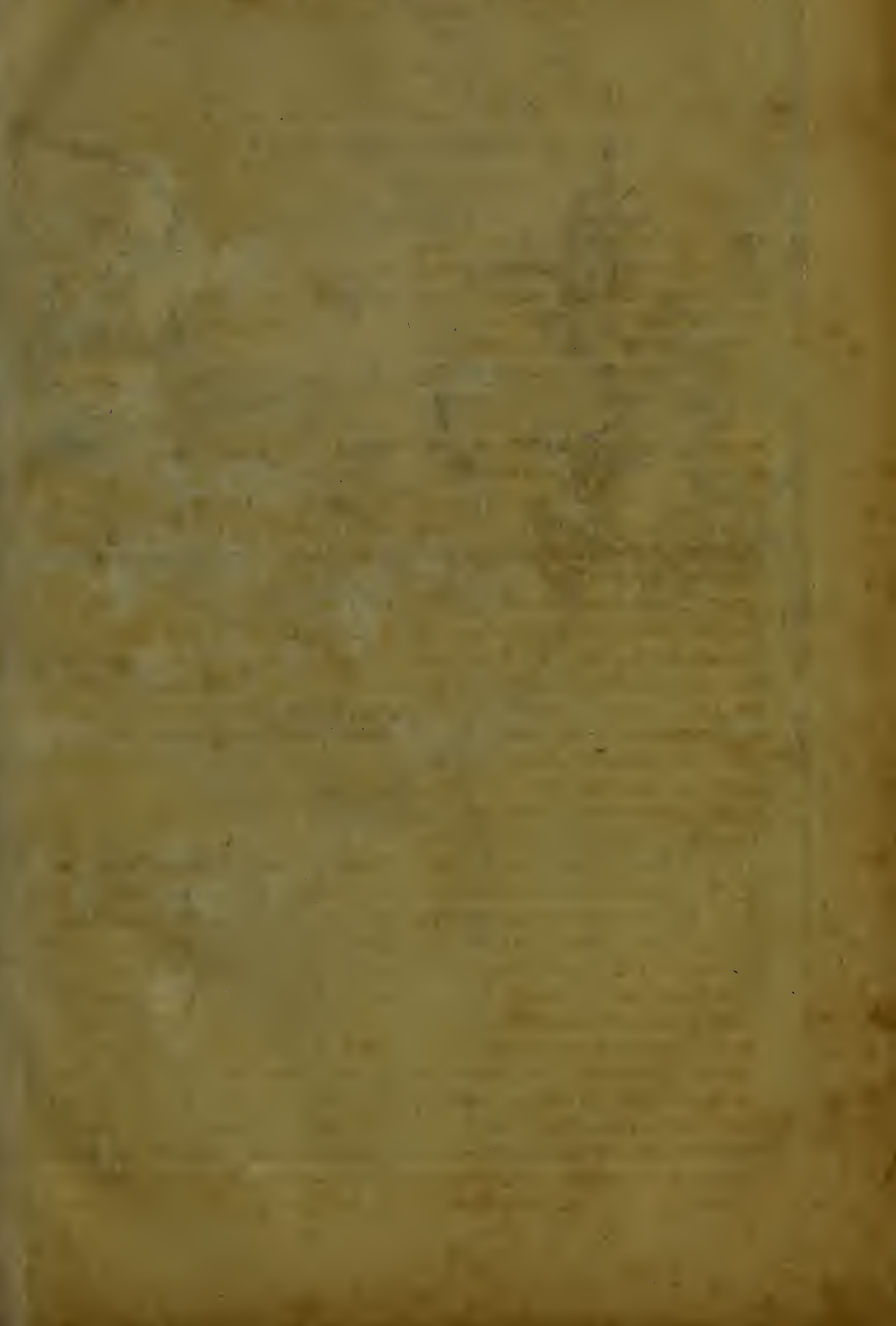




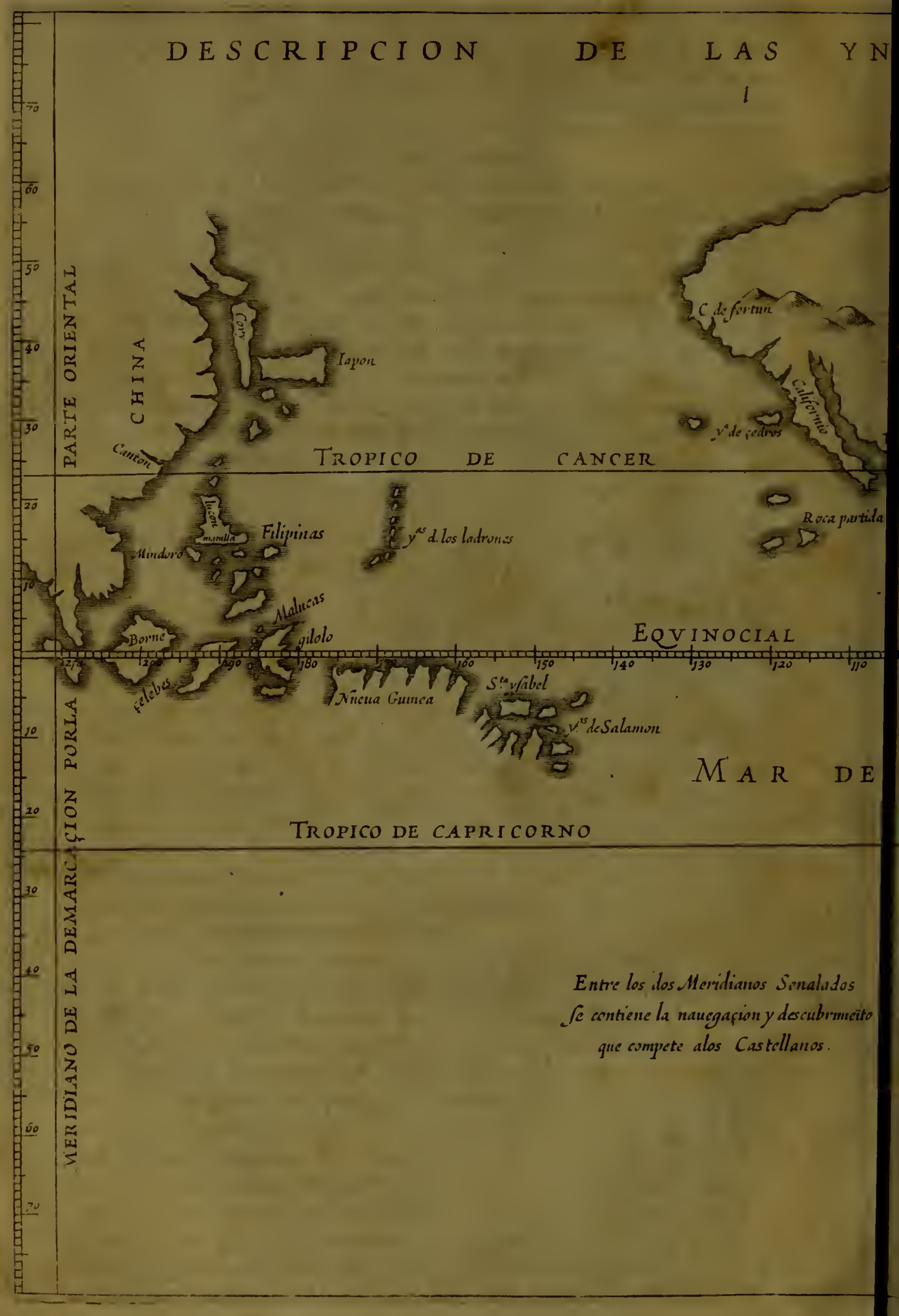




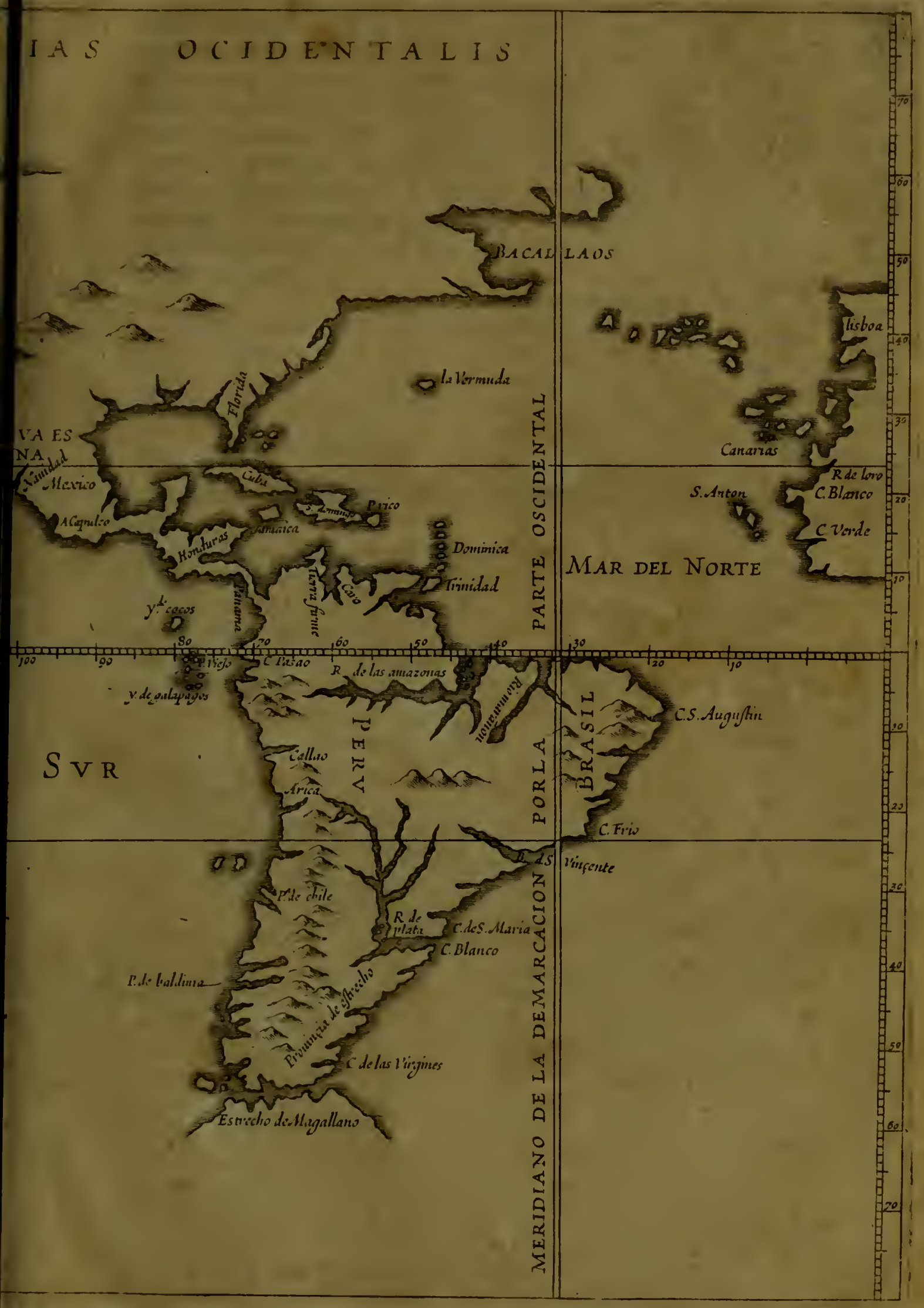




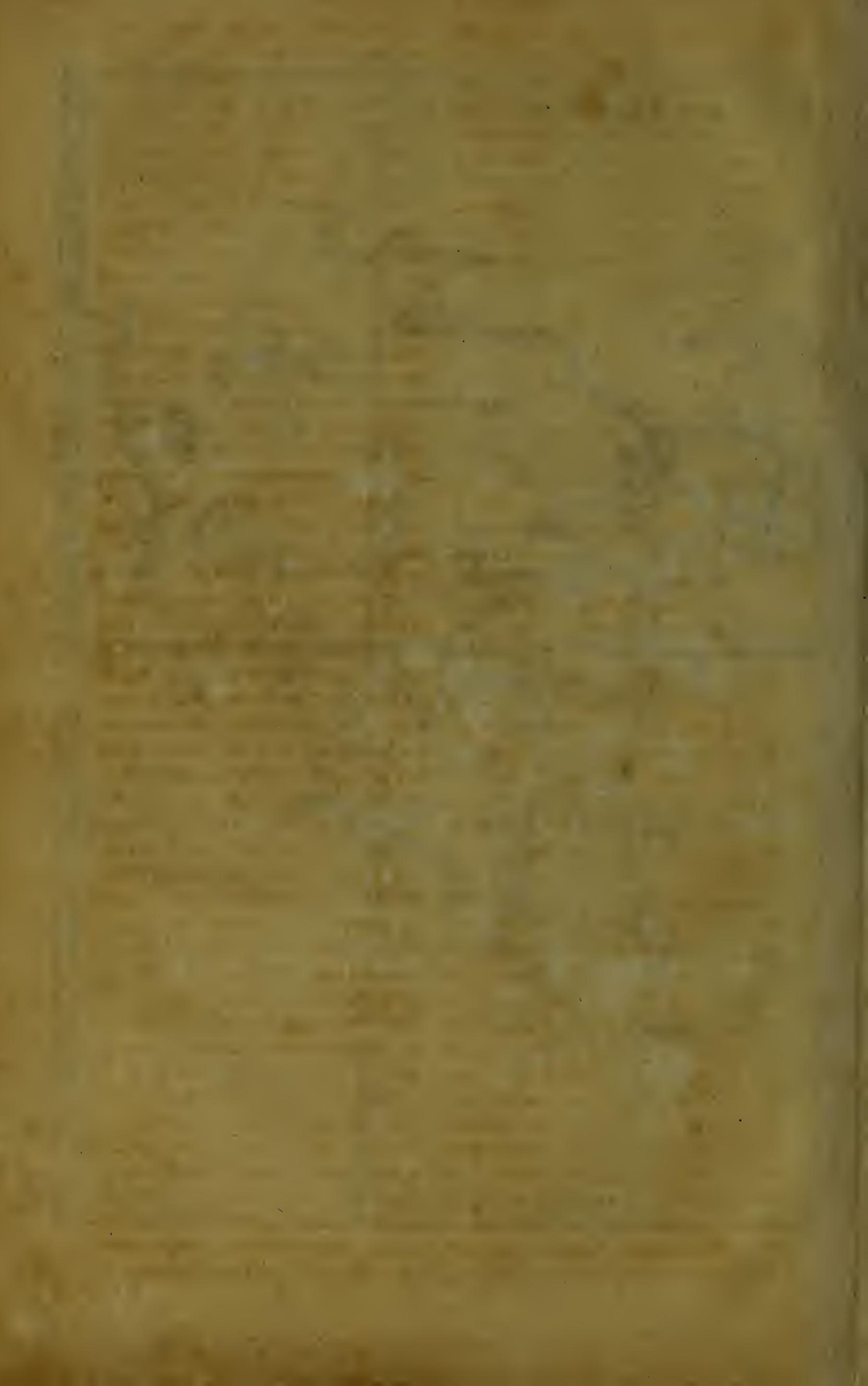




\section{uan $V V e f t$-Indier.}

Wiile Dat cell Scpl- Steen Die niet ureer weeclit dan twee ponden ma een vierendeel / en beeft be natuers liclie cracbt niet om ineer op te liclyten Dan lta oneen aljewiclitg/ it inum pecfentie ljeeft opgljelieljt vectljien pont Hifers: Defe craclit

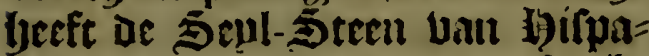
tien niet. Boozwaer tis een faethe dic de pryilofoplyen loel wat be $=$ acnetken glyett.

Hier moet comen de Caerte, Nurn. 1.

1)et 11. Capittel.

$V$ ande Reyje naer Indien.

$\mathbf{M}$ Ardel Norte wozt genaaf̈t die $\widetilde{Z} \mathrm{ec} /$ welelte int (Boltê/ en Mar del Sur, die welclie int Weetté is : Ban Defé Mar del Sur wozben befpoelt be $\mathbb{1}$ anden ban nicu 5patgien/ en Pcru, en bau de Mar del Norte, die $\mathbf{Z}$ andé die Zupt: warett vall Brafil nae de Strate toe sijn gljelegrien/ en booznamentlick Die Rogrt-watert unu Brafil na CaStilien beutu / en andere Poozder deelen jijat glyeleglieit/ welche Zeen Daer naer haer verdeulen in andere minoer Solben/ eltoe in alles beb= ben dele $\mathbb{Z}$ een vier vooznantelicke Duet sin navigatien of vaer-waters. Bie giernabiga' cerlte an de outlfe is / Die ban Cafti-

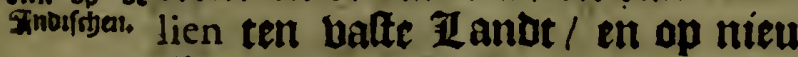
Spangien gaet. De tweede is die vail Caft lien na de Rio de la Plata, en na de Straet van Magallanes is gaende. De derde is die van de Gutten valt nieu bifpanien / van Guatamala, en Panama, naer Peru, Chili, ell de Straet is gaende: Eznde de laetite ende alder-nieu= ate is dic bun nien Spangien / op De Eplaubelt bant Hoelten / en de banoclinglye uan China is gaen= De / ghelijetsmen licticau in be ne= bentaende Caerte.
De cerlfe navigatie/ oull batfe meernte na Ineet bebaren wert / ig gljenoemt Carrera de las Indias, wert ill tween glyedect / D'ene is op De baven Juit S. lan de Vlva in uieu Span= gien. Tot welcke plactie toe van Sevilien feultmen bu uaft oulurmt at feven-Ljondert leguen / in twee भYaenden ell cell haif : m d'an= Dere uner Nombre de Dios, tun D'all= Det แa Porto Belo, twelek if int Hivelt Datunen yet Balte lant noit/ ban 1400 . Ieguen / in twee laughe S9aenden/en die bepoe loopt men inet een courfe tot datmeli comit tot de Eulanden luan de $\mathrm{P}_{0002 t}$ Zee / uan San L ucar de Barrameda, Defnartille: ban wiens barra nuen tiet mach loope: ban afloopen Conderpilote die tiundirl] is op de trille / goede wint lyeb= Gente/ en waftent Bater/en dach= licht / of Zlanteern-licht oin te fieu de mereken op de dzenupel.

De tijoen om defe nabigatien te beginnen jiju veritheuden. Dae nieu Spangien de Minter obet welende unu bet begin ban atpril/ tot dat g9ap unos bu is/ en later niet / oni Datment niet en comt aen op wat the

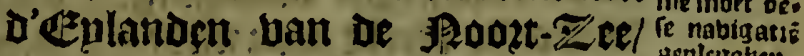
nae slugutto/alg De \$Roozde Hoin= Den Domuineren/en de Vracanen, ut: guturen / tweltek fijn omweders ban de fehertmutfinglyen ban de con= trarie minden. En nae het uafte landt / leptmen de reple aen / al eet de Hinter aenbangt/ Det gantfetjen atugutto en Septenuler / om batment can comen te Porto belo na Robember/-als wanneer om fot beginnen vande Poozde Hinden de relfoe Calte mequich ongheront ig.

Dail S. Lucar gaet men om ie Canarien aen te doen / tweltk fiin In-naelt twee boutoert en biifticly leguen feuleng/ in acljt of thien da: gljen Dooz dell Golfe de las Yeguas, Die ill de 1 Binter Daugeteus is on de oumeders / et als ju be baven yan Canaricn conuen crijghen/ full= 
Iell sli dien inloopen / of anders gaetl ju op Gomera, twele lyet balte Eulant izuan dicn.

Ban Canarien tact men ou la Deffeada, dat een Eplant is op viif: thien graden/ of een wepnich meer oljeleglyen/ oft op la Dominica, tot weltie plaetre toe Itellen ju feven Jondert leguen / dooz de groote Zec/Diemen de Oceaen feet / en Daer wert bertoeft bijf en twintich dagljen / een bepnich meer / bau welcke plaete men niet ran' we= Detliecren / ont datter ozdinariftebe

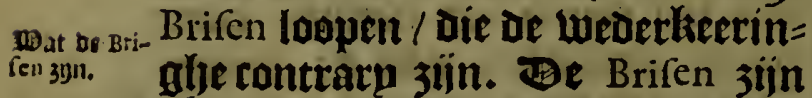
Einden/die alle de voos-berljaelde Bolfelicke winden met yaer quar= tier-Itreecken begrijpen / en zijn foo ozdinaris en balt / om dat de Inelle betweginghe ban oe erete be= roeringhe met hem weely boert ljet Element ban de locht / gbelijek als het Doet de andere bouente Orbes : en alfoo be loctjt boleljt altoog be belweginge ban den Dach/ gaen= de ban liet Boften in bet (toeften/ Conder immermeer te beranderen: en de crachtighe betorginghe ban ae zocht treckt ootk mede na hem De bampen / en exbalatien die haer unt De Zee opglyeben/en Daerom it bat de Hoint ban de Brife unt Den Ooften loopt / too gheftadich in bie quartieren. Dere vopage van Canarien tot S.Domingo, dede al= dereerft int \$aer I 5 I 4 . Pedrarias Davila, dienten noentoe den Gentil, of oen Speeloer / at: lye net een Amnat gint boo? Soulecheur/ en Capitepn Benterat ban het Tijels uan Caftilien de l'oro, Datmen nu thafte 1 ant noeme / met weleke fich feet verglyeliselit de vaerte die tot nocly toe dertwaierts wert glye= beurutkt.

Bun Datter glyetoom is / Dat $=$ men glyeen verwateringlye / noeb yout yalen fal in de Deffeada en in S. Domingo, 'oo gaet wan Daer na

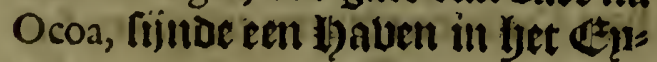

Lant Efpangola, de Bloot bart nien 1)ifpanien / cum ueruet foljunglie te nemen / en Dat jul hace Darr lanck onderlyout / is d'cokfiactlie / ont ju aldaer becomt de V racancs ban, Cuba, en uan Nueva Erpania.elgan utễt oock verberfebinglye ban mater! op let $\mathbb{C}^{z}$ plandot van Guadalupa, en albier mareken jn ljact gbereet tot de uopungit.

דe Blote ban uiru Hifpanien gaet den Caep uan Santanton, aen= Doende / De welcke is in lyet uptter = Ite ende Mettelickite orel bait lyet Ezplant Cuba, uan welclic plaetfic men leult bp naeft bijf jondert le= guen / in twintich dagben ozoma: rig/ int gheficljt ban S.Ian de Porto Rico, en ban Efpaignola, tot twee mijlen ban de mingo, loopende lanex de Culte om oe punte ban Nizao, en tufftljen de Eplanden ban Cuba, en Iamayca, gaetmen met toeficljt op de Clip= pen diemen noemt oe lardines, $\mathfrak{e f}=$ fen int midoen legglyen van de Culte uan Cuba, al waer beel Sclye= pen verlozen ziin/ palferende daer naer int gheficlyte ban't eplanbt ban Pinos, en de Caep de corrientes thaelf leguag ban de Caep uan Santanton, van meleke plactle ziiu twee paffagien naer of Vera cruz, luepar ban thien of twaelf daghen / oe ene diemen noemt als binnen doos / wan twee ljontdert en vijfrich leguag. Inde Somer-tiit ban פgay af tot Septentber / als wanneer glieen \$oozde Hinden wacpen/ die de traberten/ of win= on dwars op of 139al jiin / op oe Gulte ban lucatan, al waet uren boos bp pafteert / en oe ander die= men noemt van bupten vooz oe tiit Lin de fointer / bu naeft wain twoce yondert en tachtenticly leguas / cen wequich meer gemetê in de hoocly= te / ente oe befte nabigatie une oe mat be befit

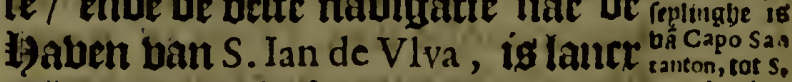
te loopen oe Culte luan Florida ent ${ }^{\text {Ian de viva. }}$ de blarkten bau Almeria om ban 
gljeme Hoinden opaljeljouben te wercoen otn de l)auen uan S. Ian dê Vlva aen te Doen / en Daer ban bo= uen point te welen.

De Bloten die te voozen gingujen ban la Dominica, en nu gaen ban Guadalupe nae bee valte lande toe/ tor waer toe ontrent bier yon= Dert Ieguen Leelys vali bifftyien Dagljen tijots/ Die gaen oul Cartagena aen te Doen lancr bet balte lamat yeen / al water de Brifen nae bu glyeffroicljen contrarp oe cours

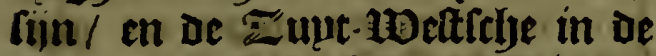
Somer / ell of Roozde-Winden in de tointer/ bie duers op de wal waepen. Esen berkêt de weeth bu de Cabo de la Vela, die tutielyen S.Martha en Venczuela is / en bu de Cabo del Aquia, ontrent Cariagena, al watr men ont ladet de Coopman= fchappen die gaen fulleu nae lyet nietwe 1iijek/en be welcke die naer Peru fullen gaen/ werden glyeboert in Portobelo, tot welclie plaetre unil Cartagena of / jijn bp naelt tne= gljenticly leguen/biet of les baghen Ifuleng / berkennende de punt ban Catina, ende van de Canarien tot Cartagena is biif en Dettich Dagljen ceplent. be bopagie - Be glyene dic gaen hae de Hondurasen Gua duras en Guatemala, gaen in ghetel= fcljap met die van nicu Gifpanien/ tot de Cabo bam Tiburon, yet al= Der Eciteliclitite uan Efpagnola, ban waet loopende lanct bet $\mathbb{C}^{\mathrm{u}}=$ lant vall Iamayca, lancr de $2002 t-$ zijde/ tot de pumt bant Negrillo, jet. uptterfte ban dien/ comen 3u upt oill âcn te Coecken de Cabo ban de Cameron/huet lueginfel han de golfe, fil de plobuncie van de Honduras, ball waer men gaet om te habenen iit Truxillo, Is. legutn int Boeften/ aen de Cabo, allwaer dat be Coop= mulnctjappen gljeloft weroen die Dace bliiven fullen / en de relte gact boazt tot De batuen de Carallos ge= maents/ oft tot de foete Golfe Dulce, lu de twall lautr bail de golfe de
Honduras, all bic te bzengyen in Guatemala.

ylut wain lat ing

$$
\text { bet I I . Capittel. }
$$

Tuvelck vervolrht de Rey/ngijen van Wert-Indien.

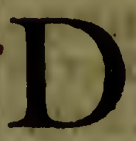

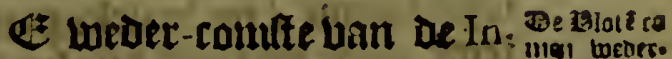
dien naer Hifpanien en call oum naer cament niet bo dienten geconié i\$/ en dacrom moet men grooter boochte nemen / loo= pende bunten de Tropicos, om te caecken de coele mintan die unt

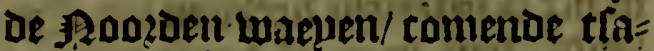
men te borglien alle de Bloten / in be Haven ban Havana, int nioden ban 3 unilig/ oun te baren naer $\mathrm{C}_{2}$ ftilien vooz de minter / ont dat be - Dofte-wint uan voozen tomt / $\mathfrak{c n}$ oe otweers-mint in be ranael is/en De Zupbe-wint is oock de obergMoint op de Culte / ban de Cabo $S$. Vincente tot aen Sint Lucar. Be Bloot ban't bafte lant bertreclit in nameer be

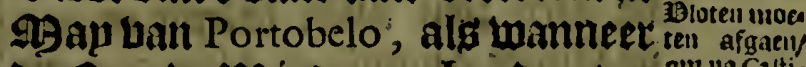

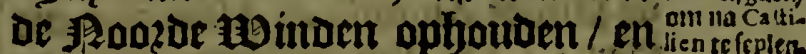
Heett weoer nae Cartagena, om té bergaderen t'glyene nen fal affen= den/als 'tSilber en tobout ban bet nieuwe tiiiek ban Granada, en oock om te inijoen or Culfe uan Veragua, en de unt-wateringlie ban Nicarahua, upt be welcke men qua lick can comen foo men taer in $g e=$ betuen wozt ball de Brifen, en de periculeule teghenttroomen/ die op de paltagie 3 iin.

Ban Cartagena gaet men ont aen 19aer heren te Doen de Cabo de Santanton, Ijet liopen uall moefterfrbe ban Cuba, bu thact gatioe. twee bonoert gisilen/ zimbe een calle bau, thien dagtjen / met op= mercli ban de ondiepten / die baet jiin in die beely. Servana en Serranilla, en Quitafvenjo, ende van de Cabo de Santanton tot Harana, siiti ontrent vijftich legutu. Oocli me:

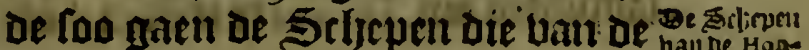
I 3 . Hondu=duraso 
Honduras comen on de Cacp ball Santanton te verkenuten.

mammer of

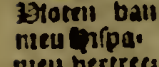
neth bersires

De Bloten bam nicu hifpanien

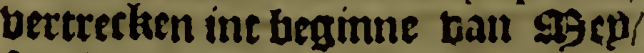

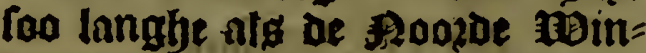
oen watpen / Die Dienen toe De om= wederkeer naer Havana, een bepmich bet in de Goochte climmende tot aen de Sonda, Diemen las Tortugas noemt / tot welckeplaet te ge replt wozt bp naett Dzie bonbert le= guen in bifthien dagen. De Sche= vain sopage par- pell van S. Martha en Venezuela, om the siv Mant- notr Caftilien te gaen loopen unt zuelas ruftelyen Cuba, en Erpagnola, om te berkennen de Cabo uan .S. Nicolas, in bet weeferidie deel ban dat $\mathbb{E} p=$ land / van waer af Dooi't midoen ban de Eplanden de los Lucayos, gaente oni te nemen de loop vail of Bloten / Dan comen ju te ver= kennen of Cabo van Santanton, en Uail Daec tot Avana, oll be thoo= bers te fryouwen.

van bopage

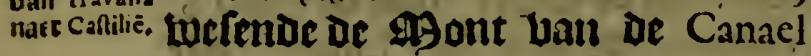
Uan Bahama uptgheloopen/De welc= ke erte uan allen beleplt is ban oc piloot Antonis de Alaminos int laer is 19 . Coo teplenien ober de golfe diemé noemt Mar del Norte of De Sargas- Zee $/$ bu naet negen bon = Dert of Dunlent leguen/of meer/met cen fenlinglie ban biff en twinticly of oerticly dagben / met ozbinarife TDinden / op tweederlepe courfen/. D'eene in oe Somer / wat meer in booclyte / tot op de acht en Derticly ofte negljeit en bertich graden te comien / op welclie hoochte de Azo: res ofte B laemirlye Eplamben leg $=$ oljen. Oe andere in de winter op Initioer boochte/ booz oolfaeclie van be reglyen-bumen die op die Atreeck altoog ballen/ dacrom werter glye: loopen tot negljen en Detticly gra= Den / cu niet loogljer / op welcke

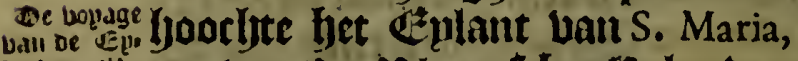
labori da, een uan de Dlacturthe Culanden

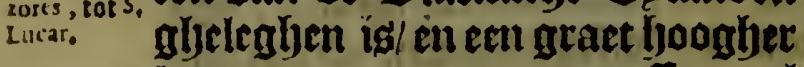
looptmen om Tcrcera te treffen / al ware altroog te bolooteli Dergate: rett oni verberfichinglye te nemten/ Conder toelaten dat pemanot ant Llande gae/ en vaul Die Ëulamden uan d'Azores tot Sint Lucar de Barrameda, teillen de Scljippers Dzie bondert temen te leplen in bilf= thien Dagben / en antore in Der= tich / om be vele Bofteliclie ADin= Den / Die in bie. Golfe ban De Azores Domineren / Lan waer men feplt tot Datmen balt op de Cuften van poztugal/ en pafteren de Cabo van Sint Vincent, en voorts int glye= ficlit bande (ufte/tot delsaben ban S. Lucar.

Be feulinglye ban Caftilien nac be boragie Rio de Plata, tot welcke plaetie toe la plasto 3iin featjien lyondert leguen / $\mathrm{m}$ to De Straet uan Magallanes, by de twee buplent / beeft meer tijtg batt nooden/ Dan be wiijote ban de Loecls frbijnt te berepatisen / om Dat lyet nopdicly is te comen in die 13zoum= cien in baer somer / welek ig ban September booz-waert:men math niet uptloopen unt Caftilien, in lulcke tiiden dat men of Equino. Ctiael in Tunio / of Alugulto Toude moeten pafteren/alt aldaer bele en groote filten regneten / cn Daetom bliffmen bijf פgaentien op oe tep= $\mathrm{re} /$ diemen can Doen in twee of Die/ foomen uan Sevilien af reuft inzlu: gulto of eeroer / en raeckenoe in de

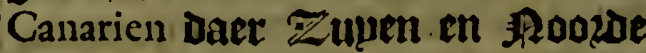
moinden regneren / tat acljt of negben graden / ober gljeen fijoe ban De Æqquinoctiael, ban Laer dam eenigbe : gaen Meft aen / oill te verkennen de Cabo uan S. Augnftijn in Brafil,en Daer naer in t'glje= ficlyt ban't il anot tot be Rio de la Plata, en be Эtraet. Atndere ziin Ilyeloopen tot be achit graden toe/! reclyt midoen booz de Zee/ Simut aen nae oe Stract toe/ al if bat= ter wepnicly belsen conmen dooz comen / vooz datter of 5omer ten cpnto liep/ Die feer cost is / ent vol ban colioe/ om datte cos boocly leut/ 


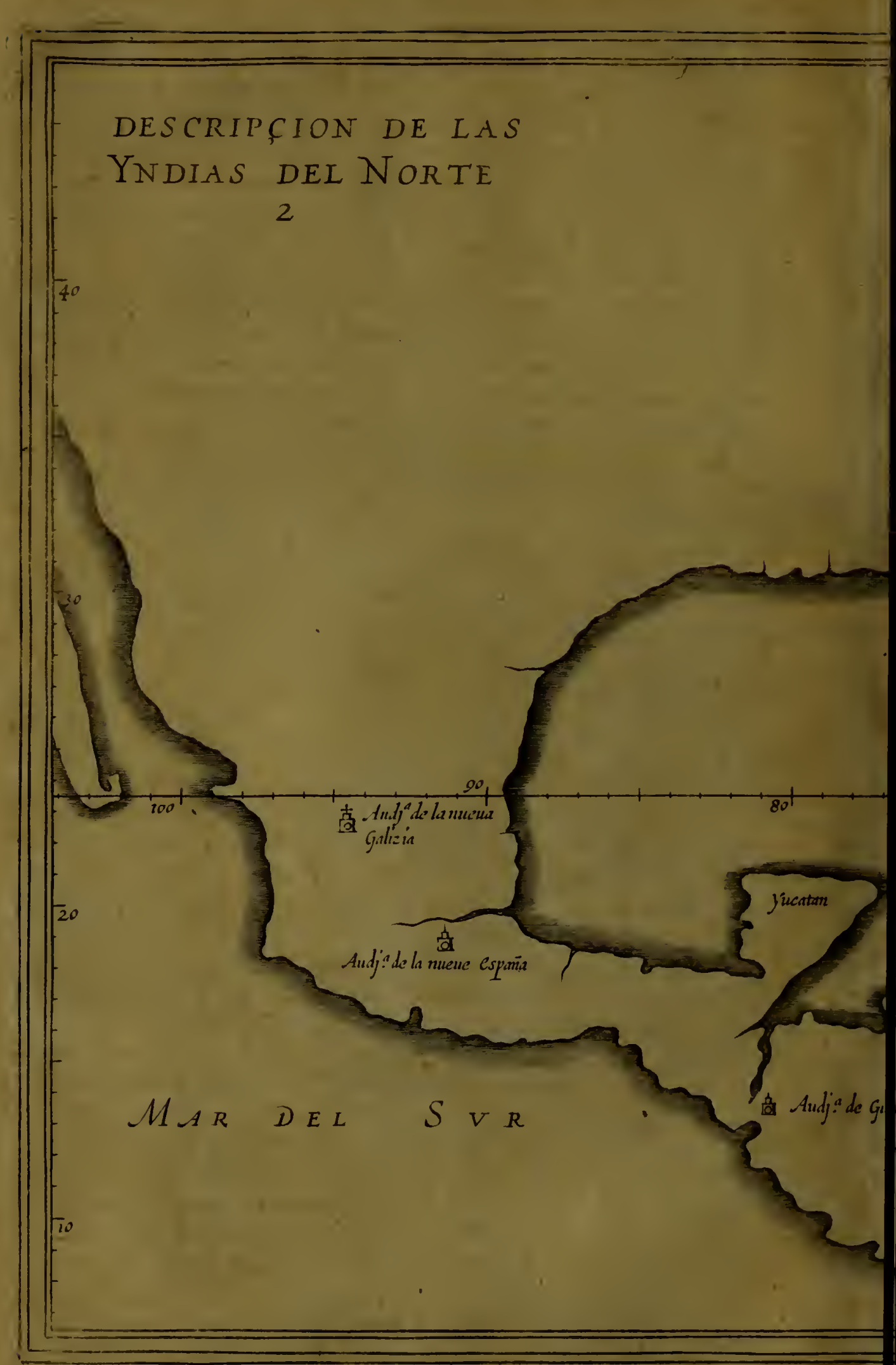

. 
om fiet welcke defe navigatic foo nwatig.

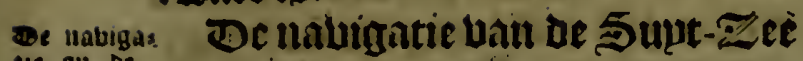
zupos-28. is ghedaren bp of twal laner/ent om dat de Supde $\mathbf{z}$ śmben daer feer continitel Lanepen / ch be opdittari= tche loop-ftroamen ban be Strtet/ mabigatie nae het Roozden. De replingbe bampe staot uan Panama, nae de Cuidad de los

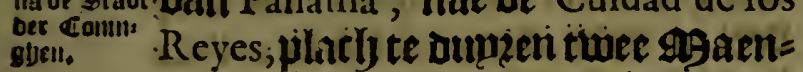
Den/el de wederconfte múnder dan Dertiely onglyen / en van glyelijeken vall de Cuidad de los Reyes, tot Chile, ban watr men nae Panama feplt negen fiondort leguas in min= ber tijot dan in twee isaehben/ sijnoer ban nooden aclit om bet waets te gaen/ beelcke nu cotter is Danfe placly te fín / om dat t'Zet= waert loopende / gljebonden wer = oen beter minden tot de repg die= nentes.

De nabigas De Denavigatienae de éntandên

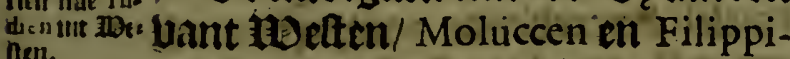
nen. nen, vancaltilien of Dooz de Straet ban Magallanes; is ban vier bup= rent leguen / en oock om datte foo lang is ? als oock oin be nwariefy hept bat tooz de Straet ban Magallanes te conien touthien bié boos feer twater om die te patietert to in Uall nova Hifpania over be theater

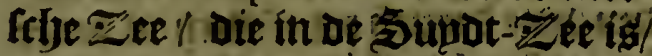
plach glje daen te wozoen die watet Lath upt of l)aben gettaenit of $\mathrm{Na}$ tividad, op de Culte uá Növa Hirpania, nu ter tije wete fo ofjedaten ván de Hauen ban Acapulco, en foo voozts tot de Moluccos; en Filippinas, ftellenie ae loop ontrent 1600 . of 1700 . leguen/ die glyefeplt two? = Den in twer 29 aenden / oft in the ende een lyalf / vertreckende in oe g)aent ban jobtuber / welcker

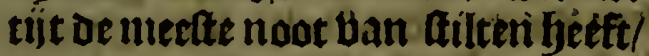
en oe bedercomitte naex Nova. Hirpania Dupet beel langlyer / oun dat: Inen niet tan wedertomê / die werb Diemeri ghegaen is/ foo is fyet nioo: bichl datmen loopt tot viegluen ent oertich gridoen / en vertreclen op
Egajo en 7 unio als de Brifen 1 B iñ Den uninft zijn/ eti met de twee dup= fent leguen die defe wech ntogben bebben/wert berloozen biergaen= Den tijts.

The

I I I . Capittel.

$V$ ân de Indiens int Noorderi.

1)edeult fiebbende natume lick in twee Deeleri de wertIndiens, Doos Den bals/ of imalte ban Portobelo tot Pànama, Itellende b'cente int poozoen / en D'andere int Supbeti / Daerom fut len bin die noenten / Indieris bant ఇoozoen/en van't Supoen. De Coninglyen ban Caftilien, en yan Leor; met advijg uan oe hooghen Thaet van oe Indiens, bebben obe= ozdinert dat in elcke beele loude

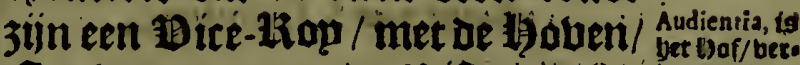
Boubernementen/en BSifoointiment/ batembe ben die daer fouben opgerecht wojoen. gaen bence.

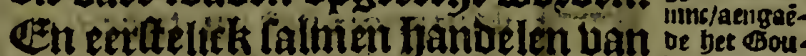

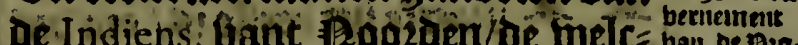

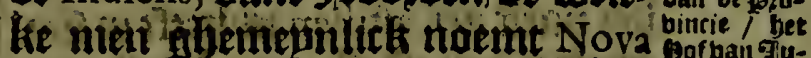
Efpania, omi bat de eerite ontoter = nitiel enve

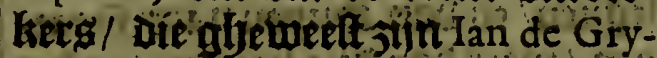
alva, en-zinn met-gberellen/ itet en badoen géfien inoe eslanoê 1) up:

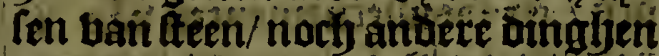
ban Sfrantien / en ál ther bonoen jo die/ met bolc bat gecteet was/ en meer ofjecibiliteert watetom su fet waerom bee

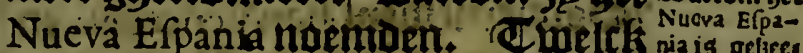
te boben gaet of andore deelen bant tel.

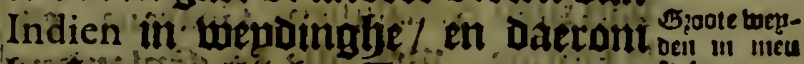

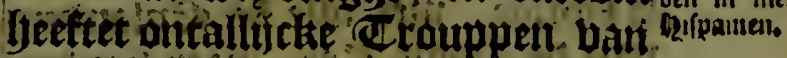
2 seeften / bain alderiene foozten/ ent obertteft oock in Lantbouwinglyel,

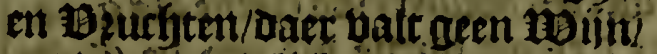
ont bàt gentetalick oe Squpue niet

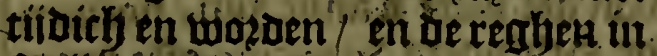
Tulio en finguito óce niet toe lact

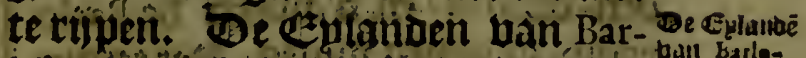

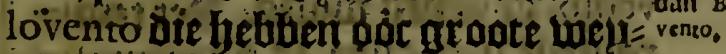
oinghen/en fetjoone oogen wenin= gle oni bat jp therle Jaet groen 
siin/ / an bloesendide met beel coelte/ Het groote 23oftrben/Dat Waran=

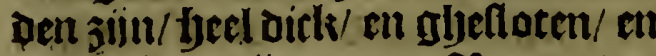
in of Uharte jiil groote 99 \&p?

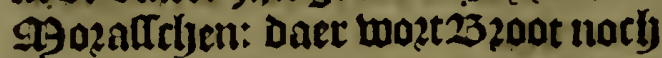
Woijn gheteelt om dat oe urelliptpt Der ghebzectken uan bet 1 lant niet toe laet Die te wewnen $/$ noct) te rii= pen: De lievieren hebbert ten meeItem beele Gout.Floride, Nicaragua, en Gua'emala aijn up-naeft van die felfoe inauier/ als meeft alle gader: Dacr int particulier op fiin placts Difriao, is cet freckitige bant lant kete linute
begrepen. van verhaelt fal weroen / ent in de uolghende Caerte fal gbefiten wozDen / Get Deftrict ban defe Indiens ban't $\Re a 0 z 0 e n$.

\section{Yot V. Capittel.}

Varibet destrit van de $A u_{-}$ dientic van S. Domingo.

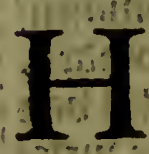
Et bof wai fet Eplandt Espagnola dat in tijot / $\mathbf{c n}$ plactre bet eertte is / om dat bet naet aêCaftilien is/betft in fiin ohelriet uan o oit naer weit s so. leguen/en ban Supen naer Rooz. Den / meer ban okie lyandert/ in bet Loelcke beni begripeti de EztanDen / en Goubernementen ban EFpagnola, Cuba, S. Iuan, en lamayca, en de. Margarite, en de Billecherije ban de peerlen / de plobíncie en tesoutuetritment uan Venezuela, $\mathrm{en}$ otn De na-up-hcpt oe \$szabintien ban nicu Andalufien, Guayana, en Ia Floride, nee alle de Eeplanten ball oe Roozt-Z̃ee / waer banber ouer oe hondert namen fíloben/ en wel res fonoert roo cleene als grootê/ en die glyene bie aen be Culte van Jet bafte Tlandot jiin/noemen De Schippers Sotavento ofte bene= Den-1Bints/en doundete oe Barlovento, of bouen wints. Het teinperamint van allen is ghemepnlick vorlytich/en upttermaten uarm/en al foe wel ju veucljtbaet zijn van Hocpoen/ en ban gljelioomiten/ foo ch jiju jp't niet uan zadé balt Caltilien, norly ban Tertue/noch Gartt! moiin / Olijuen / nocijtans beeftet beel groot en cleen Dee vãltarpany Berden / Berckeng en Srbapen/ m booz yaer pzincipale neeringlise (ijn \$) updet / en Supelker die baer beel is/ ende al yoe wel in de merte ban dien Gout is/ foo ch wert bet niet gbebeneficecrt. :o?

- Fet VI. Capitel. Van het Eylant Ejpagnola, en van Cuba.

T. T. Ett eplant van Épagnola, yebluen de ingebozenen ge= nomit Ayt', en Quifqueya, twelek beteeckende rouwichept oft groot Lant/ baer fattoen is als be Heerne ban een Galtanie/ bet lept opnegbenthien graden en een baif/ Polushoorbte / theeft in fijn on= banck ontrent vier hondert leguen/ wepnich meer/ en ftrectit hem int bacet (1) oft on zact bp-cang hon=

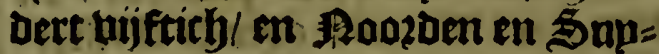
Den/ban Dertich tor tfeltich/al waer het op fijn baeeft is ; tig feer burbist= baer ban Supckerê/ Bee/ en Y uca, ban wejtkes houtelen men be $\mathrm{Ca}$ zabi maetlit/Dat fet bzopt waz ban oe Inghebozenen / ten lyeeft gheen gigaps noth cerwe / al boe wel bet nu begrint die te ghesen in be biit= nenite / en be coutite beelen. To' rijtls ban Coper 29 ijnen/en andere isetalen / en ban Gout-Xtpen / al if oat jp wepnict Daer upe getroc= ken wo2t / Dooz faulte wan Loerck = liçoen. 'theeft thien Dozpen van Caftilianen:

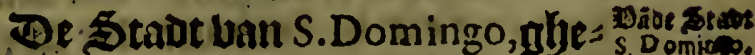
terljen aen be zee/ op De Supdt3ijoe/ aen be cant ban de ziebict Ozama, fallegghen op oe boozlende neglien: 


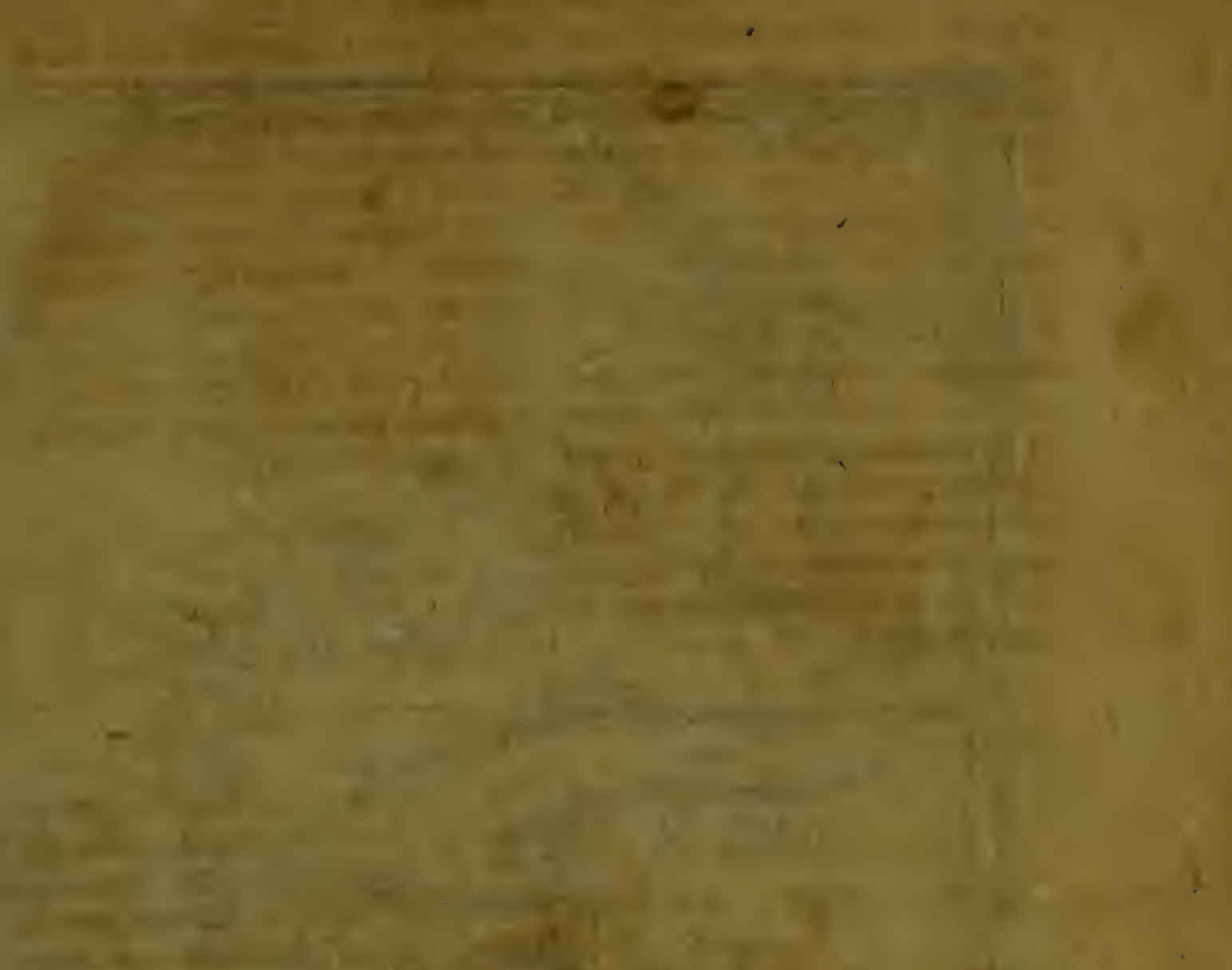

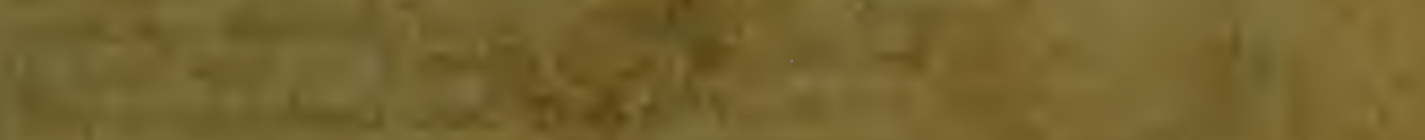

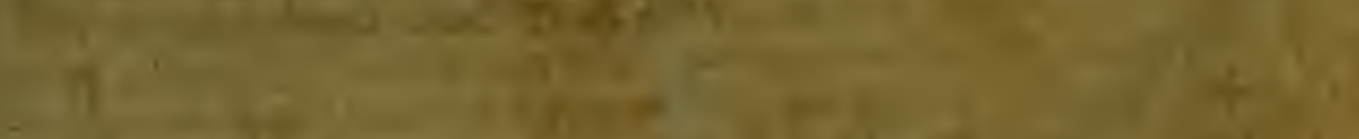

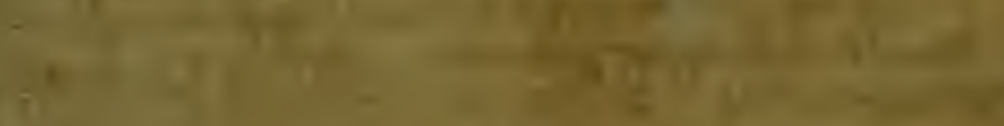

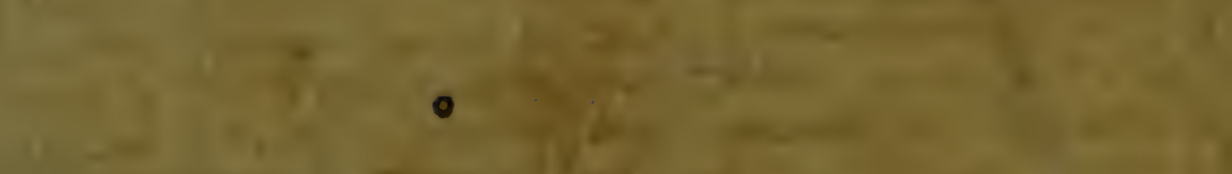

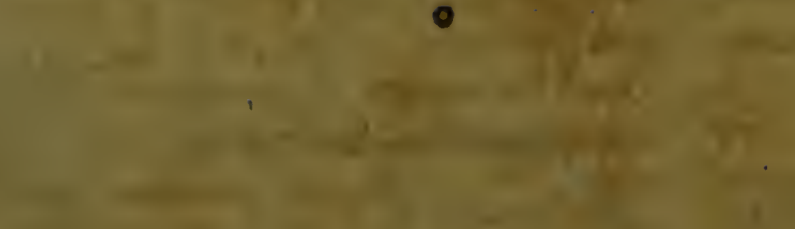

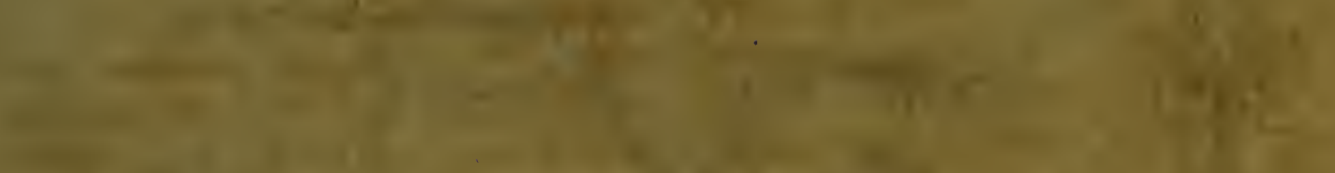

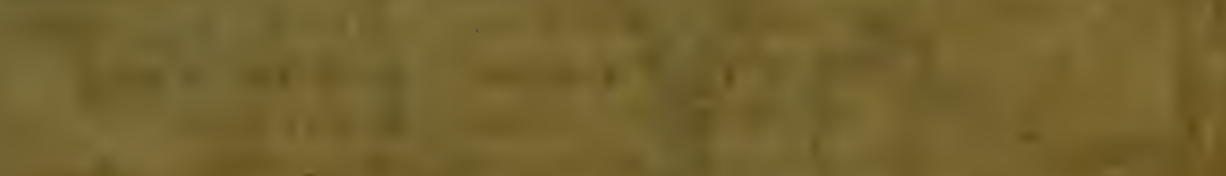

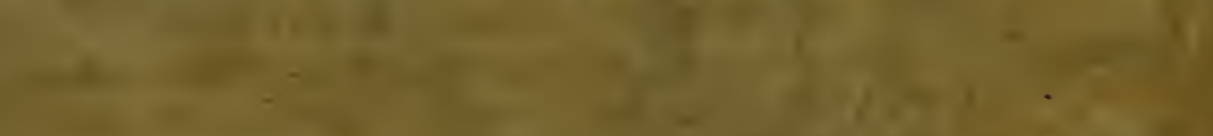

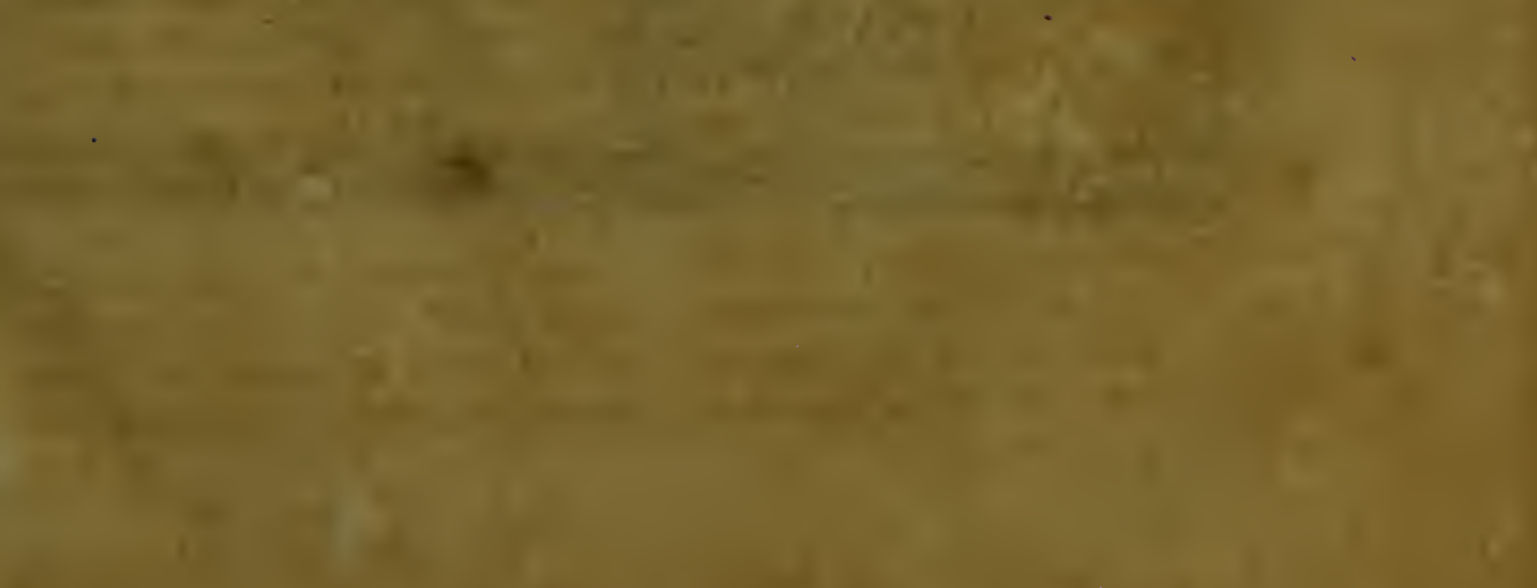




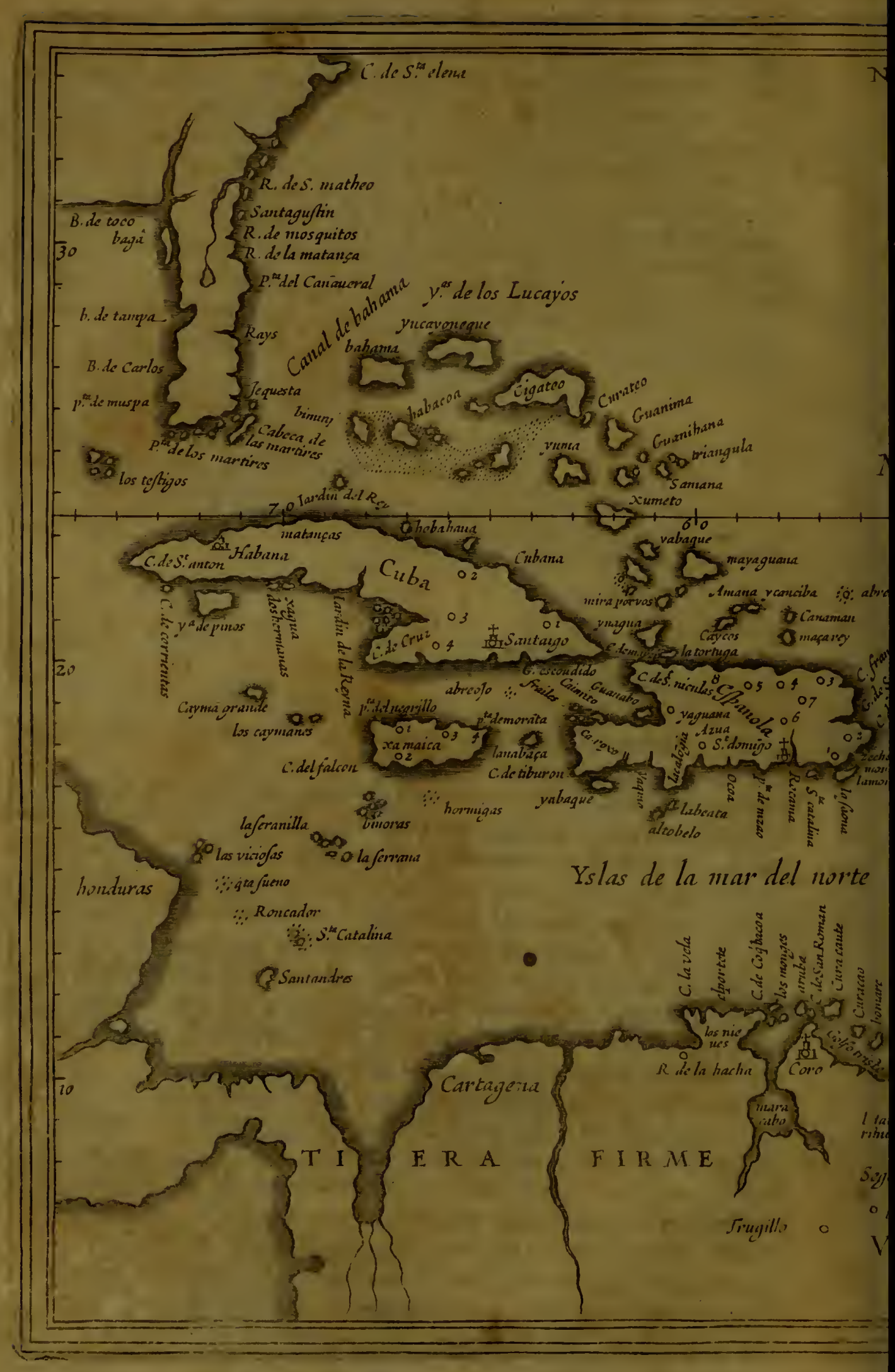





\section{van $V V_{e} f-$ indien.}

"Iețilentijicu an ecu fyalf graden/m $t$ 'ctoentich inoe langte ibeltwaert/

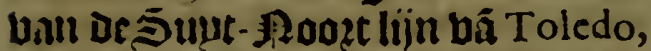
Uall warer tot Deít toe fal hebben in ecli reclite limie 1247 . I Gouen / daet fullen siju meer oan fes bouloert 230:gljers. In dere Stat refibeert bet b) of / Officiets ban lyet inco= nıen/ en of Conineklicke ílyatkift: cen Sutte/sn de Catjeozale ltert= lie / cn het sterts-siloom lyeeft tot Suffiaganen oe 23iafrboppen van be Conceptic of la Vega, out beres= meljt is met die van Santo Do. mingo. Die van S. Iàn, Cuba, Venczucla, en D’äboije ban lamayca, en in de Strat siin Cloolters / ban Dominicanetu/firancifcancu/onte I1. Dentwe ter geatien 25 zoeders; ell andere twee uan Ronnen. Een

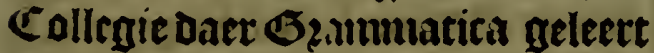
toozt / bier buplent pefen te renten bebbende/ en eell Gaft-bupg / met turinticf oupfent : De Haben bie groot is / en, bequaem booz beel Scbepen / is op de פgont Lan be Liebiet Ozama, en lyeeft in bet Roe= ften of 5tadt/ oe weleke fondeerde int Jaer 1 4.9.4. De ztonitael Don. Bartholome Colon, in bet BofterDecl/ beter en in ghefonoer plaetre/ in oe groote Cominandeur banAlcantara, Nicolacs de Ovanido, fijnue Gollverneur ban Efpagnola int jaet I s 02 . beeftle berlet daet $3 p$ nu tatet/aen oe ander fijoe ban oe the bier int Boften/ 0ooz octafie bat be 5tad vicl Dooz cen troote alert= bebingthe.

salvaleen be De Btat uan Salvaleon de. Y. syuey is ean guey, acljt en twinticly leguen ban $S$. Domingo int Boften / wan het zettg-23ifoors ís bevoldit van ot Capitepn luan de Efquivel?

De Stadt Zeybo, twintich le= gues yall S.Doningo int Boften/ nae lyet Ezplant de la Saona; popu= leeroe anck de Capitepn lan de Efquivel, in oe tijpt bant Nicolaes de Ovando.

De Stabit Cotuy, felthica lequen' (uan S.Domingo, int noozbenilyeb: bende in fijn Comarca beel collexo. Colleso is Rodrigo Mexia, de Trillo yopuleer= couparsa is De Dictr. We Stad uan Azua in Compo- als abe be

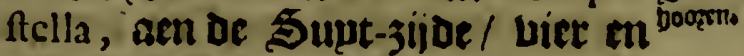
twinticls. Ieguen ban S.Domingo, int Beltē/ en in fîn Comarça heb= bende veel ingeniog ban SupekerIgolen!' is gisepopulect gbeweeft Dooz de zlonirael Dervo Velazquez; cn noemide ljet Compoftella, om een Coumandeur Gallego, die een erfe: uilfe fjadoe in die plaetfe/enAzua is de nami van yet boupjen / Dat oie Indianen albaet frieloé/ oe ertfe die in De Indiens S Supcker-2RicDen ge=

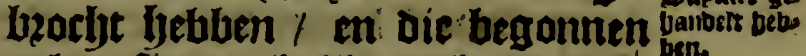
te beneficeren yebben gbeweelt $A$ tienca, en etn Bachiller Vellofa.

De Stadt Yaguana , die meñ ye zotad noent Santa Maria del Puerto, om Dat jp lept in De PBofteritye Cultel van bet Eeplant viffticly oft treftich leguen vait $S$. Domingo, bp naet tuliefen bet \$200zoen en meetten/ populecroe de grooteclommandeut Nicolaes de Ovando.

De Stabt uan de Conception ra cuncerde la Vega, in Ject Kijick ban Guarinoix, De welcke fondeectoe de eerlite atomitael Don Chriftoval Colon, ontrent de welcle fjp of groote2sac tallie won bam de Vega Real, if twintich leguen van S.Domingo int Poost-Boften / in de welclie cen Carledrale lerche is/al hoe wet 3 g geen 3 selaet beft / om dat $3 \mu$ haer gbeunieert heeft nuet oe lierc= ke ban S.Domingo. Daet is een cen pas b-

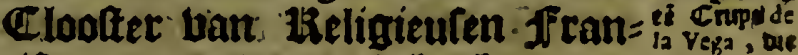
rifcaners / al waer is fjet fjout ban miranted het Crupg / Dat oe Indianen niet Ijebben connen bzanden/ affjoubé/ noch af dacn / twelck groote micas Gelen heeft gyedaen.

Sant Iago de:los Cavalle ros, thien santiaga: leguen ban De Stade de las Vega, rectse int Point Doften: / is eerat

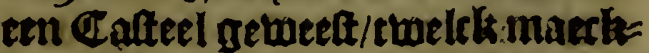
tede de ectife abmirael in de Xan: 25 Den 


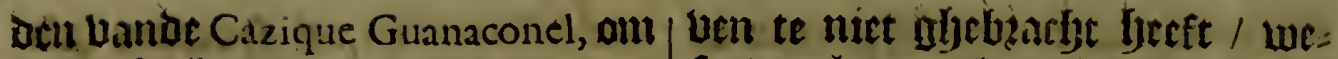

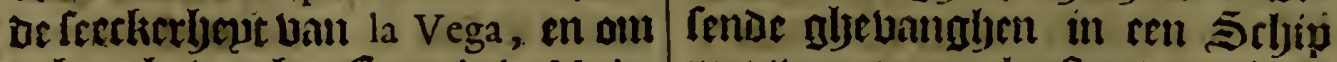
te hauthaum lyet foozt de la MadaIena, diat vier leguen daer af was.

Ee nabei Oe H)aven glyenaeme oe Plata, baupluse opide Joott-Cufte buf en Derticly of tot uecrtich leguen uan S. Domingo, populeerde de groote com= mandeur van Alcantara int 7 aer I soz: ghenaent Nicolaes de Ovando, op dat dies te aljemackelicker Couben moghen yandelen de Sche= peu ban Cä́tilien, en on niet te leg $=$ dyen berber dan thien : Ieguen ban De groote. Vega, al waer ten andere tiiden nlyeftaen beeft de Stadt Sant Iago, en tuan de Conception is bie 16: en twaelfi leguen ban oe riicke goinen ban Cibao, in was be= hoozente tot lyet 25 ifoom ban la Vega; tuele Saluen De.Plata is gyele= shen op een wepnich meer dan 20 . gtaden.

Montechrilto De.5tanot Montechrifo, aen de joopt-Cufte / beerthien leguen int Woeften uar oe fialuen de Plata, en weertich tau S. Domingo, if on= Der bet 25itoomuan Vega, "tbeeft ecn goede 1 abê/en een Soutpanne Daer in $/$. Nicolaes dè Ovando geeft Dien gutettictjtet.

San buts bag op dat deplant/ frabella en de Stabt Y fabella, die ontbolckt i\$; sodat. welcke fondectue de cerfte zaburiwael int 3laer I 493 . De Stat La Verapaz. tom Verapaz in Xaragua, die fon= Deeriel Diego Velazquez int Baer if 5 o $3: 4$ int $t$ 'felfoe Jaer is bock salvariera, beboiclit Salvatierra do la Zabana, tweltif tueteectent blackte / en velt in oe Intifetye tale/ cn die 10zobin= rie is fterften thlack. Oock wiett bebolclit tuffenen de twee machti= obe thevieren Neyba en Yaqui, de za Magunas. Stadt van S. Ian de la Maguana, int midoen unn bet Eulanot / al waet mu hocf itnet De - ltercke / en bier regeer doe Caonabo, de welcke vingh Alonzo de Ojeda, de felfoe bebolck Pillanewra tebe Villaneuva de Yaquimo, bouten Itet twee paren clunfters : an is op of Culte van't Supden / welelie of Rtominacl noentocde / if Cufte of l)auen uan Brafil.

De Statat Bonao, dicfur un Corny, es staot dien oock beualckt is dooz ure erefte Adomirael/ al waer lju mactitroe certt een foat ont oe fecckerlycubt ban de grinen/ die oe cerfte wa= ren die ulen bont in Dat Eplandt.

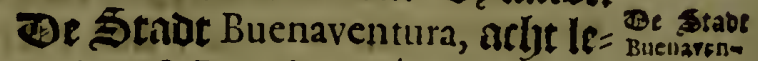

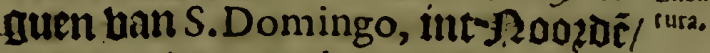

in Lares ban Guahaba, twelcit be = volcktede Nicolaes de Ovando, we= fende Commandeur te Lares. $\$ 00$ feer bloepdoed dit Geplant dat daer in waren beerchien dupfent Cafti- ouprenter $\mathrm{C}_{2}$.

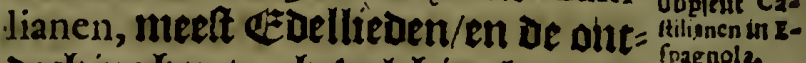
Deckinghen/en lebolckinghen ban andere deelen/ die daer naer voley = Den ontuolektedent / ont dat ban Dit/ en' bain Cuba upttrock de gant= fche fubftantie/ na de nieube $\overline{1}$ an= $\mathrm{ocn} /$ die $3 \mathrm{p}$ gingben forcken.

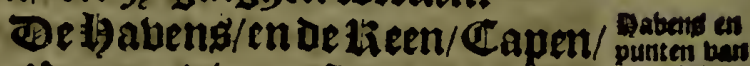
en jounten die meef notatuel $3 i j n /$ ar eplatis. en ae Ezplanden die behoozen aen De Culte van dit $\mathbb{E}$ plant/zijn op de Culte by Supuen de punte Lan $\mathrm{Ni}$ zao, so.teguen ban S. Domingo, int Hetten. Be baben ban Ocao ban Domingo 1 8. leguen / ig een bape Daer.2lncket-gront is/en be Bloten Werluerfrbinge nenten die naer niet Hifpanien gaen-/als $3 \mu$ niet ancke ren in te inwijets ban Zepezepin, die baer ditlyte bp $i s /$ oft in b'an= Dere dic sp noemen Puerto hermofo, de fthoone paben / twee leguag eermen came tot Ocao Azua de 19a= ben/.un een Dozp 24. Ieguen ober gheen lijae ban Ocao la Calongia, etn prumr bacet ober de 30. Ieguen/ rectit tegen ober uan de Gulandent Boaca, en Altobelo, biifleguen ban de Culte/ en de Beatados, Yaquimo bucans 34 . meer Teltwaert/en A. baque, een eplant ontrent de Cabo I Tiburon, jet al age- Mbefterletje ban oe Egaben al maer Ojeda, bem fel= 


\section{baut VVeflindien.}

Efpagnola. De Nabaza 10. Leguen

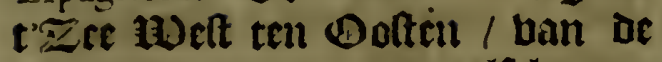
Cáep, en Cabo Royo twunelf Iemuen Uan Dace \$ooktwatert. De Roques, of oe deie geluzocders Eplandekeng outrent of Culte die wesercompt nacr't Ooften. Caymito cen ander Enlandelien tuffeljen die / en Guanabo fen ander Eulanbt ban acht legnen becet in oe inwijek ban Yaguana. Fe baben / cul Cabo ban S. Nicolaes, de alder-1Befterfrlye ban

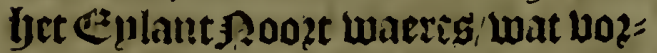
Der ig de l)aven ball de Mofquitos,

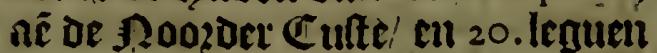
boozoer of 1 andê Valparayfo, of ban oc Conteption / Suñen en Poos= Den / met of Torttiga ctul Eulanot ontreitt oe Culte bije leguen bzect. Porto Re acl 1 z. Ieguen na t'neften vall Montechrifto, welck is eens foo vecl/ of weynich meer ban Ifabella, tu is van de loumen de Plata andere 12. Ieguen. Cabo Frances, en Cabo bun Cabron, in de Luedercomilte die langs ar Culte loopt na't Bolten/ Uoo? de Gol fe Uan Semana, die bill= nen bet Zlant loopt ; of 6 . Ieguen/ tot wetcke plaetle toe was liet oozp ne Doperer uam Santa Cruz, en in de thevier uan

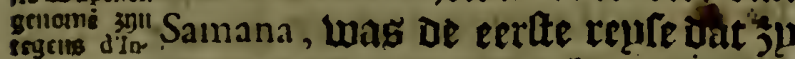

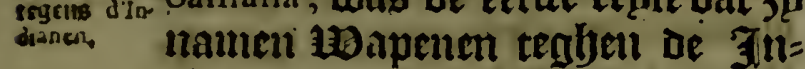
glyebozenten uan d'Indien, omidat $3 \mu$ rochten gljewelt te Doen aen Den - certten xiomirael/Cabo del Engano, bet alder-Lweitlickite wan het Eu: lait / al waer oe Cilte wederkeert baul yet Supden uae yet poeften/ int belex begintel is de Saona, bet Evlant dat de voloten verkennen als ju uertreclien/twelc wat berber fs glyelentben van S. Domingo, dan unn be Cabo del Engano. UEen an= Der $\mathbb{E}^{2}$ plandeken iffer norly op defe Culte Datnnen noent S. Catalina: alle be namen dic verljaelt ziin/ jiin gheftelt gheweeft bau de cerfte xomirae!.

bet (ez) lant Cuba, Dat ecrlt ghe $=$ naenit uas Iuana, uae de Dader/ Dou luan, ent daer nater glyeboden is te noemen Fcrnandina, nae fiin Dader / is begoft te beleplen in bet Jaer I s o 8. De Capitenn Seba. ftiaen van Ocampo, dooz laft van Nicolaes de Ovancio, om tat Ijp't tot dié tiit toe niet en yeeft conuen innentelt oun te gelooben gantrdie = licli Dat lyet een Eplande suas / is int Jaer van I 5 I I. bet Ezlanot Donzgerenft dezlomirael Diego Volazquez met dese bondert Caftilia. nen, Doos laft uan oe tweede aldoni= rael / om bat te bevzedighen thee yondert en Dertich leguen lijut ban de Cabo Sant Anton af / tot atn de punte Mayzi , oluer 2 ande te graen/ al yoe wel wet ten aentien ban Son, cul ten aenfien bande $\mathbb{Z}$ ce / loo beel beette niet en beeft/ bet sijn van de Capo de Cruzes tot de thaben Manati 45. Ieguen / en ftracr Legint Get te fmallen/en'tgaet tot de laet= Ite Cabo, oft 1Befterlelye punt / al waer yet fmat is 1 2. Iegue / wepnich meer/oft nin ban Matamano tot A. vana, fijn gelegentlyejt is biunen de Tropico Cancri, ban de 20.tot De 2 $\mathrm{I}$ : grade/bu naeft is bet gantfétye lant ulack/met bele frozeetten/ en dicke Softelyen / ban be Boftlicke punte ban Mayci , Detrich leguen beefe bet reer hooghe 25erghen/en oock zijn= le int mioden / en jp Atrecken ljaer Poostwaert/ en tot Den Supoen beeft bet feboone zaebieren met veel Dirty. Alen de Suut-fijde beeftet die Eplandetien's die de cerfte $21 \mathrm{~m}=$ miraêl noemuede / oe 25ogaert van De Coninginne / en de andere aetl oe \$Ropzt-fijde / of weleke Dirgo Volazques noemdede de\$ Conincx 25ogaect. De 250 omen sijn ban veclaerbande verfebendentheden/ en wilbemoijugaerden/too dick alg een mentrbe / bet en gheeft gheen Carbe / noch jaden ban Caftilien, dan groote oberuloet van bee / bet. beeft groote פgijlen ban Cooper/ en van Gout / en men vint bet in oe thebierê/ al boe bel lyet [rec leech van aflop $i$. 


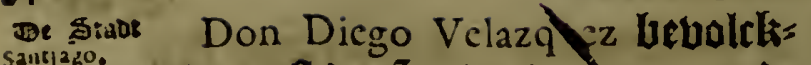
tede cerit de Stadt Sanl Nigo, op De Suut-Culte vecrtich Ieguen ban de Cabo ban Tiburon, Die in Efpagnola isl cul lept twee leguen van

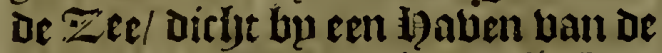
beite in te werelt / in leecketbept ell groote. De Stadt lyefteertijts twee duplent 3 itwoonders geljadt/ แu jiinder wepuich / met een I up= tenaut Goubermeur/ al lyier lit De Cathredael Capellaen van S. Domingo, an cen Cloofter ban tieli= giofe ff rancifanerg.

вaracoz. De Stede Baracoa, is een 10leckel bet oni Boft / ban het Ezplant van Cuba, op de Roozdelicker Culte/ treltich leguen van de Stadt Sant-

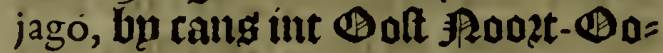
tten / Dat oock is gljebeet eetl be= bolckinge ban Den Alomirael Diego Velazquez.

Bayano.

De Stadt Bayamo, dien oock fondeerde Diego Velazquez, twin= tich leguen van Sintjago int Poozt Fbeltwaert/ is het gefontte bleck = jen uan het Geplant / en een open ztertriick / en ban froede difpolitie.

pretro det De Stadt en De labuen del Prin. cipe, op de \$20ozt-Culte / bu cans bertich leguen ban Santjago, ten

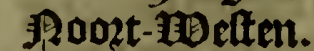

santifpiritus. De Stnot ban Santifpiritus, een liaben aen oe Sunt-fijoe/tuftehen De Trinitad, en de cayo oe Bafco, Porcallo de Figueroa, bu tans so. Ieguen ban Santjago, beuolckinglye טan Diego Velazquez.

De Stadt en laben bait Sinte El Abanz, Chriftoffel del Abana, op de \$RooztCult / bp naelt rectit ober Florida, op 22. graden en een half hoothte/ ban meer dan 600 . Jnwoonder waer de Gouberneur lijn fitplaet' y)eft / en or Conincklicke Bfficie= ren. De hauen is te betwonderen ouer liin groote / en reeckerbeuot/ bpronderlick nae dat de Conincli Philips de tweede / De Vroede toe= ghenaeme / font Maffe de Campo, lan de Texeda, en Baptifta Antoneli,
Dat 34 dien Cotion fortifierch / op dat jli aldaer foudent'fanten ber= gaderen de Bloten uan Indien, oum bebouden in Cantilien te comê. Agen noembe die certe / de faluen ban Carenjas : bu Diego Velazquez is acfondert defe 5 tadt/en alle doan= bere dic in dit Eulantot 3iin / met De luulpe vall 19ater Bartholomxi \$it is befe

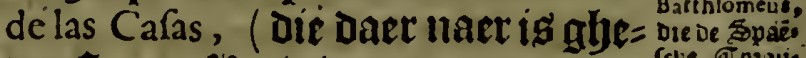
weelt een Kieligieus Dominicaen, me peefis ue en 25iffrljop ban Chiapa) gljebout.

De 1 abens / Capen, ell punten wateus $x$ van de Culte lan Cuba, en oe bp- purteil baun legabende eplanden boben de baub ort $₫ p$, booz-berfaelde die meet jijn op de Supt-Culten / als de Stadt bat Santjago op twintity graden / en bijf en twointich leguen Beltelick. De Santifpiritus, Cabo de Cruz 12. leguen boozder / en de 2Bograerden van De Coningiune / welck is een groote ondiepte en Dzoochten. De Haven ban de Trinitad op een en twuntıcly graden ontrent Detrich Ieguen luan Cabo de Cruz, en thien meer Melt-waerts /. oe golfe baut Xagua, een groote vertteck met $\mathfrak{e}=$ nighe Eulanden int nioden / en uozder de twee 25zoederg twee Ep= lanien int beginne ban de groote ondiepte Der Eplanden/en D200tlys ten / die su noemen Camarco, tur= Itben oe Culte/ en bet Eplandet van Pinos, bat tlyien leguen lanck is / en feven loeet/ twaelf leguen van de Cabo de Corrientes, Die an= Dere oock foo verde/ is ban San Anton, het alder-nortelickite ban Dat Equlant.

Op de Joozt-Culte is de ba= maerom uen ban Abana, en Derticly leguen $\begin{gathered}\text { out bet bees } \\ \text { bo be ban }\end{gathered}$ naert Boften be baven ban Matanças, allwaer cen bevolekinglye ge: wreft is / en 'twas genoemt Matanças, om dat of Indianen aldaet boot floeglyen eeniglye Caftilianen/ die in baer Camoen/ beneden de plactte bã verteeckeringe die wectyboerden aen daander lijoe / en een

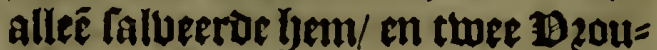




\section{bait $V V_{e f t}$-Indien.}

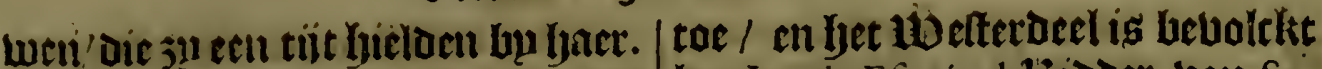
Dilli Matanças tot de H)aurn uail Yucanaca, 3ın uiiftichl legucn / tot oc lardin del Rey, Dat een ondicpte

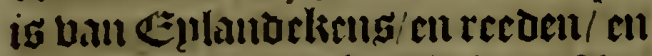
tot lyet cunde ban lyet Eplant Obahaba aclie leguen vooz of baven del Principe, vier of fes Uan Hernando Alonfo, chl Ooftwarert vall dien/feg Ieguen. Cubana twele is ect punte. Del)auen uan Varocia, bp nact twinticls leguen/ voo? of punte Lan Mayzi, lyet aloer-Hoeftelickete / van Cuba, en Puerto, of de Golfe Efcon:dida, bu ac Cabo Royo, op de SuptCufte / bur nacit twinticly leguen vall of ()aluent of Palomas welclie is bu uneft tjuch ban dir bull Santjago.

1)et VII. Capittel.

$V$ an de Eylanden, van Iamayca, S.Ian, los Lucayos, en los Canibules.

$\mathrm{H}$ Et Eulant Iamayca is foo oucrulocaich uan lijftocljt/ cul bocterl / dat lyet gheeft aroote probifie bancattoen- Doec/ Decroen/Derclseng/en Cazabi, vooz De nieute ontoeckinglye noentoe liet de cerite zumirael Santjago, als lyw het ontoecktede / en d'eerite die Daer gijegaren is om te bevolcken/ is gljeweelt Capitepu Ian de ERquivel, int Taer is o 9. dod ozdaze van de tweede ztamitacl Don Diego Colon, lyet lept op fetuentlyien en een lyalf granden hoochte / liet mid oen uan oien / en twintirh leguen ban Cuba Suutwaerts / en oork foo becl ban Efpagnola recht woet waerts / Ijet Ijeft Ijonoert bijfticly Icgucti int onleplen ban ooft nae Heit bout lyet so. en inde bzete/ twinticls leguen / t'beft in lyem daie iilla, criflan, Btcoen. Sevilla : al waer het Col= population bä I amasca. bu Ian de Efquivel liiodor ualı Sevilien. Melilla dat op oc RoostCult / veertljicn leguen uan Sevillien nae t'ooltenl lent. Oriftan, op de Sunt-Culte/ na jet lad celt sunde 14. Ieguen ban Sevilien, yet welels bebolckingljen sijn ban of Atelantado Francifco de Garay, die daer ge = gouverneert lyeft. En vande Srida de la Vega, uan lyet welcke oc 210 Imiraels l)eeren ban dit Elulaut de tiitelen van Hertogen yebluen glye= nomen ; noch ban andete thee be $=$ volckinguen in centuge Cacrten afglyetepekent lyebbe icli geen lien= nitre.

Daer is op de Culf uan dit Ej= lantot de punte uan Moralta, de ban Metilla. alder-Doitlickfte ban die aen de Roopt-Cufte / thien leguen nae het Meften/d: lsaben ban Ianta,en ro. boozwaert's de Haven ban Melilla, al waer fegglyen sp dat nenquain de ertite ztomitael / en noemtoe die Santa gloria, als ly weder quauı verlozen ban Veragua, en albier ge= urel denumterije ban los Porras de Sevilla; en ljet wag de ectite 230 e $=$ shertiicke (Bozlogitye val Indien. Cinien leguen beroer ig De lgaben gin ramagca

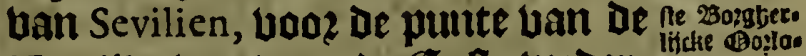
Negrillo, ban waer de Culte weder = gibe oer ca. keert tot Cabo de Falcon, ontrent de Oriftan, en gaet Supt-waert / tot de Gaven uan Guayano: en 5. Ie $=$ guen van uele Culte fiin las Hormigas, en rif dat periculeus is / all 7.Icguen ziin las Vivoras, Ẽulande= Keng befloten met riffen / at int Supden van dien is la Serrana, en cen Eylandeken ontringbelt met Clipp $\bar{e}_{\text {' }}$ met andere 4. oft s. ontrent haer / en int Boot-nelten yant Jaer/ is la Serranilla, en int welten de Roncador ent anoer Clippe / ell bu cang int Sumt-melten uan Santandros, een Ezplant omcingelt met Clippen ( Supden cn Noo?acn. Nombre de Dios, lup naelt 40 . $99 \mathrm{ij}=$ Ien Daer ban/ en ontrent ban Daer 


\section{Fol.14: 25elchaijuinghe}

cell andar ghmuent Santa Catalina, be Caymanes retht int hoeften/ van lamayca, 25 . leguen bau Negrillo, twelek fiin twee Eulandekeng/ [es leguen D'een ban D'ander legglyen= De/en be Groote Cayman een ander Celande ban 7. leguen lanck/15. Uaul De Caymanes Beftwaett/en op De Roost-Culte/ tulteben Cuba en Efpagnola, is cen ander Clippe/ die men noent Abrcio-jo.

Teplantban Sint Ian de Puerto Rico, Diat oe Indianen noent= Den Borriquen, uan twaelf tot 15 . Ieguen vail Efpagnola, ten Boften is 45 . leguen lanck van Boft nae Heit / en uan \$Roozden ten Sup= Den / uan twintich tot Dertich/is fere buchtbaer, ban t'ghene dat E[pagnola gheeft/ roo ban \&gap;/ban Iuca, en ban Gout-gitinê : De locljt is daer goet / en bp naett in een boent'ganterbe jaer/bebaljé in cmuer en 7 anuario / Dat bet weer verandert: Daer in zijn Dzie Doz= pen ban Caftilianen, met cen Gou= Uernment/ en cen 2sitbom. Bit Eplant te berkennen is gegaen be Adelantado Ian Ponce de Leon, ilit Faer i s o 8. weiende Gouberneur/ in De 13 zobincie Yguey, booz Nicolas de Ovando, in Épagnola, m quam weder int Faer is 10 . Doo? laft ban oe Coninck om bet te be= wolcken.

De 5tadt S. Ian leut int luegin=

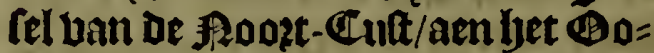
fterfelye Deel / op achthien graden foochte / en wat meer / en fu wert glyenoentt die Puerto Rico, ont de treffelickbyt ban fijn 1 aben. ant= Daer fit oe 2Biftehop / en be Gou= verneur / en de Officieren ban des Conuncr incomen/en isfuftragaen

Arscibc, ban S. Domingo, Arrecibo leppt Der = ticly leguen ten nelten ban Puerro Rico. De 5 tadt Guadjanilla, of $S$. German el nuevo, op of Culte die nae'thBeften fiet/ dacie en Dertich Ieguen ban Porto Rico ten $\mathbb{Z}$ upt= melten.
Waer was van outg in bit een ander Dozp / ont genof̂t werde Guanica, aet de Soutt-lijoe/'tepus de uan dien/al waer tul igeenlgabē ban be Mofquitos, die feer goet $i \mathfrak{z}$ / uan waer $3 \mu$ berfet is op een antock plaetfe/ op de melt-Culte/ Diement noemt El,Aguada, Inet de naent ban Sotomayor : in Dit Eplantot is cen rije ban een ghjeluerelyte/ bie dat int uidoden dooz peplt/ ban Dolt nac Hedt/tot de Zee toe/en de Comarca uan S. Germain, en bier buntmenl oe 2500m Tabernaculo, die ế Luit lyerg pe gasosn ghecti gdjelijels ald Anime, en dient olu Srbepen mede te betulen / cti oin te itcocken / en is oock medici= nael on coube upt te trecken/ en wonden te gheneín.

Daer ziil wequnich dit Ezlandt/ om Dat De gantfebe lants.

Joozté Cult feer bupl is bã Clip= pen / en 25ancken: Die Daer ziin/ ziin Boftwaert ban S.Ian, de the= bier Luyfa, ch die diemen noemtCa. noba, en la Cabefa,een punte t'alders - Ooftelickste ban liet Eulanot/ on= trent de Clippe ghenaemt los Loquillos, en Daer in een Haben / die 3u noemen S.Iago, en dxie leguen boozder een ander/ die $3 p$ noemen Yabucoa, en dzie leguen ban De $\mathscr{C}_{\mathbb{H}}$ lte/ De felfoe weeb lyeen Paffaye, een Eplandeken / en int begin ban dat ten Suptwaert/ een ander dat $3 \mathfrak{H}$ noemen Boyqui, en wat bozoer bet Eplanot Santana, Guafama is celt Gaven / en daer naer bolghen de tebieren Neabon, en Xavia, ltes le= guen eer mell conit aen de loaben uan Guadianilla, die lept twee le = muen Doftwaert ban de Aicuiere de Mofquitos, in wiens mont is be the viere diemen noent Guanica, en leg leguen ban die/is de Cabo de Roxo, de alder-neltelickife van oe SuptCulfe : en meltwaert na Efpagnola toe is ljet Eplandt uan Mona, en Daer bu \$00zden cl Monico, en Zecheo, twee andere Eplandekens. De baven de Pinus, en ban Maya- 


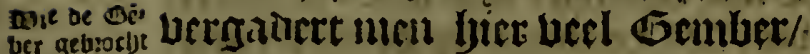

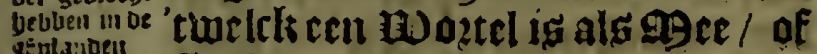

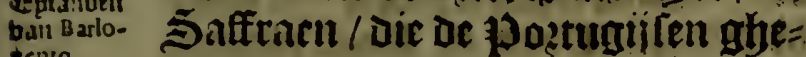
tento. bzacbt lycbuen upt ljet Baften / in Dit Eulaut van Barlovento.

De Eulanacn die benoazaen $S$. Ian, Efpagnola , en Cuba sijn / Daer Wan gljecniglje bewolrkt jiin ban oc Caftilianen, werden abenoemt De Enjarbe de los Lucayos, bet cene bet aloerlos Lucajos. \$200ztliclute / Dat coint tot 27.gra= Dat booclite / datmen unomit Lucayoneque of Yucayoneque, twelck int Doefteil Jecft Bahana een andert Eplant/op les en twintich graden en cen half/ en derticb leguen lanc/ en aclit bzect/ ban welefte fijn naen maarrat bé ncenit de canael van Bahama, tuf: Eahema is. (rij)en Florida en of Clippen de los Nimbres, wan waet unititozten de Itadiglye uloeuende ftrogmen yan be Zee / foo finel naebet Roozoen Dat al hoe bel de Boint booz?poes dicly is / Hoclytaitg en connen de Srjepen daer niet in comen / en al ljoe wel dat fp contrarij ig/ foo loopen ju upt met de Stroon.

De Clippen van Bimini per: ben alloo ghenaemt / om een ell: lant dat midoen daer in leut ban s: leguen lanck; de eerlte slomirael die nae Cuba certe mael oat bo ginck bie gaf het die naem / en tis ljet glyene dat lan Ponce ban Leon boo? nam te beuotckeren. Abacoa Is een ander Eulandit int midoen uan de glyefeqde azoochten Ianch twaelf leguen. Cigateo tan 25 . le $=$ given / Curateo ecn nnder clepnoed/ lept op fes en thuintich graden/oorla Guanima ș .leguen lanck/ en thien bzeet / en Daet neffeng Guanahani, let cerlte zanat tuiclels ontopelt= tede de ectute atamirael lan Indien, cu noentoe lyet S.Salvador. Yuma leeft tuinticl leguen / an aclit ii: De luzecte/op 24.graden en cen balf?

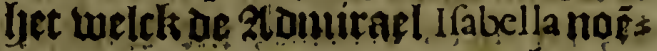
oc/ ter eecen uan oe doozluclutigbe Coninginne Bzoume Ifabella, fijt particulier belclyernutter/en dic dele ontorclingte gaf. Lunneto op dxie cn twintich araden/en een half/ $/$ jo

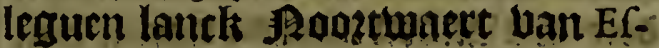
pagnolà. Samaana féuen leguen ober. olueers I tulfrljen Iumețo en Guanima. Triang!lo acht leguen lanck/: op 24. graoen. Yabaque thien les guen / op twee en twintichen eer. Jalf graben. Mira-porvos, fiin onie Eplanoekeng in een ozie fouck onccinghelt : met Dzoochten Supte waert ban lu meto. Mayaguana;20. leguen lands / en thien batec /: op Daje en tominticls geaneti. Y nagua thien leguen/ op twiaticlo en en balf graden. Los Caycos, een epa lant uan s teguen/ op cen en thoin: tich graden en aen t' Pogozoen ban bit ig zenanoer genaent Hamana, en trandexe Conciyay: Maçarey op 20. mader y becingle!t met fupa len, Abreojo is een atoofe dzo日ethte uan I s Jeguen/ Gethuoden ban oit legt op twintich graben / en onder oefe eplanaé sijn bele anoeres dis lanoekens fonder diament a : at:

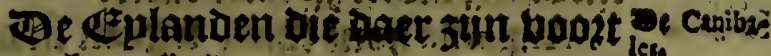
uan bet culamat s dan de Puerto Rico, Boftwaert nae de Cufte hain bet uafte I ant / meroen gtienogne Los Canibajes, on uecle Cariben?

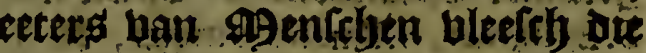
Daer in zin: en nage bat men upt: lepte in baer tael Canibal, is roo ogel Iearcanibal te fegghen / als een Aterek asenicly om dat $3 p$ booj fulcke waren abs: Gouden ban andere Indiané:'t Siin allegader Eplanden met pericu leute Dzoochten / en die naéfe aen S.lan leggljen / zijt Santa Cruz, int Supt-1Beften batt S.lan, op fectien 
en een Ialf maoew/en beeft refthien leguen / en Saba las Virgenes, twoee Eplandekeris omeingelt met bups len/ en noch andere acht of thien Eplanden/van oe welcke degroot= Ite is yaln thien leguen. Virgen gor$\mathrm{da}$, en Incos blancos, int meaten ban Virgen gorda. La Anegada if reven leguen lanck/ op achethien en cen half graben / omcinglyelt niet buplen/aljelijick als Sombrero, oock

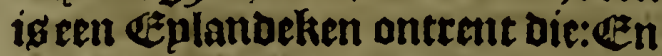
defe Eplanden biemen noemt ban Barlovente ontoeclktoede de eerlte atomirael ente int particulier die ban Santa Cruz en dandere/ bad= Den voor een coltapme te gaen ja= Qjen : פenleyen op bet Eplanot bail S.tan, oni te eten / en noch he= Den ten daglje boen die ban Dominiça: De Biouben aten ju niet? maer bieloente voos Slabinnen. Du leggê 3 norb/ bat jet wepnich. Garen zijn diat dic ban Dominica, teti gsonick grjegbeten bebben/ $\mathrm{en}$ bat bu tepoe: oat alle ofe gbene die mubleztey rouben proébent: bat jp viel camecangatighen Toutoen crij= gjen/ in êtnighe fondat tierben/en

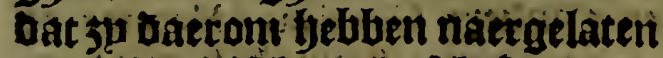
tceten as triftien bleetch/bet moet altro tieren $f^{2}$ oin bat in plaets ban

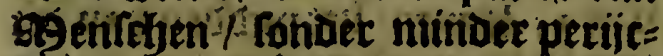

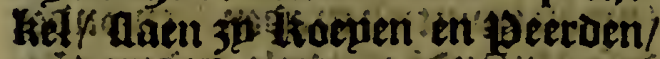
mibe beelfetet bie baet íf en baer mede verfádèt spo jare futie.

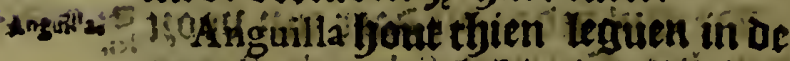
Iengte/ Iepe ópachtbien geaden. $S$ : Musten tept op rebentljien en cen balfy foceft Celthien leguen f oincin= gelt ntet epolántokeng : vp oit lept S.Étifatius', S.Bartholomeús, en S.

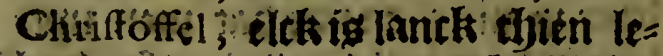
guen La Berbada op reluentluent gra atify oñtritghele inet Buplen / on = trent oe la Rédonda, tride lás Nicres, en be-Monferrate, clelt ban bijf teguen/op is.cheen talf graet. Be Antiguà, en Guádalupe, en Todos Santos, uan vèettbien tót bijfthien genterr. La Défféda aen't Bolfen isว?:19 ban Guadelupe, bị nact frg legum: yet cerite dat d'xtomiracl Don Chriit oval ontoectede/ indet twerde bullat = nie die bp naer Indien Dede / op I 4 . en cen balf grüorn / op Lelelie aen te boen altijot de Dloten gaen co= mente ban Canarien. Marigalante, oe naem ban bet Seljip Dat oen gtomicael boeroe / bijf Irguen ten Supt-merten / ban De Deffeada, cn ban de Dominica, op Derthien gra = ben / twaelf leguen lancls / al warr De B loten water en Wout nentm! om ont bet goede 1keeden beeft / al boe wel nuet perijekel ban de Cariben. Ontrent de Dominica té Sup= Den is Matinino, Santa Luzia, en los Barbudos, be welcke blijuen om of gaende Blooten aen oe flinckerhant / heblen $3 p$ die ghenoent of Eplanbem van Sotavento, be wele= ke befoozen tot be Cufte ban't va= Ite Iant/ en ban dien is bet groot= Ate/ en luet meelte/ en bet aloer-Bo=

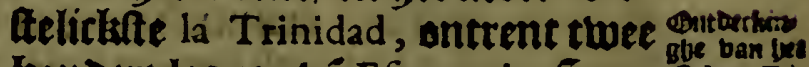
bondert leguen bã Efpagnola §up= extant TriDen en .Roozoen met Dominica, bp iars ispos vaelt treftich leguen daet of / bet is 3o. Ieguen lanck/ Ooft en $\mathrm{mBet} / \mathrm{en}$ bo naeft bertich in be beete. Int Jaer 1498 . if ontoeclit ban de zlomitael Don Chriftophor, in te beroe reple diebp bede nae Indien tóe fen noemie bet la Trinitad, om bat gy bebbende beel arbents op oe wech/baboe bilooft ter eeren 600 s bet eetite ILanot oat hy vout dien naem te gheben/ en Atrack fach of 25oot ghefel oie in of g9ars lisp/ baie boecken 3 ants / met bet wele = Fe in alder wijfe ober een quani de riaemi en de belofte. Doen ontoecktede fjp de g9onden uan Drago, $\mathrm{cu}$ ban be Seirpe, de Golfe ban Paria, en al bet vafte zandt tot Cumana toe/ teglyeng welck op het alder on-

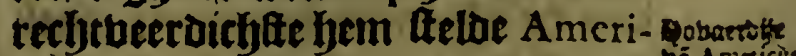
cus . Vefpucius, wieng naem onwaer - verfundtise. belick ghegheben mozot / aen bat Sumber Peninful, oft Indien bant

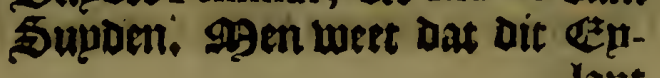


lut Trinitad glbeu goct llandt is/ al boe wel bet ueel Indianen beeft: ljet beeft 35 . Ieguen ban de lengte/ en andere regaijen dat bet meer beeft / ell 2 ;. III de bzecte / bet lept op aclist gribaell / op bet aloer-Oo= telickite Deel int froozoen ig een hoecls d:e ghenoente wert de la Galera, en Roozt warert Daer af it een Eulandeken met Eulandekeng be= cingbelt / Dat men Tabago noemt: cn aen de §upt-Cuite een caep dic ju noemlen Punta Redonda : aen het Bolterfeíse Deel/ al of punte del Anguilla, int meiten' en in De Golfe uan Paria, de weicke die Zee is die Daer is tufichen Dat Eplant/ en fet balte Zanor / t'welck narty weien ban acht leguan wijtte/ macelet een Jalue cronume Girckel / als een Croon / ende de incomite ban bet van't melten ts de meelfe engte en meette diepte/ en twee Eplanoes ketts op bet epnoe ban oe Cuit Foozot-Laets / in bet Becten/ tweiclt inten noemt ie 990 ont Del Drago, en int \$200zoen S. Vincerit, en Granade, twee andere eplan= Dekens.

1)et Eplant Margarita Dat oock gheliick als de andere frin naem glyecregben beeft ban De ztomicael Don Chriftophol Colon, De alder = batte proeve met of welcke waer glyemaeckt wozde in wat tijot bet was / confulie welende ban die ge he die ben berooften ban de eere ban de ontoectsingbe bai lyet balte Zandt/haer vien toercheijuende/ al boe wel ju de tijden berkeerden. Het leut twinticly leguen ban la Trinitad melt-waert/ en bontert treventich ban Efpagnola:bet is let= thien leguen lanck / ban Boft en Boet /en eenighe fegaben twintichy en balf roo bzeed: daet is gyeen oberbloet ban mater / al boe wel bet beuchtbaer is ban boer booz bet Bee. Daer ziin twee Pozpen op/een Dicht bp de zee/Dat lept aen een fore/ al waer do Gouberneur refidect / en fot andere twee le= guelt binnen 'gleandts / Dat $j p$ nomen de Ballepe uan S. I.uzia: bet Jeeft op lijn Culte cen goede

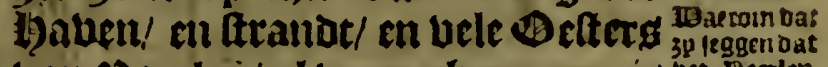
ban peerlen/al baer gljecomen is yes geetien De Biftrljinglje der peerlen / die te loope is ban

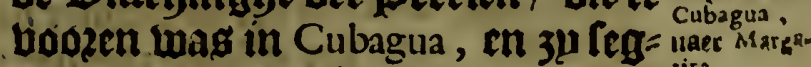
gljen Dat de. Befters vloden Daer af / oin lyet gheraes ban lyet Gbe= feljut vau alle oeschepen die dick= bills dact quamen aen dat Eplant op de laandeling: van of beerlen/ Die feer groot was.

Cubagua lent een legue uan Mar- pet Eplants guarita Dolt-waert / 'tbeeft niet een Dzoppel Materg / evenwel foo was bier glsepopuleert nieu Cadiz, en bzachten bet $39 a t e r$ leben leguen/ van de liebier Cumane. Ten $Q 0=$ Iten uan Cubagua zijn bier Geplan= Dekeng dicht aen be Culte die noe = oe de eetite atomirael los Frayles, en Bolt-waett tuateben dic en Granada, andere bier oft liif die bn noemoe los Teftigos, entueft-waert achter Cubagua, is een ander $\mathbb{E} p=$ landeken bat by noembe la Tortuga, ontrent oe punte ban Araya, ett ban bier bede bu veroer ontoeckin = ge ban Paria, welende in Efpagnola, met boomemen te bolboeten de ontideckingtse ban dit bacte ILant/ en ghelijek als bier naer gyefien fal wozDen / is daer naer guegaen tontoetken / en bont de Etplanbent De los Guanajos, en ban daet lante Veragua, tot boozbp Nombre de Dios.

In Margarita ziin oe Officiergl en be Conintklicke Stbatkilte: dic Eplant / en dat ban Cubagua, legs ghen op elf graden / een wepmict meer.

\section{Wet VIII. Capittel.}

$V$ an Venezuela, de Reviere de la Hacha, nieu Andeluzea, la Florida : eo de inwryck van niew Hijpanien, t'melck 


\section{JFal.18.}

25elchatfuinghe

bet ghene is dat overich is van bet refort van das Hof.

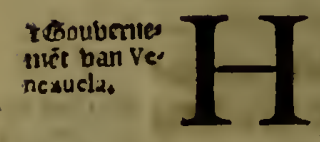

Et goubernenient ban Venezucla frljeut [ijn limi= ten op De Culte van't valte lant / iut Ooften / met nieu Andaluzia : Uam waet af tot de licbier de la Hacha, enl Jet Goubernement ban S.Marta, met tweleke bet fem voeclye int lobeten/beeftet hondert en oerticly leguen/ en Tanot-waert in bu naet tachtenticly/tot oe lant= palen ban bet nieut: taijck uau Granaden. Daer zijn in Dat YLanot beel teeckenen ban Bout/ Dat comt tot twee en twinticly en een lyalf $\mathrm{ta}=$ ract/t'is het aloerbzuchetbaerte ban Carwe / Daerom gljeft het oocks twee Dogblten int Flaer / en bet oueruloedichlte van alle foozté ban 10e/ clepnen groote / men treckt upt die 1 zobincie beel geels/25if = cuut / llaes / Speek / en beel ZLin = waet ban Cattoen : en in of Haben $^{2}$ ban Guayra, in de 13 zoluincie ban

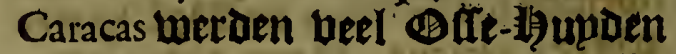
gheladen/ en farfa parilla. Daer zijn in die 19 zobincie acht Dospen ban Caftilianen,en 3 p werden gbenoent venezoun bet met ie naem bam Venezuela, om

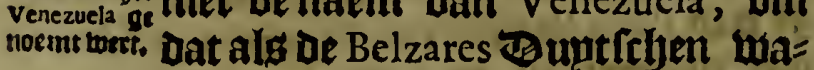
ren in defe 19zobincie om te gou= bernecen -iat 3 aer 1 s 28 . om bet zlecoost dat $3 \mathrm{p}$ maeckted on met de Hepler / uieenden ju bet te bebole = ken op een Kiotfe/en fteulte die daer is in be gront ban De 99 ewz ban Maracaybo, ban waer bet upttua= tert in De Zee / een Dozp Dat $3 p$ noemben Venezuela, Dat op acht graden lept/een wepnich meet/ en ban bier is de naem gljebleven op yet Zlantifliap. Waer pzincipaelite coro. pabt platete is de Stadt Coro, die dé Indianen noemen Coriana, diemen nu gyemepulick noemt Venezuela, 30 leut op elf graden lyoochte / en neglien en trebentich en een Derbe= Deel/van be Supt-3:2002t-2iin ban Tolcdo, Duphent en bijf jondert le= guen saer ball: al maer oe Goúverneur placlj te refideren / en ljet Cathedral is fuffaranenfrlyap van D'Aert s-23iftey op van S. Domingo. Eertt tis die Staut glyeluout uan lan de Ampues, en ftracr namten in haet bace fitplaetle de perfoonen die booz uan weghen / ban de Bclzaren waren/ De eertte is gfisweref Ambrofio Alfinger: $j u$ is loo gljefont/ en yeeft too gocden lo zochit datter gheen gisedicius uan noode jijn.

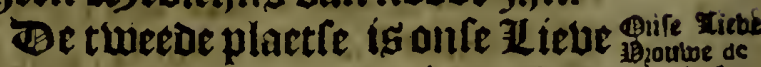
3 2outue uan Carvalleda, in ie $1320=$ carralleda vincie ban Carracas, bu oer $\mathbb{Z}$ ce tachtenticly leguen/O oftwaert valt Coro, en met een quade laven. Is bevoltkt ban Don Francifco Fajardo int $\mathbf{3}$ aet i 50 . Die Daer gbe $=$ comen is uan de Margarita af. Sant- santiago ds jago ban Leon in De boop fepde 33 20= bincie/ 7. leguen binnen 'gI andts/ en Dzie ban Carvalleda Souptwaert/ en tleventicls ban Coro, al waer meel refibeert ae Gouberneur in Die tijt. \$ieu Valentien treftich $l_{e}=$ guen van Coro, en bijf en twintich ban Santjago ban Leon, feuen ban De 1 aven ban Burbarata, is bebolct ban Capitepn Villacinda. Wet nieu Xerez bp naeft bijfthien leguen/bu= cans int Supden ban nieu Valencien, en twintich ban nieu Sego: vien, en teltich ban Coro SuntFett-waerts/ een nieu Do2p. Het nieu Segovia in de 93 zovincie ban segoric Bariquizimito, thintich 2 ij len ban Xerez Supit-waent / en thien ban Tucuyo, ent tachtentich ban Coro naer Supt-1Deften/al waer De $D F=$ ficiers ban be Coninck refioeren/ en be Boubernetit oft tijn Tute nant bu tijoen. 7 g liebolclet in Ian de Villogas int $\mathbf{3}$ aet I s s 2 . We Staut ban Tucuyo bermaetr/ ont - sucago: Dat aldaer boot gyellaglyen is oes Cpzan Lope de Aguirre, lept tbien leguen Supt-metort-waect uan Segovia, brif en tachtenticly uan Coro, is een bevolckingtue wan Capis tepun Carvajal. Truxillo, of onfe reusillat 1. $13204=$ 
2. Deaume vinde becoe/inde $p_{2} a=$ bincic ban Cuycas, bu-naett tacly= tenticly leguen/bu cants té Supden/ reeft Uan Coro, wat naet't Ooften tae/ ell 2 s. Uall Tucuyo recljt Weltwacrt.

Dit 1 and is antbeclit int 3 (aet I; 49. wefende Daer Bouberneur or $\mathbf{z}$ icentiact Tolofa, de Contador Vallejo, en int 3 aer i is 9 .ig Truxillo bevolclit ban oe Capitepn Diego Garcia de Paredes; m Daer beljoozen tot Dat Goubernement meer dim bondert dupient tribu= - gotbanith tairele Indianen, en Daer in wozden ore Indianten niet begrepen die minoer ziin dan

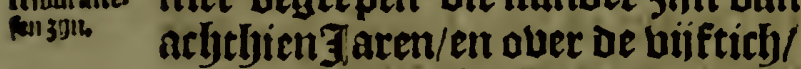
oni dat de loouge tiaed ban Indien fozglye dzaectbt / Dat in gljeen Deele ban Defe moerele/ fulcke Uri= bupt gljeben : en om dat jp alle ba= gljen walfen en berminueren in gljetale/ en can men niet peecijg feggyen / of puntuelick boe veel bacr jijn.

De mme Het 29ep? ban Maracaybo, wele=

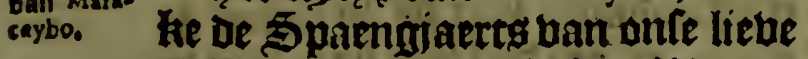
Dioulue tromen/is foet Hater/ loopt beertich leguen Zantwaerts in / uan de liee af/ bet is thien le= guen becet / en tachtentich in de omloop / mee beel Dospen op fijn Beber / aen lijn epnide comt inloo= pen een thebier die balt upt bet nieume Kijich uan Granaden, Doos be welcke' I en bock booz de asep? baren De Coopmantryappen die ju renden na bet nieube 1hijck ban Caftilien, en andere deelen: niglye 7 ngetogenen bán dit grep? leben in Barbocas; ghemaeckt yeb= bentie baér lomen in de 25 aonen int water / tri op de Lebiets-cant/ ban waer bock fiet freet den oos= Ipzontk ban hijn naem Venezuela, replende riae ectighe deelen ban bie grep? / fietmen grieen Zandt: baer tomen it Jjaer andere groate tatbiten / th 30 Hatert upt in Zee/en is een Tjalf legue inde nont wijot / en oner in is de liotre en booctyten dic voozept ziju.

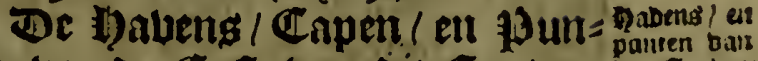

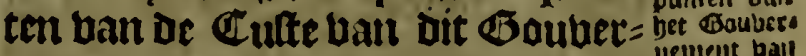
nentent / en die bu-legghende bp venezistas. baer/3ijn int Eeften/Marcapana, la Vrchila, een Eplandt tegfen ouer be Lebier Oynare, bueen ander die men nomit Roca de los Inleos, en Daer naer Cabo de la Cordera, en of 1)auen Flechado, en die ban Sardinas : ent teghen ober bet Eplanbt de Aves, booz de Burburata, een won= berlicke lyaben / Den welclie popu= lecrde de Ilicentiaet Tolofa, ende is de plaets ban oe ontladingbe vooz de goederen die gaen nae ber nieuwe Zisick / en de psobincien Lan Peru, en daer naer golfo Trifte, en in bet pooźoen ban Bonayre, een Genlant van thien leguen lant/ en acbt beet'! en baer naer punte Seca, en tegben over Curacao, en meer boowwaerts Curacaute, 14. leguen landk l' tegyen ober Cabo ban S.Roman; Supt-waert ban bet Eplandt van Aruba. S.Roman loopt twinticfi leguen in oe $2 \mathrm{ec}$ is twaelf léguent van Coro, oe India. nen noement Paràguana, baer febeele wepnich aen of t ís een Geplant/bes frecter bem int tonde 25 . Leguen/en meeft in black lant / met een 25 erch bp naeft int misoen/ die bem berde tzectwaert laet lien. Daer is int bieginfel ban de Golfe bã Venezuela be inganck encanaet ban be 29 ep? van Maracaybo, en in be inganck bâ die de 1Rebier Mitare; en int Hes tien los Monges, Dic Ėplandeken bicht bu de punte/en de caep bã Co: quiboçoa, al waer men alleenlick in gantrch Indien vint gljetwicht / on tout-5teen booz Get Bout: en daet nae Bahia honda, en de Portete, en de Cabo de la Vela, bie alloo ghenoeme werde uan De Capitepn Alonfo de Ojeda; als fju daer ginck de eerlfte rên'e met Americo Vefpucio, lanck nae die tijot dat de eerfte atoniz= rátel ontoethtede die Culte / die ap twatlf graben tept / een wepnich 
IIiecr / in is Uall oe Cabo de la Vela, / rado, in lyet Supoer-beel Uan oit tot De lietuier de la Hacha achthien legueny conter eeniglye Steen in alle bie / hoch loater/ fonoer liet lifglyenwater. Dit Gouberncment bleff verdeftecuect van oe tijot af orr Dupteflyen / om bat $\xi \mu$ bet niet bealyecroen te bebolcken / niet an= vers Dan onte lievlijten / om 5 la= uen te maecken/ en bet $x_{\text {ant }} t^{\prime}$ ont $=$ blooten uan 10 zuchten.

Aac nevicre "Eeritelick ís De thebier la Hacha; drla $H_{a}$ cha, onte Zieve Bowlue de las Nievas gljenoemt / en Daer naer de los Re. medios, Diclyt bp be $\mathbb{Z} e$, tulfelyen Venezuela, en S.Martha oltwaert Dertich leguen Daer ban/ en trelticly ban Coro 1 B bett-Laett/5upden en noozaen net oe Cabo de la Vela, niet aclit leguen begrijps / tufthen Veneziuela, en S. Martha, Conder montter ban Gout / wert gliegots= berneert booz Alcaldes, aengelenorbt aen bet lof pan Erpagnola, en int Elyeeltelick / it behoozende onder yet 2 sifoom ban S. Martha. De

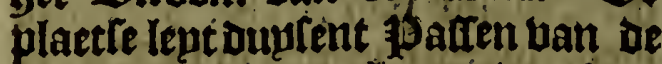
Zeel op een clepne 1 getubel : De ben Iept open voogt J Ropzoen / bet

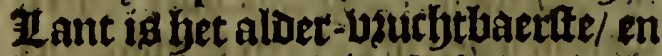
bet gheeft al Dat in Cantilien balt/ theeft veel cughers I in groote 97ofquiten / en Crocodillen in of Rebieten/bet Gouts/en gbetten= ten ban betrobepoen crathten boo? be Clalique/ oe sieren/ oe פsilten be zoop/baer ziin oot kgode lout: Hannen. Nicolas. Federman boo? De Belzares, it de beboleker gelueeft ban die plaetre.

SAleu Arda- bet Goubernement ban Serpa, tweick men noenit nieu Andalu. zia, en in ae Indirche tale la Guaya. ia, nae fet aftefyepoen wan be lant= Dalcn / Geeft van bet Eplant Margarita, tot De fieluier Maranjon, Dzie bounert leguen Dalt-waert / en ouck roo becl Supoen en 3 Ropaen ILants-baerts iu/ waer in baer be= Gupten De Indianen Omagues, ent Omigas, mict of 33 zoluincien bf DoGoituernentent / waer in valt bp oc Culte / or 13 zouincic van Maracapana, in de II antpalen uan Venezuela, bail waer af bebolclit waren de $\mathfrak{I g}$ onden van Santa $T e$, in Lelet grbuptclyap is of Morre bain Vnare, en Dat epndiclyt ế groote $\mathfrak{A g} \mathrm{eq}$ met beel Difty/ch Sout/ an oe Indianen diemen noent ball-Perito, twintich legutn binnen 'dil anots/ en De Indianen Palenques, alfoo glje= Hoent om de Staquetten Daer $3 p$ Gaer med foxtifieren/ cn het is ant Iflyap uan Cumana, Supjoel en Roozden met de Margarita, al lwaet een Dozp is ban Caftilianen, Dat ghenoemt wert bet nieu Cordoua; Dat glyefondeert is bu de Capitenn Gonfalvo de Ocampo ; als hp was abjecomen om te taltigeren de Indianeri ban Cumaua, om de verwoes tinghe baun bet Cloofter oer Heli= gieulen francilcanen : en int $B$ os Iten ban het deplant Trinitad, en De Keviere S. Ian de las Amazonas, if een 13 zovitncie ban de Indianen Aruacas, en een deel ban haer sijn de Cariben, en allegaber bellectur/ Die niet bzeedtlaem en zijn.

Daer nae is de punte tan Paria Dic op 7.graden lept / en De glont ban de Dragon, Jicft bp de Trinitad, is De punte ban Gallo, of Ane. gada, ten Supten uan de Trinitad en oe Kebiere ban Paria, of Oronico, dien eenighe noemen Yuyapari, en andere willen dat Die tweel en De Liebier S.Ian, en ban Orel: lana jpeen dinck / en oock oe Ma. ranjon, maer daer wert in gheinif. Daer 3ijn andere Rebieren in de 70zobincie van De Arvacas, Die niet beel bekient jijn: Die ban S. Ian, of ${ }_{\text {Bas bier Orelo }}^{\mathrm{B}}$ yan Orellana beeft lijm begincel in lanafparper De Andes van Peru, in de Contrepe Wan Cufco, ban waer by loopt duy= Cent en bijf hondert leguen / of ineer/ maeckente lijn keeringljen onder booz de II inie Equinoctiael, oops isanoen bie feer beboleltt ziji! 
al hor wel Dat ju noclj wiet wel ont= orclit 3114 / tot Dat $3 !$ Inaer ontladet

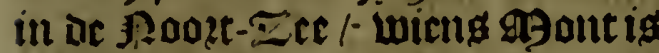
bect vifftich leguen / en int boozlte soo. Uan De פTont opwaerts / siin Uecl bebolelitedoe Ëplanden an 80. of Ijonaert leguen loet om meft Icut De lictuier Maranjon, up caing it die palen booz welcke paftect oe Thinic van de afosilingibe tur= frben Caltilien en Portugael, en is roo groote / in periculleule IReviere/ dat bu heeft vifftien leguen wiittê in oc פont / en tiin Bloet comit ban upt lyet Supoen/ en be 19 io = bincien ban Brafil, en Daer betet gibelent dat fijn begincel is bene= Den Popayan, ende de Turifoictie ban yet niculue kijick van Granaden.

ceter Eplatos bas.

Seven legien tuat Cuinaira lejt yet Eulant uan Cubagua, al waer cheweelt is gbelijck ghelept is bet nieutue Cadiz, t'welck ontbolckt werde om de faulte die daè biel int viffelyen ban de prèlen: Dit $\mathbb{C}^{*}=$ lant en freft niet een dzoppel Ha= ters datmen conde dezincken / noctj $2500 m e n /$ noch Ghedierten/om dat het gantitis Salpetrict is / bathal= uen die Berckeng die of nabel heb= ben op of thuggrate / th teniglie Conijnen / in liebibente loo grooté bcuolckingtiel gimghen 30 eben wel leven leguent tot aen de latuiet $\mathrm{Cu}$ mana on Mater/al hoe wel dat dé opinie was dat het nolcken in de Oogfien maechte. Bp defe tit: bier of Culte iff gtjebeurt int Jlace buptent bijf fonbert en bertich / op ben eeriten September/met Ietioon weder dat be zee gherefen is buer Padeine van fijn ofoinarife fooct):te / en loopende over fet Zland be=

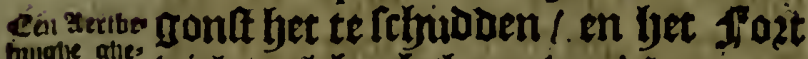
bugle gyes biel tweick ghebout was boos of =

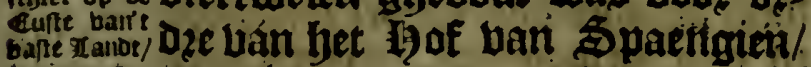
dirt jax 1530 ban Capitepn Iacomo de Caftellon; en de zierde apende fiem op bet placten/oaer upt quant biact wa ter en lwart als tint / en tfonck als
Solfer / en de 23 etgly wan de golfe van Cariaco blecf int midoen open/ afloopende beftet een groote ope=

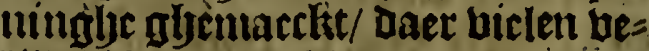

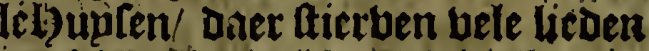

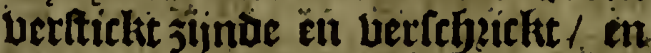
bebanghen ban de stertluebinglse. De Margarita ceñ léguie uán Cuba- be arazgas

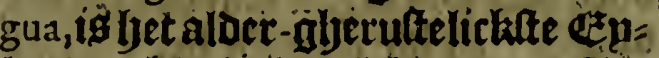
lanot / het ig bebalckt uan de $\mathrm{Xi}=$ rentiaet Marcelo de Villalobos:

De jobuncie cin'tSouberne= nient uan Floride, nae-bupktcljap, Floxide。 ban get hof bain Efpagnola ; nae oe bepalinge bain bet Souluernement ban Peromelendez, iŝ. al bat Dat ter is ban de Liétior de las Palmas; oe welck paelt met bet Bouberne: ritent uan Panuco, in nien Hirpa-

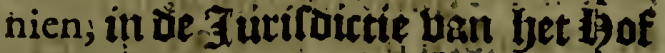
van Mexcico, te belck ke is beg oc Tropico, op twintich en twe gra Den / tot be puitte ban Bacallaos, de welcke ront té legotjen op acbt ent bcertich gratuen en een Galf / in: welcke ziin twaelf gonoett acbe en bijftich leguen Cults 1 en ban baet tot bate en trétenticligraben booch

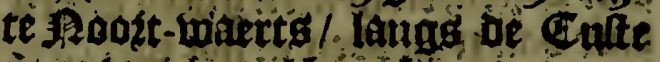
en te lant in / al bet gene oat ont= beckt is. Dit id gubecultet / en onts: beckit/ bain oe kebier de las Palmas, tot de punte bain S. Helena, en De Kéviet Iordan, thelcta jiin loo beel als tes bonoert legnen: $t$ tis baff Lant ban én goet climaet/ beucht baer/en in becl declen feer bebolets. getijet als dic weten die oerwaerts gingljén met Hernando de Soto, in

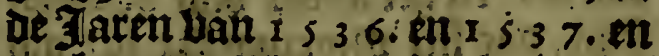
nocfj meer. en lan Pardo ingles bogen ban Cuenca, fo fo lande glye gaen ban Florida, tot in nieu Spaen gien, in min dan in tioes Jaren/ th is let aloetnaéte aen Europa; ban be Landen uan Indien, en uan be Baye bat s. Iofeph, of welcke is tachtentich leguen bain Panuco, is oock bp be felue ontócht tot Terra nova toe - en thenomen int parti rulier t'ghene bat Floride is / is dit c. 3 pintite 
punte dit Supden en \$Roozacn in Zee upt frbiet / tegljen bet Eulant Cuba ouer ban hondert legue lanc/ en 2 s. bacet boft en $\mathrm{TB}$ eft $/ \mathrm{et}$ wan= neer bet beel is/ foo if Derticl le = guen. Het is ontberkt bu Ian Ponce de Leon, int 7 aet I ; I 2. op oen Dach van paetichen ban De opltan= dinglye/ en by noembe bet Florida oni die oozfaect wille/ en daer naer quam bu wederom int 3 laer een ent twintich / en fop vertrork gljequet ta tot Cuba; al waer bp ttierf / 'tghe $=$

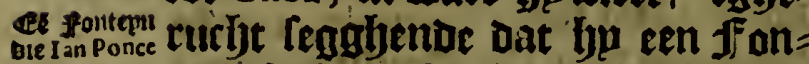
roctsis oie or tepun lochte / of de livebier / welck de maectsede. Indianen, [epDen de (B) uden Die baer baer in wiefthen / jonck te nuaes= Fen. Tele 3 zobincie is met de $\mathrm{Ca}_{2}$ ftiliaenfche ghetempertljept geliiclt/ en $3 p$ vonoen Daer beel Bzuctiten/ en 'tichijnt bat let bequaem is tot sece- en Serwe: ten beeft gbeen. Bout / ten feljijnt oock niet dat die. Indianen let trennen / en noch loo. wepnich het 5 illuer/at boe wel bat baer ghetien zijn ghetonden ghe= weet te bebben op de culte calien bain Barren; en gelt/en gheen werck Dier ban te heloben/al foe bel Hernàido de So to in het binmentte ban. De groote kebier / vont oe grootffe quantitept ban becteti.

De forten ineer dan thiee Jozten/ met Bats nitoenen/bépoe op de Crifte die nae Jet Ooften fiet/fet eene is genaemt S:Matheus, op be punte vair S. Helờa, bu carte fondert leguen bait Havana, en fret anoer ban S.AuguAtijn, Dat bet pzinciparlite is / om Dat het ent noedo loaten beeft / cn dat fjet teer oitht is bu of Canael Uan Bahama bet is bu cang ;o. le givèn bañlos Martyres; twelck beel

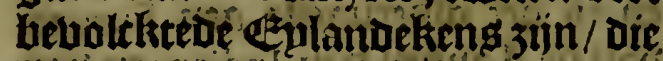

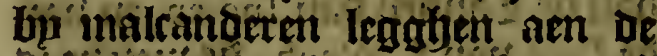
pinte bain Forida, al waer fet nuelt naoert aen fier eplant ban Cuba, cen legue $/$ of een legue en een balf

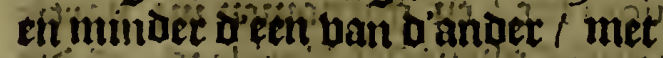
eti tpe bain ozoochten inter agon=
Den/ ban doic en twintich of vicr en twinticly Ieguen die dele Martries int langhe bebben / wieng begintel aen't Mest-epnde men noemt de B) Hute van de Martyres, entoe int Boften/bet booft van de Martyres, en alfier is eell Gulant uan $14.1 \mathrm{l}=$ guen lanck/en lect final/ lijn punte bet eertte aent valte $\mathcal{B}_{\text {ant }}$ int $B \mathrm{O}=$ fen/ lept op 24.graden/ en cen bal= ve/ al waer eeli Dozp uall oe Cafti. lianen bevolcke is dat niet is blijuen. Dupzen / ch Roozt-waert / hae bp recbt ouer de liebier Ays, ent bet Soozt-waert lept op ache en twins tich graden de punte ban Canjaveral, en bet voouts de tievier van ae Mofquites, of 99ugghen/boo? De liebier de la Matança, oie alloo ghe = noemt is om dat Petro Melendez, aldaer aenglyetroffen lyett / oe $\mathbb{C a}=$ pitepn lan Ribao, en lijn Françoyfen, en de punte ban S. Auguftijn, op neghen en twinticls griden/en ozie bierended/,en van daer 10. Ieguen is de Revier uan S. Matheus, uan waer keert oe Culte nae t'Poozt\$2onzt-Doften / altemael met ep= landen en 25apen laner de Culte tot de Cabo of punte ban S. Helena, Die op twee en dertich graden en en balf Ient / die baer ontoecktede by ghetalle int 7 aer i 520 .

In alle be refte ban de Culte/ die oe sunfebs

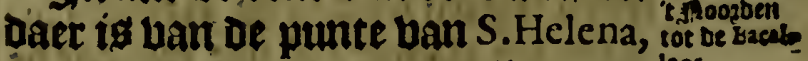
boozbp Terra nova, en Bacallaos, tot terra de labrador t'melek comt en loopt bobm de hoorbte ban $\mathbb{E}^{\mathbb{n}}=$ grbelandt/en is gheet onozp / nocli Goubernement ban of Caftilianen, boe wel op berfedsepoen tijoen / m bo verfelyepoen satien yet ont= beckt / en bereult is / en t'is bekent Datter ziin ouer de duplent leguen weechs zant conder bout / en yoe meer hoorhte/boe minoer in ocurlyt en min bewoonlick. "theeft beel Thevieren' en t)abeng / melcke on oat 3 nuiet feer belient noch glyefre= quenteert 3ijit / werter gheen gbe = wath meer af gbemaeckt ban oe Heviex 


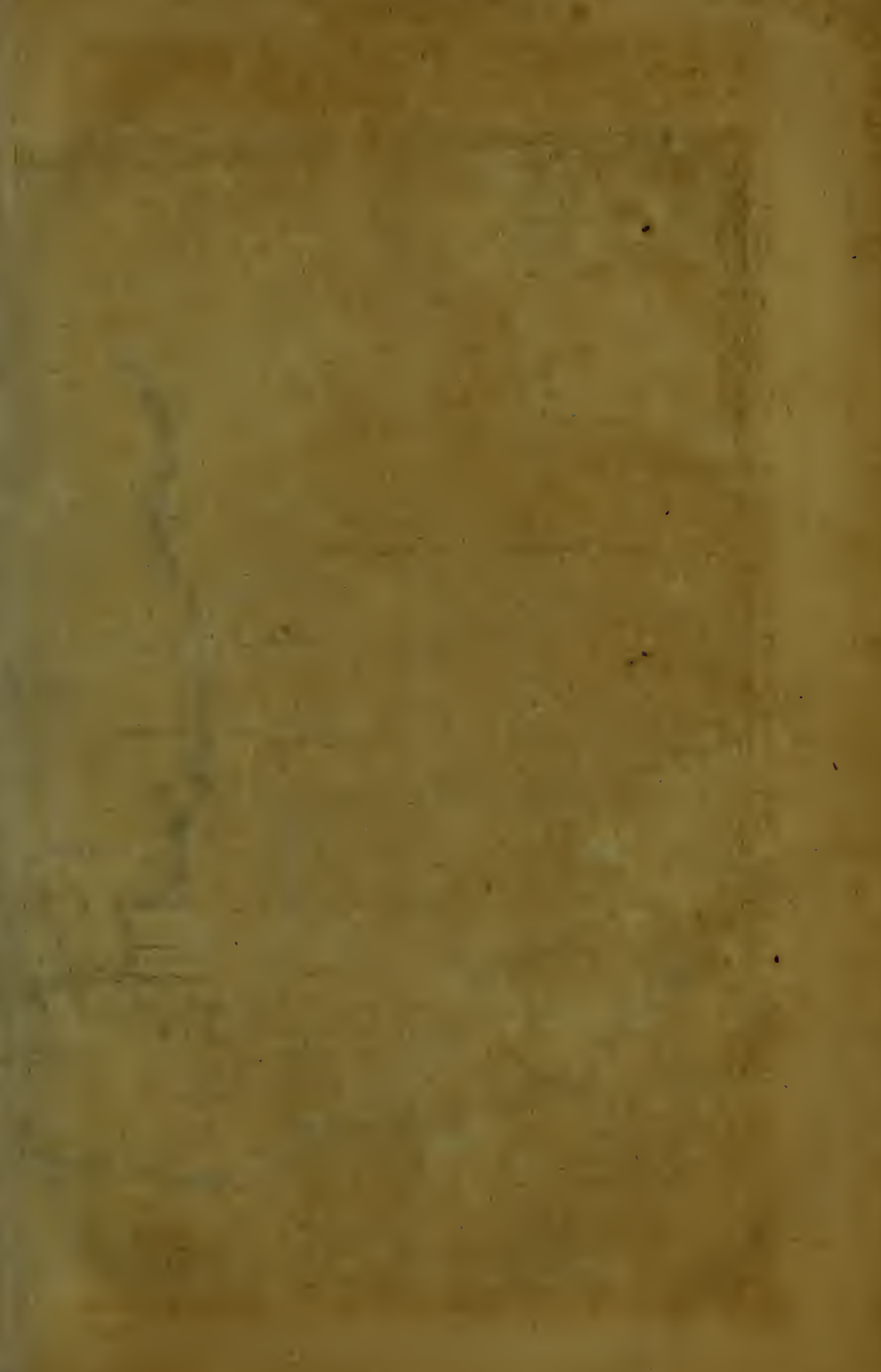




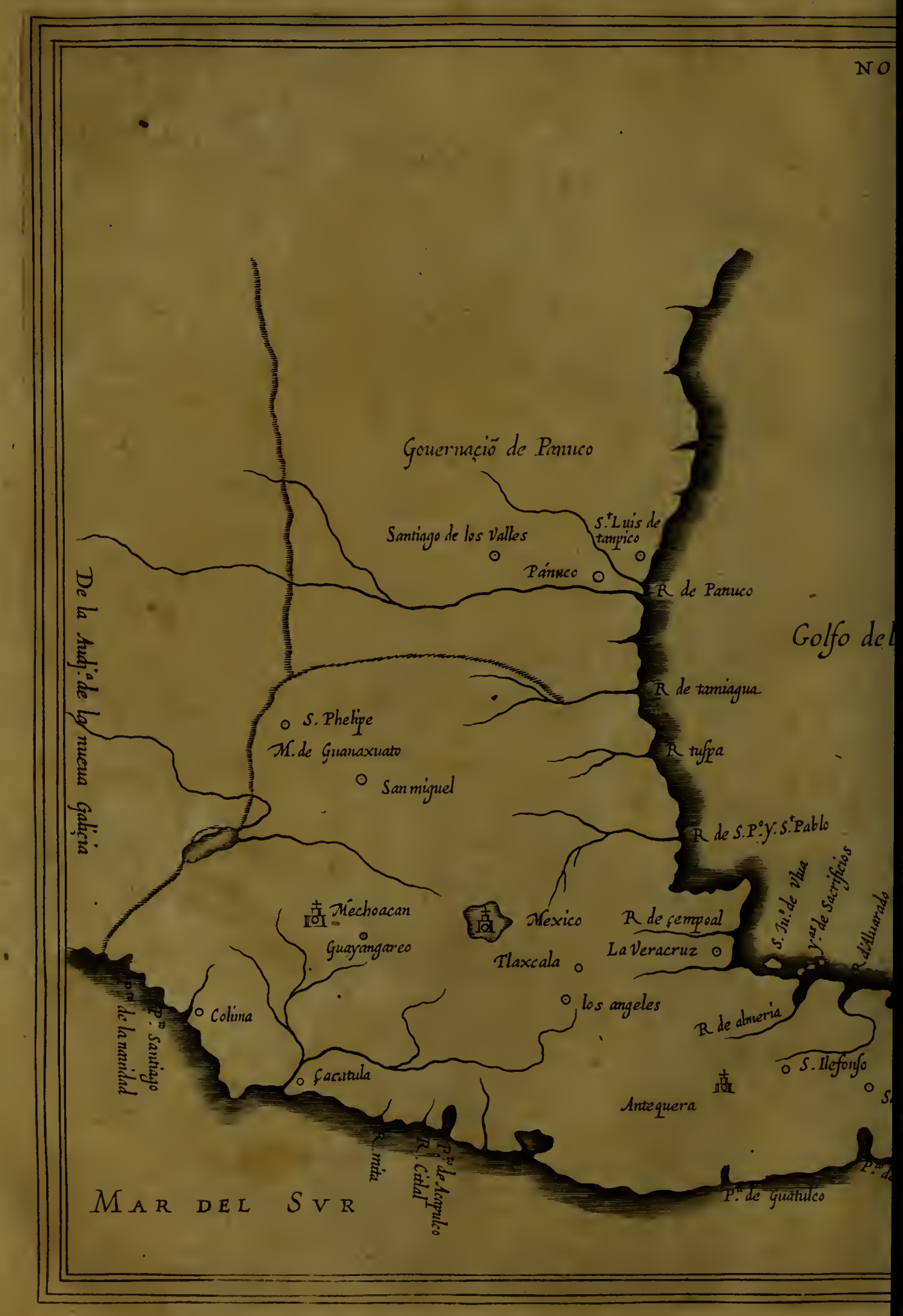



tiivice de los Gamos, anders glje a grcore nusent van S. Maria, bie feet groot Atcuer ball C.Alaria.

is/ en peticuletis / bu nact int utid = Den van de Cuite/ tulfelien de Bacallaos, ban waer ten Zande ins waretg inloopt naer't Boeftet / de groote tievier Ochelaga, die oe $\mathbf{b} k \bar{E}=$ oelinghen op bertelyepoen repten belepile heblien / ntectende te bill = bet een booz-gauck al lieer naer nicu Hifpagnien: Cen op de Culte ban Florida die nde't tielten fiet/ jiint oe Tortugas, Ceuen of acht e $\mathrm{p}=$ landelieng bp malcanoeren / en ten Joozden ualt de punte de los Martyres is la Mufpa ap bet latte lanoc/ kut meer Rookt-waetts dertljien' le= guen / is de Bahia de Carlos, wients andere naent is lan. Ponce de Leon, en noch foo veet verder de Bàhia de Tampa dzie en Dertich legueri vani De Tocobaga, antaet ghenoemt del Spiritu Santo, of de Mervelo, op nie gen en twintich geaden en een flalf bootly:e / al waec engbentlick be= gint t'obente datmen Florida noent. Deel bebluen gbemeene/ wat aen bit beel ban Florida, nae het ILant ban labrador was te binden een Straet baer bem bermengoen de Foopt en Suut-Zee/ reggbende/ ghelijet yet bebonden was int Suipoen/ bat lyet oock coo moelt welen int 30020en/maer de onderbindinglye bewijft dat int Supxen geen ftraet en is/ of ten ninlten. 'tltaet noch in twijel/ en int Poozden tot nu toe noch / al hoe wel beel het bebben bet foche/ foo en ift niet ghetonoen/ en die meelt daer in ghedaen heeft/ is ghetweelf oe Adelantado Pedro Melendez, om eenighe roniecturen.

Golfe bar - Be gantiche culte ban dat deel nieu Hifpa uien. ban Hernando de Soto, en ettelticl ami Eranf́cifo de Galay, en van bet voleli ban dit Lant int particulier? yeeft bo bertaen tat let ech leet

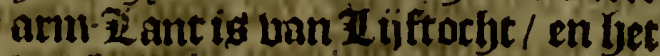
bolck ellentaich/ en boe wel daer in

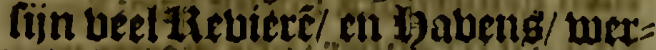
ter gbem mentie af ghenlaeck ont

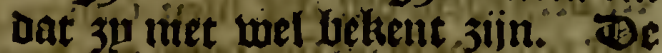
Golfe beeft twee openingtjet / Dooz o'cene conten de loop.Stroomen fect furiturelick tin/ald tultiben $\mathrm{Yu}_{\mathrm{u}}$ catan ent Cuba, enoe gaen om upt te loopen miet ineerder cracht tur= Irben fiet relfoc ceplantot ban $\mathrm{Cu}^{2}$ ba; en oe punte ban Florida, en loo= penoentaectien fies Canal ban $\mathrm{Ba}$. hama, die neenit de naem ban bec voorepde Eulant.

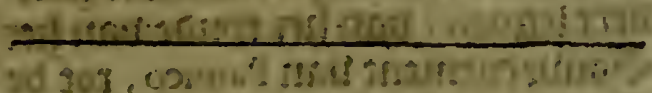

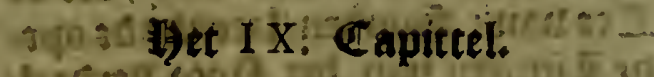
Von de lurifdictie van't Hof is van conexico:

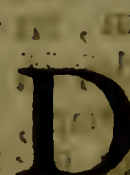
it: ! shu 'innelir? etweto Aludientie, of bof Dat gbefontrect if in Indien, ig dit ban Nova Hirpania, en fijn eertte 132 clident bag (onder foo beel die lyaer gbepzeren= teert fjebiben/ om oit Lant te gou= berneren.) Nunjo de Guzman, kios. ver ban Guadalajara, en om dat he niet gtievontien weroe bokomene bernoegingte/ is bem een ander nieu ghebiet toegberchickt/ on booz Fetiout ban dit / is ghecomen Don Sebaftiaen Ramirez, Lan Fuen-Don Sebaftileal, bie ban Oydor, ban de CLan= oydor bant

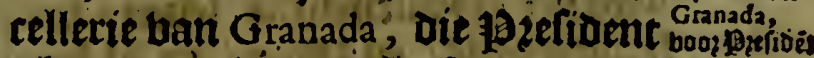

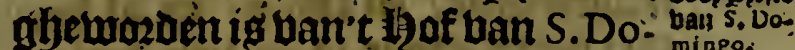
mingo, en 2Biffetjop van de Stad: Conception de la Vega, een perfoon bain groote gheleerthept/ deucht en reputatic. Isp nam tot fijn lalt bet Goubernment/ en be 3lultitie/ en de difpofitie bau oeg conincx in= comen l latende de 13apenen tot laft uan Marques del Valle, met ot: vitent ban Panuco bat ober be itie tjondert leguet is / wert ghenoemt De Golfe ban nieu Hifpanien'; in be belcke gheen bebolckinglye is bam oe Caft ilianen, al boe wel het glyegheben is tot. het Bouberne. ment ban Panfilo de Narvaez, en 


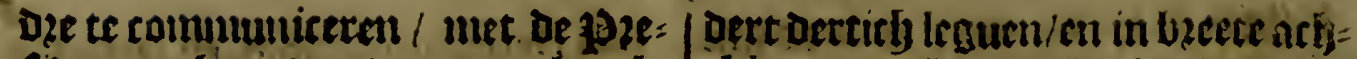
fident $t^{\prime}$ gfjent bat baer aen linck en onder foer-lieden was altoos

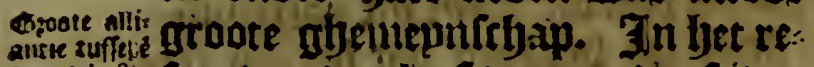

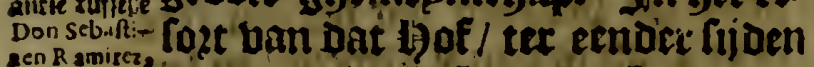

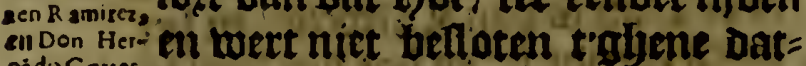
adido cores men gemeenlick noemt nieu Spaengien, en ter ander fijoe werter meer in Legrepen/om Dat nieu Galicen, Dat een tof is op lijn relfsicen beel is ban niru Spaengien. De $33_{30}=$ vincie Iucatan, die niet is ten deel tan nieu Spaenglen, balt in oe $3 u=$ rifoictie ban dit relfoe 1 gof $/$, twelcts rils nu bedentoaetbs is/ Ctrectet int lanrk boit en Roelt / bp naelf 400 . Itguen / vam bet aloer-Doftelicklte ban Iucatan, al waett bem paelt met Jet lof ban nien vializien, en 5 up: Den en \$ooken bp cans twee hon = bert leguen / ban bet epnoe ban bet Goubernement ban Panuco, tot be Zee van't Supoen/latende de ope ne zimiten ban bet joos-deroeel/ wirng pzincipale 1320 bincien 3 ijn bet Aerts-3ifoom ban Mexico, oe 3iroommien ban Mechoacam, en Die be los Angeles, of Tlafcala, en die ban Guaxaca, en die ban Chiapa, en of Goubernementem bah.Panuco, en Yucatan, met die ban $\mathrm{Ta}_{2}$. barco, en booz obebuperchap of $t^{\prime}$ la: men-bandeling oe eplandem Philippinen, en o afuozoering be van De $z e t-b a e r t$ naer China, llet nieu Spaengien is ban de befte 30 zovin = cien ban oe nieutue Borelt / en bet aloer-bewoonlickte ! in goedo glye tempertljept / oberbloet en besucht= baerbepot ban (c) ectwe / g19ap3 / en Bee/ en andere meerber dingben Loo? bet 9 senfebelicke, leben / be= balluen olie/ on 1 Bijn/ en al boe wel batter Sout is in vele beclen uan haer / too is bet Silluer norb = tans gheneenber / bau twelcke bet bett beel rchoone gsijnen.

Het Alerts-25iroom ban Mexico balt tulteben de los Angelos, en Mechoacan, $t$ tal mogben bouven in lanckte Zupuen en Ropzoen yon= thicn: welcke lyet lject tangs of

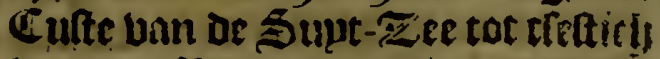
leguen s?2 ant- baert it / waer in bacr beflupten de psoviturien wan Mexico, en in Roozt-Boffai bill die Lateotlalpa, Meztitlan, Xilotepegue, en Panuco, 'twittef guelegene! endeint Teltế Matalzingo onttrnt Mexico en Cultepeque bet alderber =

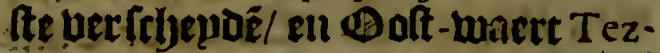
cuco diclit aen Mexico,en int SuptDeeften Chalco:; ontrent die ban Mexico, en Sunt-waert oner af Suichimilco het eertte/ cul daser naer Tlaluc, en tuftetyen Supoen en Bumt-mbeft Coyxca, en Ácápilcó bet verte gheleglyene Supt-waert. In allen dele en 3iin maer 4 . Dozs yen ban Caftilianen, al boe wel bats ter in Mexico siin beel Caftilianen woonende in be plaeté ban d'Indianen. $z$ p noemoen Mexico ban outs Tenoxtitlan, lept op negben= thien graben en ecm belft booctbel en bonoert ozie graden oer lengte!

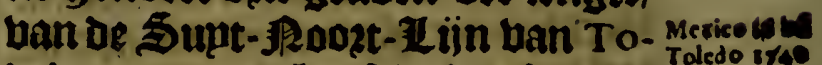
ledo, ban waer het fal af mefen met leguen. een rechte linie 1740 .leguen/ t'wals 3ijn acbt uren Sons/ bet lept mid = ben uan twee groote glgepzen die bet omcingbelen / oe cene is ban fout mater/ om oat fin gront is ban Salpeter/ of anbere is foet/ en niet Bis/Die afwatect in of Soute/ elck ban s. Ieguen bzeet/ en acje lanck:en bepoe ban bzie en dertich legum omringh. Zp conten in of Staot dooz ozic wegben ban een balf legue bzet/en baer ziin binnen 4. Dupfent 250zgbers Caftilianen, en dertich buplent thupren ban Indianen, of meer / in be bier oude quar= tieren / in oe welche de Stabt ghe= oeelt was / in be tij ot ban jet 1 ep= denoom / die bo beden noemen/ S.Ian, Santa Maria la redonda, Sine Paulo,en S.Sebaftiaen :en Tlateluloo is bet principael quartier / dat wo noemen Sant Iago. In Mexico re:

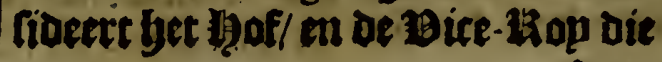




\section{Gun V V c ft-Indien.}

D.ret perfidect ell Deie Srboinen balit $\$$ of / oc officiers ball $C_{0}=$ ninct incoumen/ en oc giselt-luift: tit $t$ giet-h)uls is aldare en em ander van be 99 Mute/cil bet AertssilfehopuelickMetropolitacnfchap, tweidli beefe booz fuffraganen oe silfrijoppen bant Tlaicala, Guaxaca, Mechocan, nicu Galicien, Chiapa, lucaran, Guatemala, Verapaz, en of Philippinen : in 'twors Don Hernando Cories lie of groote leerck bace fondocerde $/$ en fteloc booz ban de Caloumen cenighe 3 selden ball వiechen bienen nocts beden fiet / en met groote brefte vozoert liin filuzijek oc 3 iactyop/s3zefioent eแ Gouberncur bã nieu Hilpanien, Don Sebartiaen Ramirez ban Fuen. lcal, wetende of extte 23 iffeljop ball Mexico, firater lan de Zumagarra ticligicus f francifinet/ feer ocuchoclick/ ett ban goce leben/ in = ghelosen ban Durango, in de beer lickljeut ban Vizcaya, wefende ooch

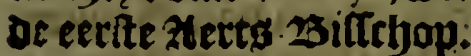

Fit Mexico siin cloofters ban Doninicanen//francifanen/ atu= guftioners/ Jefupten/Carmeliter/ oule 31. Bzoubue ter gratien / 23ar= revoeteta / en trinitatios/ thien Eloofters ban Ponneri/ een Col= legie ban jongbe Indianen, balf= Gachten / en nocb een anver ban de - Bofkeerde Sondaers / en ban die Die weder op ghenomen siin / en de Bniberfitept / al waer curieutelick en gibelecroefick geleten werden de wetenichappen: met andere $\mathbb{C}_{0}=$ legien / en biat - L) upfen / en Daer fullen ziin ontrent oe die oupfent Caftilianen dir Daer wooner in be bunzten bni toe Indianen, en BetStallinghen en oner fullen siin in De berbaeloe 10zopincien twoe bon= Dert 50. Dojpen uan Indianen, oe boildert en jo. boofden ban oe Seere/en in die/en Daer ontrent feg oupirent Bee-Stallingen/meer ban 500: buustent Indiarien cerifutati= $\mathrm{rm} / \mathrm{ck}$ meet dant boutoert on so.
Cloofters ban fitumitcanters/Da= minicaners / \&uguftinerg / een lee= ringen vari Stjecltelicken en prie= Aters / om bet Catbolicke bljeloof te anderwitifen acu be Indianen, jiinoer Comoer glietal / beljaluen De Baders of 3ctupten/ en Kte linirulen dic loon bebuen. Bork refioecre in Mexico be beplighe $\mathrm{In}_{\mathrm{n}}=$ quilitic/ ban oc welcke nen daer naer fal fpeecken.

Bp be Clute die aen oil aterts- Dabgigban 25irom grente aen oe Sunt-Zee/zee.

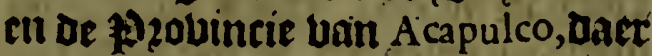
is or goede banen A capulco, op 17. graden boocljte / Ies leguen ban be Liebier de los Yopes, al waer bem boecbe bet xerts-3itoom met bet 23itoom van Tlafcala, en andé= re actse legues / meer meft-waerts is oe zevever van Citala, en andere bier die ban Milta : op oe 政oostCutte is die/ die balt in het Bous

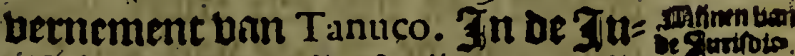

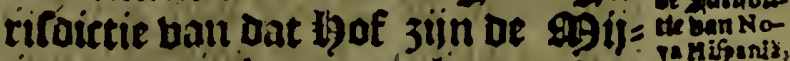
nen vall Pachuca, 14. Leguen ban Mexico, en die ban Tafco twee en twintich : Die van Yfmiquilpo die 3iin uan Xoot twee en twinticf: Die batt Ialpujava vier en twin. tict): vie ban Ténazcaltepeque acfi= thien / die van Cultepeque twee en twintith. Dic ban Zacualpa twinticly die bam z upango betrtich: die ban Guanaxato tfettich: Die ban Comania feben uan Guanaxato : Die van Achichica atbthien. Oie ban de stabe de los Angelos, en Die ban Gautla, of Zumatlan, en S.Louys de la $\mathrm{Paz}$, ent in allerr fullen meer vain 4. Dupfent Caftilianen zijin / bie met bernuft en arbept/bouben baec op die sginen die alle ban șilber 3 tin/ beflalbert ten / die too ghetent ia) ban loot is/ en bebben te be= cozglen dat be Indianen tiemende excmpel ban faet couder lecuen po= litiquelick: Etat be etertte dite met een wontoerliicke confte/' en wiifo fiepot inbzochteoe be onderwifin= gljen en bouluingben/ Die gljetweet is bet 


\section{ffol. 2 s. 25etchistuinghe}

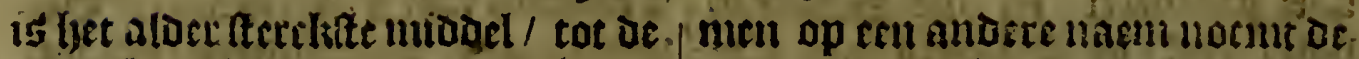

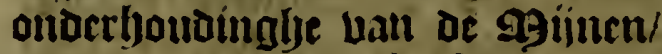
ben seballi- Wals de boozfepde thefident Don beft al oe Sebaftiaen Ronirez ban. Fucnlcàl,

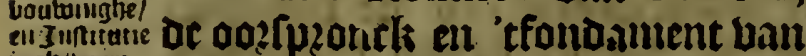
ingbevoert. be wrivaert wan defe kijelken.

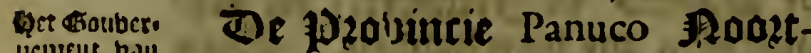
Panus. "wact bail Mexico, gouberneeroe Nunjo de Guzman, bout ; o.leguen in oc bacette 7 cn in be laugte / cn Dace naer luecde lyet gljeineospo= reet met de Jurifoictie. uan bet \$of van Mexico. Het I amt dat na nieu Spaengien fiet is bet bette $10=$ peruloedich in Tifftoelyt / en inet ccuich Bout. bet gljene dat nae Florida loopt is cllendich/wan water tot dat list comet tot fin grenfen/ heeft meet dan s. yondert leguen. Dat gouluetnetuent beeft d zic Bozs pen ban Caftilianen : Panuco op een weqnich meer dan bie en twintich graden / en met cen aniber naen gljenoemt de Stadt ban Sint Ste. ven del puerto, dien houdede Gon. falves ban Sandovial, bopz ozoze ban Marquis Don Hernando Corres, alg 3x. wareri oni teghen te Ataen Francifco de Garay, en te bebzedighen oc Indianen ban Chila, int Jlace 1,s 2a. en leut bif én treficty leguen van Mexico Roozt-Roojt-Doft-waett acht leguen ban be zec / dicht aen

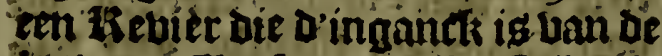
Gaben. Cis lyet grootite SchouteTrbap/ cen pzobifie booz be Bice rop ban nieul Hifpanien. De Stadit ban Saritiago de los Valles, biif en twintich leguen ban Panuco 10 altwact : De Stadt S. Louys han Tampico, acht leguen vain Panuco nae't 3oozt-Oofter dich bi de Zcel en a ljoe wel op oe cufte un oit Goulertacment beel Rebier jiin/ en ecuighe Recten/ fot be kit vier de las Palmas toel too zinife cluentwet niet belent in be Golfe ban nicu Hicpanien, dair allecnlick de Revier ban Panuco, en lijn - ho benl bie niet fyeel goest is. los Angeles, tuffenci ljet Dont ball Mexico, en ljet 23iroom: ban Guaxaca, is ban bjonocrt legucit cll meer in langte / van' or. S̈mitCulte tot de 2,oort-Culte / cul tulfeben die geculen van lyet aterts2BiDom / en dic uan Guaxaca tarlj= tentich / in oock loo vedl in lizerte op de Culte baul de noost- Frec/ cii nict neer bau arbetjien / of twin= tich op oe Supt-Zce. Tal yeeft in alles niet neer dan opte Dexpsu Caftilianen, twelck jijn de \$tilat of Dozp oe los Angeles, twee en twintich leguen bain Mexico Ooit. watrt/ een wepnicly bupgende nacx

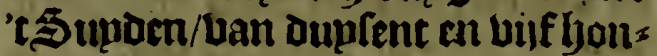
bert 2002 ghers in vier quarticrell. Daer ig bet grootlte Srljoutflyap/ al waer refideert de Cathedrael fuffragaen ban be slerts-23iatchop bail Mexico, met de Cloolters ban de Donuinicanen/ 5 rantifanter / ent Zuguiltivers/ en een ban Sonnew/ cn cen Collegie ban meer dan ;0o. Hinoeren bau d'Indianen, oul die tondertwijen met thien ounfent Befen te renten / twelek fondertoe de 25 iffchop Don Sebaftiaen Rami- Densifitap rez, die font om te fonderen offe

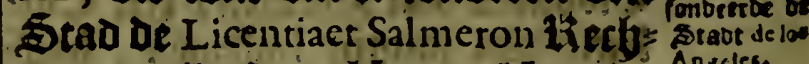
ter ban't 1 of /en al boe wel bu ghes accozbect hadoe dien te macthen in Ilafcala, liet fyet nae om de Indianen niet te difrommoderen/en yarer niet te raecken in facr erfgocdaceny oim dat bp becl lettede int generael op let welbaren ban d'Indianen, tII iit particulier op die ban Tlafeala, om dat zut wel berdient hadoé äm De Conincklicke croon.

9sen bandede cen Stadt op oc IL antuale unn Cholula, uan waet quanien 2 , Duplent ganuen ban Bozlontie./ en retteden Inet néder in cen ulaclete dienté noembe Cuerlaxcoapa bu cen Ballepe die 3 ñ noe $=$ imen Aclifeo, ap de cant ban een clepu keluier/ dic upt cé bict-23creb booat conit / al wate glyewoumen 


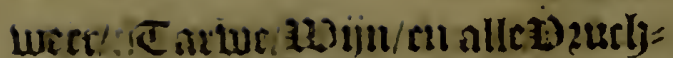

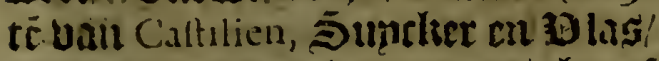
cu al Cumu-uzurlyten/om datt bet cé getcmpert zeait is/meet.warn als cont 1 - al l,oe wel de Cartue biat de llarfe landell acll de Stinat booger boo!t cant : ch cen Lespuiely verder Uimi Tlafiala , fpantern fontęuen

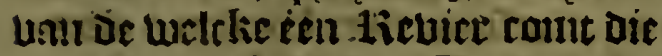
loopenide tuffriyen de Stradt ide los Angeles, th Cholula, gaet fwaetlicks:

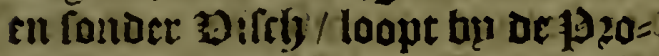
vinticul Uill Mecheocan, ml loopt uil de इult-

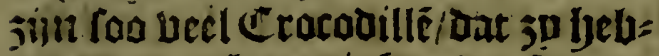
ben ontuolcht eeniglie plaetfen.

rtakats. Fn Tlafcala Rooktwaert van, los

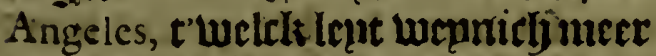
batr op 20 . gridoen yoocbre/al waer ue Cathedrale ltetclie wag/ tot dat bet int jace 1 ; 50 . Ueranderde in een Dazp. De $x$.23iffelyop was Broer Iohan Garces, en was in de Ballepe.

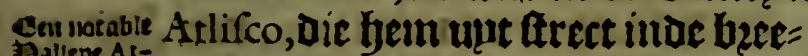
Gallepe At- te: bin naelt cen en een yalf legue/ al
buso. waer nimmermeer be Carwe be $=$ Utieft/en jp bergaderen Daer bouen Hancza, is wi 100000 . hanegen, en batet z̧iin mueet sozent ball 1000. Caftilianen die haer ber= ttacn op oelen bou/en 7 : Ieguen van De. 5 tadtooftwaerts/is oe Balepe Ec Dallepe ban Ocumba, en in de 13 zabincie ban Tepeaca, fonderdo de Marquys de Valle cen Dozp ban Caftilianen, die ghenoemt wert Segura, ct in De - palleze Balepe ban S.Paulus inogen welen

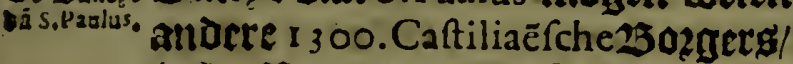

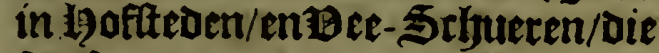
foo leer vermectderen / Datter een was die dooz twee Ouen quamnter te hebuen veertich bupfent.

De atabt De Btadt van Veracruz, lancr een wecly is 60.leguen ban Mexico, eit lamer een ander 6 s. [u is eê quar= tier ban een legue ban be $\mathbb{z} e e /$ beeft uteer dait 1200 . Caftiliaenfche $302=$ gers/Dacr í aesC oninex Schatea= unst / en th tups ban Contractien. De haben wete grienoeme S. Ian de Vlva,een maen dic glyegebenis ban deceapitęn lan de Gryalva, dic bem outdeclitede int fact $1 ; 18$, mit ir fit:

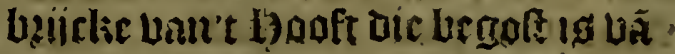
ac Dice-Lien DonA ntoniodeMens dofa:ia s. Ieguen dan de nimone dã de Heluiere de ld Voracrnz a tultelpen of

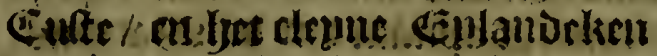
twele inde wijtte bu sang fal heblue ré Irgue/ onucingelt nce Requen/en:

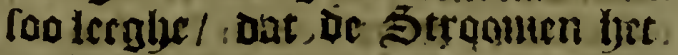
Declicil tegluen ouet ur Dentont Des. licturers ban. Sollan de Vlva, Agen

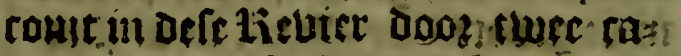
nalcn/vis neeft glyeuengalit wert ist die die de rupmefie is / gen! anantoere. noenet de caunel Gallega pic beel diepten herfe/ en duct ineeren $3 p$ des Scbepen aen een lianfonicer dan?

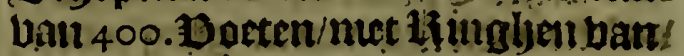

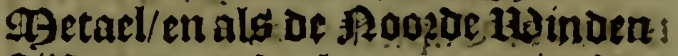
Atif wacpent foo leggen 3uniet feerreeclser. La y eracruz lept op twat's meer als 18 , maden/en altjier plach te gefrbieden of ontladinge van de: Dloten/en vertoefoen 4. of j.maen Den int ontlaften / am Dat bet Lex s.leguen ban S.Ian de Vl va, batrom Doen jul bet nu in lyet deel ban Buy-

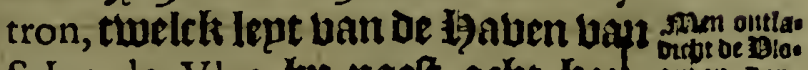
S. Ian de Vlva bu naetf acht bon= ten in Buy? dett padtei / en t'wert gljeepnoucht in een Sgaent en een balf / en men noent jet Buytron, ont een werber: gye die albiex ftont / ban een ghe= naemt Buytron, en fy grinck be bolc = ten een plaettel in defe plaets:

In dit 25itrom siin twee hout= Dert Dozpen van treffelicke Indinen, en bupfent Onderfacen/al hac wel batter siin twee fjonsert $5 \mathrm{~s}$. Dupfent er ributairite Indianen, in 36.Deplingent bande ondertwiifinge van be Gbeeltelicken/ en 30.Cloo" Iterg van oominicane/ f rancifta: uen/zlugultiters en op oeCulte die

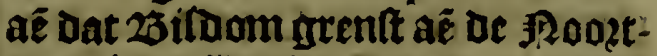
Zee/ is de thebier uan Alvarado, al waet bner boegen de laittpalen ban

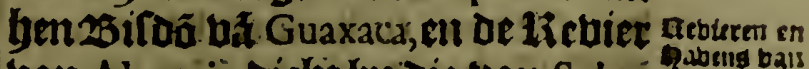

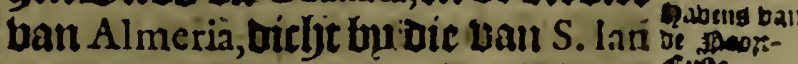
de Vlva, al waer wat bewolckt of Staot bam Medellin ban Andrieś 
ball Tapia, int $\mathbf{3}$ aer $1 ; 22$ alg be isarquis Don Hernando Cortes tont oe uoo? feude Andries ban $\mathrm{Ta}$ ? pia, en Gonzalo de Sandenal, om te bandelen met Chriftoval ban Tapia, dic marbt opnam om de $99 a r q u i s$ te ontblooten ban bet Gouberne $=$ ment ban nieu Hirpanien, en de Fator Salazar, en de Veedor Peralmindez, yebbent ontuolelst. Be Keluier ban Almeria, beeft fijn oozlpzonck in ae groote Cordillera, in de $1320=$ bincien ban be Totonaques, en Micantle, el typ gaet tulfeben de ghe= bertbeen om bem op de foozt- Tee te begbeben / en tegen ouer die ze= viex is bet Eplant de Sacrificios, fyet belcke oock fiin naen ghegheben is ban be Capitepn Ian de. Gryal va, ende be Ziebier Zempoala te. POoz= Den ban Veracruz, en wat boogber die ban S.Peeter, en S.Pau o, die int felfoe gljeberthte cprupt/en bie ban De Cazoner, en Tufpa, en Tamiagua, ontrent bet Boubernement ban Panuco.

\section{Wet X. Capittel.}

$V$ an de Bildosnmen van Guaxaca, Mechoacan, en Iucatan : en van de Provincie van Tabafco, welck oock is van de meeft verbselde Iurifdictic.

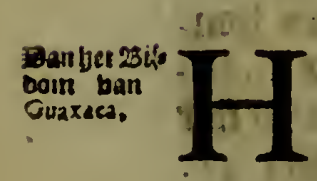

Et 2Bifom uan Guaxaca, bert alfoo ghenoemt / am De 1)2 obincie al waer bet in lept: en Antequera is de Stadt / al waet refideet bet Cathedral, in bet 20 troum be los Angeles, en $25 i f=$ Dommen ban bet 1 of ban Guate mala, het is hondert en twintich le= guen / ban D'eene Zee tot D'anber/ lanex of Gzenten uan het wifloom ban Tlafcala, en treftieb lanct die ban Chiapa, en bondert bzeet lantr be Culte van de Supt-zzee / en so. tanex fet $\$$ Roozben / waer in baec bedupten be getobincien ban Mi. ftera booch cu lecge: Ist hooghe is 40. A9ijlen uan Antequera int 30 Iten / en tjet lceglje is meer mae te Supt-Tere. De Stadt ban Antequera is ecrtt bebalclit ban Ian Nunjez Sedonjo, en Hernando de Badajoz, en daer naer quamen betoer die te bevoleken ljet eertte \$) of ban Me. xico, wefende dacr Ri erlyers allee= nich in de licentiaten Ian Ortez. ban Matuenzo, en Delgadillo, die

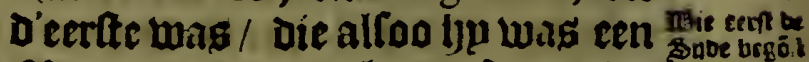
M9an uan Granada begonft te teelen of fijoe in Mexico; de Cathedrale thereke die leer goet is / begonla de prefident en Bouberneur de 23 if = f́thop Don sebaftiaen Ramirez, Iet $\mathrm{K}_{\mathrm{K} \text { amirez }}^{\mathrm{Do} \text { sece }}$ beginfel ban alle het profiit ban gounte ext: Defe kijeken: alle be pilaren ban cuarace. be liercke siin ban gharmer ban een Steen/ feer groot/ en leer dick/ en of \$tabt falmoghen bouloen 400.33orgherg/ Caftilianen.

Defe Ballepe ban Guaxaca, bain gbeefit ox waer de 9 sarquis del Valle, fün del arquis naem neeme / begint ban bet ghe = cisto berchte ban Cocola, op be Genfe ban Guaxolotitlan, daer wert ber= gaert beelSiide/Cartwe/sn פDap3/ en lyeeft be Zapoteecie tale: 'theeft gbethadt goede şijnen ban Sout. Decontrepe ban de Stadt Ante. quera (obelijck ghefept is ) noemen 3p Guaxaca, was bebolckt ban bet bolck ban Mexico, twelck was ban bet Garnifoen booz ozden ban be tweede Motezuma, ende beel Gar = níbenen die de Coningen ban Mexico bieloen dooz baet gantelye Tijck/ badoen int gemeen de Mexicaentche tale. De itebier ban die Stadt lcopt onder b'Alerde / rectjt ober Cimatlan, en comt weder upt twee 2 sillen ban fyet gyeluerchte be Coarlan, en noch twee bam.Guaxaca, en een balf grifl ban oe Stade: recbt ober cen gyebercht bie int. PRoozden is / maeclat gu een punte ban Setrezuela, en loopt ober cen Ztrm ban be Ballepe/hecl ularls tot acht leguen / twelfk is be boozber= 


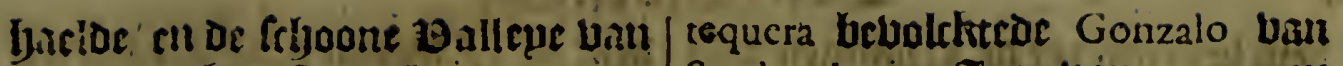

Guaxaca; glierult/cn gtjetmupert/cu ban leer gibelonde luclit / al wact oueruloedicljlick baer vooztglyeuen alderliande Coozter ban dinglen' cu bufonacrlicli oe Bzucbten vau Cattilien die feer frivacckelicli sijn. Eyecft de bzobincie wan Tutepe, que int \$unt- wedten/melcke hecft vecl Doppar lincr de zee. Cuat / en is imeer dan tfeltich leguen , en oie uali de kevier ban. Alvarado beeft lyet tudeben Roozden en Roozt. - oltew/en Die van oe Zapotecus, int Roort-Dolten bain Antequera, en Guazacoalco op deSzenlen ban Tabufco, gantict een rou lande / en cuenwel rijek uan Gotit-פgijnen? Dat nocfjtans wepunich uptglyetroc: ken wert oni de roubept / Daer jijil 4. Dozpen van Caltiliane. (Be book: teupe Stadt ban Antequera is 8.0. leguen uan Mexico ten Sitpt-Bo: Aten op de Conintelicke wech ban Chiapa, en Guatemala", D'eertete die Dasc inglyetrocken is om te bebikedi=

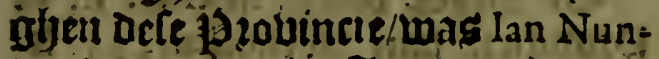
jez de Mercado uit Jaer i s 2 i: 800 ? commidtic ban Don Hernando Cor: tes, eride van daér werde oozlocht volsk ghefondan om te oienen oe Comnck Quan:imoc, tot bercher= minglie van Mexico, als Don Hernando Corces dat onderbzorbt.

sint Althor. De Créozier Alonzo de Eftrada sus selosza- Gebolefitede dien als bp in Mexico
parcess gouberneerde de Stadt ban S. Alifonfo de los Zapotecas, twintich le. guen ban A nequera, Foott. Bofte waets's $\mathfrak{p}$ ghencrender met Bout/ Cattom เH99apz/t'beeft meer Dan Dictidy Doufent Cributarifen / Indianen, het lept tuffeben de alder= hoochlte $\$$ crghen. Santiago de Nexapa twintich leguen ban Antequera Ooft waert / op de weech ban sint raceb Chiapa, enl Guatemala. De Stabt sen Nexara ban Efpiritu Santo in de pozaluincie ban Guazacoalco, op de Culte ban

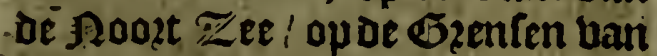
Tabarco tnegenticly leguen van Añ. Sandoral, int J Jer yain x $522.3 \mathrm{~K}$ beft bu cans $j$. Dozpen bam lndianen ctlpt aen de Dever vam be Lievier/akti ljet dect ban Ciapa: acfé Revier begint in bet obeberchte de los Mixes, sin Chontales, ontrent de Tecoancepoque, en met de Deecken van de 13 zo buncien die on ort oe $3 \mathrm{~W}=$ riforctie beboozerivan chiapa, choe de Chontales, loopt bu in oe RooztZeel dace comen in Sebepen uan joutort Daten.

In oat blyebele siroom is nict een Zheviere die glject Goue bu en bengt/en be Indiainen leben conder nootozuft too $3 \$$ begheeren to ars bepderi om dat haer niet en ont bzetkt om t'eeten/ en te clecden/ett 3p hebben Cacao, een. Bzucht als De atmandeflen / vie baer vient tot ghelt/en 3 maecken daer Boiju ald en jpeter die ghetzaden / en fous Dent boozecn groot ondetbout: Be Landen zijn gbetont/ en luffich: men teeloe te voren sujoe boos liet betnuft ban be Caftilianen met oe Ingbebozenen bant zandt/ baet baer oe Indianen mede befolpen om papiecte marcken/wanoe tweede feljozfe/en de Caftilianen yadoen geplant loo veel ban Cátilien, oat= ter ontelfaer beel bergadert wert/

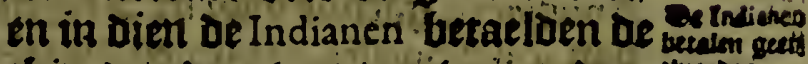
thienden daer ban/ en bain andere thentento Dingben / men coudet s.23iloom. men af connen maecke / om dac die niet betalen/ Dan de Cartilianen.

Dat ziloom beeft ozie bonbert tn so. Dozpen ban de pzincipaclife Indianen, en in die / en in dicie bon: oett Zogementen / neer dan bon= Dert so. Duplent cribuptatile In. dianen, en lyonbert twintich $\mathbb{C}$ loo. tterg ban Dominicanen / en miect tan dic lesringben in oe Brieelte: lickibepot. Be Cuite uan vit $23 i l=$ Dom / bp de Roozt- Fee / begine in dé Zlebier Uan Alvarado; die upt de dsergen de los Zapotecas Ip?upt/ en ban onocr. andere bele gaet by

$$
\text { (a) bent }
$$




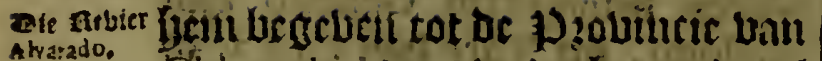
Chinantla; in wederlietrende al rruurende tet contrarie of acbet(b)= teil bain water lju getiogen is Cloopt/ of Lalt in of noopt: z et / turteljen of kebiet ban Guazcoalco, th' S. Ian de Vlva: en barr is oock oe lievier bienicn neetite ban Angualulco,

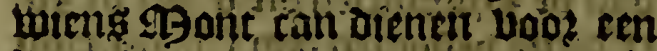
Gabcil an cen thedeuloc thot te? cen punte ban't zant/ore upt teet Wain bet gljebertbe vam S.Martin, bekent gbewozaen bodz Schepen bie Uerloozen zijn op een uerlpzen= Dinge uan blinde Clippen/en tres= ben die latigs oe Crifte legghen/ bloat's tegens oele ober op ae Cult ban ae supt-zee / heeft dit 23 if = oom oe baben ban Guatulco, op is. th een half gtade die reoot en yoet It / en baet bect ghefandelt wert. Dele 13 zobincic ba Guaulco bzocht texomirael Pcdro de Alvetado on = ber be abebotfaentyepot in tiet bu ís be lo aué lat Técoantepeque, Die oock reoclick is / en tyet Bozp is givod/ kn baer is groote Bifthe rijeban otrecten / en andete wit= Iehen bie 3 tutenthen te Guaxaca, en op oe Coninekliclie wects Cuyz: tatà is een gijne ban e tiftal beril. Tecoantepeque is 45. Itguen ban Antequera, gaende doog de 25 etgen Jan losChontales, Nixapo, en Mexalpeque, tot al warer dit 23 trom comit en stheft beet Dozpen onder fem aen be Zee-Cuft.

ant sifoam bart con

i) tet 35 itwam vam -Mechoacañ, thelck is eet gozobincie tultrben bet zlertó-bitoom unan Mexico, en betinien Galicien; bout in be byeette tainga de cult van de Sunt. Z E I bu nact tachetenticts leguen/ chitreftich linnen-flanbtal met be operts I antjalen inde Roozpet.

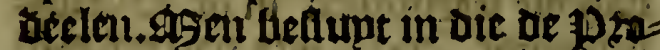
bintien batt $Z$ acatula, en batt $C o$. finia alle bepoc op de etalte var or Shlint-Zet. Déstad bã MecheoEascualo, can, oft Pazcuaro, gheleduretr op een Eepnirle mest unn neamthien ara

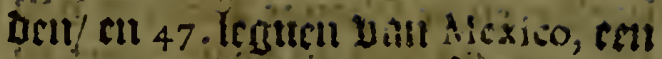

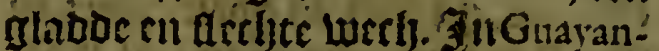
gero glbenoemt Valladolid refioeret valladolid. De Catljedsiel fuffratarm ban Me.

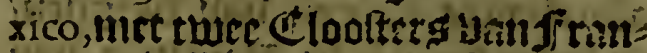
ctranen/ th zudultiners. Mechon. can fondectue - Maefle de Campo: Chriftoval de Ólid : cerft tat be Ca= theozacl tot Z inzonza, tot ben 3 ate is 44 . Dat 34 vertrock tót Pafciaro. De 23itathop Don Bafco de Quiroga hêft baer etn Cloofter ban fran: cifcaners/ en ten anoer van atugi= itiners/ en ig o. Ieguen una Mecho. can naer toofent.

De As ijnci ban Guanaxuato leg = Ouanz:anto. ghen acbtentwintich Is guen ban Mechoacan, Poozt-waetts nae Ics Zacatecas, in twelche mogljen beren bij naet fes hondert Caftilianen, in twee lienten ditfe houben met baet Bpper-Schout. Cen ticaelísem. Ift-plaetfe / ent maniex ban leben binit ofe sthene dic in be spijnen ar= bépon.

We Stade bat S. Míchiel in be s, Michla. 19zobincie ban Mechoasan, 35. Le = gueti bain Pazcuaro, Roozt-Boftwaetts/ gheletben in cen rou lant: Ezr de Staot de la Concecion de Sa- xaconnectioe laya; bie gbebout íg ban Don Martin Enriquez Ddoz laft bá oe Bice-lhop/ int zlaer I's 70 . om be wech te bets leeckeren ban de Chichimecas. De

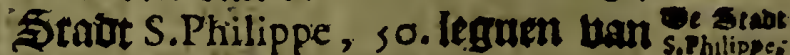
Mechoacan Roodt-wnertg/ en 62 . Teguen ban Mexico, 3oot-Boltwiretts / met ert cloufter ban alu= griftiner 9 sanicken / in cen on= bartjetuer en cout Lanot/gbefon= Deert am re berfeectieren den weeb ban te Zacatecas.

De pzotincte/ en de Stadt uan Zacatula, op be Cufte ban of SuptZefe / fept op meer dan achthien gradon/ ent 40. leguen ban Mechoàcan, ten Sunt-1ureften / ontrent de Zee / int 3aet I s 23 . tojefondert bant lan Rodriges van Villa fuerre cú Simon vari Cuenca, dele Stabt is thenhentich legexn. van Mexico. 


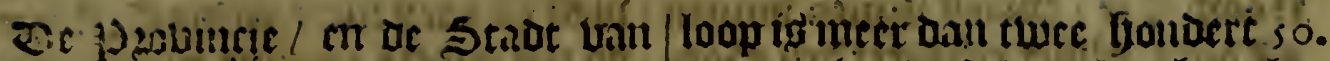

culima. Colima, op ect wewnicly meer als etljtyien grindeu op de Culte van

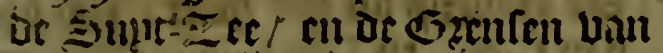

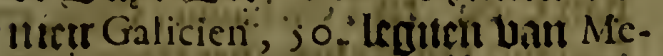
choacan Suut-10elt-whicrtg / met ren groot \$rboutrejspin can bect

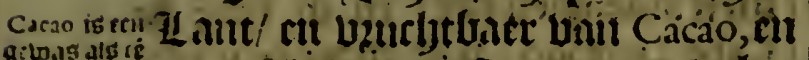

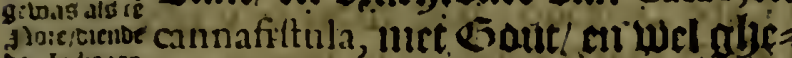
o Indanen ftelt volch. Gonzalo de Sandoval

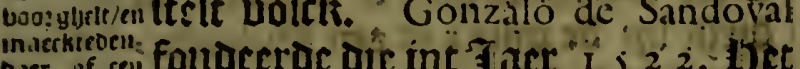

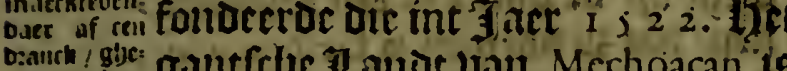

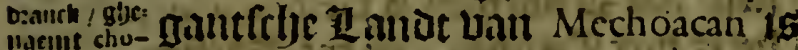
colate. bet aloer-Uzucljtbactife bati Cat - we / chi usanj/cr uati alle foózten ' Uan volititen ban Caftilien, en bau

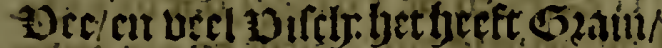
Cochrnilic Cattoen : en lset bolck is bernititidy / en otimienclit cot werclien.

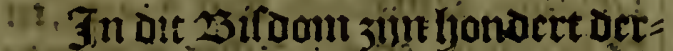

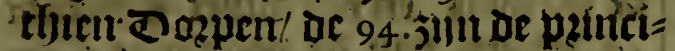
prielte/ met Scholen! cti ljet loopt tor be Culte bi de poost-aee nict aien oc Sint-lijoe lieffe ljet beal

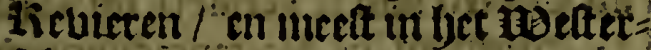

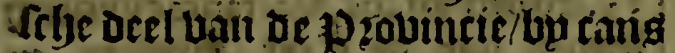
ôt de Atectk uan nileú Galicien. is ti t)auct bait Navidad, op nenten $=$ thin graử loochte Is goet en wél betaren 7 tin waer ofjedacti bert De matuigatie na de Philippinen toe/ èt bet natert Bolten Lontent be

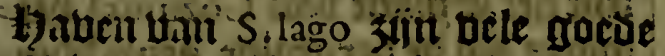
Egijnen ban Coper in oit teto 4 / birf de welcke oe Indianen maecken Woutucticte Daten $/$ om dat bet facbe is 1 m anber iater oat foo bert is ont $j u$ baer mede get zant beac=

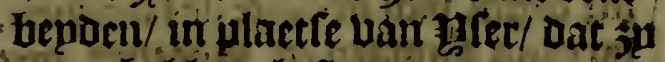
nonit bebien betheni te ooeri / tot Dat oc Cartillanen gact bet leetón. Oe pzouincie l sti tosouberne

Yucatan, Itent bar Yuçaran, twelek die viaem irectj/als Capitenti Francilco Hertnandez het ohtoertited en ontéti. - 20 mure de tos Remedios, als lañ de - Sryalva ontúectétét cozumel, waja ecrif ngetjotioen boos een Ejlant/ oun ont het bu naet met be $\approx$ ece is ointringbelt / coo bat ment een Peninful motft naeniert / en d'ont; legueti/het beeft in of hanchte gljes

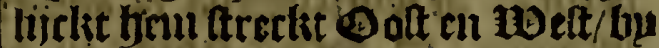

mact bonocrt leguent/ en oock foo

beel Aooat en Stunoen/ban oe Cu=

Ate dic nac' $\$$ oosoen fet / tot dical

Decle toe/ oile fuet ocel oat bem great

ittet oe 13 zobincien ban Guatcmala,

ban whiet Gee bein tecet oberower

Lu riaet $2 ;$. teguen/ riin Iocht is

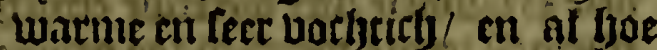
wa bet fech licliec noch loopende 10ater en feefi / gatitith Doozgaç bet IDater foo [eer joocl belende

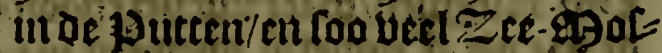
felentimiden acii of Stceneti die oace sim/ ell of culte foo lépgel theft wheglueven occafie om te ber= intococt datter beeft geweelt reni= iber tiit cen $\mathbb{Z}$ ee / tis lect beloten inet gbeberelje / 'tgeeft ooclt gluen Cosen/ of zaet bin Caftilien, t'bett oock atien ootit /noch eenich au= oer gSetacl ban ecnige lodzte/ water upe bekent wert of berfuftingéban die die repoen oat jo gtebonton

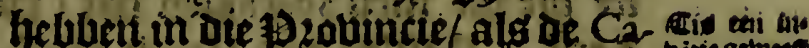
ftilianen baer in quament cruyfen bande gene ban Latoeti / ljet weletie men nopt oie froc bar

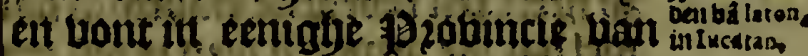
Indien, flet ig reer oberbloedictian Tathé Gitonoectic ban bercheng/

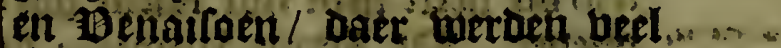
3onoecen gljetedt/men beromoert

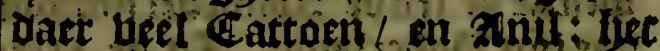
volets bementichtuldicht in lect lanek bact bag een gis an ote the comien was tot bie fjonbert Tae ren 73 fobocn aloerbanbe lootct ban Hof uan Caltilien, eti doche 13ectorit.

In dit gouberuement fonber dat ban Tabafco, théelelf met bit glyes boetif is / jiin 4. Dospen ban Ca-

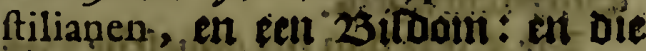
Dozpen sijn fondatien ban oen ear: Aer Soubetieut of atomitact Don Francifca de Monicjo. De Stadt

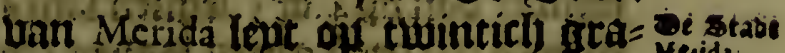

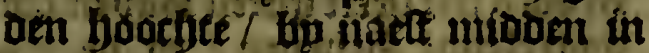

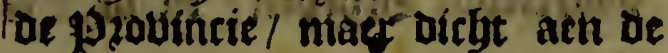
Rooit:- 


\section{Fol.32.}

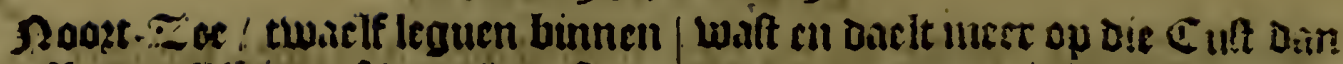
'\$X ants. Allyier refidect ben Gou= Uerncur/ de Officierg Uล̃ 'CC oniner incomen / en Srlut. Ltilts/ ell De Catbrozacl Juffrignen van Mexico, met een Cloofter ball finuteif= caners: 3 un nembent la Merida, oili begroote en olloe boubingben/Die thecft/ celijels Merida Lan Caftilien, ell t'ig te verivonberen bat $3 \mu$ in Defe provincie viet wetende eenich asctael/ conné werclien loo groote Stectien / in be weicke ju bonden gegrnueete naeckte. 95enichen met Boz-13inglien/waer upt bet thoon= of Dac bet Cempelen gheweet wa= ret//en Dat bet Lant gbeweet bect un groote feerlickhyut. De Stat

vasudid. ban Yalladolid, 31 . Ieguen ban Me. rida, up cangten Supt-Bofteni met en Cloofter wan francifea = ners heel coltelick / $\mathrm{cn}$ beeft in fijn 3ritifoictie is. Duplent ceributa $=$ rifen Indianen. De Stade en l)a

ompcter beri ban S.Francilco de Campeche, lept op twintich maden op of $\mathcal{C l}_{u}=$ te die na nieu Spaengien fiet / bp naett s0.95ijlen ban Merida maert / bupgbende nae't Supden/ 3o heeft een redelicke baben al boe wel dat bie wepnich diep is om een tupe te fiin. Don Francifco de Mon. tcjo die beeft hem fijn naem gbe samanow oujetien. De Stade Salamane de tociclae de felfoe zubmirat noembe Nactint Badeclanot. In of $130=$ - Uiticien uan Bacalar, en Chetemal, 7o.teguen lan Merida siupoen m - Boozoen / Diebt In't Borten / en toebhandere 70 ban Valladolid, on = trent de Culte ban de golfe ban de Honduras. Baer sijn in Defe $320=$ viurie thien clóplers ban fran= ciframer 23 oees $l$ en 5 cholen ban Gticeftelickljejt:

De culte van bie gantebe $1920=$ vintie is foo lainge/ oat in wepnicl] phetten men zunckeren macly op minder dan ov 4 of s.leguen lants/ en oock ten lieeft sbeen is aben dau booz clepne sebepen 7 , on oe Zee op ecen andere ectiobe placte bia de p) zovincien ban nicu Hifpanicn. De bavens die bet berft / zim dic ar paven vall Cicla, ell Telichaque, Cical, en ous moubce Cauquil, erth Hevier inet twee mon: Den/Campeche op ure Culte die nace ticu Spaengien fict: $\mathfrak{c n}$ in bast the Cabo Delgado, uan waner or Cuife Ooft-waerts begint te lieren/ cil Dicbt up of punte is een Gulanor: lketi/ oat ju noemen la Defconocida, befet met Clippen / en int werten ban dat bp naelt tbien, macbt is:guen / 15 een nnoer bat 5 n nomlen 1, Zarza,en De Triangel, Dat osie en laildeliens ziin : Dictit bp cen andorr eplandocken/onicingelt met Clip: pen/nocb featjien leguen uan Cabo Delgado, Eplanden ban Arenas, mi De Bermeja, en een ander Eplandt 30. Ieguen uan de voorfepde Caep Foort-waerta/ en be Negaillos Dzie Eplandekens met Clippen befet/ int Ooften ban la Bermeja, bp narte 35. Ieguten/en los Alacranes twinticb leguen ban of Cout Supden an Poozoen/met Merida, en Cabo van Cotoche, be Beftelicklte punte vin Yucatan : van loaer loopen 4. landekeng die $3 p$ noemen de Muge. res, Dictbt bp of Culte/ op oe weleke tept bet Eplant van Cozumel, ver = gin coaume.

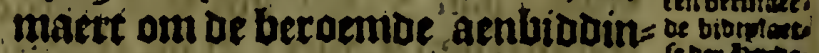
gbe ban eeri 2 seelt dat baet was/ al nath waer alle be lupoen ban bet onilig = gbende Zani in pelgrimagie qua= men; bet lept 4. leguen in be zetel int begin ban de golfe ile Honduras, bo cand Supoen en 2 Roozoen met Valladolid, en bet die golfe in fiin 3 . andere Ceplandekens met ondieptē bo canis op de eteeck ban het gsep? van Bacalat, thele is binnen ants derticly leguen ban bet $93 \mathrm{cpg}$ bai - Cheremal, opjoe Culte die befrbildert is met Éplandz/toc Salamanc. waer van de cerlte Pantoja, is dicht bp esll andicpte die sp notmen $Q$ ii. tafuenjo, en o ander Zaratä, en d'anc Der Lamanay, en oe laetfe Ylbob. $-72000$

areporar 



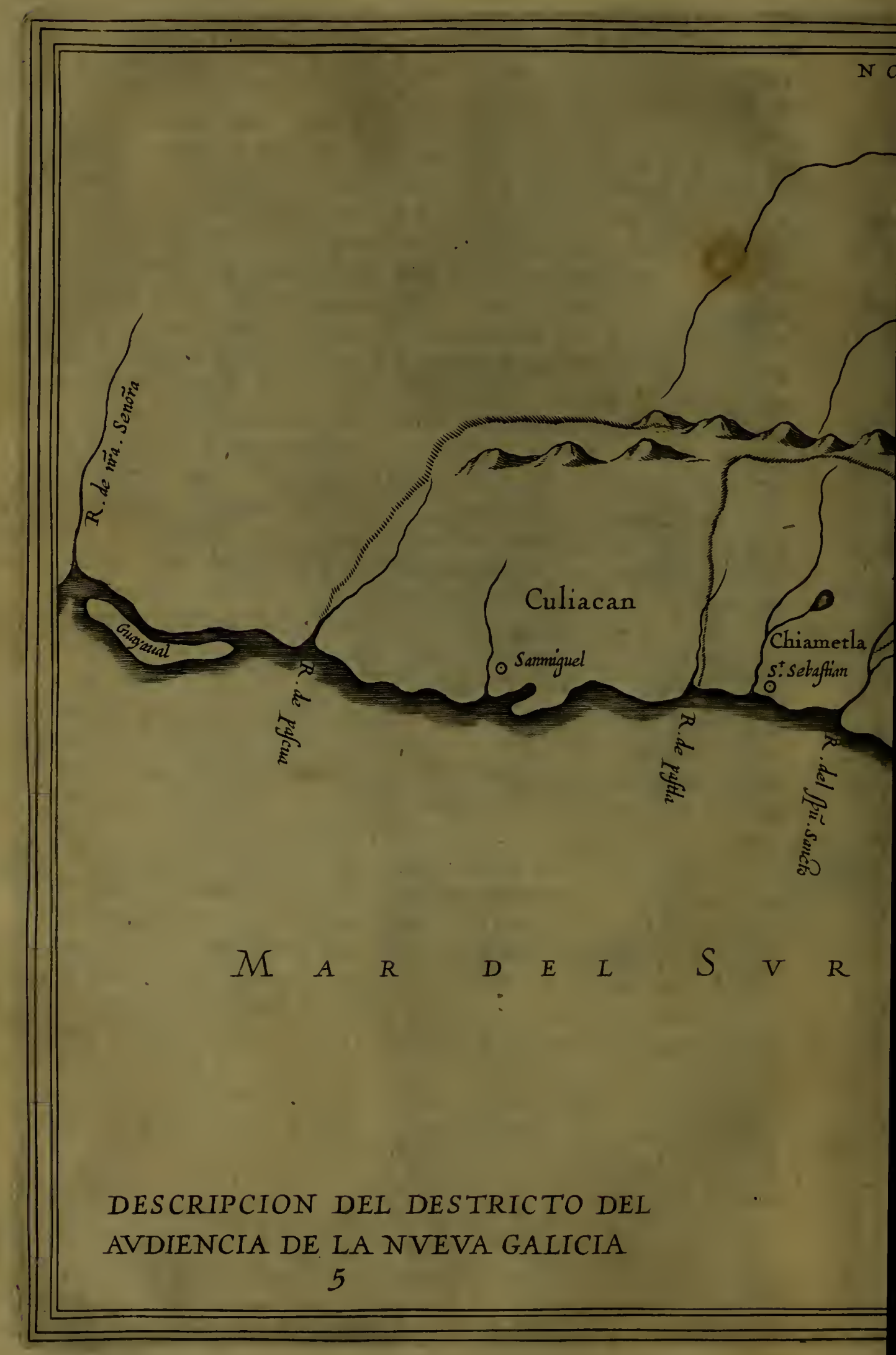



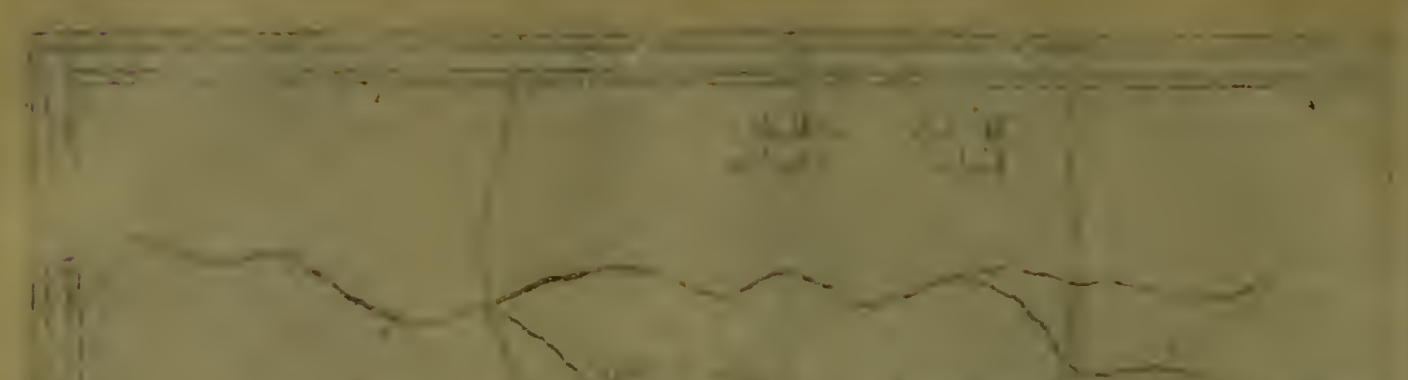

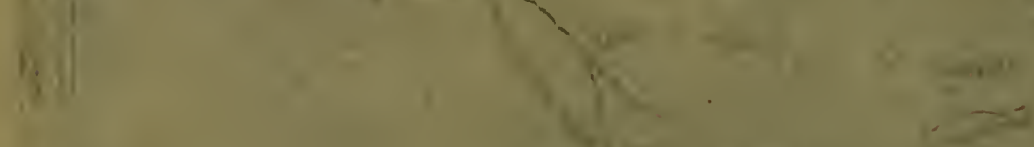

$-\quad 14$

is

18,

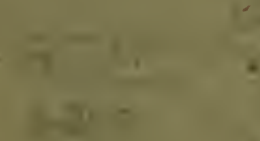
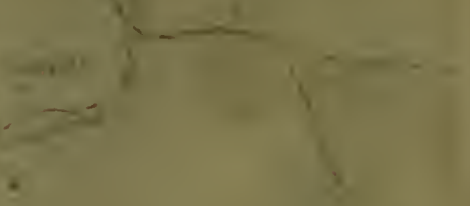

in

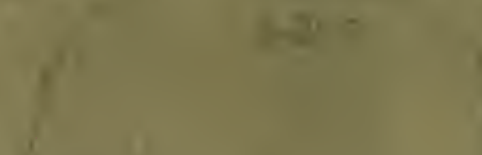

(2)

a.

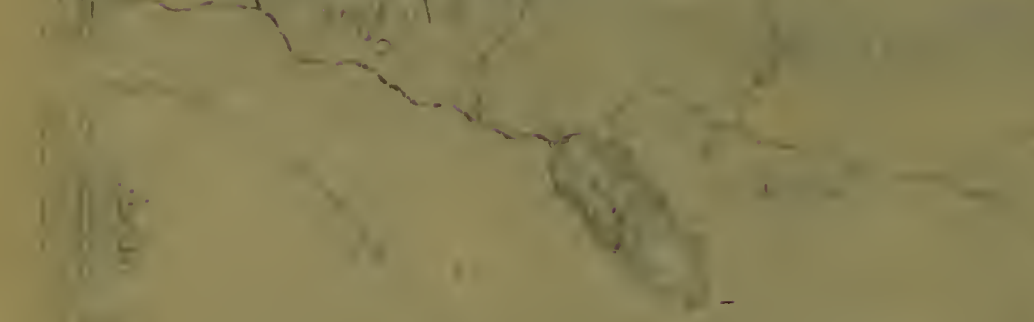

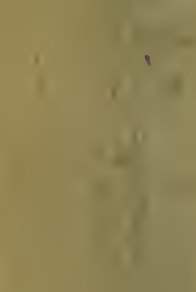

$\left\{\begin{array}{l}x=1 \\ -1\end{array}\right.$

I

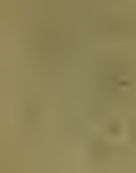
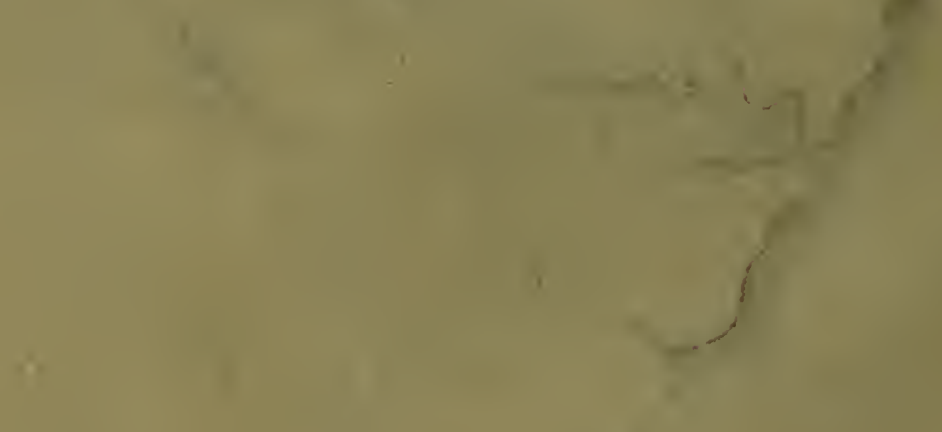

i.
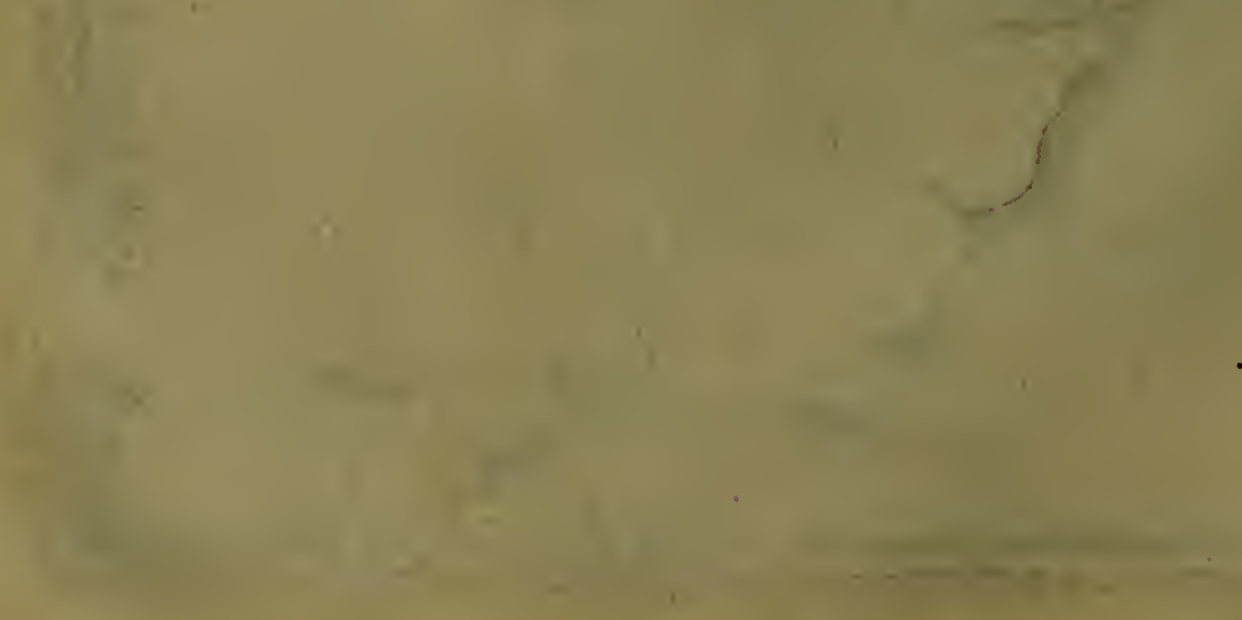


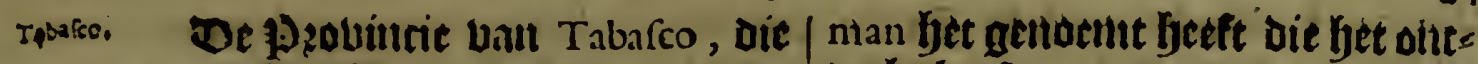
onder bet Goubet

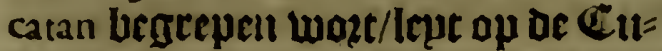
At uill of Noodt-Tee / in ofe Golfe batt uiet Spacngien, Ptretkt ljent in

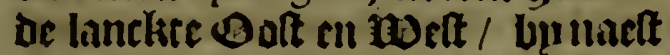
40. Ieguen van de 5 enfen ban $\mathrm{Y} u$ cutan, tot die Uait Gozzacoalco, utet yet welclie lyem voecht int Ooften/ en oock loo veel $\approx$ inuoen en Rooz=

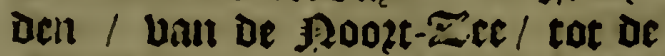
Gzenten uan Chiapa : let gantlelye laut is bol פ̧cpecn/ influmumê ban de:Zee/en beocclien/en ooc baertme Daret ouer unet 2 arquê/en Canoen; bet is feer bocbtich / en Dzoocly / en baceom leer veluchtbaer ban voetfel Looz Bee ball Biten of litoepen/ en bail $29 a y$ / en cacao, bet welck bet meelt pzofijt is uan be pzovinteie' iil bet welcles alleen cen Bozp is/ thalck is Tabafco, anders genaemt be Stadt uan onle Ziebe 1 zoutwe de la Vitoria; alfoo gljelloemt om de oberwinningbe die aloaer ljadoe van be Inoianen Don Hernando Cortes, als bp giuck une nieu Hifpa: nien, ilt F aer 1 ; 19. . Het Cribust Dat of Indianen betaclen in Dele 19zonincie/ siin twee Duplent xiquipilen ban Cacao, en elcke xiquipil is acht ouplent zAmanvelen / en cen laft matclit dzie xiquipilen, of ter-

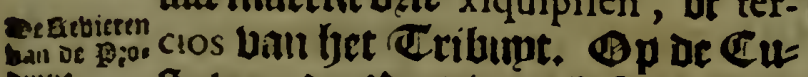

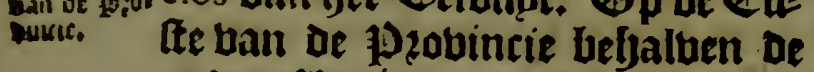
andere Hievieren/en \&Bepzen/ bant bp of $99 \mathrm{cp}$ uan Xicalango, of $\mathbb{C}_{0}=$ nincklicke baben / groot en buijt/ met twer Eplandekens inde mont/ oe Capiteun Louys Marin, Lag Die vie Dooz lât bã Don Hernando Corres, Defe probincie in bzede bzotjut.

()et X I. Capittel.

$V$ anbet RefJors behoorende onder ber gouvernemens vanGesadalajara.

F ment ban nieu Galicien, of baı Xalifco, gbjelijelt Nı ınjo de Guz. Deckt beeft/ en Don Hernando Cortes die bet dede te bozen in fijn nat̃: als Iyu Cont oe Capitepn Gonzalo de Sandoval, oul te beuzedighen los Colimas, ftoot met fijn Gzenlet aen bet Goubernement van nien Hifpanien, outrent de bauen bau Navidad, en of פgruz uan Chiapala, gaen = De \$Roozt-ooft- waertg'al waer bet int Roozoen/ en eenDeels int toe ften / Daert nocly ntet leclis ont= Deckt : beeft bet fijn ILandtpalen open / watter neer is int Bortten/ is gantich zee: bet glyene dat be = volckt is aen d'ecn fijoe of D'ander? fal comen op bondert Isguen/ waet ill begrepen wozaen oe provinien uan Guadalajara, Xalifco, los Zacatecas, Chiametla, Culiacan, Iyet nieu Bifcayen, en Cinaloa, $\mathfrak{e n}$ als ghe = bupertbap / De ontoeckingbe ban de 132oluincien bail Cibola, en ban Quibira:

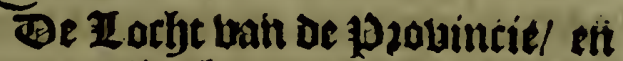
uant oniligglyende z Lant Guadala-Guadajajei jara is goet / en 'bet zertrijck is bezchtbaer uan carwe / en van פgany / en batt andere Saden ban Caftilien, en betl grincurs ban Silber / en Dozpen jijnder uain de Caftilianen. Be Stadt is lyet 190 oft ban dat zijels die bebolclat is ban Nunjo de Guzman, iltt Taer i 33 . en noemoe dat wae de naem bait fiin Baberlant/ Iept in bonbert ent res graben en een balf lengte ban de Supt en Poott-ilin ban Toledo, 1780 .leguen repifeng/ect tech= te wech / en op twintich graden en een derde-jart ljoochte / 87. Ieguen ban Mexico, tuautben 't 't Beften / meeft nelt-waert. 24 = Daer refibeert bet hof/ De (bficited uan Coninct income/ en be scbatkift/ tu be Cathedtal fuffragaen ban Mexico, ban Den Jare 1 ; 70 . Dat ecrte gheboden was te fonveeren te Compoftella : bet beeft een Cloo= Iter ban firancifraners/ en ten ans: oet ban ztugultimets / Nunjo de 
Guzman fondecrde oorlit de Stadt

El Épiritu del Efpiritu Santo ill Tepique, ell de Santa Matia, Stadt Santa Maria Uan De grseluken :

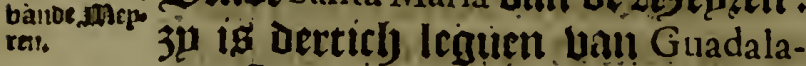
jara Supt-Laet / met eell OpperScljout/cu gebont om te verfeecke= miebrechi- ren tegljens de Indianen Chichimechimecé $3 y^{\prime \prime}$ cas, Die in de janden jiin tuffeben 't Moozaen en 'to often / een Bar = barifrly volels / die glyerpzent leben op't belt / Conaer eeniglye men= feljchickljent/en politie/in De folen/ en in de 23 afteljen als wilde $23 \mathrm{ee}=$ ften / onderfjoutoende haer met de

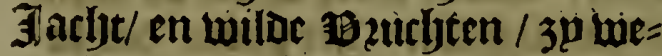
ten ban gheen tiiickdom/noch wel= lult/ $3 p$ gaen naetlt/en eeniglye be= Deckt nuet oellen ban gbedierten/ baer Wapenen ziin 23ogen en $13 i i=$

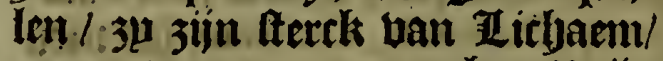
groote viaten./ $3 \mu$ matefsen zBijn

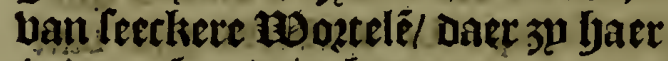
in dzoncken dzincken.

xalifco, De 10zounincie uan Xalifco is Uucbtbaerder van 99 ays dan van Scljapen / daer zijn geel peerden: theft imaec een 5 tadt Compoftela ontrent de, zee $/$ deie en Dertich le = guen ban Guadalajara Heftwaerts: al waer eerit wag bet Bouverte= ment / en bebolen te fondeeren de Gathedrale fiertiel tot bet Jaer te= fícl : wanneer ljet obergljegaen ig tọt Guadalajara om dieper te weren in bet reffozt: daer is en G loofter van 20 20erg ban S.Francifci (B)202e/. 'tip oucls bebolclst gheluect van Nunjo de Guzman int Taer $r$, 3 r. die twee jaren ginck dop? die $\mathfrak{L}_{\text {an }}=$ Den/dienten noễac bet grooteSpaen gien, Dop ialourffept vả Don Her nando Cortes; fonder dat inde gant: felje tije men uiet uat bem bernam iil Mexico. De Stadt de la Purification, ten Sumt-noeften ban Guadalajara 30 . Ieguen daer of / ontent de HaUen ban Navidad, in de Gokn= [en Lan die Jurildictic ban dat bof/ cu uan bet uan Mexico, lept in een beet iLand / en nwack / en tot lyet

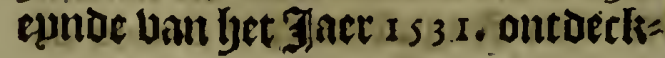

tede Nunjo de Gizman i jos leguen IL ants lancx oe Cufte bau Xalifco, dic gljelegljen is op wat meer Dan twee en tuinticl graden.

In De ADzounincit de los Zacatecas, Los Zacese-

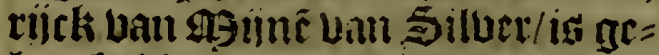
bee bebluende ban Witer/ / erwe/

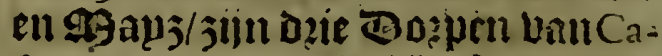
ftilianen, $\mathfrak{e n}$ 4.treffelicke fuplact

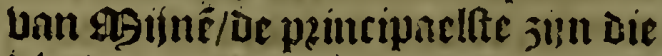
dienien noemit de los Zacatecas, 40. leguen uau Guadalajara novgut wacrt\$/ en 80: ban Mexico, ถ! Wart Desdinaris fullen ziin meer ona ; 00 . Caftilianen, s00. Slautu/ oulfrut

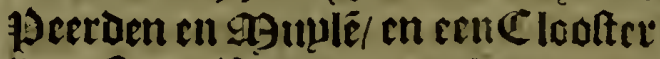
ban ffranciftaners / cu aldaer refi= Deert altossen uan oes Conume Bfficieren van Guadalajara. Boclt

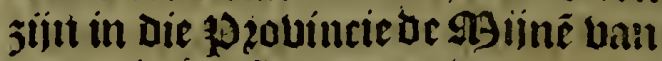
Aviño in de Contrepen tuall los $\mathrm{Za}$ catecas, en đie uã S. Martin 27. leguen uan los Zacatecas, PRoprt-Ooltwaerts/ in twelcke plegen te wefat bu naett 400 . Caftilianen, en Xerez de la Frontera, 30. leguen ban Guadalajara \$Roozt- waerts / en ro. Uail be $\mathfrak{R}$ iiné uan Zacatecas, ilt de wecly bau dien/3ijnder andere plaetfen de laegers leggen/Daer men geen glje= waclj af naeckt/om ont 30 [oo aen = unerckens baerdieljniet en sijn. (De Stadt Erena, ch de Ngijñ genaent El Ereno: del Sombrerete, 2 s. leguen uan $\mathrm{Za}$ -

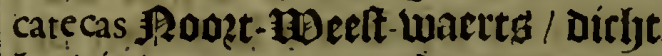
Up die Uan S.Martin \$2002t-Laerts en andere dic in die Zurifoictie šiju. Te Stad uall Nombre de Dios, 68 . Nombre di Ieguen uan or Stadt uan Guadalajara, en 10 . ban degsiinç wan S.Martin, , \$oozt-waerts/ntet eencloofter uau ffrautcifaners/ig oberblocdicly

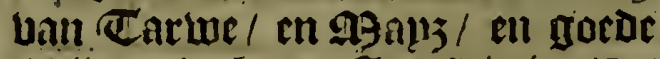
Bginen in baere Juritoictie. De Stadt Durango, iii de Intildictic ban be 9 iinen Uan S. Martin, ch de Daleue uan S.Salvador, 8. Ieguen uñ Nombie deDios, is cen grfont lant/

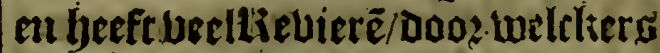
oluerlpaelinglye vergatoeren ju Lued

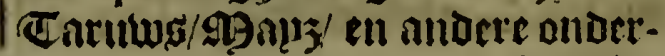




\section{ualt VVeft-Indien:}

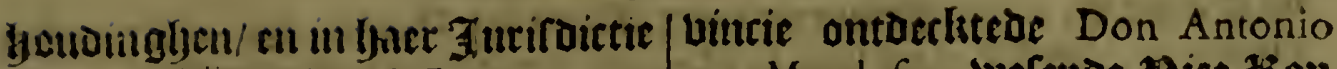
jill de biinen unil S.Lucas, enes van Mendofa, werende bice-tion

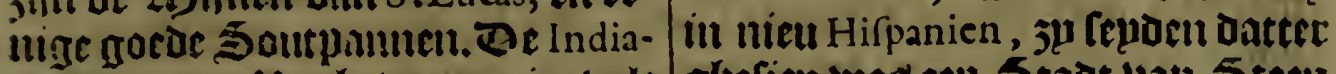

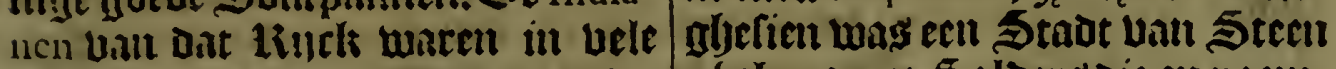
plact Ten in Dogloglye/on oe Chichimecen, ent Guachachilen deden veel friysoe op oe Luechy uan Guadalajara, ach Die ban Zacateca, ent dic Oozlo= alje was feer coltelick en lyefticly/en ju nam eunde als Dice-Liou wang De Marquis van Villamanrique. De Indianen jiin glyedenlt in die Jurit= Dictic in 100. en vier deplingljen.

sueva vize De prouincie uan meu Vizcaya, eaya, bun uaclit int Roozt-1Detten ban los Zacatecas, ; o.legutll baul die/ is em Landot bail goedoc Zliffoclyt/en veel

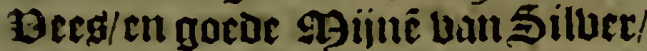
in lyaer siin die van Hindehè, Santa Barbola,en die vall S. Ian en in bie ig oe polvincic van Topia, en m defe ontdectingbe/ en bebolckinge dede Francifco de Ybarra Uele bienftê. Ter - biametla. \$) zovincic Lan Chiametla, zo.legué Ianclisen beet / op oe Culte uan be Simt-Zee/bp uaeft 4o. leguen van Xalifco, beeft veclasijnen uan Sil= uer / ch Daet leut S.Sebaftiaen, een Stadt det Caftilianen, dic eette was onder het goubernement banMexico, en lept wat boonber dan op 22 .

culacan. graden. Culiacan, if een gouberne=

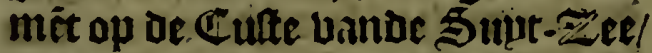
meer Boft-waet/ententeeftê bain Cniame: la, is een IL ant fece bzuclite $=$ baer ban liiftocht en: SGijnen ban -Silber/ban belcke ig cen beuolekte Reacl, bat is een lit= plactire / die str noenten dé las Virs.Micbicl. gineș: : De Stáat S. Migucl 80. It= guen uan Corri poftela, elt roo. en ozie Uail Guadalajara, is beboickt bp Nunjo de Guzman int iaer 1 s 31 .

Cinaloa. De 13 zobincie unn Cinaloa , of lactlte/ en de Roozdelicklte uan yet nieuwe tîije ปã Galicien, 42. leguen Uall . Culiacan, 1 ; o.ban Guadalajara Sopat-watert / is beboilkt in een plaet le metCaftilianen, die genoenit wert S. Ian van Cinaloa, die niet con= De bejouden werden. Defe $3 p_{20}=$ ghebout met Solbers dic jut nochle Dell Granada, ell Dat de Indianen ge= weloirly vorjsten / en Datter in oit lant groote oueruloet vall vietualie was. Quibira lept op $40.9 \mathrm{graden} / \mathrm{it}$ ect gljetcmpert Lanat / en batucht: baer. Cibola leqt 30. Ieguen valt Quibirs. Culiacan, Doozt-wacrts/en Quibira 200. Dall Cibola Boft-Lanerts / t is al arm volck / en om bat 34 glyect Cattoen vergaderen / clceden $3 p$ yacr net Bellen wan willoe Bierế/ cn hoepen ban yaer zant / die een yoorljte bebben op baer linuggheGzaet/ent grooter aly asn't book= ite deel/ de joozeng jijn elepnoer Dan D'oufe/ en in bit Bee beftaet de meefte liijckoont wan bet bolck/ont dat zp yaer met de Bellen cleeden/ en frbouen/en maecken coozdẽ /eten bet Bleetch en maecken yantwercx tupet) van De 2seenberen'/ 30 beb= ben in dete 13 zobuncie berthepdene talen/ oni dat $3 p$ wepnicb met mal= canberen banuelen.

California is ten groote punte calionsis: lants die unt loopt in be Tet/int noettlickte van nicu Hirpanien, tot op twee en twintich graden booch:-

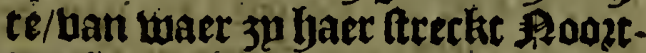
mert- waerts/ ontent 200. lefruenil al boe wel datter leer wepnich bol= comene lenniffe is / wan of as a vetis/ en Eplanden ban be Golfe uat California, bie baet is tulfetyen ve boofleube punte / en de Golfe Uatt nieu Spaerigien, die lancr daer loopt/ bp rain naert fookt-13te $=$ ftell / in be welctie al foe wel bat= 'er' siin beel tiebieren / Gapen en gounten/ en let-plaet ten / coo beeft men itotlstang gljeen par= ticuliere kenniffe baer af / om bat ju niet feet beel boozfocbt zijn. Jit begiit of dinganck uan dere Golfe is ten dzplandt ceer lauck/ encinal by de cuft langs/ m fert at 2 Diclyt 
Dicht dace by / Dat gljenoent Lert Guayaval, twelck gact ban of lie= vicrball onfe zlielue groulwe / of bill S.Scbaftiaen de Bora, tot die uan 'Pafcua in Culiacan. Bie ban de Stidut ban S. Michiel, wert ahe $=$ noemt Ciguatlan, en bet na nieub Spaengien toe / is die uan Paftla, en teglyen aber wan die is het Eulant Defierta, en aaer naer bet del Efpiritu Santo, en de haben van Xalifco, en Suyt-waet ban de punte wan California leut bet Eplanot Anjublada, en Dat uan Sine Tomas, en dat ban Flores, en een ander dat men noent las. Monjas.

\section{Wet XII. Capittel. \\ $V$ an de lurifdectie van't Hof van Sánt Ingo van Guatemala.}

$\mathrm{H}$ Et Lyof Lain S. Jago uan Guatemala werbe eeretelicli genoent het lyof wan Guatemala ban die Oimurelanden / oun Dat bevolen was oat-certet te bout=

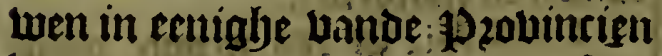
uam Nicaragua, en Guatemala, fon: oer tenoemen reeckier blecke/ bout in de lauchte / Dolt en Hoet twee bondert en 40. Iegiten / en uan ae bier en tachtichle Supt on \$2002tZijn/ tot be aclyt en tneglyentichite be langte c en ban Roozdê en Sup: oen/inae beete lyondert en taclyten= tich/uan de neglyen of oetljien gra Den boorlite/tat aclitlicn/of nega: thien / warer in mede beftoten wo?" oen de phouintien ban Guatemala, Soconurco, Chiapa, Suchitepeque, la Vcrapaz, Honduras, Y calcos, San Sal:

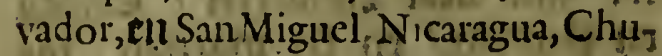
liteca Taguzgal pa, y Coltarica, en iil clck bati ute provinacien woet bee $=$

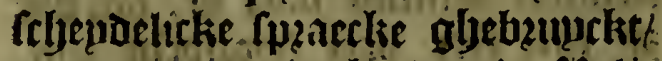
cil na oe necnimglie uan of tieli:gienten/ślyet cen lift glyeweelt ban Sc Dunuel/oin twitt te planten turs

[chen die Naticn die bloctoegeredy en warecligicrich waren.

De Depbincie / en 't Souluernes ment Linil Guatemala op de Crilte Guatemala

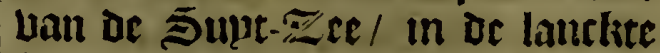
falt bu naeft fiil 70 . Ieguen / en in De becette Supden en Doozacn oer = tich: 'tigeen llant wel getcumpert/

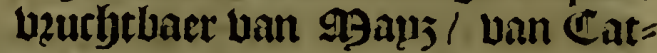
toen/ uan Carbe in ourruloet / cli andere butjecn / al ljoe weel uist bewaert wozden de jaden ban t'ee= ne Jaer tot het ander: 'thefeft wey= nich haateren/ maer als ljet regent 3iinfe feer glyctoeldict) vant pzll tot October toe: de loinden warnen Suluben en Roozden/en de Roos: De-1Biint en Duert niet ineer dan 1 s. of twinticl daglyen / en is fecr cout/ en lyeftich): Daer zïn ;. bo:= pen uan Caftilianen, gefondeert uan De Adelantado Don Pedro de Alvarado, in de Jaren vall $1 ; 24$. enl 25 . De Stadt Sant-lago, ban Guatemala, Lviens ghelegijenthepot ment noemit Cachequil, t'welcls beteer = lent cenztrent/ oul dat oe Gene= racl ban die 政atie/ als ljp upt ginc ten (Bozloctj bzocljt In een ztrent tot een fo lupm. Het is 't booft ban bet Gowwernement / al waer refi= beert bet lyof/leggende op 14 . ara= Den en een balf booclyte/ enl 93. Dan De Supt-3002t-I Iin uan Toledo, uan de welleke bet fal verfebeudorn siin op cen groote Circkel Dunfent fes hondert 60. Ieguen / en tharif uan de zise af/yet is een Dozp mist frs bondert Caftiliaenfche250?gerg/ bier refideren de Bfficiers ban des Conincr incomen/en Selyat-lifte/ bet humguan de gietinghe / m. de Cathedrael fuffragaen valt Mexico, met een Cloofter van Donuinica= nen / en een ander ban onfe zirbe Fogute ter gratien / en een Galtbuls /. en in tyaer Furifortic 25 . dupfent Tributarife Indianen, orfe 5 that is gljelealjen in een feer- lit:

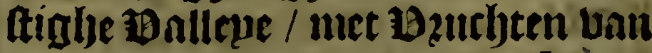
berfebelioen foosten/ en nlderbahioe 


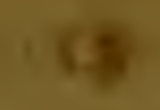

$$
x-7
$$

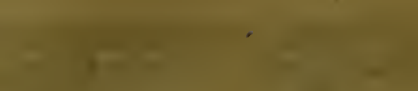

$\because$

,

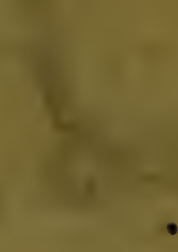

$t_{2}=8$

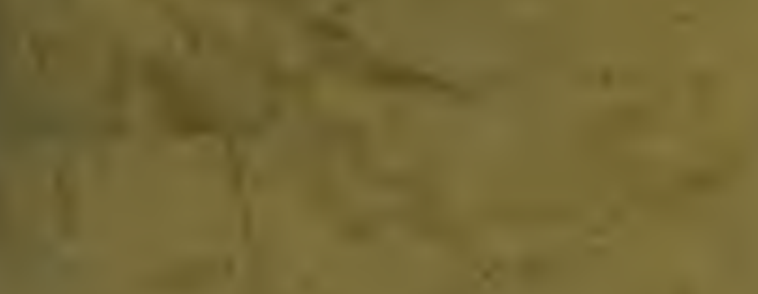

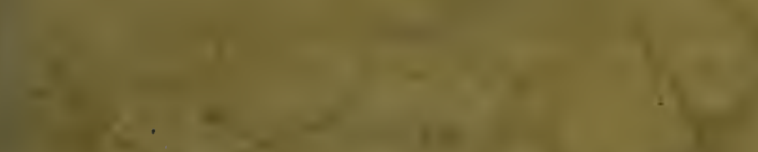

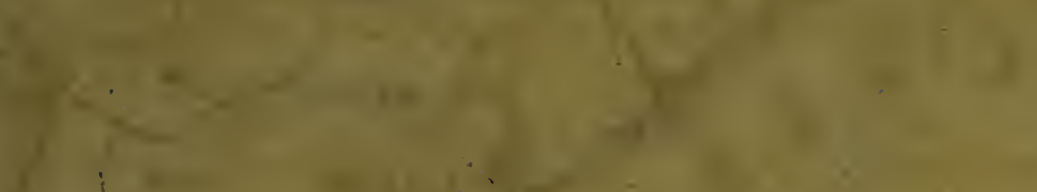

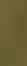

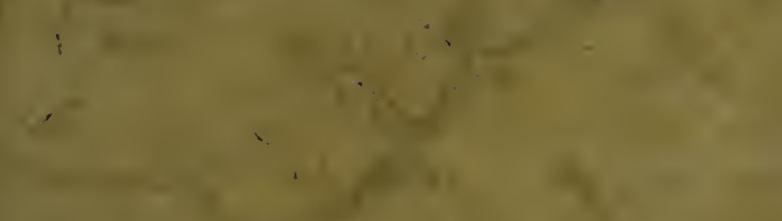




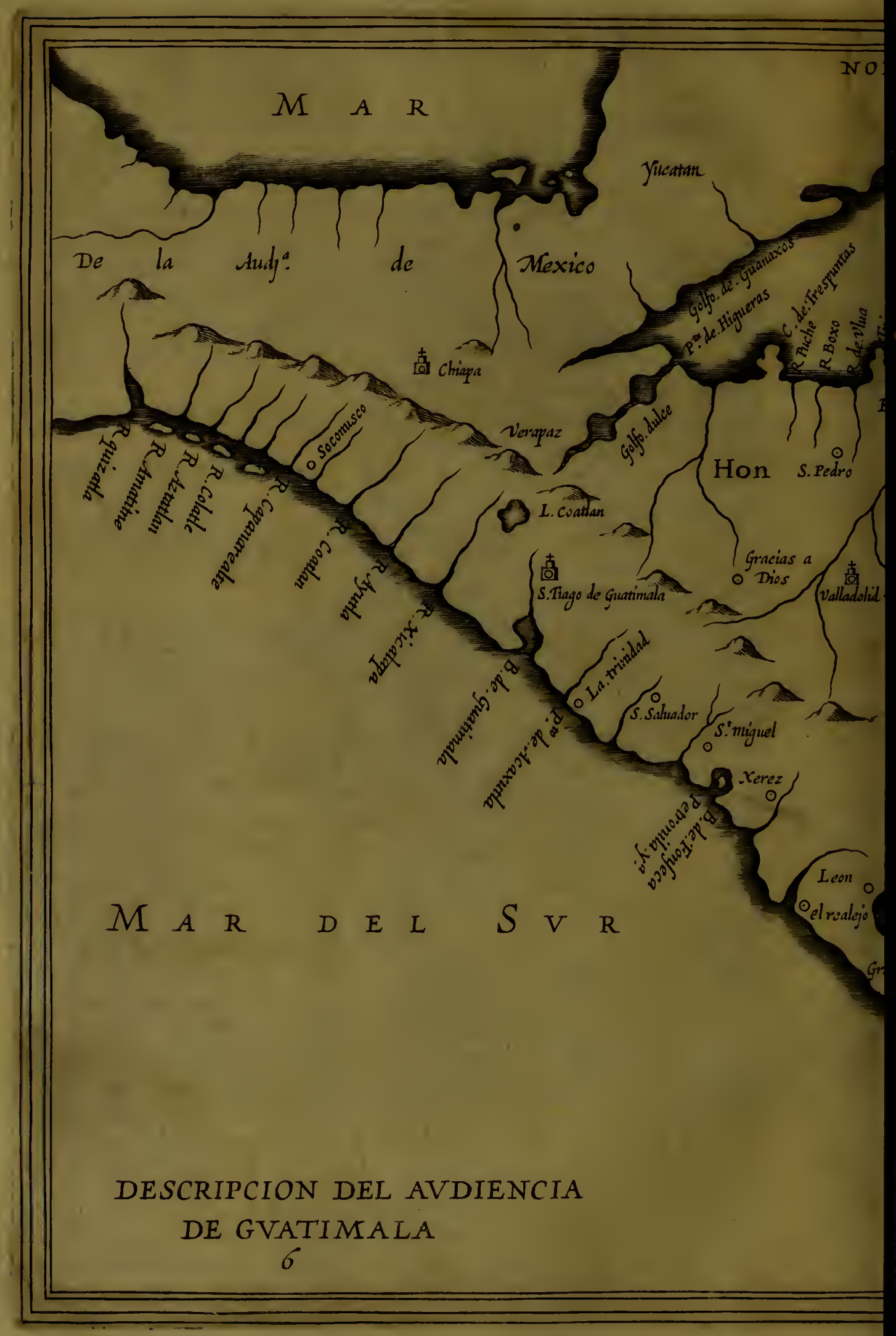




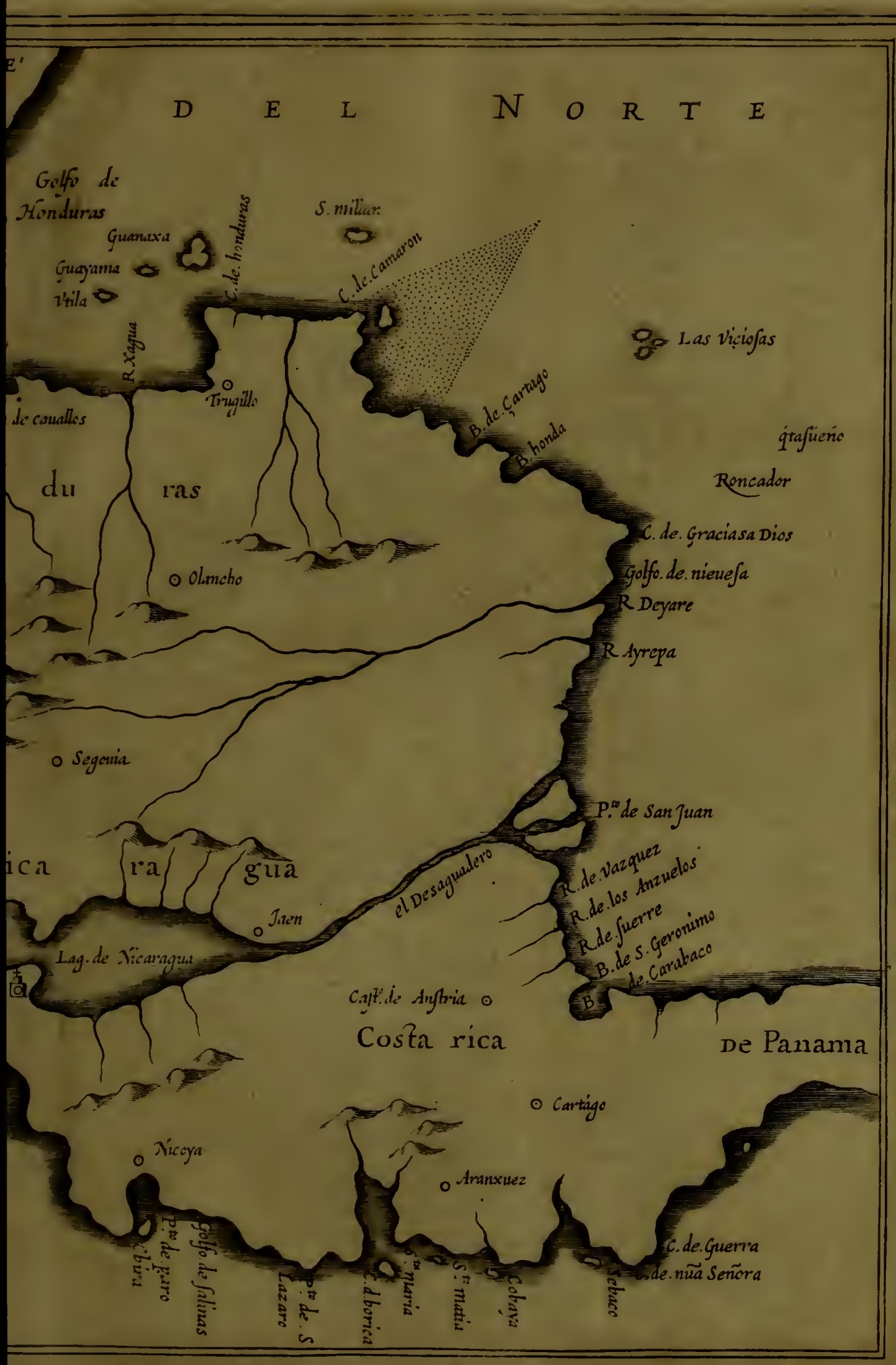




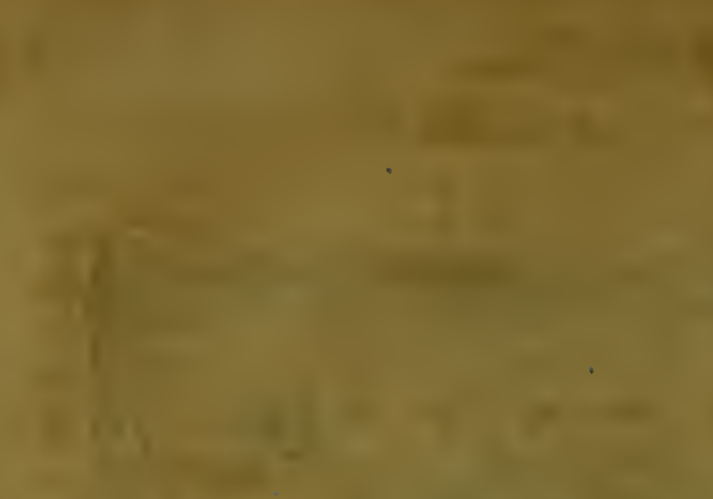

a
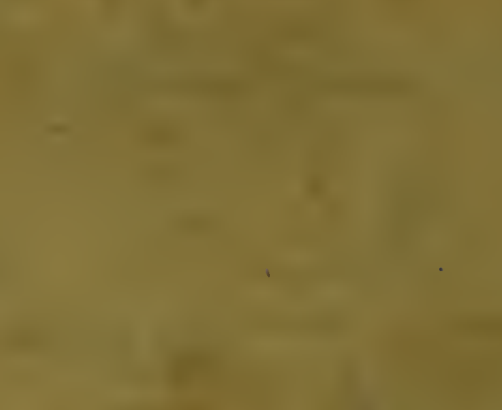

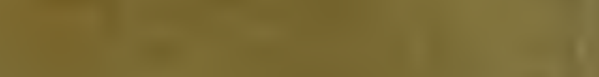

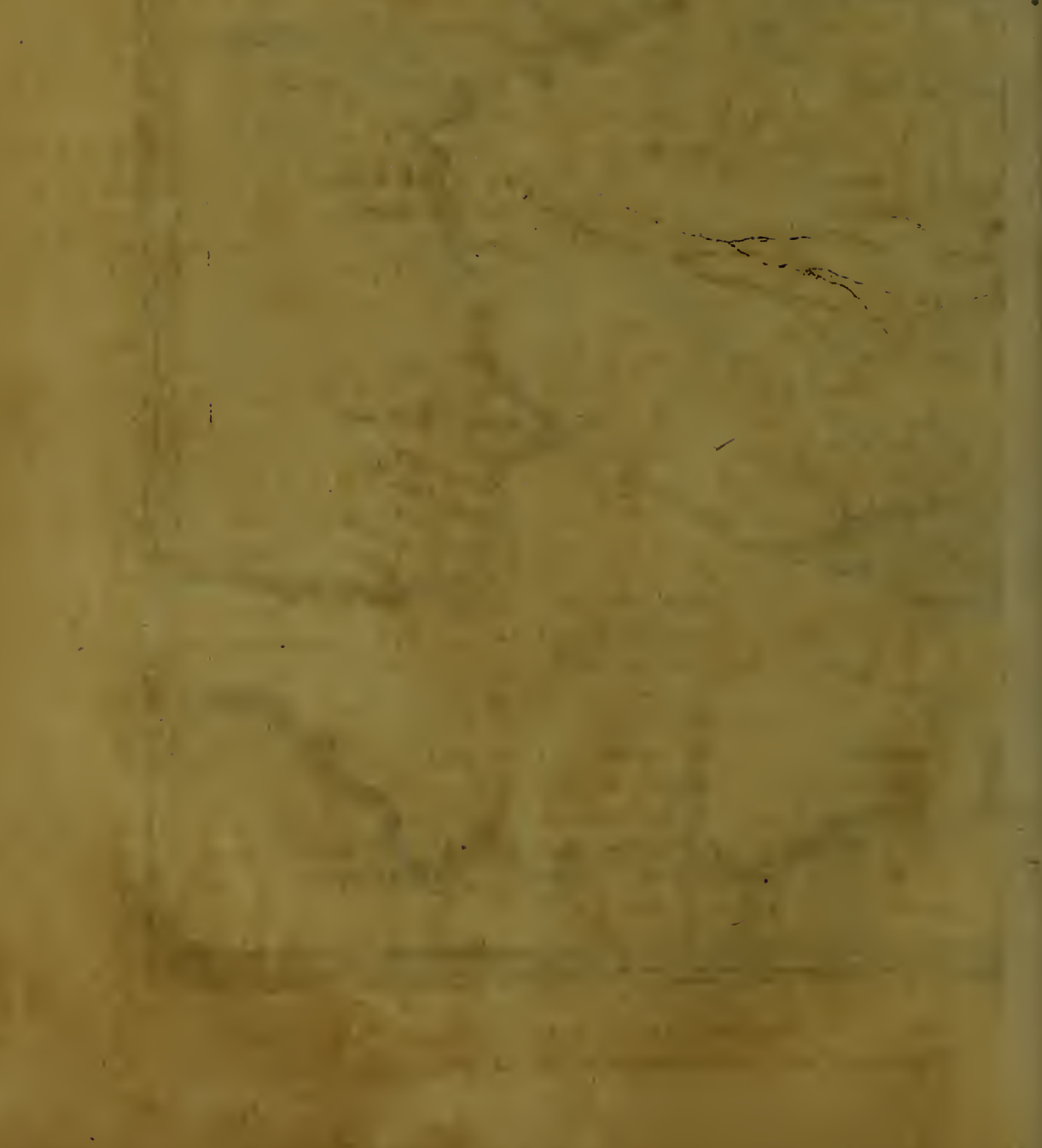




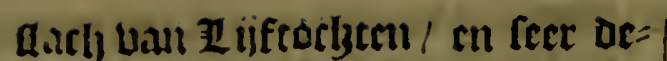
liciret.

sssalvador. Ee Staot Uan S.Salvador, due ilt oe Indiaenfehe tale glyenaent loert Cuzcatlan, 40. Ieguen uan S.lago, ten 5unt-Dotten / met een Cloo= Ater Jan Douninicancn. De 5 tritat 1. Trinidad. De la Trinidad, die op oe Indiaenfche tale ghenamut wert Conzonatc, les chl twumticly leguen wan Sant lago, Stlut-1t)eft-unerts/4. Ieguen wail De Lauen bail Axacutla, 'tis cen Bpper-5chouttrljap net oe tiitel uan fiin S9ajeltent / inet ech Cloo= Iter Der Douninteanen/in een lante= Douwe dic bachtbaer is uan $\mathrm{Ca}$ cao, en de Indianen Uan die plaetle $i$ behoozen onoer oe 3 urifoictic ban Santjago. 't 3 s een plaetre wan uele

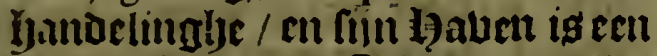
aencontere uan or Seljepen uan Peru, en uan nieu Spaengien. De sat Miguel. Stade ban S. Miguel 62 . Ienuen uan Sanijago, en twee en twinticly uan S.Salvador, Dupt-auelt-waerts/2.

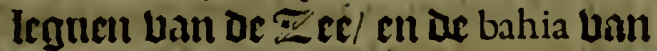
Fonfeca die baer dient tot een uen / cu Daer siin un baer begriip taclitenticls Doepen ${ }^{3}$ uan d'India-

Xerez. nicn. De Stade Xerez de la Frontera, genneme in de Indiaenfche tate Chuluteca, op de grenten uan Guatemala, en Nicaragua, tactytentitly teguen ban Santjago, ent twintieb van S. Miguel Bupt-Oolt-wact

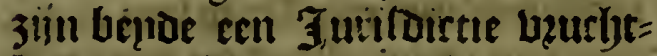
baer ban Cattoen/ cin 29 an; ! Daer Ient ontrent de Stadt Santjago, ten bzallderioe 23 ercly feer vermaert dâ Guatemala, $\mathfrak{c n}$ int ganticly Indien 3innoer veel ban oele bzanoende 23ergjen/ Imaer de vermaertte 3 iin Die ban Guaremala, dic unt gbeboz= Iten siln op Lerfelyzuden reuโen/vier tutturerpentoc Stechen / en aftelye met groote feljade bau't I ant/ ban Dic va!l Arequipa en ban Tlafcala, Quito, en andere in dat Iand 3 iin

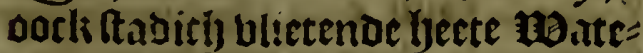
$\mathrm{rm} / \mathrm{en}$ ban uerfelienoen eughen= reljappen/Dacr jijn beeloerwen/en goede balfame die de Caftiliancn uerlient lyeblurn / Conder ant bint de Indiancis te letern/ teg? Jeng 'tgljene ecniglye atualjeur fpzecckt : cu oocls liquidambar, anime, copal, en fuchicopal, ell andere Bounuen/en daals Deruolcomentte boeljeicheden/en de gljedierten die den 23 ezinttern te: = len: ment vergnoert daer veel Cacao, dat ecn groote Rillckdomis;

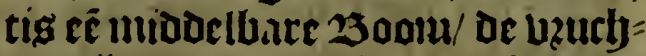
ten zijn als Caltamien / al hoe wel

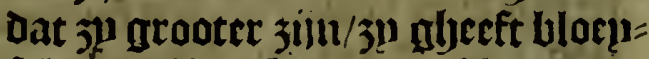
fel ende 3 zurfiten alle 29 aendacil en defgelijex uallender in dat 1 ant de Bzangienupelé/de Cacao 300 ou is meeroer bzunt Lain oe vochtich= bept/ dan ban of Soune/ cal baer= om woat fiin plante nergheng an= oers gljeitelt dan oner feljadu is."

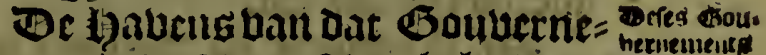

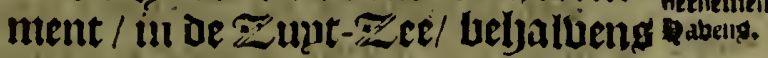

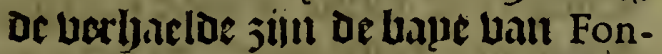
reca, Dicht ach S. Michiel op twaclf graden en cen ljalf lyooclyte, thecft fiin naeiH getregen Uam Gillis Gonzales Davila, int Faer 1522 . Uam loe:

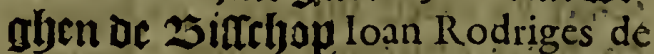
Fonfeca, Prefident ban den liacot ban Indien, en binuen in de Bahia tg een Evilandt Datumen noent Petronila; nae des 23 iffelyops Sufters Docljer. Oe Laluen uan Acaxutla, dicht aen oe Trinitad, op i 2 .geaden booclyte $/$ is lyet peincipacl ban bat

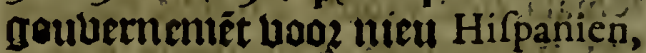
en Piru, th de 23aue ban Guatemala twaelf leguen daer ban / en oc kie= vier ban Xicalapa 7. Ieguen ban oe Bahia Weit-waert/ Pooit-waerts en raecht oefe 1 s susintic gheeniglye Culte/oul dat yet niet meer dan 40 . leguen ban Zee gaet/ tot een plaets Daerneti ont'clyecpt/dienen noent de Gaben uan de coete Golfe; ban oler gaen de Coopmantrbappen die uan Cantilien gaé/ Dooz de Golfe de Honduras, binnen glants met oe Carovanen, tot Guiateniala, ell op oe Conincklitke weds van. Mexico is bet groote פgept bait Atitlan, balt $\mathbb{E}_{3}$ tbiet 
thian leguen in oe ronte/ cal 4 .in oe bectte fondart gront.

De pjobincie en yet gouberne=

soconules, Hent bumt Soconurco, fyet aldoer-10e $=$ ftcliclifte wau Guatemala, op de $\mathbb{C}_{\mathfrak{u}}=$ fte vant of Sunt-疋ec / lanck en Jeect un maelt 34.Ieguen / uencijt= bner uan Cacao, de grootte ljande= linglye aldace/ en van al bet gljeene Datter gelaeut wort/behalbencar = we / teit beeft tuet meer dau cen Doep uan Caftilianen, twelcli gye= neenit wert Ginevetlan, een fondatie uan Don Pedro de Alvarado, al waer nu refioect oe Gouluerneur. Sare Culte die op de Sunt-Zize Iept/ begint 7. Inijlen uan be lie= bierc ban Ayutla, Melt-Liacer / en Dan de Reuieren Coatlan, Capa. nacicalte, Colatl, Haztatlan, Amatituc, en Quizatatlan.

De \$2obincie en 25iroom ban chiapa, Chiapa, is binne Zlants tuttefen Soconufco 5.ut-waert/cn bet upt terlfe ban nientue Hirpanien, melt= waert: en int \$oozoen en Oolten tultefyen Tabalco, en la Verapaz lane Boft en meef bu naelt 40.leguen/ en beet eenighe ninder! veucht= hate ban beel Earwe/en Maps/cn andere 5 aden/ct ban Bee/belyal= ben ban $5 \mathrm{cljapen} /$ dit der niet beel en zilin: 'theefteen Dozp ban $\mathrm{Ca}$ itilianen datmen noêt Ciudad Real, so leguen ban Sant Iago ban Guatematà, nat Ropzt-Boften/Dat met ecti particulier Hzivilegie ghere=

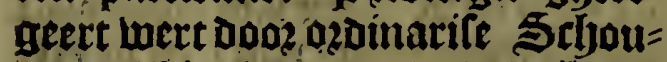
ten: al lier is de Cathedralc liere= he / met em Cloolter uan Domi= nicauers/ en ued Dozpen bain Indianen in yare 3 urifoictie. Het paincipael boup wert ghenoemt Chiapa ban Iaar/ waer ball de Djoluincie fiin wodnacrs Lueten joerden te tee= ten/ die ban baer comen ziin / zijn beter dain die ban nicu Hirpanien, 3usiin asuriickers en . Schilderg/ en leeren wel eenicly stmpt bat in confte bettace / ban outs beboen $3 !$ solit?

IIjclucet uall Nicaragua, en de Cas pitcun Dicgo de Mazaricgos, int iner 153 I. lepoe dele $\$$ tadt in cen 20 a!: $=$ Irpe al wacrfe nu is/ comot / op ren wonderliche gljelegijentyentot / op achthien graden a ca cen Inalis/ a

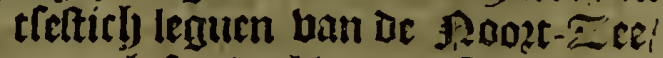

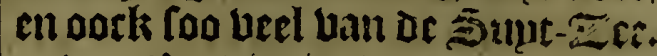

Je jozobuilic bail la Vcrapaz, Dat een naent is glyeglyedu bu be Teligieulen ball S. Dominici $B_{2}=$ Den / om dat zu bet bevzediclyt beb= Len met haer poedicatie / oocit is fugljeleglen bunnen 'flauts/ tul= fchen de $\mathbf{Z}$ antpalen ban Soconufco, Chiapa, Yucatan, Honduras, fll Guatemala, derticlj Ieguen oweers over/ en oock foo Jeel baut Sant jago de Guatemala; yet Zanot iffer uorb= ticl / en daer om iff beter booz de g9an; / die dact watt tweemacl int 3aer/Dan bool de sorbe/ cn Cat= toen/en eeniglje Cacao,en ueel bo= ghelen van die die plupmen geben ban colcuren/ boos de Schilocriien dic de Indianen maetken / t'welck is een neeringlye uan befe 19zouinciel en oe Coningfjen uan Mexico trocs ken dien / en dat wag die meetf gheachte fack die $3 p$ badden / en men oozdeclde vooz groote fonde die Boghelen Doot te daen / Dan men mochtre wel ontbeeseren/ of die nemelt. In dat Ianot is niet meer dan een Cloofter ban Dominitaners/ met een Dozp uan Indianen, ban 17 .jongheng dic dacr zijn/ al twaer de $\mathbf{3}$ negelozenen baer hebben bergadert / booz ozoze nan oe Tieligieulen om baer beter te on= Derivifen/om dat $3 p$ te boozen lecf= Den berbaerts en oerwacts/cn als Foilden/en nu leben $3 p$ a!s Cf filte tien/en int waerlick 2302 glerlicks.

In ocle 1 szovincie en is gleen Golluerneur $/$ dan allecti een $0 p=$ per-Scljout'chap/t'welck cen pza= beido is uan't hof. De tietsiere Zacatula deplt Defe 19zobincic bant Guatemala, van be welcles bet fyem unt ftecht tot aen de Golfe dolce, werwanet 


\section{batt VVeft-Indien.}

werwaerts gacnoe olli t'ontwate= ren alle lyare ticuicrê/ Doop welcke/ en oul de groote vlietingben der watercu die af-loopen uan de lyoorfitte tiotfen / was lyet Iland

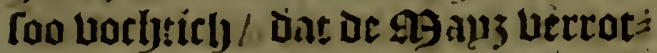
tròe / maer oc gljetemuertliepot is gheluetert/ nac dat bet afgheballen

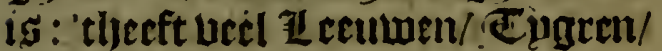
en Daffen/ Lueleker vieefely oe Indianen eten / al boe wel ljet foet is tII uials. 'twert ghenoemt Golfe dulcc om oat boos oe menichte uan de Revieren die daer lantr in Zee loopen / bet Bioater foet is: en dace siiil groote Botfelyen/ en int bplon= 3e Bas M- Der de Manati, Die oe Tee-Stitr is/

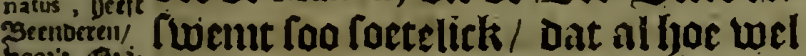
boojt $0_{0}$ ? berigoer.

bu ceer groot is / coo maeclit lyu gijeren gljetier / bliet vooz andere' cube gart te grondec / cn fyn is fut= ricus tegljens dir die hen foecken/ en lyp gljeeft groote thagljen / fiju uleetch is dicts als bai cen thoe.

1)et X I I I. Capittel.

an Honduras, Nicaragua; en Coftarica.

Honduras,

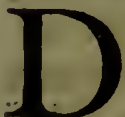

(Ez)zovincie / en 'tgouber= nentent ban de Honduras, ftreckit hem in be lanckte Ooft en meft / langs de \$2002tCulte/ meer dan I so. leguen/ en in be bzeete ban de Zee af tot de lantĐ)alen van Coftarica, en Guatemala ten beele tacljtenticly/'tljeeft veel gljeberchtec / en is vuchthaer uan 29auj/Carwe/en alderljande foo? = ten ban Bec/ cll eniglse Rgijnen ban Gout en Silluer: Daer zijn leg Doipen uam Caftilianen, en een $23 i f d o m$ / oc eerfte 23iffeliop was Broeder Ian van Talàvera, jozieur uan Prado, uan d'Ozdze uan S.Ieronimo van Spaengien.

vanesial

De Stadt uan Valladolid, in de Indiaenlelje tale glyenaemt Co- mayagua, Icpt op meer dau feltbicì graden / en is tfeftich 25 ijlen wan Sāt Iago de Guatemala; Ooftwaertg/ en by nact 40 : Lall of Poopt- Teé: De Goubcineur refidect allier / en Daer bet Cathedral vati het J Jaer 1,5 s 8. Dat fet uan Truxillo ver = trocken is/ daer bet aldereert was/ ell Dare is een Clonfter ban onfe Lielue boutue ter Glatien. Be Capitepn Alonzo de Cazeres beuze= dicljoe dat al aut / upt laft uan Don Pedro de Alvarado, lyet leve int mio= oen ban twee Zren/ en betf uan d'een tot dalloer dzie en so.leguen/ uair de louluen de Cáallos op de Poozt- Tect tot de 25aue ban Fon: feca, op dic uant Supden / en dien wech beforht oe Fingenieur Baptifta Ban be one Antonelli uit lait ban De Coninck/ banben sapoin dat $j^{p}$ baer lieten bookftaen/Dat neli. o ver dien weetj de lyandelutg lichy=

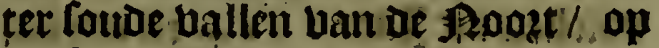
de Supt-Fe / en lyp bonot datter beel nwariclyeuts in gljelegen wag. De 5tadt Graciasa Dios, Der = Băbe zatabs tich leguen uan Valladolid; bunaeft int Poetten is bebold int Jaet I 53 o. ban de Capitepn Gabriel de Rojas, om te beneficieren de Boutggijnen/ die daex baren/en wond th groote rentontren miet de Indiänen; en bebben fyem dithuild berpzon= gen binnen int f ozt dat bu badoel nochtang ats de opubertieurguan Honduras, en Nicaragua lem te loulpe nict en quauten / om die paftie die tulfben bnex waren $/$ berliet bp bet / en int flaet is 3 6: quani oe Capitepur Gonzalo de Alyarado we= Der omte bebolcken die Stad. De Stadt S.Pedro 30 . Ieguien uan Co: s. pedro eei

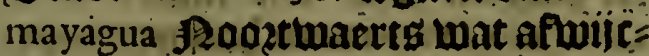
kende Mdelt-waert/ en elf ban de Hauen de Cavallos, alwaer refioe= ren de Bfficiers deg Conincx/ om Dat de gaué ban Càvallos niet goet is / al waer vertriicljemen het def pacho uan de Schepen / is glyefon: deert int jaer is 36 . van oen Adelantado Don Pedro de Alivarado: 


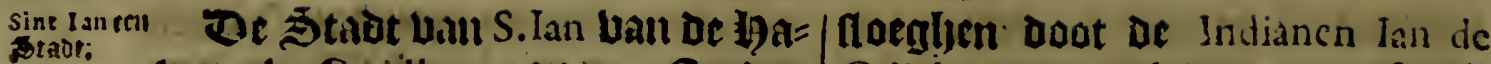
uen de Cavallos, op 1 s. Gzaden Jooefjte/ clf Ieguen van S.Pedro, en 4.unil Coniayagua, is bevolekt ban oe ffactoogd Der Coopmantelyap= jen / endoe Swarten / ont dat bet rell nniac loaben is/ al boe wel bet ren 25ape is/3p is chentwel goet: $j^{\mu}$ wert oljenoemt be bavell de $\mathrm{Ca}$ vallos, oin zatter eenigbe joacrben in $\mathbb{Z} e$ gljeballen ziin booz onwe= oet. De Staot Truxillo tretticly le= guen ban Comayagua \$oopt-Ooftminert / en 40.ball de baben de Cavallos Boft-waerts / cn een ban be 32002t-Zee/ Dace beeft be Cathedral glyeteet /sijn baven wert genaent Ian Gillis, 30 is goet/ al loe wel bet een doooe 23ape is/cn gljelloten/al wher eertelick or Schepen aenco= men die nae Guatemala gaen. Francifco de las Cafas begonft dien te be= volcken int 7 act I 524 . en om dat bet al bolck was bait Eftremadura nombe by bien Truxillo, en Don Hernando Cortes epnoighede dien te bebolcken / als bu Luas bp de Y. Be start bueras. Be. Stabt ban S. lorge van silorge. Olancho, 40. Ieguen ban Comayagua bolt-waert/ban $40.2302 g$ bers/ en in fijn Furifoictie refthien du!!= rent Indianen Cributarifen/en beel Gouts / bufonderlick in de 17ebier Guayape, twaclf leguen ban befe Stadt. Defe Ballepe uan Olancho is feer lutticly en pzofiitelick/ en men trechter veel Gouts upt: en be Gouberneurs uan Honduras, en Nicaraguia bondent op andere tijoen groote differenten/ont dat een poer bien begbeerde in liin Turildictie/ en baerom walt bat alljier Gillis Gonfalez Davilantam bondert twin = ticly Duprent pelen Souts ban Hernando de Soro, ent by berliet lyet voleli dat Jem ghegljeben wag uan Pedrarias Davila, tot befefermingel en fier bereljertude Gabriel de Ro. jas Den inganek/ Juan Gonzales de Sandoval, die bu conoe Don Her-

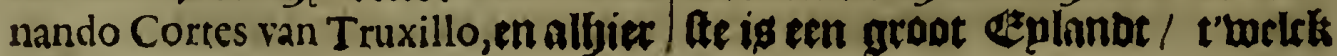
Grijalva, een wel birmtaert Capi= tequ/ en nocls anderc.

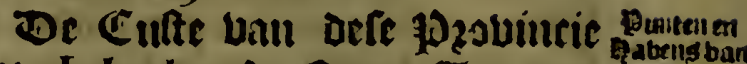

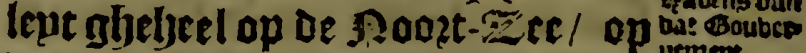
be golfe die genoemit uert de Honduras, ont ac ljeele Tec-5trant is tulteben defeppolvincie/ell Yacatan, tot waerfe t'famien coinen uiet la Verapaz, al waer bie wert gencallie de Golfe van Guanajos, de ecrfte punte is dic ban Ybueras, alloo glise: noemt om dat be eerite vonten beel

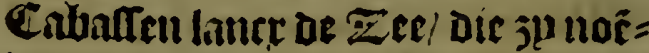
den Ybueras, in bet Spaculedy/ fp lept op I 6.genden lyoocljec/ diclit aen de golfe Dulce, een finden vooz Guatemala, al waer werde bebolekit S.Gillis van Buenavifta, dieljt bn de Cabo vall Tres puntas Ooft-Laerts vail de Golfe dulce, en vertae ghe fondeert int Taer i 2 4. ban Gillis Gonfalez Davila, en meer Boft. warert wert ghelunden be siebier Piche, en de zaebier Baxo, en die ban Vlva met eel ander naem Balaha ma vooz de Haben ban Cavallos, die op 1;. graben Itpt / en Daer naer de Liebier/ en punte luan la Sal, en el Triumfo de la Cruz, een Cabo ban Dzie punten/al baer int 3lace is 24 . bebollktede Maeffe de Campo, Chriftoval de Olid, be ticuier Hulma, of de Xagua, en rootr-waerts ban fiin Sont bet Eplandt ban Vtila, en Poozt. Boft- waett Guayava, He. len, en Guanaja , en S. Francifcus Supden en Poojoen/ met de pun= te ban Truxillo, dat de Eplanden ziin ban de Guanajos, of voozep = De punte wert gbenornt met cen ander naem Cabo Delgado, of uan oc Honduras, van waer af tot aen Cabo del Camaron, in wieng glje= biet bet gnet tot aen Iamayca, beef= tet dertbien tievieren / en op of punte vall de Cabo beeftet een groote ondiepte / meer dall twin= tich leguen Eee-waerts in / en int midoen ban Jaer dicht aen be $\mathbb{C} 11=$ :- 


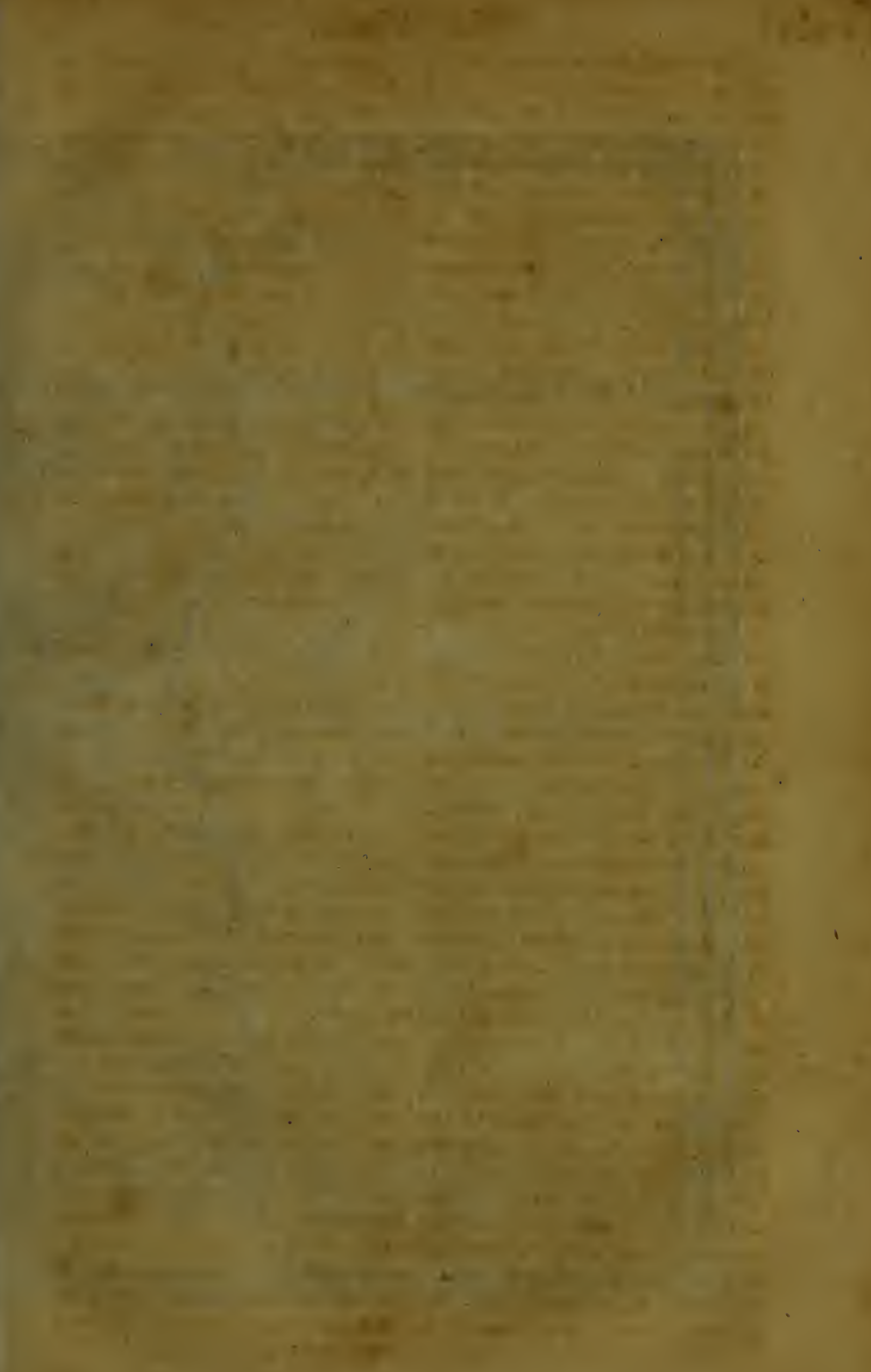




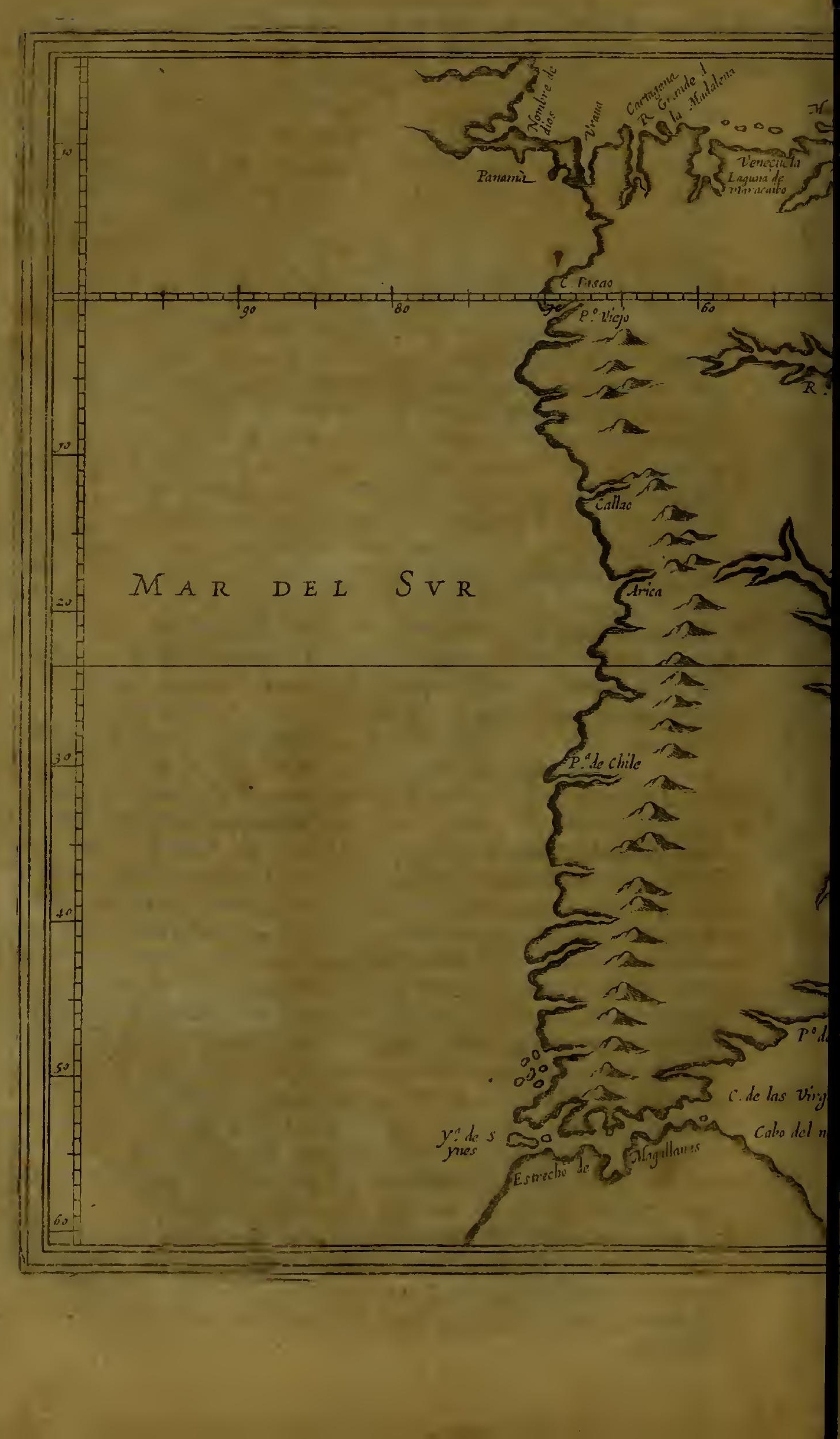


ECVINOCIAL

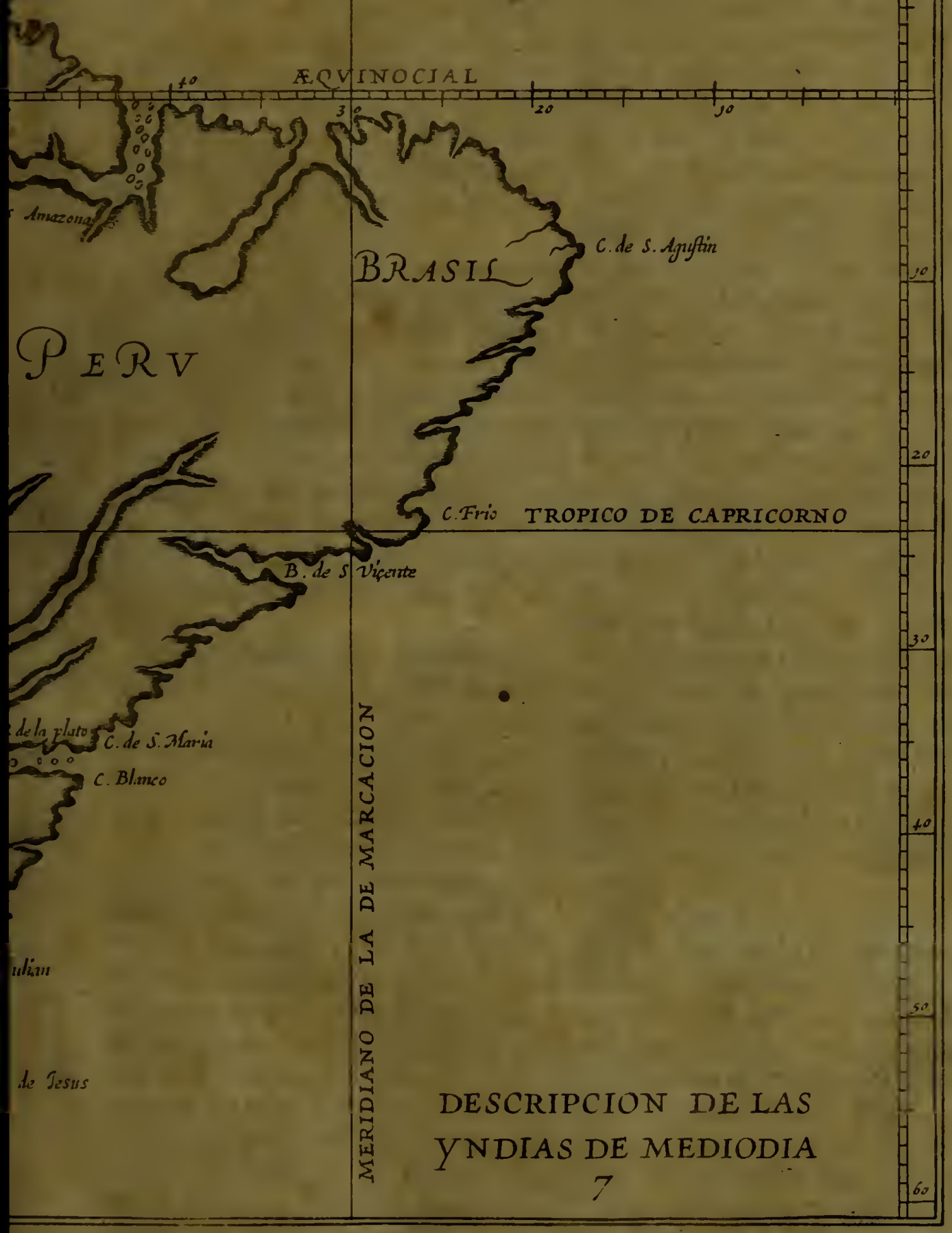



3!) nocmetil de los Baxos, ell cen an= ber Reort-tonertg ontrent de Clip= pe gemaemut San Millan, en gljepar= Ceert jilloe de Clippe/de 23 ąue bail Carrago: cul de 20 ane Honda, vooz oc Cabo Uall Gracias a Dios dic op 14. Irinden lepte / en een Derdenderl/

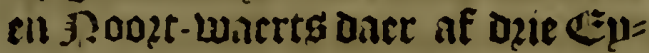
lantorlietus dic j!l nocmen las Viciofas, ent Quytafuenjo, ent Roncador, tuet periculeule Clippen/ en aljepalfeert jijnde oc Cabo, oe golfe ban Nicucfa, al waner lyu ber= loozen gincls int Taer 1 ; 10 . Eut De tieluter baul Yare op Dertljien graden / al warer lyace t fimten voc= ghen de Goubermementen wan de Honduras en Nicaragua.

De Eplanden vall los Guanajos, Die de gljene ziin baer af verbaelt

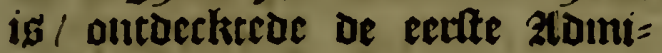
racl Don Chriftoval Colon, in't Faer buurent biff bondert twee in tiju laetfe Bopagie die ly dede in Indien, als bp ontoecktede lyet ba Ite $\mathbb{Z}$ andot / op de Culte van Veragua, Laer Doos gn bekent woz t om Dat by was te Veragua, was aen D'ander fijoe ontoeclit jet nienwe Spaengien.

Tne 13zouncic en 'toouberne= Nicaragua, ei lutent baul Nicaragua, bet welche Xanuf(rbay. Don Diego Lopez ban Salzedo, noenide lyet niewwe täij ban Leon, nofett-waert boerbt bem met Guateniala, en foott-waerts met Hònduras, en Suut-waents met Coftarica, is van hoindert biiftich leguen Ooft en Helticn tachtentict Sup= Den en Joozdell. "TIs cen Xandt. beuchibaer ban, lunnz/ cacao / Cat $=$ toeni / uet bee / Conder Cerbe/ en Sclyapeu / hat heeft viff Doz= pet Lan Caftilianen. Leon van $\mathrm{Ni}$ caragua is Uant Sant-jago. de Guatemala hollocrt uier legué/ bu naelt int \$upt-Doffen / ch twaclf bain be Sunt-Ze l diclyt lp be groote I9ely vall Nicaragua : al waer refi $=$ Deert de Gouverneur/be Conincl: = Licke Dfficiers / en de Catbedzale

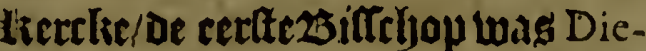
go deAlvares Offorio,tbeft s. Cloo= Iters bau oule Zlieve voguure ter Gzaticn/an in fiin 3uritoittie yon= Dert en twinticly ouwlent Cribu= taurile Indiancn. De \$tad uau Granada létlien leguen ban Lcon, oe welckic aljelijek oork Leon fon= decroe de Capitepn Francifo Her-

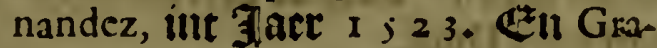
nada is bu cans int Suut-metten/ act be callt ban lyet groote 29 qu/ en vicr en twinticls leguen ban or Gauen Realejo, en noch oock op ljet groote socpz / is dic uan Lindiri, en dic merckelicke bzandende 3erch uan Manlayatan. Het gróte Agenz eft en vlocut / theeft veel Eulanden/ tot aen de Paopt-Zeel tot aen de thevier diemen noemt el Defaguadero, theeft ueel biffey/ en Capmannen / twee leguen Daer van daen / en leven van Granaden is een groote baandéde 23 erch van Mombacho, wiens boachte groot is/ en met veel 230 ontell ban beel Buchten / van of weiclie of $1320=$ vincie leer is berfien. Een Plie? Iter gaf te berltaen / dat oe malle van bier bie in foo beel Jaren fon= Der berteeren/ bzandenta bumen. oc lizandenden $25 e r c h$ uan Maffaya, was Gout / bp macelkte of met eel reeckere conite- 1 een letel met fiin lietingen oni fet te bifrchen/maer al ear die ketel met ze leten aen'c biee quamen waren $3 \mu$ gbefmolten als IL oot.

Het nieu Segovien twelcls wag segorb; : 'thegin ban oe bevolekinghe ban Pedrarias, lept Dertich leguen ban Leon \{oozat-waerts / en oock loo veel ball Granada, oock bp canta int \$20020en/in wicitg Furifoictie men Uecl Bouts unt faclt. EII Iaen een Taen \&:13 Stabt dertich legué ban be RooztZee / in lyct cunde van bet groote agep / al waer unt vliet trie tae vier die 34 noenien Defaguadero, cu su varen met baer Coopman= Irbappen baer boos met Earioen/ 
dic jil uzenghen uan Nombre. de Dios, en mu wan Portobelo. De El Realejo. Stadot Rcalcjo een Iegue ban de Gaven uan Poffeffion, dic 3 gtye= metunlich nomutu del Realejo, Itet op elf graben en cen lyalue / ent $t$ ts ren ban be belte baljeng/ en ban oe reerlierlte die op die ganterfje Culte jiin / in be welche j! bouken goe = oe Scljepen/ dooz de berepoingtse ban goct bout.

3il Dit Boubernement siin beel Doxpen uan Indianen, Daer groo= te quantitepe ban Tributairifen in jijn/ en op oe Gzenfen ban Jet Gouberneinent / en batn Coftarica, en ban Nicoya acbtenvectticty leguen ban Granada op oe Culte ban't \$ 5 moen $/$ een rectst plegin= ghe / in bet welclie/ en op het Ep= lanot ban la Chira, bat van nin $\mathbf{3} u=$ rifoictie is acht leguten in oe ziin beel dributariten Indiane van ac Conineklicke Croon/dic te voo= ren teonden onder til of ban $\mathrm{P}_{2}$ nama, tot oen Jlare y 563 . dat Coftarica gbeincozpozeert wozde / wieng Boubernetre fteloe een Stee-1)ou= Der / en be 23iffefop uan Nicaragua eth Vicaris, baet is een redelicke Taben : Op oeCufte uan bele $3920=$ vincie ban be zee ban't beefe bet na de liebier Yare, die bet rebept ban be Honduras at/ die ban Yairepa, booz de Zevier en ban S.Ian, die zp noemen be Defaguadero, met een groote tiebier in de sgont/en daer naer eenighe an= bere 1Revieren ghemepn met Coftapunim on rica. Bp de Supt-zijoe beeft bet babens ban nae dic than Realejo be S.Iago, voos die van la Chira, en oe lo aven ván Paro, teghen over ban Nicoya, in die golfe dien $3 p$ noemen de Salinas; voot oe punte ban San Lazaro, en Cabo de Borica, aen wieng Boft-liiar jiin of eteplanaen uan S. Maria, en S. Martha, Cobaya, en Sebaco, dielyt aen de Lantpa= Ien baut Veragua, objemeerimet Co. "Atarica.
Jne 3 aer $1 ; 29$. wat bevolclit op oc $\mathcal{C}_{\text {utte ball Nicaragua, aen or }}$ Supt-filde/ de Stadt Brufelas, en Diego Lopez bu Salcedo ontualcli= tede dien weder / am dat 3 ! aldaner yndoen ontfangrizen Pedro de los Rios, Soutuerneur uan Caftilia del Oro, die ginelt ont te nemen lyet Gouvernement ban Nicaragua, al waner lyem badoe aljeltelt de Salcedo, en gaf ban Honduras af lyem fiin goubernement. Fnt jact is 24 . bevolektede dien de Capitenn Francifco Hernandez, in ecn twiffelach $=$ tighe Straet / cn in or gljelegljent= bept ban Vritina, en aen d'eene fii= de fjaboe bet oe zec/en nen d'anf oer be blackten / en vooz het oerde/ bet gljeberebte ban de gaijen / ca in dere gantrtye werelt / en siin glyeen Indianen meer erperter in oe Caftiliaenfche tale / dan die ban $\mathrm{Ni}$ caragua.

De Drobincie / en 'tgonberne = ment van Coftarica, lyet alder- $B D=$

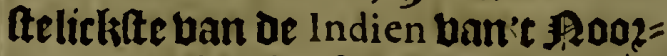
oen / en lyet lof ban Guatemala, freclut lyem Dof en welt in de lengte t'negbentich leguen/ ban be palen ban Veragua, tot die ban $\mathrm{Ni}$ caragua, met bet weleke het $\mathbf{h e m}$

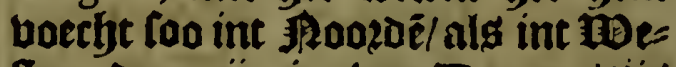
Aten : Daer zijn in twee Dorpen/t'is een groot zanot / met becl g9on= ters van Bout/en eenige van Sil= uer. Cen ban de Jozpen is de aranjues. Stadt Aranjuez, viif leguen ban ue Indianen Chomes, volcls ban de Juritoictie van Nicoya. De Stnot Cartago veertich Ieguen van Nicoya, en twintich ban oe Iee / bu naeff int midom ban of 1 pobincie/ twelcke maeckt een l)aven / en af= rchepinglye op de Culte uan oe Sunt-Zee / en de Roopt-Zee / in Jet weleke jiin eenighe lievieren tultelyen Nicaragua, en Veragua, gbe = mepn met dat Boubernement/ en oe ssapen uan S. Hicronymo, al ban Caribaco, ontrent oe I imitei van Veragira.
Erufelas. Coftaries carango: Carrago: bete 


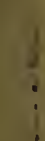

\section{in 8}

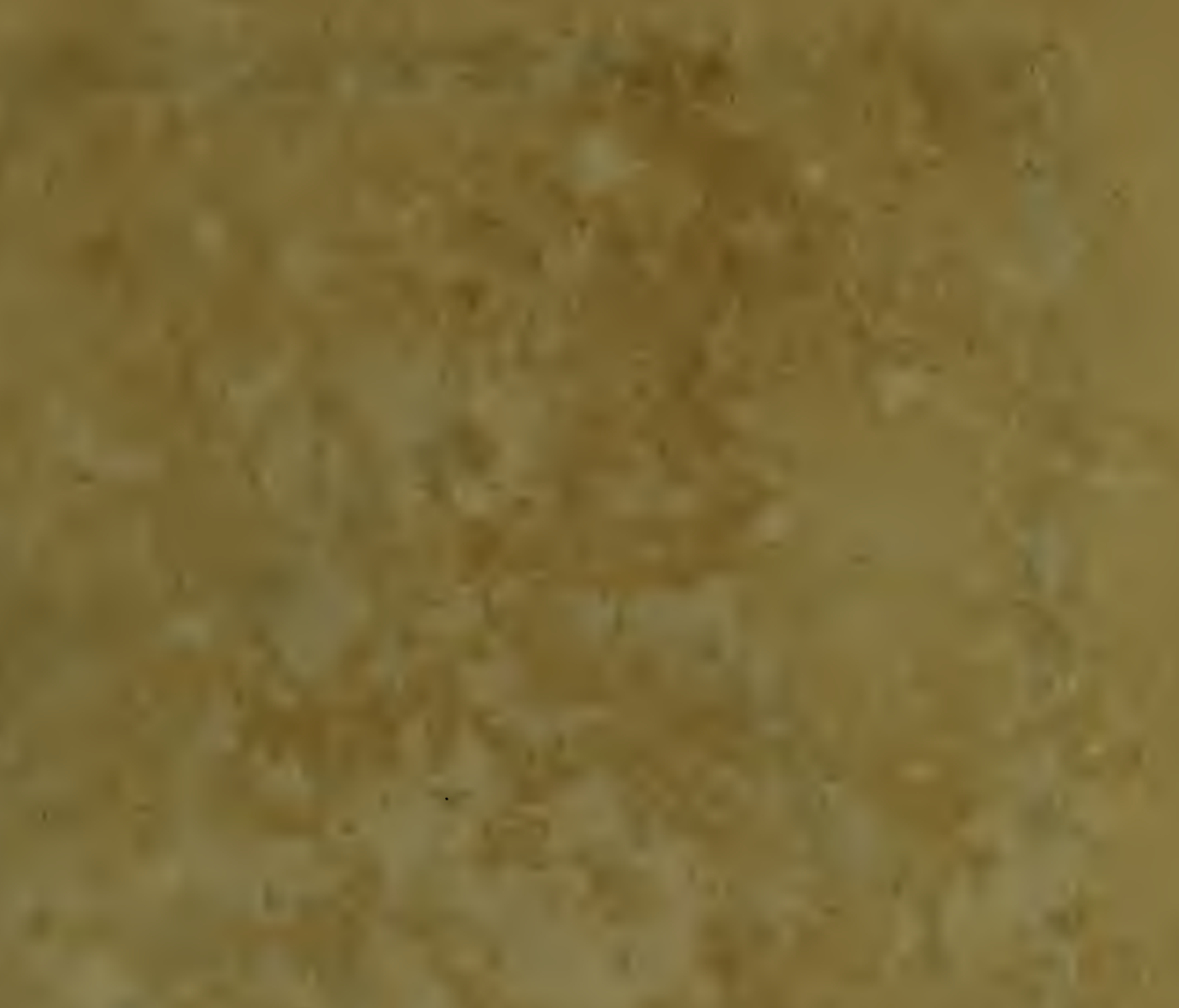

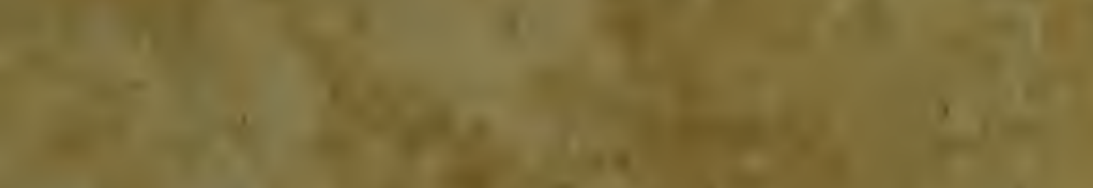

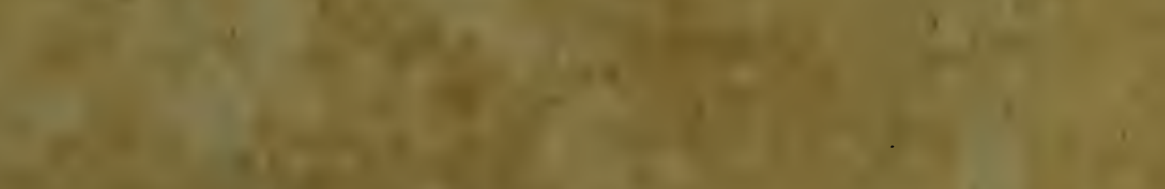




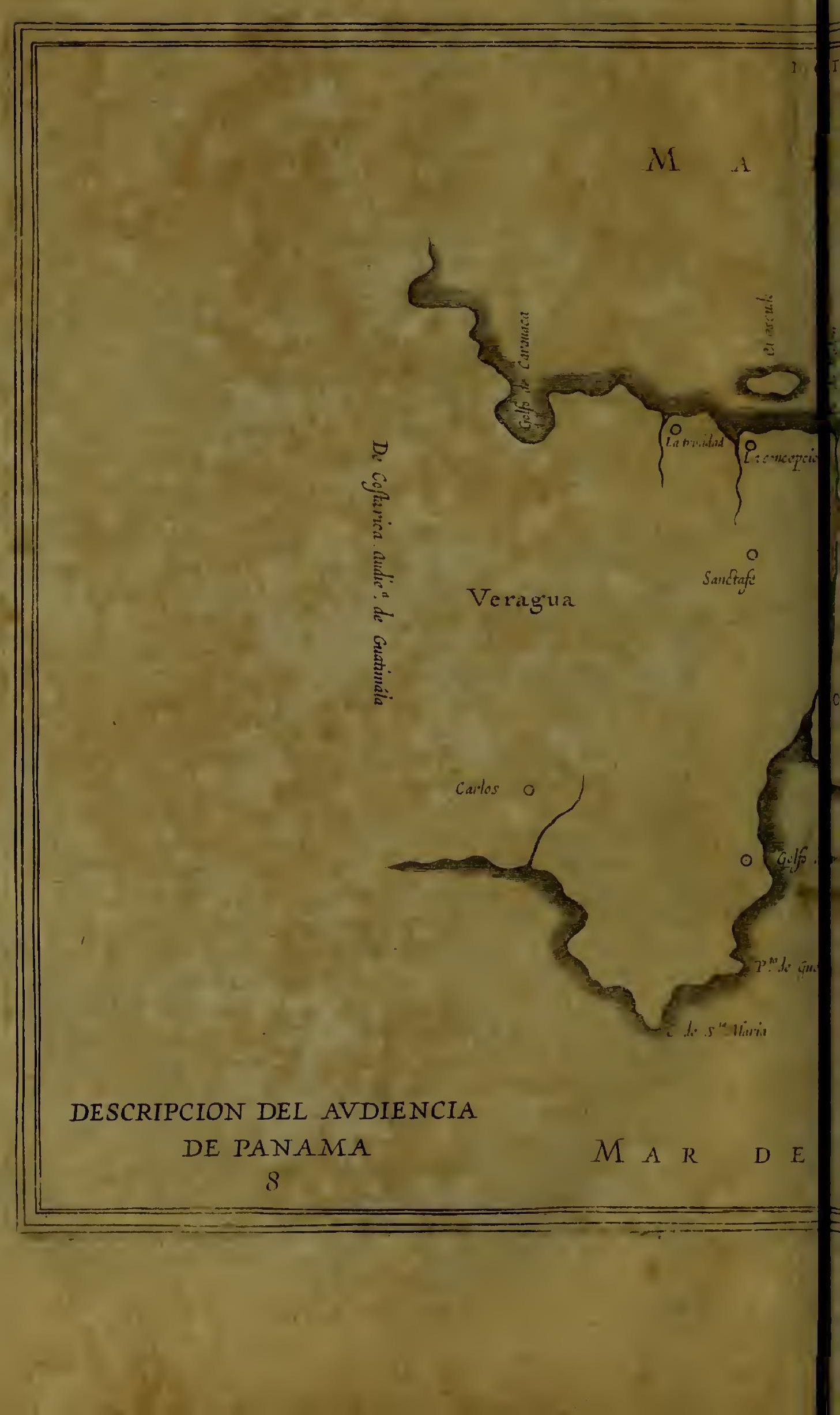




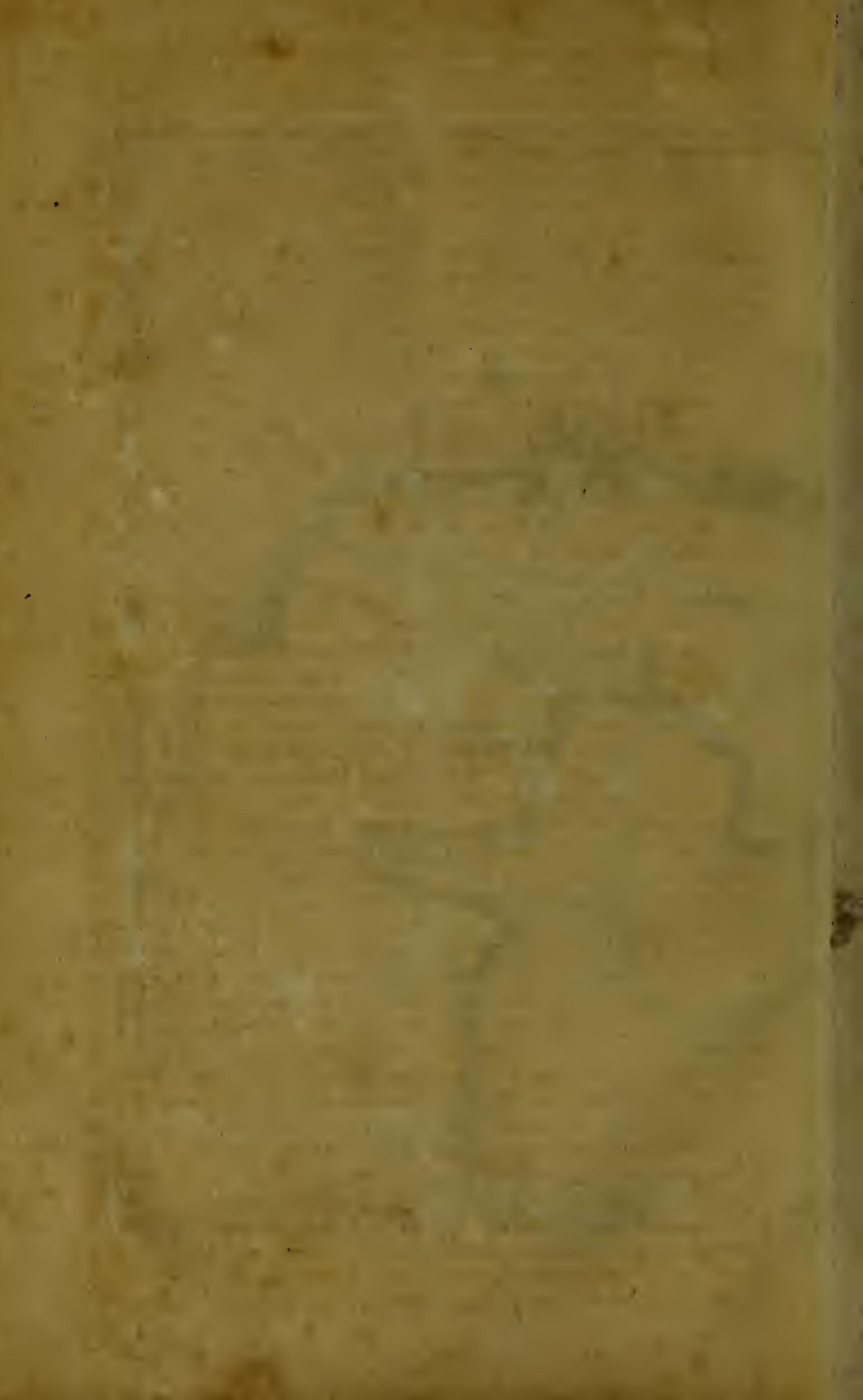


1)et XIV. Capittel.

\section{$V$ an Indien int Suyden.}

$\mathrm{H}$ Cet Deel uan Indiē int Șu= Den/onrecljtelick gljenoenut America, í de freele ontoet = limabe ban Nombre de Dios af en Panama ten Sunoen / waer in be= floten werden bet vafte Lanbt / oe livicten van Peru; en Peru Chili; t'welck be Indianen noemen Chille: De plovincten uan de 5traet ! tu oe tieviere de la Plata, eti Brafil; al waser 5. Hoven jüin / dat van Pana= ma, lyet nietibe Zijick ban Granada, S. Francifcus de Quito, Lima, los Charcas; en elf Bouuernementem/ cen oecl ban laer Culte raeckt aen

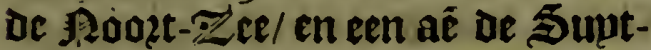
Fee/ in de welcke ten meetten deele regeert de Sunden en Supt-nes ite-1Bint / die tegljens fiju natuere is allgier loct / en de groote ljitte tê= pert / waer dooz nen can betwoo= tuen dit Tant / al boe wel dat bet nimumer en reghent/ noch faghelt/ beljalben in leet clepine dittantie/ Les coxdile en de twee Cordilleras die Dooz defe

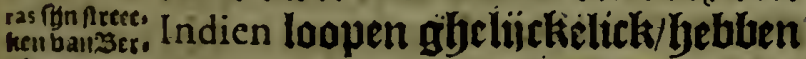

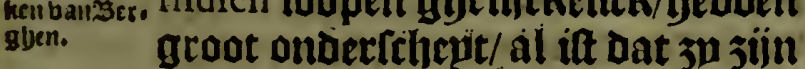
op eenparige hooclite ban oe $\$$ ool/ om dat oe cene is teer becleet met 250 ffelyen/en daer regent yet altijt/ en is ljeet/ oe andere is ganfely fon= oer gheboonte / en igcout in fiin Be Cordile- Sonter en Mainter:bere Cordilleras

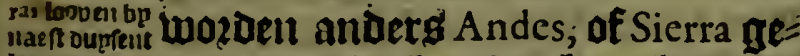

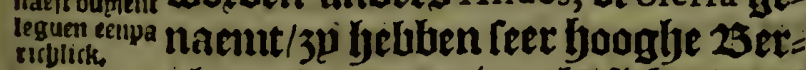
(r)jen : en loopen int gbefichte d'eeri baut d'ander dupfent leguen) bp nareit eenparicly. Op lyet gebertyte teelen verfeljepden gljedierten / en in de deelen oaer $3 \mathrm{p}$ baer openen/ Daer maecken $3 \mathrm{p}$ Ballenen ! baer wel te woonen is / gljeliick die van Xa:ıxa, Andaguaylas, en Yucav: $\mathfrak{I n}$ De Andes werden oock gfyeteelt ber = Icljepdeute beetten / en vootun de Stadt vall Cuzco gaen defe cordil. leras baer bertyzepoen/en nemente int mibden ect groot Delt/Dwelelt

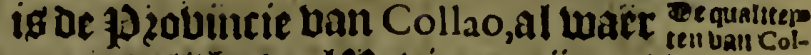
onfundelicke beel lievieren $3 i j n / e n$ ino.

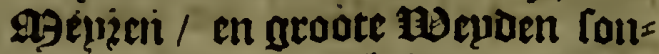
ber 23oftrlyen / nocly bout / ant de onglbetempertijent ban't Landt/al boe wel dat lyet gljeront is / en feet bebolckt. Sigue actiter dére 19 zovin tie ban los Charcas, warm / en ban groote bauchtbaerjent / met roume fentuelen / met groote Kiickbou ban grijnen. liset fatloen ban dic Indien lietmen in be boozgaende. caette. if , 1142 $\div+11$.

Het X V. Capittel.

Van bet Reffort van't Hoff van Panama.

T Et reffozt ban bet jof ban Panama, t'welckeerlt gbe: naent ig Caftilla del Oro, en baer naer 'tbalte zland $/$ is feer clepn/ om dat pzincipalick albier refibeert bet 1 of /om bet delpactiees ren ban de Bloten / en Coopman= Itbappen die gaen en comen naer. Peru, betbeeft in be langhte bant Boft en noeft / bp naeft tnegentich leguen / tuan de Gzenten bañ gec Foubetnement ban Cartagena, on Popayan, tot aen Cấtillo van Ve. ragua, en inde berette ban de suntZee tot aen of Poozot-Zee ginf waert ober de trefticf leguen $/$ en bier benedé tot achtliten ban Nơm bre de Deos; of Portobelo, en hae Panama, het Ianot is generaliuck gantech roubu ban gheberclite / vor ban gozaltijen / en gbeflootent loclyt met dampen/wochtich en lyeet/ en dat is doorlaecke ban beel fiec= te / ban gsep tot - Robember toel 'tis een on uevichtbaete zlerde / ent Daer gljebsectien bele bingben/om

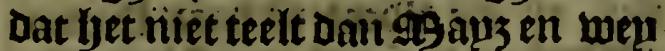
nicly / theeft ebeniwel goede $13 \mathrm{ep}=$ aen loos de Hoenen / en bee-tees $f_{2}$ lingly. 


\section{Fol.44: 2belchativinghe}

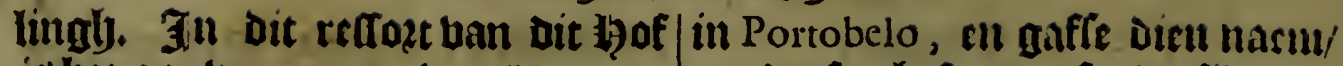
is bet gouvernement ban Veragua, om datle ghefonder foube fijn/ en en in dit / en in t'shene van $\mathrm{Pa}$ - bequanter tot be ladiugte en ont: nama jijn be Dozpen die nae bol= aben.

- arara. De Striot uan Panama, op oe Culte vande Supt-Zee/Dicht Dact aell / op negben graden bzeette/ en twee en tachtentich langte ban of Supt-\$oozt-liin Lan Toledo, ban waer bet af lept lanex een reebte Tinie is 60 . Leguen / t'is een Jozp met les hondert 230 aglers / t'mee = Itendeel Coopliedé/ en bandelaers/ en albier neffens het lyof/ refideren albier / De (Bfficiers van 'rConincr incomen/ en De Sclyat-ktilte / Die Gract gaé na Portobelo, alg de 1 blo= ten daer ziin / op de defpalthe van Dien/ootk is lyier hetCathedrale fuffragaēfchap van het Zlert's-25iroom de los Reyes, en Dzie Cloolters van Dominicanen / ffranciftanen / $\mathrm{en}$

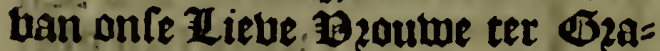
tien. De lgaven ban de Stadt i\$ redelick / bat al boe wel oe - Sche= pen by ebbe op dzooch vallen / en derbalven in be Sonter comen $3 p$ booz de llaep/en in de PBinter / in oe liaven ban Perico twee leguen ban de Stadt/ Pedrariras Davila be= bolcktede de plaetle / als bu Gou= berneur was uan Caftilien del Oro, tegheng de wille van de 23 ozgherg ban bet oudeS. Maria de! Darien, int Jaet I s I 9.:en een mepnich baer naer quam aldaer de Cathedrale litereke/ en mochte wel beter litplaetre bebben / en ghelonder / en gbeleghenoer on redenen vall de contractaticn van de Supt-Zeel Conder groote aftebeudingbe van de plaetfe / al waer nu defe Stadt íg.

De Stabt uan Nombre de Dios, is eerlt glyefet int flaer I 5 : $\circ$. ban Diego de Nicuefa, en Daer naer ban Diego Albitez, Dooz lalt ban Pedrarias, en die aldereert bare 1 auen ontberktedel is obetweelt de eerte sumirael/ en fp is getranlposteet

ladiuge van be 19 loten/ en oul bare leeckerbepot / cul ball de nicuwe Stadt van S. Philippe, die aldact gbefondect is/ lyeeft de Jugenicur Baprifta Antonęli een Cafteellien ghemaectat/ en een ander abedefig= neett fiost aen Dander fijoe van De Laben / om dat 30 detl inganef couben belwaren. asen baengbt ore Coopmantrbappen vall Portobelo tot Panama dooz twee wegen/D'cene ober landt in/ met Carouanen ', van waer het is achetbien leguen / van minder difficultept dan dooz de wech ban Nombre de Dios, en D'an. Der is ober Zee / en be Fieviere de Chagre, wiens mont is achthien le= guen ban Portobelo TBelt-waerts/ op de weleke de Coopmiantebap comen / als het water rijlt tot de venta de Cruzes, en ban daer gaen $3 \mathrm{p}$ met Carouanen s. leguen tot $\mathrm{P} 2$ nama toc.

Te Stadt Nata is Dertich leguen sant lago a uan Panama Meet-waert / op de Culte van de Supt-Zzel de Cas pitepn Fráncifco Compagnon be bolcktede dien booz laft ban Pedrarias, tot den Bozloch met den Cazique Vrraca. De bebolekinglye ban Acla begonte te fondeeren de $\mathbb{C}_{a}=$ Ads. pitepn Gabriel de Rojas, Doo? lalt uan Pedrarias, op de Culte ban de \$Roort-Zee / en den inganck ban ae golfe uan Vraba, teghen ober ban het eplant de Pinos, van fet luele= he in teglyenwoozat glyeen gljelyeu= obenife neer af en is / t'ledert bat aldaer ftierf de bermaerde Capi= tepn (wieng ghedenckeniffe altoos Cal bupzen.) De Adelantado Bafco Nunjez ban Balvoa, en van fiin me= Deghelellen. In de cloobe van de 23 erch A lmagro, en int beginne bant De Keviere Chiepo is Gout / all it voozgaende tij ố trockmtender beel upt: in alle oe thevieren siin beel Capmannen / en feer groot / die de 


\section{van VVeft-Indien.}

erte olutoecliers 1 chl bevedoigtiers ban ljet il ant lteloen/groote moey= ten bjebuen achglyedacu / en aten

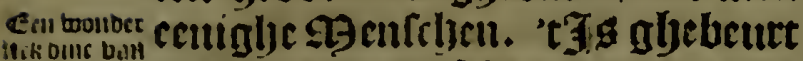

eai co, pan. Datt.

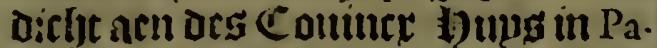
nama, en cen Cauman roofde bem ban lyet arijterte buat' Soljip/ en barrde lyeill oill te ectell of cen liocte / ch 'tgljelucurde als lje be=

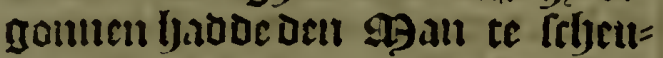
ren unidoen Doos / dat ju bem feljo= ten uset cen 1Rocr/ cn Doodedé henu/ cil 'tDede dit usozoeel Dit oe asent= felje fteruenoe / ontfincli de Sacra = unenten uan oe liereke.

De provincic ban Veragua, dic op wat meer onu eljien graden lept/ parelt met Coftarica, int woeften/ itreclit yem in de lauclite Boft ent Helt so. legueal/ en in de brette 25 .

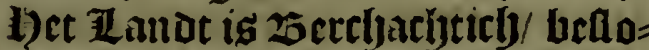
ten met Doonne bofrchê en Strunbellen / fonder $23 \mathrm{ccm}$ den/Bee: Ta rume/ en Garlt/weunich $\mathfrak{N a n y / c u l}$ Cun-Crupoeren/noclytang DoD: = loapen met Bout / en met becl $\mathfrak{A l e}=$ ren bact buts / cul rucke Agijnen in de ticuicen en clouen wan oe $25 e r=$ ojuen / en Indianen vie un Oozloglje

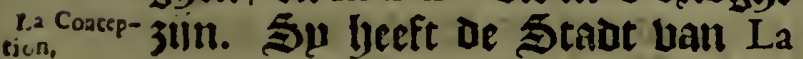
Conception 40.Iequen ban Nombre de Dios Weft wacet/ al wact refi= oeert de Gouluetneur/ en de Offi= ciers die beden gljeltelt werden van $\iota_{2}$ Trinida'. dic Uin Panama. Dt Stadt Trinidad ts 6. Ieguen ooft-wactts uan oe Conception ober $\mathbb{Z}$ ee / om Dat= ment te lande niet gaen rau / dicht aen de licbier Belen, 3. leguen ban De Zee. Oe Stadt van Santa Fc; 12. Ieguen bau be Conceptic Stupt-

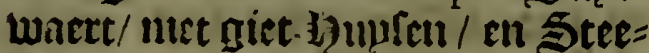
boutuers van oe officiers. De cartos, Stadt Carlos op ove Culte uan de

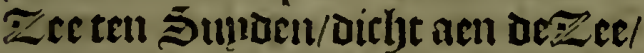
ro.leguen van de 5 tadt uan San Fe 3ecit waerts. Zulle de Indianen ban dit goubernement siin in $B 0 z=$ loghe teghens de 5 paengiaerden.

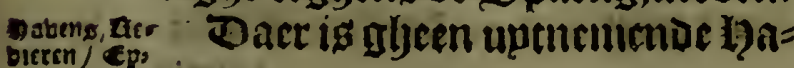

bepot Culten foo $\Rightarrow$ utut als ballonten balt \$0020en/ van dit 5oubernement/ ous wfoato cn int gantiche reftoet ban dit bof: 'tlject acfenabolghende 1ieviereny Dabens / ch Dumten. Je Bape uan Carabaco, of ban S.Ieronymo, op de Cufte van oe \$oozt. Zee / en de ILantpalen uan Veragua, Ooltwaert ban yaer / en de Ziebier ban la Trinidad, en die van de Concep. tic, en die unn Belen, al waer de certte bevalckingbe bag die ac eer. ite ztomitacl dede int valte landt/ van bele ganteche werelt int Taer I 503. die nict en bleef dupzen : en vooz de Efcudo cen Eulantat/en be Lievier de Chagre, en wat Boftwaert een legue de Portete, tot waet de somirael ghecomen is ontoes= fiende int felfoe jaer/ en de labueng Wan Langoftas twatlf leguen ban Nombre de Dios meft-waerts / en die uan de Gallinas negben graden/ cn die van Buenaven tura feg/ Portobelo s. en baer teghen ouer oe $\mathbb{E}^{z} \mathbf{p}=$ landen de las Miras, en die van de Baftimentos, en twee leguen voozbn Nombre de Dios, de lievier vall Sardinilla, en die van Sardina 4. It guen/en die ban פ9apz/ en Die uan Culebras, ell die van Francifco atjit leguetr / en in den inganck ban oe golfe ban Vraba, al waet int Jaer I s09. fondeerde de Bachiller Enciro de Stadt van Santa Maria oe onde del Datien. 'troag die Bachiller En. cifo die vooz gaf Datment in ot 1) 2ovintie Caftilia del Oro plaet'rn waren / al waer men yet Bout bi= Iteden niet Setten / ' welck cozagie gaf aen beel liedent te gaen naer In. dien , int Jaer $151 \%$. met Pedraria Davila, en int is 1 3. ta upt Darier gfettotken de Adelantado, Bafco Nunjez de Balboa, om op te foectien de Supt-ZZEe/die hp oork ontoeck= tede. De jounte uan fiet ceplandt de Cativa, leut recht vooz de Clip= pent uan S. Blas, en bet eplant van Comagre, en dat van Pinos, bet de golfe ban V-raba ini./ en in bet bints ff 3 nenlte 
itenfte uan diell oe haven van Nilcos, ontrent de 2 gont Dan de the biere del Darien, die de le imiten Deplt ban dat loof / en die ban ljet Goubernement/ ban Cartagena; en aldaer ig bet Culata Uan Vraba; al waer int iaer i s io. Alonžo de Ojeda, S.Sebaftiaen ban Vraba betbolelt= tede. Defte golfe lept op ẩt grta= ocn/cn beteft I 4. Ie guen baì lẩnclitê binnen thaitts-waerts/en inden in= gancli betft lyet fes in of beette/ en bet boozts s. en op'tepnoe 4. en 5. leguen innelwaerts / is abeweet ot Stadt uan S.Maria de oube del Darien.

Bpde Supt-Zee is of Cabos. Maria, en de 13unte de Guerra, en vooz Panama óe golfe uan Parita, of Paris, al maer Nata is/oe punte ban Chiame, yet Zant ban be Cazique Chiapes, een bzint uan Báfco Nưn. jez de Balboa, die hom hiétp in fiin ontoeckinglye: en booktiv oe ligaven uan Panama De Levier Coquira, of Chepo, en die de la Balfa, of de Congos, in liet binnente ban do golfe

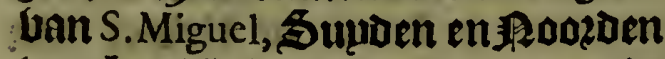
van bet Uizlanot de Perlas, en de 3unte of liaven de Pinjas, in oen inganck van oe golfe aen oe Suptjijoc/ Dwelck is biffticlj leguen ban Panama; en twinticl owers ober tot Vraba, en oe lgaven Quemado; diclyt bp oe Cabo de Corrientes, op bif graden ban de \$oozoer pools joochte.

bet X V 1. Capittel.

$V$ an bet RefJort van bet Hof Sania Fe dé Bogota, twielck is bet nieuwe Rÿck Granaden.

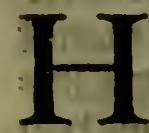

Et tieftozt uair't of ban nien Granaden ftreckt ill be lanckte Boit en toelt daie bonoert leguen/en oock loo ueel ten

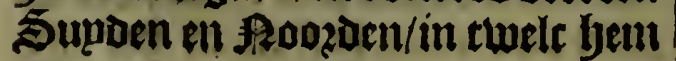

beflupten of provincien ban bet Iiculue Zivick ac Goubernementen van S: Marra en Carragena, en efa Decl ban Popayàn, enl onl oc $\mathfrak{d y e}=$ bupzeftyap de plovincien del Dorado, of nieu Eftremadura : De $\$ 2) 20=$ vincie ban lyet nicutwe Ritic/ tureleli is 'tgene dat lyet l)of regeert/Atreclit hem int langle ban't bolten m't Doeften i 4. Irguen / en tacbtentich in oe bectte Supoen en \$200zoen/ ban een effen beubelicly 21 andot/ meettendeel met Balleinen en glie= berchten : Lan gocde nocuben booz alle Coozten ban bee / Dat Daer iil abondantic is / en in veel deelen ig Carwe / Sganz / en Batelyten wan Caftilien, en geutepnlic veel bouts beel fijn / en פgiinen ban Coper en ajur/ en de Ingeloosenen żin mee= Itendeel feer habil/ groote ljande= laérs/en cleeden hate met Cattoen. De Dozpen die in dit 1hijet zijn ban oe Caftilianen jijn ocfe. We Stadt van Santa Fe oe Bogota die santafe. ghefondeet is aen de boet ban yet gbeberchte Bogota, alfoo gyenoemt uan de Cazique, die ghenoemt was Bogota, twelek bebolcktede oe Adelantado Gonżalo Ximénez de Que. fada; on gaf die Stadt Dien nam! en bet kiick / oul bat ypeen natu= reel was uan Granada, nocljtans in oe ontoeckinge fjadoe een deel de Adelanitado Belalcazar; en Nicolas Federman : Ip leut op $>2$.graden eu én falf op oe langte ban de Supt

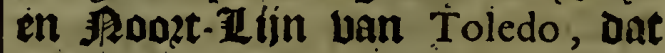
op een groote Circkel fal fiju 1440 . leguen / en 4.graden oluer defe fijoe ban of Æquinoctiael. Daer ziin meer dan fes lyondert 2302gljers: en Daer refideert lyet ly of / en be Bfficiers ban fConincr incomeny en de Schat-tifte/en bet Giethups be Cathedrale Metropolitaen, wients Suffragaen ziin 7 Popayan, Caragena, en S.Marta unet een Cloofter vau Joominicamen / en een anoer bau ffranciftaners / en in fijne Jurif= dictie meer oan so. duplent ciribu= 


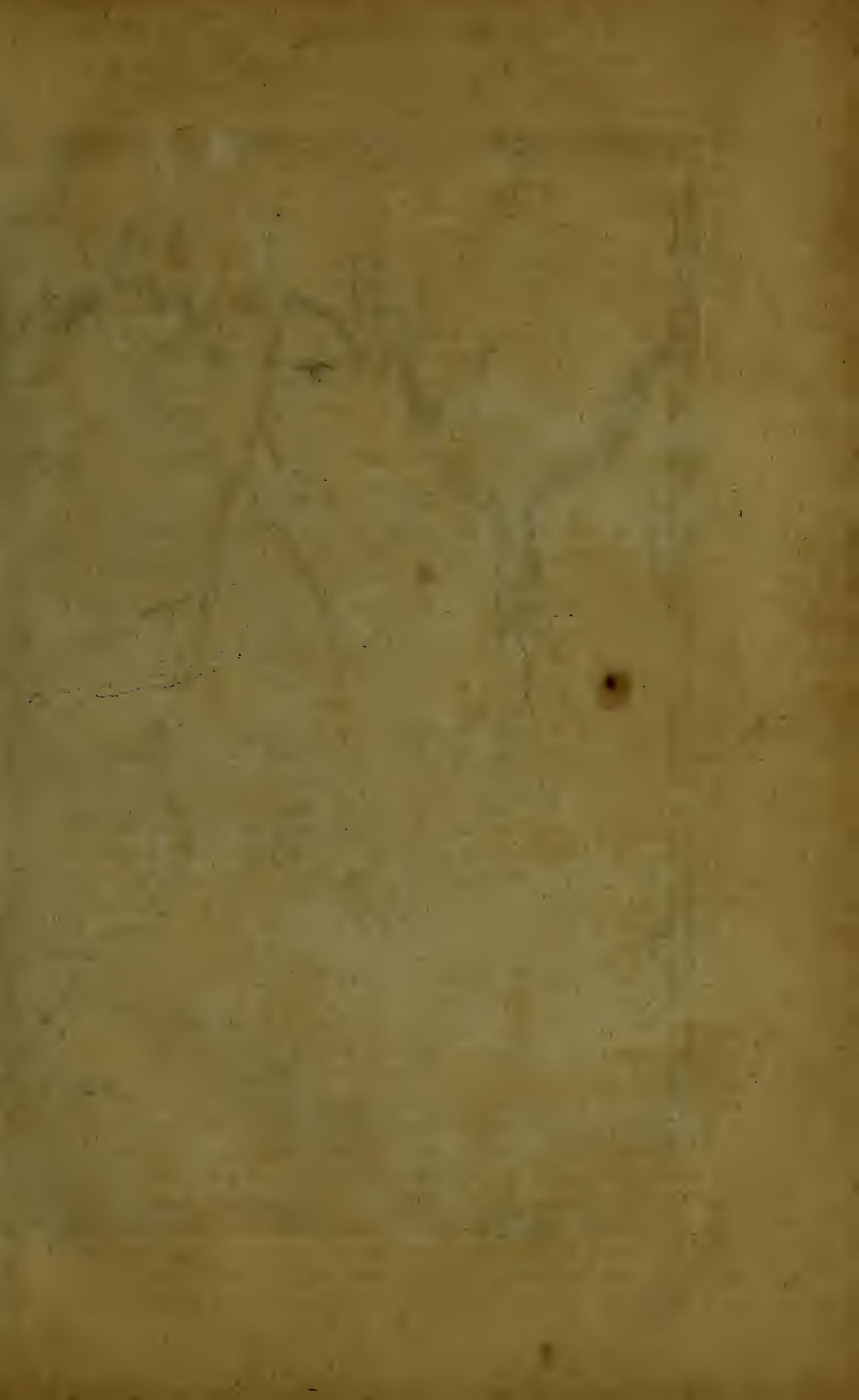




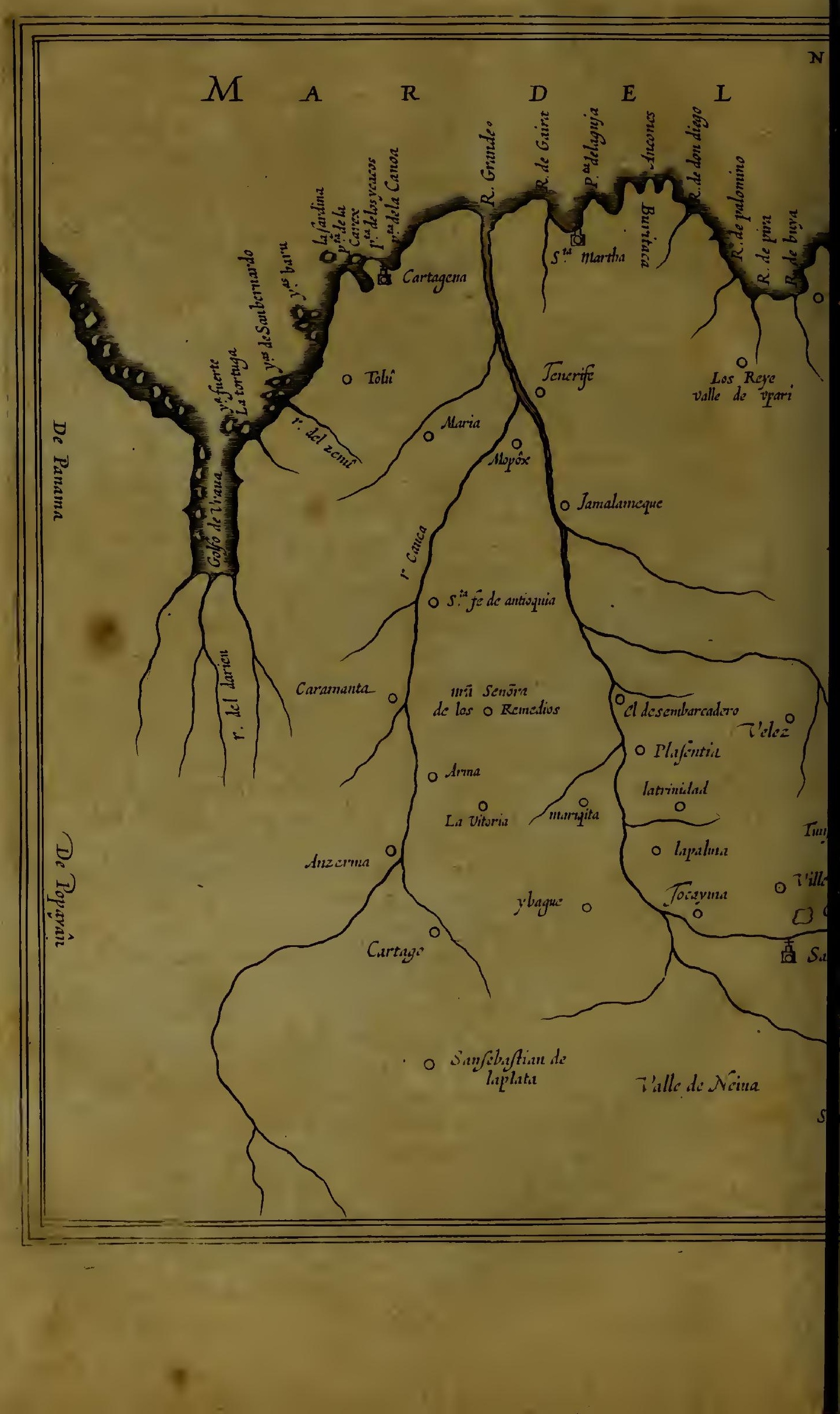




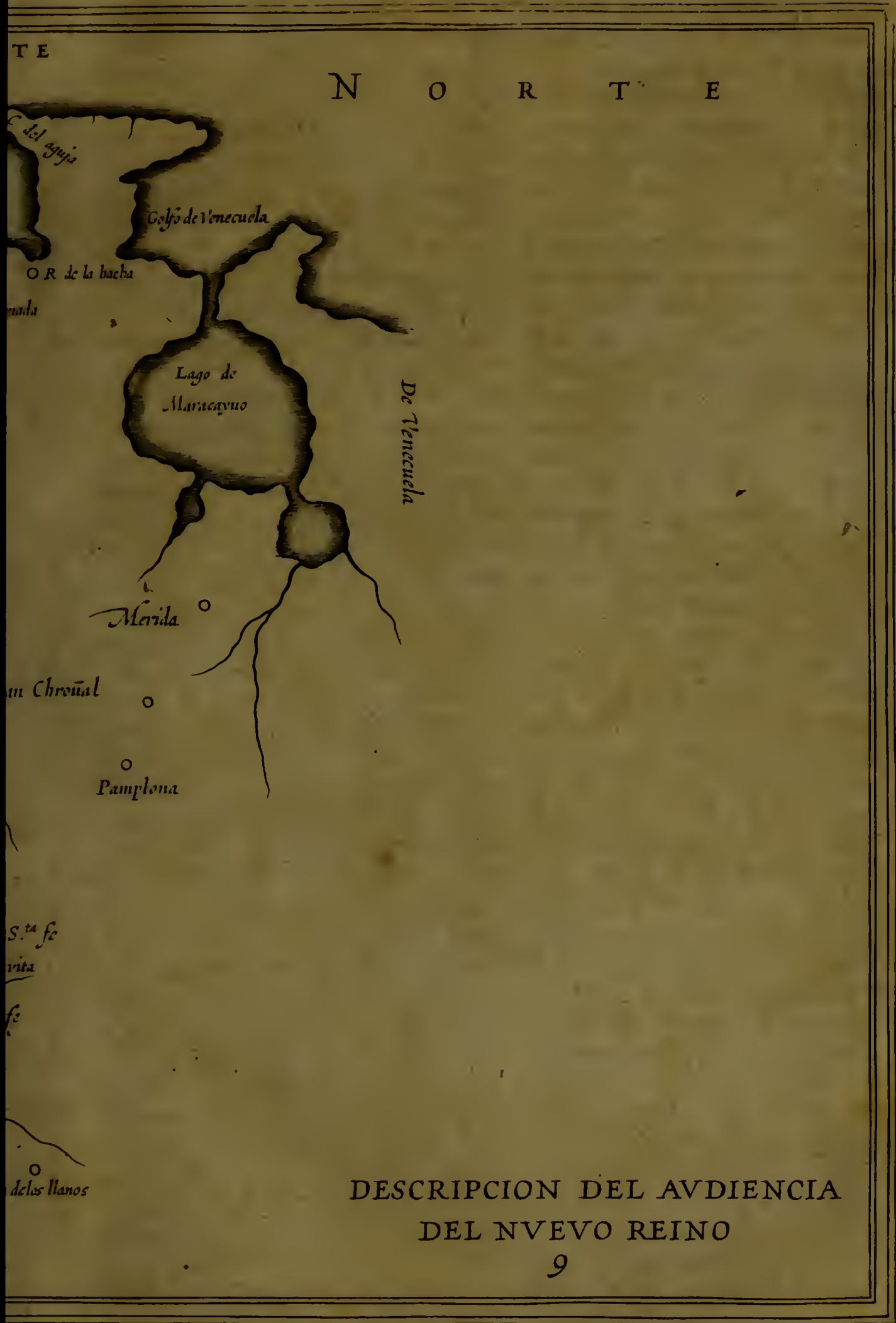


$-$

I

1

ats

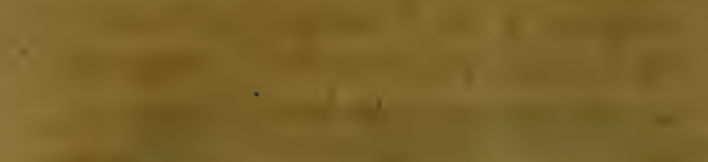

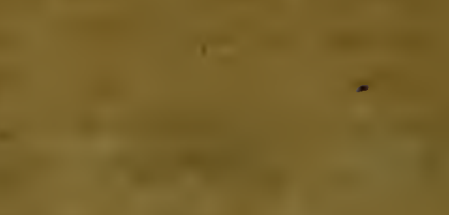

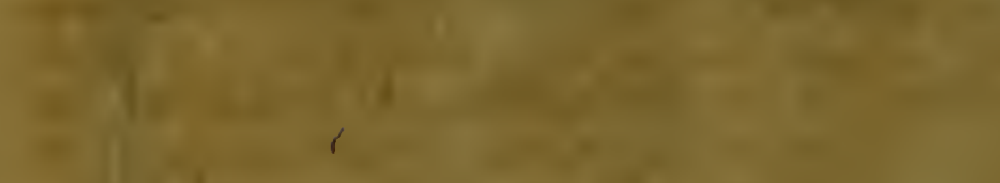

$-1$

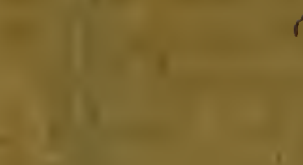

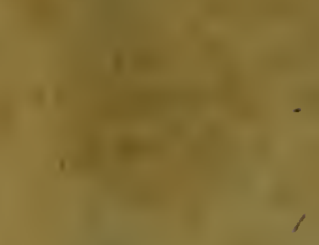

$\cdot$

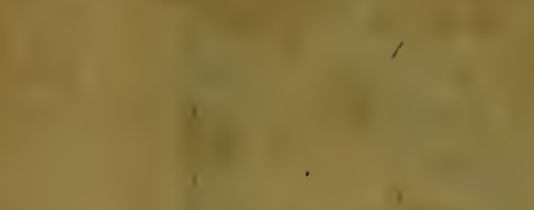

1.

(1)

$<$

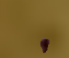

$$
\text { Pa: }
$$

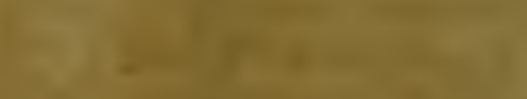
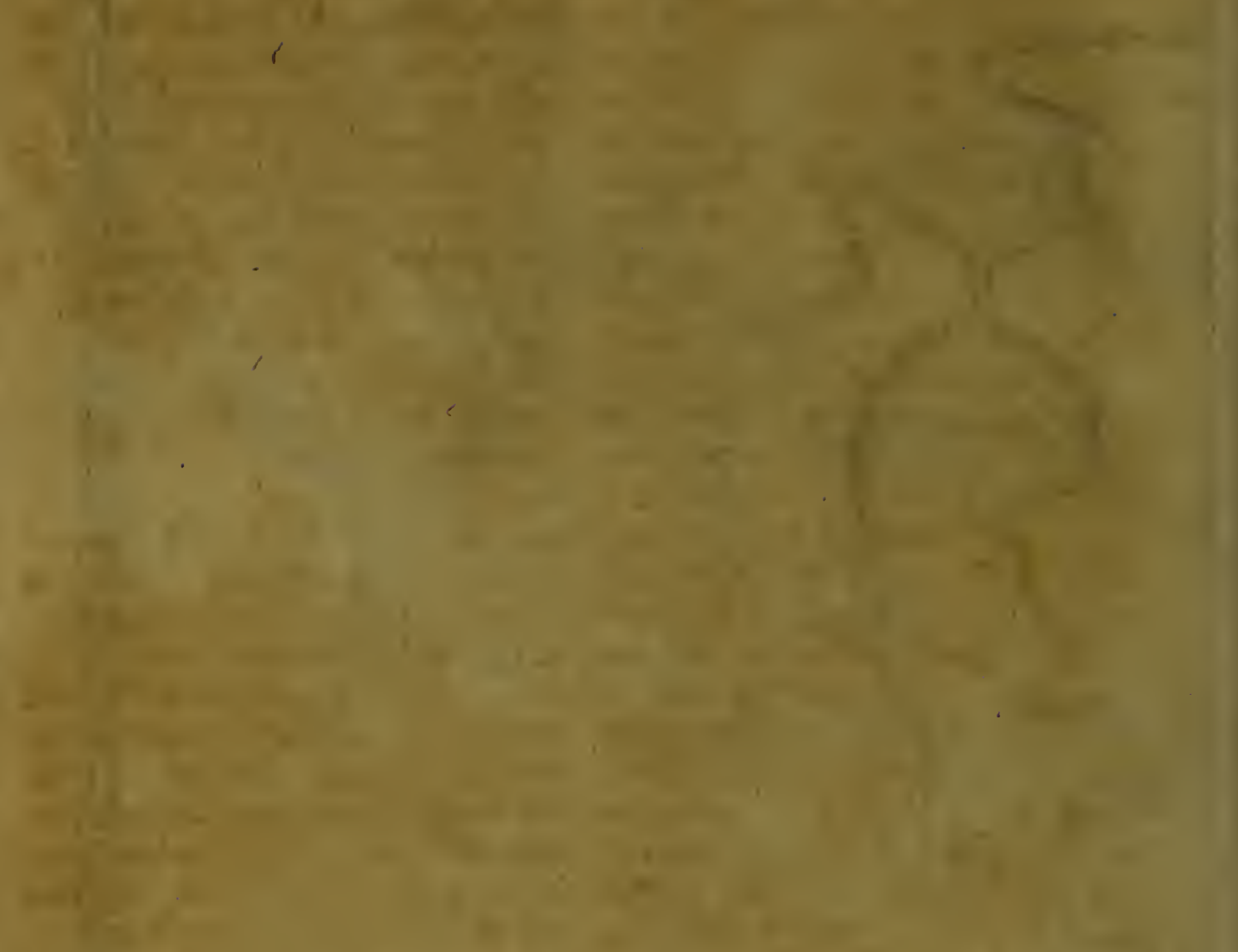

1.
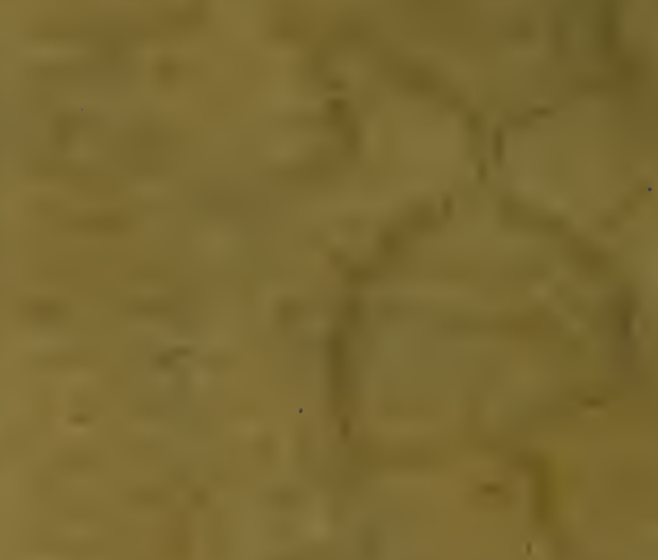
anis 19

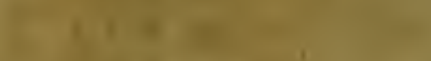
$x+y+\infty$

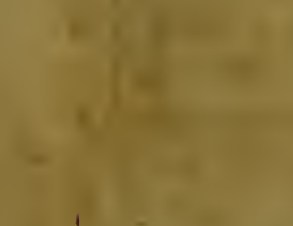

$(1$

$$
\text { titing }
$$

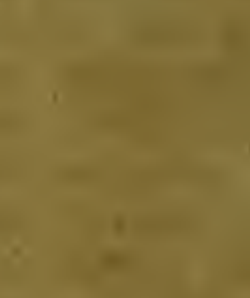<smiles>C1CCCCC1</smiles>

ran
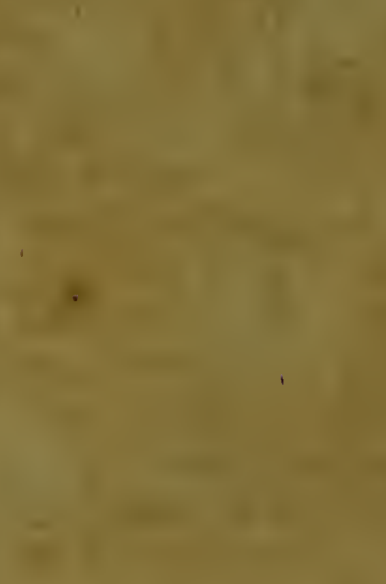
อ: Mex: ail Gudtaquart deḍe te giach uare Santa Fe dat coltt it. De Striot Tocayma, i s.les gucul ban Santa Fe Boelt-waerts/ wat uelugljente uire ljet Roozoen/ inet ecti Cloolter ball Domiuica = new/werbe bevalcht int 3 aer I 545 . Dooz De Capiremu Hernando Vanegas op be cillt ban be paincipale lievicre Pati, die baer ontwatert in buc uan Mugdalena; lyu ljecft glyeen Gout / ell is be aloer-wertmite / en Inachts en balter gljeen boww in. In tyet gantiche tiijeti en is glyeen alghemeene tale / en bie nen neet verlatet is die uan be Panches. In de 13zoluincie ban los Mufos en Colimas, Dienten met cen âliber name noemt Canapeis, twelck beeft - 25 . legutn in be lanckte / en Dertlyien in de beette foot-Boft-waerts ban Bogota, cen rouwd Lant / ghe tont/buchebace bantioepoen/gout t. Trinidad. en efmerauden / De Stadt Trinidad jiin twee bebolckingljen / twintich

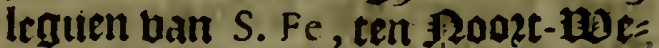
Iten / De belcke bebolchtede de Capiteun Louys Lanchero, int 3 laer is 82 . als fyp ginck ten oploghe met de Indianen Mofcas, een loos bolek/en bicelaclutichs: en in dic $\mathbf{3}$ it. riroictic is oc riicke grine ban be Ermerauden/met veel Indianen, bie nochniet bebzediclst en sijn / in in

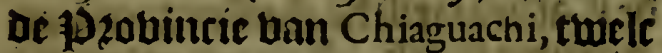
betceckent Caracoles; om batter uecl jiin.

is palma. We Stadt uan la Palma, in los Colimas, ban nteet hitte dan toubel bijftlien leguen ban $S$ : $F e$, naert
Noozt-10rtten is gytuout int iar I 57 2. ball de Capitepn Don Gutiere de Ovalle it de Bzobincic ban Tunja; die liju ment crijelst bau ftin Cazique, nae bipecljt int \$ool= oeH Uall Bogota toe/en uar bu op de frlfoe wiile als is oe Staot ban Tunja, twee m twinticly Irguen wan S.Fe, bl cans noodt-o oft-waerts! op een booglie 25 ercly am te wefen cen fterclie plaetle / liebben $3 p$ die Daer glyenateckt tot Den Doilocls tegeng de Indianen. Die benglien op vali die Stradt twee bontert te goeert / ende is de oberuloedichete bau ll iff-tocljten ban oefe gantíflye Turifoictic / ct baet wert ghedaen De grootte Coopmanfebap ban bet

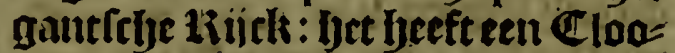
fter bant Dominicanm/ ell een an= aer ban Jf tanciftanet: de Capitepit Gonzala Xuarez Rondon bebolek = teoe dien/boóz Den Adelāiado Gonzalo Ximenez de Pulfada. Be Stabt ban Pamplona trefticly leguen bait Pamploan Santa Fe, ten Joozt-Ooften : Géft een Cloofter ban Dominicanen/ baer wert beel Gouts uptdibetrocs Iten / en t'feeft beel Bees: Be Li centiaet Michiel Diaz de Armenda. riz, gljzboode dien te fonderen. Be Stadt ban S.Chriftoval 13. Ieguen s. chitionst van Pampelona Jooktwaerts/wer be bebolekt bain Capitejin Fran: cifco ban Cacires; ontrent bet Lant Itfjap de la Grita, alloo ghenoemt/ ont dat de Indianen upt quamen on be 2 erghen om te ofteben een abs: tier op de Cátilianen, en dan oo or te Gaen: Daet wert vergaêert foent nich Soutg / en 'thedft groote glys = Ieglitenthent om bet tê teelen. De Staot ván Merida in de laht: Meilds palen bain bet Sowbernement bar Venécuela, en 'tnieube Riijelt/.40. leguen ban Pamplona I Poozt-Boft: waert\$ / is een buchthaer Xanot

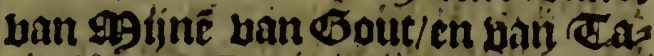
ritwe. Oe Stadt bat Belez dertich leguen uan Santa Fe Roost-waettíl en is.tan Tunia, feeft een cloofter 
Uan firallcilcauterg / Je Capitepn Gonzalo Ximenez Rondon bebole= tede dien / is een $\mathfrak{z}$ antat daer beel Dondertagljen pleglien te ballew/ maer IIu vallender loo beel niet/ ait bat baer in te bulpe comt het aloerbenliclute 5acrament Des $24=$ taerg: Daer is ế bzandende 2serch die veel Steenen upt werpt. De

sariguice Stadot ban Mariquita yan Ybague, anders ghenasint S. Sebaftiaen del Oro, 40. Ieguen ban S. Fe $\$$ Rop2twort waerts /-werde bebolclit int Faćr I s s 1. ban of Capitepn Pedrofo, in een Belt dicht onder bet gljeberches : Gact glyetemperthept

rague is leet beet. De Staut Ybague Dertich leguen ban S.Fe, bp naed int Hoeten / bet ecrite Dozp ban Jet nieutwe thijck/ Dat acn Popayan paclt / or Capitepun Andreas Lopez de Galarza bevolckecde dien int jaer A S, 1. Doop laft van't thof om te bergoeden de fobaden die de Indianen oeden aen die ban Tocayma en Cartago, en oin te opener een weeb tot fet gouvernement dan Popayan, beeft een Clooiter van Domini= caners.

envicotias

Oe- Stadot de la Vitoria de los Remedios, so, Irgurn van Santa $\mathrm{Fe}$, ten Roozt-10elten/is leterijck ban Siran de loo Bofinen. De Strat ban S. Ian de los Llanos so. leguen van Santa Fe ten Fupden t'is een Z and Daer bet 5 outs is. In dat Gouberne= ment is ontbolctet oe Staot ban Tudela dien fonbeerde de Capi= ten Pedro de Yrfua, ooos laft uan be 4 icentiát Don Miguel Diaz van Arméndáriz om dat de Indianen Moxeas Dater rchaoe ban badoen:

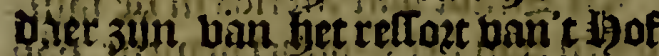
Un in thicune Lilick $\%>$. \$orpen ban Het Gouluenenent ban $\mathrm{Po}$ plyan, Sania Fe pun Antiochia, Cafanán ta, Arma, Anzerma, Cartago, en S. Sebatiaen de la Plata, en S. Vincent de los Pazes.

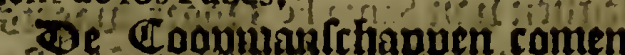
inoat trijel lailgs of groote ate vire de la Macialena, ball de squmuel van Malambo af ill De 3 uríioictit van Carragena, ell of ecrite die glye= Conden lyecft on te ontiocken iefi tievicr/was Garcia de Lerma, Gouverneur uan Santa Martha, int $\mathbf{Z}$, aer i s 31 . 54 loopt in oe $\mathrm{Eec} /$ coo bzeet/en treffelitis/Dat als oe serile= pen daer boozbu liepen oat $3 \mathrm{p}$ ill periickel waren / in Dicn jp Dare niet beró af baren/ om de tach uan de vloct / en bzectien bande Zer/t'beft ech Eplant in de mont wert befeple meer dan bondert so. leguen / en uteer dan date fyotideit leguch oppacert is fn onder gront/ Ip Ipzupt bouen Popayan viust twece fontepnen/die 4o. Icguen berffhey= ben ziin / en baer famen uoegbende maeckenfe een ticbier : fp wert ge = noeme de ia Madalena, oll bat op dien bach de mont bande felfoe tie= bier is ontoeckt op twaelf graben foocijte / en fes en twinticly leguen ban Carthagena.

De 1520)incie en 'tgoubernemêt ban Santa Marta, op de Cufte ban't 5,Narsha, balte Zanat/70. leguen lanck en bzeet tulteben Cartagena, en de LiE= bier de la Hache : is etn 2 and vzurlytbaer ban 99ap3/ en 23ata= tas / veel Bouts/ en Coper / en ee= nigbe Etimerauloen / en andere Steenen : daer zijn s. Dozpen uan Caftilianen, en beel natureelen/ meet lupoen ban d Bozloghe. Die Stadt uan Santa Martha be= bolclitede de Adelantado Baftidas, uit Faer I s.2 s. Dirbt aen De Zeel op thien graden bzectte i en vier ett ticuenticls lancte/Duplent bier fon= Dert twintich Iegum uan Toledo, al waer refideert be Gouberneur/ en be Coninicklicke Dfficiers / $\mathrm{m}$ be Cathedaelirbe Suffragaen ban 'nieutue kiick, (c) is een redelicke Galien. Dit Gowuernement lecft 4. Bzovincien/Pozigua, Berona, Chimica, en T ayrona, twelck beteeclient cen. Sornais/ en met reden/ onn dar in oit 3 ande is ean onepnoelicli 


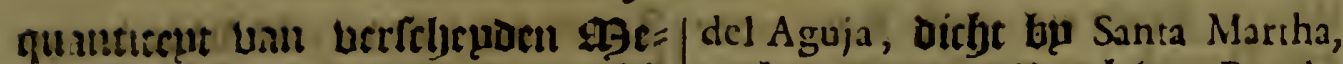

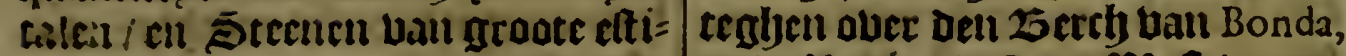
tite/ of wirerje.

Eenerife.

Tenerife, bll oe groote lieduiere uan la Madalena, 40. Ieguen vall Sanca Martha, bu uacit ten BuluatWibitm' cenfoecls te Wider/ eare= oecls te Lanoc. Francifo Henri. quez bebo!cktede dicri/doos laft ball Gonzalvo Perez, die gonvertiecroe in Santa Martha, osoe laft uan oe Adclan ado Lugo. Tamalameque, oft D: Stant de las Palmas, 6 ;. Ie = guen. Uan Santa Martha tell Sup= Den/en tbitutidy uan Tenerife : twee leguen ban oe grootezievier/werbe bejoleter int I wer i ;61. van Capi= trun Bartholo ine Dalva. De Stadt ros Reyes. bam los Reyes, in de Dallepe van Vpari, uzuclitbace ban E⿴anj' IL tiftocht en Dee / en van Ueel Coper) ten 5 sut Doften uan Sanca Martha so. Irguen Daer af / m Dertich uan de Reviere la Hacha, bejolelktede de Capitelin Santana cooz latt uan de Ticentiaet Miguel Diaz, tn te bozen

La Ramada. Laas bevolelit la Ramada, Dat eecet ghenaent wart uieu Salamanca, 40 . teguen Uan Santa Martha, Boft. wa.aetts / cu acht uau de ziebier la Plata, aen de loopenoe 3 eecken ban het glyeberchte Nevada, Dat in De Dallepie uan V pari leut/al waer foo beel Coper is als Iten. Be Coop= manteljappen ban dat Gouberne= uneit comen int nieulwe Kiick/boo? liet Slick uan be Stadt / bue acht Iegues leut uan bace ouer Zee / en boots twaelf tot de decinpel uan Melambo, in de groote lictuier. Dock Ient in Dat Goubernement Ocanja, twelck bevoletrtede int jaer I 72 . De Capiteun Francifco Hernandez, en werde eertt ghenoent Santa Ana. Op de Culte ban dat Goubernement is oock de Reuier Buhia, dicht aell Ramada, en die uan Piras, en die ban Palamino, al waer oe Capiteun ban bic naem verdonck : en die van Don Diego, los Ancones de Buritaca, ente Cabo ch de kevier de Gayra Helt- waert.

De Dzaluincie en toouberne= ment uain Cartagena op de Cufte caragenae wati t bafte laut / en be Sopat-Zee') fal hyem ftecliten in de lanclate opot en weft / wan oe tieviere de ta Madalcna, tot de kietuier del $\mathrm{D}_{2}$ rien, tachstentich leguen Sugnoen en Roozoen / en oock loo - veel tot de Lantpalen Uan't nicuberiaick / al joe wel bat of weeb wozt ghefent Hecerder te fiín. Het is ant is berchs= actiticy / vol thotren en Ballepent wan boogbe 3 oomen / reghenach: ticly / en vochtech , de Saben van Caltilien gheven gheen Iart upt/ Daer is gheen Carulwe/noch Sout/ ban ill enighe Contreqen. Baet werden ghenlaetkt veel barfen in cenigbe Bergben van dit Souber= nement/cn aromiatique Bommen/ ch andere vachticheden die $\mathbf{5 p}$ unt oc 33oomen trecken / en groote quantitept uan Dzaecken-25loet/ en cen wel-rieckende 23alfem / en ban groote crachten.

De Stadt ban Cartagena is bicht bet fravt aen of Zee / twee leguen ban be genaso

Punte de la Canoa int Buelter / op tljien graden uan bzeette/en 76. pan oe lanckte dupfent bier bondert tfe. tich leguen uan Toledo : van meer dan s.jonbert 23 azglyers/en onder die meer dan twee bupfent Bzous wen. Allfier refideert be Gouber= neur/ D'Officters van des Conincr incomen/en de Schat-laifte / in be Cathedrael Suffragaen ban't nieube Tiick / met een Cloofter ban Ba: minicaners en f rancitcanerg. gljelegbentbept is black/en bprang als ecn Eplandt/ teghend 't $\$ 200 z=$ Den/ al daet ontrent de $\mathbb{Z}$ Ee/ en t' is een woelte Culte/ en leer ondiep/en bp't Zanot isten ztrm ban de Zzet die vergadert een $95000 e r /$ cat een פgepz is bie ghenoemt wert de $\mathrm{C}_{2}$ napote, dic uloept en ebe/ met be ¿ee/en op de felfoe upge: men gaet 
vin oe 5tadt ou't balte Zant/ober cell 23 zugh en op oe ninirice ban ecn gleplaucpoe Lueris die ontrent niarlj weien uan twee hondert so. pacten. De Stad is ghefondecrt ap Sant 1 bace 3iln Fnljammen Dace unen foet toater bunbet / Dorl] ishet fomitits ongljecont/nochtans Too lecr niet als de Cllite van Nom: bre de Dios, oun dat de $\mathbb{Z}$ oefjen ten refpect bail oe agopder-plagben piinen te uerog? laecken / mochtants if teh meerten-deel abrout. De baven ig bande belten van Indien, alloe uel pe groote Schepen, uer $=$ be legglien vin of Staot als jp ach comen : 'tbect in oen inganch ceir Culunot alg oat uan Efcombiera in Carthagena uan Caftilien, Doch grooter, Hoacrom fu bet nố Den vorli Carragena, eni het Eplant itocticos zy vin Codego, en wert un olienoemu ban Caxex, bet fal sijn tuie leguen ine lantlis en fencu: nicls meer alg Jilf foo bzet: lyet plach belwoont te zily uan d'Indi:

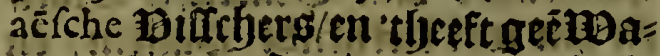
ter. "Oe certte dic Carcagena lach/ int Tner a 502 , was Rodrigo de Baftidas, en inf 1 net dupfent vif honDert Lier quim op't Llandat Ian de to Cóf, cu uonot Louys Guerta, an Die warell a cerife die d Boplogbe begonurn teghens ac Indianen; foobacturich en ucricert uernicleos 95 annen en 10 zoulucin net vergif= tige 1 Silen ôner naer quã Alonzo dc $O$ jeda ureder / met lan de Ja Cofa, boo opper-forloot en Americus Vecpucius baot Schipuer : ctl ceni=

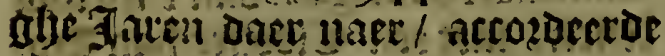
Gregorio Hernandez de Obicdo te bejolcken Cartagena, en lju ginci

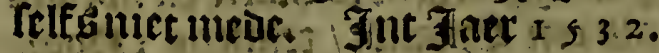
was Daer Don Pedro van Hcredia gljebozen ban Madrid, en lju bebolelite / en bebzedichoe cen groot Decl ban't tant / al hoe wel nict arbest cn liet / om dat let was cen bolliccus Dolck. ch Daer wing ecil Degulue unu lop-rans acljtljen
Faren out/ oe welclie vooz dat $3 n$ yaer vingen boodede met faer $250=$ ghe ache Cantilianen.

Oe Strat Sant-Iago van Tolu, sant Iago de

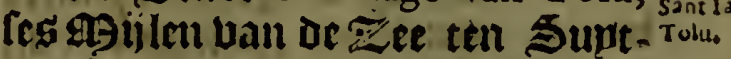
10eftell ball Cariagena, twacif le= auen daer af $t$ eentoeels ouer Zeel om datuien te lande niet Ean gaenil en eenloeds dooz de $\mathfrak{a y 0 0 0 e r}$ en glyeberchte/ is een ghefont Ilanbt; yan Dee-teclinghe/en Tlandt-bous winghe / en Bzuchten dam Cantilicin, 'tuerde beuolekt ban de Adelantado Don Pedro de Heredia. Oe Stadt ball Maria twee en Detrich Maria $^{\mathrm{L} 2 \text { vilte de }}$ leguen van Carthagena ten Sula: oen / ig oocls de bevolrtsinghe ban Don Pedro de Heredia int Taer bup: fent uiff hondert bier en dertich: De Stadr Santa Cruz de Mopox, theluenticls Ieguen ban Carthagena oluer $\approx \mathfrak{z} /$ ell or ticbier de la $\mathrm{Ma}$. dalena, op wichs cante dat (u leut/ cil al waer op om-loopt bp naedt wan de midoen ocs bechls is olls

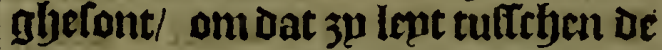
990zafthen. Een Capitepn van Don Pedro de Heredia bevolcktede dien int Taer ouplent biif fyondert

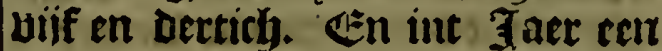
Duprent vijf hondert nealjen. $\mathrm{Ba}$ chiller Encifo (als glyefeut is) lue= bolelitede Santa Maria de oude del Darien, die in bet felfor Gouluet: nmucht is : te niet doende de Stadt Sinte Scbaftiaen de Buena Vifta, bet welcli beluolekt haboe 'ticifoe Jaer or Capitem Alonfo de $O$. jeda, ill den intwijels unn Vraba, cil Dacr nace quan weder om te bebolrken Sinte Sebaftiaen, de Ca pitevil A lonzo de Heredia, vooz or Adelantado liin 25zacber / in ecll cicpin locubeliten / bu cants cen balf legue uan de $\mathbb{Z} e c$. $\mathbb{C E}^{z}$ int $\mathbf{3}$ aet Dumfent biff yondert feluen en der = ticli numm van Sinte Sebaftiaen de Zirentiact Ian de Vadillo, met een goct gljetal Soldaten / en lijoende grooten arbelzot / too op de aloer= rouette Clippen / als doos De ucel- 


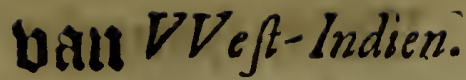

Jeflid Der sicrglyen / qunu in oe Strlot Antiochisit lyet Gonvernement Luan Popayan, en lyn baadoe een -5olonet dic uau Dace quam tot ant ir Stader ar la Plata,elt los Charcas, t'urleli stun Dunfent en twee IjonDert Iegitin.

Lo Bast mas L.a Barranca van Melambo, twuelelt is crul 1) ults al waer uten de 5 elje= pen anthit om aen Col welte Doen betillen / is una de Jurifdictie bau Cartagena, Derticly leguch Daer af: op De rant wal De groote Revier/ cul twinticly ual Santa Marra, en les

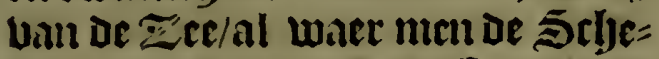
pen ontlaftet / van De Coopman= febaupen/ Die ment boert ober Bant iin't nicuwe Liiiels / ent ban De Barranca gael ju op oe kievier unet $C_{a}=$ noen.MBat leeger ball Mopox loopt be licuiere Cauca, in Die ban Mada. Icna, Die oock fiju op? [pzonck wecmt Goven Popavan : en meer une Cartagena, en 1 bett-waert ig be (eljoone Morro, en De J Junte Zamba, en de Buhio el Gato, of De Arboleda, en De 7. Buhios, ent de puate uan la $\mathrm{Ca}$ noa, twee leguen ball Cartagena, en de puttet Lun los Ycacos in Den in= gancli van De loaven / recht tegeng oluer bet Eulande Lait Carex, en De punte baul la Nao op liet valte laut: acu Den anderen cleque inganck

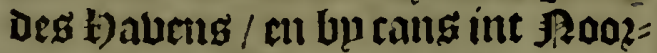
Dentisteell Epiandeken Dat ju noe= men Sardina, ell op oe Culte uan Tolu zijn De Eplanden unn Baro, Die fess jijn / $\mathrm{cm}$ in Den ingancli van be golfe de Vraba die les die ju noe= men dic uan S. Bernardo, tegtyeng olver De Lieluier Zenu, en meer de golfe intwewaent is het iterelf $\mathbb{E} 1=$ landt / en la Tortnga. De Haven bau Zenu is 25 . Ieguen van Cartagena, t'is cen groote Sape die fijn utgancli ljeeft unil't Ooffen / fu ig beplich: hier wett beel Souts glye= maeclit / En heeft fiin naem ghecre= ghell bau't Dozp uan Zenu oat op De kicuiere wais.

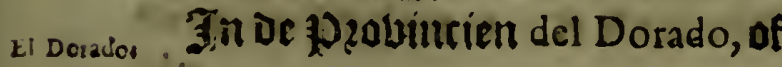

nicu Eftremadirra, of welclic ollit de mabunzerclyap befjoozen tot fyet tiels loet ban fyet bof baut' niculwe rites

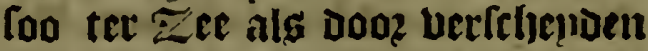
Declen bault ILand / jiin Dacr in getteden bele Capitepunen/en beb: ben niet beromen os Riiickionumen Die Jet stjerucjete beloofde. Oefe p) zouincien ronen te Ieggijen aen dander fijoe vall de vievier S.Ian de las Amazonas, anders ghenaemt Orellana, Dat ecnigbe met minuer= Itaut Luilien Dat lyet De Maranjon [n/ en alljier isj de Orónico; an andere eroote Rebieren $/$ en oe Golfe van Paria-, De welcke maeclit bet valte lant/'met het Eplanot van la Trinidad, met de gronden vall la Sierpe, en De Drago, wietden alloo gljenoent ban de cartte ztDinirael / al waer fjp lyem in groot perticliel fach Dooz het ltrij Den Dat aldaer geflyiet unin De Coctellaaterê net de Couten/ ban lyet weldk te woozen geen lien= nifte en was: an al frier begint de Luaftiuglje bau de Zze reer groot te wozaen tot de Straet ban Magellanes toe/en gatt alfoo contimues rende dooz de gantrfye Culte balt Peru, en nieulwe Spaengien.

$$
\text { PIR V. }
$$

E Probincien van Peru, (weiclec naen bem nu wijDet uut (treckt/als te bozen) befunten bet ganticlise: 1iijck van De:Ingas, ofte nteer : Als bet gbe= wonnen wettoe/ werde bet ghedeplt ill twee 5otwernementen/Dat ban Don Francifco Pizarro, ghenoeme nieu Caftilien, ban Quito af/ tot Curco toe / trefticly leguen beneden Chinca, en Dat unu Don Diego de Almagro, gettaenat fiet nieube Toledo, twee houtdert leguen tot aen de Straet toe ban Chinca af : Lelc = lse Eothertiennenten waren ber fifjenden / tot dat ljet bof de los 
Reyes, ghefonteert werde / m ver= Dat begint bau Sinte Francifco del fien werden met een Dice-12op uan be liijcken ban Peru : in welcke Goubernement baer beflutieten. Het Wof Jan S.Francifco del Quito, Dat uan Lima, of de los Reyes, het de los Chatcas, bet goubernement ban Chile,ent de Yan aen bande Straet De Céplandé banSalomon int ter $E$ Iten/en om dé tialuupefrbap de $1920=$ bitucien uan Rio de la Plata, en' $\operatorname{tg} e=$ ne dat bu bepalinge begrepen wogt onder het Boubernement ban de Bice-1Rop: 't 5 al bem trecken in of lanckte Supden en Poozden olier Duplent leguen ! en Boft en Hisct / 'tolyene dat ontoeckt ig ban be Suut-Zee af/ tot aen de \$ooptTete. Dook alle de jozouincien ban ae a.cordil Peru loopen / De twee Cordilleras,

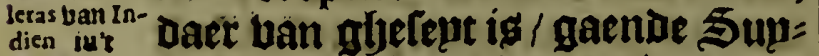
Suppent. oen en Poozoen die van die Andes Lan Popayan af/en tod eeniglye wil= len ban fyet balte lant $/$ en niet Hifpanien af tot datmen comt tot in de Straet/ en De andere niet loo verre van Quito af tot Chile, langs oe Culte twaelf Ieguen daer ban inne= waert\$/wepnity minder of meer.

Cuffenen de twee gbebercliten gaen be twee wegben / de eene die 5u noemoen de los Yngas, Dooz de Andes uan Pafto tot Chile toe, dat utegen honbert leguen in be lantktê yeeft/ en 25. Boeten gbejlabent/ en ban 4. cot 4. Leguen Itiucen feet coltelick/ die $3 \mathrm{u}$ noemen Tàmbos, iin oe. welcke provifie wag van eten en cleedinge/.en alle Iralbe leguen lup= oen die op are bolten ftonden / oin te benghen enighe aoviten/en oz= Dounantien ban lyant tot jant. De altoere weels ginclt dob? 'tmioden van de ulackte / in oe lanckte van oc. Culfe/25. Boeten bzet/tufferen twee Handen een badem boocly. 1)et ftaet te bedencken/Datniet ver = ftaen nuoeten lueroé ondor de naen peru is bet ban Peru al de Indien ban't Suy=

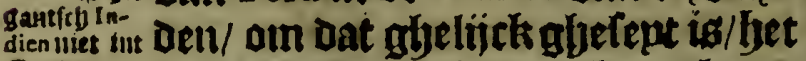
Siluphen. nist anders en is oam bet ghene/ Quito, dat beneden De midael zilin is/ en loopt lanckte tot Chile, loo= pende bupten de Tropicos, twelek fal siin fes bondert leguen / $\mathrm{cn}$ buf= ticly in de beette/al boe wel bet wii= Der is tot de Chachapoyas, en 1 s glye= oeelt in Dzie orelen; de blatiten die tJien legtien in de brectte silil / ell op lommighe Steden mikder/ die zijn de Cuten uan de $\approx e e . ~ B e$ gbebercljten die fullen twintich le= guen zijn/ en 'tsiin alljoocljten unct eenigbe Ballewen / ende de Andes fullen andere twintich houden / die de alderdicljlte 23 affeljen / en $33 \mathrm{er}=$ gljen $3 i j$ / $\mathrm{en}$ in loo clcene diftuntie bu naeft so.Ieguen/ cum glielisrs verfebenoen jijtide tuan oe Iiniel en oe Pole: Daer ig foo groote ver = lebepdenbepat / Dat bet in d'ecne Decle altoos kegbent: en in D'an= ber bpeang nimmermeer / t'welck oe Cufte is/en in fet derde' ant ljet glyelereflyte is/ twelck balt int mid= Den ban de twee uptterlten / D'eene tijot regbent ljet en d'ander tijot niet / om dat bet boudet ziin \$o= mer en Binter als in Caftilien : en de oozfaecke waeroull bet niet re= ghent op de Culte/ en bat bet in of Andes reglyent / is ghefept int be= ginlel van of siltozie.

Het X V I1. Capittel.
Van bet refJort van't Hof van S. Francijso del Quiro.

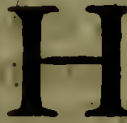
Cet keltazt of de Jurifdic: tie ban bet of oat welck in Quito refiocert / cu paelt int poozoen met lyet uan Panama, in oe Gaben uall la Buenaventura, en int joozt- Doften met het niens we 1Rijets/ en int 5oupden met bet ban Lima, Calmogben ftreclien ii be lanchte in de Cufte uan't Su!n= den/twelc igal waer bet hem meel upt 


\section{DESCRIPCION DEL AVDIENCIA \\ DEL QVITO}

$\begin{array}{llllllllllll}M & A & R & & D & E & L & & S & V & R\end{array}$ 


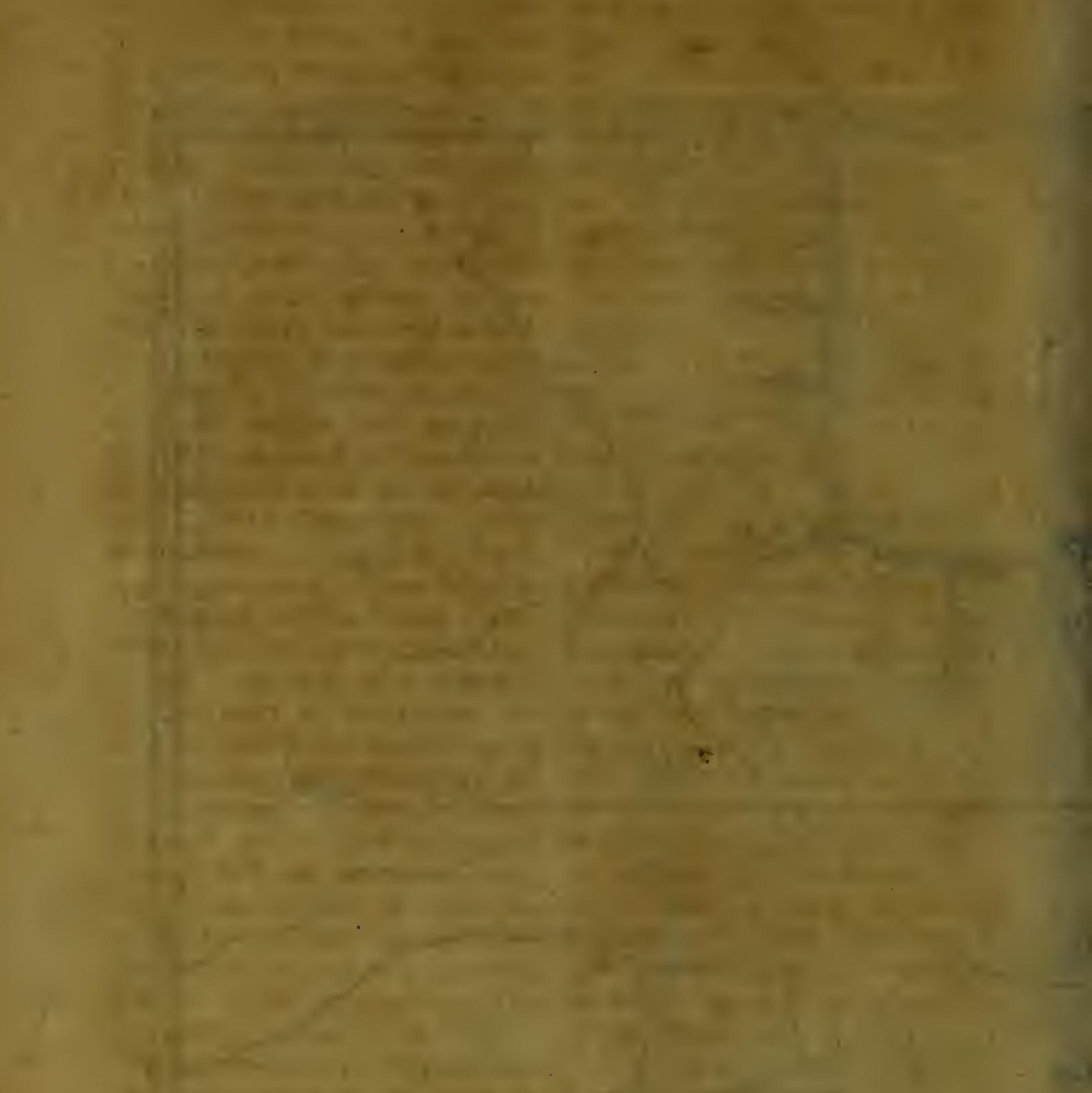

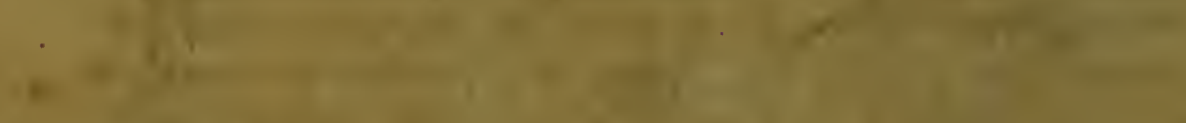

*⿻一𠃋十

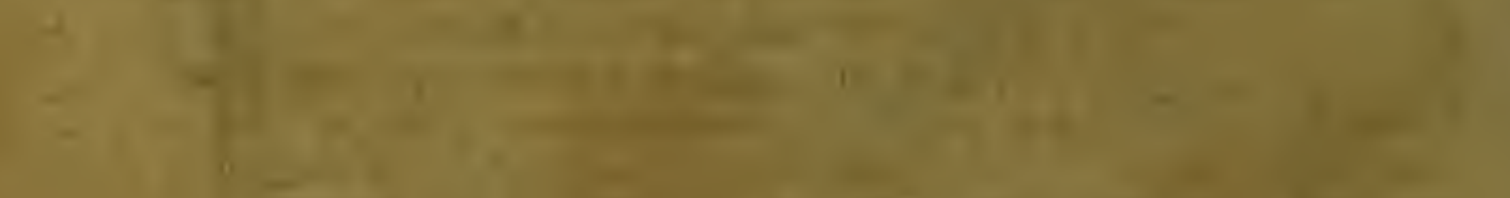

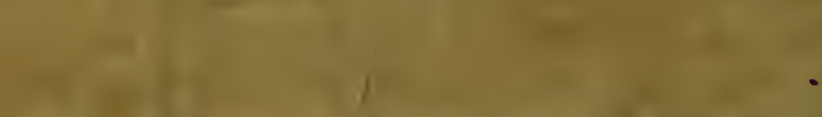

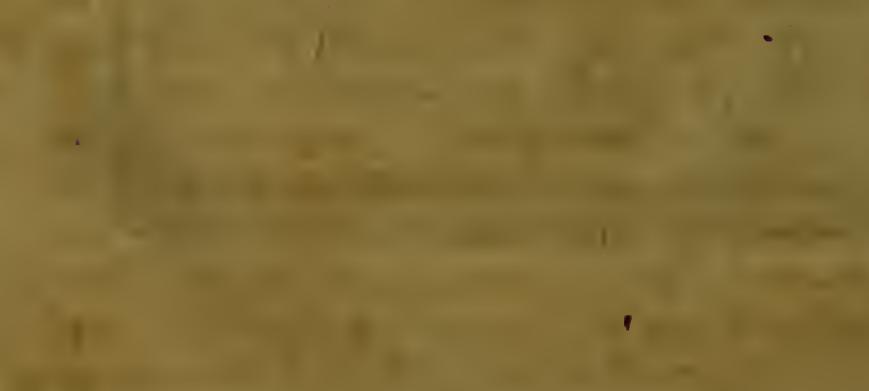




\section{Uan $V V_{e} f-$ Indien.}

aut ftreclit bu cans twee lyondert Ieguen/ Uan de b)auen uan Buenaventura, die ill de golfe ban Panama is/ of ban S. Miguel, we L)aben ban Payta op de rulte uan Piru, en ban Dare Dueers ouer tot lyet uutterlte viln Popayan, meer dail nocly twee bohtoert jo. leguen/ unt Tonderende Die opene Ziluten aen lyet Dofter $=$ febe deel / beflutende oner in dacie Goubernementen/ Tonder Dic ban't Lof/Lelelie zijn Popayā, los Quixos, enl la Canella, en Datt uan lan de Salinas, ball los Pacamoros; $\mathfrak{e n}$ Gualfongo, oljedeplt in twee 2sitoon= men.

De g zolvincie en 'toboubernes. ment ban Quito, nwelck is t'aljene Dat lyet lof regeert / fal moghen Atrecké tacbtétićt) leguen in lencte/ diebt bn Detl Æquinoctiael na daan= Der fijoe / en daer in żijn Defe nae= volgenbe Dospen ban Caltilianen. Sijn 1 entel en bet iLandat al boe wel bet onder de Zinie is / is bp cang ghelijek met die uan Caftilien, beloer en frboon / neer cout Dan heet / en in fom lteden al waer ot Sneeu bet ghebeele 3 laer duyzt. Get reghent Daer ban Ortober! tot ga artium toe/dat $3 y$ noemen de Hinter/ en in de andere 9 aenden gSaeptmen en vergadertmen. yet Szaș / t'welek al boe wel bet niet reer beel is / dient boos bet Bee ban Caftilien t'belck daer beel is / en beel Carutwe / en Garti / ell Gout op eenige plaetlen/ en in dit zant $=$ tclyap leeft-men feer bzesdament= lick / Daer ouer datter gyeen dinck is ban meerder plaplier booz bet menirbelicke leven/dan luft te beb= ben in een gefonde en luftige locht/ oun bat ju glyeen Bointer belbent. bie haer quelt met coude/ noch $50=$ mer die bereert met . Jitte: - De be= bolckinghen ziin dele. De Stadt ban S. Francifco del Quito, al waer ghelioozen wata Athavalpa keurex ban Peru, fp lept op een balue graet hoocije van de Equinoctiael, en twee en taclyentiely uan de Sunt en S, oort-PLin bar Toledo, op een groote Circkel duulent feg hondert feg en taclytenticly leguen baet af/ en 6o. van de Supt-Tee: 'tljeeft s. hontart 23ozgers: Dner refideert bet 1 of om faecken ban Fultitiel oui Dat dic van't Gonbernement siin tot lafte van de Diceron. Def= gbelijer refideren daer oorls in die Staot / ac offiriets ban ljet inco.

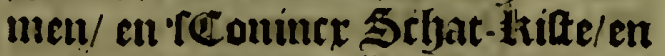
oe Catjebzale fierclie ban bet 23if= Dou/ lyet Suffragaenfibap van de Alertg-13iffejop uan los Reyes: Dite Cloofters ban jominitaners/ jirancif́tamers/ en 919etceniers : elt in lyaer Jurtroictie 87. Dozpen/ of partijftyappen bain Indianen. Bp defeplaetle al waet defe Stadt is ghefondeert / was een groote liger bergbe bien be Coninek Topaynga boudede / en die berfjeerliclite fiin Soan Guaynacapa, en noemie vien Quito, baer ve Stadt fijn naem af nam: of Adelantado Sebaltiaen de Belalcazar beluoletede dien/ welende een Soldaet ban Don F rancifco Pizarro,etn man die de Coninte glje: trou wag/ bu laft van de Adelan. tado Don : Diego de Almagro, Die Gem nam booz Bouberneux ban Defe 10 zountiel als in baer geepn= Dicht toas ire twilt met den Adelantado Don Pedro de Alvarado.

Riobamba lept in oe 19zobincie Riobamis, van de Purvaes, t'is een llandt ghe= liick Caftilien, in de getemperthept ban Gas: / Bloemen / en anbert dingben /. Date is een Jozp van (jerders/'tlept 2 s.leguen té Supt's Beften ban S. Francifco del Quito. op ae weth de los. Reyes, in lyet welcke zijn by-naeft 40. Duplent booforn uan bee / Jetmeefte necl 3iin Stljapen / al warer Ingen bad: den een Coninctilicke becherghe! en alfrier hadoe oe Adelantado $\mathrm{Be}$ lalcazar eem pzofijtelicke 25 ataille met de Indianen, en bu bertwontel en in die placter was de boostepoe 


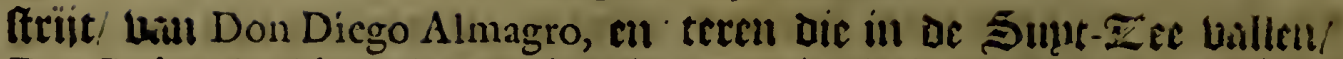
Don Pedro de Alvarado, en in die bant die die in ve nodet- Tee bal= was ertitelick gefondect destnot uall Quito.

crenas.

De Stridt van Cuenca welcke glyeboodt tefonderen de 9 arquis ban Canjete, wetende bice-1iop ban Piru, die op eet ander. naem gijetromut was Bamba, s I. leguet ban Quito Supt-waerts / ig een reclyt-pleginglye vooz een pozouific yan de Bice. lion/met een Cloolter ban Dominicamen / en andere ban S. Francifi Bzoze : in hare $\mathbf{F}$ urit= Dictie/3ijn rijctse gsijuế Lat Gout/ ceniglje ban Sillers/ en rijtk van Quicklilber / Coper / Herer / en or

Loxs. Jteen A3ufte. Or Stadt Loxa, anders gljenaent la Zarça, tartyten = tich leguett ban de Stadt Quito, bu naeft ten \$updé/ en dertictj van Cuenca, is een recht-plegingtje/ een provifie wook oe tice-tiop : $3 p$ beeft Cloolters vall Dominitanen en francífauers/'tlept op oe weely Die loopt ban Cuzco tot Quito; waer af lyet lept tachtentich leguen/in of fcljoone Ballepe van Cuxibamba, tufthen twee kevieren: : De $\mathbb{C}_{a}=$ piteun Anthonio de Mercadillo fon= Deerde dien int Jaer I 4 6. om die Inghebozenen te bewedigljen dic ibealtereett waren.

zanora, De Staot.Zamora; die $j \mathrm{u}$ noe= men de los Alcaydes, t'neglyentichy Inguen van Quito, bij-naeft ten Supt:-Boften $/$ pafferende la Cordillèra de los Andes, is een rechtplegindue / pzovifie voos de Bice:

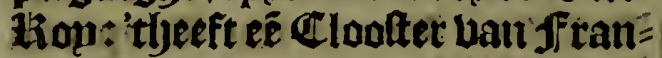
ciframers/ Daer wert geen cartwe gljewonnetr / om dat yet Lanot al te borfytich is/:tljeeft rilcke gsijnen

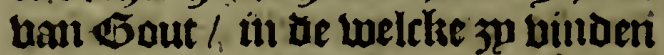
6zmuen ban vier joonden ghe wirljts/ en meer. Be Capitepu Meicadillo bebolektede dien int jaet 1; 49. Doos ober-een-coliunge net oe Capitepm Benavēte : 'tlept twin = ticly leguen ban Loxa, over de Cordiltera, die deplt de loopende $\mathrm{AB} a=$

Iell / De Indianen noellidefe Zamora : die glyeleglyantlyond of Contrene wert glychochut Poroanca, twelcti beteckent Indianen ball Gozlorly : daer wart veel Gouts untglietror = ken/ en daet jiin gljebzorljt acn fiil

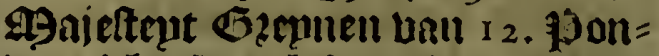
Den/ theeft oock Cout-g)anuch ban Cout-Mater.

De Stadt van Iaen is. Irguell van Loxa, m derticly uanl los Chachapoyas, follderde int $\mathbf{Z}$ aer 1549 . of Cnuiteun Diego Palomino, in de pozovincitn uan Chuquimayo, in die ban Chacaynga. De Striot ual

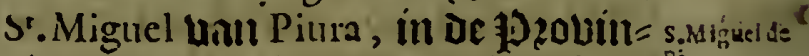
rie unin Chila, yonoert twintuly Ie = guen ban Quito, bn cangten SintOolten / en 2s, van de Laven van Payta,al waer epndiglyet lyet reffo?t van Dat 1 of / 'tis een reclit-plegin= ghe/ taende aen be provilie wan de Dice-1inou: Het lyeeft een Cloolter ban onfe zieve Bowuwe ter Gza= tien/en al loe wel lyet in dit 71 ande niet en reghent / Dan met ver won= Deringlye: foo beeft lyet gaede be= Douwiughe/ waer ban het wel /oa= ruwe gheeft / en 29anly / en de sa = den en ozuchten ban Cáftilien. Int de Juriroictie uan dele Stadt igde Gauen ban Payta, op s.graden beSupaen de XL unie/die goet is/groot en vepliclj. All waer inloopen die Scljepen die uan Guatemala gaent naer Pcru. De S9arquis Don Francifco Pizarro fondeerde defe Strat

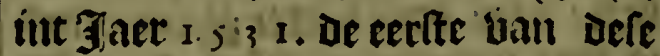
Cominckiticlten / al waer gljeliout iz de ecrite Kerclise ter eeren Godts/

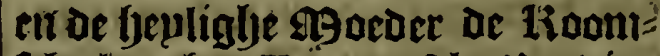

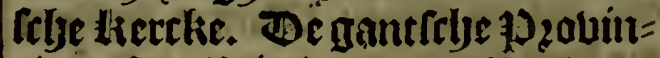
rie en Jurifaictie/ ban de ballewen de Tumbez, is Dzocly / en de Ca níncklicke weely / uau de Ingas gnet Dooz dic Balleuen uan Piura, turs" frfen glueboonten / en caelten / eit binnen de psincipale balleye voe glyen lyaer t'laulen twec of dzie ke. 


\section{טan $V V e f=$ Indicn.}

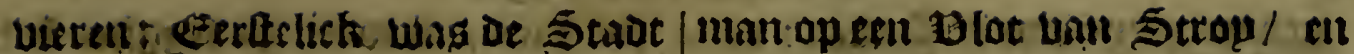
ahefoltoect in. Tangaz̧ala, ban wact ju oluergljefet is om pat lyet ech, on= ghe Coude. Contrute buas / mu nu is

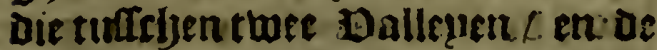
gantclues wecls is aat .ongherant/

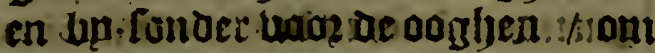

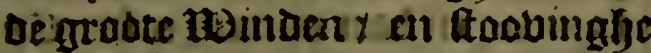

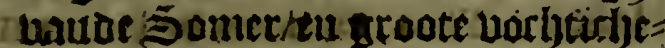
oentuan ae 10inter. . . : is 4 :Jut (Be) Strot Lan:iSant-Iago: Ląn

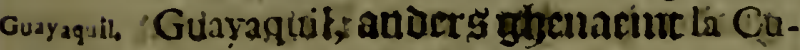

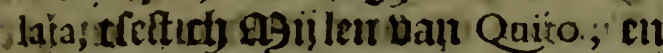

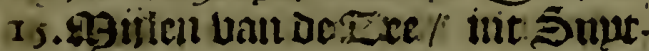

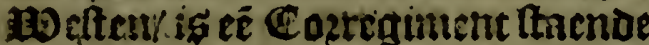
Eencoser

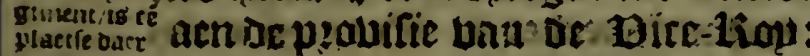

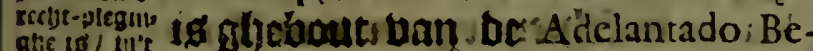

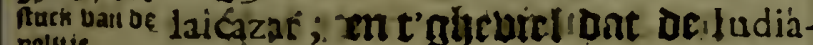
potutie.

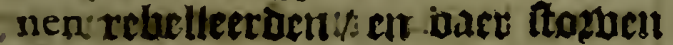
sucel sprangiactoenaine 3 aer i 537 . quimt oe Capitcpit Prancifco d' $\bigcirc$ -

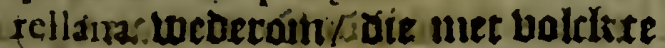
befteten. blyec ist het aloer uzuclyt = baietfalent/cn Leneotfacm/en met vect (jonich in be bollicljeden van af 23 sonmen. Be 1kevier die by naied loopen ban on = Der oe 2 inie: Equinoctiael wozben

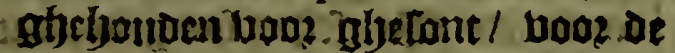

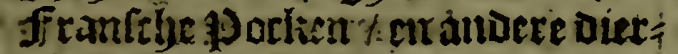
- aljelicke, quellinghent:s oaer gact veel boler ila of liebier / on baer aljefoutijeniot to crighen / Doos de Ueelyent Lan de 10 sitelen uan Saifaparilla, die in die Licuier.jin. thy is oock fis lyecl acoat nitt/ m bie

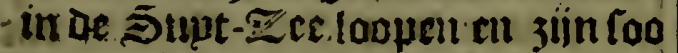
- grootuiet/ als ote bie tyaer ontla=

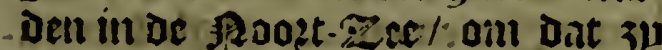
loopen doos cent clepne [patie/. en - noclitams ziin fuctoer /, cn met bae= ftiglje neder-bloepinge out bet bal= Ien bass fjet. oljeberdjte. De Indianen njebzupcken vecl yzactijcken om de 13evierent te profeecen: 31 bouder op etuige placteu cen lta bel owers otier Je lievier / en aen dien cen cosf/en tellen in die den

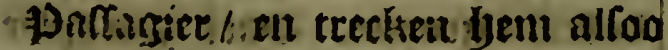
buer de ziebier. Bp andere kie= ftele de .joaltagiet op fiju o!n te paffecen. In andere plaetfat Gouden: 3n cen groote 23ack ban

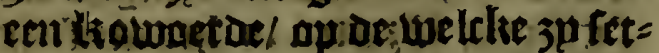

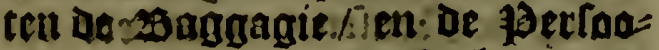
ren/en oe Indianeñ balt gljmaatit jijnoe met cen Codzoe / gaen al fuenmehoe en tectkmoe als 13 eer =

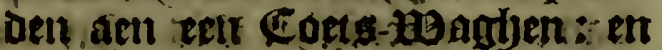
attoere:Dutent pzactiflaen / oble= beupcken 34 din og:zhebieren obet te comen. Be Hacben ban bie Stadt istoicljt int lact / om dat oc licvier fret bzectis/3002 De belcke op-counen de doopniantchappen uptter Zec/ent datt duec 2 ant gaen naet Qirito. Int Jatr a 568. po $=$ pulector of Eapitenin Contero, de Stadt Caftro, in dis Ballepe ban Vili, ; wefentoe O5oubernette van de Hiicken lom Peru, de 1 icentiaet Lope Garcia ban Caftio vili is in of 12 zobincien ban Bunigando, Im-

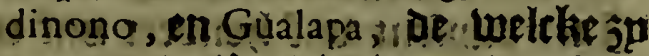
noemen de Dzoluitcie hat de Efmerauden; in fen vertroff lia die ban Guayaquil; on ontoeclttede alle die 3) 20 butcien/ban Paffo af tot of the = biece ban S.Ian, Die ill de Sulutzee valt;

Oe Staut uan Porto vicjo, is bu Puerio viejo. cang tarbenticls legucn uan Sinte Francifco del Quito, in't Harften/ bochnet ban een effen idoech: eil andere luiffrich uati. Sant-Iago de Guayaquil, al waer men gart bait Quito: in Gare lieflogt is Paffao, dat de eerte foben is uan bet Zandt bau Piru, cn uan de thevier Santjago af begint lyet Gomberne= ment Lan oen 9 arquig Don Francifco Pifarro, en om dat ljet Janot coo diclut bn oell Equinoctiael Zl $i=$ nic is / welende op een graet / niec: nen eeniglye oat ljet onghefont is/ nocljtang op altoecesplateten foo nae bu oc linie leefunen met groo= ce oljefontjepat / butbtbaerbeque! en overbloet ban alle : vingljen tac

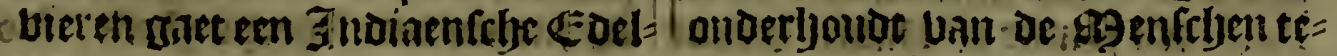




\section{Fol 56}

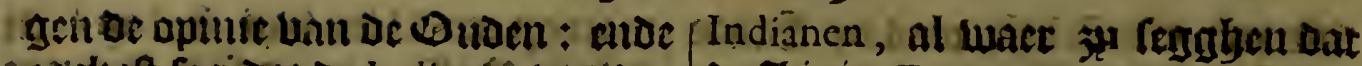
coock ift foo dat de Indianén ban dat

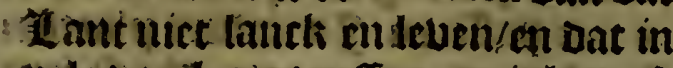
velc banilyace waden etnighe ror= - raclutigije statten in ber:aenítictyt/ -culop oc Baculen / en antece dee= ten cil alloo bet een nuaret gbe= I beck is / milmacckt bet buet feec/ - riv tioost gbelooft dat bet conipt

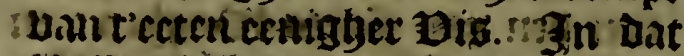

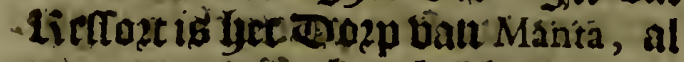
waer $j$ p wercitegton bebben groote

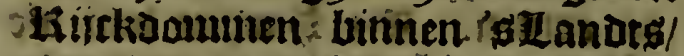
fiscu bout booz feecket dat al bier

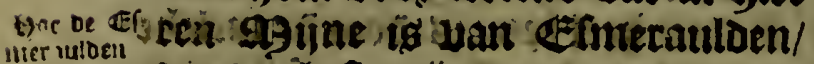

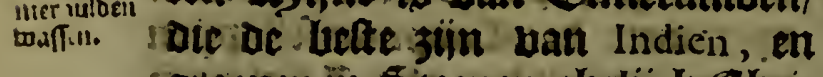
stospen in Steenen glyalizls chai= Ital / en 3 s tyandelente : als $29 a x=$ skiue-Stech $/$ allenctkens af-trec= liende / crísualtopende be Jelft wit/ de belft groen / gaen gु? die rijp Inaekton / en crivghen baer bolco= :mentljopt. De Capitepn Francifco - Pacheco bejolétitede dié Stadt in't 3aer Duinfent wilf yondert bijf en - bertich/ upet lalt ban Don Diego de Almagro : 'tjeeft ueel Indianen ban - ozlo ocly/Daer is een Cloolter ban onfe Iiesie 1Bzoume ter Gzatien: Dar woat gheen claruwe ghewonnen/ om dat yet daer reghent de acht 9 aenden int Iaer uan ben - Ortober boozwaerts/ daer zijn ooc goede ganlters ban Gout.

auten ar Bp be Culte van dit Gouber-

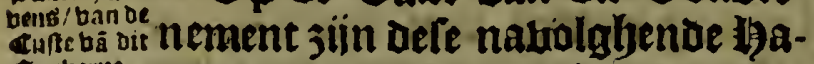

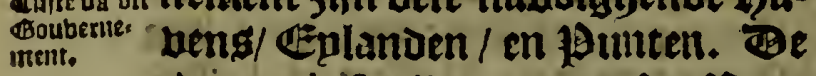
Aricon de Sardinas, booz de Bape ban Sant-Iago, die lept vijftbien leguen van de jounte dan Manglares ten Suuden / en Itrace die uan Sinte Matheo, en baer naer oe Cabo Jian Sinte Francifco: en daer voozbp de Quiximies, vier Zlebieren cer men compt tot Porrete, al wirer be 5 werten oie haer falbeer oen ban eer 5 chip Dat omabewoepen was / baer beblen ghe. boerlyt met de Indianen, en beb. ben cen Jozp ghenuarkt : en de Panso cent gunte of haben vall de De Linic Equinoatiael boog loopt! ontrent ljet gyeberciste van Queaque, oork de 25 ape ban Cara, die ljocz de Pucrtó vicjo is / ech graft bes Supaen ae zinie/ mi uif leguen luan daet is de Cabo dinn.Sinte Laurens, elt dafe ontrent ig lyet cep. lande de Plata, en boote de liaueng uan Callo, en Calango, vooz de Punte ban Sinte Helena, op twee graom joochte/ en de tievier ban Tumbez op bier graden / ien lyet ezplandt uan la Puna, daer. ontrent / en uat van Sinte, Clará, con wepunich verder in 2 ee/ en: Cabo blanco viifetyien leguen wait Tumbez ten Supoen/: en ftrace ot $\$$ bunte Lañ Parina, en Supt-waert Yjet Eplanot van Lobos vier leguen ban de Gauen ban Payta boozfept/ cri la Silla booz de J Bunte vanAguja, en de 1 aben ban Tangora.

De Ingelogenen ban bat Lant me newren regghen / dat in boozleden triben bant s, Hio daer quamen ober Eee op polotcen/ oat $3 p$ beel bouten t'fanten gbevoeche en ghefoten aen malcanderen/ ghenicben foo groot dat uan baer linpen tot beneden toes maren 30 grooter ban andere ofDinarife goncrben bet gantrobe Zichaem was / en dat 32 maeckte. Den een feer diepe zout op eé fotyerpe Hotfe / die men noch beoen fiet met feer berfify en foet fBater / op de Bunte ban Sinte Helena, dat een werckis ban groote berwonderingbe/ en om dat $3 p$ gbebzupckteden groubelijeke fonden / biel yet Bper upe den fismel dat batr alle-gaeder berbzandende / en na binden $j p$ op die plaetfe de aldergrootite 25 eenderen yan 29 entchéf en tucken ban canden die beetthien Oncen begben / en in nieuwe Hirpanien, eta in't kactozt bant Tlafcala vintmen 25 eenderen vair de felfoe grootte. Baer sijn op de pounte ban Sint Helena gaeten en

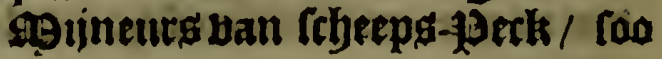




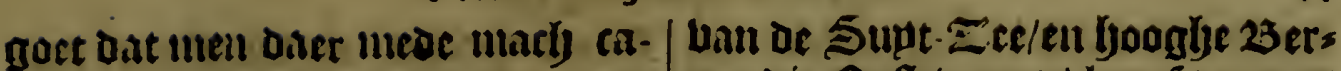
lafateren / en ljet cont עooz den gen die Ooft-waerts loopê/ en aetı dacly feer warm.

1)et X V I I I. Capittel.

Van Popayan,los Quixos,enla Canela, Pacanoros, en Gualforgo, twelck de reste is van bet refort van't Hof van 2 uiilo.

Popiyan,

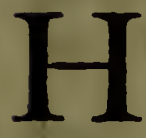

Ctgoubcrinethent wan Popayan, yondert enzo. leguen Simben en poozocn ban be Genten ban de pzobincie Quito, olther be Equinoctiael, tot bie bain Carthagena \$ookt-waerts / en andere bondert ban die ban't nieu= we kiijels int Bolten/ tot aen de Supt-Jee / in liet welcke enigbe Dozpé der Caftilianen ziin/ int beel ban't bof uan Quito, en bet beel ban t'nientwe tiuck is cen $\mathcal{L}_{\text {anot }}$ Doozgaeng rou/en leer vocbticly/en Daerom beeft bet wepnich 9 apj ent min Caculue/en niet veel Beeg/ maer het is rijels ban Bout-9sij= nen / en ocle navolghende beboic= Kingljen jijnoer.

De Stadt Popayan lept op twee graden en cen lyalf be-Poozden die Jinie Equinoetiael, ent op acht en trefticly cu cen balbe van lancletel buplent viif bondert tachtentich $l e=$ guen uan Toledo: al baer retioeert een I uptenant Bouberneur/bet Catledzacl/ en een Cloolter ban onfe Ileve Bzoume ter Szatie. Be Adelantado Sebaftiaen de Belalcazar beboletede die Stadt int jaer 1537 . bet Bolet ban die pzobincien is feer vertehepoen van dat van Piru, om dat het met leter beritant be= gacft is / en in meerder politie leef= de/ mo de uan dit Goubernement lecfoen als in carauanen. Het wozt gljenoenit Popayan, om dat alfoo genaent was de Cazique, die 1 ieer Daer af: 'tleeft een deel op de Culte b'anaer lijoen la Cordillera de los Andes, ent uut befoc deelen (pzun)= ten bele Revicren/en ectl is die bail Madalena, die inde \$oozt ₹re ben ontwatert / in dit Goubernement is coel en glyefont Bolck/ en de ant= ocre warm en lwatk.

De Stabt Cali lept op 4.grituent. en twee en twinticls leguen wan Popayan, en achtljien van oe SuptTee/ ju is bevolekt int $\mathbf{T}$ aer i 537 . Dooz de Capitepn Miguel Munjoz, ent eertelicli dede dat Scbaitiaen de Belalcazar, III de Dozpen die nuen nocuit los Gorrones : t'is gljetegent in een blacke Ballequ/ birlyt aen oe Ooet van een $23 \mathrm{ergl}$ / oe li ocht is aldaer yeet: aldaer refideert de Souverneur / en of Officiers ban het incomen / en' 'CConinct Schatfrifte / Dacr is een Giet-1) ups / een Cloofter ban onfe Tiche souto ter Gzatie/en een ander ban $\mathrm{S}^{\prime}$.Au. guftijn. Dr bauen ban Buenaven. tura lept in back Turibictie op dzie en cen balf graden foochte:'al daer refioeren eenighe $250 z$ ghers om te ontfangen of Coopmantebappen. De Indianen ban Cali zijn ban goes oe contitic en gocae Cyoiftenen: haer oube 1 eer wozt gbenaenit $\mathrm{Li}$ fupete. Je Stadt St.Fe ban Anti- Antochiai ochia meer dan fondert leguien ban

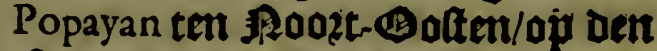
Beber van de kebiere Cauca, ban het reflozt oes bofs ban't nieutwe kijck / en 't2sínom ban Popayan, rijck ban Gout-פgijnen en bithe

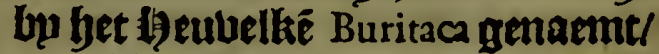
omal bet Bout Dat oaer in was/ t'ig bebolckt in een blackte: Be in = gbebozenen zijn goet solick / ban goede Zichamen/ en blanck / en be Tocht is alfoo dat zp aapen bup= ten de buufen/ Conder dat de coelte baer pet beichadiche/daer wert Bee gheteelt/en beel Buchten/en Bif= icben jijnoer in de letereren / en de 10oelen. De Capitepn Gafpar de
Call. 


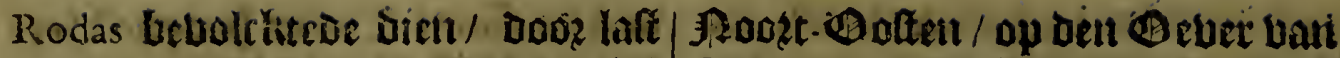
nan De Adclantado Belalcazar, int Jaer I $54 \mathrm{i}$..

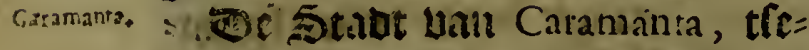
(tich) of tfeumtity legucn wan Popayan tell moots Ooften/ biclit ach Oe ntoote lievier Cauca, unit lyat

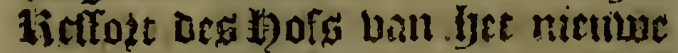
Hijels/ het Gouluentument ch $315=$ Dont ban Popayan, is ouretulactich ban 95 ay/ an ander ghezact / fon=

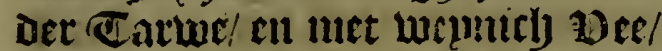
at hoe wel batter uecl Dereliclis ziin: men gaet lancr oe Bicluier in 5. of fes tween tot Antiochia toc / al lyoe tuel Dat liet 50. Iegmen siju / $\mathbf{t}^{\prime}$ ig all Dat liset ltif booet gaet: 'ris een bevalclimge ban oe Adelantado Belalcizar:

Arma. - Or 5 tadt van Sant-jago de Atma, yecte vele Gout-2gijnen / so. qgijlen uan Popayan, ten fpoote Goiten / afwiicliende na't Boften/

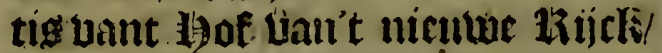

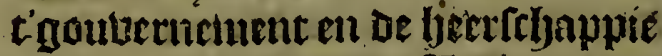
van Popayai, fonder earibue/ en gefact banCátilien, nochtanis ouer= vloeuchbe ban die uan yet 3antot. - De Adclantado Belalcazar lievolek = tear die Stnut/en hict bede bu ont= halfen De Marifchal Iorge Robledo, fulept feltbien. Ieguen wan Ancer-

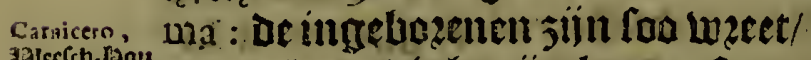

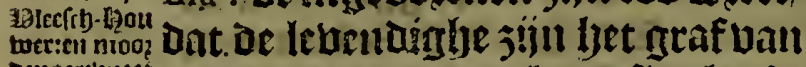

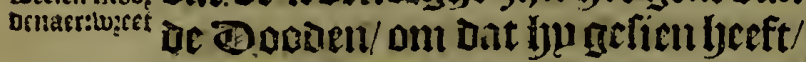

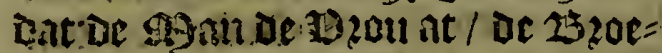
Der de Siffer $/$ ar Sodn fin bus Der / en'trilyelueutoe als juy uat glje= mataclit lavodenecu glyebangen/ op Dien Dach als zulyem [outuen cetcu/ beachen fu hem voost met bee! glye = fancy/en ue lorer glyeluoade/ent ren Indiaen yenu Coube af caupen elelt

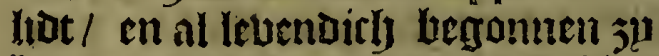
bem te eten/en tledert ae beuolclitil= glye uan Arna Gebloen ju glyrgeten meer dän aclit buplent Indiance en eenighe Caftilinen ljebluen geleden Defe unatelifatie.

Ancerina, : De Stadt vail S. Ana Uã Ancerma biffticly leguen bant Popayan ten Cauca, wail ljet bjof ban't nieutue Ziijcli / t'Soubernentent en t'25iF Dolli uail Popayan, [onder Bee/noeb) Caruwe / fecr gljequelt uan Don detflagen. Te Eapitem Iorge Robledo fontectue dien/ doos laft uan Lorenzo de Aldano. Bock ziin die

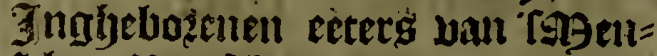
[rlien-Bblectely / 3n gaen naeck / jul bebluen glicen Leelaen / of cenich

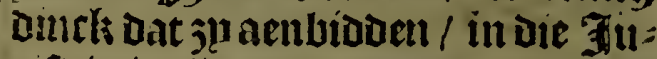
rifdictie jiln goede Gout-gsinine

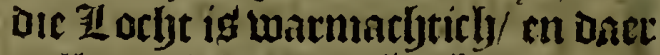
Lhallen bele Donderdagljen.

Wre Stad Cartago, 25 . Itgumi cartago. uan Popayan lup narft ten RooztOoften/ Lan bet bof/wan't nieubr Liijet: / Goubernement en 'tsif = Dom san Popayan, fonder carbue norf gluclinet uan Caftilien, is eet gljetenuert lant en gljefont ! ban wennich Goit / Jet teglyent ved/ loct en teelt ghecli ander bee Dan Itocuen / En 25ocken: 'theft beel

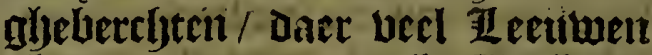
gljeteclt wosoch / oock Fugljerg/

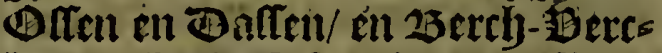
king: 'tletft é C loofter ban fitan= ciffalters / Dr. Capitepn lorge Robledo beboleltede dien/ en noernde yet Carrago, ont bat alle be bebole= lietg waren van Cartagena:

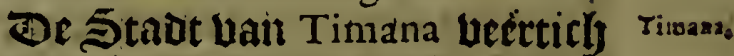
Itguen unin Popayan, tên SuptOoften / ent tretticis van Santa Fe de Bogota, ent 30 . van de 13tovincien del Dorado, al hier is ecn II lutte= nant/Gouberncuir/dic ootli tot lijn

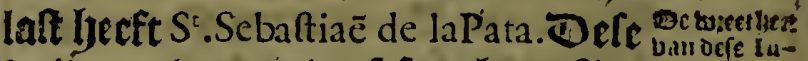

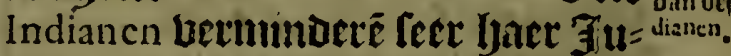
rifoictie/ ont ant ju foo onmentelye $=$ licl: jijn dat 3 u in ved plaét en lyeli= ben opentiare Blecfely-1) wuren ban

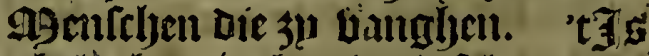
gfibleglien in bet beginet van oc Dallene de Neyva, ent de 2 orljt if = fer [eer warm/ binmen yaer llantpaelen is ean fenbel/ upt ae

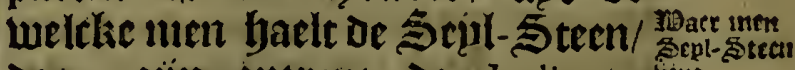
vart jijn astrent of Indianen bint. 


\section{úan VVeft-Indien.}

Paczes, en oe Pixaos, dic oock Cari- gefit bet deפgap3 tweemael giaers

ben jiin.

De Stact ban Guadalajara ban

Buga Buga, I s. Ieguen barn Popayan ten nookt-Bolten / is int lieflort bau yet hof Uan Quito, en oe beerftyap= piie Uan Poparan:

s.sebastizen De 5 tadt Uan St.Scbaftiaen de la dela Plats. Plata, op de Eokenfen bian dat Goul= bernenient/3 s.legum Lan Popayan, en Derticly uan Santa Fe ten Dupt-

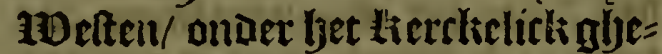
biet ban Popayan, al warer bele $\mathfrak{i}$ ij $=$ uen ban Siluer jiin/en in farer $\mathbf{3}$ !ll= rifoictie 24 . Devlitighen: 'tlent dacie Ieguen van de haunu uni Onda, op De groote kevier uan la Madalena, al waer ontladen ju dic ban Cartagena comen : 3 ! is betuolcht in oe ulackte / Diche bp or lievier Guali: in bet lat ziin ueel febudoingen/ en in be nointer ift neer warmban cont. De 7 nugonoc:s die uermin = Deren/om Dat die ghegeten werden Uan be Cariben, die ju noemen ban Rincon,en hebben openbare 19 leet= Gurfen daer ban / fonder dat het ran gljeremedieert wozden. Dien Staot bevolcktede de Adelantado Belalcazar.

almaguer. De Stadt van Almagucr twin= ticly leguen ban Popayan ten SuptOolten / is jutulbtbarer uan Carn= we/ I9anj / cul ander gljefaep / en Fice/ $\mathrm{m}$ 'therft Goust. Oe Capi= trun Alonzo van Fuen-major be $=$ bolcktede dien/ Dooz laft van den 2lirentiaet Brizenjo \$ontuerneur/ en Lieclyter ban de refiocntie ban Popayan : t'is ghelegthen op eenen

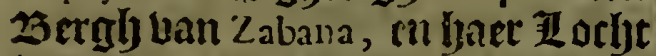
is coel en t tralck gaet gliecleet nuet Kocken ban Cattoen.

Truxillo.

S. Ian ban Truxillo, en anders obenacme Y fance : Dertich leguen ban Popayan, ten Supt-Doften. Medriga!. $\mathbb{E e}$ Staot uan Madrigal, of Cha. panchics, 3s. Lan Popayan bu naelt int \$unben/cen rou is ont/cn daet mengizen Carloe en bergadert/ nocly gljeen bee en teelt / noclitans in yare 7 urifoictie / en om de rou= lyept baut $t$ and siju de Intwoon= oers quaet om te bevzedighen/en in vic Stndt / ban Agreda, en Almaguer 3iin פgijnen ban Gout. Agreda anders genaemet Malaga, lept 45 . leguen uan Popayan tel SupotMöetten. BE Stadt van St.Ian de Pafto, palfo, alloo glyenoemt / om dat yet een ZLanat is bol ban Mepdinghe / is so.legueit uan Popayan bu naelt tell Supt-zoelten / en nocly loo beel Uan Quito, bu cans ten \$ROozt- $-00=$ ften / en een graet ban oen Æquinoctiael, inde liercltelick glyebiedt ban Quito, in een goet Yland/ ban goear jocht / ouerbloedich uan Sgau3 / en anoere 3 iiftorlyten/met Gout-gginen / in fijn Turidictie 24. Duplent Indiane onder eet com= manderie/dant geen Cariben en ziin/ dan alleenlick quaet ban glseficljte। bupl/ en therijt/ ju en bonaender gyeen 3 eelden in De tijot ban baer fepdenirbap / $3 p$ glyelooben Dat jui tae de boot comen fullen en leven in beel lultigher plaetfen. De Xies bier die $3 p$ noemen de lyeete / alye leglien tulithen Pafto en Popayan, beeft leer delicaet 13 9ater/ en ouer. die lievier is een $25 \mathrm{ergl}$ al waer Gonfales Pizarro de 1Bice- Liou Blafco Nunjez Vela boleljdede/ ent tot de tievier Angafmayo, Die in die $1320=$ vincie is/ quam de Conincli Guaynacapa. Ouer de jeete Rielvicre op Date Detban

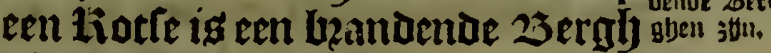
Dieen groote quantiteut ban dam= pen upt werpe / en unt blaelt vait onde tijden af/als de $\mathbf{I n g e b o z e n e n}$ Lan't Zant fegglien. Deflyilofo= phen willende verclaren wat oe bzandende 23 erghen 3 iin/fegghen/ gheliick als in Ijet \&tertrijck vlaet= fen zijn die een cradist bebluen out aen te teecken Damuighe ftoffe $/ \mathrm{cm}$ Dic in 13ater te beranderen / ban waer tomen de fontęmen/ die al= toog blieten : alfoo ooth zimber 1) 2 plaet'en
Agred2, 
plaetfen die aen trecken bzooglye die ban Zedros, lept op twee graden Daunen/ en becte/ Due lyer beran= Deren in BDper/en walnten/en die met glietoele vain oien / werpen unt oocligrove inaterie / dic baer refol =

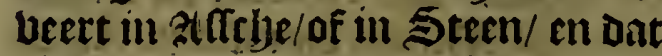
ziin de bzalloende 25etglen. S'. Ian de Paftos beeft ciloolterg uan Do= minicanen/firaucifanen / en oufe Zleve Bontue ter Gantien: 'tis ech cout il andt/met overvloet vall etex/tlject ingenios bau \$uleker/ en beel beuchten bau lyet lo ant/en ball Caftilien : als de Capiteun Lo. renzo de Aldana dicn beuolclitede iit Jaer 153 9: noentoe baer Villa viciofa de Pafto: fu lept 40 . Lemuent vall de Supt-Zee/ nae lyet ceplant Gorgona toe.

פgen yeeft antualdit/en bermun = Dert in die pozobincie de Stnot wan Antioquia, De Stadt ban Neyva in de Ballepe uan Neyva,twaintich le= guen vall Tumana, en jet was om de Itrengichenot van oe Indianen Paezes, en Pixaos, en om de Manipos, tin de Ballepe Saldanja, en de 5 tadt uan $S^{i}$. Vincēte de los Paezes, trefticly leguen ban $S^{r}$.Ian de los I lanos, op De Gzenten van Popayan, Dien foll= deerde Domingo Lozano; en de Stadt los Angeles, twee entwin= ticly leguen ban Tocayma, en negen ban Neyva.

Febiern, ca De Culte die dit Ganbernemtent

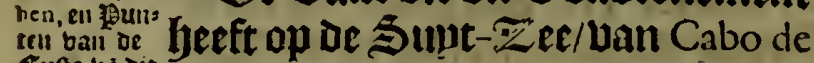
Euffe batbit Corrientes af die of s. graden be $=$ Joozoen De llinie leut / is de tie = vier bau Salinas tufrelyen oe Cabo de Corrientes, en bet Enplandt de las Palmas, op 4.griaden en cen derden= Deel/ en op oe Culte die is tot la Gorgona, Iept de Reviere uanSt.Ian, onder andosere bele / Die lyet llandot feer R9ozaftich maecken/en teghen ober De mont ljet Eulant Gorgona, twee leguen in omganck / al waer Don Francifco Pizarro verlaten wag ban fijn volck / met Derthien ban fiin glyefelfelyap. De liebier wan S'.Lucar, chl die uan Nicardo, booz

van de Ilinie/ op de weleke lept bet Eplanot van Gallo, en Daer naer de liguen ban Cruz, en de gounte ban Manglares, al waer de Culte ban Quiro begint.

Ban bet Goubernentent uan los Quixos, en la Canela, en beeft menl Lor Qnixos, met meer kennulfe dan dat bet walt upt den Dolten ban de pozobincie van Quito, en het deel wã bet Sup= Den / nae lyet Goubernement toe yau lan de Salinas, Daer in zijn Dzie Dozpen uan Caftilianen, met een Gounerncur die de Bice-kinp ban Piru beltelt / on int Giyeeltelick is het bant 2sifoom ban Quito: lyet Zlandt is cou / en 23erch-achticly/

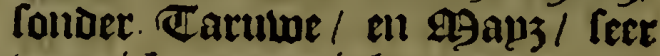
weunicly/met eeniglje 25oomen die frbijuel yau Canzel. Het extle Dozp is Bacza,achthıen leguen ban S. Francifco del Quito, bu maeft tent Sult-Doften/ al waer refideret de Gouberneur. De Stadt uan Ar. chidona ig twintich leguen berder Dan Baeza. De Stant yan Avila is iitt \$opzen vall Archidona.

Het Soubernement an de $1020=$ uincie yan Pacamoros, en Ygual-pacemoror fongo, of Luan lan de Salinas, wiengs fongos Pimiten en Dalen jiin bondett le= guen/Die genomen wetaen int Bo= ften / ball Direr 20. Leguen boozts Jail de 5 taut lian Zamora, in de Cordillera de los Andes, $\mathfrak{e n}$ oock too עeel Suldan en Roozacu / is em goet ILamot / in ILoslyt en glyetent= pertljent/ Hooz Caruwe/ t'gljefaet/ en 1Bee / Wail riiclic Gout-9giinen/ in de welcke ju binden feer groote Gzapuen : Daer in jiln t. Jozpent ban lyet 3irdom duan Quito, de welcke fondererde de Cinpiteun Ian de Salinas.

De Stadt una Valladolid op 7. valladelid graden boorbte / Iept twintich le= guen uan Loxa ten Sult-Boften/ ober de Cordillera van Piru. Je Stadt uan Loyola, of Cunbinanja it feltyien leguen oolt-waert wan 


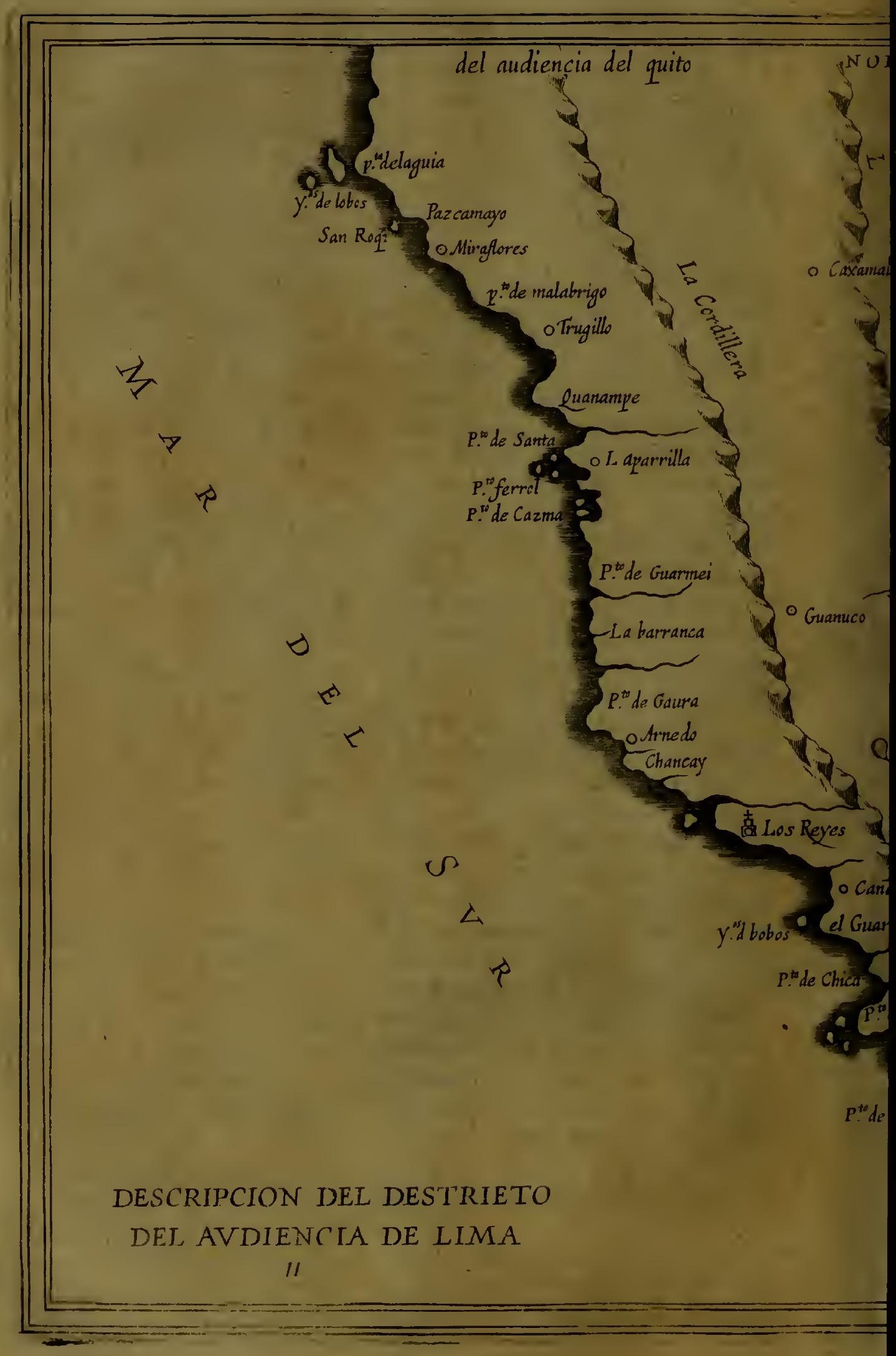


and ne

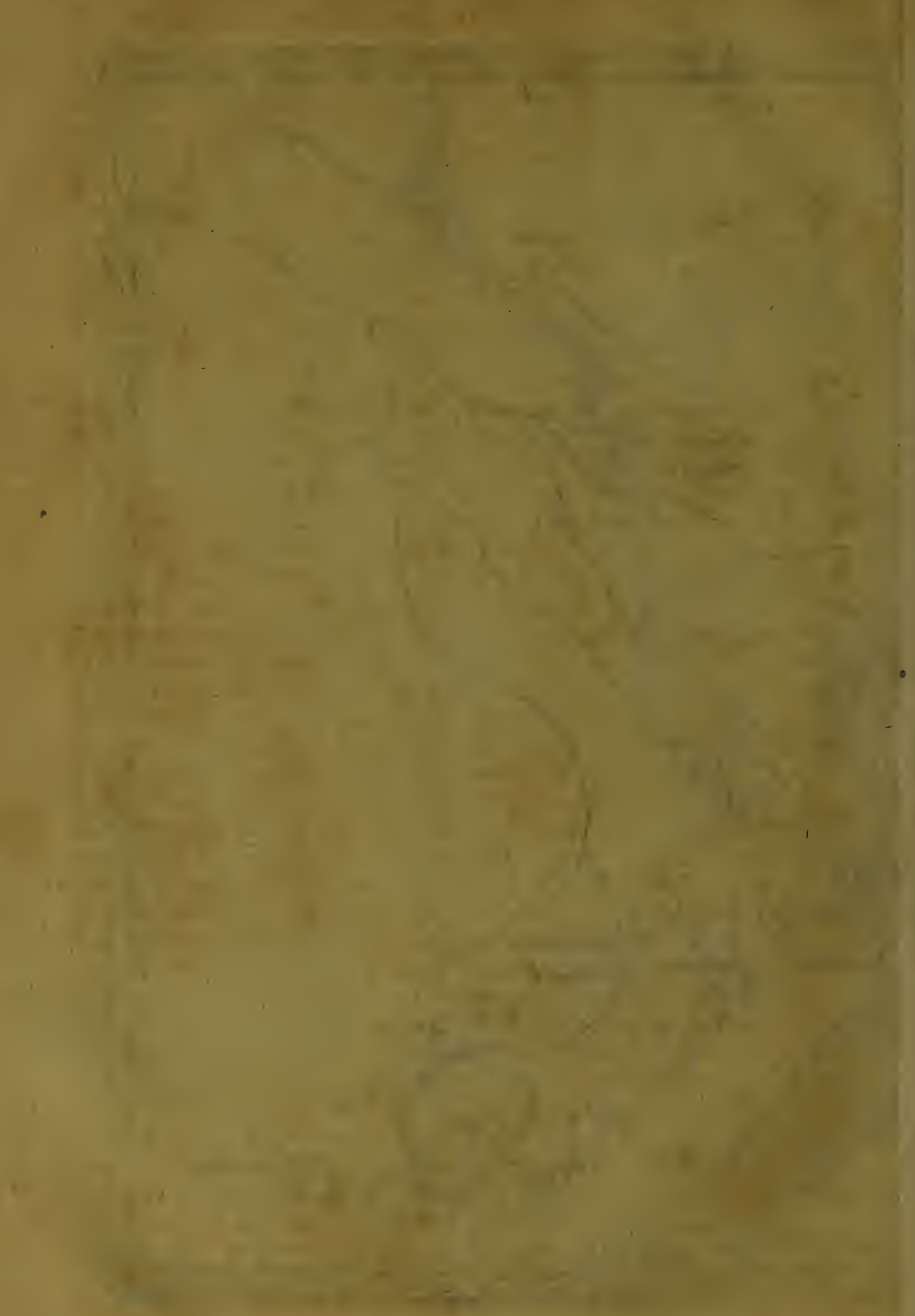


Valladolid. De Stadt van $S^{\mathrm{c}}$. Iago ban oe gljeberchtell / is ; o.leguen ban Loyola, bu naelt int Oolten/en in lyace 3 urifoictie is veel Goutg/ culyzoch vall allou / en foo beel lyet

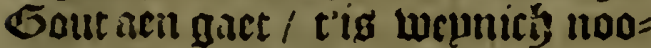
dicly te bandelen ban lyare booztref= felicilięet / nae dien bet beul bout

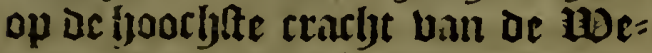
Dop lioe teet relt. âsen treclit het unt in die Ingrut it i- diens op deicocrlen manieren : de (t) piar to fuiken fonder mengunge van an= Der 9 yetacl'ch dic niet ball nooden yeduen Datnenle beneficieert Doost B)y:c/ en die Gzanen jijn ozoinap= relicli alsetn lieerue ban een Ca laburfe, enceniglise reperen grooter, ban fulchen Gout vintment wen= necly/teat refpecte van de refte. De tweede maniere is in ecn Dteen/ twelclieen Steen is dic in oe felue Steen walt / en die Stecnen butt= men m bet Gouvernement Uan lan de Salinas feer groot / ganticl] Dooz= loopen unet Sout / en eeniglye Dic balf Gout jiin/het welcke ment vint in 1)utten eil agijuen/ en is leer Cwaer ont te arbeplaen. Het ocrde trecltamen uet iset ftof/en is de ures= ite quantitipt i cult men biut bet in Iscuisten of plaeten / al waer chie $=$ patreere beeft ecmeb loopentoe tio $a=$ ter / ear of Rievieren ban die Indiens fievbent ueel/ bulonderticl in de Gitirken ban Chile, ban Quito, $\mathfrak{e n}$ 'titisu've hiiick Uan Granaden, $\mathfrak{c}$ ta de begiufelen uan oe ontoeckin= glje warender urel in de Eplanden Lan Barlovento. bet ljoochfte Gout is dat Uam Carabaya ill Piru, en dat ban Baldivia en Chile, onl ont het cont tot date et twintich quilaten, en ecl: balue/ en noch yooglyer.

Get X1X. Capittel. $V$ an bet reffort van't Hof van
Los Reyes.
7 , Shene wat epgbentlick en particulierlick genoît wert Piru, is bet rellozt uan't bof de los Reyes, mi beflupt fich ban Supden en Roozden $/$. uan de feg tot feven graden ljoochte be-5ulu= ocn oe I inie/dat jijn twee bondert twintich leguen / al boe wel ju out te repren ltellé ozie lyondert leguen/ bail de punte Aguja, ober Payta, al waer lyet lyen voeclyt met lyet 1 of uan Quito, tot gepaffect bebuende of Stadt en Haben ban Arequipa, al waer begint die ban los Charcas, Boft en melt. Het beuolckte van Dat Lof fal mogtyen ftrecken bunaelt tot I oo. Ieguen / van de Culte

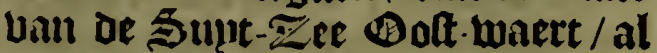
waer de Zimiten open blijuen tot de jozovincien uan de lievier de la Plata, en van Brafil, t'gbene twelek is wan de Cordillera of / al mact af glyeleut is dat bet ftadich reglyent/ tot aen de $\bar{z} e$ / noemen $3 \mu$ de ulac $=$ ten ban Piru, in Dwelcke ljet niet en waet ent regies

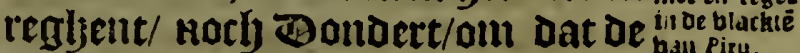
groote lyoorlyten batl ljet geberchte bedeclit de blackten / alroo dat die nict torlaten dat oe nomt upt yet Zambt Glacit waerom de EBint upt ter æee regueert / Die gljeen tegen= wint beblende/dauclit upt oe dan= pen die haer opgeven/ waerom dat ju reghen fonden inaccken/ foo dat de fuptinglje van lyet aljeberchte Gelet Dat de Dampen dick werden/ cn de fante Uan teffe cauleert dat in die Culte die dampen too oun jijn/Dat jun niet en maecken Dan een vochtiglie \$evel/ die pzofiiteliclis is on te temperen lyet gefinende/t'wele litet in en lyecft Conder de neluel/ dooz al bet mater van bedontwin= glye bat lyet lject. bet gantldye

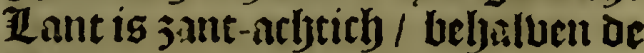
Fallepen die dact jiin bu de loo= pinglyen ban de liebieren / die ban bet geluerchte neder ftozten/al warer gl)elwonnen wert dooz de bedon= winglie veel 25200ts/ Wiin/Oliel Stlpelier / en lyet oljefacpt / en de b) 3 vatucy= 
Cualitenten
ban ber gus:

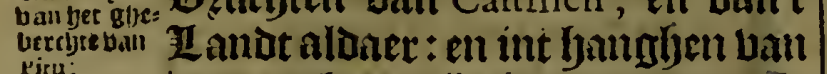

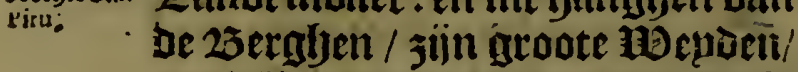
en teelingen ban oee / en de 2 orlyt is berfelepiden / alfmen can wen= Ichen/ on dat jet boory cout is/ en: bet lagije beet/en de nuidoens deel= achticly uan De tutterlten/mae Dat $=$ nten min of meer naer baer coint. Het Goubernement ban Dat of/ êt Dat uatl Quito, ent los Charcas, ig tot lalt ban oe bice-1Roy. Eên it jet reftozt ban dit bof jijn oefe na= volghende Steden.

De Stat - De Stadt uan los Reyes, of Libã los Reyes, ma, oull kat foo gljenoent wer = de die Balleue / die de naen yadde ban fiju Cazique, en ljet is de groot= Ite Balleye/ en be bzectite/ ban alle vie glyene die Daer $\mathbf{j}$ iju Lan Tu mbez

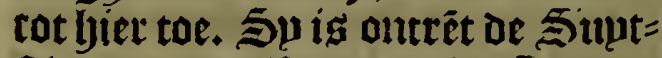
Tee/ op twaelf graden be-5upden de Iinie/ en twee en tachtentich bat be Suut-joost- 1 ijn ban Toledo, Lan waer fy af lept bu naeft Dument arfyt bondert twinticly le= guen / op een groote Circkel/ op foeft meer dan daie Dumlent $250 \%=$ gyers / or Marquis Don Francifco Pizarro fonderte tien int begin!le ban't 3 aer i 53 . om dat bu wer = moede Dat de Adelantado Don Pcdro de Alvarado foute Dalen op ise

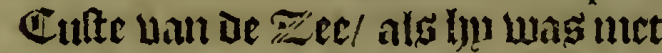

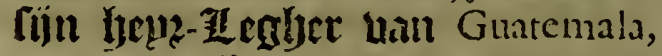
bacr-en-tuffelyen dan Don Diego Almagro guncli teglyen te itach De Poovincien un Quito, is oljeco= men in te nemen de gon氏agic uan oc Zce. Ticlyt by oc Stadt / act de Bolt-jijie / loopt ect hicuicr uan de wilckic 1 bater ontfinglyen alle de bunfen / cu fjace of lummen/

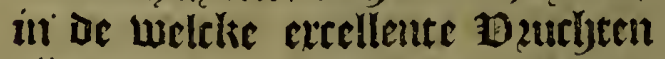

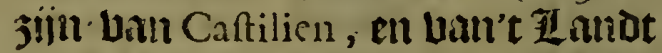
aldaer / en Daet igen uan be befte Lacijen vall oe Bberelt / midta baer glycen bougijer[-noat is/nacl] 10rite / noclitieglyen / nody Don = bet / neclj Daet ballent DonoerAaglyen / noch 23litem/ foo ont be Dumfent Swerten.

Fin defe Stadt refidert be Biceliop/ cul bet Coninckliclie bof/ent Sale san 1Bzovooften van't 1 of/ de Officiers ban bet incomen / an 'TConintr Schat-lifte: De Bules frlyare ban de beulighe en aldye= meene 3 nquifitie / die glbefonderert werde op de lelfoe tijot als die valt nienwe Hifpanien, we'entar $\mathbf{3}$ tuqui= liteur Generael in die Lisi;cken / de Cardunal Don Diego de Efpinofa Biftryop van Siguenza in die face = le gepzocedect bebuende met ripe deliberatie / tot vermeerderinglize van ons beulicy Catbolijex ghe loof/cn Clyettelicke Religie. Oock is ill de felfoe Stadt een Dniwerfi= tent/al waer de wetenfrbaypen feer gljelectedick glyelefen werden / en onderbuiffmgljen van uerfetjęroen Calen ban oe Indianen, wacr in feer neertich ziin de paters batr

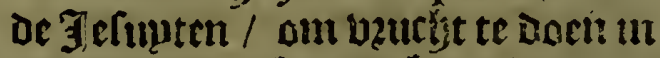
de verrondunge bân liet Ebange= lium. Oock refioeert in dic Strot of Metropolitaen Aerts-2iffthop/ wiens Buffragmen siln oc $2 \mathfrak{i}=$ frboppen vanChile, Charcas, Cuzco, Quito, Panama, Nicaragua, sn be lie=

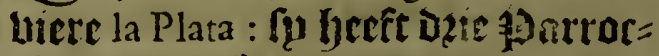
rlyial/ cu s. Cloalters ban ic $4.02=$ Dens' en ban de 3 efunten / cn tluec ban fRonten. Jataer is Callao dat baet baben is / twee leguen bact af / groot / rumm / eth fert goet / al waer groote neniclyte ban Hufer is / can Srljout / en ect l) ung unt De Adrana, een Lietefte/en cen Cleo= Ater unn theligiculen / Bommita= thers: allyiar hesf men gefien ghe fet te wozden ont te bercoelen bet mater of de wijn in Eleften / in de $\mathbb{Z} \mathfrak{c e}:$ wact upt nten beflut inat deOceaen yeeft de eracht oin te tem= peren en te bereaclen de ouerma= tiglye hitte: vele affirmeeren bat in Die Stadt silu 12. Duprent 10zon= wen van alle fatien / en twinticly 


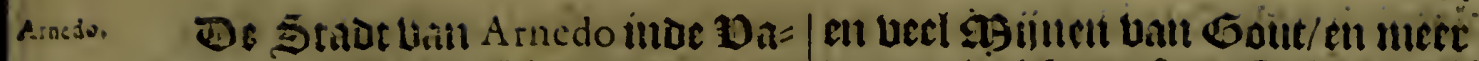
Iepe bin Chancai, tyien leguen ban Dan twinticly otufent Cributarifé

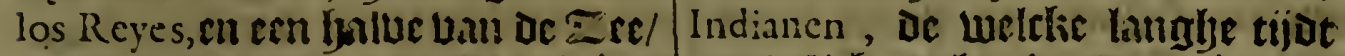

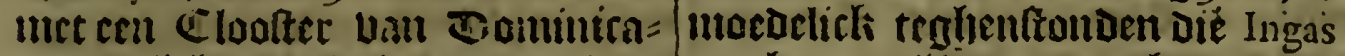

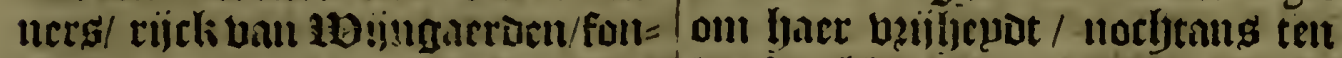
Deerde de Conde van Nicva. De laerfen bleben jlu olierwonuen / ent Strod uan la Parrilla, of Santa, din de Dallepe al wier juis'; j. Ieguen unn los Reycs, cll i j. Uasil Truxillo

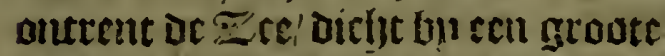

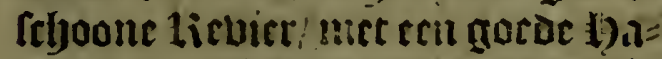
bet!/ op neglien grindet/ al waer in loopen dic ङchepen dic op ac $\mathbb{C} u=$ ften unu Piru fowlen.

bele oll Denterite rulte bã't ILaint/ beorlytan jijoer te Cufco, an bebole= teden ech betiuellen sat jit noe men Carrienga. Oat jijn Indiarieit dic blaucker jijn/cu ban beter gra= the als alle vaul ganteld Indien, eti?

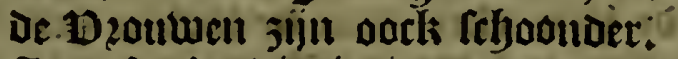
In vefe probintie quam de Mariichal Alonzo ball Alvarado, int lact De Stad ban Truxillo it de Dallefue uall Chimo, die cerit due= grecp Don Dicgo de Almagro, ent daer naer fouberde de agarquis Don Francifco Pizarro, int jact i 533

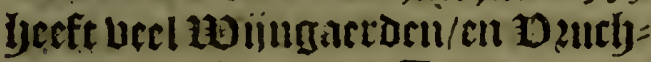

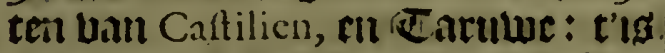
al bedoutuingiye / cu ect gljeront Zinot / en liecft groote b?angieAtupelen / a teelinglye ban b) oen= Deren : leut op feluen graden/en cent balue / tachtenticli legus! hat $\mathrm{Li}$ ma, dicht aet de $\overline{x e e} /$ met Cloo= Iterg uan Domunicamen / fintrif = caller / cn \$'.Âtuguftiners / en een alloer ban onfe Z ieve Bo zoutue ter Glatien / ell Conincklicke Offi=: crers/ plovifién booz oc Dire-Kiop/

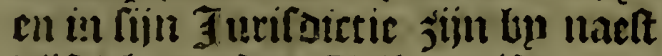
biiftely Dunifent Cributarife Indianen, it tluce on beatticly ofulin= gijen. Oe loatuen is tuce leguen bath dones/ ill reli 2 Jụe die heel opê leut / Die qaact is / en in de weiclic

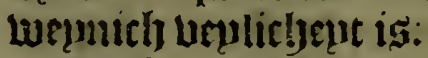

siiatines. De Stader Wan Miraflores, in de Ballene bianZina, is 95. Ieguten ban de Stadt ban los Reyes; aen liet Poopare Deel/ en ontrent ae Tee.

chachapoyas . Oe 5 tadt ball Chachapoyas, of Se.Jan de lá Frontera bu naeft you= dert twintich leguen van oc Stadt bail los Reyes, , een Cloofter van onte Z ielie 1 2ou = bje ter Gratim / ende een ander

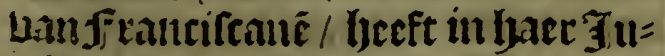

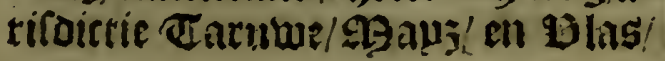
i 53 6.000: laft van Bent 9lsarnitis

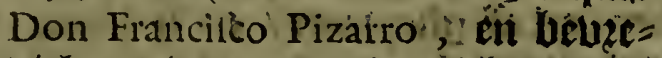

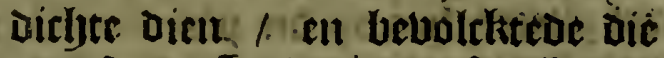
voozteude 5 tabt/ ineen ftercke ge= Irgfiene placté gljenocnit Levanto, enl voozts ginte hy boo it tot de Pzoluincic uan los Guaricas!

De Staut vall Sant-Iago de los los valleso Valles, of Moyobauba, imeet dant Gondert leguten uall los Reyes; bu naelt Noost-oolt-waerts / en is: Uam $S^{t}$. Ian de la Frontera, levet in een plaetfe vie yeel reglyenacljticly ig/ en oberuldedich wan Ber.

Dé Strat .uan Leon del Gua-Lcondelguä nuco, biff lonoett legnen van be Stad van los Reyes int Poozaent/ ontrent de Eoninelilieke weely bait los Ingas, whet aftuijcltende nae't Ooften / beeft beel Cloofterg ban Domuitianely / f rancifanen/en

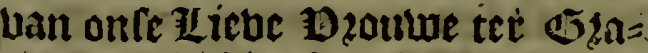

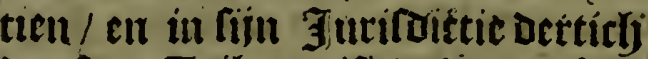
ouplent Critutarife Indianen. Flut Faet is 39 . ollt den oozloch die de Tinan Yllotopa aen dede die pló villcie/ Cont oe 99 arquis Don Francifco Pizarro Den Capitęun Gomez Uan Alvarado, die dien fondeerde/ en buer naer ontuolektedere/ en Pcdro Barrofo lyerbondofe / ell alg de 5ataille uan Chupas gyepaffert was / oe Z licentlaet Vača de Cáftro; Cont de Capiteun Pedro de Puelles; die untuoerde dat 3 accozderden. Su is glyelegien op efr bequame. platetel 
plact e/oic glyefont is/oueruloedicts

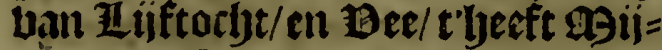
nen uall Silluer / ell 'tuolck is leer redelick; daet wert beel ceamue gljewanuen / om dat oe Indianen abeleert bebluen goede atrbejoers te jiitl/ont dat bu naelt in de Indiens niet en was noch Caruwe / noch Panick, ifee $\mathbf{G a r l t} /$ nocly inixo,nocb panizo, norb बEets. enich 3 let ban let 25 zoot ban Europa, en ju conten geen ander foos= ten l'an $\mathfrak{B}_{\text {zaunen/nocly anderg dan }}$ de Ro:telen/ wan de welcke fyet pzincipacl wag de 99 apz / die ghe $=$ uonden wert bp naeft in ganticty Indien : de Tughebozenen bebben: ber veel af gheproeft / en hebbent wel ghecregben op die plaetten al aequatisens waer bet vooat quam: oum dat lyet maps. agay3 loo fterck niet i\$ / en gljeeft alfulcken fubltantie niet als of fer $a=$ ruwe / $3 p$ is dicker en warmer / en matelat bloet/ en waft op Rieden/ fp bzengt boozt een oft twee atpzen en eenighe Caftilianen etenfe/ waer niet anders ten betten is:

Guamnga, De Stadt ban Guainanga, of $S^{2}$. Iuan de la Vitoria, treftich leguen ban Lima, \$utut-Bolt-waett op de wech ban be Ingas, een 23 iroom van Cuzco, beeft Cloofters ban Dominicanen/en francifaners/ en van onfe liebe Boume ter Geatien / en een ban zonnen / en in fijn 3 urifoictie meer dan Dertich dupfent Indianen die onder tribupt ziin : is feer bzuchthaer ban oa ruwe / en TBiin / en rijck uan Sil. ber 9 gijnê/de 29 arquis Don Francifco Pizarro fondectoe die Stadt int 3)aer is 39. eeritelick teldede lyp dien in een Tozp ban die Indianen glyenoemt Guamanga, ontrent de groate Cordillera de los Andes, en nami tot fijn $\mathcal{L}$ uptenant ote Capitepn Francifco de Cardenas, en daer naer is fp uetlettet ter plaetfe waer fy nu is/ t'welck is een blackte ontrent een cleen glyeberchte aen be Supt-zijoe / ontrent een 25 eeche ban gaet mater: in dele Stadeziin be belte 13 eiulen lan Piru ban fteen! ende cichelen : fp is glyeleghen op een gefonde placte/ ont nocly Son/ nocb lacht/noch coelte/ noch woctst/ norb wernte lyaer beiclyadiclyt/Daer uert glyebonaen in baer 7 mifoictie cen groot glyebou/ twelck oc Indianen Teggben dat ghebout bebben eeniglye lotte zl upden/ ende glbebaerde/ die aldaer quament booz de Ingas, feer verfflyepden bail bet fatroen uan dien die de Ingas boulDeden : de meefte 3 ngelsozenen van Dit $\mathcal{Z}$ ant jiin de Mitimacs, 'twelek te feggben is / de uerplantede / om dat de Ingas gebzupeteden tot meerDer berfeeckeringe yan yaer kiiicly/ te trecken upt D'eene 10 zobintic bet Bolck daer $3 p$ baer niet op at betroubeden/ en dif te fenden oll te leven in een ander.

- Guainanga is 'tfecticly Ieguen ban Cuzco, m op die wech plach te ziin Stier-Jacht in de blacte ban Chupas, al waer floegen Vaca de Caltro, en Don Diego de Almagro de 7 on. ghtelinck/en wat woazoer op oe $\mathfrak{C}_{0}$ ninclicke weeb zijn de edificien ban Vilcafin Andabaylas, elf leguen ban Guamanga, $t$ 'melek was het miboel: punt ban betiaijck vatt oe Ingas, ent algier was de grootelkercke dan de Son/en de 13 zobincie bã los Andabaylas ig groot ban incomfte / beeft beel ligupaick Bee / en beel gebou. wen/ uan hier comt men tot of thevier uan Abancay 9.leguen natroer Cuzco, al twaer de Adelantado Don Diego de Almago de Bude / lloech/ en nam gevangen Alonzo de Alvarajo, de Generael ban פgarquig Don Francifco Pizarra.

Bolebt de Tevier Jan A purima, acht leguen ban de boozgaende/ Die roer groot is/ en ltract oe 2 bergh uan Vilcaconga, al waer be boozrepoe Adelantado Almagro won em groote 23 ataille teglyens de Indianen, eer dat bp Cuzco won/en daer ontrent is de Ballepe ban Xaquixa- guana tuafeben twee Cordilleras uan 


\section{uauVVeft-Indien.}

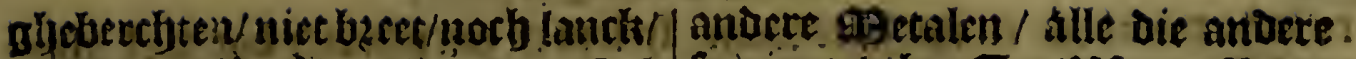
al waer ac pzelident Predro la Caf

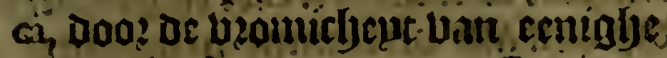
coutagienfe/sil trambe. Capitel: neil Ynojóa, Pedro de Valdivia, Gabriel de Royas, Alonzo de Alvarado; cil andere / Wan ore welcke op fiin plaetle particuilicre mentic fal ghe = utaecke weraeiv doech Gonzalo Pizarro, en bem uinglgenien van defe Ballene tot de Stad van Cuzco, rijn vif legtien : in die Dallepe ualt vecl carulos/ch dact is beel oeeg.

Fit X X. Capittel.

Van't gene dat overich is, van bet Hiof van los Reyes.

A. Cljt of thien leguet ban de Staot bun Guamanga, bp cans tên \$oozt-10 elten ziin De aghínen ban Guancabeiica, Die spu naemen el Affiento de Oropefa, al waet osdithaerlick refideren neer Dall ozie yondert Caftilianen; oe Indian $n$ bineficcerden die oul unt te trecken dic gheberfoe Steenen/om bact te [etjitaeren/ en befmecten/ als $3 \mu$ in or tijot ban baer thentien= bom ten Dozloch ginglyen/ t'welek is Dernicilloèn/ En goutwernerende in let Piru de ilicentiaet Lope Garcia uan Caftro,int 3 aec i s 6 6. Cen 13oztugiis gyenacmt Enrique Garces, merckende dátmen van ioer= meilloen treckit yet quick-5ilber/ bede een wrocur / en vont fiet alloo te weten. Dit Metacl bzochten de tiomeynen bänCantiien in Steen/ en trocktern baer upt ljet quick-Sil= ber / en ju ljielaent lioos een groo= ee rijekdom/ de Indianen en hen= Dent niet / en berforbten nietmert

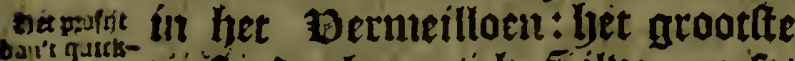
auluswo piofit dat lyet quicli-Silber geeft/ is'te fupueren lyet Silluer/cul frjoon te maectien ban de Alerde/ Zloot/ Coper/ Daett bu grocut / en bair of

en alloo bzengen ju bet in Bellew/ of Steene Baten : oock dient bet quick-Silluer tot antaere merckin=? ghen/ an tot Agedicijnen:

In De Contrepe uan Guania V.clica, is een ffontepne/ be weicke alds fu vlietet/bet 1 bater clontert in een witte Rotiteen / uan de welcke $\jmath^{\mu}$ grer baufen boumen/en foo 99 en:

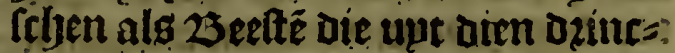
Ken/ tteruen / om Dat ljet Boater.in bet Licljaem yem oock in Steen verandert: In de 25aderi die 34 noes men van Inga, ontrent Cuzco; juit thoce loop-gaten ban moater/beene Dicht bu dander / bet cerlte is beet/ bet theede is cout / foo dat fchiint Date Goot gheltelt beeft allyter/om te temperen : en in't relfobe shebiet is cen fonteyne / die als $3 p$ utictet verandert in goet en wit 5out : op befe placte beginnsn lyaer te vet fpeepen de twee groote Cordilleras; en maccken int miboen cen groote openinglye die jp noemen / of qa20= vinciét ban Collao, al waet veel lie vieren $3 i \mathrm{jn} / \mathrm{en}$ de groote lieviere Titicaca is, en groote I Landen / ent wespoen/en al hoe wel bet black landet is/lyeeft yet de felfoe ljooclytel. en onghetenyerthenot ban de ghes berclyten/ daer werben gheen 230 fs felyagien \& nocly bott gljeteelt : het 23zoot bat pacr gljegeten wort 3 lin moztelen/ die jp noenten Papas, Die oock waden in gantfely Pirer, tris een ghelont $\mathbf{z}$ ant en feer bebolctat on twelct aen-walt alle daech t: 'theeft veet Befs ban aldertjantore roozten.

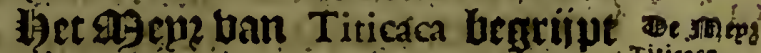
iactitentich leguren/ en op ecnighe plaet lén! it taebtenticl Baemen diep/en daer ont/en ont de Golven dic haex op gheben als het wacpt/ fchiint cenich bocht te stin bain de Zee / ban de welcfeljet wefen fat ontrent t'tefticls leguen. $3 \mathrm{n}$ dite loopen twaelf of bertlien lieluterê/: 
tn bele $25 e c c k e n /$ en upt haer loopt ren Hevier/ in een ander 99 eq/ $/$ die ju noemen los Aulagas, die obeen uptwateringhe en becft/ nochtans om dat ju glyefien bebben gaten ban Hater / die onder de zterde gaen/ oul te loopen in TEe/oozoce= len jp dat het meren mach / Dat het mater ban die 9 gepzen theopent beeft een wech ooos bet binnenlte

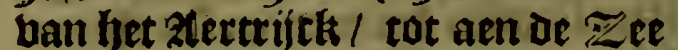
toe: Daer jijn ontrent Den ombanck ban defe groote agepz veel Doz= pen / en in die jijnder Eulanden/ en beel ghefaep-inge/ en allier ple= ghen oe Indianen te betwaren bare coltelickste dingen/tot meerder ber = ceeckertfept.

ElCuzco. Te groote Stadt ban Cuzco, een Conincklicke lit-plaetre ban de Ingas, en'thooft ban't tiitck/en nu ban oe Coninckrijcken van Piru, nae de Clijtel dat het Daer af dzaecht ban ot Coninghen ban Caftilien en Leon, lept op Dettljien graden en een balf boochte / op 78 . ban de langte / jondert 2 s. leguen uan de Stadt de los Reyes, ten Stut-Dolten / van meet ban duy= fent Caftiliaen fche 20 ozglyers. De H9arquis Don Francifco Pizarro fondertue dien / tu feeft acht pa= tothien / en bier Clooftetg bán de vier Bpoens / ban S. Doninico/

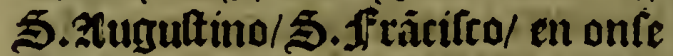
Zliebe Bzoulwe ter gratien: en oer Gefupten / en een van geonicken: albier refideert DeCathed rael Suffragaen, bant alertg-2Birbom ban los Reyes daer gaen ban de tiit vall de Ingas af/ uä de gerct delegs Stadt\$ bier Conincklicke weglen / uae de bier deelen Deg 1Berelts/De cene die 3p noemen Chinchafuyo Poozotwaerts / mae De blackten toe / eil de

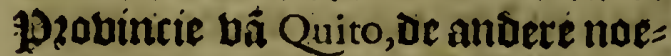
men 3 Condefuyo 10elt-waerts/ naZze:dé derde Collaofuyo, Suptwaert/en na Chile:de vierde Goftwaerts/ Andefuyo, Die na de Andes loopt/en t'afgaen ban't gyebercyte:
If Iept in ect elrebiet bat wel glie=: tempert ismatelick cool on gefont: vap Lan alle uerniinige gewozmeé? en bruebtbaer ban alle oingen met ouplent foozten uan Cáltiliaen rche Dzuchten/en Grupden/en weIrice= liende 25 loemen/ op alle tiloen fert uermaeclielick. Tn bare 3 urirotette Ient de Ballepe Toyma, en alloere/ die beel pzofijts gheben doo? be $\mathrm{CO}_{-}-1$. ca,en ga onlters unt Gout! Silber! quick-Silber/ tn lu bert in barc Furifoictie 1000 co. contribusternoe Indianen. In alle dic Deelen ban die Indiens $\mathfrak{y}$ ebben de Indiane greote luft te jebben in ae $\mathfrak{A g}$ ont 0 ?di= naerlick / poptelen / a ooten / of Crupden en 'tghene dat 313 meeft ghebrupcken in gantfrh Piru, tw $D e$ Coca, om dat $\mathfrak{j}$ - (loo ju (rgabm) wepnich bonglyer voelen / en baer bebinden tect wacker altoos bat

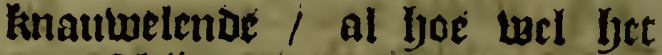
meer frlyint een coftumme/ oft ecr. oljerft abebieck: Defe Coca wert glicplant / in gheeft clepne \$3oout = leng die su boutwen cu carelteeren en gbevent oochies als oft isyete wacte / en glocozoocht in Cozben/ benghent su te coop / en crijgender vecl ghelts af.

In de Eallepe uan Vilcabamba, s. Prancitco, lept s'. Francifco de la Vitoria, in An? defuyo, tuiltelyen la Cordillera uan los Andes; bumaelt twanticly leguen Jan Cufco : aen Oofteri / met een Gotiuernerir / finde cêt pzolvifie

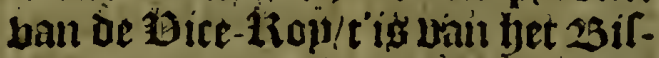
Domban Cuzco, in een rotiwe ons trepur/en met groote 3icrgben.

De Strat ban $\mathrm{S}^{2}$. Iuan del Oro, s.lendés in de 19:ouincie ont Carabaya tach tentich Iegruen ban Cuzca, ten Boft Sinnt-Boiten / en Derticty

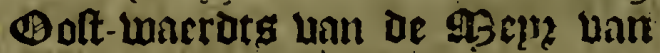
Colfao, en thozt ghentocmit del O. ro, om datter beel Wouts is in die Contreple.

We Sthot ban Arequipa, is op Arequipa.

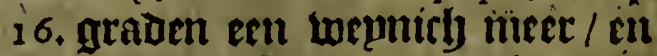
I 0. Iegnen bả delos Reyes, Lu nacít 


\section{แลเ $V V_{e j t}$-Indïen:}

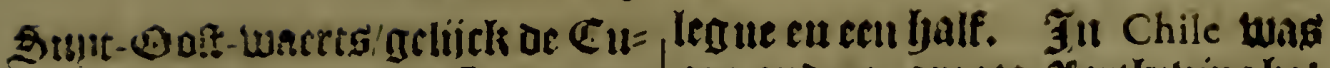

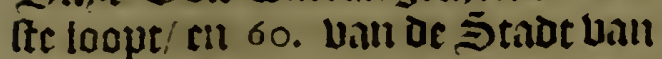

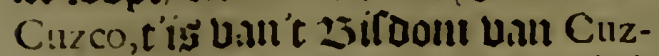
co, thiferit Clusters bam Domini= cancu/firancifinten/ten onle zictue D2oulue ter Santich : tljesft ;0000. Indianen dec conttulueren/lyet is de

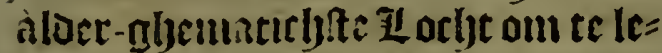
. Lect / Jum aile die it Piru ziili: : ech aroote menichte bail HBin werter Theiunomen un hare Furthoictie; $\mathfrak{c n}$ Tarwe/ die barben is in dell in= gancli bant oe lieviere Chile, die loopt dicift acil of \$taot / al warer unen De Coopunatrisappen out= linit.

Arcquipa Inte in de Dalleue yan Quilca, 14.leguch uan of $\mathbb{E} \mathfrak{e r} /$ in

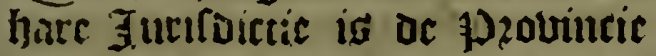
bal Condefiyo, ac Dozpen umi los Hubinas, Collaguas, Chiquiguanita,

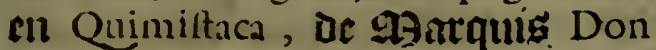
Francico Pizarro fondectoe dien int

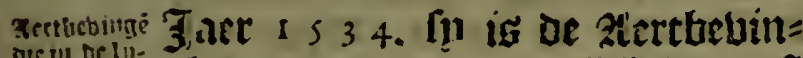

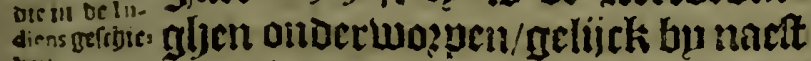
conit.

ech andere groote atertlueluinglyel dic uedervelocoe glyeberchten / en Dallepen/ Root de 3 eeclien uan de lievieren / en trocli de zete unt fiin Zliniten senighe Ieguen / betwoeItede gantefebe oozpen / en bzocht om den fals cen groote quantitepe

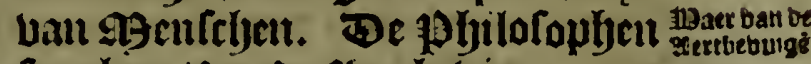
fegghen / Dat De zlertbeluinge comt comen. van De hitte Des 马ous/ cn Uall nul-

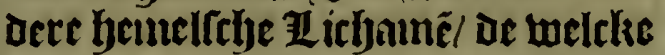
niet alleculick trecken tot yaer de crijalatic en daunpe vall de blackte

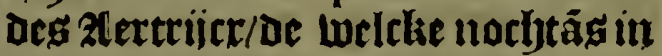
bet binuêtte bun die zijn/dic booltcomentue baren Hoinden/ en regen/ en in dien bet gebente dat de xerde alfoo glyedoten / en dicli is / Dat de uptoanuinge can in gheenen dee $=$ It boozts-comen / Dan beweeche fu haer ball d'ecne deel tot d'ambere/ Dooz be openiughen uet groot ghewelt / Icljickento uut te Larften / etr boozt te romé als bet 250 ate - Crupt alle dic Indicns, en luprontorlick dic ach oe Fere legem: int Ziner Is 82 . wattex cen fehnodinge dic bun naet olll woep oir Statat / cn mul wafter esil aitoer/ en cen barftinglye baut een biandentor 5ergly ban de welc= ke j! luanderlicke oingtyen bertel= Ie!t / in' J Jer I ; 86. Lualter een alloer in de Stnat de los Reyes, Den

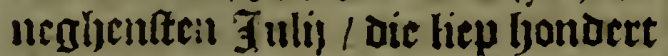
tféticly lesuen it oe lanckte lamex ie Cuite/cu visftich binnens flants wirerts/ ju! rulueerde de Stand/ wepuicly uolcli quan daer int glye $=$ bacr/ oin Dat j!l opt belt licpen/en té wennich dace naer als de fethuo= dinglie ouer wass/ liep ae $\Xi$ ee unt

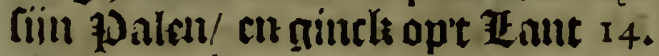
Dindenen lyoorly : noclf fulteten an= dere beturginglye wanter int uol: gljenoe Jare in Quito, in or Staot la Paz, IIt Jaer is 8 r. tenl ball De aloerijooclifte Iiotren llam ban be= nederit uetly ueel Indianen die $\mathcal{L}_{00}=$

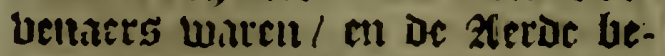
werijucue! die hem omluiery cen ban cen grine/feljeturende inet foo furieuren gijeluclt / Dat de alerde Lerlt / Dacet is / en bact opent met groote furie aen wect om upt te gaen/en loo beel heftigher ig lp yoe de extyalatie die befoten ist grooter ig.

De Stadot ban San Miguel de s.Miguel de la Ribera les leguen van de Zee in de Ballepe uan Camana, is bondert Derthien leguen ban los Reyes, twee en twintich wan Arequipa beSumben de Aqquinoctiael, $t^{\prime}$ í ban het 23iroom unat Cuzco. De 5tadt Valyerde, in or Balleue $\mathrm{Yca}, 3 \mathrm{~s}$. Valrerds. legumt uan de Stadt wan los Reyes, thim lemicn / wan de ven uat Sangalla, is batt de Turifdietic ban los Reyes, ent ban het zlerts-Difbon / hare contrepe is

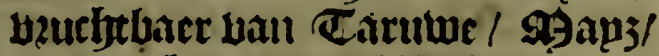
en Buchten/en ucel Hoijus.

De Stadt bail Canjete, of uan Guarco, aen de Dallewe / al waetfe Guaceo; Ient/istuar de los Reyes 25 .leguen! Ŝupt-wanert/ế legue en té halle van 32 De $\mathbb{Z} \mathfrak{E}:$ 
De ₹et: baer Contrepe is buthe = bact bail 23 zoot dant het befte is baul

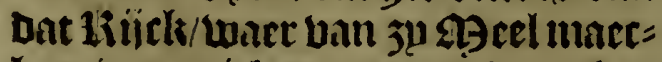
lien in menichte / all te becnghen nate 'tbafte landt / eermen comit tot ocle Dallepe jijnoer ballewen ban Chilca, en Mala, in D'eerfte reglyent bet nimumermeer/ Daer en loopt gế lievier doos / noch 23eeck / en men Dzhiucliter uut Dutten / t'is bol ban befacluinglien/en 10 zuchten/alleen = lick boos de bedauwinglye ban den Heinel : jul viffeljen veel Sardinen in De Ece $/$ en nuet elcli Ganuntie ban gisaus werpen $3 \mu$ oer een in een booze / al water nede 3 jaepen een of twee loofaen/ met welcke boch= richept walten $3 \mu$ wel. Fn oe 1 bal= Ieqe ban Mala was bet: al waer by Den anderen quamen Don Diego ban Almagro, en Don Francifco Pizarro, en de pater Bovadilla, ban de Bzaze ban onfe Zleve Bzouwe ter Gzatien/alg felyepelman / lepoe ter Heder baner differenten: Gen groote kievier loopt dooz de ballepe/ ban waer $3 \mu$ Jebben veel verbertehin= ghe/ uif leguen voozoer / is oe 1Re= vier Guarco, in fiin 1Ballepe alber= upuchtbacette uan alle dingben / al waer de Jingebozenen fyebben glye= leden 4 . Taren lanck den borloglye tegljend oe Ingas : en om lyet te bejouden Jeluben $j^{p}$ boen bebolcken eenstade/en bebben dien nenoemt Cuzco,en getimmert tot een trium= plye van de victoric / op een theubel cen groor Cafteel / Dat de Golven uan ve Zee faen tegeng de Crap= pen. Ses Iemen van Guarco is de Dallewe van Chincha vermaert/ bermaectrelick / en feer oberuloe= dicly/ al Daner is ect Cloofter gbe= tiumert uan Heligienfen Donumi= canen / in oe plaete ban de lierck uan de Sonne/ die de lndiane yno= aen: inet bet Cloolter de las Vir. gines.

Stract volelyt oe Ballepe van Y ca, die niet cleunder $i$ / / noch min = dec ouerbloedicly/en baer upt coment af of ballepen/an is Zicuictm uan la Nafca : en or psincipaclte bert gibenoemt Caxamalca, al waner $\mathfrak{t x}=$ rellente Doijnen gemaeclit werden. GGen gaet ban be ballepent tot dic vall Hacari, Dare jiju Ocanja, Camana, en Quilca, Dat de l)atum is van de §tadt vall Arequipa, fll bookts jill or Dallewen van C huli, . en Tampobolla, ent uan Deylo, sil die van Tarapaca, hievieren/en ouer = vlocdicly met gocde \$gijuch bull Silber/en ontrent of $\underset{Z}{E x}$. De Indianen ban de Eulanoen ban Lobos marinos bzengen beel aset ball Boghelen over haer Erflanoen/ met de welelie $3 p$ bet onburtytbaet lant batuebtbaer Inareken.

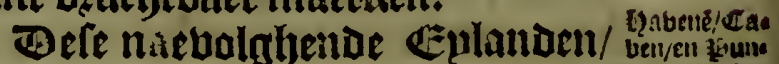
baluenen/ 1 S unten ziin op de Culte ien vali yee ban dit bof / wan de jounte ban woof ban de Aguja, al waex 3 tyaer voedyt met Die yan Quito, op 6.geaden ljoochte be-Supden de Ilinie. Jaer jijn twee eplanden/ die 3 noemen Lo. bos marinos, op 7.graden : de ecne lept 4. Ieguen wan de Culfe / en de anderemeer in zet : ell voozts leyter en ander die 3 p noemen bait San Roque, ten Sumt-Doften / on= trent de Paźcamayo, ell wookts is de Gauen ban mal Abrigo, tien leguen booz die Jan Truxillo, op 7. en eent balf graden: en 7.leguen ban die ban Guanape, en die ban Santa op neghen graden / en s.leguen ban oaer Stut waerts is de hauen ban Fen rol, en les Lan die/ is dic Lan Cazma, en acht legucll ltooz= Der if die Dan Guarmay it de 2 Sont ban een Ziebier / en die Barranca is twintich leguen Supt-waets / $\mathfrak{\text { sll }}$ die ban Gaura al waer ech oucr= Uloedigbe 5out-goanne is / end dic obergedaren ziinde/wolclit lyet $\mathbb{C}^{*}$ = lanot yan Lima, in oen ingancli yan de haven ban Collao, en twin= tich leguen Sunt waerts / is be Poutute van Guarco, en ecll Eulant Jan ie Lobos Marinos dicljt bu dic/ cn oe Bunte wan Chica, lept op 15 . 



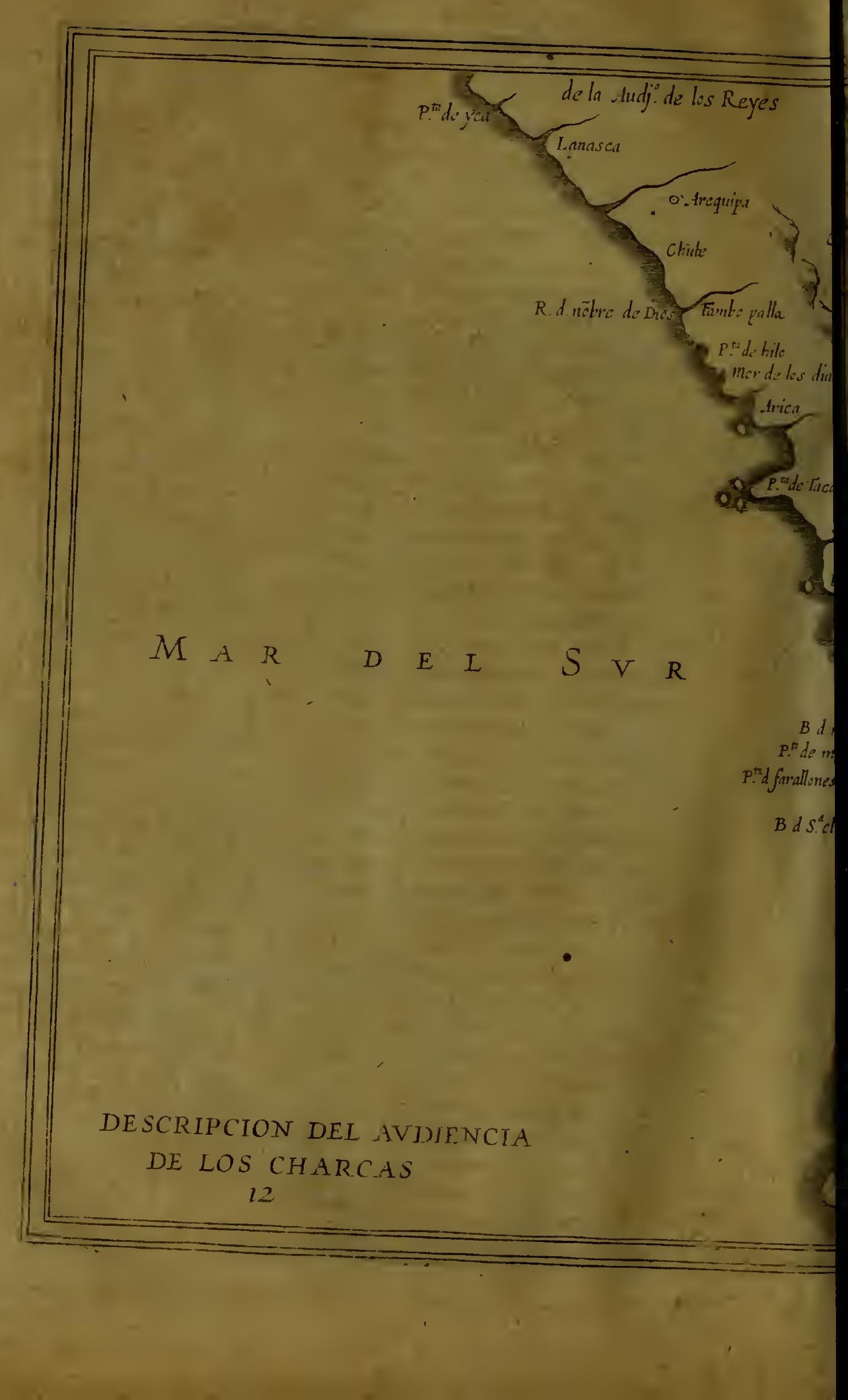


C $\backslash R T E$
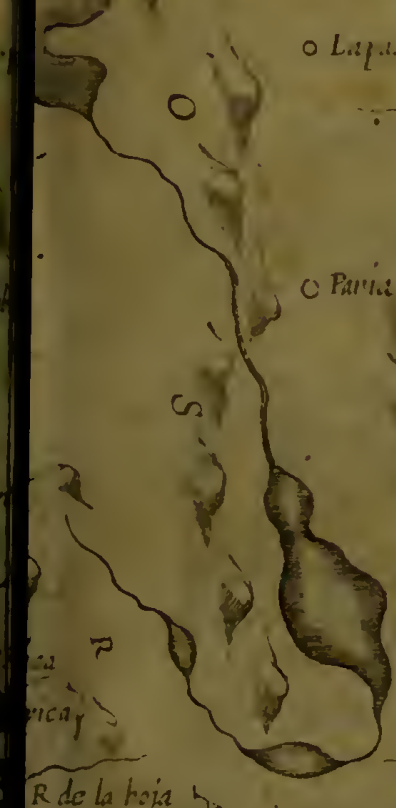

R de la toin $p$

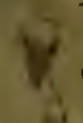

$y_{-\infty}^{z} z^{-}$

$y$ m

$y=3$

42

3

Santiago

ก simmign, i

TVCVMA N

- Falanern
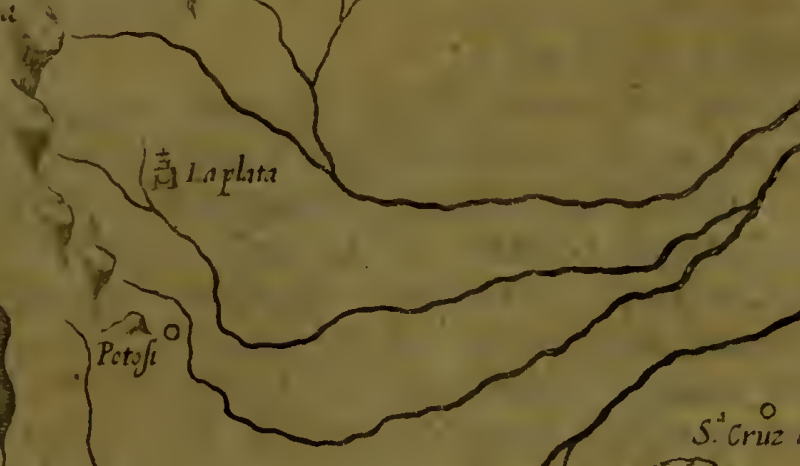

S.' Cruz de la frerra

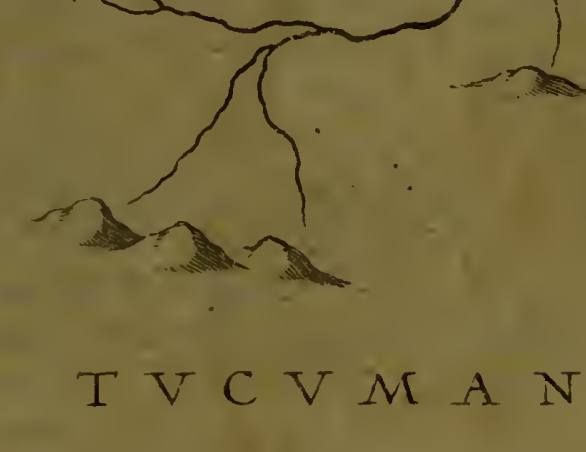

$-2-2$
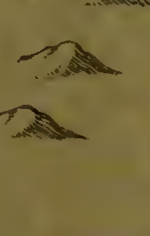


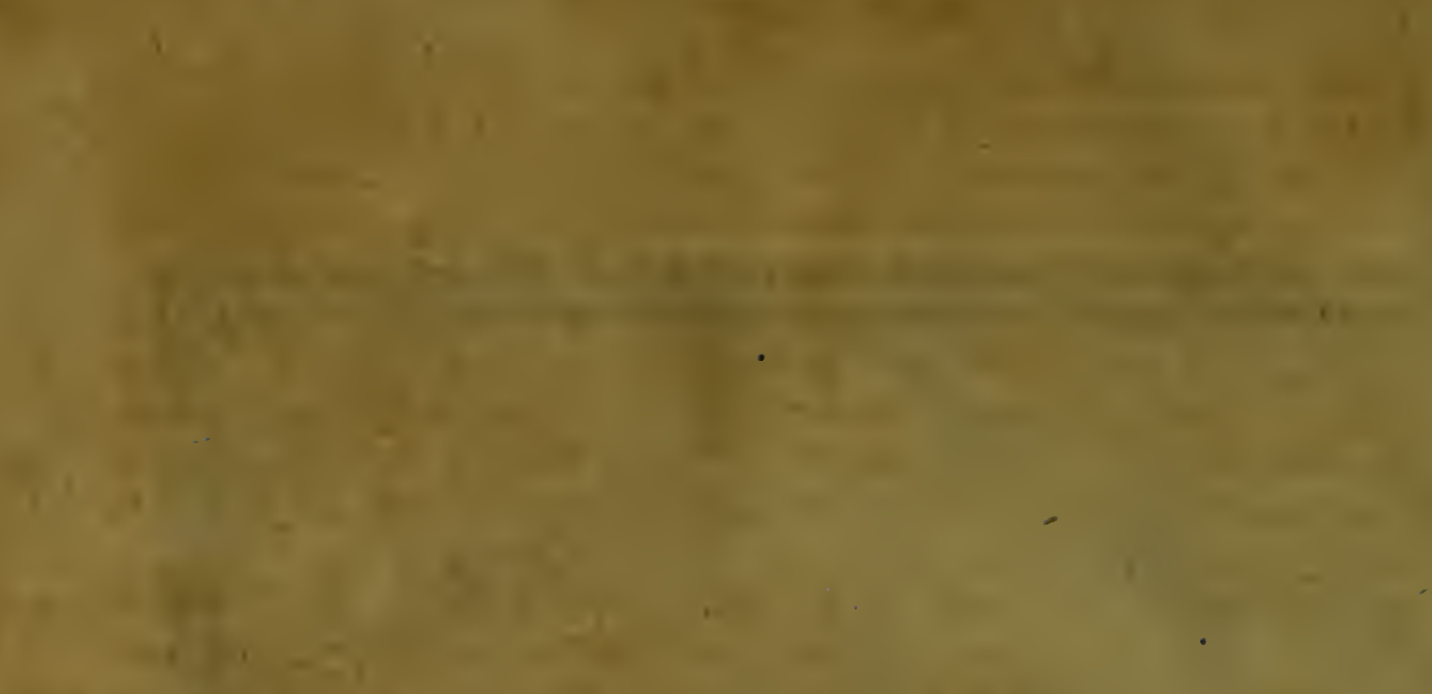

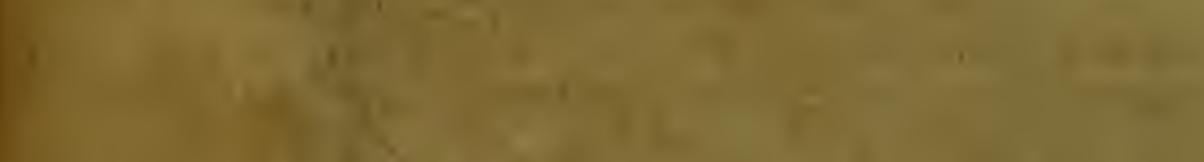
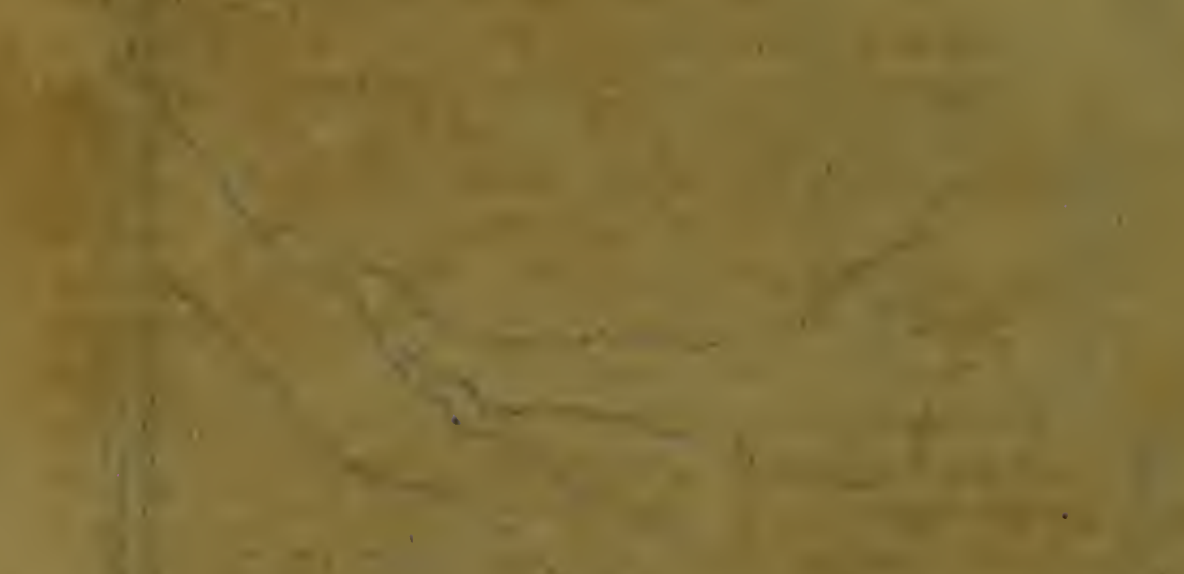

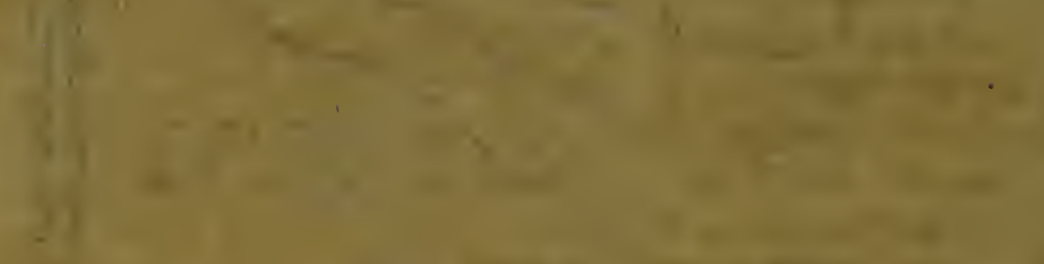

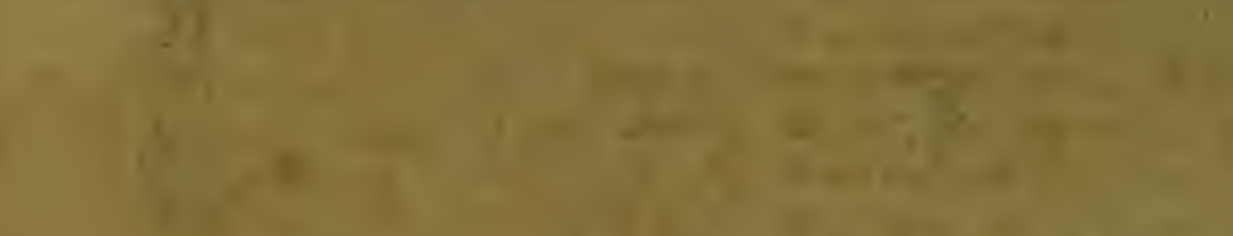

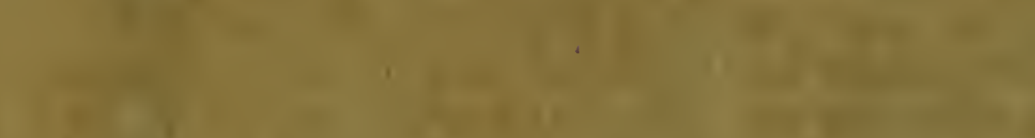

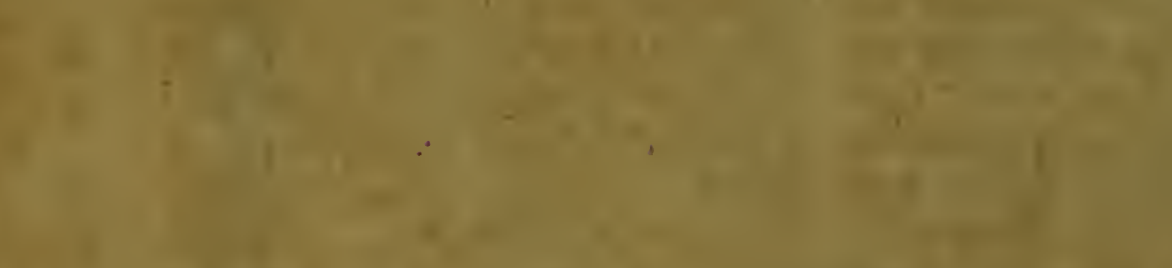

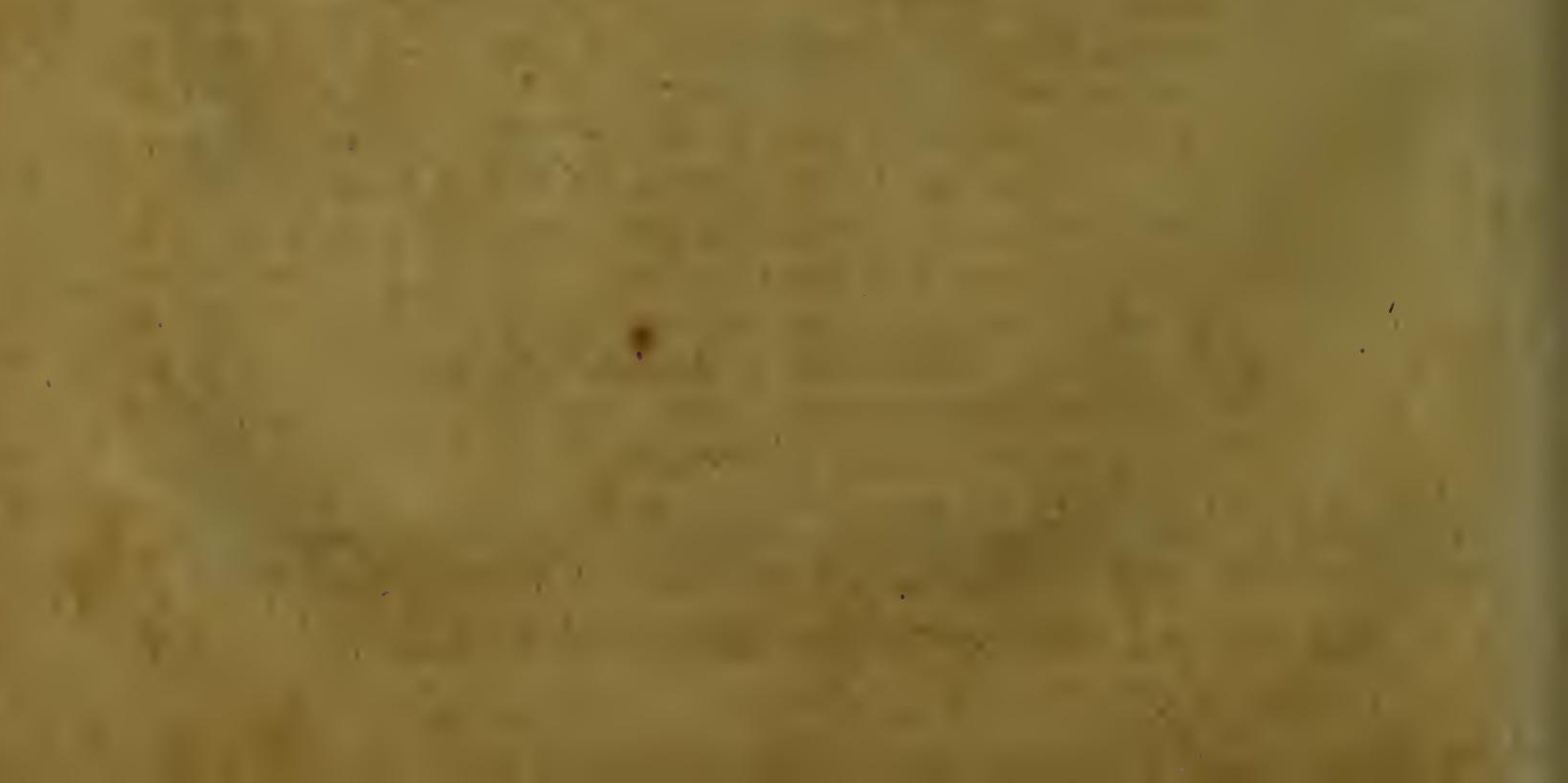




\section{ualt $V V_{e} f$-Indien.}

oriban / en bicht bu die leut Nafca:

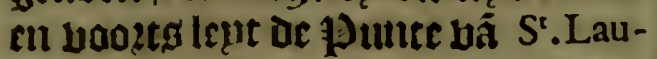
rentz, in or Dallewe ban Quilca, ent Dicltt bu of Eievier ban Arequipa, m be Caleta bau Chulien : en Daner mae lequt oe lievier wan Nombre de Dios, al wiate yaer thamenlueghen lancr de Culte oe reffozten wail liet yof uan los Reyes, m ban los Charcas.

bet $\times \times 1$. Capittel.

Van bet refjort des Hofs de la Plat a, of de los Charcas.

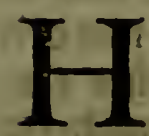
Et licflout ban bet bof um los Charcas, t'welck oçult fiin Xantpalen net dir van de los Reyes, Int op $17 \%$ graden en een half be- 5 upoen oe Zlinie: Ju or tievier ban Nombre de Dias; cul lat beginne ban de I9epg van Collad, feet ltreckt hem unt int tauck dzie bondert legueti/ tot of Aallepe. Coplapo, bet begin ban ac 1Dzouincie ban Chile, ap 28 . graden hoochte / al hoe wel dat $3 x$ tellen dat bet is 400. leguen rep= [eng/ en Ooft en 3torth / t'ghene is tudechen de Cufte: ban of SuptZee / en ban de Zute ban't \$oos= orn/ of \$upen/ welclese relponotert op ar 13 zoluncien ban oe tiebier de la Plata, welcke at hoe wel yet t'ee = nemael niet is ontoedlic/ wert wet taen / om dat fret ganteche Z Zanot ban dit 1) of ghelentyen is op beel booclyte/ meec caut dan warm.

bet Gonbernesnent bant dit 1 of is tot laft Jan of bice-liop bain Piru, en dic ban't ty of dan Quito, en bie vint los Reyes : 'tljeft tweet Gouberneurs / en twa 23ifoomIInen/ dic uan los Charcas, en Tucuman : De 1 zouintie wan los Charcas, de welike wert gljenoemt ghemepnlick tglyene bat is yan waer 'tlitb woeclyt niet het tieftost ban't
Hof unn los Reyes, tot vaoulbu Po. tofi, en ttrectit bem inde lanclute Supden en \$oozoen (bu cang hyon. Dert so. leguen / en noch loo beel Doft en Xaeft: lyet Landet is niet feer vzuclitbaer / al ift dat bet ober-

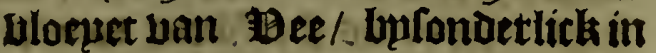
Collio, dat is ban be פep? ban Titicaca, tot ie Charcas toe / al waet ae Winter en de Sonter liin beel contrarii glyelijek in Europa : bet Qland is bol Bers / m bufonder ban \$chapen / die Daer beel zijn/ m baglyen leet fije wolle:

In oufe 9 gobincie zijn 4. Caiti- De stabtde liaenfche Steden/ monor een $23 i$ Dom/ wetefizijn de Staot de la $\mathrm{Pla}$. ta, dien beboleltstere de Capitepn Peranzurez;int Tate 1 \% 83.0002 latt uan de 29 arquig Don Francico $\mathrm{Pi}$ zarro, Iept op 72 .graden / lanckte uan de Suut-Roox--ilin uan Toledo, al buacr bet fal afieggben op

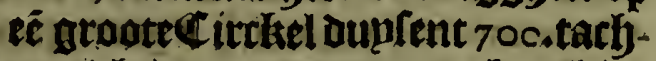
tenticls leguen / en op negbenthien graden han de bzeette bjondert 75 . leguen ban Cuzco, aen de fijoe bant Supen of to Polo Antaretico, binnen de:Tropicos, in bet laetfe Dan de Zona Torrida. Taer fullen 3ijn in de 3 urifoictic van die Stadt in Charcaras, Dat erfeliche I of - Steden 3iin/ acht yontuert: 23 ozghers Cantlianen : tris een feer cout Lant / Dat beboozoe ghetemipect te welen/ of warm/ tonfozm be foochte wan of Pool daer het onder lemt. In dere Stabt refineert bet bof en de $\mathrm{Ca}$ tedrale laercke/en de o toolters ban oe Dominicamen / ffranciflanerg/ atuguftiuers/en die ban onfe I ietu Dzoulwe ter Gzatien / $\mathrm{en}$ in fine Juridoietie ziin 60. Dupufent Cributarife Indianen, glyeaeplt in neghent en twintich oeplingben / 'tgheeft wel de zade uan Caftilien,en 'tljeeft bet. Dijnen ban rijck silluer.

De Stadt ban Nueftra Senjora, Nuefra Seno de la Paz, anders ghenaemt Pucblo paz nuevo, en Chuquiabo; int midden loan Collao, 1oo.leguen van Cuzco, I 3 en tach- 


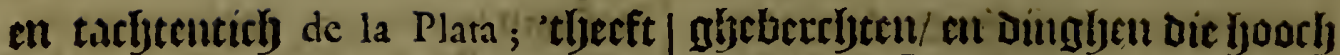

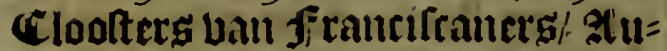
gultiners/en bau oule Zl ieve 1 20u= weter Gzatien/ met beel poijns en Dees : lp is aljefondeert ban de Capitepur Alonzo de Mendoza, int Faer i 49 . als bzelident was de Goubeuneur van Piru, de Ticell= tiatt Pedro de la Gafca, 25íteljop una Siguença, die fju Daer naer is glye= weelt:

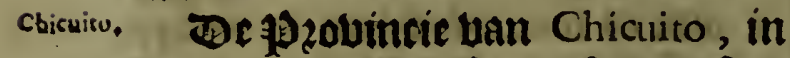

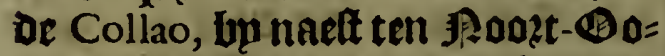
Iten ban la Plata, meer ban fjondert Ieguen Daer af / ig een bebolckinglje uan de Indianen, met een Caftiliaen. fche zectyt-pleather / van het $23 i$ if = oom uan'los Charcas, en ế Cloolter Lail de 5ominicaners/[u beeft veel Beeg / en is gelegen op de cant ban

oropera, Titicaca. Oropefa, is em bebolckill= ghe vall de Bice-thon Don Francifco de Toledo, in be ballepe uan Cochabamba, twintich leguen luan De Stadt uail ia Plata, yare into= nien ban dedarulwe/ig baer wintt/

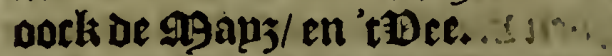

Poton. De ktequeticke Stadt uan Potofl, op ueglyenthien graden booch = te / achthien leguen van of Stadt la Plata, en bo naeft acht Lan de ATeug/van los Aullagas int Boften/ int afgaen van de 25 ercy uan $P o-$ tom, Die nae't, Rookt-Bolten liet: jeeft s. bonoert Junlen der /Caftilianen, die op oe פgijnen letten / en Der Cooplupioen/en so.duplent Indianen ordinaris/gaende en comen= be op de neeringhe waul bet Silver/ mal hoe wel lyet een Stnot is op ficly felfs/ foo en lyceft fe glyeen $\$ 0=$ litique tieclyter / dan bien die ban be Stadt la Plata:allyier fitté de of = ficiers/ en be Coninclilicke SrhatLifte is hier van de 1 gobincie / om de 23 ijnen van de 25 ergh uan Potofli; die een. Caftiliaen thenaent Villa-roel, alltoetktede / Doo? mid= ael Lan eenighe Indianen, int $\mathbf{Y}$ aer I s 4 sien wert glyenoemt Potoffi, oin Dat de Indianen alfoo noemen die 3iln: cti 't weet / Dat unt ghetroclien Lucroc in De begrinlelen / ant int 3 aed is 49 . alle 5ateruaglyen als jul mooten/ doegen ball 25, tot Derticly dumlent pefos : Tiealen ban vijuen. Je $25 e r g b$ isflecht/en gelat : De Loon: = plactfe is dzoocty/en cout/en ganfely olluzuctjtbaer / die gljecn Bomeljt voortuzenght / noch Szaen / noch 5zas/ all on't Siluers wille/ i ît meeft bebolcht ban ganttch Indien, cn ftreckt betu twee leguen int rout= be/ al waer men bint allerljande Coozte ban delicatefre/ en ouerbloet/ al yoe wel bat yet met de Harre gheboert wert. De coleur ban de Zlerde deg 23ergdja / trecht nae den lioffen / Donclieraclytich : lyet fat= foet istals een 23200t-Sunctiers/ Dominerende ouer de andere $23 \mathrm{er}=$ ghen die daer ontrent ttaen. De boorbte is rou/al hoe wel datmenfe te paert gaet/een legue in ongane/ en van fín pounte tot aen be Boet fait fiin een quartier ban een legue: topheeft nen alle beelen feer rijcke adoren uan Silber/ban bouen tot beneben: m de aben ball Arica, ban Laer de Coopmiantebappen gheronaen werden / en jet siluer Dat daer woat uptglyetrocken ban Dien $25 e r g y$ / lal Daer af fiin the gbentich leguen lop naeft in't WE: fiten.

Seg Ieguen vall de vogrentide 3ergly / op de weely ban oz ball Arica, is de 23erglj Uall Porco, Po \$rco. een onde placte uan Sillure-99ii= nen / ban waer wert gljefeut dat De Ingas muttrocket bet mectte decl unn't Silver dat in De lerets uan de Son uan Curianche wag / van waer de gant'tye wech lance sil= ver untghetrocken wert / en men nocls upterecken fal veel Silvers.

Santa Cruz de la Sicria Icpt bouls dert leguen uan los Charcas, Boftwaerts / op oe wech al waer men gaet baul los Charcas, tot Affumtion, 


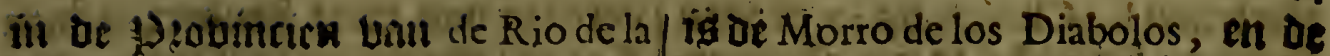
pláta, tor wher toe 3 il itellen dyie bondert leguten rep!chs / met ech Eoubertecur/een probifit booz de

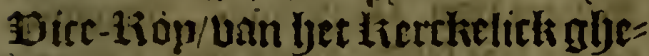
biet wan los Charcas, en ect Cloo=

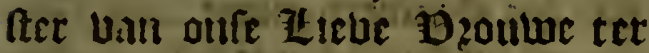
Gsatien ; it lyet 1 ant bater 10 ater gifedzeck is / al boe wol bet butht $=$ baer is bat cartwe/ toijn/en

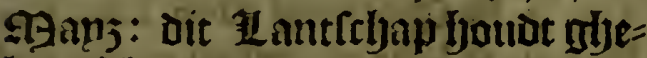
bu!nefliap/met vele Natien van oughrloouighe Indianen, en ued ban oien bebben bace Doen Dod= peiv/cul d'erite die ouder oe $\mathbf{3}$ nghes bozench or of botie ltelde / was een Ciltiliantehe Soldaet Die anoer fyner ginck/1)lutitende oun (ijn nuif= beurclich/ dic oum dat ju groote be= uautheuat yadoen ban ghebeeck bail Water/baer naecktede cen groot ertusp/ en bact wijg maetk= tede dat ju bet aenbaoen met oevo= tie/ ch Hater rotiden Lenteeten/en Godt Dooz fiin barmbertichepot verhoo?dere/en gaf grooten regen/ int 3 aci i $; 60$. wettroclt de Capi tcpil Nufflo de Chaves, vã de Stadt ban Afcenfion nae oe ticbier de la Plata oun te ontoccken/en hu quam te Santa Cruz de la Sierra, flyediaent bebben de groote ontoerkingtyen; beeft bet wolek haer wederom ghe= wozpen / en feljiinende dat lyp wag ontront die ban Piru, luas lyp in die Btad ban, los Reyes, al watr Di= te-1ion Lang or A9arquig ban Canjete en met liin ozoze / als II tujte= mant unu fiin \$oou Don Garcia de Mendoza, is uuederom ghefreert of Capitepur Nufflo de Chaves, omte Eebolcken dicn Stndt / al boe wel In uertrock tuit de kievier de la $\mathrm{Pla}$ ta, to: Dim eninde ant Ijp foube ant= Deckeri de pzobincion ban Dorado.

oy oc C ufte van oat bof / oat

in De Iicuter de la Plata; of Tampo. balla, is de baven uan Hilo dicht bu cen ticuicr op achefien graden en rell ljalf/en ureer Smut-waerts Gaben ban Arica Iept op negbena thien graden en een deroenteel / ett die van Tacama op een en twinticls tII de josunte ban Tarapaca te Sup= Den / en bet bootts lept de thebiet ban Pica, en Die uan la Hoja, en ban Montela, of lyaben bain Mexillones, en nteer Supt-waerts is de Punte ban los Farollones; of Morro Moreno; vooz de Bahia, en be kes vier/ St. Ciara, en bet Supt-waerts la quebrada, ent be Bunte Blanca, en Die quebrada Honda, en te hebier ban Santa Clara, bp naet oetticf le: guen ban be Liebier Copjago, al waer de Cuifte van Chile begint/en die ban los Charcas tuntoigtet.

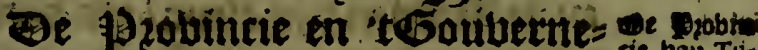
ment uan Tucaman, is beél binnit- caman!

lants / baec reflozt fteckt bain bet Zandt de los Chichas, bie onder be Juritoictie zijn ban orie tetererlicke Stabt uan Potolli, is ghelegben op oe Areecke ban oe staot ban Affumcium, ban be Lieviet de la Plata, un naelf bondert leguen! ban be Cuft van oe Supt-izec/ arenrenide

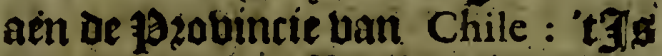
een glyetempert Jant/ midoelnas ticly vetichethaex / en tot nu toe con= Der gginen ban Silber / en Bout/. daer ziin eeniglje Jozpen van $\mathrm{Ca}_{\text {- }}$ tiliane in een 2 siloom/ oik zijn van ve Stadt Santjago dél Eltero; die $3 \mu_{\text {Entero }}^{\text {sanjago del }}$ in oen beginne noemoèden del Varco, of 28. graden fyootjte / bondert 85 . Irguen vã Potoffi Supt waett/ ế wequich wijetien oe o oft-waert: all lier refideert de Gouberneur/ be Officiers ban bet incomen / en de Conincklicke Scljat-ftilte/en bier if 23 ifoomi an of Catbiozacle iterclie.

De Stad uan Santa Maria dé Talavera, if 45. leguien van Sant- Talaverai jago Poout waert / en 40.van Potoff, op feg en twintich graden.

De Stadt ban S. Miguel de. Tu- San Miguel: cuman, is arbt en twintich leguen ban Santjago ten melten / op ben 
uech uan los Charcas, op, 27, gradon Th.werde lievolckt in oat bouber = neulent a Mendoza alg liin Bader ot $\mathfrak{A a r}$ quid de Canjere Bice-thop was. Dacer igoct nieutoe Londres, en Chalcaque idat jo noemioen niculw Cordura, in De pepoincie bait Los Iurias, en D raguitas, bie ontbolckt 3in binnen wennicj 3 aren. Bet 3olck uan dit I ant gaet met $\mathrm{zool}$ Gheclect/en met lieer gebuzocht lecr 20 ogherlicls/op de nuanier ban de Capillerie Unir Cantilicn, daer woat Ueel Bees aticinonnen op't I anot oni oer Holle willé / aaer 3 p profijt

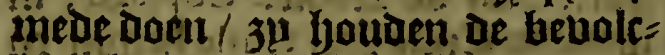
Kinghen feer dicht bu ben anderen/ en be Dozpen jijn clepu/, om datter niet meer dain een ghedlacht in elek ig/3p ziin int ront bedoten niet to: Aften als caerom/en $0002 n e-2500=$ men l om de Dozloghen die onder baer waren: "tzijn cloectie strbey= Ders / en t'is Bolek Dat niet dzone= Fen en ozinckt/om bat $3 p$ baer daex toe niet beglieven / als andere s $^{2}=$ tien van be Indiens; in oie $1920=$ butcie zijh 7.groote kiebieren/ en Meer dan tachtentich 25 eecken ban goet Dater / en groote Hatuden. Inde Hinter en de Somer siin de tijoen als in Cantilien : $t^{\prime}$ is cen ghe Cont Lanot/ wel ghetempert/en oe cerfte die daer in quamen waren de Capiteunen Diego de Rojas, Philippe Guticrraz, en Nicolas de Heredia, en quamen t'ontoecten aen bie zijoe ban de liebier de. la Plata, tot oe forterelte van Gaboto.

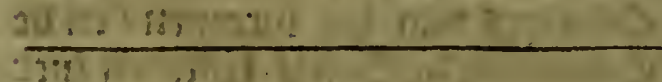

Set XXII. Capittel.

$V$ an bet refjort vain bet Rück van cbile.

TRt Tatr 1534 . is in Toledo 1 gepzobiderert ghelueelt Don Die. go de Alnagro, om te weten Gak= berneur ban twee bondert leguen J ants / glyeleghen ueel verder dan ljet Sovivernement uan de $\mathfrak{9 a r}=$ qiuis Don Francifco Pizarro, nae ofe Straet van Magallanes, intitule $=$ rende dat felfue decl/ inet oenune van bet Bontuernentent ban Toledo, an Iy ginck on te belazcaighat bat 2 andot / en om dat bie bet niet en volfzorfit / wert bet int Flace Is 3: De פararquis Don-Francifco Pizarro, te lalte gljegeben en ljp gaf Daer ban ljet bevel int Jaer I 50 . aen aen Capitepn Pedro de Valdivia, en fug gincker met hondert so. Caftilianen. Iulle de Yanden uan dit tiijeksiju int Stupen van of Zunie Equinoctiael, op meer S.un= declicker loochte / dan lyet Litick uan Piru, en bare 132obincien bin= nen die Torrida, ban den ÆquinoCtiael, tot Den Tropico ban Capricornio, die loopt doo? een ontwolcts= tede plaetfe / die 34 noemen A tacama, die leyt ban 23, tot 26.graben/ en ttracr begint het Wiijct bau Chile, Dat De Indianen noement Chille, en booz het bevolekte 1 ant lept op 23.graden en derie quart de kevier Uan la Sal, dic bunt Bolten ban de Cordillera ig/cnde loopt melt been na de $\mathbb{Z} e$ / dooz een feer diepe Bal= lepe/ en cbenwel geft het feer claer 1BDater voo? De Deerdé om te Drint= Ken / efi doo? be lyitte uan de 5on= ne / wozt lyet water foo pupz tout/ ontment uiet en can ghedzuncheni en op de Oeluerg is een liebire eer Datmen in cont in d'cerffe pzovin= cie van Chile, twee en twintity le= guen landel / al waer ie Xagneycs stin / dat ziul water-putten / on! datter olyeen ander en is in oe 22 . leguen / en dat gant frbe tiijck lept binten be Zona, die de Buben naé = ben Defierta, die feer be wolct is bati witte Indianen, en is ghelrglyen op

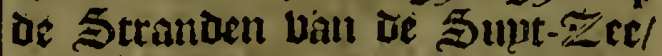
twelck is de groote : 2 ed / die beflo= ten wert tulfichen defe culte/ en die van China.

Dit 


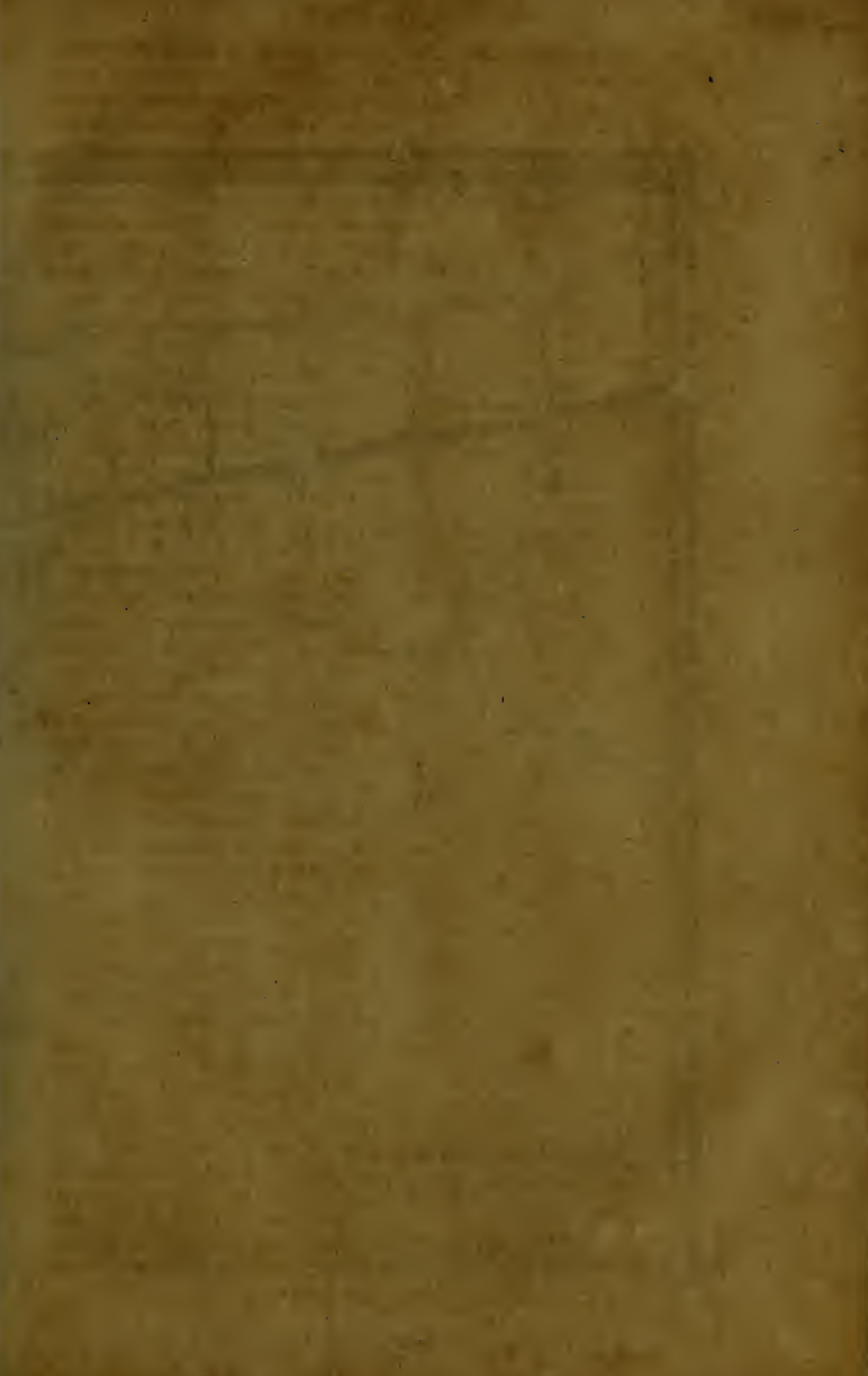




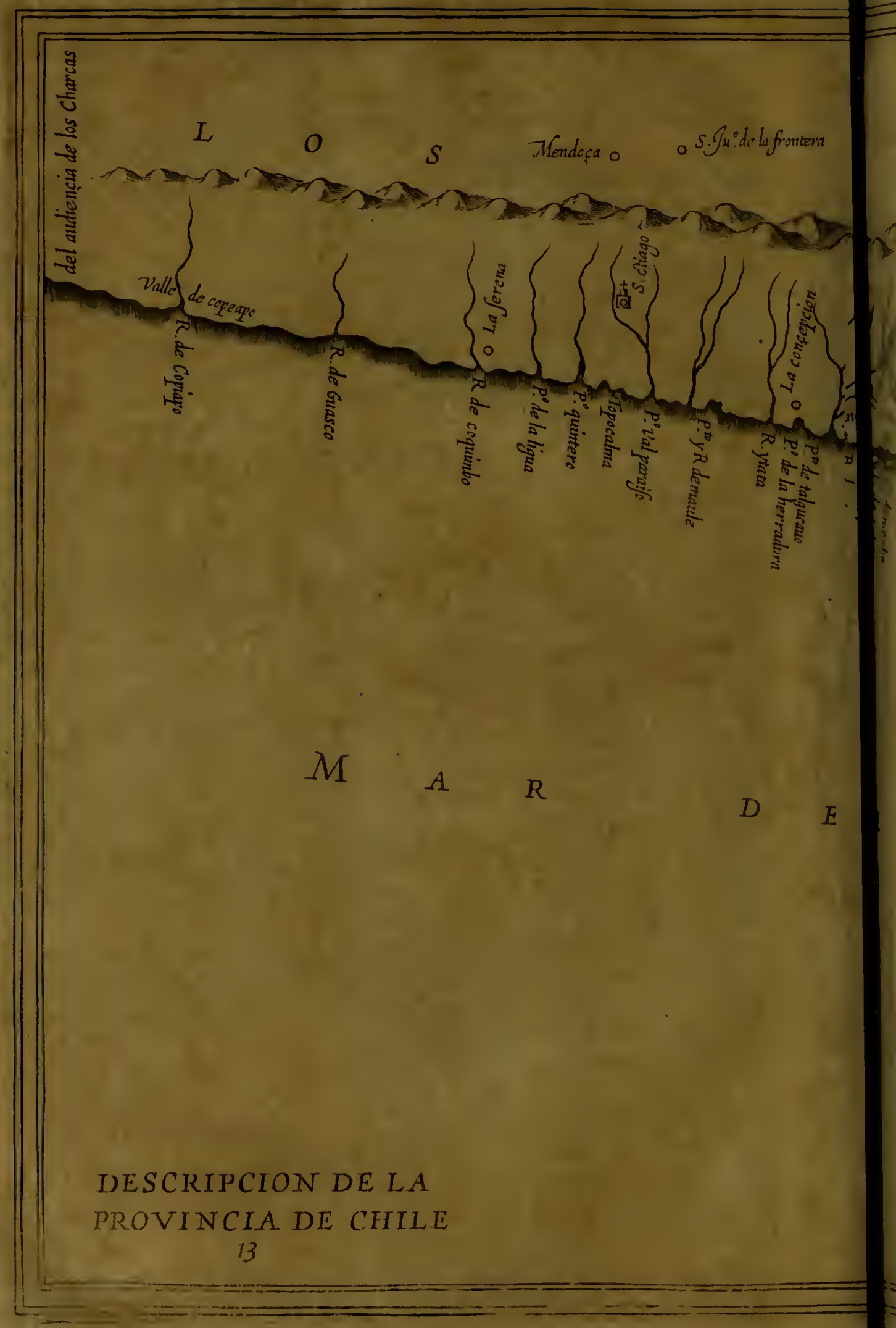




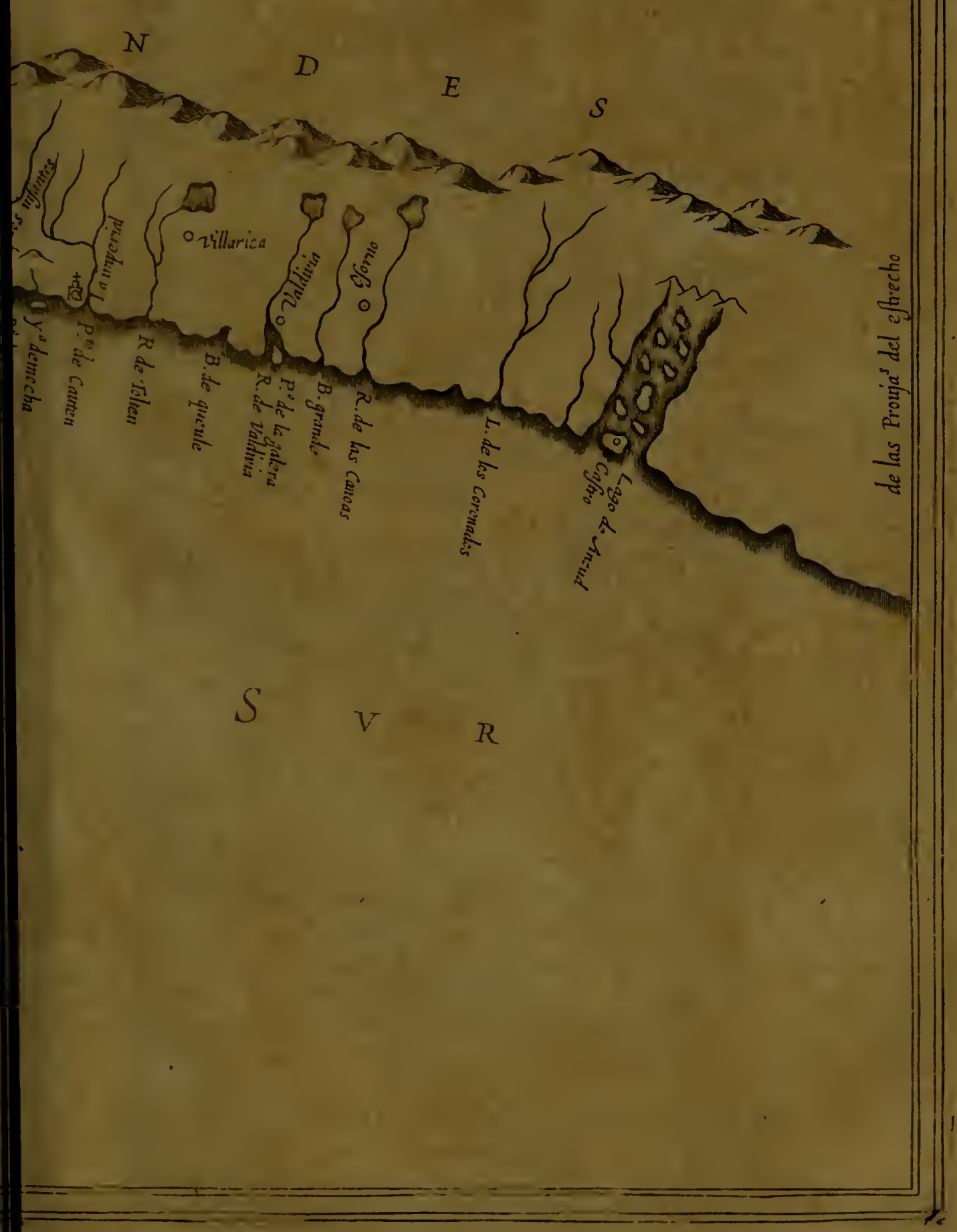


r $x^{2}$

$4 y=$

8

$4+1$ 
Dic Souvernetuent rupu glje $\mid$ cecion, enl los Confines, th bet Imnomen tot De Straet toe/ beeft bujf Gondert leguen int langb 5 upuen en foozocu i uan de Dallęe van Copiapo af/ unit waer 3 ! begint op feven en twuticly graden / en in de beette Dolt en welt/vande SuptZee tot die \$oozt- Zee / vall vier Jondert tot s.Jjondert leguen lants but nochte bebsediglien is/Dat bem gaet veroumuen/ tot dat lyet glye= keert wert bain oe Straet tot the= gljenticly of bonoert leguell. bevolcktede bail dat Gouberne= ment fal 3iin Dzie bondert leguen/ lance de Culfe uan oe \$oost ₹ee/ ent de beeite uan dat / fal zijn twee honoert leguen / en minder tot de Cordillera Ualt los Andes, die eun= Digbet outrent De Straet/ en loopt Dooz dat Ziiicli leer loocb / en bp= cang altoog beorickt met Sneew/ t'is gant'fly cen ulack Lanot / ten miniten fonoer eenigyemerckelicke roubwichent/ belyoubeng al warer oe Cordillera ban Piru begint/Die hem begheeft twee of daie leguen lancx be Cutt.

De gluetentpertlyent en qualitẹt van lyaer / al boe Leel ju niet en igs Conoer ecniglje berfebepoentyepot/ om de beramderinge ban de joorly= teti/it de welclie ju gheljeel in ghe = leghen is / jul ig baul bet belte / en belwoonlickite Datter in Indien is in glyeteunsertljept / ftrectiende nae die van Caftiliē, op weickers hoocly= te lyet bu rang contrarie conit te legghen/oork in oberbloet/en goet=

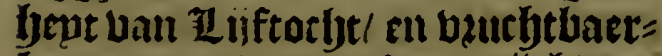
bepat un alle Dutghen / rijclioom baul פgijnen/enפgetalen/et lterck= te en warkerbept ban de $\mathbf{I}$ ngebo renen / want oun dat ju altoos foo betliceur jiill glyeweelt/siin ju qua= lick te lebzesigljen glyeweedt/en int ghelerchte / en aen oe fijoen bati't epmoe uan de Cordillera, it de 39 ? $=$ viucien ban A rauco, Tucapel, en De Ballepe van Puren Zantpalen en Zurinittie tulteben de Stabt Conperial, fijnoer ueel dieder norb bof loglye voeren.

Eaer zuin in Dat Soubernenient elf -oozpen ban Caftilianen; met cen Bouberncur / gbefnbltitueert in oe faecken ban liet Boubetne= ment by of bice kop/en 't ban Piru, naer dat bet bof te niet ginck dat in Dat Zanot was: en Daer in jijn twee 23 iroommê/Sufs fragneniftyappen ban bet atert523ifoomi van los Reyes.

In het 25itoeni van Sant-jago sensizo: 3ijn vier 5teden. De \$tadt ban Sant-jago in tue tijot de eerfe be= volckingbe ban Chile, de Capi= tepn Pedro de Valdivia fondeerde Dien) int 3 aer duplent biff bondert eenen beetticly / lept op vier en ders tich gradé en een bierendeel hooch. te/reben en tfeluentich ban de langs te / ouprent neglyen bondert tachs tentich leguen ban Toledo, op een reclyte linie/bijftbien leguen ban de Zee / en tljien verder van de Bals lepe van Chile, dien $3 p$ inden bes ginne noemoen Nuevo-itremo. 2Al bier refioeert de Catjyedzale lierc= he! met Cloofters ban Dominica= nen / firancifaners / en ban onfe Tieve Boube ter Gzaticn/ in een Contrepe die bzuchtbaer is ban Cartube/ en HDiin/en andere din = gljen / en uan Ieer rijcke stijnen ban Gout / en in bare Turitdictie 3iin meer dan tacljtentich Duprent Indianen, in les en twinticly repar= titien. Bete Stad wert nhedient met de loaven uan Valparayfo, op be gsont uan de tievier Topocalma, Die bieft daet by benen loopt.

De Capitepn Valdivia beboletts tede oock la Serena, int \$aer bup = La serme rent biif bondert bier en veertichy viclit bu een goede baben / let is bet eerlfe bozp ban de Caftilianen, iti Den inganck van Chile treftich leguen ban be Stadt Sant-jago, ba cauts Pookt-waert / wiickende nae twelten/ dicht aen of Zee in oe 
7. allepe unu Coquimbo, met cloo= Aters vail finncifaners / en ban onite $\mathbf{1}$. Dzoulue ter Gzatien. Cen regbent ljiet utet dan bie oft vier macl int jaer/en in de 3 Landen ober becs lijoe vant dace en reglyent bet nimunermect. De Gaven die $3 \mu$ noemen uan Coquimbo leut op 32 . graden / is een goede inloiick / al muer de Scliepen ban Piru aeneo= inen. Jin de 13zovincie uan Chucuito, die aen d'ander fijoe ban de Cordillera de los Andes is/ in een cout en onbzuclytbaer I andt / ziin Mendora. De 5 teden ban Mendoza, en die

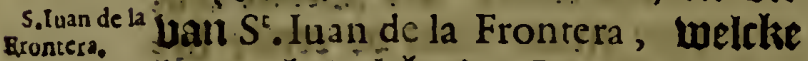
hepoe bevoicliteide Don Garcia de Mendoza. De Staot Jan Mendoza, in ope Contrepe uan Santjago, bp naelt 40 . leguen/ een moepelictse werb om lyet Sneeu dat in de Andes is. De Stadt ban s'.luan de la Frontera, lept 3 upt-Luacrt ban die han Mendoza.

Szet:3iroom

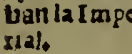

In het Sirdon van la Imperial jiin reuen Stcden der Caftilianen, twelck fiin/ de Stadt uan la Concecion, op 37.graden bopchte/tie= bentich leguen Suut-waert bau Sant jago diclut bu de $\mathbb{Z} e$ ed Pedro de Valdivia bevolclitede dien int 7 aer

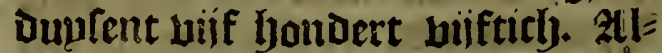
bier refivertoen de Gouberneurs/ tredert dat bet bof verlaten is dat aldaer bis wan Den Tare duprent biif ljondert fesuen en treltich tor Get Flaer duplent duif hondert wier en tínentich. Het becft Cloofters Dan Dominicanen/fPrancifaners en ban onfe $\mathbb{1}$. Bzombe ter $152 a=$ tien. De baven van de Stade is eeu inwijck met befeljut ban een Eulandt. De Stadt Villaneuva de los Infantes, of de los Confines, be $=$ bolcktede Don Garcia de Mendoza, en ae Gotiverneur Villagran bljeboot datle gljenoemt foude werden de los Confines: Sulept leathien Ieguen ban Concecion nae de fijoe jan de \$traet $/$ acht leguen ban oe Cordillera de los Andes; en vier
Daer af bie loope nae ie Culte. Sp becft cen Cloofter Jan Dominicanen / en een ander ban ffranciftanen. Zlen de $\mathbb{1}$ antpalen ban die Stadt romen of Indianen dic Bozloch voeren / en de treffelicke RieUier Biobio loopter dooz / $\mathrm{en}$ andere die in die loopen/en de tieluier Nivequeten.

Pedro de Valdivia bevolclitede de Stadt la Imperial int $\mathbf{3}$ aer ouutent Ia Imperal. Jiif yondert een cn viffticly / die op neglyen en Derticly graden yooclyte Ient/ negben en Derticly leguen ban oe Concecion, uae de fijoe ban de Straet/ Dzie leguen dan de ₹ee! al waer de Cathedral refideert/ met een Cloolter yan franciftaners/ en een ander Dan onfe $\mathbb{Z}_{\text {iclue }}$ B 20 we ter Gratien / en in haet begrijp heeftre neer dan tarbtentich dup. fent Indianen, met andere meer die norly in Bozlody ziin / in lyaer Zlandpalen. De Kevier Cauten loopt diclyt bp haer beenen/diemen bebaeren mach tot aet De $\mathbb{Z} \mathfrak{e} /$ al foe wel de lgaven niet goet/nocly ueplich is. Je Capitepu Baldivia beuolefitede oocli de Stridt Vil- villaricas larica, feftljien Ieguen ban la lm-

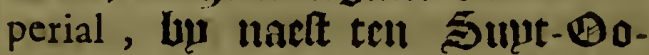
(ten / en bu cans vier en becrticts uan de Concccioin, dicht bu de Cordillera Nevada, met cen Cloofter yali franciftancrs/ ent een ander ban onfe Ielue Dzontue ter Es:atien int cout $\mathcal{L}$ ant/en onduzuclytbacr uan \$zoot en miin.

De Stant Valdivia ig twee le guen van de $\mathbb{E}$ ec/ en vifftich ban la Concecion, aen de fijoc tran de Straet. De Capitenn Valdivia be= uolektede dien oock / met oc Cloo= fterg uan de Dominicanci / f ran= ciftaners/ en.oufe Zl iebe bionue ter Gzatien / in rell chewerte dat beuchtbacr is uall carwe / cll $5 \mathrm{a}=$ den / en op entighe placten goede

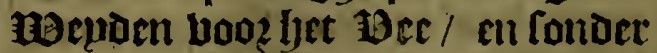
Mijnen. Br Coopmantrlyappen romen vooz oc kievier ban Valdivia, 


\section{uan $V V e f t-I n d i e n$.}

bie Dicht bu fyaer fyeen loopt / en De / ten ander tiebier/ tn Coquimbo op bebcil ta in be gont than die/ op uecticly graden ljooctre.

oonno De Strdor vall Oforno, dic Garcia de Alendoza bevolelitede / ig t tes atich leguen of niecr ban la Concecion, naer de jijde ball de Straet/ feucil leguen uan De $\approx$ ce. Sul lecft cen Cloofter uan Dominicanen/ en ecll ander van francifanety/ ulet eell ander ban Ronnelu/in een cout ILand / Conber oberbloet van ilif:toctse / maer van beel Goutd/ en in fact begrip siin twee hon= oert Duplent Indiaenen in bupt= Crljappen.

Eatro. De Sradt uan Caftro werde be bolckt als de Zicentiate Lope Garcia de Caftro Gontuerneur was ban De hiijcken uan Piru, de welelte in be Znotrlye cale Chiluc genaemt wert/ Dat de lelte is baul oc bevolck= teden in Chile, in cen Ezlanot ban Die die baer zijn in de glepz ban Ancud, of Chilue, en die Archipelago leut op Dzie ent veerticly graden hoochee / cell en weertich teguen be-Supden Oforno, met een Cloo= Iter ban firanciftaners met twaelf Duplent Indianen, in bupertigap= pert / twelck een Ezplandt is ban biifticly leguen lanck / en ban twee tot neghen beecot / die de zee macckt / met nach andere groote/ bececkende bet Yanot tot aen de Cordillera de los Andes: 't $t$ to een 23 erglyaclytich zande / en fieubes lich / Blucljtbaer uan carmwe/ en פgap3 / en goiinen ban marty/ Gout/ op de Stranden / een caecke bie toequich is glycfien.

Gabms en De Eouberneur Villagran quit= ait Bouber tectoe oe naem uan de 5 tadt fian reuremo Canjetc, Dien Don Garcia Lan Mendoza bebolektede / en betalte the = noemt te wozbon Tucapel, en baér nate ontuolckte jp die. Tn bit goubernemêt dat op twee en twin= titlygraden lept / is oe baben en thebier ban Copeago,en acht $\$$ un= Den ban bace / is die ban Guafco; twee en Derdich gracoen / an die gyepaftert zijnde / is die vall la Cigua, in die tarbier / ent die vali

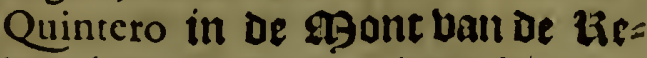
vier ban Concagua, booz die van Sant-jago, of Valparayfo ; cul dic uatt Topocalma , in de Revier uall Maypa, en olyepnatert zijtloe de ties bier van Maule, is de la Herradura in de $\mathfrak{A g o n d}$ uan $\mathbf{b} \dot{\varepsilon}$ Reluicr Itata, voos oue uan de 12 Concecion, ill de grodte zievier Bobio Rooat-waett / is bet (Eplanit Santa Maria, recljt ouer de Straet ban Arauco, en de 19 aluen tan Canjete, en bet Eplandt ban Mocha merr Supt-waett / en de Gaben wan Cauten, $t$ 'belck die is ban oe Imperial, en Supt-waert! De ontla-placts / en de thebier van Tolten, booz be Haben uan Valdivia, en die obepaltert zijndel coint la punta de la galera, en meer Supt-wart lept oe groote Bahia, of bauen ban Oforno, in of thes vier de las Canoas, en ten Sonpuer dace af by naeft bertich leguen is De 99 euz Lan los Coranados, De alo Der-bzettite Lebier van bit Kiicks en int epnoe ban dit sijck is de ģ⿻上丨 Ancud.

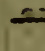

19et XX111: Gapittel.

Van de Provincien van de straes van cMagallanes:

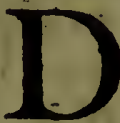
进 1 zobinción băde Straet ban Magallanes wozt ghe noemt al bet gbene dat bant ae Rebiere de la Plata is tot de Supt-zee / van bet upttérite ban Chile, tot de Straet/ twee bondert leguen eetmen baer toe comt / batt twee en vectich of dxie en beer tich graden boochte / tot $s i$, ofte meer / De welcke al hoe bel bu bep de zeen sijn op betrfiepioen revis 
fen beluaren/ tu darer Bolckeren in Lazarus, op ; 2 .geaden/ net tes cas

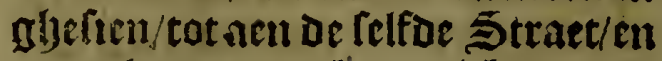
is nochtans met bebedicht / uoch ceniglie populatie glisuneclit/ Dan Die due d Ftrmade une liet die Diego de Valdes beotyt in de $\mathfrak{B S}$ ont van - de Stract/aen de \$odet-liide / int Sart I 8 2. Die ghendemt wert be Stndt ban S. Philippe, welcke om Dat jul op foo groote lyoochte lacly/ en morbt niet belyotiden berden om de groate cottoe / ell Daerom ís Die nautgatie fwater / en peritulets omi be gljeftadigbe temipeetten / en groote Boinden bie Dacr altoog siin.

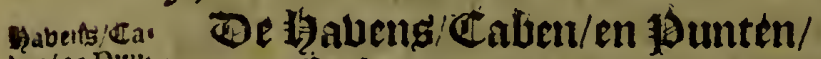

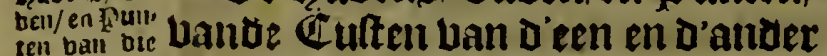
swee Cufsê. FZee / tot aep de Straet / al hoe woel Dat jp ueel jiin/en eenighe gheteet= kent in de Zef-Cacrten/ nochtang eit blijekt lyet niet gljebeelick wat Liebieren bạtter ziin / twoch Daer ig ban allen gheen leeclier berbael. Ende De nueet bekende van be $\mathbb{C}_{\mathfrak{u}}=$ tte / van Chile af tot aen be Straet toe / De welclte loopt ban Valdivia bu naelt 100. Ieguen melt SuptBelt / liin de Caep van $S^{t}$. Andres, op 47.graden/: uan maer de Culte wederkeert recht ten Supden / tat De Straet / tot aen de Cabo lan $S^{c}$. Roman, op 48 . uraben / en ontrent het Eplandt van $S^{\prime}$. Catalina vicht bu de groote Bahia, die ju noemten De Alcarchofada, en binnen die is de

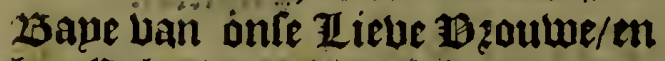
het ezplandt baii $S^{t}$. Barbora, en wat meer vozoer zin oe thaueng ban Hernangallego op acht en weertich graden / cn thee derdentoel/ en de 25 ape Uan los Reyes, achtien leguen Uan de Haten-Hernangaltego, eth de Saupe vau $S$. Luan, op vifftich gra= Den en cen Derdendeel / en ae aebo ban St. Francifco is op si. al waet iiloopen ecrifige canalen int Zant/ kit bet eitlaut uan la Campana. elf Ieguiei ban S. Francirco, oock met cenige canalen/ die niet innewaerts bercult anjijn/cn de 23aue ban Sint nalen ter jijoen / die feer bzeet en lanck in loopen/ aen D'een en D'an= Der zijbe / die leer wepnich berevit 3ijn / en ten Archipelago ban E్ lanoen/Die altoog gljefept wort dat $3 \mu$ is aen de 5upt-zijoe ban de Straet / bet welcke Don Richardo Aquines milfaeckt/ Datmen aen Dié giont uan de Straet aen de Suptjijde niet meer en bint ban vier Eplandekeng/ en een int midoen op oe nanier als ent Supcker232000t / en Dat ju ten minften af= wiickende ziin fes Ieguen wail of ggont van de Straet/en Dat de Archipelago lept aen een tijde/ en Gp bout booz feecker / Dat lyet glje= ne is bat men fept / Dat het valte lantot ig ban de Subt-zijoe ban de Straet/en Datter gljecn vaft landt en ig.

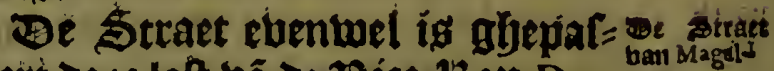
feert Dooz lalt bã de Dice-2k op Don tanes. Fráncifco de Toledo, van de Supt3iide/ tot aen De \$oozt-zijde/ Pedro Sarmiento,en Antonio Pablo Corfo, en men weet dat $3 \mu$ is gfteleghen van twee en bujftich tot ozie en vijf $=$ ticly graden boochte / al waer baer alder-Supt-licklte is opghelegew en bat $3 p$ in de langte hout hondert thien leguen/ tot hondert biffthien/ wat min of meer / en in de laeette ban een tot thien/nopt en beeftmen begonnen te leplen van de Supt-

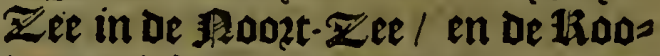
verg diefe ban't \$oozden tot int Supden ghepalteert bebben / beeft men nopt veritaen dat $3 p$ doos dien teederghekeert bebben. De wooz= [enue Richardo Aquines fept/Dat bu veel daglyen glyeloopen beeft booz oe Straet-m lyp bebelficht dat let gantfrbe Ianot aen de Sunt-fijoe gheen baft Tanot en is/ Dan allent vele Eplanden / die op less en vijf= tichgraden loopen/twelck bu conde weten / om datfyu liep tot de ghe= lende leg en bijfticls graden / nuid: Den Dooz de Eplanden/ en alg hy fach 


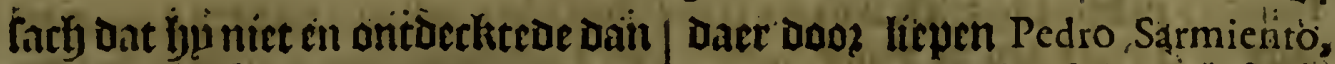
Zee/ quam Inu wedecom om te bol= en Anton Pablo Corfo dooe lalt die กlyen fin cours die dooz de Straet lep: en dat lyet uiet anders can we= fen / om de verfeljejoentljeden ban De Bloedeit die veroolaedit wer= Dein / Dooz de beellyepot der ingan= ghen/ Dic Daer zim tulteben oe Eju= lanoen/en but be bewooningle ban

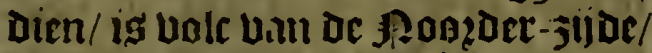
Dir tot De boozerude Evilanden co= men / oun te onderbauden de $19 i \mathrm{f}=$ frberije / en op lyacr tiiden weder= keeren nae haer Iant toe/en dat 3 u begreep veel Caeclien int bu[onder/ inies gijelien te lyebben balte bebole= limghe/ Dam atleenlick eenige but= lseng ơte öe Indianen baer yaneftelick op flaen/ lyet felfae leut Francifcó Draque, oun dat lyet ghélueurae als bu de Stwaet pafterde/ int Jast I 7 9. Dat nae dien ly unte quam

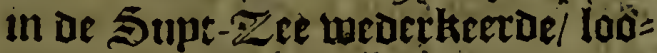
pende met fortupri / tontom Dele Archipelago, tot aen'ae gsont ban ae 3200at-Zee / en Doos de felfibe werh die bu gljeloopen badac/ dede by fijn nabigatic conoer cwarich= lept tot in of Suut-Zzer.

De bulonderlte deelen ban be Stract in Den inganck ban't Siug

Cabo Defea. Den jiin De Cabo Deffeado, op Dzie enl bisftich graden en de Canal ban Todos Sanios, twee en twintich les guten uan de gyont feer bzeet en lanck / en die ghepaltert wetendel De L)aué uzn la Traycion, en boozts etr andere groote Canal bie lanck is / Die ten Rooit-Waelten loapt/ ende la Campana de Roldan een groote tiotte int midónen ban't begin van een Canal:welcke die naen ghegleven ig om dat al baer was/ om die te verfiennen / een ban de medr-glirfellen yan Magallanes ghenamit Roldan, die een 5 offetrieter was: voltht de pjunte van la Por; feffion, die vier leguen lept ban de Cabo de las Virgines in Den iuganck

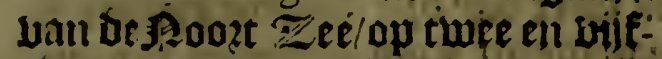

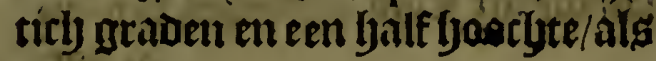
jucregien out de Straet te ontaerken / om dat baer gheboden was grondich t'onoerfoecken/ ofter beel lichtect nabigatie was tot oe SuptZee / Dan die ban Panama : $3 \mu$ berkenden de twee engten die baer $3 i j i$ aen de Podat-zijae/en bet Docht Pedro Sarmiento bat d'eene, foo nauto wag dat ju met ghefefut conde bewaert werden/en hp perfuadeerdet coo (eer / Dat al hoe bel de t)ertorly ban ztlda altoog affirmeerde dat bet onmogrjelick was/dact buchtteloos gefonden is die xirmade ban Diego Flores, en ten laetten fach hu Dat aloaer ên ghebaerlick vaerToater is/en dat be waftinghe van be twee \%en / dic int midoen ban de Straet fjaer t'famen boegê/ / bertrecken met fulcken furie/ balknde opeeniglyeplaetten meer dan thettich ellen/bat wanneer ae Schepen niet en bzocten weel Cabeld omilyaer te wel te atricketen / $3 \mu$ en fouden die niet conneti beleplety fijno wel ghy éballatt.

In de 400 . leguen welcke dace 3ijn op oe Culte ban oe gront ban De Ftraet tot aen de Revier ban la Plata, die gantfty loopt Poot- Bof en Supt, Bett/ rijn de kebier van $S^{5}$.llefonfo, twaelf leguen ban ie Cabo de las Virgines, ae likebier Gallego; en de bape uan Sant-iago, beetthien leguen van de kevier pan Sánta Cruz, op vijftich graden / en aen de gisont is een eplant dat $3 p$. noemen de los Leones, de Gaven ban $S^{c}$. Iulián op negben en veertich graden/eti de Zebier ban Iuan Serrano Supt-Laetr van de Eplanden de los Patro op reben en beertich graden l de kelsier Cananor op

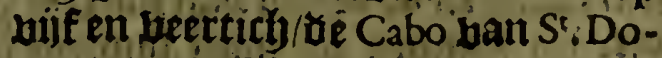
mingo, vooj pie Cabo de tres Puntas,? en t $t$ Lant de los Humos op atjien derticly graden. Or punte bat $S^{c}$. Elena, efl ban St:A polonia op retien en Dertity graden / boos de Cabo

$$
\text { it blanco, }
$$




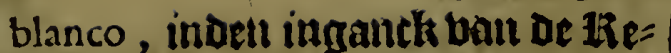
vier de la Plata aen De supt-zijoe.

Sobaflian Gaboto mes be tube we gifellman, gaenoe met eet surmato

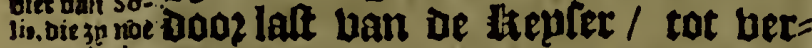

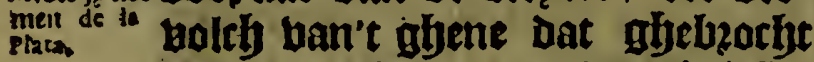
Jaboe de Commendadoz jzieftet Garcia de Loyafa tot De Eeplanden van de Malucos, en bet febeen dat fjp tot die niet conde conten / beeft bp boozgbenomen pet in te ne= tilen/ Dat tot pzofiit diende / en is baer inghecomen int Jaer i s 29 . ontbeckente de kevier ban la Plata, al waler hp was bp cang Dzie jaren/ en als bg gheen vervoleb en creech op bet rappost ban't gene bu jadoe ubevonden / is bp wedergbekeert in Caftilien, gbebaren bebbenoe bien bele legnen opwaerts / bont Silber bp de Indiane van die Con= trẹien/twelek bere Indianen bequa= men in de Oojlogen die $3 p$ badoen met Die ban of Iijetten uan Piru, en dat vaer af be kebier ghenaemt wert de la Plata, om dat die te vozen gifernoent weroe de kebier Salis. Oefe 3 zobincien voegen baer met bie ban Brafil, bu de 3ic ienie ban de afoeplinglye/en $3 \mu$ bouden aen geen oper Tinititen die ghedetermineert siin/ Dat allế laner de Culte die in oe foopt zee bait / en den inganc bain de letevier de la Plata, ban de welthe bet 1 aut rijn naem ghecre =

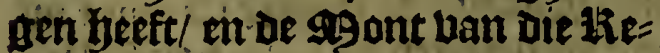
bier fal legoben bu naelt oupfent Téd honoert leguen ban oe loaven bati San Lucar de Barrameda.

3e beuclite batchept bat ce Probili: cioi ban to
Suprker / ch alle andere aefacnen/R to de en 1 gucliten uan Cantilien die tie= ren Daer wel/ ju ljebben oroote moeyroen voo? alle foozté ban beel die feer bermenichuuldidje 3ijn/ en bufonterlick de jaerden/en al boe wel bet ghelweeft is beel Taren/fonc Der Dencken datter gainen van Gout waten/ of ban Silber/ en nu Geblen $3 \mu$ stgonlters ban die gbe= bonden/ en ban Coper/en Her/en een ban de Stễ Ainietilte fert bol= comen. salle defe lozobincien siint ban een Goubernement / met De Conintklícke orijtel / obefubozdi= neert om de nabupzicheut aen den Bice-2Rop uan Piru, met een $23 i$ s Dom in te welcke ozie Dozpen ban Caftilianen zijn / en een groote me= nichte ban 3 ngebozenen met groo= te Zirhamen / en wel gheconpitio neert: Deplaeten zijn otre. Pe Stadt uan onie zielue Bzontwe del Affumcion, de eerlte bebolckingl;el a a aras en 't l'pooft valt die pobincie/ lept op 2 s. graden en een balf foorbte: de Capitepn Iuan. de Salazar fon: deerde dien / Dooz late van de bous berneurDon Pedro de Mendoza, $\mathfrak{y a}$ = re plaetle werbe eerlt glenoemt Gurambare, Brafil lept aen De rech $=$ terbant twee bondert tachtentich leguen daet af/en in die Ineitoictic heeft bet tachtentich leguen tot $\mathrm{Ci}$ vidareael, Dat de Indianen Guayra noemen / en aen de sijoe ban Piru, die (B) olt en melt lept/beeft bet biet yomiert 8o. Ieguen de Stadt van la Plata, en op twee bondert tactiten= ticy! De Stadt ban Santa Cruz de la Sierra, de welcke Nuflo de Chaves bebolcktede aen de Suut-zijde / Dic nae de Straet ban Magallanesig: theeft feer groote en tijcke 3 an= den / en de Stadt lept dzie hondert leguen ban de 9 ont uande zheniet dela Plata, oujefondeert dicht bu de Kelvier Paraguay, aen de Bolt-zijiel

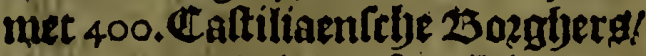
en nueer dan Dzie dup fent thinderen lan bie int Zant ghebozen zijn/Dic zp noes 



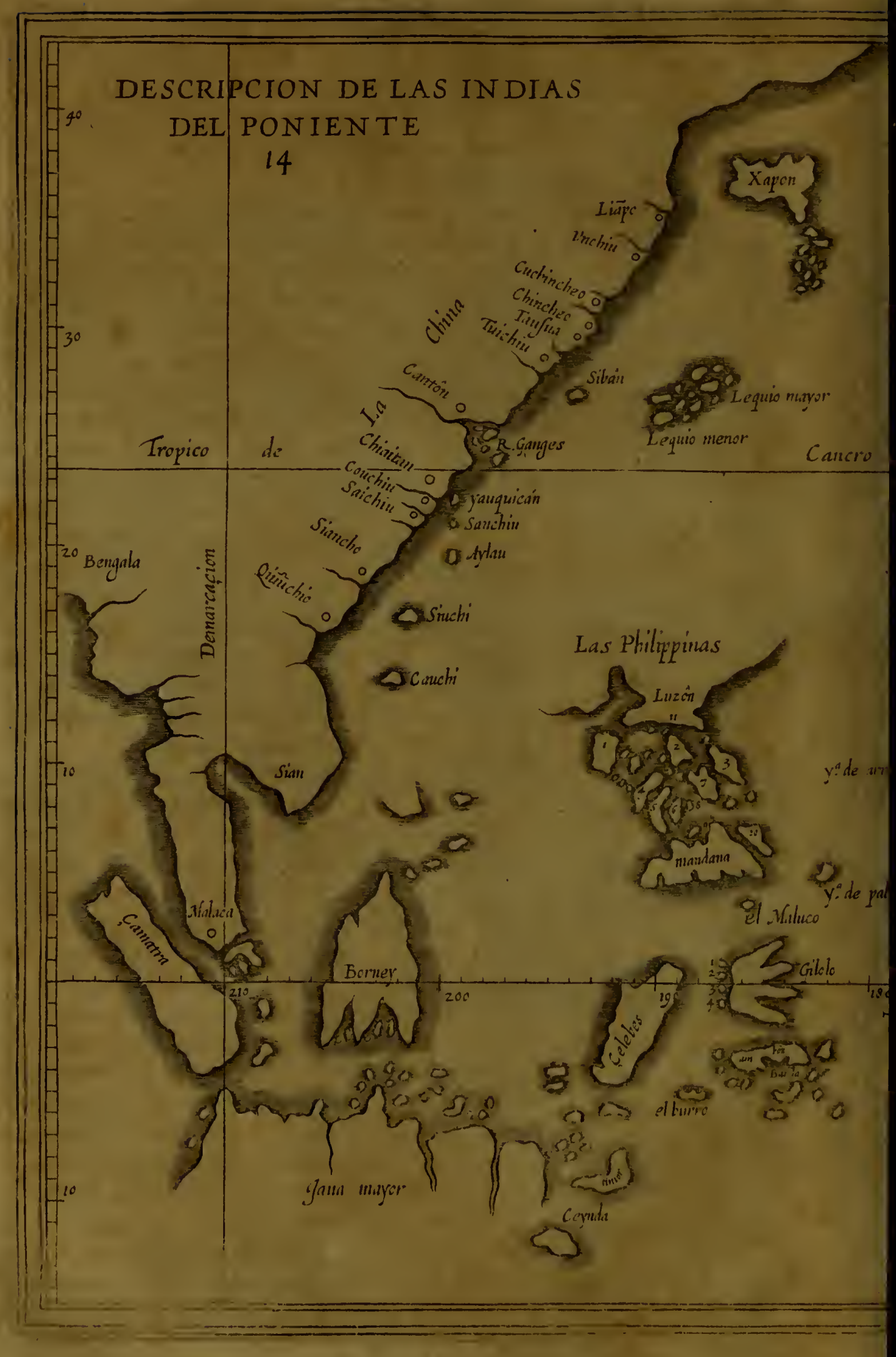




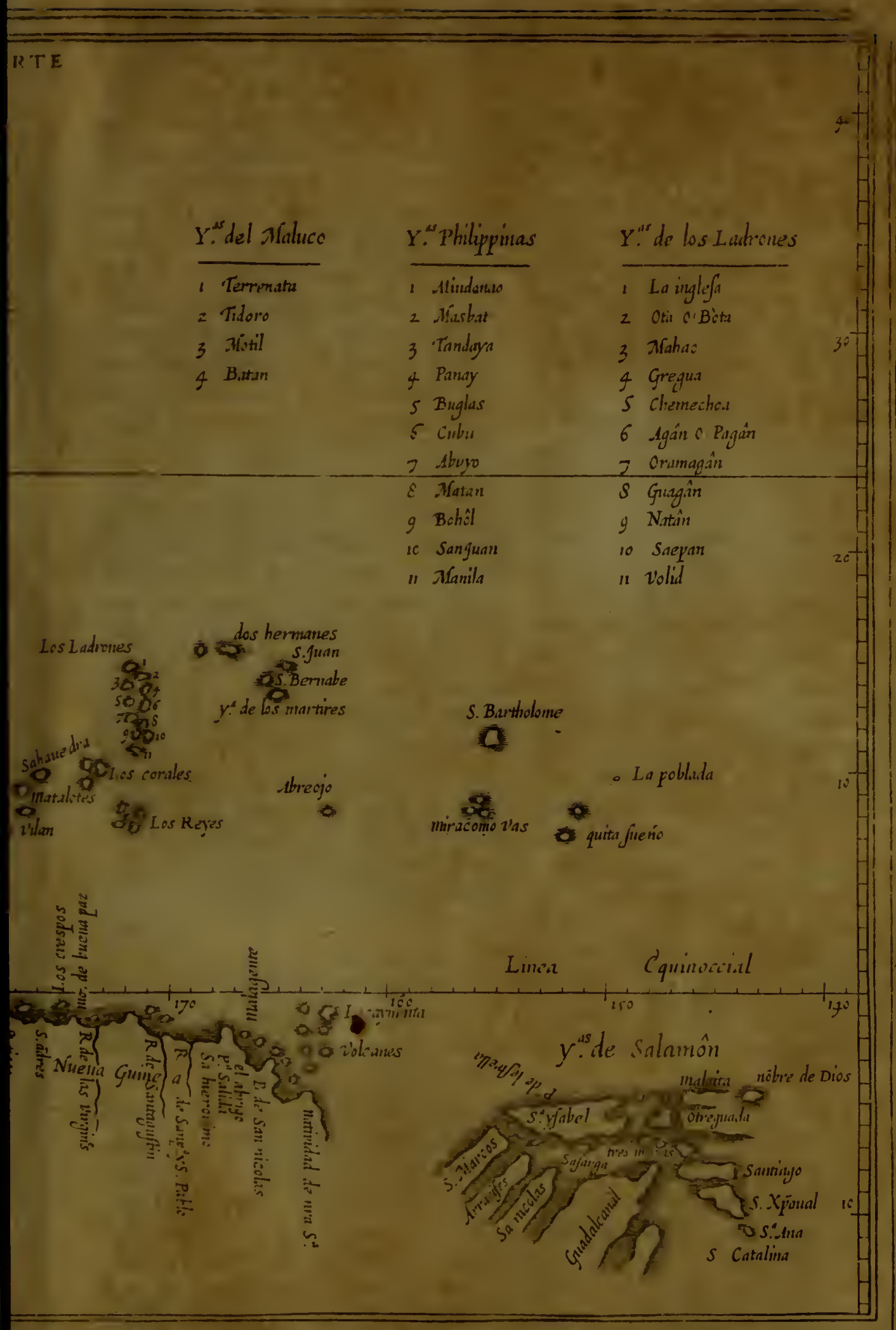




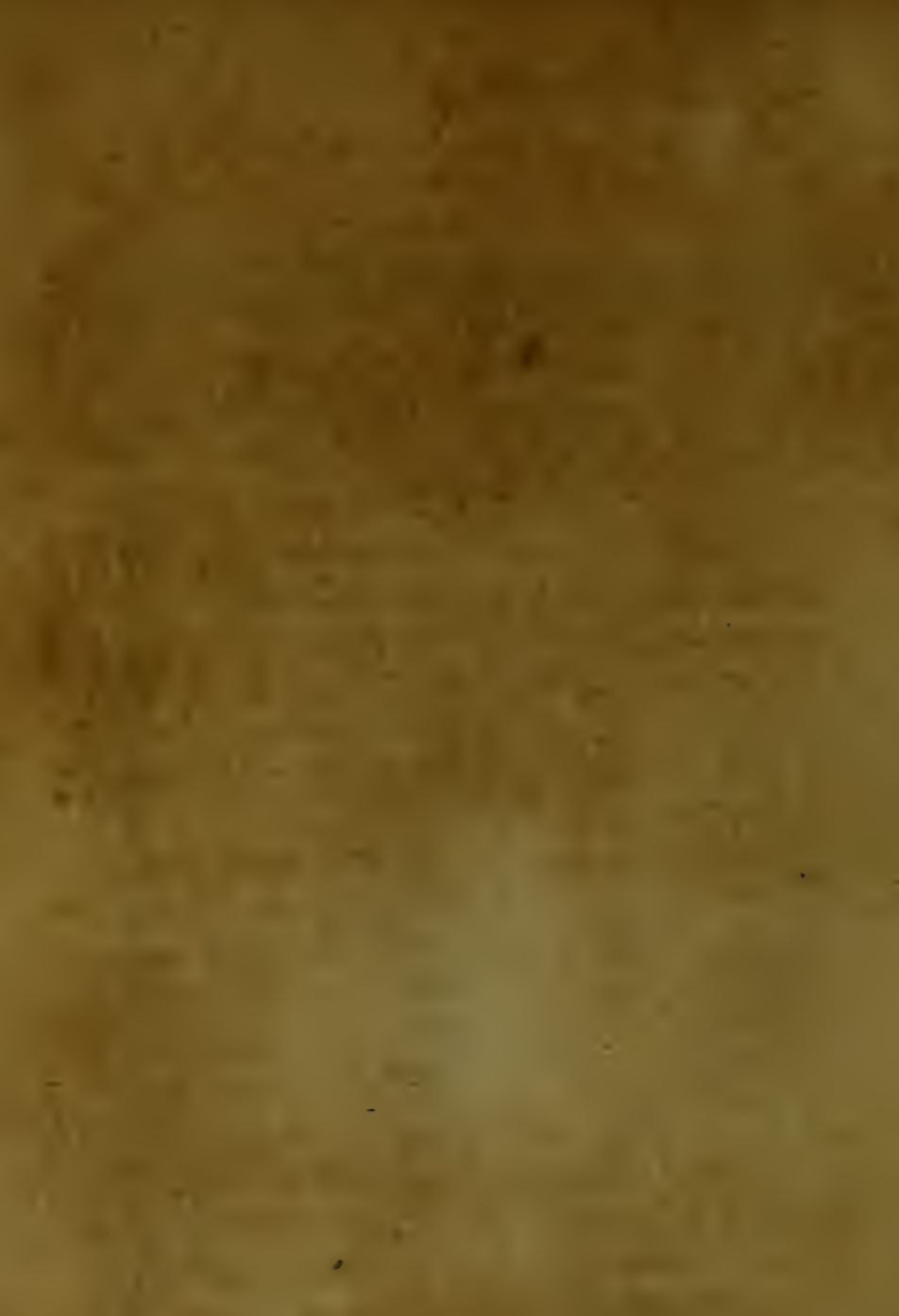


5u nocmen meftizos: in ode Stadt refiocert of Goubernetur / en Co= nincklicke officicrs / cu de Cathedral, die j! noemen lyet 23 iloan טan la Plata, Sttffrngaen uan de aertg-23illeljou uan los Reyes, en itu liin $\mathbf{J}$ uriroictic jiin meer Dan bier fonbert outulent Indianen, Die belcke beginnen te bernterderen.

ciudadua. De Stad Ciudadreal attoers ge= nacme Ontiveros, beuolct Dooz Ruidiaz de Melgarejo, lept tacljtentich leguen Lan Affumcion ten RoostDoften nae bet $\mathcal{Z}$ anot Jall Brafil : dicft In de 1ieviex Parana, t' is in cen goet gljeweft ban 1 iiftorht/en ban PBijngaeroen / etl ban beel foet Coperg / ell een goet gljetal Indianen die oock feer vermenichumbi= gljen : in de keluict Parana dicbt bp Ciudadreal, is een groot $\mathbf{B}$ ater-ual Dat gheen 9 sentely lyeeft connen op twee boutoet paften daer bp comen om bet groot gherupg/ cnoe Jebel ban bet Hater/ en lancr oe liebier met gheen Canoas / of 25 arke/ op een legue/ out dat de furie ban't 3Bater yaer lottoe omwerpê/ twelc comt jooz de afual / welcke fal jijn uan twee ljonoert Dademen / ftepl nfgefneaen tiote/ en is alfoo nau/ en bet 3 ater loopt foo Dicht op malcander/ Dat ljet feljijnt / Dat ljet Itreloer blicalyt alseen 1 ijl upt een 20ogije.

werowayses Buenos ayres is eell Stad die ban outs ontuoleltt weroe/ ontrent ter plaetle lwaer [1] dace nae iq ghes plant/ it de plobincie uan los Morocotes op de Deluerg ban de $1 \mathrm{k} e=$ vicr de la Plata, in eet bzuchtbaex Zant / en al waet alle de 13 zuchten ban Caftilien wel groepen/Desau= berneur Don Pedro de Mendoza Je = bolcktede dien int 3 aer i 53 s. welc = Fe oede ontoecken/als Gaboto daer ban beefe afoljelaten. Dit $\mathcal{L}$ ant ig Doozgaens cffen / om dat beljalben De Cordiileras, die aen de Eulte uan oezee siin/vie twintich leguen ful= Ien zijn tot Brafil, yoo?tg loopende/ omcinglyelende bet ganticfye $x_{\text {ant }}$ tot de lievier Maranjon, en oe Cordilleras uan de Lijeken uan Piru, bet gantlelye I ant black is bebou= oens cenighe cleyne 23 erchjeng.

Bp de CUlte uall die 13 zobincien mabems en ban het I ant ban Brafil af/ tot ic un cure: lievier ban la Plata zijn bekent uijf of fes redelicke babens: Ðe tha= uen uan $S^{t}$. Vincente op dote en Der= tich graden hoochre/ cetjt ober bet Eplant Buenabrigo, Dooz ljet welc fre oe Zinic ban de afoeplinglje/en leg leguen Supt-waet is oe Hevict Vbay, en De bauen/ en fet Gelant Lan la Cananca op 35 . mraden / en Joozts of Liebier de la Barca, booz oe 1 aben uan oe 23aue / of oe kies vier uan $S^{t}$. Francifco, en het \&zlant ban Santa Catalina, anders genaemt Puerto de Vera, of Puerto de Pa os, en die ban Don Rodrigo twintich leguen Supt-maert/ uan Santa $\mathrm{Ca}$ talina, ên Eéplant 29.gradê en meer Suut-waett/;. Ieguê is Puerto cerrado,en vijftichriopoblado,en oact foo ueel ban die Bahiaonda, en De liebier Tiraqueri Iept of twee cn oerticly graden en een lyalf booz de Cabo Jan Santa Maria, die op unf en Derticly graden lept/in dentuganck ban oe Licbier de la Plata.

Dele lieluier bert ghendemt in oe Indiftye cale Paranaguazu, en gljemeenlick Parana, Jyeft lyaren in= ganclt of 9 gont op De Eec van yet Supoen ban de viif en oerticly gra: oen/tat 36. graden hoocjte/tultebet de Caben ban S.Maria, en Cabo blanco, die uan d'cene $\mathfrak{B g}$ ont tot D'ander fal Gebben dertich legueit/ en ban daer binnewaertg nocly 10 . leguen in oe bzette / met beel Eus lanten int midoen / en beel lievie= ren feer groot/en treffelick/dic Daer in loopen yan fet Balten en 'twBe= Iten / tot de bauen de los Reyes, t'welclicen aroote $\mathscr{A}$ euz is / die j! noente dc los Xarayes, wepnichmiı dan ozic ljondert leguen ban detice bier de la Plata, al waer uecl thevies 
ren inloopen/ be weletie comen ban / balt ten Botten ban de zinie/ of De 20 eeckien uan los Andes, of $\mathbf{3 p}$ moeten fijn ban De kicuieren die uptloopen unt oe pozouincien ban jas Charcas, en Cuzco, die nae bet \$0ozoen loopen / al waer een an= Der boclyt in loopt in ae boozentoe

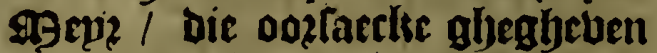
beeft te dencken dat diekevier yem menglyt miet die ban $S^{t}$.luan de las Amazonas, en andere feggljen int bp fpetupt van oe $29 \mathrm{ep}_{2}$ del Dorado, Die biiftich Dach-repleng lept van die ban los Xarayes, al boe wel dat= ter meningen jijn Datter geen Dorado $\mathfrak{c n}$ is. quam bp nacbteen opgher als een Caftiliaen wag in tiin Camer met fiinl)uptuzou/in be buptenlte tijoe/ en gaf de agan een Gacly/waer me= be bp bem doot aoedy / en lyp wert wecb gheboert/en glyegheten in een Wiet-25oleb. De Capitepn Salazar sinck unt in de Dagljeraet om te foecken Den (Tugljer met so. Sol= Daten / eil gaende dooz een 250 trh Ichepde fyu alleen af dooz een wecty/ en ontaeckende den Eugljer/als ljp was verf́tbeurende / licp bu tegleng Gem aen / en op de tijot dat lye op= Geftede fiju lyant oin hem te aaen/ feboot oe Cauitenn fijn tioer loz/ en feljoot den Coegbel in bet bert ban de Cugljer / Dat Ip boot viel. Gen fept ball é groot gemoet/ vail groote confloeratie / cu derteritept.

咆et X X V. Capittel.

$V$ an de Provincien en't Lant van Brafil.

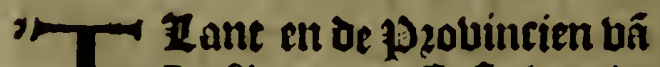
Brafil op oe Cufte ban oc -3002t-Zee en't valte lant/ beroen gljenoeme / al 'tobene dat Meridiaen ban de afoeplinghe/ uan neghen en twintich graden lanckte van de Siupt en \$oort-1 lijn bait Toledo, tot negljen en dertich / bat siin twee Jondert leguen uâ't $B_{0}=$ Get tot bet Baflen/en bier bondert so. Supden en \$oozoen/van twee graden ljoocbte be-5upoen/ ober de Cabo yan Humos, tot 25. ober bet $\mathbb{E}$ plant bau Buenabrigo, en van be eertte bebolckinglue/tot de laetite bain Brafil fijnt Dzie bonaert biffticly Ieguen.

Vincent Yanjez Pinzon ontDeck $=$ Yincenc $Y$ Yan-

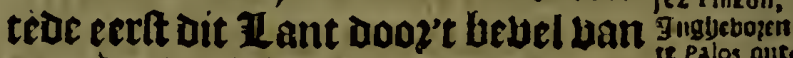
De Catholijeke Coninghen / en vestecer Bea Itrart Daer naer Diego de Lepe, int ooct Diege Taer is o o. en leg פaenden Daer desepe. naer berbiel bu gheval daer op Pe. ralvarez Cabral, gaende niet $\mathfrak{e} \tilde{c}$ BDoz= tugaelfebe Alrumate naer Indien, oe welcke omte mijen de Calte ban Guinea, too berbe bet1 in $\mathfrak{Z} e \mathfrak{b e}=$ gaf / Dat ljp dit ZLand bont / en bu noemde bet Santa Cruz, oni dat ljp Jet op dien dacly ontdecktede / lyet ig al beel wamv/moe tointer en in oe Somer / en t'is [eet reglyentinty= tich/ en ouncinglyelt met vecl 250 of reljen / en פgitten / beel onglyénout/ en bol ban cruppende feniniglye

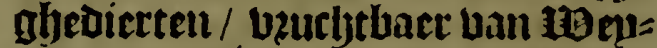

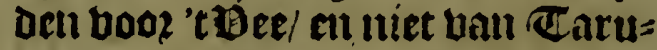
we / noch g9ap; / daex siln beel Agonfters van Silver en Sout/ norhtaus al boe wel de 13 aztugii glycen pzofijt achten of 9 siinen te benificiereu/ noclytans jijn jijoer.

Het peintipael onderbout van Dit Ilant is de Cazabi, dic $3 n$ van Yuca meneclien/en oe grootiteC oop. mantelyap is oe Supeker / ctl Cattocn/en 23 zafiliell- i) out / Dat oock 'talyene is wat lyet oe naem glyeghe= wen beeft: ittoe gantrobe pzobin= cie fijnoer neglien Boubernemen= tan/ Die ju Capitęnurdyappcn noe= men/ en in die ziin 17 . Dozpen van

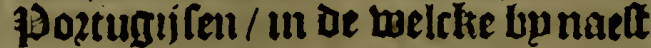
buie buplent ozic yontoert $250 z$ gers 


\section{baut $V V_{e} f$-Indien.}

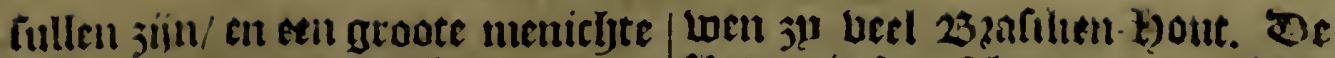
bun Indianen Die cloerk ten Ooz= loch iiin/ ote oe joattugijen glyeen placter ghegbeluen beluben ont te uruoleket anders Dan ou de Cu= lte op welclie beel Kiebieren jiin/ en phetter diser men af-[eljepen marly ell ueel h)abens die (eer bę1licly jiun / ili de belclie connen iiltoopen aldertyande כ̈etjepen boe Groot datte oock jiin.

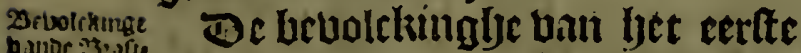
irctor Ëufr. Comitcuntelyap/ en lyet aloeroutite is Tamaraca, de tweroe ig Pernambuco, viif Iegueti ban Tamaraca, Sunt-unacrt op oe Joochte van aclit graden / en Dacr is ecn l)upgs bau de Compaignie van lefus: liet Derde is / Lant Todos los Santos yontidert Ieguen van Pernambuco, op orrthien graden / cn alhier refiuert De Gouberneur/en de 2jiffeljop/ en de Ztuditeur Benerael bau de gantidlye Culte/ 'tlyeft oocle cen Collegie van de Comipaignie ban lefus, het vterde Capitepntelyap ban los Incos, Iept Derticly leguen ban de Bahia de todos los fantos, op veertbien gradew/ en Date Detoendeclet / 'tljeeft en hups ban oc Compaignie ban lefus : In Dat Capitepniclyap bert gevoniden eell 250 om van de weleke ghetrocken wert coftelicke 25al(eni. Het Capiteunfelyap ban Porrofeguro Iept Dertitly Irguen wan los Incos op reitlyien gradé en een balf/ 'theeft date bebolckinaljen / en een bups ban oe Compaignie bah le. fus. Het Capiteunfelyap lan del ESpiritu fanto bijfticly leguen ban Puertofeguro lept op twinticls ara=

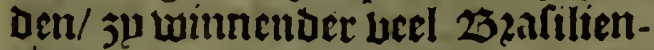

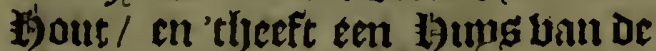
Commaignie. L)et Capitepnftyap Jan de Ke vier ball Genero lept op t'fettich Ieguen / cu Uan Efpiritu Santo op oxie en twinticly grizen m cen DetdmDeel/ met een t)uus ban de Daders yail oc Comunigme/ til ils bare Juriraictie bauLicluter is feer feljoon / met frbjoo= ne en profiiteliclie Oebers. Wet alacr-lette Captenulebap is $S^{\prime}$. Vicents treftich leamen wan de ke= vier ban Genero op biet en twill tít) grinden / 'tyeeft ecn forte refre op cen culandet tot befefyeruunglje tegljen or Indianen an or tioovers / en ern Collegie vall de voos [clear Daders / dic aroot pzo= fiit gljeanen bebuen in be bebole= kinglye van dat 21 andt / en bekes= rimglec uan de Indianen, $\mathfrak{e n}$ in lyaer

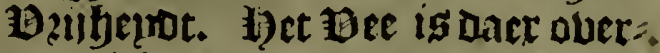
vloedicly/ ch daer is oocl groote opbocdingtie ban Derckeng/ en hoenderent / \# crijglyender beel ambar, dat de $\bar{Z}$ ee upt werpe met chwrocr als de pateren haer bewegtjen / en beel joerfoonen yeb= bender Iacr mede verrijckt. De Somer-tiid ifler ban Soptem: ber tot jebzuario toe) en oe 3 Bin= ter ban $\mathfrak{A g}$ artio / tot Zlugulto. Be Daghen ziju bp nact foo lanck alg oe natjten / afleenlick cen upze waften $3 \mu$ en verminderente. Tn de Hainter-tijot loopt de BBindt altoos Supten en 5 upt-Balt? ill de Souner Roert - Boft/en BoffRoost Bolt.

Altht of thien pzincipaelfe $a=0$

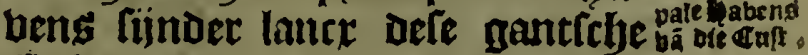
Cufte. Daer is de thebier ban Santo Doiningo, en uan las Virtudes, ten \$Rookt-Bolten ban Pernambuco, ent Tamaraca, dat een $\mathbb{E} \mathbf{z}=$ lanat is / ghclijck als ghelept is/ de ertite bebolckinghen / en vooz De Cabo van San Auguftin, Die op neghen graden lept. De liebier ban San Francifco op tyien graden en een half / die groot is / m treffe. lick. De 25ape ban Todos Santos Dzie leguen beet/ en dertbien lanch binuen s'landts tat San Salvador toe. De taeluier en bauen Trenado al waer be 5 chepen inloopen die op Dele reug gaetl. Oock is hice de lacbier Canamun op bers

$$
\text { tl tbirat }
$$


thich en een yalf aradat. Oe $\mathfrak{k} \mathfrak{s}=$ vier uan las Cucntas, of bam San Auguftin op beerthien graden en ech Iyalf: cu be kieluer ban las Virgincs op feitjien geaden: Puertofeguro op 17. gradell. Oe Kievier la Parayva op 20 .ontretut Die van Santi-fpiritus, Detiebier Genero, on= trent San Sebaftian, $\mathfrak{n}$ Cabofrio op 23.graden/vooz San Vincenie.

\section{loet XXV V. I. Capittel.}

$V$ an't ghene men noemt Indien van't Westen, $t^{\prime}$ welck zÿn de Eylanden van de Specerien, Filippinen, de Cusste van Chima, Iapon, en los Lequios.

I

Ndien uant Heften/ ziin alle De Ceplanden / $\mathfrak{k n}$ 'tuafte lantot befloten bannen de afoevlin: ghe van de Croon uan Caftilien,en van Lcon, in bet Wefterfehe deel ban ar uooktoemoe afochlimgthe

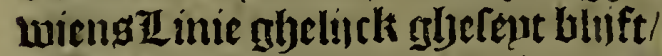
paffert dooz lyet ander becl wan oc

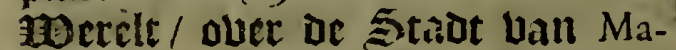
laca, Lan waer af Ooft-waert / ew nae Nueva Efpania, is eth grcote golfe tan unepnoslicke groote $\mathfrak{c n}$ r!cune Etilanoen / in urel ftuclien

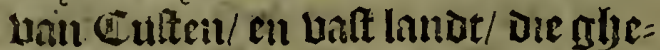

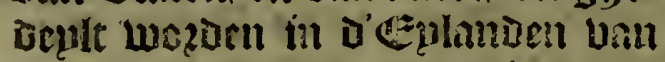
Specerie, of los Malucos, D' $u$ ulam = Den Uan Filipinas, De Cufte Uan la China, De Enlintuen Uan los Lequios, $\mathfrak{n}$ lapones, ine Culte uan Nova Güinea, de Etulanden bam Salamon, en die ban los Ladrones. Het temperament ban alle Die Eeplan= Den / en zlanden int generael is uochticly/ at maticly wartm/ uzudlyt= baer ban is ifftocht / en ghedeierten/ met cenich Gout/Dan fiechte allow/ Daer is gantfrls geen siluer / veel

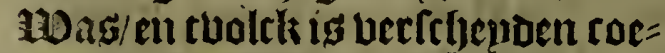
Ieureal / etl ożuituaris als Indianen,

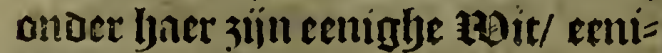
glye nuart gibeluerft.

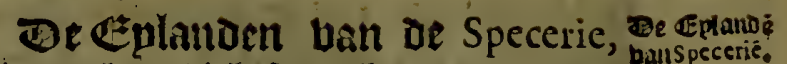
die englyentlick too glyenoent wer= Den / om dat Dare uptgljetrocken

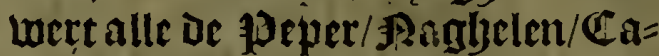

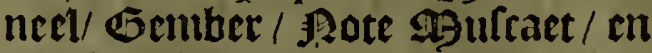
Sgaftic Dir in Eiropa vertect wer = oen/ ziju Leel int grjetal/al yjoe wel de paincipaelfte daer bau s.in gye= tale zim en rleph/onder De ÆquinoCtiacl, op 1 94-graden uan de SuptRoort-ilin van Toledo, befloten in lyet onderpant / Dit deder de Ketpler Carolug be viifoe/ acn de Coniutls ban Porrugael bocz Deie yondert uifficy dupfent Curaten. Defe $3 i j n$ Ternate ban arlit of $n e=$ ghen leguen int ront / met ech tha= uell die $\mathfrak{u}^{\mu}$ noemen Talangame, $\mathfrak{n}$ Daer regeerde Corala, Die fich de naem gaf booz ecn Baffal ban ie Coninck ban Caftilien, als oe Frbepen die oberbleuen / van oe ZTrmade ban Magailanes Die En= landen bonden: Het Eplandt balt Tidore Icut cen legue be- $\mathbb{Z}$ upDen Terrenate, yefft tbien leguen mi ronoc: datt unat Matil, of Moutier ts bier leguen int ronbt / en lept reclyt ondaer de Equinoctiael : Eet Maquian lept opie ieguen Supotwaerts / en loeft felien leguen wit de ronte/ en thien Ieguen ten \$uporn uan Batan, of Baquian/ vã twintich lrgutn int ront : $\mathbf{3}$. Tidorc re gerroe Almanzor, die oock Irude Dat lyp en Bafal was van de $\mathbb{C}_{0}$ Initrli ban Caftilicn, Dic/ en cock Corala felten gljerdyzeduen/en glieconden yare 23 zieuen met Iuan Sebaftian del Cano, inglyebozen uaim de Stadt van Guitarca in de $1 p_{20-}$ vineie van Guipuzcoa, De welclic gandbe uan Tidore int $\mathbf{3}$ aer een Duplent viff yondert twee en twinticly/uerviel opt Ezplaut ban Zama. tia, $\mathfrak{k l l}$ comende ontrent de 42 .grab

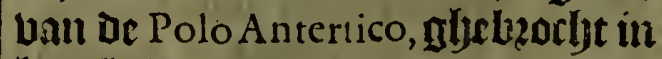
Ijet $\mathbb{E}^{z}$ plant van Santjago bun Caboverde ch vall daer te Sevilia met ljet Srhip Vitoria, verfleten lyetrbende 7. Aganden tijots toęunich min 
min int conten Lum Tidor of. Patian igs lyet unfore Cillandot van de felfor mance als dimoere / 'twas

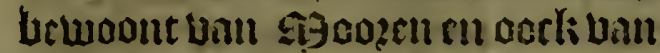

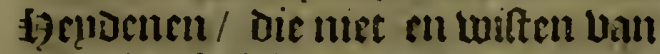
Or anterffeliclifenat ics jicls / cn

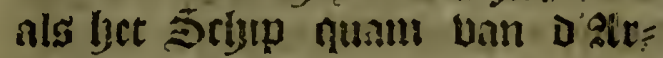
Imnde ball Garcia de Loyafa, re= secroe it Tidore Rajami out lijn= de Dertlyicu Taren/ ch ban nicus gaf Iy beu tot glycljoozfacmlyent Des Canuek uan Caftilien, Defolye= tiitr aotli of Conincli uan Gilolo, die alyenoentit Lietde Sultan Abderra menjami, fll ju locueltiefjoe= Den met ecue.

Giloo. Gilolo is ecn Cenlant uan onder de Zilitic bier leguen berfflyenden

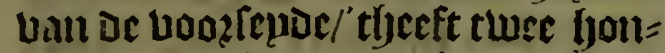
Dere leguen int ronde/ Conder $5 p e=$

Ambon. ceric. Het Eplant uan Ainbon, op Dic gliadu en een halue tet Suy= Den / van bet Eulanot Uan Gilolo, Condier Specerie. De Eulmoen Laal Bandan leggben op bier gra= Den ljoorlte bi-Supden oc 1 inic/ al warer bergavert Lert de pote OSurcaet/a l umas. Cende lyet Burro, Dat betweltall Ambon lent/ en Timor enn aniser Eulamot / ten Stiut-10riten uan Bandan bu naet fombert leguen fonder Specerient unare linels bai Sandalhout. Zeinda Itur wijficly legustl van Tinor ten Noost-Hociten / leeft beerticly leguich utt ronide / leer oberbloe= birly van jaeper/ en bet Evelandat Celebes, Dat feer groot is cllde Borncy major, ontrent of Straet ban Malaca, dat ficly glyeeft tuffedyen bet Inet valte 1 andor! en Zamatia, oat ji baul outs gljenoente bebluen Trapóbana : en meer groote Éulan= oen / en clestie / vie acn de Suptsilide $3 i j u$ / outrent Iava mayor $\mathfrak{c n}$ menor, ell ilit Ropoen yoor de Cuafe bat China. Oe joatugij? cen ljebluen een. 5 terchte in Terrahate, en ect alloer il oe staot baik Malacca, in oen inganck ban DE Archipelago: -2xe

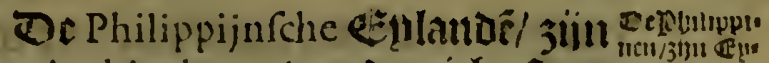
cell Archipelago, vall berticly of 40.6 . groote Cislanden / fomber amare mere clentle wie by een legnlyen in de limger ban ture bondert leguen

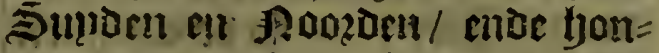
Dart ul de cuglite tuftryen nueva Efpania, su de golfo uan Vengala, m jijn ben nast Denefnt feurn hondert Ieguen ban de l)aben uan De Navi-. dad in nueva Efpania, uan fes tot 1 5. ofe feftjien graben ljoorljte beNoasdell de: I inie / Lan redelick

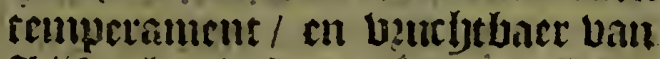
iliftorlje / Lufonderling ban thita/ cu cenicly aecht Gout fil cenighe Declen/ en in centge Eulanden ji) 30onten uan Cancel/en lange pe= per. Oe Jnglyebozenen ziin bat gocoe taille/en in eenige Culandetr. 3ijn §uerten / en dic int midadifte ball't lant wamen jijn witter dant Die ghcne die aen oe ₹er-cant ziin: therft goedo bauené en lyout boos oc Sclyepen. De maenten ban de boaziacmite Eulander "siin Mindanaa, dat lyet grontfte is; Mindanas, ch 'tsuyoclickate bondert teguen in oe lanckte/ cat dite bondert in de ronte/tis ecn roum LLant/ maer puerblocuicly uan. A9aus/ WOag/ liijs/ Genuer/en entels Cancel/ en Mgiinen Uan Gout / nuct frljoo: ne 1 abens / en Tieen. Het Eeplant ban Buenas fenjales, ent S. Ituan, vicht bu Mindanao aent Boften ig 20. leguen lancli Supden en $200 z=$ Den. Behol tljien leguen nae Min; danao, aen ar \$ookt:jijoe negben= thien leguen lanck.

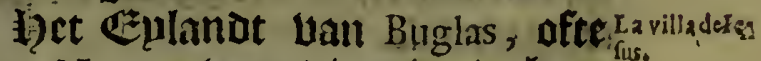
uan Negros ban dic die in yacr 3iin/ int mioden van de Archipelago (B)eft-waert/ is ban veerticly leguen lanck Supden en Roozacn/ en uecrtyien in oc berete/ en op fiin Cult lyecft bet eeniglye poeclen. het (E) vall allen van oerticly leguen in de lanclute Sumoen en RoDzden/lyeeft ecn Dopj yan Caftilianen; dat 34 站 2 Haenien 
noemen de 5radt ban Iefus, aen de ban t'welcke alle baer taken trij= Ooft-jijoe inet eall goeac baucil/ Conder entiglie andere Die int $\mathbb{E} \mathfrak{p}=$ lant jiin. Oe Capitepn Hernando de Magallanes ontoeclitede dat 追p= lant/ int $\mathbf{J}$ aer 1520 , en de Coninck

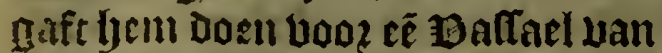
De Croom luan Caftilien; en te voo= ien ontoeclitede lop de ejelanden Die bu moemoe bain las Velantatinas, am Dat juleploen in haer 5 rhepen met \$rplen Lan 2 jiefen uan palue

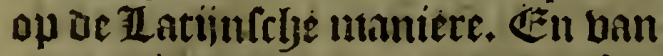

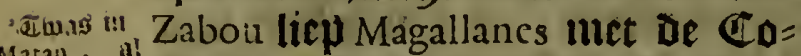

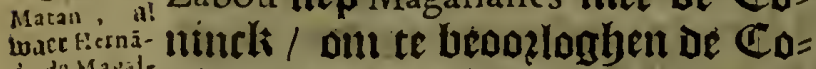

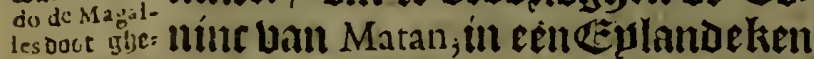

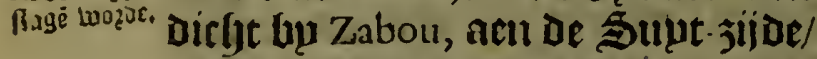
al warer om dat 3 l Laten ouerma= tich fterck/ en moedich/ a oeghen $3 \mu$ Ifen doot: cul de Caftilianen baer bopagie uerdolglyende in verboley vair De Evilanden bait Specerie/ antorckteden Quepindo, en Puluan, en Burney; dat een groot Eulandat is / ch ruicls/en oberbloedende van

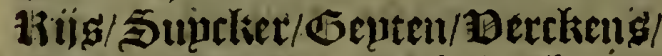
Cameelen : Diner is glyebeck ban

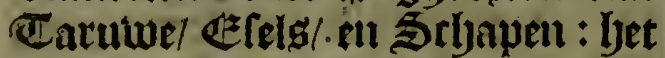
baenght Gember / en Canuber/ ISpabolanen/en andere dzoogen. Alvaro de Saavadera ontoecktede vock de Philippinas, en nam uan die de befittimglye int $\mathbf{3}$ aer is 2 7. booz De Croout uam Caftilien, Die tooz latt ban de g9arquig uan Valle liep unt te folven luan Civatlanejo in néva Efpaija , int verboledy uan de Eplanden ban Specerije / en par= ticulierlick Laas Iju in Minidanao, ent wie untloopen is niet gbearen buy= tell propooft.

Wedarkecrende tot de ozoze vain las Filipinas, volcht de voodyaelde

вabay. Abuyo, of Babay Derticly leguen iiti de lanthte SI11) thien in be ligette/inde welcke goe: De Agiinell siln / en oock peerlen tandayals, op oe Culfe. Tandayala isflyet ber= maertite ban alled / thelels oun dat fiet Luas het alderecrfte dat ontoect werde/ werde fyet genoenit Filipina, gljen tot gljeljengljeniffe bande on= wuinliclie Conumeli Don Felipe de tweede / torgltenacint de Vroede:

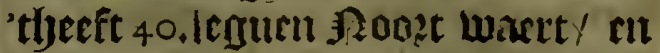
Ooft-waert en Pooat noort- Doft-

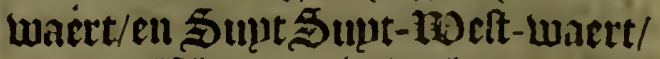
en twaelf Ieguen in de berette / op cenige plaetfen meerder/met goede Galuens en ticen. Mafbateen mid= Dellaner Ẽulandat PBett-wacrt ban Tandaya, feftljien leguen lanck. $\mathrm{Pa}$ nay 40. Ieguien lanck/ anl 1 j.bect. Mindoro, 2 s. Ieguen lanck Supoen

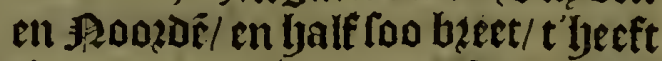
peper/en Agiinen ban 6out. Het Ẽplant van Luzon, genacmit nuèva Caftilla, foo groot / of grooter dan

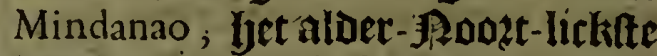
uan De Filipinas, in lyet welcke is be = voltlit Manila; een Stadt van de Caftilianen, met een tijtel ban tref= felick: al waer de Gouberneur $\mathfrak{r e}=$ fioeert / $\mathrm{en}$ De Officiers Uan't inco=

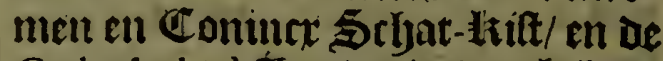
Cathedral! 't thandt is Lutuchtbaer Uan lijftorlyt / met berl Gout-99ij= uen: De haben ban de Stadt is quaet / ende fiju becle ghenteringfye Ifaet op De groote hanoelingle met Die Lan China, die Daten uan porcclana bengyen/ Doeck/gbeluerfoe fijoen/en andere Cramerien/Luzon fal ftrecken inde lauckte twee yon= oert leguen/ en yet ia feer bzeet.

$\mathfrak{I}$ nalle dic Evlanden jijn veel Mgalyumetiften / al waer ju glyeco= men $3 i i n$ Dooz Ooft-Indien, en ljeb= ben liebtelic in oe blinde ligendencut gljeplant baer mis-gljeloof. De Capitepin Magallanes ontoeclitede Defe Eulanden / alfoo glyefept is int $\mathbf{7}$ aer I 520 . gaunde nuet rent Oloot uan weghen de Crootre ualr Caftilien, om te roecken de $\mathbb{E}$ ulan= den ban de Malucos, en nant de be= fittunglye van yaer/ en daer naer uolbzoclje die t'ontoeclien Migucl Lopez Lan Legazpi, int \$ace s 564 . met een Afrmade die upt nueva Ef fpanja, met nam/ Doos latt yan of 
Bice-liopls Don Luis de Velafco, pen/ en politiicli volck/ cu altocg 'twogt gljeoogecelt wan of Cinlan= acu / Dat ju loopen tot clf lyouacrt/ tutrebu groote cu clęunen/ jumoé-

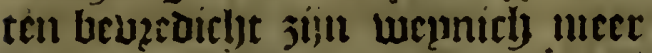
Dan 40. e! beticert mect dań ecu SHillioan yau $\mathfrak{M e n f e l y e n ~ t o t ~ l y e t ~}$ Catboliclie gbelooue / met groote colten uam DeConinchliclie Croon/ Conder ectuicly p? afijt tot un toe gefuat te fjeblon van oat Zant / Dat elch Hicligicur oic icewaerts aljeftiert is gewert / cofteoe tot de bül. frut Ducaten / die glyene die tot nu bouluen dicu Hoijugact met de aloergrootite upucljt uande jielen ziin oeticligicufulals Doumicancrs/

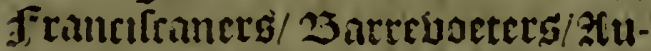
guítincts/ en ban de Co:mpaignie yan Ielus, eแ ban dic Ezplanden af/ Ijeeftuten begonnen en bitte werpen in ac \$ont van de ałahome. tilten / vie lanct oe Cult dian A Gen ginghen/allenfkens tutlterkento Doos Die / en andere Culanden / en bock tot dic uan China; ell Iapan. De postugijfen fouden al lanck uerlozen bebben die eplanden uan Molucken, imdien de Caftilianen uan De pzoluntien Jan oe Filipinen Die nict en Jadoen glyeljolpen / en butamt glyedacti uerfelyeuoen rejen op uecloerjande manicten.

De n:obu: \$u beeftmen beel beloerer kencomurribes nilfe van China, doo? be onderban: banchma, oclimglje yan oe Filipinas, en baer rijckidom / en bzuchtbaerljent wert betient / en oe nuachtigfje tiucken uan ie 3 secelt / cil ban Manila ral zim tot oe Culte uan China 300 . le. gucu/ aryt dagen requlens: oc Culte Wan Dat $\mathbb{Z}$ andt loopt bpcang ten Doo2t-Dolten / meer oan 700. leguen/ ban ecrien twintich tot twee cul twinticls graden / ban ot breette bu Roozocn om looch/ en Daer wo?t gljenteent dat owerg Dooz lyet Ianot is cen bopagic ban s. of res M3acnocn/ tot Dat ljet paclt tegeng Tartarien : bet gantfile 0 and is yol groote Steden / met becl Dopwarertento op bare befrljcimumge/ al boe Lel onbequaem tê ooglocly/ ell de Culte to leer bejolelit met treffelicle licuieren/ en goede bauens / Areckt bent unt Stmuen eut

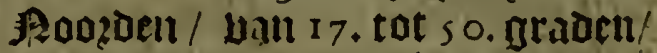
en Doit en melt bellaet fjet twec cut twiuticly. De Ŝtadt Paquin, al partia wact of Coninctilijin bof houbt lęut op 48 .graden uan onfe Pool,

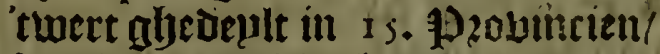
feg die aen de ₹ee leggben / en uesljen die binnen-\{lants jijn / en $3 i$ jijn gevenlit D'een ban o'anoer met een glyebercljte gljelick oe Pirineen liin/en daer jijn niet ban twee pars fagien / wooz de welefie ju t ot malcanderen cament.

Tomas Perez zimbathadcut van Berenee bain oe Coniuck uan $9 s$ oztugael/ vertorfue op de wech uan Cantan tot Nanqui 4.9Haenden/altoog reurtuoe Roozt-Laett: : cn tot de groat-

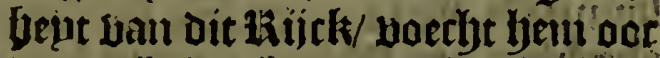
oe bzucljtbaerjept ban bet I lanot/ die reer beel is/ wace toe ooct dient de beellyent oer theuieren dic replbaer zijn / en jie jet bebarbtighen/ dooz bet belcke bet frbijut te zijt een uermacekelick fozet / of blonen = de 25ogaert / en oe aberuloet bet oubbelt ljen oj beeloeclyanoe ma nieren / oun dat oe Conmglyen niet toe laten eeniclj beoenf / maer dat het aen alle canten bewatert iérty [nijoenio groote 2 bergen/, en Arefy tenoe groote Balleuen / batter nergheng olyeen barmte en ont: beeecke / on dat bn naelt ljet gantfclje Zant lent onder de Lona temperata, oocls gljeen baclite oill oe lacbtichepot ban oe natuere oes Zanots / en boogt vernuft ban oe $\mathfrak{a g e n f}$ fyen bermenichfuldicht yet allefins / wefende allegader beficl am te betmeetberen / iffer niet cent palme lants fonoer pobfiit:

Daer wozt bertelt onder antate monterbed notabele oingljen/bat te Cantan een groot ghetal ban 25 linocn is/ dic य 3 aftutil. 


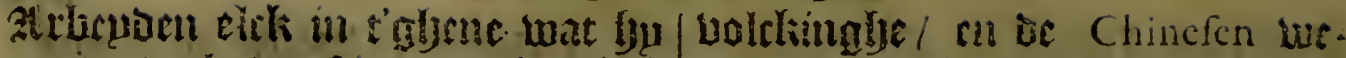

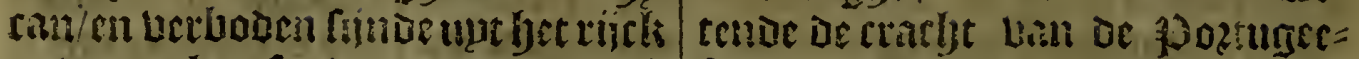

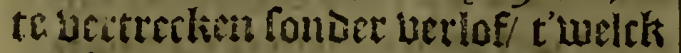
corli uice socablelaten wert/Dan tot Eetl gliselimiecedoe tiit / is noot faec = kelicli dont ghefradelicli de propa $=$ gatic tuaftentie/ t'getal ban't volck foivoer ennase is/bulanderlick altoo ju bae? [ecclier Gouden , Datter in placter ban les joetropent die daner Ateruen / reuen abeliozen werden/ Dact 1 poch fulchen fuluerijepot

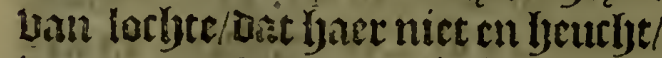
Dater eat algeineene pefte is glye= werdf en met alle orfe welitamoen 5.jobeit ju oock bare nuaricheden/ ou datter stert lebinglyen uoozual=

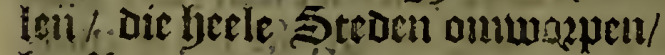
lyet ulant vertwoeften / en ie ande - Lievieren ltoppen/en andere weder ontiecken / Herbten oock joogfye ghebercliten / inet aroote angeluck bain dat volck.

BD: barfinge will Chinas Jut Jaer is s o. bouft unt bet biumente vante stertrijcl fulchen óberbloct bau Inater /. Dat een Lpa = tig da treltich leguen gantrely inum= deerve/ en deftruecto 7. Steden neet yare \$urifoictien. Soo vecl iffer tain bet valte lant ban China.

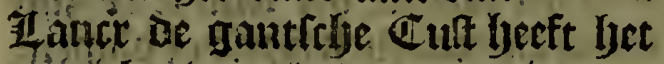
meniclyte Lain Enlanden/ Die Mee= tentoel ous onbelient jiin/ontent ve Staur Nanqui is oe Cabo de Lanipo, of enlanben uan Aveniga, Abarda, Sumbur, Lanqui, en die ban Cayailos, uan oe uelclie men gracen

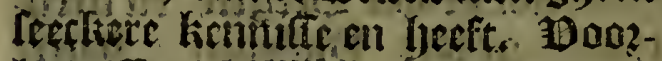
Gupafrecrinde of atad ban Chinch fo leut Lamao, en meli fiet en quac ghetal van Eulnuben on = trent de licede ban $C$ antan, or ver = illacitite ziju Lantao, Macaó, Veniaga, al waer oe thauen is van Taino, I ampacao, Sancoan, ill wele= lie alle de Cemincl van China gar = nirocil bout te Boater en te 3 ande/ bebătuet in Lampacao, dat niet be= volclat is.

Macao, 7II Macao, anders glyenaemt Ma. can, Jebluen De Doptugeten cen Le=

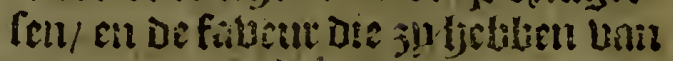
ac Caftiliane dic inde Filipinas jiin/ connenfe nict wel lurantigen/ mbat

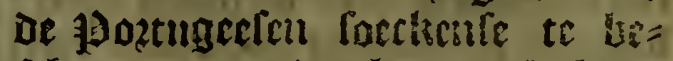
fefjermien / oul dat bacr becl aijeic= glyen is aen Den lyandet bail China. Sancon leut Dcrticy Irgura? ba:? Cantan, al wact Dicktuils or $130 ?=$ tugeren te lande genacu jijn/ Dacr is glyeen Eulandt bam China bin grooter impoztamtic Dan Anjam, in be gront uan De Golfe that Cau. chinchina s. leguen vam bet wafte lant/en yondert en taclytcicirly bat De Filipinas, en t'is loo groot / Dat ghefeut wozt Dat het Dertich tterif: ten yeeft/ Daer is etu bifrberije van eleun poerlen/bet is oberuloe = Dicly uan Bictualie/ ch tuan becle Bzuchten/ onder lyet Gouberne ntent uan Cantan, en ig het puincipael decl van China, de 7 merbeloo= renen jîn groue 3 Lugen/ en boer = achtich.

Iapon, twelck jiin Let bertelyew: dene Eplanden met clene inljam: men Deg zecs / glyedeplt in date en Derticls kiiscken / wieng ljooft is de Stad Meaco, Ieqt vertrlyeubclicli yau China, en $\mathfrak{j}^{\mathrm{p}}$ tellen ban yetep= lant Goto in Iapon, tot Liampo in China trefticly Isgum / t'welcli lyet aldernaelte is: Oe laponefon mia= lefteren leet de Ċincefen, viterer met

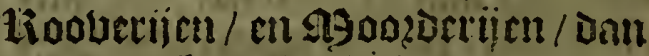
vitet opzeclyte Oazlorls/ Dill dat lapon gljebenit fiinde in varthersen

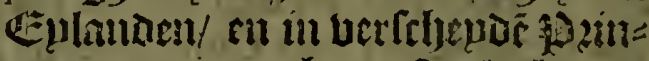
cen/connen ju theen obalorlj boe= ren tegljens die van China, dau met rwacke crachten / su zij! be= quamer ten Obalaclsen mordiglyer bimt de Chincefen 't 2 amdet is gibe=

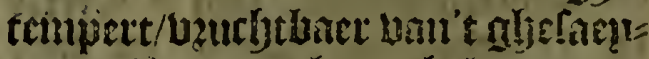
de/ cll bec/ ell aljeboglielt Ln Eu-

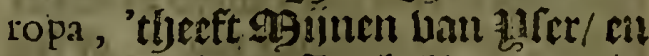

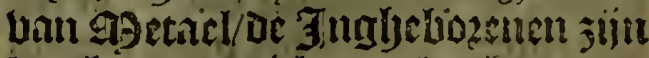
lanck / moedicli ch cloers rot at = beut. Of Eplamden lenglien op 
Uiif cis sertich graden boorlyte / cen wecunich meer of miin/ te gljelticken met De diltantie van elch Ẽlant: De laponeien en verftaen uiet de Chinefen, dan Dooz glyefrlyeift / oull Dat bet gbelisclie figueren jiin / of Zletteren int gheljeel/ en al boe wel ju bet felfoe beteceliencu / coo en theblen ju de feclfoe namen niet/om Dar ju dienen allean oun te beteec=

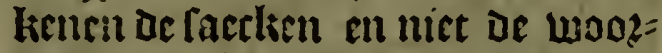
uen / glyeliick lyet is met oe glyeta= Ien van Atrithnetica / gijelijet in Dical icli atelle 9. eetr frumlman/ Caftiliacn, cen Eutyelfutan falt veritaen / Dat ljet 9. beteeckent/ nocljtang cen peder noent het op (1) m manier.

De gelegchits bept ball oc banca, aen ponefen met ie Filipinen, uan waec D'ander iDDe,

ju banaclmglye bebben niet baer/ cil bet glyecft grooten uuer ue ba= vers ban de 3 cluyten/ die in de be= Kecrunglye watu bolek ban de Co=

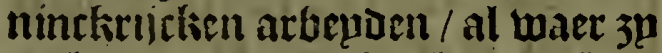
Dock notable Bzusbt glyedacn yeb= Uen / Dooz twelclie Dic Eplanden feer uermaert jiin dooz de Boerelt/ en doo? de coinfte ban de Ztnilsaffa= Deurs ban dat nicuwe Chyilten= dom tot den paus' en de Coninck Felipe de tweede de Vroede/en glje= litck als ae natuer lyaer gettelt lieeft in een afghelonderde glycleglyent=

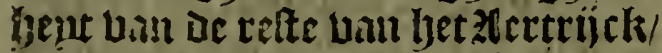

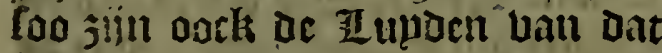

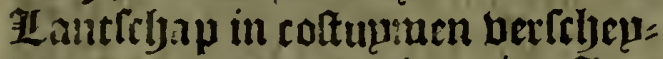
Den van audere Ratien / int $\mathbf{3}$ aer i 5.92 . Nobunanga, die lient arde nocenc liculer van lapon, befprong suet aclut bonaert Scljepel / cn chl thuee jondert dupfent gisannen/ eciz feether 10 sobuncie dat tributa= ris wiss uan China, en lyp wan yet.

1.ss Lequas. Doan het Eplandt vall Simo, t'basich lyet gljene ig / out ae groote bect ban lapon, ftreclit hen tul= Fifjen 'toolten eir 't $\$$ oozden een Cordillera uan cleune đEplandeltês/ Die ju noemen los Lequios, en $\mathfrak{j p}$ loopen bier/ftreckende tot de Culte
Lam China, tot de thuse groorfer ban oe we!clac elekeru is limis biff= thim of thumetidy lequen/ onezsut de Cufte ball Iapon normers jut I equiomayor, ell af nnere twee ooeli groot/mater foo met/ asc op bet çu= de jiin wan ie Cordillera, slecumen ju Lequiomenor : emiglie jim be= bolelit met een wal glicsirponest Bolck / ont wit is/met goríc pol: = tie/ wel gheclect/tot D'Do?!och be = quacria / ban gocde rescu / ju jill oberuloedigyer van Gout Dan ant= dere uan die $\mathbb{E}$ ec / sin met minter ban Bittualie/ frtupten/ en ban gort Bater : dirlyt by Lequiomenor lept lyet Eulaint Hermofa, Dat ae lelf de boedanicljeach lyeeft / ec? niglje legohen dat let foo groot is als Sicilien.

\section{Het X X V I I. Capittel.}

Van't nievore Guinea, de Ey: landen van Salomon, en de Ladrones, met inelcke eyndighet $t$ 'ghene dat $z \dot{j}$ noemen Indies vanit Westen.

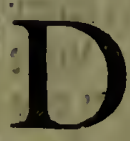

E Culfe ban neuva Guinea, Niea Guibegint bondert leguen int Oolten / wan bet Eplanat ban Giloio, op een graet booclje/ wequiry nteer / ban D'ander juide ban De Equinoctiael, uan waer fu

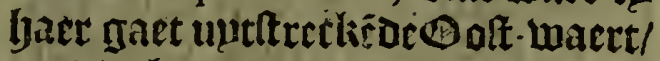
tot Diteljondert leguen / tot Dat lyet loopt tot s. of fes graden : loo dat = men abethuiffelt becfe tot nu toe/ of ljet eplant is of valt lant: om dat bet foo verte is afglieléglen : in foo groote vopagie van ben te mogen boegben met bet laut ban oe $\mathbb{C}^{z} p=$ landen ban Salomon, of de \$Bzos vincien uall de Straet ban Magallanes, aen de Supt-zijde / maer die twijfel blifft afghedaen / aooz yet ghene dat die bebeltiglyen / die gloloopen beblum aen de Suptziide ban de Straet Magallanes, dat 
Ijet gigen bult lant is/ Dum alleen outdecktede wask Alvaro de Save-

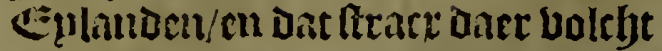

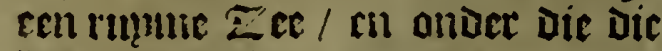
Ifet bsucilingen / is Don Ricardo

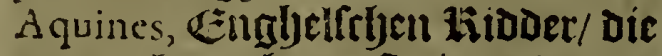
45. Darghen ghelueet is onder de

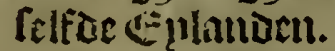

Eulanei /en Dan De Wabcus vü Lan De Cult ban Guinea foet / en Guinca. Die Fughebozentm die aljefien șin gljeureit/ jiin glinmende swar= ten / op De Culte siin beel Eplan= Dew/met felyoune hien en lyabens/ wan or belcke gljech particuliere fenmiffeis/ ombatte bepuicly be= varen is: Dieuten lint in eenighe Cacrten/zijn El Aguada ljet BO Iteliclifte / 3 s. Ieguen wan bet ecrite landt/ Dat een graet be-Supden de zinie lept: en arbthien verder is De Yauen Sant-jago, en ljet Geplant van los Crefpos, wan reltien teguen lanck dicljt bu de Culte/ recht ober de andu ban San Andres uectich Iegum ban dis uan Sant-jago, en meer nae 'toblten is de Hebier de las Virgines, en De Vallena een $\mathbb{E} \mathfrak{p}=$ hanoelien uooz de thevier van San Agultin, Dic uncans welen fal so. Ieguen ban de Haben ban San An. dres, en ontrent die ig de kebier uan San Pedro, en San Pablo, vougz oe Gauen ban San leronimo, en een Eplandeken ifler ontrent de Puntafalid2, veertich leguen van San Aguftin, Dat 34 noemen de Buenapaz, en meer vozoer daee af is Abrigo en Malagente, twet Eplande = kens / en de Bahia ban San Nicolas so. leguen uan Puntafalida, en on = Der andere Eplandan ifler cen met wit volek / en be la Madre de Dios book de Buenavaya, en die ban la Natividad de nueitra Senjora De laetfte Wan het ontocelste / cn bp naelt Roozt-mierts af uan dit / is Caymana, ten Eulant Dat bupten in De $\approx$ ere lent $/$ onder andere die fon= Der gfyetil jiin.

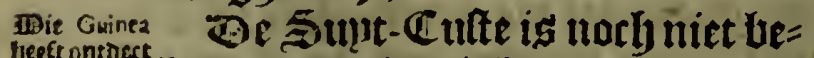
beffontosct Ytent: De ecrute dic ljet nucva Guinea dra uerloozen gaende ntet ueel on= wederg/ wederliectende ban Nueva

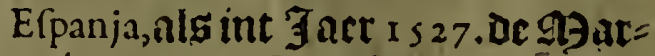
quist del Valle lyent font / oat bju in

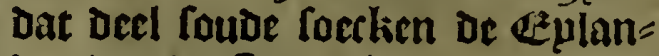
Den ban de Speceric.

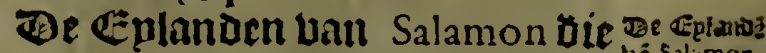
ache yonoert leguen jijn ban Pirụ, Geblen dien naem gljectegen doo? De meenmghe die nien ljecft baแ yaer Hiijckdommen / De weltke ju Ipecialick noemen ban't EBeften!

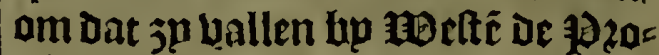
vimcien van Piru, van waer A lvaro de Mendoza dede de ontocrkingle ban haer / Dooz laft ban de $\mathbb{1}$ icen= tiaet Lope Garcia de Caftro, fiin Retf Gouberneur welende ban D: Coninckrijcken ban Piru, int Taer 1; 67. Een jonghen ghenaent Trejo mas d'eertte die eerft fach ljet lant ban die Eplanden / Die in een Mars wan een Schip was / $3 p$ leg = ghen ban leben tot twaelf graden hoorbte be-Supden de Equinoctiael, up naeft ouplent vijf bon= Dert leguen ban oe Staut de los Reyes : fijn ueel int ghetal/ $\mathfrak{e n}$ in groote/ en arbthien zijnoex de pzins cipaelete / eenigbe ban dzif bonder: Irguen int ront / en twee van twee Gondert / en ban Gondert / en ban bijftich / en ban wat noch minoer/ foutoer beel die niet beel om geleplt en ziin. Zp legghen dat fu fouden connen jijn cen valt lanot / met of Culte ban ljet nueva Guinea, en be Landen die nocly t'ontdecken zijn aen de aldeft-zijoe ban de Stract: alle de Eulanden frhijnen te zijn wel ghetempert / en betwoonlick/ Wetuchthaer wan lijftorht / $\mathrm{en}$ Bet! Daer werden in gbebonden eeniglye Bzucljter als die ban Caftilicn, Berckeng/ \$oenderé bp menichte/ Inwoonders ban liclyte rwerte co= leur / cenighe ziin blancls / en rors arbticly/ en geelachticly / twelck cen argument is dat jp valt jiin mat de Xanoen ban aicu Guinea, baur 


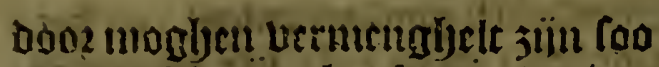

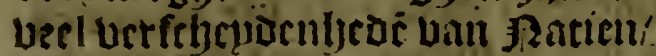
than ote Dic remen yantarlen ith of Elylandern batt oc Spectrien.

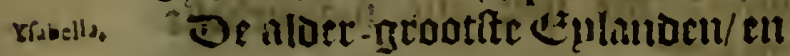
treffelickifte jlin die bail Santa Y Falwl, wal arlie tot negher gravon foorijec / be tedte ban ljondert bije= tuls leguen lancli/cu actithien beet/ c: 1 Dier is sen goede batach / dic ju. nachen de la Eftrella, Silorge, of Borbi ten ecu legue cu sca lyalf / uan oerticy - Itguten in't roiloc. Oork San' Marcos, of Sin Nicolas Uan fyondoert le= ginen int roube Sunt-Ooft uait Ariaz:fs" Sania Yfibel, "en liet Eylandot-Arrazifes foo groot als bet voozgact = oc Stmiot-watrt ban Ýfabcl: $\mathfrak{k n}$ Bof-waere lept San Geronimo vall Gonoert leguen int rontoc/cu \$uptEefit-waert lent Guadalcanal :groo= ter dan alle antere/ en toett-waet uan Santa Yrabel Ient lyet Eplandt Uan Buenavifta, en San Dimas, ent Get Eeplantot la Florida elck twin= tich leguen int ronde / en Bolt Loacer ban dat is lyet Eulanut Ramos twee fondert legutil int ront/ cn vicht bu dit is Malaita, $\mathfrak{c n}$ Atreguada ball Dertich / en die dzie Marien cenighe Eplandekeng/ en dar van San Inan ban twaelf leguen int ront/ tuffeljen ae Atreguada, en die uall Sant jago ten S1upden van Malaita ban boutoert leguen int ront/ elt sunt-Boft-waert daer af is San Chriftoval, loo groot als 'tuooz= gurchor/ cu Santa Ana, en Santa Catalyna, twere clęune Euhandekens/ dicht bu dic : Dacr Iejt oock Nombre de Dios en cleputeplandelten/ berfelyepoen ban dandere viiftich icguen / op feuen graden lyoocljte/ en op oe relue hoochte Roote-waert ban Santa Yfabel, jiin or Clippen dic ju nocmen la Candelaria.

Dare is in de Dopargie bie ghe= Daen wert bart Piru af, 'llae de Ent= lantour buit Salamon, tent ęulanot Dat $3 p$ uccmen trin San Pablo op oc foochte bait utjetfich gradocti tcuei: jondert legmen lan Pirt, all op vieGljentjien graden bair jondert le= guen 1 andts jijnori allocre bie conne weren die/ die su cerlt noc!n

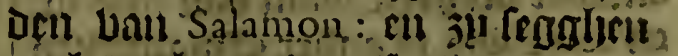
bocli dat het aubere lotuden commen. jiin die ech weunicls ach gech lijoe.

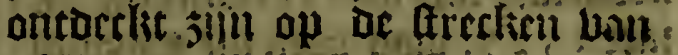
Chile.

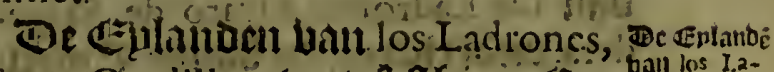

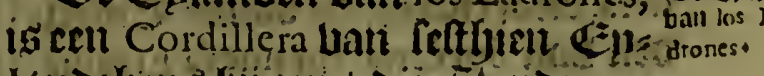

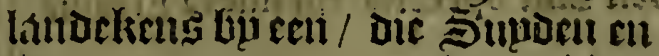
\$oozden loopen/ utet bet midom baur de Culte bail Guinea, ball 12 .

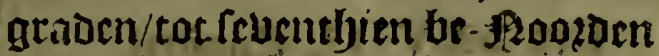
ac Initie / of neer / niet verre ban of Filipinas Ooft-waert / let gaut felue. IL and is onluuchtbacr / en el=

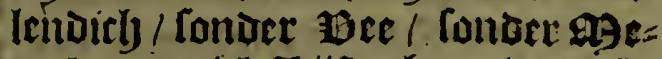
tacl/ wepnich Jifftoclyts / beboicht ban arme zl upden/ die wel difpooft ziiiz/ en feet glyenegben tot lteelen! jae tot lorely nemtulis toe of 5 piic ters lan de Scljepen / die tot haec: comen / Daeroni noemacte Magal-1 lanes los Ladroncs, utt 3 (aet i s 20 . als ljy tot Jaer quam/ gaende in. ueruoled ban de Specerien : haer. tiacuren siin la Inglefa, bet ßooztlicklte/ en acliter Dat Ota Mao, Che-mechoa, Gírgua, Agan of Pagal, Orantagan, Guguan, Charcguan, $\mathrm{Na}$ tan, Saepan, Bota, Volia. Onder Die Filipine Eylanden zijnber an= dere acljthie of twintich bie ju noe= menl de los Reyes, Arrzipielago, of zos Reges: d'eplanuen uan Coral, en los lar- coral, dines, een andere quantitept ban Eplanden : Dater is oock Pialogo, San Vilan, ecn ander etulant biclyt bu de lardines, ent 'tabene ball los Matalotes, en dat bain Arracifes, en vail $S^{t}$. Iuan, of dc Palmas, ontrent de Malucos, tit acht Poozacrued uan los Ladrones, unif of frs Eulan= artens Diclut bu eat Dí ju thormen Volcanes, al waet urel Cochinilla yoteanes, id/ en Malpelo ect ander clęur En= Maletos

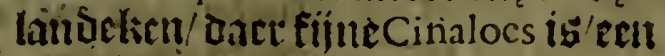
aen do ofof fribe bail los Ladrones 


\section{Fol.yo.}

zijn be dos Hermanas, twee Eulan= 5.Battolome. Diekteng op thien graden/en San Bartoloine op I 4. en meer/ ban nueva Efpania de Clippen Miracomovas, Quitefvenjo, of Catanoduerinas, $\mathfrak{e n}$ Martin, ontrent die ljeteplant ban Martin, 5.pablo. en San Pablo, ế ander Éplandekten met Clippen/en la Poblada het $B 0=$ fterlickete nae nucva Efpania toe/int jâet I s 2 7. Was oock int Eeplandt Uan los Ladrones Alvaro de Saave. dera, wederkeerende bande Eplan= den ban Speceria. na nueva Efpania.

解et X X VII. Capittel.

Van de Hoochfte Raet van In. dien, en bet gheestelicke Gouvernement, en de Conincklicke befcherminghe van -de Kerske.

Qes beaznes ime oer stocs Eonlingluen.<smiles>C[14CH2][14CH2]</smiles>
EZ Catholijeke Coninghen ban CaRilien, en ban Leon booztbarende in baer oune en Chriftelicke Godtotuchtichept/ ftract nae dat haer ontoec kt waren ocfe niellwe is anden/ en gheluaecht bp de. Ichoone Monarchie, bebben $3 \mu$ belozcht in die te planten / ett te contenteren be Catbolijeke keli= aie/ en de tijdelicke politie/ met loo grooten lozghe en accoozt van de alderwiifte פ9annen ban die ziije fren / oat met de antoeckinghen/ oock wietten de Cooplyandelingen/ bebben $\mathfrak{j}$ gljefozmeert een particu= lieren liaet/ met een ploetioent / en liaetlieden/ Dat jp yjaer niet louJen becommeren iil andere dingen met meerder neerltichept/ten dien = Ate Godts onfes Hetel en tgoet se aaetbr Goubernement van die merelt:en indiem

om datmen baer naer mentie fal maetken van die perfoonen / die uan ben beginne af gljearbept en alyedient lyebluen in usn oppertten Kiaet ban be Indiens, die die is die gheleden ljeeft loo grooten laft tot un toe; fal eert gefept wozaen lyet
Gyeeftelick goubernement / m bet tijoelick / en de refte die waerditl] is om te weten/ Dooz yet waelcke imen lal verttaen de ozoje ban die Bonarchie met alle moghelictie cottyept.

De eerlfe faecte die defe fodt= \$e lafs ber: bzucturighe Coningben belate en bevolen beuben de eertte ontier. hen / en van hant tot bant belaft 3ijn gheweeft de relte ban de ont= Deckers / en Gouberneurs ban die nieuwe merelt / met beel itrichte ozoonnantien was / dat 34 be[ozcl] $\mathrm{den} /$ dat bet bole dat jp daer bzocb= ten / met een Cbsiftelick leven / en inet faer goede coultupmen looda = nighen erempel gaven de Indianen, dat jijfe noodigen fouden om baer naer te bolghen / en die aen baer berbonden: eert ingaenae / con= foem de cavangelifebe Het/ dat oien de Religieulen fouben 13 zedic= ken oul batmeer met foetíchent van dien/ Dan met ghewelt en gheramt inel van mapenen de aengbenos men werde / en dat de oljetech. tichept bedient werde net fulcken inoulgentie aet allen/ dat $j \mathrm{p}$ meeff gheactit/ en gherelpecteert werde.

Zulleg was maftende/als de bole = oo bea

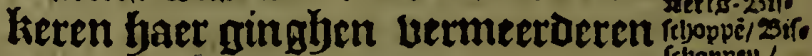
op alfulcke naniere/Dat $j \mu$ met Den ciropenters, puer van de dienlt Godts / en wel- Sidveleru in vaert ber פgenfelyen / quamen tot dien epnde/ dat men yeden binar glyefondeert in die gantflye $20 \mathrm{r}=$ relt / thedert dat die Croon befeten is/datmen daer naęr gljelien ljecft. Bijf Zuerts-25ifooiumen / 27. 25it= Dounmen / twee treffeliclie Bniber = ritenten / al wacr met groote glye= leertljęut gfjelefen wozaen alle bue = tenfrljappen/ meer dan 400 . Cloo $=$ Iters ban Religieuren / Dominica= ners/ ffrancifeanerg/ Alugultinerg/ onle 更itue Bzou ter Gratien/ baul Jefunten / met eenighe Cloo= Iterg uan ßonnew / en Collegien/ onepnoeliclie veel Saft-buufen/en \$5zocderf(j)appen/ ontallicke vele belorchoe 


\section{valtVVeft-Indien:}

befoectjoe benteficion / dic $3 n$ noe $=$ Imen dotrinas, out te leeren be atn= comrude Ellaittenen / en de ber= miten / cll. 23artDoenders dic fon= ber gijetal jiin. All bet welcke be= goumen is tot colten uan dic Croo= IIe/ ell noch bedell boozt gaet / al if ontter uiet gevonden wert/ Daer oe coftell mede geloetet meroen. Tn effect die Catholisclie Gobtuzuch = ticheut / gact D'ect Detulyt tot D'antDer/Doo? oe barmberticlyept Goos/ uermeerento met fulckie eerbicdic= beut ell cere Godts/ Dat in sheen Decl bant De Cljiftenljcpt gearbent wert net miectoer accast en cozage/ Dooz midoel van de boochlte liaet cof ore ta ban De Indien, waer uyt befloten

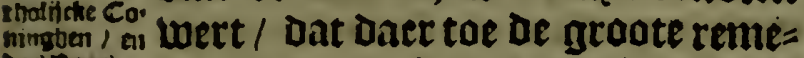
inspause.

bie is glyetweet/ oc toelatinghe/ die ae bepliglye Alpoltolifelye Bamepn= fclje Stoel bede aen oc Croone ban Caftilien en Leon, van de thereltelicke betchetmunghe bes nieumen merelts / in de welcke Sodt onte Seere / als dic alleen is die fiet / en booz berept alle toecomende din= glyen/ljeeft gedaen een dinck weer= Dicly fiine grootlyept / en daer naer

- beefí glyetljoont de onderbindin: abe / Dat foo bet gljegouberneert wareghelueet op ecn andere $\cdot \mathrm{ma}=$ niere / Dat het onmoghelick hadoc ghetucelt / Dat oe faecken louden boozegijegaen ljebben met alfulclien t)armonie/en cenftemmichent; roo egual/als ban Kieligie/gherect)ticljepat / en Goubernenient net groote alieljooźaenibent / en rufte.

Doe dat oe De Itercliselicke beftyerminglye

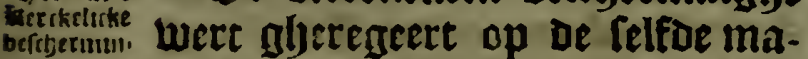
ge gereysert nier als int liiicli van Granaden, De Catholiicke Coninghen peerenterende de paus alleentic be atert 25illchoppen/ en 23illchoppen/ oun Dat $3 p$ louden ontfanghen ban fiii banden diefozelaetlehappen/en bet Detpaceeren van fijn 23 ultē/altoog Leftellende dat bet perfoonê fouben ziin uan $e^{e}$ religicur levê / cn groote geleertbept. Oe retterēoe bignitep. tê/m beneficien fijn probitien boo? ben Coninck / met arn boorbeten tiaet ban Indicn, en $\hat{\jmath}$ en gacli niet naer kiodmen om 23 ullen / ent baer renten beftaen in be thienderi cu cerft gljewaftel / die van de Ca. ftiliacnfche bebulclieris ecmen: ism bat int meelte deel vall defe Indicn; oe 3ngliebozenen dic tlyetiden niet en betalen/en al waer die tlyienuen ontbeecken / uerbult vie tet'feo. ninex incomen: nopenter oc thien. den en eerlte-gljewaften / hoe die betaelt moeten werden/giin Ber vele ozdonnanticn / cu Tiegifters ólse. maeckt / confom met oe ttîl bar de Lijcken ban ljerwactis oler/ om dat ju werende ljaer tielinge/ent tolonie/ ia recljt tat jp volsen harê abebzuytien en coftummen. En al boe wel de Conúgé van Cáftilien, en Leon, jiill Qseefters bân de tienden Doo? do zt polfoliftle toetalinge/ enta die couden mestsen nemen toet haer/ballende al waer't ontluecet/ met fet gene dát op andere plactfe obericty is/foo werden die nochtans gelaten aé de 192elaten/entseretren/" boosfiende van: riju Coninclick in comen/ntet miloicljęt uart fon uee? Catfyolicke punce/ / op alle noo:fi-

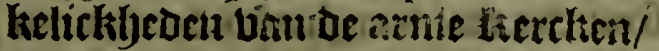
geluende ele cen dist opgeliont werde vannicus lyet grootlte ocel dat glyeröfameert wert in liaer fabaiic/ net

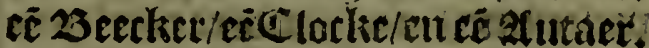
Op dat dic untoevlitíghe bait Ban be ós

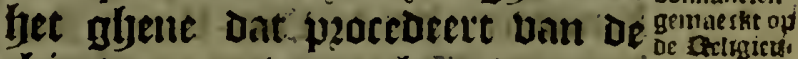
thienden / en ban't bheite ont cant fen. fumeert bost ban fiet Conincklicke intonten/ tot lyet onderhonden binn de 1Declaetfelje weerdiclieden / cit Canonifuen uan de Catyedzalen en ae gliebencficieeroen p aftoozen. en Berfoonen die befoigneren unde Godts diente / en int onoetwilen ban de Indianen, bae lyet mett veuclyt gtferchiede/en confornt met ac hejt: liglie intentie ban de Caluinghen/ heeft oe ljooghe linet beel gaede oz= Dinânticn gljemarelkt. Eerteclick: 


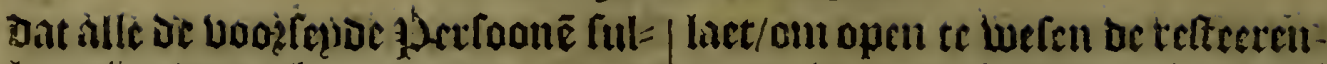
len siin ban gljewoonte / en ban ech goet Ietuen / en bufonderlicli die vie bace beglichen fullen tot de on= Decwijfinglich / cerft geeramineert

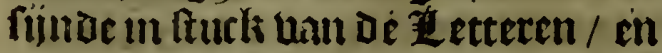
voozts in oe Jnoilche tale / om dat let wepnicl fal te bedunden lyeb= ben dat de leer-jouglyers baergese = fter niet en ueritaen / en dat $j^{\mu}$ ita $=$ Dicl) bu Loonen/ cut daf glyeen par= Itooz of al ceraer macty yelsben twee Uenificien / ell ap Dat ait gibene die ban bele derlen fullen naer Indien gaen/bett fullen onberloclit siin/itt gljebioden / Dat gljeen clercls ber = treclien soude conder conlent ban fijn prelaet / in uan be Coninck/ en foo aldaer ren ghetuonorn fal Wozoer lonber Dat bef́clyepot / Dat yp Itach fal beocrom gljefonden werden inat Caftilien.

Servortb; Enombatment beter berftae de inanier hoe dat thegonuerneert wazt ae Conincklititke befrlyermain = gye/ mae dien lyet belyooit tot die Groone am die outorekt te bebben/ en vercetghen déte nietwe Boerelt/ en gebout/en Dater in begifticht ban 'TCaninct incanten/ foo beel laterlien/en Elaofters/als dooz de zlpoAalijelie toelatiuglie/ Contor dat oni ghem oozfaer tuille bet voozfepde 3patroonfelyap / noch eeniglye deele ban Dich/noch Dooj coltupme/noch pzéteriptie/ nocly andere tijtel ljem can refjepoen uan die : foo is gljeozDonneert / Dat de Bite-liop / ZlitDientien/Goubernetirs / en Cozrigadozs forghe moetén uooz dzagen/. op de Itraffé die de obertreders' uerDienen. Eerftelick Dat niet ingeftelt wert tenighe Catbedzale ltercke/ nocly qDarochiale/ woeh Cloolter/: noch l) ofpitacl / mocly liercke ban belofte fonder confent ban de $\mathbb{C}_{0}$. ninck. Dat alfe in oe Cathediale: sercken niet en fullen heliber bier gelseneficiecroe refioenten / verfien booz de Conincklíke pzefentatie/ m Canouicke pzobifie ban ot $192 e-$ De pzourniden of abfent jijn merer Dan act) 9 Bach

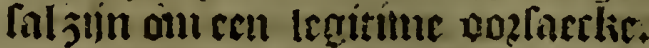
De voozfeurac jozenet foo langlie als dectoninck fal pecfenteten/ urrkieft tot verbullinglye bau De uter Gherftelicken / bouen die Die noo?fien warén/refidentelt/bau de uteclf bequaenite die baer hallen pzerenteren / fonder ont fulclie jpovifie $3 \mathrm{u}$ in de Ceiitel de torlelie lupt : Si non ad nutum a mobile, miet ae melcke $\mathbf{j t}^{\mathrm{z}}$ gljeen fitplaetle contoen beluben in De Chooze/noch ftenme uil de Coulfittozie. Dat gheen 3 zeclact mach maecken eenighe Canoniticlie inftitutie / nocts podarfie glueben bait eenighe pzobende/ of ueneficie/roneer Councklicke peefentatie/en ix fulckeh gljeballe dat jp. fontaer bertreck os pzauifie gyelen / en gebie: oen te ontfangben met be bucly= ten. Dat in alle Digmitenten / $\mathrm{en}$ peluenden/de geleerden fullen ghe: pzefereent wozden/ boben dien die het niet en fullen zijn / en die die glyedient beblien in de Catjedzale Lierclten.uan Caftilien; oeffent fullen sijn in te dient una het Cyooz / beben bie dic daer in niet gedient yebluen. Dat ten minaten gepzelenterert werde voo? eleke Catljedzale terclie / cen gepzomobeert Itirift en en dyeologant/ Die het pulpitum belube / met lyet berbant luclcke in bie liijclien yeb. ben de Camaniicken / Doctozen/

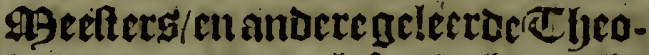
loganeen/ am te lefen de yeuligbe Stlyift: of een ander 3 uritt / of Chêlogant tot lyetCanonijerthap una geanitentien/ confozal met de - Becreten ban lyet beuliglise Concilium uan Crestetn. Dat alle auDere benificien/ Poaftopsflyappan/ fimpele / warerliclie en Glyeettlicke! en alle litereltelielse dienlten dic vaceren fullen/of uan nicus te uartien. fullen sijit 7 op oat bet glyedaen werbe nuet os minte untefellingtis 
en of Conititeliclic beferyerminglye beifutiden weroe/ is liet aljeboden Dat ber ghedacn werde op acle na= valghentac manicte.

Enat bactende cenitye than de bousu bethaclac Lencficion/ of offi= cicn/ of 3 selinet ghebiet bat uren ftellan fal sjilletten / met cell be= Jjoosliclie tijt en Der glyene die ach = comen oill hater look tè ftelten te cruminct / $/$ all geinfozuncert siinde wan lyier coltumuen/Dat lyg noche/ twee dac oe befte sillu / en be witeLion/ bet hof/ of oc Goutuerncut

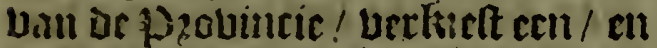
ftiere de clectic act de pielaet / om oat lji ac piobifie doe / en de colla= tie' cu oe Canonuslise inftitutie/ bu weslien van coinuniftic / cn niet op cen ciitel van gifyeom12icly/ maex

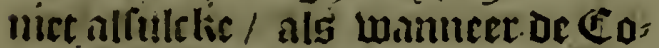
nimeli oc pacentatic fal Dociu/ en in Die une geoziuct ral fium Dat de colla= tie fal gefelicoel onuer een gebuit= righe tijtel: De Canonijcke inftitu= tie fal be lacttte welen inde e eijtel/ ent nict in commulfie/en Dat de gente Die gljepicfenteert siin Uod de Ca: ituick / altoos fillen ghepzeferert ziin uoos bact asiulterg.

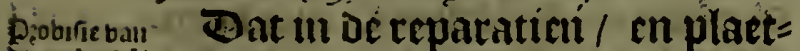
be vobentori Ien ball Indien, en andere oeclen/al waer men ghecn beneficie hebben fril/oml Dat te Kiefery/ of een nianier on te ftellen dien dic de Sarramen= tell bedienc/lact oe b)zelaté befozgế Datter esn ju die dacr ondertwile unet of lecringlye itellende ech 25 il = Itt. cn gemfegmeert zijnde ban sijn urquacmiscut/ en goctlyeit / ont lyp fiende or nominatie acit de $\mathbb{C}_{0}=$ IIncklicke Dienaces/op dat jo pie= fenteren erin ban De twee ghetioem= oe/ en foo der maer een is/ en doos eract)t van alfulclic pectentatie/linet oc Djelact de p:obific natecken/ inleciende inftructic hoe dat bn be floost te leeren / en gll)ebicartide lyet t'ontfanglien met of pzofiiten. Dat in de perfentaticn bain alle of dig= nitcuten/ officien/, cn bencficien/ berficn fullan ziju/ die fyet huect wel uetbiculthebluen/ en bic meet berieb jiil ghelueref in de beliecriil= glye vant de lindianen; en urdieniu= gibe uan de Barramenten / in Die beft fullen commen oe Indifelye talef fullen gepzefeteert jill boben dallocre. Dat dic gljeen die fullen to= nien of gljefontuen werden aen fiju signicftent / Dat hy pesfentect tot calliglse dignitept / anipt / of bene = ficie/ lact bju collupareren booz de Mginilter ban of pzobincis. / en verclatende fiju petitie / ban de informatie de genere; 25ietien / $\mathbb{C}_{\mathfrak{a}}=$ Atimen / en lifficientie / en ander lact ljet or is inilter Doen ban fijn officie / ch opj fijn Dunclien laet bju lyet fendorn / en Dat bock die pieten= ocett $/$ neme nppzobatie ban fïn 15zelact $/$ pon bat conder al bele Doens / en fullent bie gljeen bie to= men nict toenthelaten wozaen:

Dat nitmanot can berceijgberi twee beneficien of dimnitejten in cen literds/of in betebendene. (Dat niet comparctende de abeperten terde : binmen den beftemben tiit in de pzefentatic loof de jozelaet/ van gljecnoer waet de fal fijn : en vatter ayeen Canoniicke inltellin: ohe mach ghematckt werden;

Get X X I X. Capittel:

$V$ an bet Gbeestelicke Gouver. nement: van der overledenen goederen: : van de ghetrouden.

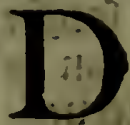

e refte van liet verhaelde is de pzobific / oat niet abe conlenteett wert/Dat nljecn die een Dzebende ljeete / in de cas thedale trertken/gheniete de ren= ten ban dien/ foo fypereniet en lyett bedient / en bu glyewoont / en dat de beneficien van dic Indiaenen, nict ntoghen werem/ Daftomzfchap= pen / Cappellaenfebappen / m bat 
in of nientwe ontdeckinglen / en / tels / die die oxie boben bechaelde bevolekimghen die gyedatn fullen

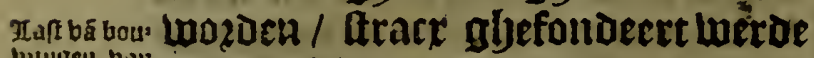

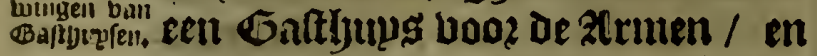
nect cranckljeden beladen / die niet befmetteliclien ziin / t'welck ghe= bout fal mozoen oicht bp ore lterck/ sil tot een befloten plaetre van de Sieclien uan befmettelicke qualê/ falder gyebout werden $/$ het Gaft= buus / in eell plaetfe / daer gheen ftbndeliclie Roint Doot baepenoe/ quete de reft ban de beusolckinghe/ en dat fet gheliout wert op en lier= bevene plartfe fal bet beft wefen. En otm dat of Coninck gheinfoz= merert zinde / dat de goederen van De porfoanen / die in die plaetfen cainill te lteruen! niet becomen en weroen foo ghebeelick als $3 \mu \mathrm{mo}=$ aben / ende foo gherect in handen yan de Gerfonamen/bp teftament/ of ab inteftato, om foodaniglye glye= Itozuenew/ on bele oozfaectien : ban bet welcke grojote febaede quam booz De Eerfobenaenen / en Dat de teltamenten niet bolbzocht en woroen: oil t'welck te remedie = ren / Luert voosfien / Dat wat Caftiliacn die coomen fal tot eenighe Strot / of plaetie ban die desleu/ Dat ly hem pzelenteere, voos de

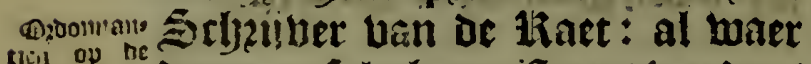

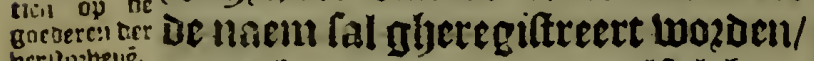
berlluibelis.

en ocek de toenaem ban alfulcken/ en ban waer bj ghebozen ig/ om Dat als by quame te. Aterien / men nitil weten twaer te vinben fullen wefen oie van yem fullen bebben te eruen. Bat de ozoinarile Infititic/ met oe outite Gouberneur/ en te Scljiijuer ban de thast lozglye Dzaglen ban oe goederen uan de 30) erfoonen die comen te ftetuen/ endar ju[r op cen 7 nuentaris ftelIen / Wooz ar Schjeituer en adjetupgisen / en oe fellulden die hu rebul Dicl) is / en wat ly hadoe in (Sont/ Silver/ Quick-Silluer / en anbere dinglyen : an datment vercoope / an

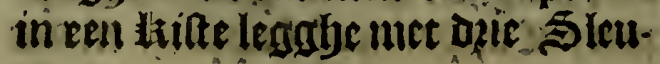
fullen bebben. Dat de goederen vertorht werden in een openbare opbeplinglye / met trouto ban die Srbeniver/ die nootfaeckelick fijnbe tot befchermingle ban die boozrepde goederen / bem itelle als 13zocuratoz. Dat de voorfepoe $7 u=$ atitic reeckeninglye nente van alle die ghene die laft bebuen ban der berttoguenen gorderen / en dat $3 \mu$ vercrighen afueerdinglye conder Lelet'el van atppal/ en Dat legghen in be thift van oe date Sleutels. Dat jebuende het teltament van oe aflijuiglye al waer lyp gyeftoz= ben jy/ en Daer de Eerfoljenamen of executeurs al daer maren ! fal de Tultitie gautlely yaer baer niet me= De bemoepen / en lyet goet niet nes men / nemenoe alleenlick oe ken= nitte / wie die jiin die Eenfgbena= meu fullen wefrn van oe verftos: bene. Dat de uoorlepoe Jultitien/ liegenten / en Sehneijuers tenden fullen aeu bet Hupg uan Contrac= tien ban Sevilicn, al yet glyene dat ju fullen Lecomen/van de goederen des afgyeitozuene/ verclarende fijn naen ent toenacm/ en woonplaetfe van elel afiglectorbene / neet de co-

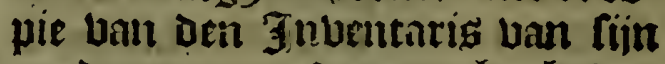
goederen / op dat ju graegbeluen weroe fiin erfighenamen/ op de ogocn/ die deg acngaende / ghegbe= uen is. Dat int nenten ban de reeckeninglye / vall dic glyene die gbeyouben hrbuen vie gororen ban Den afgljeftozuenen/ gefonden werben arn ben lyoorbften liaet ban Indien, met feer particuliere belderbeut / en reden ban alles. Dat be Zuftitien yaec Doen infermeeren met forghe/ loo de lyenders ban die goederen der afgheftozucticn beb. ben albedaen eenich bedzorly / of feljade aen de goederen die $p p$ in baec macht gljebadt helben/en fmben aen be liaet reoen oner af. Dat of befitters reeclicuinghe ghe: belt miet rétomipente / Dic voostrpoe 
Tuttitic. खatect alle Jace reectie= inuglye glyegljeben / cu gljetfoont werde acil den Gouverneur van't Sant oe nemozie ban be afglyeftoz= benen die datt Jaer gljebeet jijil en bun baer grocderen die ju bad= Detl/ op dat dic abefonoen werde naer Sevilien, en outle glyeglyeben wozoe bare Carfobenamen / en de teltamenten verbult werden / tu in die goede reeckeniugle $j \mu /$ en re Den/Dic behoozt glyebzupclit te woz= Den. Dat in elcli bof een rechte $3 p$

- van de gocieren ber afglyeltozue= nen/een uan de Hectjters / fuccede= rende b'en nae d'ander / van de

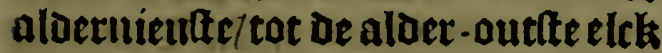
op fiin tiit dic fiin coinmulfariffen fal lenden/lancr bet reftozt om reec= kruingbe te nemen / ban die ghene oucle beblien/ en loo daer in aof= fichept is / bat oe tiectycers belaft werde oe onderfoeckinghe te boen/ en Dat 31 die opnemen/ th oock te waren als bet baelt is.

Dan vegbes Be Catljolitcte Coningen gbe. souben. informeert zijnde dat in be Indien waren vele ghetroude Caftilianen, die welelte leefoen afghefchepden van hare boutwen / Doo? welcke Joben de offenlie die bout den beere daer mede aenghedaen wert/ volgben groote itconvenienten in De bejolelinghe van die zanden/ om Dat aloner gljeen bafte woon= plaet te bebbende / dace nict ghe $=$ aumuirlylick en bletuen / noch en trachtender oni Daer boutwen/plan= ten / te teelen / te zatpen / nocli an= bere binglyen te boen / die de goede Levolckers pleghen te Doen / waer dooz de Dozpen niet en bermeer= deren als beljoost/ altt welen foude too zn leefoen unet baer Bzouten/ en Sonen / en opzechte 23ozghers; willende bet bouen g!lefeude reme= dicren/ hebben gheboden / Dat alle en wat jerfoonen batter gljevon= oen weroen / Dat gbetrout cullen siin / oft belooft in Dele Liijcken/ Dat $3 p$ lertwaetts oluercomen oin yaer Dzontwen / en bat $j^{n}$ nict we $=$ berkecren louben nae de Indiens Conder haer / of met balte bewiifiugben dat ju boot jijn. De frlfoe ozde werde ofegheben Dooz alle de liiicken/ ban de nienwe me = relt / en $3 \mathrm{p}$ is op verfelyepden rep= cen berbattet / en gbeboden datfe gljecrecutcert foude werden met ruare traffen.

Booztgaende banden jare 1000. Ban let ber.

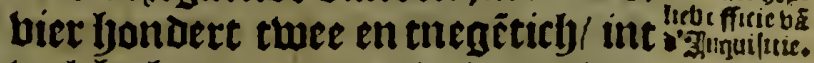
welcke begonnen werde de ontoec= Kinge ban die merelt/in bet Glyee= Atelick Eoubernentent op de weely te baenghen en te ftabilieren / glye= lijck ghefien is glyebeeft met de aldergrootlte perfectie / en glyedumrichept van dien. De Catboliicke Coninck Don Philips De twecde/ genatme de Vroede, merckende dat onder de gronte weldaden die de Indianen bebluen outfanghen / bet grootte is gheweet yaerlieder verlichtinghe om te ontfungben de

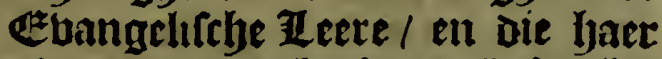
al vooztgaende beeft uptgheftreckt en gyelet fijnoe oock op de finguliere gratie die Goot glyeb?uuckt Geeft nebens baer booz fin barmbertichept / Dat ly baer gheglyeben beeft / ae kennifte van ons beplich Catholijck glyeloove / en Dat bet noodich was conderlinglye forghe te dzagben / op oe beljonoinge van de deuotie/ en reputatie ban de $\mathrm{Ca}$ ftilianen beluolckers / en bevzedighers / bie inet foo groote moepte verfozcht hebben be vermerderinglye ban de Religie / en de grontlegginge ban bet Catjoliiclie gheloobe' in welelie deelen 3 u gliednen bebluen als gbetroutwe / en Catboliicke Clyiftenen / en goede glyenatureerde / en opeecljte Caftilianen, en gbefien fyebuende / dat dic die bunten be gljeboo? faentyepdet uan bet Catholiiclie gycloove / en de zedpolicke koomflye liecrke liju/ obltinaet en bertneclistl jiin ! in yaer owaclinglyen / en Ketterijen/ 
altoas beulnten an te ver!epden de

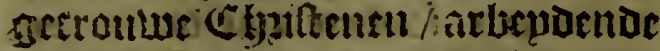
vie te bestugen tot lyate wallidye opi-

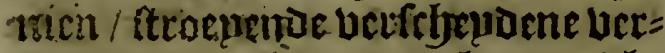
bovenc soerlieny nae hat welcke grode fehade is glycuolcht in onfe Ireptigite licligie / en bebuenoe feer - rocue atperientic/ oat de belte mio: tôt om usoozite comen ocfe: qualen/ bef act int Wechuenuen bau be com= municatie net de letterfelje coonen/Ctriffende hare dwalingen/ confoummet de difpotic uan de lyen= ligbe Callones! cn ar metten ban Die Riiicken / ae welclie Dooz die lycplige miodel dooz oc Godorlicke barnilyetticljeut bewaret fullen fijn ban Die alderfimite befmettinglye/ elt Dace wert gehoopt/Dat ju bookts bewaert fullen weroen/Dat die relt fulclien icljade nist en crijgbe/ al waer oc bevolekers ban die 2 ij $=$ ken/glyegtigeuen ljebluen coo goeden exmipel uan of Clyiffelickfiept/ $\mathrm{ct}$ de Jughejozenen baer niet bekecrt tot dwaelende Ieeringhen wan de Fetteren. Set yeeft goet glyedocht Lin Dsaieftent/ met toeltemminghe uan de Cardinael Don Diego de Efpinofa, 23ifteljop van Siguenza, Generakl $\mathbf{3}$ nquifiteur in die Liic = Ken/ren $\mathscr{Q g}$ an van groote wijf(tent/ en ban vele en rare deucljoen bp nneit waer Dooz verltiefinge ig ghe= frfyiet טã fiin perloon/ Dat lyn Coude beluen dzage den lalt uan foo gron= tetiidrtict / all Signozicn / ende de liaden van de frepligle en algbemecne 3uquifitie/ en uã bat hoochfte bande lidiens, ant in Mexico be= jocfoe giveplant te wozden ecn bof ban loct beuliglie Bfficic / booz be Aiitelien baul neuva Efpania, en of relt vau of Indiens int $\$$ Roozden/en ecn ander in or Stand de los Reyes, booz de kiifelien ban Piru, en lyaren aenthauts / die sn noetnen bet In. dicn int $\Xi$ untinen / net oe autharitept dic oe holuen van oele łaijelien / by de welclise nict nu bekent unerden de faecletr vande Indianen,
Dam alleen der Cantilianch, en ai: Dere Ratien die gevanton weraeni iil die Indiens, fll met de welelit Couach camen ic Xppellen tot de hoorbife Ract / Die ut out th of refibect / ghelijef glycoaen wert in Spangien, en tot berbullingte ban dien/lyeft int jaet 1570 . De Conint Don Philippe oe twerde/gljenoent De Vroede, een generale macju gije: gheven in de Stadt Lan Madril, op Den felthirndẽ Itotide Inqufitcurs/die genoemt foude werden als doen / en booptaens teglyens de geretiienue trouloofyept/m afbal / en dat be officiers/ en nootlacekelicke Dienaers ban dit beplich :tmpt/ gbeboorn wert haer neder te fetten in de Steben uan Mexico, en de los Reyes, dat jp oeffenen ende thebzupeken fullen Gaer \&lmpten / en Conincklicke probitien : tot bet welcke/Don Martin Enriquez, en Don Francifco de Toledo bice-2hopg/ en generale Capitepnễ inockijicken ban Nueva Efpania, en ban Piru, en de lisoben en \$ultitien / en Gouberneurg/ en andere wat 3 Berfoone dat bet zijn/ fullen betloonen alle bulp en gunff dit fenlighe Officio, en fullen ghenomt weroen Jinquifitenss/ en Officiers / ofyelijek op fiin rechte placte beter af fal gbebanoelt wozaen.

\section{1)et XXX. Capittel.}

$V$ an de forme des Gouvernemenss van de boosbfe Ract van de in. diens, en van de infersingbe der Ho. ven, en Consncklicke Cancelerien in die Contreyen.

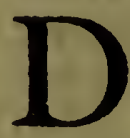

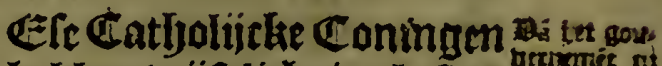
hebuen wij relick ingbettelt isudiea. Den boochlen tiaet van de Indiens, op oat 3 baer foutoen bel: pen dzagen loogrooten late alg bet 


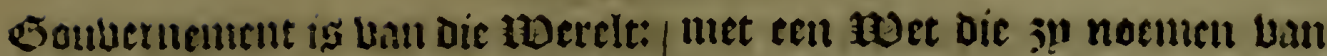

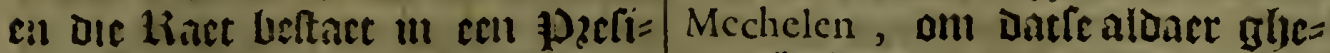
oent/ ell aclit of mere liatetlieden/

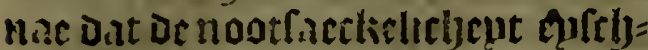

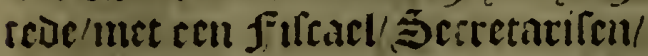

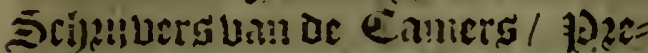
hatents / cl! audere Officters / ech Canter van reeckemungen/ al waer glieboutacil wert oe reeclienumglse

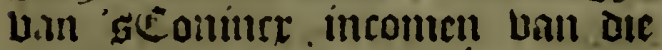
placten / ca op dat vooitghegacn conve wozach / confozm de reglyel gl

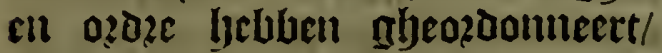
eerifelicli dat dic liaet vergadere dzic tupect alle tacelys '(ntozglyens! en twee uyzall t'fubonts/ Date da= glyen in oe weeclie/ alt gijeen beu=liglye dacly is/ en dat oe pzouifien gljeftelt werden / die betnelt fullen werden in die liiicken / norbtans Dat die glyele die Daer buten in de Indiens, fouden ueteriygijen de Ca: ninckliclie patente/ cu dat ju ull die plactle teinen fouden oe oppertte Liecht-wlegingtie / an conben mo= ghen Hetten inluecken/en Ozaon= "natien fien en cramineren alle $\mathfrak{t t a}^{2}=$ tuten/ conftituticn uan 19 zelatein/ Capittels/ en Conbenten Der łie= ligicit / en uall de Bice- Hopgs / to o = bell en Liriden / ent dat $z^{\prime \prime}$ in oc Indiens, en in Die Aiiickely/ en in face= lien dieg aengaende/ glyeljook facunt Touben siin: Dat bet Gonuerne= niciit uail de Indicns $3 \mathrm{p} /$ als ljet van Defe liticken / en dat meer ut par= ticulier / dan in ceniglye andere raeken befoingmeren oc liact in faccken La!t't Satuernement / en dat de pzocedten gheremitteert aen díc bau ce Counelklicke Rinet/ ful= Ien comen glyeuen hace ftemmen aell die ban de Indiens, en Dat jul bait die twee ftenmmen fententie glyeuen op. De pjaceften minder Dan ban unf lyandert pefan : Dat de tuecde fupplicatie 3 u ban thien Duurent pele!!. Batter nict bekent wout reparatic ban Indiens, in dien Deelé/Dan alleen ie gefuluntancicer=

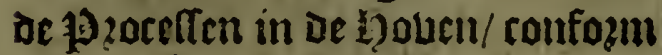
maeclit is / dat bie comen tot deit loochiften thact. Oe abdes Diemien yonoch fal in of infozmation var De bedirninglien / dat oe gautfebe Liact Die lie/ ent int ltuck ban de be= loomunlje glyefrlicoc toljene dat oe mesite decl fal determumeren/en dat in die ju de fupplicatic / en Dat gljech gecrpediecror de derde reure gljefien werde / en dat in faecken met alle coattjept gerefolbert luex = De / dat oe laiten gljegumt werden dien diele beft ueroient hebben / cut datfe niet gljeglyeluen werden die berbondenen / of vienden ball de linet: cul dat dic nict en fullen co= men Sollicietcutg / noch psocn= tentsjun / ch dat in of pzobifien van de Officicu geen loon tuffetyen come: en dat die van de liaet geen Indianen bu verdeulinglye en frebs Len / cn dic oplyouden in bare lous: [en/ onl dat $y^{\prime \prime}$ in die die binden Couiden die negatieren / als jp nict te ract clu gacn/en dat in alleg gboc warfyt werde het tecreet ghedenlt/ cul bouen al / Dat de Ranet Tonder= lingh forglye dzaghen wan de beker= ringbe ell goede onderbuifinge ber Indianen, en ban $\mathfrak{y e t}$ Gljeeltelick goubernement/en Dat ban res fon = Dert duulent $\mathfrak{A g}$ arabidífen neder. waert/comic bet ztppel tot De Haet/ dat gleappelleett can weroen ban oe Cententien in oe bijf glyeuallen van de nattmeslicke doot / of beroo= bingfie ban leden / of andere lichas= melickeltraffe openbaere befegaes minglye of pijninglye/ en dat de appellen fullen comen tot de raet/met andere bele en priiclicke Osoonnantien / Die oin de coztlycut nagbegficlaten mozoen.

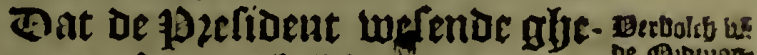
lcert een ftenulue ljeblue in de faeclien van goubernemét/ ban gunltel ban bedaunckinghen/ bifien cur refiDenticu/ cn niet in pleptch / op Dat bu macly meer bag fijn tot bet gou- 


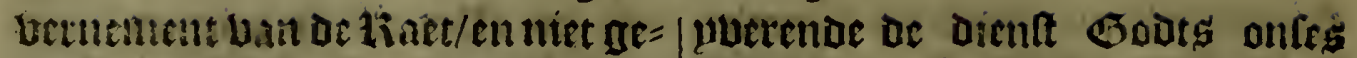
fert finitue / laet lyu glyecu ftemme bebba: / beinlben in faeclien ball ganite / Entuementente / en be= dancliunghe/ en Dat by cau de thact

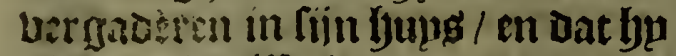
glyelycugyenifte boude ban de ne= goten/ cat dat dic vall be tiaet baer met aecomyagneerem met oe nego= thanten / ch daterom frlyint het een Hoa:fiacticlicke liache te lim oat

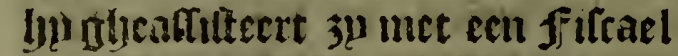
in oe liace / Datter glyeboven wert Dat lus lyet felfoe lalaris lyebbe oat de liaetfiecten bebuen/en dat gije= tevert werden oe oelpartog van bet Oficio, dat ly foeglye dzaglye om te weten ljoe dat uerbult werden de Heguifie doo? de Indien, dat hem glyegeben werden de papieren die noodich ziin tot fiin stimpt/ Dat bp arvifien fie / al eer ac lhaet die letet: cat bu een Doecli belobe in ae wele= lie oe capitulaticn ttaen/ oie ghe= monten fullen weroen ntet de $\mathbb{C}=$ minck / cen ander in de welcke deg fificacls plepten fullen itaen/ Dat hu oc plepten nict untftelle/dat fiin brebeetren/ of die yaer teghens die fullen itellen giveaomitteert woz= oen / foo lyet oe liaet fal believen: bat lyn 23 octiolyoude/uan bet ghene dat ghedepefereert fal werden ban te faccken/ Dat ly fozgle ozaglye te weten belcte Officters/nalaten te fenden oe relatie alle Jaeren aen De traet.

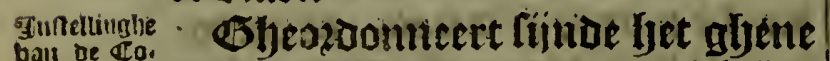

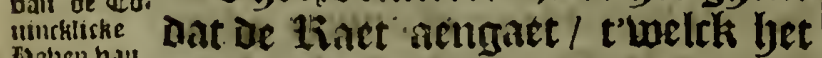
hasuen ball
Endien.

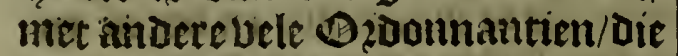
nitet berlinelt werden om de cort= Ijet wille moeten gifeozoonneert werden in alle ae 1 \$obincien ban Indien, oc lacileen ban \$tuttitie/ ofeclick als be nootlaeckelickbent

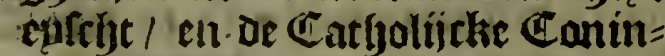
dilen betacme / tot het glyemeene belte ban dienieute Ferelt / op Dat haer Onderfaten die $\mathbf{Y}$ ultitie fullen beglyeeren/te bererijoben/ lgeress / weltant ende yzofiut / cu coulancment bimbe uoo?lepar on= aerfaten / en tot viedoc , en gherult= bept una oc volcketen/ gljelijck de Coninckaen Sodt is verbonorn/ cn aen baer / ont te berbullen met lyaer bet Anut dat fye op oer Ater = ben Dzaecht : beeft glyeaccozoecrt loven te Itellen / en Conincls!lclie Cancelerij / bie ghelept ziju datter in Indien zijn/nuet de ltatuluten ende ozoonmantien die glyegheben jiln/ op dat de Ditnaers haer ampe fou= oen Doen/en de glyerechtichept / of Fultitie wel bedient $\mathbf{j u}$ / ent dat oe lupaen betomen de lueneficie die ban baer glycpketendecet wozt.

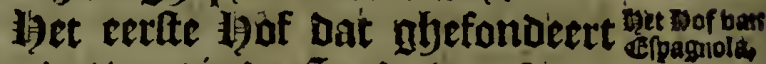
worde/ was in de Stadt van Sànto Domingo, in het eplantst Efpagnola met en geleerde jozefident/al boe wel dat bu mu om d'oozlochs wille en Soldaet is / met oe tijtel ban Capitepn General $/ \mathrm{en}$ vier Hechers/die thoeden dzaghen/alg Alcaldes; en draghen kennis ban bet 25 orgherlick/ en 'terimineel/in taet van zlppel / èn in de eertte int tantie in Caecken van't l)of: en bet goubernement met fijn beftrite is bevolen de 13 zefidont alleen bie nti $1 \mathfrak{s}$ Antonio Oforio.

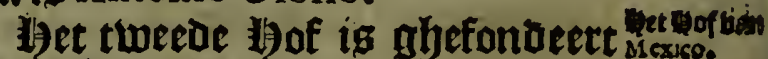
in oe Stabt ban Mexico, in nueva Efpania: De eerlte 10 zeft toent die daet lwag / wag Nunjo de Guzman, bu badoe glyeen acnfien.

In dat tweede l) of mas booz jozeribent dezsiffryop D. Sebaftiaen Ramirez, die Doen was in het gof ban Efpagnola en lyadoe het gouberne= ment ban die zijeken/en de hoorlyIte autboatent / en liet oe faecken in goedeftant / met bet glyene dat baer toe behoogt/ en be Intetitie/ gljeliick die teghenwoozdelick is: De lozerident ban dit hof ig de bire-tiop die nu is de Conde ban Monterrey, hectt acht haetffeeren/ die Kenniffe bzaglyen ban $250 z g y e t-$ lijefie 


\section{Ualt VVeft-Indien.}

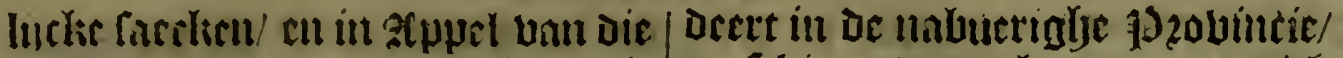
bant gouturancment theleli de $\mathrm{D} i=$

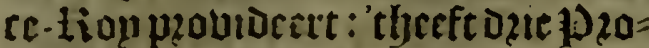

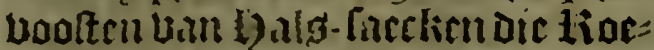

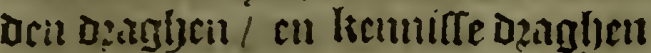
bin crimuncle faeclien/on tuee fif if:

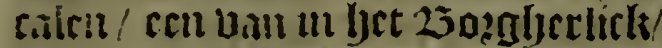
D'anter in lyet crimumed cat Lerden bogefien de recljt-plegmalyen Die ture bewaret wozach joo? be $\mathfrak{C a}=$ mincli' cul or andere officien/ ch be $=$ fulpunglyen bain ailcolten in qui= tecten / cit bacatien we refort bail Dat bof ban Mexico, ch in dat ban neuva Galicia. cu [eljunchoc dat lyet met noodicly was/ berlicp lict / $\mathrm{cm}$ int Tacr aun= cent biif bonoret tecuctitidy berer Dender wedergljelieert oul te fon= Deren un de \$tadt Sant-jago, ban't Liijcli biun Guatemala, Daner ig cen 1) zeliachit / 1 welclt is oe Doctoz Crado Uanl Caftilla, vier liacts. Herten met hoeden/en ech fifeacl dic licnuts Dzaglien ban bet $2 \lessgtr 0 z=$ bijerlick en 'termunecl / in attplicl cu in de certte inftantic : in fareclien

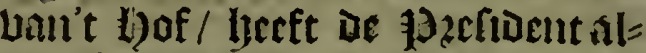
Icen bet Goubernement / al 'tbe= grtuen ban oe Indiens : Iju befelyiclit de recljt-pleginglyen/ cn andere tij= Deliclic Bfficion.

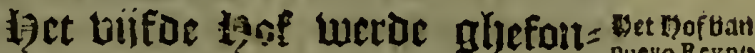
deert in de Stadt valn Santa Fc de Bogata, it lyet nuevo Reyno de Gra-

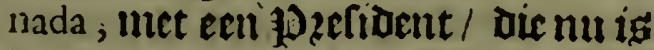
De Doctoz Francifoo de Sande, Luier fiactg-l)etreil met hiocden/en een filfael met de lelfoe autlyogitept ałt de vouzgaende.

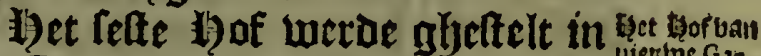
oe 5 tadt ban Guadalajarn, wain het licier. nictue taijck van Galicien, met

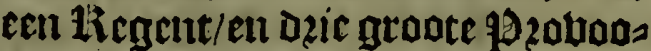
Iten/ en defpaclyeerden langlyen tijt [ondacr 5 eghel / ent tiegilter/ en de negatien waffende werder ljet $5 e=$ gljel gljeglyeben / en 'tliegifter / en Daer werde esn 1)zeliaent glyeftelt/ Die nu is de Doctoz Sant-jago de Vera, tul duie liaets-lyeeren met de thoede/ en cen fiffacl/ en oc Bicetiop ban nucva Efpania ljecft lyet goubernement.

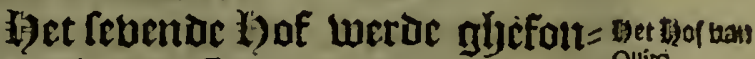
Deelt in ar Striat $S^{t}$. Francifco del Quito. Quito, uan de J)toluincien ban $\mathrm{Pi}$ ru, al waner oock ent liegent was/ en hooglie pozounotten/ fonder $5 e=$ oljel / en daer naer torcde liet bofbefet met eet pzefident oat nu is de Zientiact Migucl de Yvarra, met Daic liechters met oe liarde/ en een fif ifacl unet de felfoe macht als die yall Guadalajara dic oljeluetide lyet

het vierde bof werde glyston= $R_{2}$ goubers 
Ifol:oo.

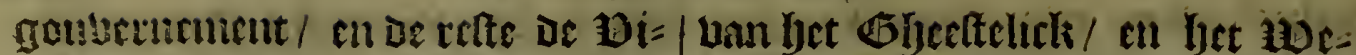
ce lisuluall Piru, als bouen gye= โคนtเ

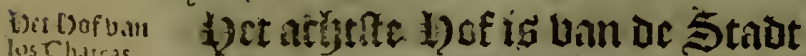
de la Plata, ill ae pzoumcie uan los Charcas, ntet ecu liegent/ en groo= te pzousuften / ch daer naer twer = Der rem bzeniont ghe?telt / en vier lieclytersinet liocuen/ een ffifael/ cru 5eglyel/ al cen liegifter/ mu is gorfiont oe licentiact Cepeda, met referbatic ball de jozobifie ban lieilyeuingljen/ en de relte tot de Dice-liou uan Piru.

Det befbam l)et neglyenoc l) of is ant alyefon=

13nagn, Dreet is in oe Struat uan Panama, met cen 19?efident / met een 99 an = trl: en cen liapicr / om Oozlochgfirecken wille/die n! i\$ Don Alonfo de Sotomayor, met ae Ciitel Capi= tcun generael bau fyet bafte lant/ 'djeeft daie Hiecljterg met tioeden/ Die kemuife daaglien op de atppel= Ien ban 230 afyerlicke queltien / en Lan criminclen/ cu ter eerlter ill= Itantie van queltien ban't 1 of / bp yeeft allecn het goubernement.

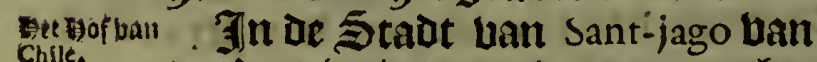
ae 13 zobintie ban Chile werde glye= fondect het thicnde ligof / en om dat fyet fefyeen dat lyet niet noodicl wasa/ too berliep het/ en daer Lerde beltelt een Gouluerneur die aen de Dice-Lion bain Piru hinck.

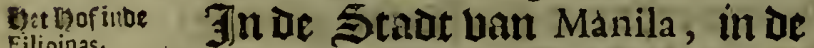
Eilipinas. Filipinas was ect lof / en lyet ber = Dueen / an dat het febeen dat het niet noodicly was/ tis Lemuich ia= ren gheleaen / Dat wedorghelieert wozae oun dat te herboulwen met en Capiteun Genctael/ welclitis Don Pedro de Acunja, aie pozeli= Dent is / en bier liecljeers / en een frifeacl/ met oe felfoe authozitent als ac anvere bouen $/$ om dat de Catyoliiclie Coninglyen bar Caftilien met lyet aduiis ban de booglye tiaet bau Indien, berogghen altoog met een opzerlyt an rechtbeerdity herte 'tgljene dat bequacm is tot befantocniffe : an bermeeraeringbe reltlick ban dic deelen/fonoer te let= ten op colten/ of auluept : en elck bof is confozm lyet ghelbetueli uan oef Liiiclen / val Sclyuluerster Ca= meren/lielateurs/ Bookwarturts/ Bargeantch/en Officiers dic nog= duch ziju.

\section{and
den is}

Wet XXX I. Capittel.

ande faeckè die voor fiè uvorden in de boochfte Raet van de Indiens, met Raet van de Cosinghen. authoxitept afyegheven is die ghes noeclyfaem is naee reden ban ftaet! alsnae de reputatie ban be 㐓ulti= tie gijedocht beeft te betamen / beyouathae booz ds honghe Obetbeut tglyene bat gljeoozaeelt wert nootlaceltelick te fijn tot lyare authoziteut / Daerom booz de Bice. liops / en poefioenten / op dat 314 Couden voldoen die gbene die't wel uerdient yebben/ en $3 p$ meerder gerefpecteert fouden jiin/ zijn haet untaljerondert Bfficien die ju conuen probideren/en dinghen dact $3^{u}$ in connen baientichap doen / en tot prouilie van de conincklicke $13 e r$ foon / met raet wan den lyoochltent tiact wan Indien jijn gebleben defé nabolghende officicn.

Int Coninckrijck ban Chile De goutber

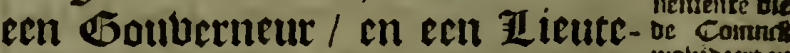
nant die gijeleett is / met mach)t te se Indicos, conmuahueren oluer d'Indianen: andere in Tucaman, met de lelfue macht : cen ander boo? de \$3zobincien uan del rio de ia Plata, vooz Popayan, Santa Marta , Cartagena, en Veragua, met fijt Gouluerneur ecn yghelick met aefelfoe macht. In De Jjobincien unn Nicaragua, en Coftarica efll. $\mathfrak{Z}$ nl het $\mathbb{E}$ plant 


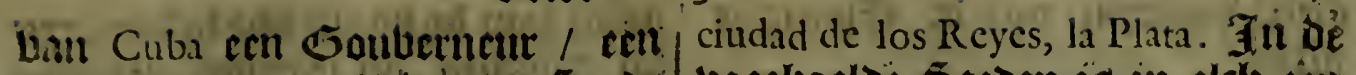
Copitem generacl die in oe Stadt u.uli Se. Chrittoval del Avana reli= Deret Daer jait meer goubermenten=

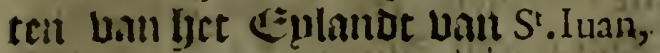
uall Puertorico, Veneztiela, Soconufco, Yucatan, Cózuncl, en Tabafco, die tramen èn fouberné nicut unaclien / unet oe authostept uan d'Indianen te Legfieben. 't $\mathbf{3}$ ifil oack beglycuingfjen ban fijn $19 a=$

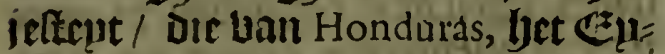
lant Margarita, la Florida, la nueva Vizcaya, el Dorado, los del nuevo Rcyno de Leon, en die uan Pacamoros; Yguallongo, die fiun ghtaup rende bet leven en get felfoe in de 9) obuincicn ban Choco, Quixos, ent. la Canela, of equanoen uan Salamon, Santa Criz de la'Sicria; ell lyet. latette ig/ Dat ban nieu Andalufia:

sare terbeple' gingben bie yrobloectr.

Dock wereden oete nabolghen = ae reclje-pleginglyen gljeptobidect ban of Coninek. Ue Cuzco, ae Stadt uni la Plata, en de fitplaetré uau or agijnen ban Pótoffi, en de Psobincie Uan Chicuito, of Andes van Cuzco, ố Stadt vani Truxillo, Arequipa, Sant-jago biul Gnayaquil, Guamanga, De Stadt vait la Paz, Chiquiabo, $S^{\circ}$. Iuan de la Frontera, Leon de Guanuco, puerto Viejo, Zamora, oc bevolekingle uan oe Minas de los Zacatccas, In nueva Galicia, Cuenca, Loxa, Tunia, de $\mathbf{S t n d t}$ Unil Mcxico, De Staut uan los Reyes, De Dzoumeic uan Nicoya. De alder grootlfe lpzowoolt-leljap= pen jill die van de 5 tadot ban Salvador, ban oe jo zounincic uan Gua. tcmala, enl lyet binuentete ban yet Eulantat Efpagnola, Nombre de Dios, oe Stridt uan Chuluteca, de B? ouincie Uan Chiapa, Zapotitlan, de Sctidt ban Nata, Santa Maria de. la Viroria ult Tabafco, en De groottte Drarwarerset-frisuppen siin dic/ in de \$tadt baln $S^{t}$. Doniningo in Mexico, in Guadalajaro, Sant-jago de Guatemala, Panama, Santa Fe ide Logota, St.Francifco dẹl Quito, la vootjaclac Steden is in clck ren cen aroote Jerurwartock / dic eell ftem hecft in oe Raet als tiegeut! chl de voosnoembe il isutenanten! tot glyebanek unu ajn ztupt $/$ ent in elcli lof is een ander grooti Jieurwaetder net 'matjit ont té nocmen twet andore zlicutenantô. In yet goubernetment ban ' $\mathrm{CC}_{0}=$ mine incomen weroen urefien bai liiil gsajcltept : met aduijs uan beit boocljten thate van die van Indien, beil Officiers/ finctotisl Crelozicrs/ Recelienmecters / at Conmuttáriften / die alle $230 i g l y e$ ftellen in Cattilien, en in Indien; binn goede en tronbe bedieninglyel en om dar oefe nicute republijelie alfoo yoost gincls in vernieeroe = ringlye / becft liet glyelicft te ber= unlien / tot dientt ban Gode / in des conínte \% dien neer te ber= coelen en "tautljoziferen / met lyet Itellen bait twee Oice-liopl\$/ ren in nueva:Erpania, en een amber in oe kiijcken van Piru, op dat $3 \mu$ ii de naent lan of Coninel gouluer tieren / en provideren fouben de faecken to concerneeren tot beit Dienft Soots/ en Des Coninct / en tot belectingye en onderwiifighe ban de lidianen, foo oock tot on= oerboudinglise / gheouericlsept / bes volekingtye / an veredelinghe van

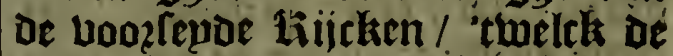
onderbindinglye ljeeft ghethoont/ dat betamelick en bondicly ig gbes weeft / welcke Dice-liopen gheglje= uen wozoen leer particuliere in = Itructien van het vookfyaelde / en op dat $3 p$ in befflyermingye coriden Ijouden ljet beplich Officium batt D' Inquifitie / en net haer ftercke macht Conden bercljutten en beicljerinen / om dat bet is confoim/ cen llacektent owangly / en of fuebere / en Lare bewaringlje dan de Gljectelicke en mereltlicke taet/ t'welek is of belte en alaer-waer. rachticlyte reghel ban lact / ent A 3 nuecti 
meti coufozill met ide Elanulyelifrije.

mant ne Du Cock wert De Bice-tiouts/ cul

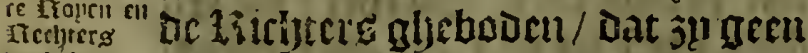
urrheden uertict.

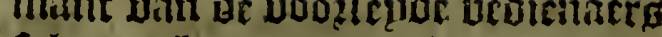
fal moglien op-legofyen canghe Sebntemglye of andere vinglyen/ nocly dat tjes glyedient werde ban de Indianen, tell 3 uie betalende. Bat aljeen ziduotact / 5 clyziluer/ noch Ticlatcur toone mi huls van

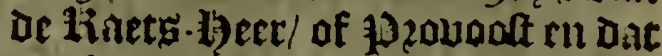
De jolepters nict dichen de tieclyters. Dat de liacts. Hecten ban. Panama, hict naer laten te uerghe[elfrlyappen de Cooplicoch /ch geen plaetle gbeven die gluene die bare Dooulen accompagucerch. Eet bat glyen liacts-beren luan alle de Hoben / houben uecl conmunuicatie met de jolepters / zubuocaten/ \$ozocuccurg / en dat in binnculfe ban't bof niet ghegacn wert / tot ondertrou / begravingfe / of trouwinglyen/, ten ju dat bet een nootCaeckelicke faecke $\mathfrak{j} \mathrm{p} / \mathrm{en}$ dat $\mathrm{j}^{\mathrm{p}} \mathrm{geen}$ b) untoectringite op oe 230 ger maglyen anen op eenighe laecke. Bat 30 haer nict ftellen in factken ban De kepubliicke I bat oork glyeen Znaets-l)eer/ noch ecnich antoer beDienaer yin't lof Daer twee officien yebbe. ên bootts foo ill anaree vele Oroonmantien en goede TOetten / die aljiin Itreckende tor

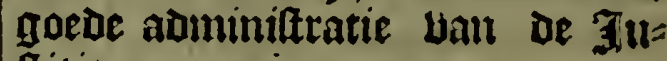
ifitic.

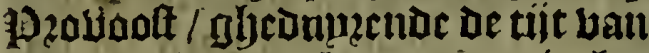
(iili officie / madb tromben in lyet refloat uan fiin $\mathbf{3}$ urifuictie. Dat

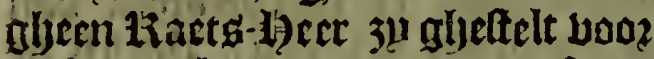
recht-plegljer: end dat de dooderpoe

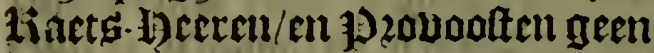
lalt dzaghen uan't glyene 3 te doen beturen in abrentic ban yare Offi-

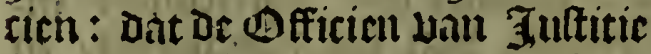
niet gepzodideert werden/aen lyaer Solnen / of Dochters-29alts/ of frloon-23raciocrs / of 5 waglyers bau de pefiuenten / Lỉatts l)eeren fifralcn/nocly aen de Officicrs ban' 'tliof/ of yan oc Ontfangljers ban' 'sctoniner incoumen / Wecl min aen lyacr Dienaers/ of Dic acn fyaer uerboden zijn: en 'trelfoe locet de

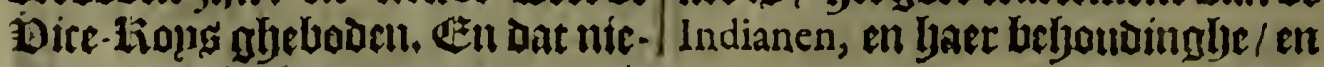

1) X X XII. Capittel.

Vervolćn van bet goede Gous: vernement van Indien.

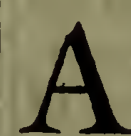

Xloo Defe Catliolijelie $\mathbb{C}_{\text {a }}$ niugben nict fycblen naergelaten cenicly dincli twelel: ju nict woorficljtelick woosfien fyelsben Dat confoem lang lyare plicht: De eerlfe laeclte dic $3 \mathfrak{y}$ ajeltoden laner Bice-liopg / cn alle be- Bienaers int algyenteen / an particulier is / get goet tractement ban de Dever: 
Se verumllinglye ban dic ozdonman= tien Die ju dire op ghemaecke beb= ben/om te Atraffen uiter groote ften= gichent oc ouertreders / en alloo de Indianen Ieeren de Cáftiliaenfelie po = litie/ cul bae: beclicly connen Doen/ ell Leten in wat farcisen fact ober = lalt glyerebict / oul baer grooter fo= lagentent iffer boosficn/Datter geen plact $3 u$ / in De we lelie in is plep= tell tuffelyen de Indianen en yacr oz=

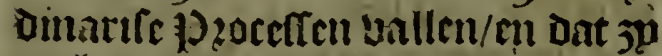
oock tuiet lunck rijt / gbelijck bet placly te gisebeuren doo? de boof= ycut uan ecntglye sidvocaten / cn

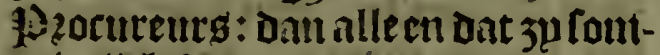
nicrlick fullen gedetermineett fiju/ boudende lyare ghebaupeken en co= ftuminen / miet finde tiennelick on =

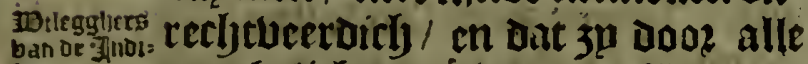

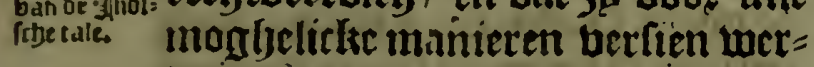

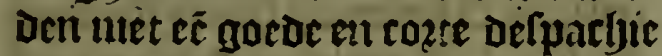
ual dien. En ghebeuren de Datmen wift dat in or upelegginglye van de Indiaenlebe talen eenicls bedzoch was om alles boos te comer / wozt gheozdonneert / dat die uptleggin= glie ghedaen wo:de Dop? twee upt leggliers/om gheen vergaderinghe temaecken op oe uptlesgingte ban bet Indifch : en aat te uoozen eer $j^{\mu}$ ontfanghen fullen zijn tot het ghe= beupcli ban bet :tnupt/ oat baet af= glyenomen luerde de eet van trou= we bedicninglye / en dat 5 n uiet ne= menfullen ecuiglie giften ban de pleptende Indianen, noch uan an= Dete die het fullen connen welen. Dat $j \mu$ aftitteren fullen oe accoz= ben / De Wouen / en de vifiten van oeglyebanckenilte. Dat $3 p$ de Indianen niet berbooten in baer hupg Dan dic olyente die 34 vooz't hoff bengluen. Dat oe untlegebers gecu Sollicitateurs of pzocurents Lan De Indiancn fullen siju / Dat $3 \mathrm{y}$ ban baer nict begleeren. En tot he: groostte uoozoeel uan haer is bettele/oat de fifeacl bande tyooch $=$ Iten liaet ban Indien, $3 \mu$ betcber $=$ uter ban oe Indianen, en baer de= fenderc in lyaer plenten / al begecte in De kiaet alle bet gljene bat noo= Dicls is/tot baer leere/belyoudinglyel en politijels Icuen / en till jtinder p) zotecteuts giljecolent in oc litije= lich ban Píru, en nueva Épania, met misule ogsonthanticn/tot bact bet thaictentent.

Daer is oock gryeuoben / Dat 5rboulcugheltelt wozden ban de Caftiliaenche tale / Dat de Indianen die Iecren / cll Dat 3!l liet Doen van jonex af / en Dat ghern vagabont Caftiliacri leere / nocly $3 p$ in oc oo $=$ uet ban de Indianen, en daer oocle bimuen cri come : foo contratte glie= feljiet/ fillen de Bite- Tiops/cn l)o = uen alle neerfichelyot Doen ifut te tocpen affulctie tune lyet zant / die affelsepcriende nact Caftilien, eti Dat de'Meftizen aljedwongljen ful= Ien siin te biench en te leeren oe dicniten. Bock if glyeojoonnect alle jozelnten / ald oock alle BiceLoug lisoue//5ouluerneurs/ hechtpleghers / en alle andere boedani= ghe Jultitien lyet siin / Dat $3 n$ lon= Derlinglye coaglye fullen Dzaghen/ dat ju baer Devoir boen om booz te comen / dat opljouden die verttooringlie die zu onfen iscere acnighedaen feblen / int trouwen bam de jonglye frinderen tip ie lindianen: conder aenfien ban ofudetiom/ dat ên Cazique niet meer ball ế troumen fal/ al boe wel jp angheloovicls $3 p$ : te beletten dat alder een Cazique fterft / Dat 3 Dan een auoer Doot flaen / om die te begraben net dien/en andere alfulcke grouwelicke mifbzupclken. Jat be Indianen toeghelaten werde dat $3 p$ moghen doen dat $3 \mu$ willen / alg Waije portoonen / en vasu ban alle foozten uan arbeut / al yoe wel dat bettelt moet berden dat $3 \mu$ arbep-

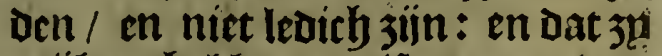
vaijtyept bebben te dínoneren vait haer incomen / baenglyende ecrlteliclt in oe openbare uut-keep oertich dagben lyaer Eerfooederen/ en 
yaer racendide gocderen neglyen da= glyen/ Dare is Thefititic teglyenwooz-

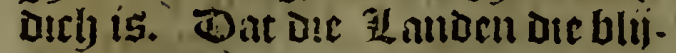
Uen fullen bam Di ifglieftozuene Indianen ab inteltato, fullen blijuen bu de Dozpen van de welcke $3 \mu$ obcbun!en jijn : Dat ju uaijelick fullen yaer Cooplyandel deziiben/en lyaer uercoopen baere Coopmanregappen. Enat baer goede ghebzuprlien en coftumment glyepzeŕn werden. Oat ju mogljen fenden in de kiirlisn Indirche jogecureurs booz dzic Jaren. Wat de refidenrien vercondicht wetdé in de plaetfen ban de Indianen, om te fien of ceniglje begherren bet reclit te currchen. Dat in cleli Indifch Dozp sll cen Galtbung / met nootfaeclielicke peouifie.

Datter geen Eslabé fullen ij,

Jn yet ltucli bande Indifche 51 laven / wat in orn beginne ban de antorekingben verfrisepdene meeniughen en ogdonnantien/goubernerende confozm foo de tact ban Taecken eprthtede / nocbtans nae Dat ald pozefident van nueva Hif- pania quam de 23 iftchop Don Sebaftiaen Ramirez uerde abroluutelick onthaglyen Dat glyeluzuck/ niet belettende dat oude dat de Indianen hadoen/ D'een Den allder tot Slatien te maecken: Dat jp oock ban ljacr nict uroglyen gecocht wozden/ noch die ontfanglyen/Dat oock gheen 1 serfoom mach bzenghen in Dele Lijiclien cenicly Indiaen onoer de ciitel ban Slacf $/$ al boe bel dat lyu glyenomen wert in de recht= veerdiglyen ooflocl : en daer op 3iin gheglyeben foo ftricte wetern/ en ofoonnantien / die puntuelick fullen glyehouden werden / en bock Datter niet fullen ziin in blyecnen

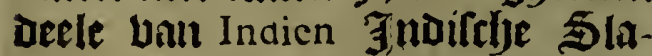
ven / al waer bet roo dat $3 p$ waren ban Lumten de tococulingye ban Caftilien en Icon. $\mathbb{E}^{\mathfrak{n}}$ am meer Looz te comen Dit inconvenient / ift verboorn de Indianen in Defe Contreuten te bzenglyen/ onder wat pze- text of oodarcke yat ju.

En allon boven al moodicty is ofouman.

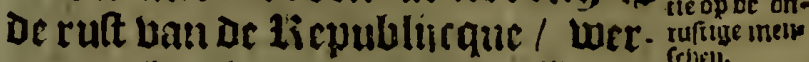
ter macht gyegeuen De Dice Aiopg/ p)efidenten en bouberneurg / $\mathfrak{c n}$ andere Fuftiticu / Dat $3 p$ monyen upt De Indiens wospen / cul tult bet ILant Deiiven die profoonen Die yaer fullea frbiinen onruftich / en die nae defe tijiclien toe te [endari/ oozbeclende dat lyet alfoo noeniey is om de rult vall lyaer / noctytans Dat lyet niet en $j$ ! dooz lyate of paffie / noch om Diergbeliclit andere redente. Ell op Datter ecmiglye toom jy in die oberlte / wert ljacr glycluoDen dat $j !$ nienant beletten lyet feljziiven aen of Coninck / of aen fijn tiate / of aen andere berloo. nen / 'tgliene ju! willen fullen / Dat 3e glyect paacketten nemen / noch cenighe 23zicuen op cware Itraffen/ en dat jure toclaten gacu m $c 0=$ men dooz't gantiche lant tot dic gljene dic $3 p$ fullen begerten/ fchic= Fende datter weglien en 23zugraen gljemackt wozoen op die plaetfe Dactle niet en ziin. \$oo beel die faecken ban D'Oozloghe aen gate / ift oock befryiclit met groote fozglye/ op dat oe biceTopg louden ronnen ziin in groo= ter authogitept / Dat haer glyeglye= ven werde maefte om wacht te hou= Den te voet en te joaert: te inaec= Gen Giet-buten ban frtillerijel en Coeghels/en Xtmununitie-13un= fen/ bolcli te lichten/ Schepent'ar= meeren / en fortificatien te maec= ken / en be relte te boozfien / Dat noodich ig tot Dic Hijcken : $\mathfrak{m}$ glyelijelumen moet booz-comen de ouerlalten aer §oldaten / Coo bel op't lant/ alg ter $\approx$ ee/ en die glyene die op de Blooten gaen/ is abe= Dzdonneert wien dat de ftraffe ball dien fal toebeljoozen / onl unt te boeren de colmpetentien ban Dit liecht.

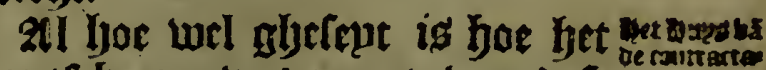
gantfrbe gouberthement bar defe te vam ses 


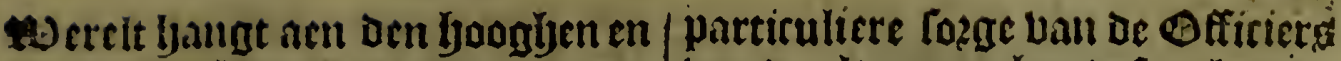
Conincliliclien liact uan Indien, Die baer relidentic beeft ontrent de Conincliticke perfoon / noebtans alfoo moodich is / Dat de executien ball Die plact fen cozrefpondentie boudorn niet die/en oock datter biet 3iin Dif berfozglien om t'effectuie= ren tobene dat bebookt tot conler $=$ batic ban't gisene aldaer / is noo = dich glyewerft te Itellen in sevillien een Conincklick bups ban b:are contrachatic / al wher coegle obediaget wert booz alle de Coop= bandelinglje ban Indien, en dat = men Der neet anders in beficly $3 \mathrm{p}$ / Dan op de beftellingle uan dien Ifandel/en dieg aengaende. Son= ber Dat glseen perloon/ noeli alufi= tie / bent menglye onder de raecken die die negotien aen gaen van Indien,en bet is in Der baet een Hier= febaer van groote authozitept / in oe weleke een pletident is / Dat nu is Don Barnardino Delgadillo de Avellaneda， een Kiceclienmeetter/ cen Crelozicr/ een fiactoos/ ozie gbeleetue tiechteers/ een fitcael/

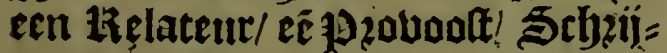
bers/ een Deurwaerder / een Calls celier/en andere officiers. In be Eplanden van Tenerife, en la Palma fullen geltelt werden twee gije Irerde Ziechters / die ju Conintr Bfficieren noentē/ of tiechters van de tiegifters / om dat ju doen lou= Den de Opdonnantien die gljeglye= uen jiin booz yet cargazon, ent ke= gitters ban Die Enlanden/ en be= feplinglie van dat baer-macter. bet loups uan Contratacion lyeeft Gare inftructie/ en ozoonnantien/ boe dat bet gljegounerneert fal wozden/ en oeffenen lyare $\mathbf{3}$ urif= bictie / en de gheleerde 1eeclyters weten oock wan baer glyebzupek en oeffeninglye / houdende in bet opfien ban de jlenten tuftehen partict/ de ozdinantie die gljebou= ben wert in de bouen van Valla. dolid, en Granada, en bierom is De ban dat f)ups op bet defpaclyeren bail de Dlotell an Atrmaden / dat 3u uýt-loopen op verfelycpoen tij= Denl / dat $\jmath^{\mu}$ baet in befoignecreh met De aldermerfec ineerltichejt/ en te ontfangijen die glyene die daci comen/ cin legghen in verfectie= ringhe het Gout / t'Silver / Baecr= len en andere dinglien die comen! met onderfryept vann gyeluicht / en Wet : lalt aljeluende ban al 'tofyent

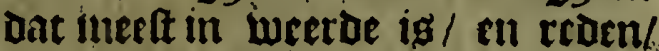
en ont provifien te doen ban de Blooten en Ftruaden. Soo goede ogsonnantien zijnaer gljeghcben/ op dait de Dienaers niet en louden te buuten gaen en de Onbetdanen gljecn rüarichept en crijghen/ bat alles paffere ljeel confozm de in= tentie ban de Godtburbtighe en Catbolijeke Coninglyen/met bulp yan de groote neerlfichende ban ofe gooctjote likat baul Indien.

En om dat alfoo feer bermeer = Inferustio oert zijn de negotien ban Indien, ball be Ge dat de joochlte kiat die niet can Defpacheeren met een cozthept die bequaem is tot lyet goede goubers nement ban dien merelt $/$ en toc Dienlf ban de negotianten/met lota ghe Uan or 13 efident / en den yoos: ghe kaet : too beeft de Coninck Don Filippe de derde onle loerel nabolglyende fiin Catholijcke en Bodtuzuchtiglye voozganghers/tot grooter welbaren ban fijn toalla= len/ ingljeftelt een Camer-thaet/al waer bebetticht en ghederpacheert werden alle negotien ban Gyees. Atelicke provifien. / en wereltlickie gratien/ en Dantken: en boué dien/ yebluen $3 p$ inghettelt twee Salen/ al wace op reeckere dagljen ghe= yandeit fal wozoen van materie

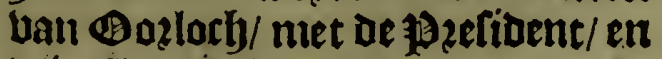
die latats.lieden van Indien, $\mathfrak{e n}$ twee of dzie ban de thaet ban oozs. loch / en op anbere daghen/ van de Jandel ban des Conincx inconten/ bu de bzefident en kats-19eren 


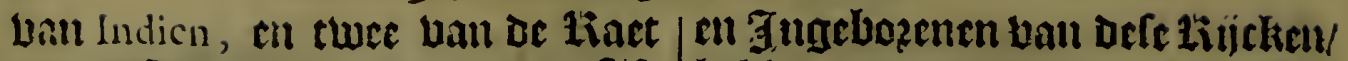
bant geì oniner incomen/ ch de ffifcarl/ cll de Scereraris ban de Het Uall Indien, noentende ten gozeltDent die gijene die ljact fal glyelicusu.

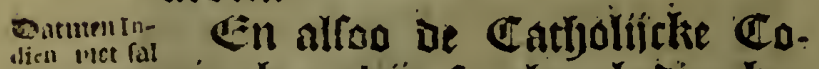

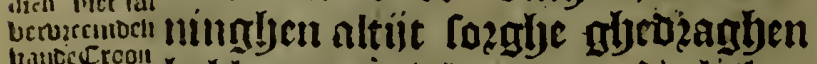
banspangici If loben oull wel te doen of lieden

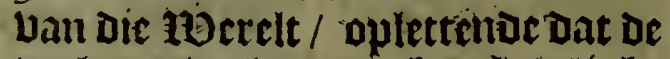
verberyoinghe van bet tefulighe cLangelium/in geenen wijle voozfpocdiger foude connen booktgaen Dau book bare handen / nocly ftreelien tat yaer lietyoubinglye/ en om oe conqueltecraers / en bebolcliers van die deelen te voldoen! nae dien su allegader fiin fijne onderfaten//

Jebien $3 \mu$ uerclaert bu ljaer $C_{0}$ ninckliclic pzovilien/gibrgeben unt jaer buplent buf bolloert twintich/ ill Valladolid, en int jaer Duplent vijf honorert ozic en twintich in Pamplona, Dat hare 29 ajeftepten noch ceniglye ban bare Erfgtjenamen/ te gheenen tijot fullen berJeenioen wan de Conincklijeke Croon uan Caftilien en Leon, de Eplanden en 19zobincien ban lndien, nocb Jozpen / nocf ernich Deel ban Dien/ en beblen dat oork belooft en gherept. met haer Coninckliicke woozden: 


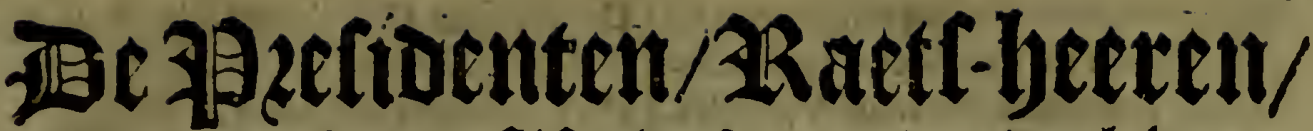 Secretaxifen en ffifcalen die tot aen dach ban beden toe bebjen ghedient en diench / in de boo =

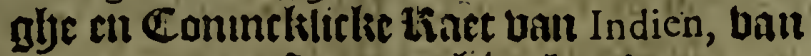 De eertite ontocktiugle af.}

\section{$P R E S I D E N T E N$}

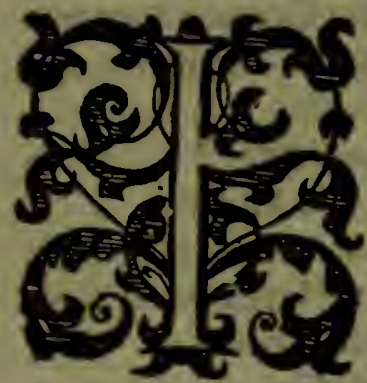

Van Rodriguez vā Fonfeca, Broeder van de $\mathrm{Hecr}$ de Coca, en $A$ lacios, Aerts-Bífchop van Rofano,en Biffchop van Burgos, wefende Deecken van Sevilien, gouverneerde hy't ghene dat aen ginck tot defpache van de Vloten, en d'Armaden van Indien, tot dat dic Catholijcke Coninck, Ferdinand de vijfde, hem riep dathy in fijn : Hof prefideren loude op de negotien van Indien, en hy dede dat tot dat deKeyfer quam te regeeren, die geboodt dat de Doctor Mercurino Gatinara; fifn groote Cancellier fuperintendent foude fijn van alle fiju Raden, en door fijn hant ginghen alle de defpachen, en quam in alle dic vergaderinghen, die daer ghchouden werden.

Prielter Garcia de Loayfa Generael van de ardre van $S^{5}$. Dómingo, conferfeur van de Keyfér, Biffchop vän Ofma, dic Aerts-Biffchop was ran Sevillien, en Cardinacl.-

Don Garcia Manrique, Graef van Oforno, die tot hulp quam van Sevilien, en prefideerde foo lang tot dat de Cardinacl wedcrkecrde van Roomen.

Doń Lowijs Hurrado de Ḿridöza, Marquiz de Mondejar, die daer naer Conincklicke Prefident is gewcelt, en Rátivan de hooge-Ract van Caftilien.

De Licentiact Bön Franci $\dot{c} c 0$ Tello de Sandoval, die geweet hébbende. zan de Raet van Indien; worde
Prefident vande Coninclicke Cancellcric van Granaita, en dacr naer quam hy te prefideren in de Raet van Indien.

'Dé Licentiaé Don Iuan Sarmiento was oock van de Raet van Indien, cn daer naer quam hy te prefideren in de Coninclicke Cancellerie van Granada, ván waer hy weder quam oin te wefen Prefident, van de Conincklicke en hooghe 'Raedr van Indien.

Louys Quixada Heer van Villagarcia, en van de Crijchs-Raet.

De Licentiact Iuan van Obando, van den hooghen Ract, en de heylighe en generale Inquilitie, prefideerde in de Raedt van Indien, en 'sConincx incomen.

Dé Licentiact Don Aritonía de $\mathrm{Pa}$. dilla, van de Conincklicke èn hooghe Raet van Caftilien, was Prefident van de Raet der Orderen, en daer naer in hóogeRaet far Indié;

Dé Licentiáet Hêrnando de Vega, en Fonfeca, ginck van deritiooghen Raet van de héylighe én generale Inquifitic tot de Ract van des Conincx incómẹ, en van dien, tot de Conincklicke en hooghe Ract van Indien.

De Licentiaet Don Pedro Moya, de Contreras, de eerfte Inquifiteur dré te Mexico was, oin te ftellen in die Stadt hêt heylige Officinm, was Aerts-Biffchóp van die Stadt cn. Prefident vañ dén hooghen Raedt van Indien.:

De Licentiaet Paulo de Laguna, ginck van de Conincklicke en hooghe "Raet van Caltilicn, en van de hey- 
lighe en generale Inquifitie, om te prefideren in de Raedt van "sConincx incomen, en hare Vierfcharen, en daer naer voor Prefident van de hooghe Raet van Indien, en in tijn tujt begon de Coninck. licke Raet, die ghenoemt werde de la Camara.

\section{Raèts-Heeren.}

$\mathrm{H}$ Ernando de Véga, Heer van Grajal, die was groot Cómandeur van Leon, en Prefident van de Raet van de ODdren.

De Licentiaet Lauys Zapata.

De Licentuaet Moxica.

De Doctor Sántjago.

De Doctor Palacios Rubios.

De Doctor Gonzalo Maldonado, die Biffchop was vã de Stadt Rodrigo.

De Meefter Louys Vaca, Biffchop van Canarià.

De Dochtor Aguirre.

De Doctor Mota, Biflchop van Badajoz:

De Doctor Sora:

De Doctor Pedro Martir de Angleria, Abt van Iamayca:

Mofiut de Laffao, $)$ Van de Raet des Keylers en Raet van Staet.

De Licentiact Garcí de Padilla i en yan de Ridder chap van Calaurava.

De Doator Beltran

De Doctor Galindegz van Carvajal.

De Dóctor Berialo

De Licentiaet Pedro Manuel.

De Licentiaet Rodrigo de la Corre.

De Lisentiaet Montoya. anni

De Licentiaet Mercada o c.. I i

De Licentiaet luan de Y (unga. )

DéLiccntiaet Xuarez de Caryajal.

De Licentiact Alvarode Loayfa

De I icentiaet Gutiere V elazquez.

De Licenciaet Gregorio Lopez

De Licentiaet Don Francifco Tello

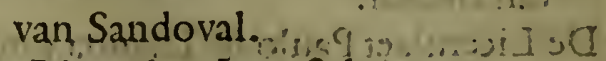

De Licentiact Iuan Salmeron.

De Doctor Hernă Perez de la Fuente.
De Doctor Garcilopez de Ribadeneyra.

De Licentiaet Birviefca.

De Licentiaet Gutierne Lopez.

De Licentiaet Don Iuan Sarmiento.

De Doctor Iuan Vazquez Arze.

De Licentiaet Viliagomez.

De Liccntiäet Martin Ruyz Agreda. De Licêtiaet Lope Garcia de Caftro.

De Licentiaet Xarava.

De Licentiaet Valderrama.

De Licentiact Don Gomez Zapata.

De Doctor Francifco Hernandez de

Liebama.

De Licentiaet Munjoz.

De Doctor Louys de Malina.

De Licentiaet Antonió dé Aguilera,

De Licểilaet Don Hernādo de Salas.

De Licentiaet luan Tomas.

De Doctor Villafanje.

De Licentiaet Botello Maldonado.

De Licentiaet Otalora.

De Licétiaet Diego Gafca de Salazar.

De Licentiact Gamboa.

De Doctor Gomez de Santillana.

De Licentiaet Efpadero.

De Licẹtiaet Don Diego de Zuniga:

De Licentiaet Lopez de Sarria.

De Licentiaet Enao.

De Doctor Lope de Bayllo:

De Licentiaet Gedeon de Ynojofa, van de Ridderfchap van Santjago:

De Licentiact Villafanje.

De Doctor Antonio Gonzalez:

De Licentiaet Francifco Balcazar,

De Licentiaet Medina de Sarauz.

De Licếtiaet Don Loys de Mercado.

De Doetor Pedro Guticrrez Flores.

De Lịcntiact Pedro Diaz van. Tuo 1) danç.: :

De Licẹtiaet Benito Rodriguez Yaltodano:?

De Iticentiaet Aguftin Alvarez de Toledo, en de la Camara.

De Doçọ or Dan Rodrigo Zapata. :

De Licentiaet Pedro Bravo van Sotomayorg it

De Licentiaet Molina de Medrano, van de Ridderfchap van Santiago, en. de la Camara, Commiffaris van defe Hiftoric.

De Lisentiaer Diego deArmenteros. 
De Licétiaet Alonfo Pérez de Sakizar.

De Licentiaet Conzalo de Aponte, en de la Camara.

De Licentiaet Don Iuan-de Ocon, van de Ridderfchap van Calatrava.

De LicentiaetHernando de Saavedra:

De Licentiaet Don Tomas Ximeneż Ortiz.

De Licentiaet Eugcniơvin Zalažat:

De Licentiace Don. Francifco Arigs Maldonádo.

De Licentiact Andres de Ayala.

De Licētiaet Benavēéc de Benavides: De Licentiaet Roque van Villagutiedi ne.Chumazero.

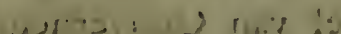

\section{Secretarifen.}

\section{Van Goloma:}

Miguel Perez de Almazan:

Garparde Gricio. 1:L?

De Cómandeur Lope deConchillos: Francif́́ de los Coboss groot Cóm-

mandeut van Leon:

Iuan de Samano.

.
De Commandeur Frăcifco de Erafo. Antonio de Erafo.

De Commandeur Iuan de Ybarra:

$$
\text { Fifcalen. }
$$

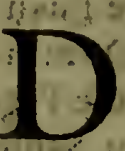

E Licétiaet Francifco de. Ver.<smiles></smiles>

De Licentiaet Prado:

De Licentiaet Martin Ruyz Agreda:

De Doctor Francifco Hernandez de Liebana:

De Licentiaet Geronimo de Viloa.

De Licentiaet Gamboa.

De Licentiaer Lopez de Sarria.

De Licentiaet Scipion Antolinez,

De Licentiąt Negron:

De Dostor Valenzuela.

De Doctor Marcos Caro.

De' Yicêtiaet Benito Rodrlguez Val. todanosis is.

De Licentiaet Alonfó Penez de Salazar.is a.

De Licentiaet Roque deVillagutieme Chumazero. $\therefore$ Y jis i rioca

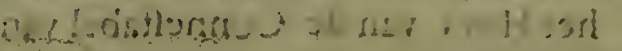

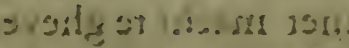

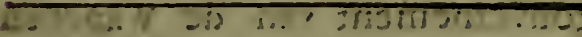

TRe Gouberneuts en alite

likpg / Die totnuhebben ghegouber $=$.

neter oe kijeken van nueva

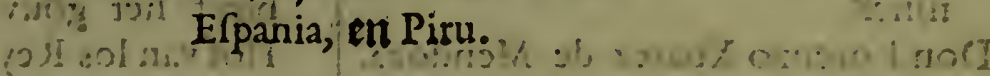

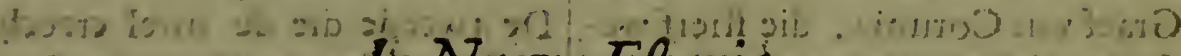

In Nueva ESpania.

(1)

On Hernando Cortes,
Marques del Valle,
Gouverneur, hooge
Rechue, en Capireyn
Generael. De Licentiaet Louys Ponce, van den Huýc vari den Hertoch, Darcos, Rechrer vall de Refidentia', met macht om aen te nemen het Gouvcrnement, en om fijn aflijvén, was in die plaçte gheftelt zijn Lieutenant de Licentiact Marces de Aguilar, Ingheboren van de
Stadt Ezya, en om dat fijn doot volchde binnen twee Maenden. fubftitueerde hy infijn dingen de Treforier; Alonfo de Eftrada ja. geborẽ. vande Giudad Real:en geweten fijnde de doodt van Iauys Ponce in Caftilien, werde, yerfien dat Marcos de A guilas gouverneerde , en in fijn abfentie Alonfo de Eftrada, tor dat het Hof goct vondt w met ordre dat daer in prefideren foude Nunjo de Guzman, Ridderiyan Guadalaiara, Gouver: 
- near van Panuco, ter tijdt toc datter cen Pyefident quam, en om dat het betaemt te verlaeten dic Rechters, werdender andere ghe- fonden in fijn plaetfe, en voor Prefident in het generale gouvernement van nueva Efpania quam Don Sebaftian Ramirez de Fuen. leál, Biffchop van Santo Domingo, en de la Concećion, die Prefident was van het Hof van Santo Domingó een wijs Mañ : en die nae veel digniteyten ftierf in $\mathrm{Ca}$ ftilien Biffchop van Cuenca, en nit wert het ghegeven op een nicu de Marquys Dón Hernando Cortes de laft van het generael Capiteynfchap, op dat hy gouverncere de faecken vän dén Oorloghe, met het believen van Dón Sebaltiaen Ramirez.

Déeerfte die de rijtel hadde van Vice-Roy of Capiteyn Generael van s' neuiva Efpania was; Don Antonio de Mendoza, Broeder van de Mararsquys van Mondéjar.:.....

Don Louys de Velafco, Ridder van het Huys van de Conneftabel van Cantilien.

Don Gafton de Peralta, Marquyz de Falces.

Don Martin Enriquez de Almanfa, Broeder vàn dé Marquizi de Alcanizes, Hofmeefter van de Coninck.

Don Lorenzo Xuarez de Méndoza, Graef van Corunja, die ftierf wefende gheprovideert om te gaen naer Piru, en om fijn doot, gousockerticerde daerentufrétien Dón Pe"tl dró Moyá de Contreras Aérts-Bif-

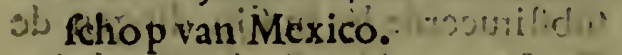

Don' Alvaro Manrique de Zuni- Jga Marqúuiz de Villa / manique, aviBroeder : vañ den Hertoch van

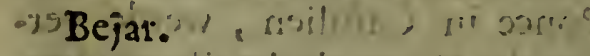

Donk Lohilys de Velafco, Soon van de olvoórfetirévén Don Louys' de Ve. "xulco, die ginck te gouverneeren - - Ue R ijcken van Piru, al waer hy te"nghenwoordich is.

Don Gâfpar dézuniga, en Fonfeca, แืว?
Gracf van Monterrey, die heden regecrt.)

\section{In de Rücken van Piru.}

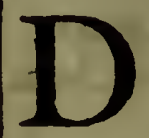

On Francifco Pizarro Marquiz de los Charcas, Gouverneur, hooghe lufticier, en Capiteýn Generael.

De Licentiaet Vaca deCaftro van het Ridderfchap van Santjago, vin de hooghe Raet van Caftilien, creech de tijtel van Gouverneur Gene. rael.

Blafco Nunjez Vela, Ridder van $\Lambda$. vila, was d'eerfte die de Tijtel creech van Vice-Roy, en Capiteyn Generael van de Rijcken van Piru.

De Licéntiaét Diebo de la Gafa, van de Raet van de heylighe en gereràle Inquifitie, creech de tijtel van Prefident van het nieuwe Hof, dat ghefonden werde nae de Stadt van los Reyes, en van Gouverneur Generael, met macht te gheven het gouvernement van de Wapenen aen die diet hem beliefde : de Biffchop van Siguença @tierf, en fijn begraeffeniffe, en tropheen werden ghefien in Madalena van Valladolid, en om fijn abfentic bleef het gouvernement by het Hof van los Reyes.

De tweede die de tijtel creech ran Vice-Roy, en Capiteyn Generael was Don Antonio de Mendoza, dic de Rijcken van Nucva Épania gouverneerde.

Don Andres Hurtado de Mendoza, Marquiz de Canjete.

Don Diego de Zuniga, en Velarco, "19 Graef van' Nieva.

De Licentiaet Lope Garcia de C2. Aro, van de Conincklicke ea hoo"! ghie Raet vañ Indien, creech de rijtel van Prefident, en Gouver. (i) neur venerael.

Don Francifco de Toledo, Broeder van de Graef van Oropefa, Hofmeefter 
meefter van de Coninck.

Don Martin Enriquez, van de laift van nueva Efpania, ginck hy te gouverneren de Rijcken van Piru.

Don Garcia de Mendoza Marquiz van Canjete.

Don Louys de Velafco; van de laft van nucva Efpania ginck nae de Rijcken van Piru, al wacr hy nu is: en door oorfaecke, foo het werck hem laet aenfien, is gefchict als Vice-Roy, en Capiteyn Generael van die Rijcken Don Iuan P.a. checo, Heitoch van Efcalona:

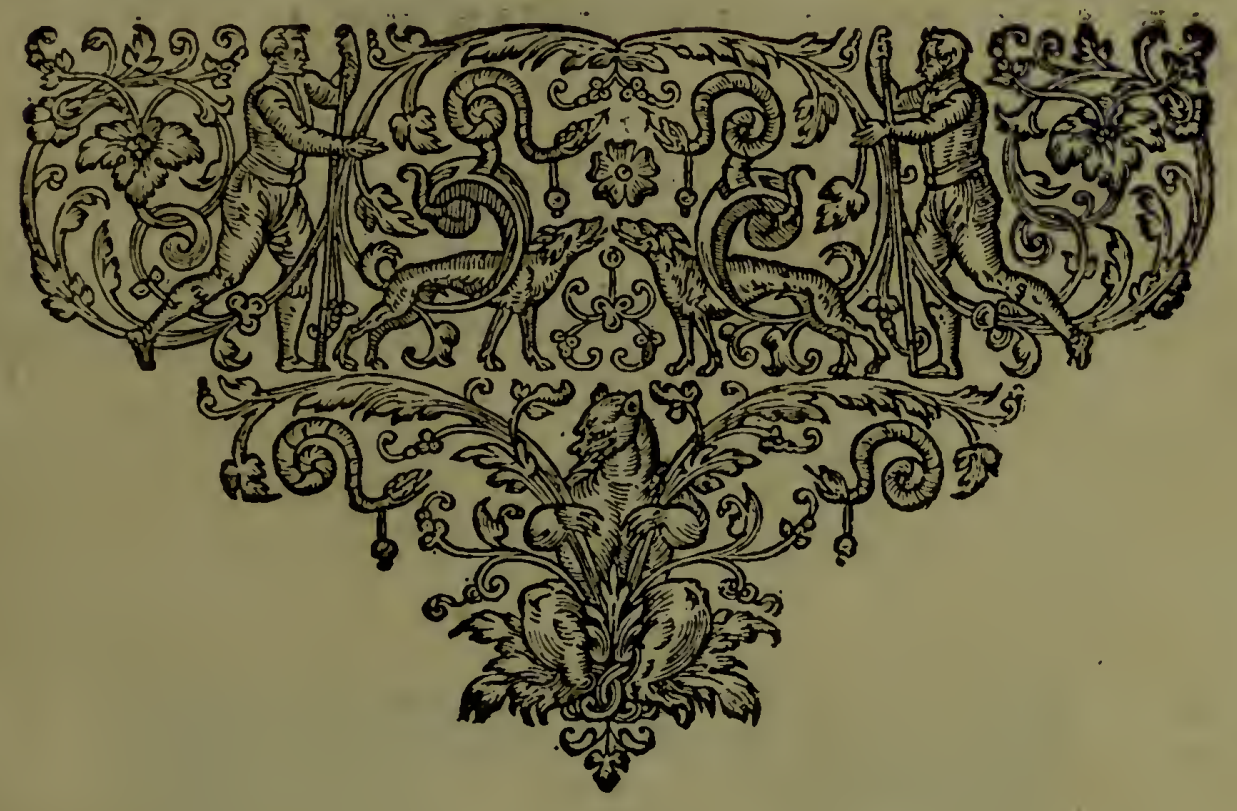



Eyghentlijcke Befchryvinghe

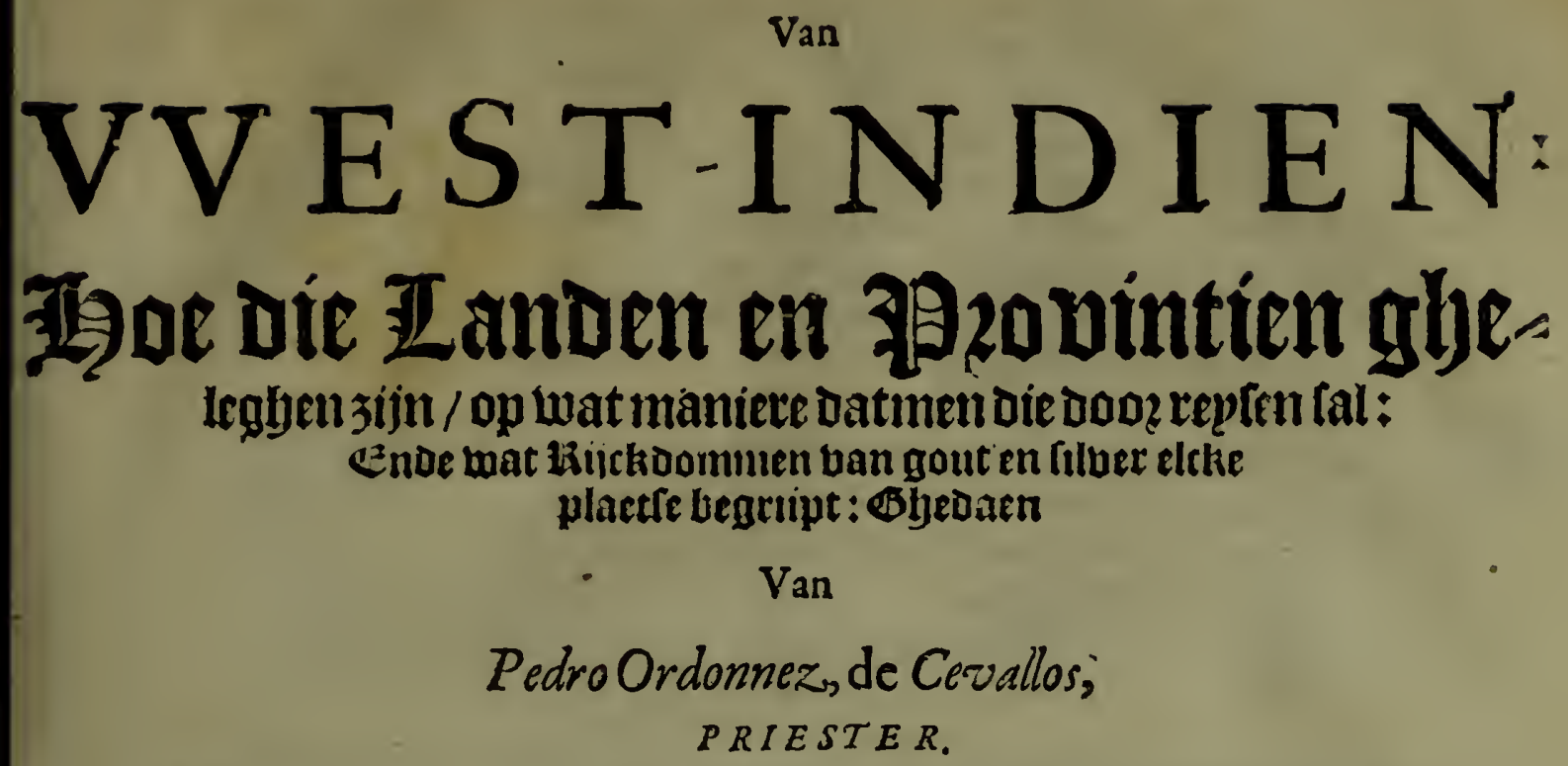

PedroOrdonnez, de Cervallos;

PRIESTER.

Die de zanden net groote neerffichept dooztepet en befichticht beef.

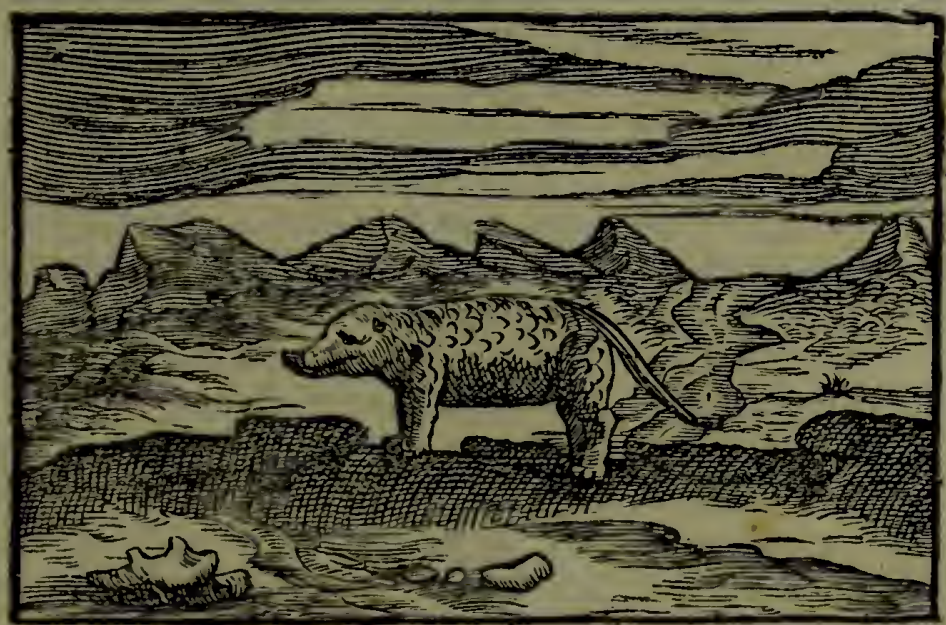

TOT AMSTERDAM,

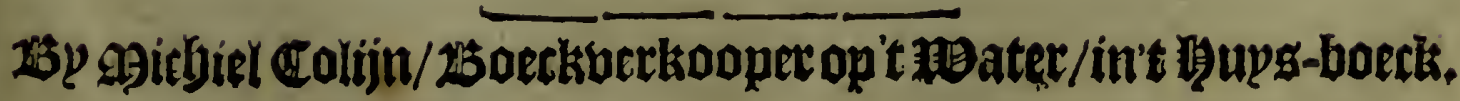



Eyghentlijcke befchryvinge vanVVeft-Indien.

Hoe die Landen en Provintien gelegen zijn,op vvar maniere, datmen die door reyfen fal: En vvar rijckdommen van goudr en filver elcke plaetfe begrijpt.

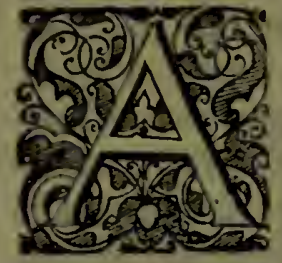

Zloo dact gljeen onredelucker Dunck ig dan de ondanck. baerbeut/ en bu alle pljulofophen/ja alle naetuerlijcke uer = ftanden milp2elen wert/ loo ift dat ick Pedro de Ordonnez uan natue= ren altoos dien ghelyaet hebbe / en noch niet en bemune/en ic oanck. baethept toonen wil acn mijnevê naeften / voos die ghenade die unp Boot bebelen beeft in mijn repie oooz de gant'flye Herelt/ en wil oe kenniffe van Dien aen alle menftyê meoedeelen: bulonoerlijcken ban melt-3ndien; die Derwaerts wil replen fal bier op moeten letten / Dat De bauen ban San Lucar de Barrameda, Iept uan de Stadt Cadiz viif leguen: Ban eell ban Defe twee bauens loopé de Blooten en Galeoenen unt / nae de VVeft-Indiens, en de $\mathbb{U}^{\mathbb{E}}$ planoen uan Santo Domingo,en Havana,en Cuba, en lo voozts. Su legglyen op de ljoochte van 37.graden. De Eulanden van Canarien legghen uan bier twee hondert en dertich leguen / op de 9)alcaerte jupD-welt waert: fu plegen op de repte te berfupmen acht oft thien dagljen. De Zee is feer ontltumich om lijn groufaembent cn winoé / waer doo? lyu meer hem ontlettet dan anoere. Een oor wert ar colfe bantlas Yeguras. ghenoeme / Dat bet glyeen golfe en is, Dic uan las Yeguas. Het 3 iin fetuen Eplanden/Groot Canaricn, Tenerife, Gomera, Hierro, la Palma, Lançerote,en Fuerte-ventura. En altyoe.

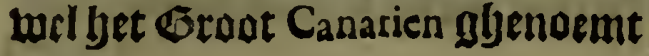

wert/baerom iff geen grooter $\mathbb{E}$ lant/maer bet ljooft uan alle b'an. Dere. Tenerife ts bet grootlte. De dzie uan dien jijn Scigneurien: La Gomera is eé Srazffeyap : en Lançarotte en Fucrte-ventura 3 ॥ln een ander Graeffrbap: De vict taen onder be Coning. In Groot Cana. rien tig het bof en de Gouberneur/

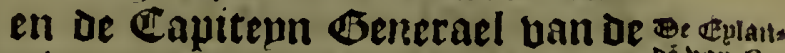

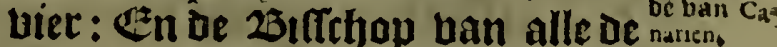
feuene. Centglic van die 3in leer vzuchtbaer / en bet meelte is Tenerife : $\$$ u legglyen op acht entwin. tuch graden febaers. Sp wierden in vooztuden gbenocmit las Fortunadas.

In dat Eplantiseen $25 \mathrm{ercb} /$ oie ghelien werdt theventich leguen in de $\mathbb{Z} \mathfrak{e c} / \mathrm{en}$ ooc tg bet het eerfie dat= ter gbefien bert :en wert genoeint Pico de Tereue : lap is het gantíthe Jaer buit befnecut : $29 e n$ beeft dzie of bier dagben ban noode om bos ben te comen/ alwaer een Gleene plaetfe tz/van waer gefien werden de andere eplanden/die leer klepn frbpuen / alboewel batter een Ejp. lant loo groot is als dit/ 'twelck is del Hierro. Mat oit lianot treckts men al de folffer Dtemen weet / en boozt de ligertogé ban Maqueda toe. Ben boom In bet Eulandt del Hierro ghe $=$ retuls. naemit / alboewel bet loo groot is/ to beeft bet geen water: zalleenliick is Daer ern boom nict bekent/nock gefien in andipre declē uan de 1 Be: relt. 2Bouen dien is ozoinaris een wolck / twelck een ooffaecke ts dar bare bladerê altoog groen ziin / Die cleen en becet $3 i j n / e n$ diltilleeren io beel waters / datter ghenoecl is vooz alle be Inmoonters van dit Cplant/ t welck een ozoinaris 99 rakel is. Aten oe recljter bant uan die Eplanden juinoer andere/Die ip noemé die uan San Borondon : Een wonderliicke laecke Dat.fis bp tp= bant wutrea aen glyetien wozoen/en lupiasen ble bergaen waren op vertebepdế tp= oen zijnoer op geweeft/en alfmien. 
fe focht/ en bondtmenfe niet. Wet moet een groot lecreet jiin / alleenlick gyelaten vooz Bodt/ Diefe fal ontoecken 'tjinoer tijot. $5 \mathrm{p}$ legghen dat lyet een veuchtbaer zant is/en Dat of luwdê Chziltenen zijn.

alle Defe feuen éplanoen stin reer olveruloedich / waer booz de liftocht aloaer beter coop $i \$ /$ als in Spaengien/en ooc De Spaenjaer. Den/en Guancha ban bet lande le= uen weetoelijck. Oaer jiin ontal= liicke beel Cameelen/en Supcker. Ban bier boeren wh af in oe Bloo: te die booz de Generael Francifco be Noba, en oe submirael Don Francifco oe Valverde ginck/en wp lepl, den op De felfoe ftreeck / feven en twintich Dagen/en la Defeada wer De ontoeckt / dat van Groot Canarien acht-bandert en bertich legus en affept/en Die/en andere beele die fier ziin/legghen op bifftbien gra= Den. Ia Dominica, en Matalina, en andere zijn van oe Indianẽ betuolet/ Die top noemben Caribes of Gimarrones, een bolck dat meniche bleeg cet/ en oock om op't lant te fprin. gen wachteden baer be onte/fchie. tende beel ftucken los op bet bofth oat op't geberchte lach/en oe Sol= Daten van de 1 Barlst [pzonge upt/ om dat als ip nitt op haer hoede waren/namen fure wech. Haplie. pen nae Matalino, en Daer biel boo? cen notable faeck $/$ / at wp bonoen in een bocht een groote floot/ en in Die twee Slangen Dick / Datmen't niet can bertellen / Conder groote uermonoeringbe : En lebietende met de grofnuetten om dic te Doo= ben / waren de blafmghen die fp maecken foo groot/ Dat fn De $\mathbb{E} p_{\text {p. }}$ Ianden ber'flyzickteden/en veel Indianen bielpen oock/en'twas noo= bich Dat alg [p vergaocrden / Dat = men alle de ftucken afficljoot. Een Sgan/lijnoe een Spaenjaert/ Die mp in luede ghemoetende/berteloe ons/Dat in Dit $\mathbb{C}^{z}$ plant / en in D an= Drre betl Spaenjacrts waren/en
Bzoumen/en Dat fpre nu niet eten/ om Datter eenigbe met de Indiaenfche gyetrout 3ijn / en andere inet de Spaenirbe/en dat fu Sonen ge. teelt bebben/en Dat fp cenige vzou. wen Cbziltenen gbemaeckt yad= Den/en noch anoere/en Dat bet een lichte faeck foude 3 iin yaer allega= der te bekeeren. Qalboewel ick bet mp laet uod?faê/ Dat onfe Catboliicke Coningê bुet niet en Dô̂/ nabien het licht foude zim om dic te beuolcken/of dat lp glyebieden louoen datmenfe al oinbenghen fouoe / op dat albier eenicl laetters vpanot niet ontfangen weroe/ als gbefclytet is in Florida : Een Dat Gyp hier bem fortifieere / Dat groote Íthade foube weten: Uen melende in Bozloge/ Dat fu fouden beichermenfaer Zanot ban dic. 1 prep. oe bat bet een langlje tijot ghele. oen cuas / oat fp gljeen 13zietters noch 30zouwen ghegeten badoen. De Bzouwen/om dat lp begbeerden pzofit met baer te boen / om atercke Sonen ban haer te hrughen : En be 19zieltetg/ om Dat lpder een ghegeten badoen/en alg ta aten/ luvallen en bozten al bie gees ne die ban bem gbegeten yadoen. Ban de Eulanden werter glyes carrasena: repltin acht of thien daghen tot De iinue Indi: Staot Cartagena, een Haben / alwaer de Salioenen/en oe Blooten van bet balte $\mathbb{I}$ ander bergaderen/ het booft uan de regeeringbe die Daer is ban veele 19 zobintien ban lndien. 't $7 g$ een thadt ban groote trafijeq en coophandel/am oe belbept ban de Srbepen die bu baer vergaderen / en om lyet nieutoe Zaijck uan Granaden. 't l'seft onder hem de Stadt Mompox,en die ban Tenerife, en die uan Tolu 5 parnjaerden: (jet plach oock te beerfebe ober oe Steoen ban Maria, en Die van la Conception, Die van Santiago de los Cavalleros, die ontuoltkt wierden/oun dat De Indianen revolteerdé / en de Spaenjaerden doot- 
De groote Itibur la Isadalena.

soamm bร Balreul.
Uoeglyen om quacot regiment bat fu haer aendeden.

't 7 s uan de Eulanden van Dominica tot Cartagena, meer dan Datie honoert leguen weechs: al eer bat fu komen aen oe monde ban de grooteliviuitr Uã la Madalena wach = ten fu baer van die merr dan dyie legucu ' $\approx$ ecwaert / en Dacr werdt gevoelt oe fnelle bloet Die lyem op: geeft / en men leefter gefien dat de Soljepen groote rufingben Doen/ en dact wardet gljelepot / bat meer Dan twer leguen' $t$ zeelwaert/y,m [et loo't water vergadert beeft. Dit goubernement fal hem ltrec: lien meer dan tweeljondert leguen in oe bzeette/cn in De ronte ontrent be urif bondert t iseeft ecn groot I and oill te conquefteren/ en'tals Der-rijcklte / en cen oncpndelijck bolck tot aen oe gheberchten toe טã Tolu, Ilrava, en Carquana; en an= Dere nieute 19?ouintien/get alderruckfte Zlanot ban goudt. In de Stadt ban Tolu, fiin 2bergen ban ueele leguen/die allegader boomen ziin van oe aloer rijcklte $23 a l l e m$. De Indianen jijn feer onoermozpe en pzofutelijck vooz baer Ouer = heerell. De Indianen hebben oe ttadt Mompox in/ bue gheben uooz tribupt lpu[e/ en de Canoen die be Indiaen beeft/'tmelck oe Buertte 'sjaerlijex waerdety is / fonoer pet wat feglyeuen uan ziin bupg/ biff= tich ducaté bzp. Be Carares uan de oroote Libuer wierpen haer op/en begaben haer op oe wegl / in de Tinier reebt over de Tamalame, Dat een ltabt ban oe Spaenjaerts is/ ban bet gouvernement ban Santa Marta, bet waren Onderfaten ban Mompox. Oin te varen op dere groote Zibier / boeghen baer $t$ ' $\mathrm{Ta}=$ mî de Cauben bande Spaenithe Cooplieden / en met baer langbe gittolenpafteerentp be wegh ban Armadilla. $5 p$ bebben groote lebade gyedaen in de Canoen al: Ieen / en oit Conincklijck (bof / en gigaer nu if yatr belet/ foo dats men fonder bzeefe repit.

Relaes van de Provintien van Santa Maria, van het nieuvve Rijck van Granada, van het gouvernement van Popayan, en het Refort van Ruito, met zijn Bifdommen.

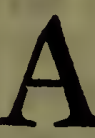

ZThoctwel bet be waethepdt is / oat oe vopagie of wegly om een tour te doen om de merelt/niet noodich is oin te gaen nae Cartagena ban de Indiens, om Dat De rechte wegh is van oe las Dominicas, en om dat ick gheko. men ben in mijnen reps Doo? Die wegh/en mijn tour genomen bebs he 0002 Piru, fal oock booztgaen met miun relaes / reeckenirbap ge: vende ban faecken. Ezn guelijck ick ghefepot bebbe van yet boubernement / en 't 23iroom van Cartagena, fal ick ban oe refte fpzelten) boe ick die reploe. Ban Cartagena ginchick nae bet Boubernement van Santa Martha, 't welcke bet 1)ooft ig,ende albier in die Stadt/ ig eenen Bouberneur eneen 2sit: feljop : Tat een is ban be buf Stes Den. Santa Marta, Salamanca, la Ra- sanca Mazz mada, dat bp naeft al bu een is/ oe ${ }^{\text {ta. }}$ Ballepe van llpar, en Tamalameque. Salamanca ende Ramada jijn oe Steden/alwaer de peinicipaelfte. 5ozgerien jun van de Smarten' die de peerlen vitachen: Ẽnde la Ramada jijn. haer Zogementen. 't 7 s een ban de grootlte Rilicks Dommen ban de nerelot/ om dat. ter Jaren zijn / datter gbevileljt meroen eenen grooten quantitept van Ducaten aen pertlen: de weles

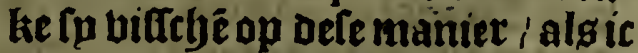
nu fal leggen. Be Smarten gaen in yaer Canoen/ oe welcke zijn ees nen loozte van 2 artken/ende alle van eenen Itoffe/ ende fp bebben I 3 baex 
baer Capitepntchap. fet sijn oupe. kers die gewoup ${ }^{2}$ wozzen in t'wa= tcr/en gaen tot op oe gront ban de Zee/en fy nemé eenige Lacken / Die blafen fu op/ en uollen die met $B e$ : aterg/ en met die fpungben fu in de zlanaen/en daet openen fp die $B e$. Iters / onder oe welcke zijn peerlen Dte in groote toraerde plegen te fpn/ en dan keeren fure wederom / ain Die weder litt water te woepen: Baer jiin groote Dupckers die neel dieper in oe zee gewoupe wo? = pen, Die jijn die/die meelt den adem in bouden / en be belte peerlen bif. frben. Ilck legge wederom Dat het een groote rijckDom is / lole berle= fert werdt. Doen was allyier de \$9aerichalck/een groot Spaenlely Soldaet/ Commanoeur bã de In. Diens / en oe liseer $23 i$ tithop Don Sebaftiaen oe (Oquendo die in een Iaer upt trocken bondert duplent Ducaten aen peerlen.

Tairomas,

Bat goubernement yeeft een qroote quantitept ban de Indiens, conoer conquelteren /en oe alder = Iterckefe Ratien van alle of Indianen, Dat Taironas zijn/en zijn too tractytich als bie ban Chile, een volck Daermé op betrouwé mach. Dat 23icoom beeft in de ronte nuet jet 9 gep? De Maracoibo meer dan feg bonaert leguen.

't piteume raitck ban Granada.

Het nieu Thijck van Granada, is een zlerte-hiloom / bet Hooft is be Itadt uan Santa Fè de Bogota: theeft een bof/cñ lijn reloet is dat ge beele lupg/en he goubernementen wan Carragena, Santa Marta,en Popajã, en het coerenginuent vấ Tunia, en' tan= Der van Mariquita, en'tgoubertes ment uain lós Mulos, cis een ltadt Die fier wel uerfien is wan bzoodt/ bleerty ent uzuchten: De wijn contt uan Spagnien: fu beeft filuer / en groote quantitept gouts / als ghe rept fal berden in fijn tteden. Het goubernement uan los Mufos beeft Sau de EF- twee Itedé/ die ban Mufo ban waer merauden, meraudé die dooz de gantiche toes rclt gefien merden/en oocli uie ban De vier boecken ban oe 3 orerelt tos men / maer uan fjier kiomen de bes Ite / en meer dan in die derie andere

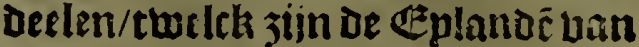
uan Ceilan, en Sumarria, en Vicipuri van Cuchincina. Butgin groote eftime / Derbaluen lo lu vergelelien werat met een groote cul lect fune glyalté veel. Daer is ern liotí foo groot/ die net gecunoirljt fal wo:Den Co lang de nerelt traet/altyoes wat lp thienmael co veel volex bad: Den/fo bet ghedaen mo: ffe jitin wan oe Swartell / niots dat dacr gles baeck is ban of Intwoonderen. vijtar decl coude zijn gBajeltept ế groote fonme gyeloen. Hier bont een Indiaen van een Commandeur een vermaerde Steen / dir is die gheleut is in bet Coffer van bet al Der-bcplichite Sacrament van't Efcurial, die dien gaf de Coninck Don Philippe De tweede/en De dzous we 19uncelfe Clara Eugenia vã Auftria żin Dochecr / Dtcfe ghelonden merde te pzefenteren : Ễ als june Igajelteut vzaechoe mat's gelooft werde / kanden alle be Jumelicrs dien op gheen paijs ttellen/en tekir

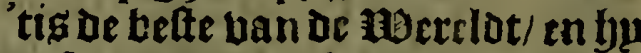
gaf yem lijn bephepot ien yp dede yem andere belooningben.

De andere Stadt if dic banla Palma, van waet uptgbetrocken werdeen groote quantitept ban Pita, ban aloechande lach. Defé pirac is als Stadt is gljelegben in De rectjtple= ree oaer in gingbe uan Tunia, due leer goet ig. Sp touden vooz cencu treffelijck dinck de Beftingben ban aeroe/ be welcke ghemaeckt zijnoe / cu gela= ten ziinde datter een 13 inter voo:s bu gaet dicle uan boben bereghent be atate en glat maeckt/ int epnoigben ban let regenen doo? de Ulackte/ sillite fo Iterck Datter met ecn lpurlier in en kan/en oocli met kispunc onco: Aten feefte bermaerde geboumer. De ftadt uan Vclez, die uan Pam. 
plona, Ocanja Merida, la Grita, die bã / fo bit voltk/bat ick nict en lath et. Santiago, De Strden van S. Chrifto. ral,en Leyva, en de ttadt uan be bas ben ban Ocania, dat is al goet lant/ cn in bele declen van dien werter gout untgyetrocken/en in de reffe glyeben De Indianen Deeckeng tot tributut / en tu begeben gacr tot be lapingé vande Ouertjectde/en opt Bee / oat in groote quantitept 1 . Dat jiin Ouerlyeerde die twintich buufent (B) Ten hebben/en nocl) foo

teftad Mariquita.

In Santa Astueda it Aluer. becl uant kleen Bee. Be teclit-ple. ginge van Mariquira, beft be ftadt uan Tocaima, en Die ban Ybague, la Santa Agueda, alwaer groote פsij= nen banfiluer $3 i j n /$ al bet gene bat iit Nuevo Regno is / Dat werut buer untgbetrocken. In De Stadt uan In Remedi- los Rensedios, ell dic van Zaragofa, gofa, balt trecktment een groote quantitept gotat.

ban goudt unt/om datter troupen ban Swarten zijn in groote quan. titepot / om Dat die meer zijn oan beerthien Duplent/ die'tgoudt upttrecken : twelck en laeck is / Daet ouer barwondert wert. 'tl'seft de baren van Onda, De welcke is die alwaer al oe Canoen en Fregaten bergaderen/ Die opeomen wan Cartagena lanca, oe groote kibier ban Madalena, en neoercomt foo groote quantitcut ban żifftocht / ban Egecl / bammen / toalen / Conler: ven/Confitup?en/en Pita, en Deet: lieng/tzabelg/ Re Derbalen / / Tou: wen/20arbeslen/en Bintuanck cels $^{2}$ van bututê laften/ Bilber en Gout/ en écimeraldé/oie een groote Ítyat waeroich 3iin / en als fu twederkes= ren neinen fu mede EDijn / en al oe relte dat ban Spacngien comt : In to groote menicl)te/Dat bet \{p vooz Dat ganticbe Rijek in fijn StcDeli/ glycljoudeu op lyondert ouptent/en twee ljonocrt ouplent oucaten.

De ftadt van Sanra Fé, is ílo ge. [ept is) het looft : Subect twee andere fteden / twelck 3 lj n die ban Suma paz,en San luan van los Ilanos. In die ftaot is een generatie ban nich fo blanc als Dat. In Dat gant:

Irbe kijck jain oe mecte Đozpen van or Indianen, een legut / of tmee ucrfclyeyoen: fo dat was oin dattcr roo vecl Indianen waren / die Dlie= Eliegteits ghen noembeden.

\$et gouvernement uan Popayan, berfe de ltadt die bet looft ig; tjeeft dic uan Pafto, die uan Almague, Neiva, Calocoto, Cali, Buga, To-bernsutent ro, Carthago, Ancerma, Arma, Cara- balt Popasa manta, Mocoa : In alle oe Dozpen ban Dat gouvernement faloer upt: getrocken wozdé de grootltenuan. titept uan gout. Daer zijn wep; nich Indianen die bet upt trecken/ Daer 3ijn oock gbeen Smarten/m. dien fu die badoen/Daer lonoe tien. mael foo beel uptglyetrocken woz= Den. Ẽn indien 3 lin 29 ajeftept fal gyebieden/ Dat fu louden bergade= ren in dit goubernement/en in dat van Antiochien, twelck ick ftrackg fal tegghen: Seg of acht ouulent Swarten / ende dat haer betrout mocht wozben in elck Do?p / nat ware gberechtityept die beboozac/ bet loube leer yzofuteliick fiin. Bat 2Bifoom van Popajan; beeft oock relfs een ander goubernement ban Santa Fè oe Antioquia, twelck paelt santa Fe aen een lube ban het / en met het cozreginuent ban Marequita, en met bet goubernemene uan Cartagena. De ltadt vã Antioquia heeft die Lã Rodas, en die ban San luan. Tis een leer berchacbtich 10 ant/ cn daer 19 beel in te bevoliken: 'theeft beel. gouts / en ghelizesk ban bolck dat Daer is uan Popayan : lget 2sifoon falyem ftecken verder dan bute= bandert legien unt ronde.

De groote 7 zobintie ban San Francifco del Ruito, bet mectite oeel ban't thant ig leer goet/om dat dit ban Quito ts / dat het is ban de grootheden uan oe mectelt. De ge-

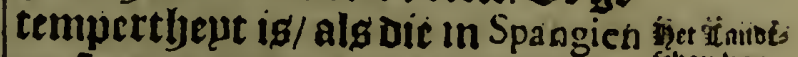

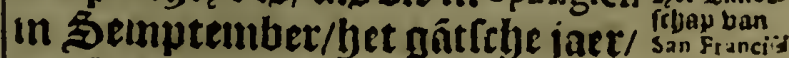

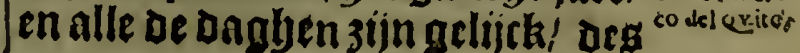


Fol.8. Eyghentljicke Befchryvinghe

fmozglieng begintmen te refien en epindighen fauondts te felfen. Be puincipale Itadt i\$/San Francifco del Quito, altwaer het Conincklijek \$of is / en een politiicke Kechter/ en een 25iftelyop: t Geeft de gouluer. nementen / en cozreginuenten die ick lal leagben. De goubernemen= té van Salinas, bie anoerg genaemt Yaguarfon- werden Yaguarfongo, ziju looft is ro. Santiago de las Monranjas, Valladolid, Zamora, en Sanja, 'theeft veel gouts: yet goubernement ban Loja beeft de Stadt/en die uan Piura, en Puerto de Santa. Bet gouberne= ment ban los Quijos, De ftabt van Baefa, Die ban Avila, Dir van Archidona, Die uan Sevella del oro, en Die van Loxibaros, het cozreginuent vã Guayaquil, oe Stadt los Puertos de Manta, la Puna, de ftadt uan Puerto viejo. In la Puna is een groote tainier / grooter Dan Die ban la Madalena, langs welck opvaert alle oe vzacbt De van Spaengien gaet naer Panama, en van baer na Guayaquil,en nae Quito, en nae alle $r e=$ Aterende goubernementê/ en rcthtplegingtyen / Die in groote quantitelut ziin / $\mathrm{cn}$ van Daer kont goubt af/en filber/en fpure/en andere din: ghen / als glefept is ban 'tnieutwe Riick / met het welcke het trafi. queert/en groote rijckdom kriiclit. De recht-uleginele ban Merica van de livier Bamba, oeltadt van Villar don Pardo. De recht-plegin= glye uan Cuença, dat een 5 tadt is. De recljt-plegingbe van de Stndt ban Tacunga, en andere bele rechtpleginghen int ghetaliban ooapen Der Indianen, elck een wan twintich of dertich dozpê/bie leer beel maer. dirb jiin / en alwaer veele Spaen= jaerden rijck wozoen.

In oe nozpen uan be Indianen, behbenoer beele Sparniaerts be: bolckt / met groote riickeoinimen bã het Belt/ houé en Bee/groot en cleen/in groote quantitept/en noch gbelt een Srbaep uier realen/een
Hoe vier en twintich / een gl;elubt Bercken yeel bet/oock To Leel/ een Scute twee en Dertich / fen iongh 19aert lettyien / een Isuul om te vellen vooz thien : En daer wers denoer foo beel ghellaghen/ Dat in Otobalo een rectyt-pleginge van de Indianen bem een Sparnjaert ob= ligeerde / Dat bp baer alle Faers foude berepden fefthien Dupient/ en'tulees wert baer verlozen gelas ten int Delt. 't beeft groote bereps. dingyen ban Zlakenen/ferfapen? Bajettê/ Catclongnicn/ Sargieu/ en fp gheloen een redelijeke paijs. Ban defe pzoluintie werden ghes bzocht groote bach-wercken / Dan al het gljene dat geleut is te Lima, in Cufco, en Charcas, gbelt alles leer weunich / om ae groote beelfepdt die Daer is : Qfebt bzooden ban een pont werden ghegheven boor een reael / een loinne gbelot oock coo beel / en een Capoen / twee Conp: nen / en oock deis en gbeloen ntet meer dan een reael / en andere dins ghen siln fo goet koop/alleen twee bouden yaer waerde/'tweltk is de Spaentrbe twijn / Die ban be $1920=$ Uintic van Lima komit / gljelt acljt realen een quartillo / ende dic ban Spaengien komt twaelf : oer dat op een yooghe jeijg blijft/ 3ijn de Ė te gheloen dupfent en bijfyondert peros/[oo hu goet is: om oe groote quantitept ban de \$9erruen die daer ig. Az oork legghen fp ozdi= naerlick : Mat is bet Diextfe te Quito? en baer wert geantwoost: Je $\mathbb{E}^{2}$ ?cls.

Van de Bifdommen, en Provintien van Lima, Cuzco, enChar-cas, ende de refterende Provintien van Piru.

Iru beeft beel 1920uintien in yem/en eenighe lo groot/ dat fu Liijcken foudê connen jijn/ en oock wert bet gehouden/ en ge: 
hoesmit bin òc Stadt Pafto af / tot Chile toe/al Piru, glyclijckmen fept טaี Spacngien / Dat bet in beu beflut ueele tiijcken: $\mathbb{C}^{2}$ wu oock ghefeue bebben in het boozgaende Cauittel vã de 1)zobintien en 23ironinmế uan San Francifco del Qujto. En onl boote te gaen/feghick/ bat lyet Hooft uan gantfely Piru, is In Rejes etri ber: maerbe fabr/sboof oe trabt uan los Reyes, anoers ghenaemt Lima. theeft oc Dicerop/en bet is een ban oe uermaer de chargers die onfe Coninch probidert/ het meette dat bu bout / is/ bat bu psoviderert de Commufien van In. dien, Uan Quito, tot Chile, Zancien en tioers: recljt-plegingen en goubernementen/bedieningen van ge meenfrbappen uan oe Indiens, en ban de werckinglyen / befeberminghen van be Indiens, en andere offi-

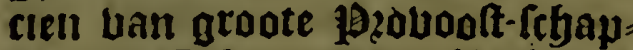
pen / cn Sclyzubers / en Zaethters/ en Eminentiat/2senefitien/C ura= teurtclyappen/an Capellaen/chap wé / het Bollochl-yolck fo ter 疋e/ als te lande. Ban de Benerael

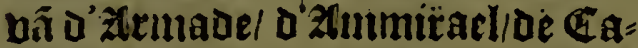
pitepnen / en boots alle of offi= ciers en Soldaten /en alle oe aunp= ten uan oe Galioenen die bet fil. ber afbzengben ! De Generael van De Galepen / en uan de andere $B \mathrm{f}=$ ficiers / be Bentrat ban Callao, Eapiteunen / cn andere bfficters/ twelck al een groot wetck is/ cn op alles probioeert by/en bp geeft De Indiens ban de 9 iinen / Dat de grootfte laeck is / van waer wel wat te legghen was / en oock gbe: noech op te letten/ foo oe Vice-roys Teer begbeericly waren. allyoewel Dooz be bermberticbepot uan de beer / bucaus alle oe meelte bzin= cen oie Daer gaen/ 3 iin goede of $y z i=$ Itenen : En foo Datr pemant is die sijn yanden glyelent beeft onite beruullen ziin asalen met 23 atenfilucrs, Itract weten bet oe Catljo= lijche Coningen / en fu remedieren Jjet, èn De agajeftept van oc!n be= mel is gedient/bit berbuatrtg gaeu Vice-roys die loo heulinge en goede Cyzittenen 3ijn/alg Don Lovvijs Don rawijt oe Velafco, ban de melcke ick te vozen ueel af gheleuot bebbe / Dat= men loude konnen ban ziju leben fcljepuen/ een groot tractaet. En Don Fernando de Torres, en Porru- Don Fernane gal, Szate uan Villar, Don Paxdo, ics, en Poringbetozen ban Iaen, inelck ver, maert buys/en foo oud gbeflacht komt af / Dooz een rechte linie ban de Coningen uan Portugael: Bocks bielt ocek fog goedt reclyt tot die laijken en Bonninien : Bock is daer een van de booznoemoe een gljeweeft / Die een groot Cyziften was/wiis en wetende/ en een trou Dactacl ban zijn Coninck welen: de/abandonneeroe bp zijn recht op stine Agajeltept of Coninck 3 hi: lips/ ende gy fepoe wijftelijck/ Dat alg yju befitten loude die tiijcken ban poztugael / bat bu vie aban: bonneren lotiog aen eenen bulen en Catbolijcken Coninck. Enoe om dat Ick op een ander plactle regghenmacb uan de grootyeden van foo grooten kidoner/fal ick die nu naelaten / berbolgbende mijn \$iltozie:

Dé Stadt Lima, yeeft ectien alert[-2siffchop / D' Jnquilitie / bet Hof/ be Cancellerpe / enoe de Coz= regioos / Die aloaer altoog eenen groot tiidoer is: 'tlyeeft een Vni-. verfiteyr, ende foo berwaert als fy publijck is'/ enoe ick gijeef gbeloof oatmen beeft boezen leggben bats oe alder-grootlte glycleerden/ oock Cheologanten / alg oock ban ane oere faculteuten / Dat het eene is ban de goederen die de morreloe beden beett.

Defe Staot heeft eenen notro oaer bibs bel binck / batter nergheng nhee = geen vaken Inen Dack is / alljoewel Datter ber: maerde gljebouten 3 ijn/ om dat bet niet noodich is / ende dit is de reden / bat het nopt en reghent. 't Js eenen Staot van of groot 


\section{Eyghentlijcke Berchryvinghe}

Ate rijckoom ban be Berelt. Bat De Blooten/en nu be Gaterenen/ Qlerte bifoom beeft be ltaot Truxillo in oe vlackten: 't Heeit Chacapoyas, Gusncavalica, ban waet uut= Ean bet aljetrockell wozt alle lyet quickfil=

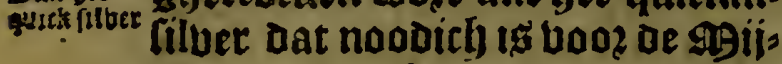
nen ban Potofi, dat é groote rijck= Dom ig. Guamanja twelck maken fu oock é looft ban bet 23iroomu/ en gebent andere fteden/en Dozpen uan de Spaenjacrden die naebu= rich zijn/en beel blecken van natu. tele Indianen.

Get 25ituont van Cuzco is nu bet befte ban Piru, die vermaerde Strat heeft cen dincls/'twelcke al: boewel bet niet feer groot is/itt een

De ftabt ban $\mathrm{Cuze}_{\text {, }}$ rijck oinck /.om oe groote boutht baerlyent ban zanden en 18zobun: tien die bet tjeeft. Su deplen oock mu ban bat 2Bifoom een ander oat วlin liboft Arequiqua is. 't 5 elfate beft oock anoere fteden/en Doz? jen ban Spaenjaerden / en blec. lien van naturele nagebulnen / $\mathrm{De}=$ welcke alg fp gedeplt wozoen / als ghetepot ig/ wan bie daie 23iloon! nuen / van Truxillo, Guamanga, en Ariquipa, ban Lima, Cuzco,en Quito, bebben [p allegaocr gbenoech: famie renten.

Las Charchas, ell Potea.

Las Charcas is nu een aliertf-tir Dout / in'tplach te welen een 2Bif= bom/het is fjet rijcktte vanoe noe $^{2}=$ relat / en tisen Atertt-bitoom ges maeckt / en oair fullen twee 23 is= Dommen ban ghemaeckt wozben/ van die ban la Paz, en uan die van Sierra : en Dat ban la Paz is lo goet/ en uan la groote rente/ Dat yet om lijn beteríclyap de 2lertr bificlyop uan Santo Donjingo veranderen Conde. $\mathfrak{Z}$ las Charcas is een Co: ninckliick bof / 'twe Ick bergadert uail Cuzco af / tot Chile toe / en de Hibier de la Plata, Dat cen oneun= ocliick Guch landts bethaet.

Ban hier tot Potoli ilt achthien

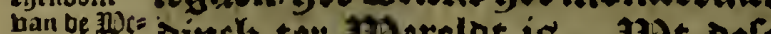
relt. die gheladen komen met filuer/ en bier bliickt eene grootbepot / en wonoer van oe Godtlisclie vooz= fienichept/ Dat Goot gheltelt berft bouen Den 23 erch een wolcl / oie altoog Daer bu ig / en werot gefien खetwolsk op feboone dagben / veel leguen tofien Poo van bier.' $t$ Tg foo ueel te legglyen: Gier is de rijckdoun. De 23 rech is op de manier vaneen bzoodt fupes ker/en lo booch dat die op zijn top fal klimmen / waer op inen ban Hlimmen/fal daie leguen gaen: is feer koubt / aen De voet lepot oe Stabt Potofi, Daet Dzomacrlicts 3ijn twintuch ouplēt Spaenjaerts: aicht of thien Dupfent Bzoutwen' enoe foo neel Swarten/en \$wars tinnell; en meer dan buer bondert buprent Indianen. 't ż een beel groot atuck / dat gbenocmt han

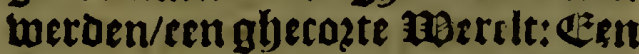
Zlanat Dat in bem niet en berft oin mineralen te 3 tjn: Srs leguen in de ronte sffer gtjeen grag / en Dat: ter is/ig berb2anot/tisifo betli'tges ne Dat baer in ko!nt/ Dat bet plach te ghelaen t'eenige replen / fo wep= nic's/als in bet 3Lanot oaer von fn bet benghen. Agen beefter gefien op een Dacb thatlf of feltyien rea. Ien / een banega meels / cn faracks een andir hondert realen/ noe op en ander tracks bederkeeren/ te gbelden als bet eertte; en bock ift van de meelte faecken / 'tggebzeck wan 'tyout/ en placls te gheloen bp tpden bat bet te betwonderen ig/ en op andere tpden ift feer goedt koop.

Datr zijn repen / bat aleer dat Ip die aengaen / Doen fp baer upt. waert / enoe legghen yare 9101 ten/ gbelijck of fu fterben fouben / am Dat tuden suepnitis wedetkeeren. allyortuel Datter eengroot Glye. bout is / dat of Gzaef uan Villar maecktede / Dat alle oe Indianen die beglyeerden te woonen in Potofi, dat fu datr wounen 
conoen/en oangelijet winnen bier realen / en bifí s'nacits / alboetwet oat fet in oe gsijnen / nadien (u fo oriep jijt / altijot nacht is / en met licht ghewzocht weedt met oefe vlijot / ende met bet ghene dat fp fteelen / if toa bebolckt / en die ar: bepoen met gbetelt : Dat fp toes telijck wzochten oner fouben too veel I dianen nist fteruen/maer die berce zijn/ en niet willen gaen/ftet: len andere voo? gaer; betalende oic dien reatl en een balf vo0z elcken Dacly / naer bet welcke fu baet bet. loffen ban fo exceffiben arbept / en oncolten ban'tgaen en weoerkee: ren/en op dàt men niet foude op: Gousen uut te trecken eleke Jaer fo veel filvers/'twelck moet woten acbe of thien sillioenen /'twelck gbeeft Interelt be Cozone uan Spaengien ban fo betl quinten/en foo ueel recbtg ban de Coopman= frbappen bert bet gbelommeert/ 'twelck een groote faeth is. Ban twelck een groote ooslake lig gbe= weelt Don Fernando de Torres, en Portugal, een Soon van océele en treffelucke ftadt ban laen:

Inde 19zobiutie ban Chile sijn twee 2bifoommen / oat ban Chile, en ban Santjago : bet Zlandt is bet vzucljtbaerfte van alle biemen ino: Berelt weet / na dien de vzucbten ban Spaengien die in Die 3ljn/ foo groot siin / oat cp die andere te bo: ben gaen.

Dat gantché Zuant/ en of $1920=$ bintie beeft foo beel grijnen ban goubt / Datmer unt foude kannen treckeneen groote fonumê / lo $3 p n t$ 29ajeltept geboodt te winnen die ganticbe generatie van oe Atercke Indianen van Arauco, die loo beel quaetg Doet, en gedaé berft/twelck liclyt am ooen loude jijn. 't Soude ban groote confioetatien jtin i en ick foube wel konnen regghen de ootaken/maer ick fal die reggben op zijn plaets. 't theeft twiet anoer 20 foommen / bie fy noemen ban
Tucuman, en uan Paragual, teti decl.

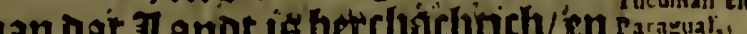
Jan oat Lartot is betch Daer wetc in teetkt / albotwet oat bet is uan veel lifftocbts / en mid. oelmatich Laijck/en uan trafijck in kleedinge des̆ aettrijer/ van cato toen/ Deeckens/en kleeré bèr vzou. wen / als boben en beneden rocks iens/die baer kléedingbie $31 j n / c n$ de frapde fupers/dat rieme zujin/daté (p baer meoe gozoen. 't t)eet gigij= nen ban gouot/oie mbepelijckjtjn om te bearbepuon.

Van de Reyfe nàe de Eylan:den van Cuba, hare befchryvinge, en de refte tor Acapulco, en het beginne van de Zuyd zeê.

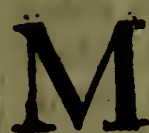

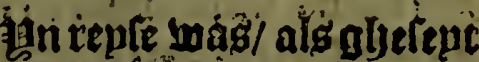
is/ in oe twee boozgaende Capittelen/Dooz oit lant van Piru, tot Chile toé / en weders gelieert zijnoe tol dé pjobintie uã Quito, vàn baer ick ecploece but beoeit te. Komen in Spacengien/ quam tck te Cártägena, ban wa aer ic te tebepe ginck/om te looperi opj oe Cabo San Anton, allwaer ick fcljip. becuck ledehbelijek obetepot is in oe Biltozie in sijn plaets. Daer na ben ick gewrelt in Cuba, en toefier. kerende tot de ejulanden Dereáda. Máralino, ent Dominica, ban waer ick bettrock hà Cártagenà, lénǵ be ick / Dat foo als aen o'eene 3 poe als diandet beel. Eulandeñ śtin / ban oe welcke icle oatt nater tal (pizeers. lien: Ein om dat ick gécoinen wa tot ofe bath Cuba, en tin zin ber. maerde baven / oie be belé l' bari

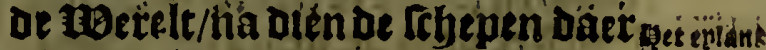
binnen liggben bip vain alle tifico ban Cubao uan be Zee / ea ban ppanden omi oattet eeh fojt is thoen inganck/ oat gljeriaent bett el Morto, fo ich meene/en oock leggê be gitoote cat pitepneen soloate/bat bet bantio 
belte is bienten vinoen can / om de / Baluidreben / en men placht oaer groete gelegéthept / en alle Daechs gaen lu wercten aen be 23 ol were. then/en bet ghefebut te planten/en aen oe zanot 3 pue is een gracht/ Die'voltopt zijnoe/met bieljonocrt igan ban binnen/en lifftoryt/ (oni Dat bet water beeft al dat het noo= Dich beeft, if niet moghelijck dat bet gewonnen wozde. Dit eplant is tweebondert en bijfentwintich leguen lang / en beet leven en Der: tich. 't theffeen $23 i$ toom / Daer Cuba bet 1 ooft af ig. Jaer is eell Gouberneur/en een Capitenn ge= nerael/oie retioeert in oe ftabt van San Chriftoffel oe la Havana, om dat bet oe groottte is/en alwaer tot bet vertreck be Blooten vergaderen ban nien Spaengien / en int we= berkeeren alle oe Blooten en Gas lioenen/en al Dat vanuieu Suaens gien komt / wert allyier in de lgabê gebzocljt/in een lterck bups/Dat op het ffozt van Morro is / als Gout/ Silber/Corhinille/Quickfilber/en De Galioenen die tot Cartagena toe Ioopen/en Puerto Velo, aen bet va. At Lant: int mederkeeren ontfan= ihen fp dat al i en met be rijckboin Die fu bzengen/kome cp in Spaen=

De rijeks bom is in Moiro.

encyen pupoer.

gien. Bet is bet aloerb?uchtbaer. Ite Eplanot van veuchten van Dat Zant/ban grapg/ en anoere wo?: telen / en ban sseelen / en andere Dingben bie van bupten komen. Daer is beel bouts / leer fun gu= achapil/Dat in'twater nizt en ber Derfe / en baer fuijckers 3ijn beter als van per / om bat het bem ber eenicht / en gbeen water en treckt. Daer is beel Loejien. vlees / maer fp toeroen gbeflagen alleen ombet bel. - Baer jijn veel geluboe Berc= kens/en tulees is lect ercellentina. dien bet oock de krancken ghege= ben wort.

De Cabo uan S. Anton, leudt op

Det Ambar, twinticly graden ien la Havana op Dezie en twintich lanck / Die Zee if rer onepnoelijcke menichte ban te vinoen eell groote quantitent uan Ambar, 'twelck fu fegglyen te ziin bet fchupmien buplicbept van De Malutityen.

() et Eulant Puertorico Iept Uan net Eptant laDominica vijfentwintich leguen/ op achtbien graden. it To lancli vectich leguen/ en beet twintich/ en inde ronte mect ban bonoert en vijtitich.theeft veel Bee\$/ Bupcker en Ozangie boomê/ Citroenen/en Timoenen uan aloerbanoe foozte. gsen [apter tarwe/en alle dinglen van Spaengien/en Daer is groote quantitept ban gout/ende Dacr is niemant die bet unt-treckt/twolck een faute is ban alle oe refterenoe Ezplanden/ die ghebletuen jijn Con= Der ingebozenâ/ Daerder ecn groote menicbte was als fp ontoect wier = Den / en loo lu legghen alleen oen azem uan oe Spaenfchen doodt baer/en ick legglje/ Dat bet quacoc tractement/en exceffive atbept oir Get gout'tfelfoe doen: twelck blije = ken fal met een exempel.

$\mathbb{E}_{\mathfrak{e n}}$ Cazique oft Coninckgken cé notabn van een uan die Ezplanorn weten= =uck. De dat Daer be \$paenjacrts qua. men / vergaoeroe al zijn volck / en lecrde baer een pzactijeque/Teggen. oe: Dat de Bod oer Spaenjact was bet goubt/ en dat [p oock Dat louden vergaderen / ea merpent in De kibier / gbelijck tu oock yet wierpen in de Havana, Dit Coninct. ken Hatvey, dic alfo genoent wer: De / oede fweeren al jijn volch/ Dat alboewel Dat f $p$ allegador fierben louoen/ dat fp niet leggben louden oat fu get baoden/maoien [y om te vindé lyaer 500 baddê uptgecoept alle oe Jngebozenê uan Altim, Dat Jet Eplant ban Santo Domingo Is. loct Eplandt uan Pueriorico befft viet Steden en een 25iffebop/daer wert veel gembers uergadert /'tig t'cenemacl een 230omigaert; 'tlept van Santo Dorningo vall punt tot nes eptens punt twaelf leguen/en wan baven tot 
tot Gaven tacjtenticly. Die van punte uau Sananton; werter een Santo Domingo is leer groot/ lepot op achtien graden; yet was bet cerfte Kanat dat ontoeckt weroe inde ganticlye Indiens, en oock beb: ben lu bet gbenoent Elpagnola, ell van yaer ig ontorckt gewozoen ế co groote merelt:' $t$ Is beuclytbaer in bingen ban' taertriick/ bet vee; als Oufen ifter oberdadicly/Daer is glyeen 3 tugebozen gbebleben/ ende baer warê + gigiltioenen menícbế/ theeft vele kivieren/en [eer groot/ en ta meer dan leg Jondert leguen int ronde / en de andere refferende Rituieren zijn met gout/[u leggben batter gljecuonden merden fucken Coo fijn/ Dat lyet niet noodicls was Dat fugegaten wierden/ en weecb: Den meer dan Dzie oupfent bucatî: Baer is ontallick becl foet ricot/ waer af beel fupckers gljemaeckt wert : Daer is gember/ en Caffia-fiftola, veel bees dat bap? beeft; foo Dat Ẽ plant volck yadoe/Daer fou. be ueel gouts untgbetrocken loer= oen/en oock p:crlen. Het bzoot ban 'tzant is Yuca, bat wu nớmî $\mathrm{Ca}$ zavii, daet bert bzoodt gljebzoclyt ban bet valte Zandr/uan bet gouvernement ban.Venezuela, 'tis een warm 1 andet/en oock ift goet boo? De Stwarten/'twelck bet volck is Dat nu Gaeft in Dat Eulanot / al. buater nu wel siin twintich Duprent in gbetale. In De Stadt van Santo Domingo is een alertf. uuftchop/ en een Conincklijck bof. Int die Zee sinn Palvifreben/en o'aldergroot. Ite Zee-bonden. Ban lier werdt ontoeckt in 2.Dagen ban't Eplant ner cptant uan Navaza, een kleun Eulant op Navaza, en 17.graden/ en diclut bu dat is bet Lanzijca.

Epland ban Lamaica: $\mathfrak{A g e n}$ moet cozgbe dzagbente repren ban dat Eplanot op tuoen i om datter on= epnoeliicke veel ftosmen uan con= trarie tumben ziin / en bpcans op De ftreeck is bet uoozfepoe Eplant van Cuba, of Havana, foo bet nu ge= nacmit werdt / en antoeckende de rechte wegly geloopen/tot Datmen ontoecht ljet Eplandt ban Campeche, 'twelck lept ontrent bet ba. wet eplam Ite Zanda / 'tis Dzieljondert leguen int ronde/alle de Jingebozenen ziin nut Cysiftenen : Baer is een $23 i T_{3}$ Dom en Gouberneur/tis bet alDer. uatuchtbactlte zlandt/ na wepnich Dagen bert ontoeckt/en men count tot San Iuan oe Lua, 'twelck de $19 a=\operatorname{san}$ Iman uen ts uan Riel bifpanien / oaer zijn ueel buple leen in / en Daer 3 iin oock Diloten/die de fchepé inbzen. gben :'t (5eeft een bermaerde yen in 3ee. Het Tant Daer binnen de stats is de Stadt Vera-cruz, altwaer nu cruz.

al de bandelinghe is/alboewel bet cen feet Eplanot is: : Dan Dacr is oe Gaven nae de groote ftadt van Merico, Die met een rechte tptel ङegroote macly ghenoemt werden de groote fico. atadt/ nadié lqu beel grooter is ban Sevilla, fu beeft dertich ounlent Spaengiaerts/ en meer vzouwen/ en twee-bondert Duplent Indiane, en meer Indiaen fche Doumen / en twintich buplent Swarten. Het is bet booft van alle die uptgtyes baeude kijcken/ alwatr een Xertrbiătbop i\$/de Vice-roy, en bet $\mathbb{C}_{a}$ ? nuncklijek bof/D' 1 nquifitie en beel vermaerde Convinten en laerckêl als in de bulonoerlfe 5 tadt ban oe Merelot: bacr gbetenuertbept en oberuloet is als in Piru(als glje. leut is) Get Zlanot is lo vermaert/ an de ¥ngebozenê fo leerfaem / Dat ick eenigbe particularitepten legs glien [al.

Wet cerlfe is / oat fu groote eere Je Indians

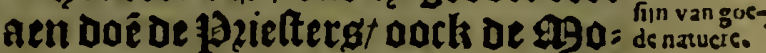
nicken / en Dic in eenige o?oze zij/ twelck gbetelyirt alter beel bolex vergaert / en fu de klocken boozen Isleppen / al eer dat fp vergaderen; en men lacb uan d'eene boeck tot D'anoer loopen de Jongers/en ee. nige Indianen, cn maké een Grupg/ en in be pzoreffien lefende de gebes oen/konie fp als bukang bet volck 233 upt: 
iutgaet/liatn to baer bootoen nes oet / legobenoe: sheloofe lu onte

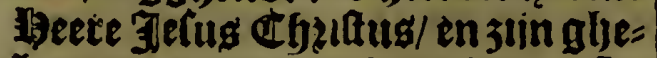
benedune $290 e 0$ et Santa Maria. So ceut de pelefter indet eenwichept/ en lu leggben/atnien. Een oock ac= compagneré Tu bem tot De lactck/ alwaet tp biooen / en bienen bem in al tabene dat bp besbett/entp gbrien getoote aelmoeffen / oatter replen ziin dat bet bolck mp gaf booz oe silten bonoert oucaten: den van al Dat was d'ookfake oie groote Chzilten Don Martin Cortes, Marquiz del Yalle, bit in bat ganticbe Ianot ghebied badoe: Ban bie extelente / en groote Sols Dact Chyifti wert gberept / Dat als by tegben quam een jzielter die quateot of goet was/ bleef bp taen op de atraet / en becktede bem niet voos dat hp vooz bp thas/en alte. met ginels bp te boet/en batte de de knie/ en kultede bem de banot / en bem nauolgboéde doê de 3 ngebo. renen/ be welcke teggben/ oat na= Dien be onftetffelijtete Viceroy bat beoe (Dien lo allo noemoen olì 3 lijn gtoote macbt) oat niet veel en ig/ Doen fu bat: : En voozwaer 'tis cen wonoetlijek dinck te booken ban be Fingbeborenen ban Dat Zanot/ bie dinghen die baer boozoubers ontfangben bebben Doos ttaditie/ gan de greotbeoé ban dele groot= moedighe en aloer-rbultelijekte

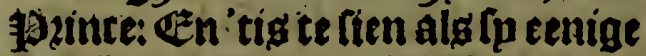
binghen boen / foo lu gbebzaecht meroen/mie haer oat geleert heeft/ fegghen lp: De groote Capitepn Martijin Cortez beeft ljet alro gebo= oen / en alboetwel eenigbe Viceroys yebben billen afooen eenigbe oin= Glyen/ Co ljebben?uniet gekannen/ oin dat het ban ote maten/ die die goede Marquiz baode gbebodet/ phrticulierliick fo als het was ban te koment to de dienti uan be 1 plie:

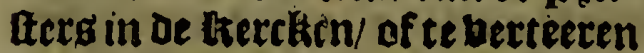
haer welbaren in de kofalijekbept batt of atercken.
Su maken oe felfoonite betloen of betibes ban pilupunen / bat ban eenige bie pringen van baer zijn ghekomen/ ghefien sbenceetho waren dingen die leer oelieaet wo: revi/fo oat fu baer bernuft toonen? oock ban oe plupunen ban klepne vogeltics maken $1 \mathrm{pecn}$ beelot van een rofectanfken / met al stjn bij milterien foo bleen/Dat fig om to fchilderen loudê een fetyoone frbils: octue weren/ en pp maken anoere aenfichten/en liebamen/en rleedins gben geljeel perfect/oat fo men bet net gbefien badoe/ men toube bat niet kannen gbeloolsen.

(9et niell Spaengucn is ech Icer Dart nist groot Lanot/ bant gbene dat ont= Deckt is/en daer wert ghelept/Dat= ter nocly t'ontoecken is fes niael fa. veel/nadien in onie tijt oe Antonio Efpe 0 , $\mathfrak{e}$ Luermaert Capitepniont. vecktede Liiftlyien 19zouintien/ Die roo Jeel Lanot bebuen / als twee Spaingens : Fp vonot baer poli: tijck bolck/en groote bruolckingef met ljuulen ban fteen / ban dzie of vier boochten / en bp maeckteder ecnighe op bplonoere plaetien/omi bolck tontoecken / en met alle die beeft bp ontoeckt thien Bzobina tien/ban welcke eenigbe foo groot 3ijn/alg beel Spaengicn/batr nas inen zimn/Merico, Muchoacan, Nuevagalicia, Nuevabifcaya ; en Guadia-' na; Honduras; Guatimala, Campeche, Chiapa, Guayaca, tnonder die ziin: vet andere elf / en bet Nuevo-Merico, en Nueva-francia, be welcke too Daer \$od mede gbedient is/ fullen bebolcht weroen ban be spaen. jactoen / alg oock al be reffe siju. Daer zitn oule Conincklijeke 190. ben/en Gorberneurs/ en Corregi: Doozg/al Spaenjaertg/en in anoes te bozpen zinn groote $39 z 0 \mathrm{booten}$ en in be bozpen ban de Indianen 3iingroote 19 zoboofté in of refloz: ten: 't $31 \mathrm{~g}$ al meelt gbefont zanot/ om oat bet roo getenupert is/Doozcake is bet regbenen ozoinaerlije in de \$gaenoéchan Iunio, lulio, $\mathrm{Au}$ gufto; 
gufto, cul Septembre, en Dacr loopt altoos een maitfon uan ecn windes hen foo froel; bat al tet oat oe son frljiint / cu bat in eć ferr beet zlant is/ [oo berter glyeen bette gevoelt/ en oe narbten 3 ijn kout ot / out haer cuglienc oo? fake / om dat de Son ontbzectit/en om bet moilfon.

Het is bet aloer-rijekfte panot

Broote gualtitue. van gout en filluer/om dat bet vecl פriinen beeft/ell ban Cocljinulle: baer werdt een groote quantiteut fuoe gletwecht/van China getroc= ken: Boic Spacniacroen Doê groo= ten hanoel/ en trafiquecren van D'cene placts tot $D^{\prime}$ ander/bzengen= be van elex wat Datter gljebzeckt/ en tgljene dat obertrfyiet boeren fu op een ander / baet Lan fy rifck wozden in kazten tijot: Oock 'tn Dit Lant; als ntede in Piru, ollt bat De wintten groot $3 \mathrm{im} /$ is oe bertes ringlye groot : De ectuare waer is goet hoop : en boben al oat yet een bolck is ban groote waerbepot/cn Dat D'een d'ander. groate troutue hout / men beeft glyefien dat bier imenfeljen fer boos quamen/ia als äoozdenaers / en bier foo veran= beroen / Dat fu nict alleen niet en ftalen/am Dat bet niet gbebzupekt wert in Dut Tanot/maer een grou= wel oaer af kregben in ben boorb. fen gratt. Dit to te berfaen ban ue Spaenjaeroen / oie baer altoos logeeren bu de Intianen, alg Con: quefteerbers / enl netnen wecly'tge: ne fu febben/alboecwel fu felfs dat gheven van loutere verefe / dat foo fu bet niet en gebé dat fp yet ebert= wel quiteren moeten/en alloo foes: fell fp vicentfejap te winnen met hacr midodelen. Fch fal een cafum bertellen / Die pacteerde tulfichen ế Cacique, genaemt Don' Gabricl ban Carabajal, uan Carargue, 'twelck ế Dozp is van Otabalo. Hu vzaech = or in oe tegentwoozoichept ban oe

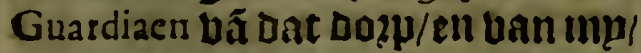
cu anocre perfoonen / Bozielters en zeecken/Dec apitepn Pedro de Lo- melin, Signos /in be grootite ffe = ect vaacts

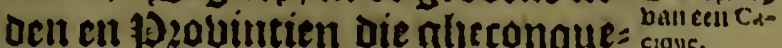
ltecrt siill in oe Derelt / boe lance Deurie de pilleringe? hu antwooz= dede ; Cacique, in elcke Stadt une oat fu groot was/ buteroefe acljt of thien Dagen : So kecrt lju weder = oun te fegghen. Weft bet fo wey. nicls tijots aloaer gljeduert? naat is een plonderingtye ban acht of thien dagben / onder de ellenoighe Indianen? loe laughe fal dan Dups ren bet bulwoonen/meer dan yon. Dett Jaren juzéfe ick.

In Dat lanot ts for veel bets! dat feer gaede fmactk yeeft/datter een petfoon is die fact tlyien Duls fent otuck Bees/uan Bffen in ooc fo beel van 20orken/ allecnlijck om De bupoen te ftierell naer Spaen. gien/en Datr igueel Lanots toe/cn fadich groene twepde. Daer tg ueel spaenirbe Cartwe/en פgap3/ en Beuchten / die van Spaengien Giar gbebacbt jijn/ als ban ecn Zlanot / alwaer ip yeel fimaliclick 3 iin. Daer is een boom/ genoemt os boon Maguey, of Cabuya, fo pzofutelitcti/ dat ick gbelien bebbe daet af mas ken/ oinghen die te verwonderen

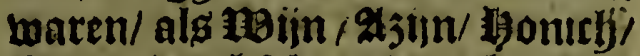
Garen/Deckirts/en dat ghenatpt wierdêmet de punten van oe 2 bla=

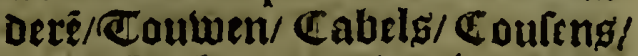
en Dat bet butang Dient in alles in cen bupg tot hootoxuftige oingen/ als tot leften/ đafels/en Earen/ om alles balt temaken; en be blaa Deren tot ticbelen/en andere bins ghen van de sgedicpnen / datmen gljelien beeft merckelijcke turen/ met be knoppen boen. Ez daer. om foube konnen gemaerkt woz= oen van or groote oungen van dat Hijck Lan Nueraespanja , een lyifto = rie fo groot / als oe grootfte die tot Jeden toe gljedutkt is: Jck fal't nemen Dat bet onmogelick is Dat= nent al leggben kan / meettendeel al/ fo bet uan mujn fiftozir nueten is/egundigbenoe inet ist guene/dat 
Fol.16.

$$
\text { Eyghentijicke Berchryvinghe }
$$

uan be groote Staot Merico, tot voo? fuffraganen of 23ittchoppen aen de Gaven ban Acapuleo. jijn uan Cuzco, Quito, Panama, twee c'neglyentith leguen ILanots/al be= ban Chile, fn Nicaragua. bolckt en bzedich / en 'tig een Ha. ven in De $\mathbb{Z}$ upd-zee , gbelijck als San luan oe Lua ig/in of Roott-3eel op $19 \cdot g r a b e n$.

Befchryvinghe van het Rijck Piru, "tvafte Landt Chili, en andere Provintien

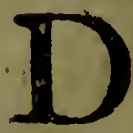
Etadt be los Reyes; an= orrg ghenaemt Lima, lepdt op 12 graden van de linie:

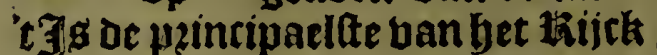
uan Piru, alwaer bet $190 \mathrm{f}$ is / om Dat in baer is de lit-plaet fe van de Vice-roy, en hier is gefonoeert bes Tonincx Laret / en d'Jnquilitie/en Metropolijt likertke / en d'Vniverfiteyt. De Vice-roy is de 19 zedirent uan bit of / Gouverneur en $\mathcal{C}_{\mathrm{a}}=$ pitepn Generael ban hace retroatl en uan ie Goven uan las Charcas, en van Quiro, en alles loo veel als raect tot bet gonbernement lo oos= loge/en be helooninghen. 'theft veretich ouplent ouraten tot fala= ris / en loo veele de Gosloghe aen. gaet/ cn bat baer toe hoogt/ famen De Bienaers ; ende Conincklitke Beffitiers / weroen betaelt unt oe

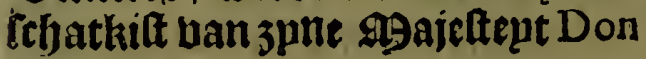
Philippe.

Jn bet Jof / en Cancelletpe van

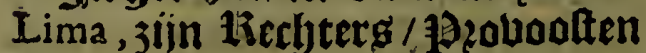
ban'thof/twee ffifcalen/een groo= te Deur-waerder/en Officiers/een Bierflyaer uan oe groote lieerkemeetters / ende een ander ban $\mathbb{C}_{0}$. numckliiche Officterg Leclyters: $: \mathfrak{e} \hat{\varepsilon}$ ander raeckenoe aen oe Cruzada, uan waer een Comuntlaris 1 / d die

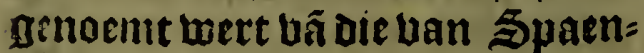
gien / oie provideert andere in alle oie 2 sifoommen / quiterende oe ju.

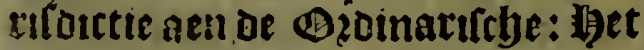
relto?t ban bet bof ban Lima be= Dact Deichonoert leguen / en beeft

cwee leguen ban Lima, eenen Tande Teer vlack / lepot de Gaven van Callao, dat de peintipaelfte ig an te bes van de $Z$ upd 3 ee/ alwaer of Viceroys gheluonoen werden om te de: pareberé de blooten met de fryat/ dre in \$aengien kontt/en in andes re octafien ban oozlogben / en fes courlen: Gier komen t'famen beel frlbepen van alle Contrepen ban oe zupd-jec / en oe rrafijek / ban= Delinge/ en comercie is daer groot. Fn de Stadt de la Plata, han De Danbe oe 3Bzouintie ban Charcas, is bet ratad. Hof/en De Cantellerpe/ alwaer de 19zedifent i\$/ ende de Rerbters/dic oock 39zoboolten zijn van 'tlof/ ffittael/en Coninclijcke officierg: Albier is de Cathedrale tercke met een zertf-bilfcljap/ Deetken/ en't capittel. Ban Charcas tot Li. ma toe / stin datebonoert leguen. Bm Dat het $25 i f o o m$ ban los Charcas rijck en groot foube 3i)n/en Dat bet renten foude bebben vall der: tich Duplent pefos; en merr / herft spne 19 ajeffept dat gbeoeple / met tonlent en welgheballen val den jBaus / Daer af makende dzle 23if= Dommen/las Charcas; Chuquiago, en Santacruz de la Sierra, oock deplende oe renten op defe maniere / oat die uan Charcas, Gem contentecre met bijftbien / die ban Chuqujaco met thien/ en die ball Santa-cruz de la Sierra, met die ouerigbe bijf.

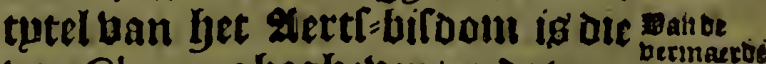
van Charcas gljegbeben/ende voo? srabe Pee Suffraganen, Chuquiago, Santacruz ${ }^{\text {tofi. }}$ oe la Sierra, el Tecuman, enel rio de Ia Plata.

- Stadt Potofi lept atythien leguen uan de Seadt oe la Plata, alwaer oe groote berch leudt/ die oe ganteflye BeEelt met filuer ber. uult beeft / en ban waer men bet noch beden trecke / als op den eer. Iten dasb/alboebel met grooter arbent? 


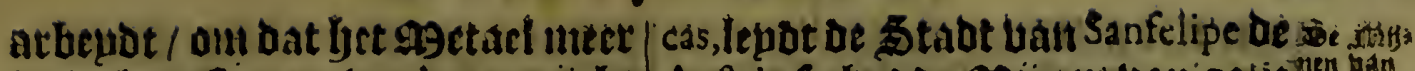
is in het Center ban't acrtrijck/ tot tweeljondert itadien mun ofte meer. Potofi ig die placts uan lyet meefte bolck van 't tiijck / oock tande Spaetiartden/als 3ngle= bozenen. De Indianen gingen Ders waerts te vozen Conder loon / enoe als inet ghewelt om te wetcken in oie grijnen/ ban uctrticly/t'lettich/ tachtenticly/bondert mule weech \$/ oock uan bonbert ende vijftieb/ min of incer / maer nabten dat of toat goets Lermaerde Bonde del Villar, Don

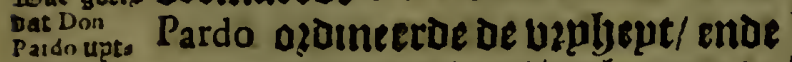
recbeteces aenwas van oe betalimghe van de Potofi,

Indionen, is alles met winlt vookt = glyegaen/als gyeleptot fal wozoen/ als wh 3ijn bermaert leven be: fclyepuen.

Het goeut boedel uan 'tfiluer/en oin dat bet daer veel is/ epfeyt oat oe wateren uan ben Hentel veoech beginnen i van liatftijt/ want met mannet die malen los ingenios oe ageta: wertck balt Ien : Bp Dic tijot gaet naer Potofi Mil Potofis.

ae jecrident uan las Charcas, of een Ziecljtit oin htulp te gljeben aen 'tghene te malen is/ en tot bet goet aouijg inct of Indianen, om dat de g9aent van febenario ende 99 ar= tio/bat is / als bet filber neber-ge= bzacht werot uan Potofi naet $\mathrm{Li}$ ma, if goedt oefpicheren / 'twelck beltaet in twee oinglien: teererte in oe wateren/('twelck ick nu ges reuot beblue) bet tweede in bet nuickfrlber / om dat booz yaer toes Doen beel filver uptgetrocké bert/ ende fonder dat ifter beel minoer. Eenigbe rewien ift lact gbelateni Dooz faute ban'tghene dat gbelzpt is/ ende ntet groote neerfticbept if

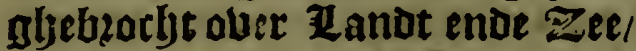

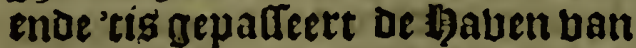
Arica, tot Callao de Lima, ende van bier be anoere reple / nae Panama, ende ball Panama tot Puerro belo, albaer be Galioenen legghen ban

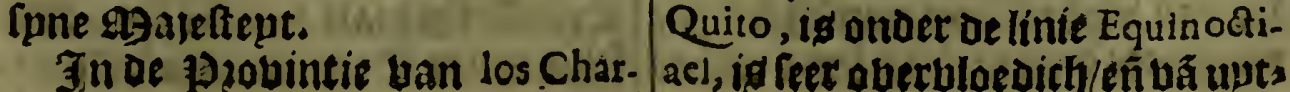
Auftria, [y Jadoe 29 ijnen ban gotit in oe tijot van Inga , oe welcke ges' heneficert zijn in feg Jaten in bed Cen oeele / nte toe bulpe/Fauenr/ eit. vetmuft bart Don Emanuel oc Ca. ftro,en Padilla, Zaeclyter vã las Char cas, dat yp niti te te Lima, enoe hy beeft uptgbetracken ech groote quantitept van filuer/uat ghegaen is tot bettminostingtbe lende net

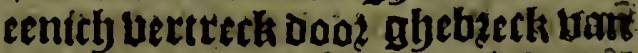
quickfiluet 7 :twelcks bet paincipact fondainent is / om bet: filuer var bet metael te trecken: gandere leg? glyen/Dat die ghene die dete faecke Cotide vozberen / ende fabozileren/ gaven de Indianen thiet boot bent arbepot ende bet benificeren bau oe Sijnen/ente d'Ingenios, enoe Dat fu dat oeden om Potofi te bes botiden dat fot niet foude onc: volikt twetoen / om dat oe rijck dodm vain. Oruiro ontultéte feer dic van Potoli:...

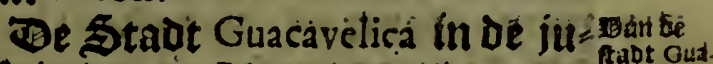
rifoictie ban Lima, ban baer be cavclica. rijcke agijnen zijn ban quick-cil's ber/ende daet to arfi met nuenich té van oati gbebaclt is inect oan. acht ouprent quintalen int Jaer: toan eenighe in dien decle ts bet gbefaelt / om datter centghe $2 \mathrm{Bet}$. gben ingbeballen waren/ bat ber blinot oen arbepot / ende beeft he ganticy Piru in groote lozgbe gbes ateit.

De Marquiz uan Montefclaros riende een foo al-gbemeene ende foo wichtigbe frbade / quam bet te bulpe al eet bp int goubertuement quam / ende was aen be voo? fepde Sginen/ ende met oe neerltebepot Die ljp bede / beeft bu den arbepot berbetert / ende Daer betot upt: gyetrocken met boope / dat bet wederom homen fal tot bet oftot weten.

Oe Stadt ban San Francirco de pauter 


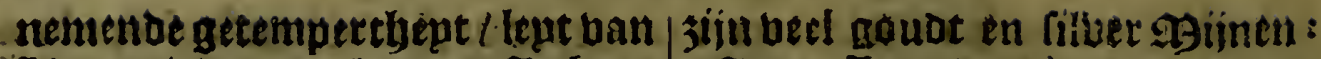
Irima Driehondert leguen: In lyaet is ghefondeert een eancellarie/ met een 19zefident/ ende Recbters/ bie oock waoboolten stin ban bet bof / de filcal / een gropte Beuc waeroer / en Coninckliche Offt ciers/'thett cen Catbedzale kere. beien 2 Giffopop/een Deecken tea pittel/ met een Collegie bat cen Semianum is : loaer refiost uan th of ereckt bem upt tweebondert en treftich leguert: Onttent of $\Omega$ tadt 3iin ueele bzandende 25ergen / Lan cuecuen uper: gen weet ban geen Gongher in oat Zanot/ baet boo? Feer bermeetdert werdt de genera: tue ban de zingehozenen / waer toe beft de meelte uzucht ghegbeben be pecaicatie uan bet begligbe $\mathbb{B}^{2}$. uangelium.

Santz Ez ve $\rightarrow$ In de Stadot van Santafe de BoBogota gota, ban't mienwe 'Rijck ban Gra. nada, ig een Cancellerpe/albaer be 1)zedifent Bouberneur is/ en $\mathrm{Ca}=$ piteqn Generael / die be bant bout aen de recompenfin /'t goubernement / en de ?ultuie. Bacr sijn oock kechtets / 'tzijn 13zovoolten yan't bof / een ffifical / een groote Deurwaerder/ende Bfficier\$/ een Me tropolijt Lercke / een atert-bir= [chop/een Becken/en tCapittel. 'i Jis'een particulier Clima, tweite inbzengt orfferenten / en diffentien onoer De loofoen/en in dertich of nieer Jaren in dat deel / en weraen fu niet gbefien in bet 1 of / of bu de Biflateurs/ten [p Doot/ghe hoept/ en met fchandael / cn in al ote tijot beeft oe Hact geondonneert/datter onderfoech gedaen werde ban oat 1)of/ renoenoe derbaerts ueel per= coonen/ en bebben bet niet uerhes: ghen/oin de voosfeude moozden en gebanclieniften. Sp beeft in haer reffort oe gropte kibier van la $\mathrm{Ma}_{2}$ dalen , van waer fp nederbaerts baren nae Cartagena, en Do02 die hoinen op de Coopmantrhappen/ en anoere dingben: In baer refrost 't 3 sen lant baer' tgoet koop t\&/ en uan goede ghetempertljeudt/en in de 19zolintie bar los Mufos jiin De 29 inen uan Efneraulden,'thefft voos Suffraganen de 23iftchoppen mic dit uan Bopayan, Cartagena, en Santa ${ }_{\text {dcic }}^{\text {Xnd }}$. Marta. De Staor bivolchtede en gern gyes conquelteerdoed/met bet gantfobe gerfs. nieubur thijck ban Granada, of dte. lantado Donl Gonzalo Ximenez, de Quofada, Zngebozen van Granado. In het liiick uan Tierra firme, is oe Stadt van Panama, alwaet de cancellerue ix / en de 192sfibent is Soubermer / en Capittpn (6e: nerael. 't loeft tiechters oat 19 ? voalten uan't thaf siin/ cmoe fils= cael/ente Goninstslicke officierg: 't loefteen Catbedzale lierclie/cen 26iffchap en Beecken / cn't E apit teli't I Iand is feer bect ende vorby tich. De Zuub-jee flatt baer te: glyens aen / ende tis uan waer de Glooten kouten met be febat ban Piru, van waetfe affrbept / ende werdot te Portcbslo ghebzocht / als waer of Balioenen zin / acbtbien leguen ziinder tuffelyen oe zupd3ee/ende de froozt-3ee/ de fimlte wrgb van de Karclot/ende ban Puerio Belo loopen de Galioenen nae Cartagena, ende ban bier doen (p yaer Bopagie nae oe Havana, uopt nae Suarngien.

De Staot uan Cartagena is int Dancaras

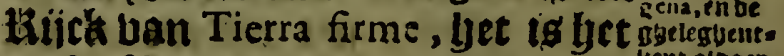
eertte Zlanot ban de Indiens, al: beptabsacrs

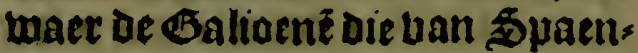
gien kamen bauenen/ende alwaer eenroeels fp ontlaben de Coops manfchappen die fu beengetw/ oock booz dele Stadt/als vooz yet nteu: we tiijch ban Granada, Die glyes bzacht werden tot oe groute zh. vier banl la Madalena. Batr is ecn Gouverneur / enbe Capitepn Ges nerael/met latichr-uolch/en bar = nifoen tot macljt uan de Stadt: Een Eatlyedzale thercke / een 2016 Icljop/enoe Derken/snde'te apit: 


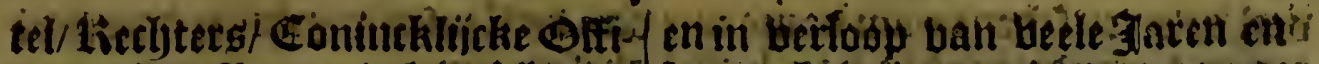
ciers. bet zande is bebolcket toet Chile toe/ ouphent en tweeljonectt Ieguten.

fronoe fiet niet getchíteoizent woss? oen/tot dat het Sod glyclieft beeft/ Datter ofe fefoltitie genionten unitus?

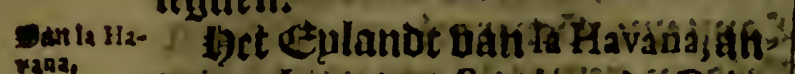
oets ghenotnint Santjago dé Cába--

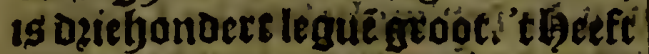

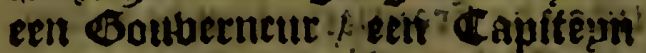
Benetael/ Toniscklijcke Offitie: ren / e rijelse voldk/ / en Barnifoen/ iterclite Caiteleni: Baet is ein eras thedzale ltercke/en' 25itfebop/een Bectien / en tCapitcel. Fin Defe Galuen ban la Havana- Komen be Saléocrien/en Dlooten/-efi.Sche: pen bail gantity tódíen ; où baet te uretialieten / en te calafatrecen/ om baer bopagie van b́ter te doa nace Spaengien'tis een repre ban twee gatenden / nuin of mecr / fón = Deraen Lanót te doen? Dan algfy op Terçera vertuallevi/tiointen fu ouî oat op te toen/en ouckbulg loopen Ip dat booz-bu 7 -ende verkennen Spaenguen. Dertich Icguen ban - ceaneel la Havana, begint de cancel van mas saba- Bahama, en jijn groote Areomen/ Cetr periculcus/ in oien bet Daer in be wint is / aen d'auber fude jo bet Lanot uan Florida, alwaer een Souberneur is/en volck wan 0 o:logbe.

out eqlans In fet Culandt ban Santó DoExazıo.2* mingo; glyenasmt la Efpagnola, is cen Cantellerpe / die pzifiaene is Gouberneur / en Capitepn Eene= rael $/ e^{2 n}$ tbolck int Barnitoen ren Caltelen. Daer jtin tiechters die

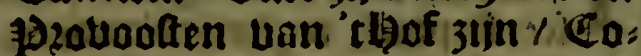
nuncklijcke officiers/ enoe antere Dienaers. Baer 1 ten $\operatorname{cet}$ etropo: litt lkerclie met een zutetr-biffetgop/ Beecken/en t Gapittcl: thecft boo? Suffraganen, aen be 20iflchoppen ban la Havana, Puertorico, en Venezuela, 'tis cen warm 3 ant / en van alle boecken berooft van oe Inge= bozenen / fu werden ghedient ban Swarten. Be Engeifelyen en fila= mingen plagben te gaen tantlon: neren get uolek ban bet Gulanot/ de $/$ de bebolekingtheri te beranoes?

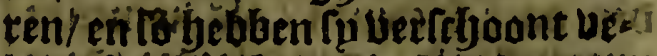

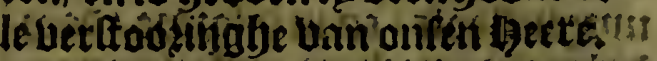

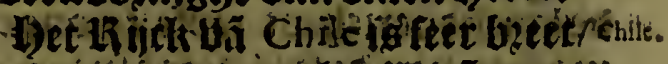

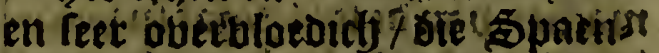

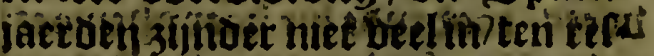
pecte bant of Indiaben! oie in contes:

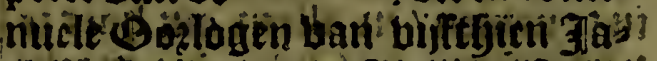

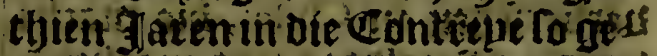
oeffent 3 in

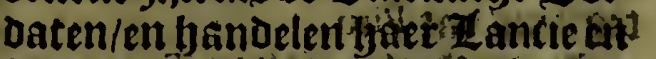

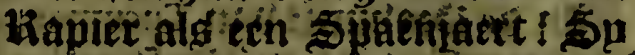
weroef ghefolpen mitet alle twapes

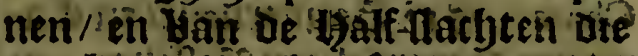
met ljacr buerglog détit waren / tu hebberi en groote b́boj paecoen/ en in êtn Erquádron atellentif fes of

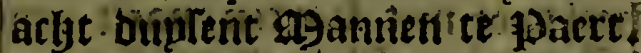
Daet is een Bzelioetit bain thof!

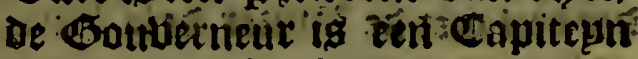
Genera!len Hechtert/ente onincs

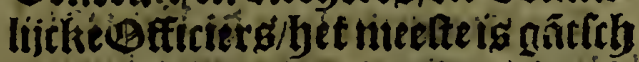
tat ben Gotloely gebzocbt: 'tlyeeft twee 25ifoommen/eeti in oe Stad uan Santjagó, en D'ander in la Concepion; het volck ont ghebzodit werde tot bulp rjaetlije na Chili, gaet ontwillitj en geowongen/om dat cu gheen bzubejot febben oni wever te keeren als fp willen / en yaer wett dock geen alteentic glyeo geben \% ent bebben gbeleden groote noot / foos beel angiet oe betaltngen en belfuilpen/ en to 1 ant is leer arm / cn fp'braffen thiet beel / meeff ote daet oienê zijn Blamingen om oat oe thoepten bie fp lpoen on epnoelijck nj /en oock ?tRifico, th 3ijn betopt/en oact is fualijck te eten / en noch diminer onoerbolts binglje / maét beelfyept ban bpans den/en minate befebermunge : 1)et

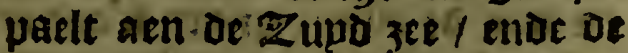
Stract ban Magellánes, ende als ee. nighe zoouers geconien sijw Do0 de Straet (bet weleke ig met aroot Rifico, cnoe moente / herliefenoie of bet 
beel volcx/ertelyepen/am.oemoep= ten en ueranderinghen uan lucb. teri) is dat uan Chile, etr ban fyet eerite Zant bat-fp ber kennen/bier ballen funaer Piru. Salgre buan. oen Daer incoulen / als Gomende. uptoljefloget / en in difozoze 10002 - De lanckouericliept/reple/en onge maclien van be ftract/gaé $(j$ booz= boets / roobenoe die baer gbentoet in be Zee conder eenigbe louen. in te nemen en alleen am baer te berberichen in Chile, ole macht ball de Boerclot is net maç grich tegheng dat Piru.

Eetlanbt: rspap rucucown.

In ve $18 z 0$ bintie ban- Tucuman, bat bet reltoxt is ban thof uan los Charcas, is ee Bouterneuic en $\sigma a=$ piteun Generael/een.2siffebop / en Cathedrale litercke/'tis een arm Dand/berlfecht/en unen repet bet beel boos met larren/paelt aen tolanot uan Chile, en aen bet an Der deel met oe zituier de la Plata, en Buenosayres.

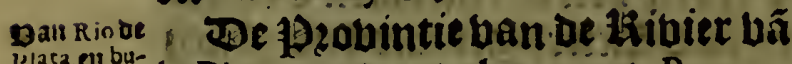
caes ayreso la Plata, anders ghenaemt Buenos ayres, heeft een Gouberneur/en cen Gapiteun Generael/ een Cathedrale likercke/ể 23it́tlyop ten Coninc= lijclie Bfficiers. Be haveri ban del Rio, de la Plata, is in be \$200zt-3ee/ altwaer veel felyepen komen ban Iiboa, ende ban Brafil : uan Lißboa wert fert goede bopagie ghedaen/ en feer kozt / en om niet te dif́com: moderen de cours uan de Indiens, werter glyeen ILitentie gljegbeuen yooz of Srbepen ien o coopman= frlyapuen. Ban Rio de la Plata tot Potofi, en las Charcas, zijn vierboll. Dert leguen / paelt aen Chile te 3atiot/en ban Dien ligaen werter abefepult booz de ftraet uan Magallanes, om dat aen de 3 poe van oe \$.2002t-3ee is Die kituier/ueel groo: ter dan be Nilus, en aen o'anber gubeis de ₹upt-jee / en bet eerite balte 1 and is Chile: (Bock is aen D'anoer kant bet zlanot oat de atact omuangt / Dat is bet ganot Inot

$\therefore 2$.
Lan de tieufen/van wace baer beel gljefien zijn.

In de 19zolintic ban Santacruz santaciuz, de la Sierra, is een Bouberncur/en Capitepn-Generael / nu tfer een zsifuam gemaeckt/een ban be deic uan de depling be uan las Cbarcas, tig op de f rentieren van of Indien van (Bozloge roeene zijn genoemt Chiriguanaes, en viandere Mojos, die aen o'anocre spoe ban oe cordillera leggben. De 2siftegou is in bat Kanot niet gyegaen / en beeft oock de catbedale tertie ntet gbefonoeert / 'tigeen arm Lanot/ en. met de Bozlogen werter veel: ellenotg, glyeleden / en fp fallentueel becoienen dic daer dienen i foo wel be lgofoen als de onderfaten.

In bet goubernement Uan Po- Popayar; payan, dat tuffeljen Quito is / ende tmeube laijck Uan Granaden, is

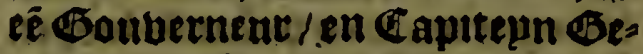
nerael/een Clathedzale isercke/ eent 2Biffchop / bet llant lyeett givinen ban goubt / en wepnich zingeboze: nen: teept op oe frontieren ban

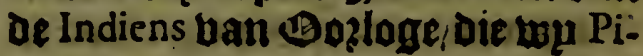
raos noemen.

Relaes van generale faecken, fonder te handelen yet int particulier.

T Re Indiens jiin twee Hepu' oefpaent blijcken/Die d'eene regeeren fljn - feer Atpoicly tegbens danoere.

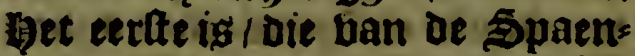
iaetcs/ de weicke gljebutucken yet goet politijek gouturnement van Spaengien/en fọ becommerç lyaer in be beoieninglie / en 'tbenificeren ban lyaer incomen ien opboedin. ghen/en boutwingben / waer toe trelpt oe diente en arfepot uan be Ingeletenen / om bat be Sppaens iacts in oe Indiens, nuet en ploe. gewnoch en mijnen/als in Sopaen gien/ booz al nemen fu booz niet te bienen 
bienten in vic Indiens, alwace fp ges is het getal ber talen outeunbelifek.

tranoelt weroen als kiobers / of edetlieben / en nautwelijctax faloer ghevonoen weroen een lackepe/ ofe Spaeniche 13aggie / oie fal kons nen pecienteten eenige treffelijcke pctenngie/oan alleen de Vice-roy, our ljet ampe dat bu beeft. Su weroen geappliceert bu cooplies ben en banoelaerg ren em te bous octr twinckels uan oingen onteten/ en bankleederen uan Caftilier, en van'tzant/ en am te bandelen/en contratteré onder de ingebozenen of om te weien overlte nanio'm: conteng/ en quartieren ber soldat: tew/en in be gout enftlber gisituen/ en in d'ingenios, en de reoen ban oie aenlepoingbe is / oat als fraer gijenegentljepe / en indimatie bacx baet toe bzengt/ oat fp baer betrije ken/keeren fu weoer naer spoen. gien met baex incomien / ban lwex = Den fu geappliceete tot de officien cn beoientrigben / die ban oock oe imeste coinmoditept bebben ain viel te taintretr.

De aevu: oljeke bail be Zngert trilen.

Be tweede Ziepullijeke is ban os Indianen, oe ibelicke ontfangben yebben met goedelienteeckenen oe predicatiel en olloerwofingbe van tyer fjecligbe Cuangelitum / en met ocuotic en perferktigept in be fiere ken haer begeben an onderberen te zijn/en gljelectt/en tot al bet dat De GoDg-Dicklt aêgaet/maer meer in b'cene posumtie als in o'ander/ Dooz de fozglse ban de 2biftejap/en noede Dienacrs/ bequaem tot de predicatie/in de moederlijcke talé. Enalljoetwef oatter in oat Tiijck cene algljentene is/Dat be tale ban oe Ingats/oie eninc was van die Riijclielt / als onoer ons be latijn. febe tale; foo it bat die Ileraers uict alleen oien behoozen te weten/ alsoock oe moederlijeke tale ban iske plartfe/ om te mogljen biect). ten en dienit te Doen: En allooder alfulcke 3 ?ouintien en 3 andé beel $31 j$ /en co uerte gbelegen ban een/.
De Indianen zijn ecu flecht uole/ pet geboer. ban klecnecouragie/tuepuicly aen, sutbus fients/en actiifbeut/nocly ellendiger ban be in als or Joben/ tis wonoer dat fué oinck wel Doen. Jneenighe otn: gben toonim in baet / als ine celes beten ban Sacraments Dacb/uan Foefictjen / en Sint Iâns Dach / met groote wackerhepot Gan danfen; mutric en praceffuen / en in be laets: ken gebsupeken fu alle bie bingent om bet feed meer te folemmiferen. Bp oe feplighe Donoerdach wer. oen fu generaliick gbecaftijt / fp onderbouden wel baer sheb?oe: dettebapjen / en op ben wach uan reeckeming be boen ty een al-glyes meene bekentenuffe / uan loo bcel goets fu in baer bupen en veloen bebbev: Suj begraben hare doode met efferljandé en 2 sillen. Taet= entesen febben fp dace andere/bie reet psetudoiciabel jiin.

Znt generad jtin fp feer bernuf', seat baes

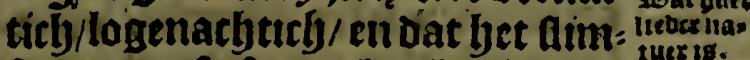
fte is / oat [p leer gebzecketijek zijn int Dzincken/ en baer bol te fupuế fonoer dat boos febade te bouden, bet welckefy beftellen bat fp bers gaberen in bepmelizke plaetfeny alwaer [y bluben een bach / twee) orie / en etn weecls / jae oock meer/ etende en ozinckende aberdadiget dan de Juptichen / bp gaer bou oende baer bzouben/ende bocks: ters / op bat fp fouben bzengen pet om te eten / of te ozincken / en op baer tiit naer batr bupfen fouben gaen/ en alfo fp bier barb en nacht 3ijn/en berlielen al baer oozoeel/ berballen (p in groote conoen / cn bet is bet gljene / daer die ban de Futtitie die meete moepten am doen om te remedicren/om Dat bst gbefrhiet op die boet/als oe $\$ 900=$ ren baer bancketten bouden/airoo oock op de folfoe manier/zijn fu veel dagben met malcanderen/fin. gbende en banfende/en feggen bat bet gyebeden zijn/ die fu boo? Jaer ${ }_{3}$ Dooden 
uooden doen/en ick fegghe dat bet Jet eertte leten ban de perfoon/bie Tchandelijelie Offertianden 3 itin! die fy aen [jace libende lichamen Doen/ waer medefufe bederuen/en inakenle gherect tot alle fieckten/ nls ghercet is.

3aat reabe, 2ulto de Indianen yaer orcuperen Dalter riffi met eten en ofincken/en appliques viist bane girb bane ren fp Gacr tiet tot den arbejoty want be Ind-boos dat bet een epnot beeft / en de

noot baec daingt i of tot bat [u siin gheozeuen/en gbepart wan hare Oberitei. Hat ampten of hon= iten bat fu glyehzuucken / Doen fp ilit groote vernufticheyt/oock lyet teien/en'tteljeppen/maermen beeft Jet yaer niet toegbelaten te leeren. Onoer de Indianen jiin bele felya: Deliicke mentchen / als Salf-alach: ten en Swarten/en 99 ulaten/on! bet quaet tractement datme baer Deet/en alljoe bel bet berboden ig/ ôijnocr die al baer leluen lanck baer qualiick fullen tracteten.

t): tribuest or lndianen gijn beebonoen te

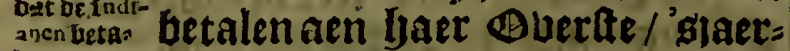
tar. .... lijer een leecker tribupt ban filuer/ rockloen ell andere dinglyen con= fozm met oen tacki al oie (joben yebben: en de belft dienen/Dat is twee $\mathfrak{A g}$ ainden in 'tjaer/ In de per= Tanele Diêt / in fulcke Contrêne als Jet of fal ozdonneten/ to wel o'ế als banoer betaelt van zijn ach= thien Jaren af tot uiftuch/ on bat fij te bazen / en Daer naer niet ber= bonoen zijn. De Coinmanocur trect die rence/ of tributat / Dat bemi gljegeven wert boo? twee levens: tot vergbeloinge uan3ijn dienctety imet lafe / oat lyu ljace noet onaer: houdort ím oe jecr / en betalen den

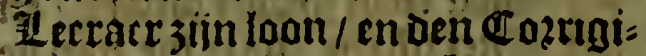
Dooz jutu falar(s/ cn de tbienden in te kerck/ oat alles glyeozoonneert eli berclaet is bu de ligeten lop de tact die daer gljegreten wost.

Bergut: Indiens Leroen gecanman=

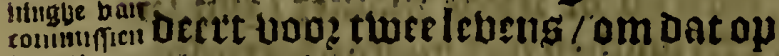

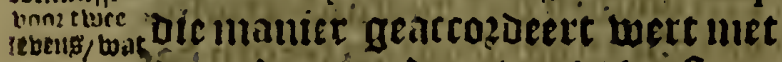
Let 16. of onden / en bacr werdet werfaen oe Deulinghe ban be Indiens bela= ftet wort/en bet tweede is wan jijn Soon oft grootlte Docliter / die daer infuccedert/en loo Daer geen Bzontue ig/ nadiê dat die twre les beng berbult 3 iin / keert het weoer tot fine grajefteut/en de befittinge werdt abenomen op sijn naent/ en wert gbeleut in siin Conincklicke fclyat-kuft/ tot datter ế ander per = foon gecomimandeert werot / ende oat raeckt de Viceroy, in al bet ges ne bat in 3ijn refloo?t Uaceert / en aen andere 5ouberneurs/en be: nerale capitepnen in bare jueis. Dictien/lo ber niet en is/oat [y dooz oe hate gecommandeert wozden/ bet welcke hoult te boozen oe toes Iepoingbe ban impoet antie/ met Cedullen ban verfoeck / met bet wetcke de Bice-rop/ ende de Gous verneur niet en konnen difpone= ren ban die toelepdingbe / om dat bet berfocht is. Sijn g9ajelfept en de taet doen beientfrijap vooz eenighe lebens meer / aen oe $50=$ nen en be klepnen wan de gbecon= quelteeroen/ oaer in gebzupckenoe bare goedertictentbept.

De contractatien en winningen wante

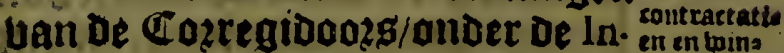
dianen zijn leer dayper berboden/ ninggeis om dat fu fo contrart en febadelijck ziin voo? yaer bekeeringe en leere' om dat fp ozomaterlic baer becom: ineren met bleeden te inaken/en in andere mercken en arbepoen t $\boldsymbol{\mu}$ : ghentlijck vooz of Cozregiooozs/ muttenoe de tijt om te maken baer werckê/en incomens/ oiu Gact tri = buyt te betalen/Dat een oo? [ake is/ Dat lu ulieden / en ablenteren baer ban Jare Dospen/en kaulen te be: latten die / in de lwelcke fp blutuen/ niet alleenlijets in be períonele ar. beuot / dan oock am te betalen oe tribupt vooz de abfentê. De liaet/ en de Biceroug bebbê 19erten ge= maeclit / en Ozoounantien / die fp booz alle dingen belweeren / te oul= actibat: 
van VVE eft-Indiets.

Dethouden/en tha te roment.

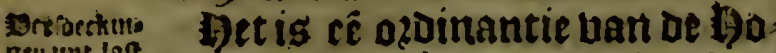

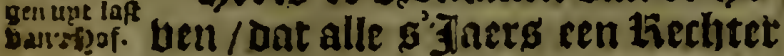
utut-treckt te berfoeckê zijn rellogtt on te befoecken be deplingen ban oe Indianen, en fien boe fu getarcert siin / en wat tribuptel! [q] Getalen/ en oftp meet of min konnen beta= Ien / conform ittet de natuetliiclie en felfs gepzactifeet oe butteljten die lu bebben/ en oock oin t'extuleren een algfiemeene gravainen, bat of levenoighe niet en betalen booz oc booben / ae ablenten / en die belet 3ijn'en ouden booz ianghers/ oock om af te Doen oe gravamina die fu hebben ouer oe Sparnjaerts / en om te ueritaen yaefu onderweten sijn/en gheleet/ en hoe bare open. bare tonden glyettraft jiin. Te Viceroy ain 'tgouuernement plafls te fenoen perfoonen die or werclien vifteten/en oc glyenteenfchappen/ en ont dat de Jngehozenen louden ontlati wozôि. EEn tot het lot uan Quirofenot De Marquiz ban Montes claros, Don Diego, Vaca de Vega, cen perfoon uan voldeeninglye en veritant in alleg.

wan be ' $\mathbf{J}$ iseen laeclite on op te letten/ sit. ten / kinderé ban de §paenjaert\$ en or Indifche B gouben / bic in bat 3laot loopen als Bagebenden/een vertosc hoop / Die hem ntet begeeft oin te bienen/ nocb oock on eenige Machanique ampten aen te vatten. Radien be ledichept is oe 29 oeder ban alle ghebzeken / foude bet een Taecke weren daer veel aengelegen is / Dat fu ghedwongen Laaren bat fp wertkitedê / en Dat fp baer biga bé/of inde boutwerpe / of in publije: Ke ampten te Doke /ont dat fp Loozts nadien fp in yare brkommeringbe siju / fouden kounen afoljelepdet weroen van quade inclinatien/ Die de Bagebonden bouben in lo nicu loe llanden / bet foutde wefen een goet goubernement van be Riepu: blijcken te bebben/meer officien mn
- Ofticicts / als tot tentaas bain het Litick/ en die gene die dact in 50003 nen : Fiet in wert niet geoaen vait

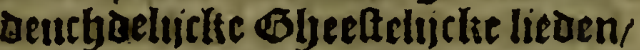
en oock wacrlickser/om dat f $\mu$ niet en bandelen ndeb bact becomume. ren in errliicke beoieninghen/ onet tp bel wat fouden berdicuen / als ip wilden berbonden zijn.

De Dsajeltept beeft gljebzont: oun be

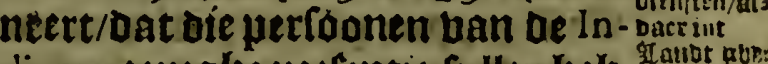

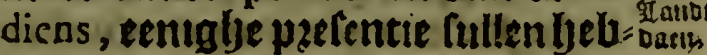
ben/ bie fullen cpefeben in oe $190=$ bet/ / en doen haer belwijs net cita. tue van de ffiftacl/ han baer bet. diencten/ qualitepten / en dienten/ en bat bet loof infoemure van hacts ampt fecretelitck / intet die perfoo: nen/oie baer frbpuen fullen van be meefte qualitent en troume te jiiny om te veten de dienltê ban de ver: loonen/of fp gbedient bebben bare grgajeltept in etnighe occalie / mot lyet welch confozm zujnde / fendet jet boes dat het lecreten befloten blutue in oe thaedt / ban oc be!oo= nunghe/ die ghedaen maet weroen aen alfulcke perfoonen / bie bet bes yoozt / ban beradet de liaedt met fijn gagactept / op be maniere ald blijtke. Die ghene die ban Indien komen in Spaengien/hebben pzes tentien/ lu replen over lianot ende Zee/azie dupient lequen/ vele ver: anoeringhen ban lacht bebbendel met een grout rifico ban bet leven/ en met exceffibe oncoften ban bast midoden.

Get Concilum te Lima gebou. Figet giteta den/int Jact 1583.in de derde artie/ katrs: int tweentwintich fte Capittel/Dat gbeconfurmeret is bp zijn teplic: bepot/an zijn glajeftept ghelooden beeft te erecuteren / Jeeft geboden/ oat alwaer tweefonocrt Indiche tributarifen fullen zijn / oat fu met yaer Buders / Sonen en bious. wen / Duplent zílen 3tin / Datmest Daer ftelicetn Xeerare/of foliterer/ en oat weter bolbzocht in ernighe 3) 


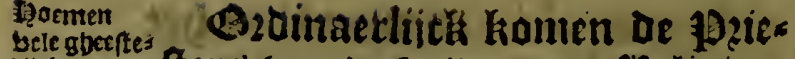
yjeks yers tters tuan be Indiens, om Religicu= roanert bers fen mede te nemen/op de hoftê ban willal. Iijn \&Gajelteut/en bet is bet aduiig ban bele 2Biúcboppen/oat de kaet handele niet de adoers ban be $\exists e=$ hupten/datter veel berwaerts gaen in elcke Bloate/ om dat het groote arbepders jijn in de wijngaert des Heeren / en ban oe belcke meelt de 2Jilteboppen ghebolpen wozden/ in be pzedicatie/leeringe/en onder wufingbe ban be Ingebozenen/ en bie oock meelt bolghen de 7 ngebo= -renen Spaengiaerts / in Sermoe= nen/in 2siechten/ out den poet unet De velcke fu baer appliceren tot de bekeeringe van oe Indianen, en tot be anberwuringhe/en opbotoinge uan De Sonen ban de Spaengi: aert\$ / en met de lichtichept Dat โu de tale leeren/en de overbloedighe butbt die fp ghedaen bebben / in alle plaeten waet fy zijn. 3ck en wil in tegenwoozoichept niet upt: Aupten de groote verdienlten die andere refterende theligieulen ver= Dienen in baer ampt/ van de zielen tot Bod te bzenghen/nadien die fo profutelijck zijn/en ick getien $\mathfrak{b} e b=$ be mannen/bie fo bolcomen en van too groote aentien waren als $1110=$ gbelijek wag.

Van de Bifdommen, en andere vele faken concernerende tot het gouvernement.

Sanke kerckeligcke cettuett.

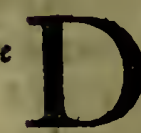

eEterckelijcke renten ban de 2 Siloommen / ae thiendê werden int gros gémaeckt en werden in vieren ghedeelt. Wet eerlte beboost conform oe oyzecly= tingen/aen oe 23tffejop: Het twee: De/ae Deetken en bet Capittel/bet welck gbedeplt wert op oe gewen= be abewoonte/dat bet de Deecken ontfaugt/ten tefpette van bonoert enviffich / en tot de dignitepten/ ban ljondert en oertich/en tot de
Canonicken ban yonotrt. Dan bie andere tbuec declen / van die boos. fepue vict / uan bet grog/ weerdicly negben deelen gemaeckt/Diefp novenos noemen / oe twee eerften be: yoozen tot june gajajetept/ uan os welcke hu plach belooninglien te boen aen oe efatbedzalen tot lyare wercken : Het neglyende decl en oe helft werdt gbenomen tot be fia bijick/en d ander negbende oeel en oe belft tot oe yolpitalen / oe bier oberighe neghende declen werden ghefmolten int betalen van oe $\mathbb{C} a$ pellanen van de Cathedzale terc= he/tul de Sacriftie, Muficiens, Orgeliften, Dienaers/ en Jnectjten van De ferck: De oberigbe weroen ge= appliceett tot of frab?iick / al oit wert betaelt natr de (O) Dommantie uan be zoiftrboppen.

Eenvan de faken die meeft noo. oberteg:

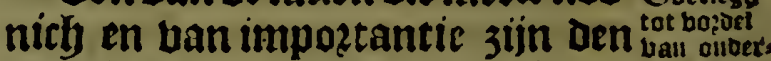
Ingebozenen/ ig/ datter 3 ijn in bet tupduge os principael ban elck 25: $100 \mathrm{~m} / \mathrm{Semi}$. narien uan ber Caciquen Solnen/ en lan oe pinciparlfte Indianen, tot yare natuerlijcke Bolítie / tnoe ouer-natuerlijcke / en tot bare bes kecringe/ en bier upt fal uptfpeup= ten meerder kennitte/en liefoe uan Get bepligbe Eutangel!um : buron: Derliick fo baer lieder inftructie en onderwplingbe bebolen werot be Baders van de Zlefupten / om dat fp een Conderlingbe gave beblen van onfe beer tot de diente. De fundatie/en tobene dat noodich is tot anderbout / coude konnen kos men uan de incomeng ban de gbe mepnifhappen/of uan oe Caciquen relfs / fonder bat oe nudoelen ban be Coninck noodicl $3 i j n$ / op dat. ter nect ontb?eke / of pet wat daer af berteert berde.

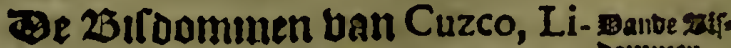
ma, en Quito zijn leer groot/ en leg = gen feer verae uptoefpzept cll ver: f́ljepden / Dacr sijn leer quade to $\varepsilon=$ ghen / Dat het onmogbeluck ts oie te gouberneren/ on onl te gấ boDz 


\section{van VVejt-Indierr.}

een prelact. Wat ban Cuzco, beeft [ [ebop van Quito, om bat vic ltadt meer ban tweebonoert leguen / en it be beete meer dan t'febentich. Dat ban Lima nocly eeng foo beel/ Dat ban Quito oocki lo teelen meer/ en om dier oofaectie wille/tyebben De 2Biff bjoppen bant De Indiens aen

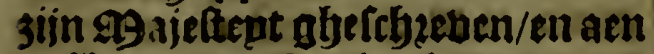
be thaedt/ boe Teer bet fretaemt Dat fu ghedeult en gerchepden worden/

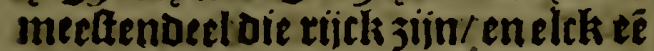
fal komen te bluben op twinticls oupient pefos, Eurilo tyet een lake is bet goubernemêt aégaende / wil ic Daer op niet blplue / alto Dat mijn

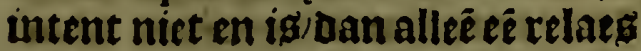
te geve ban al het gene in Die beplê.

Daut be Zurfoo beteen Bodlijets recht isl tyruber. en gljeftelt / oat alle be echziftenen tyjienoen betalen uan oe ueucbten Die lyaer $\$ 0 D$ geeft/ ban bare bou= wingen/en arbepoen :'tz's alreede cen coltupine in de Indiens, in be meefte 1 souintien / en in de 25 if = Dommen te betalen ae thienden/ of be belft uan bien / alfoetwel lp bet laten nae baer wille/ bat coo booch loopt / als die gljene leggen die bet niet $\mathrm{en}$ betalen/ om dat bet loo een 2Barbarifth volck is/bchooztmen te glyebreden dat de coltupme on: berhouden werde / en dat fp gene= ralick gfiecregten wozden van be Indianen, alfe ontfanghen werden vall oe Spaentche/Dooz bet welcke De faeclien uan be leeckeien be be: dienunge uan de Gooliicke dienta gaen fal tot grooter aenwaftingye en vermerrarringhe.

Din oe De 13zouinciale Conctlien wer: contilin. aen getelebzeert in Piru, van reven tot feben faren/op belcke be $9 \mathrm{e}$ -

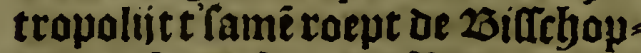
pen/en \{p reulen ober Dant en ZZZe vire en Cefbondert leguen / alfulcke weghen / Daer noch koetren nocly Zitjers te yaffe komen / fu tepten ulet grooté arbent/coften/en rifico.

canbe vni- In eenige beclen ban be Indiens, jijn Vniverfiteyten vertoclje glye $=$

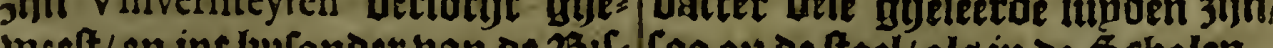
lept dzichonoett legatn ban Lima, en in baer refropt beel $\mathcal{L}_{\text {ando }}$ be: llact / en iilt particulier oe gottuet nementen en Pzobintien uan Quito af tot bet ualte lant toe/ ch Nuevo Reyno, en dat bet een ghetont Lant is / bã goede getempertbept/ reer bequaem om te tuderé/en t' ar: bepoen/ ende dat int kilick alle lif. tacht aloerbeft te koop ig/ende als niet gefondeert en wierdé alle wet: tenichapuen $;$ bebbê fp begeert ten minften oe Grammaticam, oe linuns Aten/en de Cefyeologie/ de cafos ban be confrientie/ en be tale uan oe In. dianen : De welcke met viet of unit ouplent oucaten aen renten / in oe Indiens, die aphouben fouben in die 1) zobintie/foude konnê onderfycus dé weró al lyet gene dat gefent is̆:

De Viceroy Don Francifco de To-

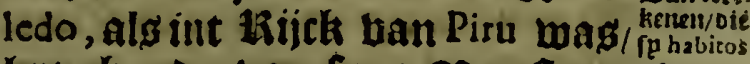
kreech ozoze ban fpne g9aleftept/ nuemen: in oe gbelegentheoen uan confides. ratien/ fo wel in 1)aps als in $B$ of: loge / eenige pertoonen die bet ber= dienden baer te eeren met teeckene of habitos ban be ozie ozoenen / om Dat oock die van die lfe dé alg pzin. cipaelfte ban Lima, Quito, Cuzco, Charcas, La paz,en Potofi, en ban al. le de reften dat uerbolgé met baet berbintenutfen/ende de relfe geani: meert werdé tot deeere om te die: nen/en berdienen lo groote eer/ na diē bet leecker ís / Dat de beloonin. glye is een prickel ban oe Deucht.

Ban De Ecclefiaftique ftact/ oock Dan tserss iu De 19tiefters/als in die/Die eenige deugderts Diboze bebben jijn bele ende gaede fubiecten ober al/alwaet gbepzofi. teett werden beele wetenfichaypen en Detuchoê / en alfoetwel ozoinaet: lijck bet frbijnt een routwe fake/ten tefpecte van wertentrbappê / in bet bandelt ban oe Indiens, to ift noch: tand wat groots boos die gene bie bet niet gefien bebben :'t Datter bele gbeleetoe linoen $3 i j$ /

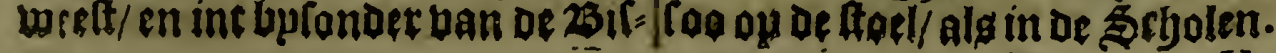


Foli26. Eyghentlijeke Befchryvinghe

Van de grootheyt, rijckdom, en de groote Chriftelijckheys van nieu Spaengien.

xall Aicu ลẹatigié.

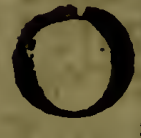
פ) op een niea nu te Gande: len uan De rijckbom/grooto bepot en Cbzilteliickbepot ban bet Nueva Efpania, tal bet noo. Dich sijn Dat ic mu upefpzepe/ meer Dan ban Piru, om dat oe 3 ingebos renen jijn ban grooter inclinatiel meer leerfaem en habil, dan die ban Piru, nadien bet ghefien wozot in dingen die ball daer gebzocht woz: ben / ende laet booz exempel sijn be beeloen uan plupuen (als ghefept is) die oin be berwonderinge moes ten berwondert werden/en ic ach te booz $\mathrm{mu} / \mathrm{dat}$ bet een werck ig ban'smenicben banoen/bet ts bet treffelijckte binck van allen / om batmen ban plupmen alleen / lonDer eenighe berwen baer bp te boes ghen/maken lu aenlichten/ en met alfulcke bolcomentbepot/roomen't int bleen befichticht / men louber ober vermonoeren / en 't toube bus: Fants ongelooftijek zijn/alimen bet fiet. Daer naer te regghen/Dat bet fo grooten 2 Landt tg/allulcke Zije ken en 19 zovintien / en Dat fulcken getal ban balck go geconquelteert beeft met fo wepnich Spaenjaer Den/ int' twele de bermaerde 19 zin: ce Ferdinant Cortez oock ts te bers wonoeren / alboewel ziin groote Eljzittelijekbepot/ ende puer tot de Dientte बrods / en zijng Coninn is een bekende fake; dat be Alimach. tigbe hem verlichte met 3 ijn $600=$ liske wille. En oock regghe ick reflogt beel leguen yeeft/ en berga. Dert bele 19zobintien: Lals die 3ijn uan Merico, dat bet pzincipale is/ Honduras, Campeche, Ciniapa , Gua. jaca, Mecheocan, Nuevagalicia, Nuevavizcaya, Guadiana, Guatimala, en andere beele / die ick om be lanck: bepot te blieden niet en berbalef maer batfe meett alle ziin bebolet/ en be alder-bzuchloaerite / en alles goet koop / gbelijth te bozen gbes centig.

In Guatimala is een Conincle ssectore lijck 15 of / en baer 19zentoent is een wuatime. Gouberntur / en Capitepn Bene: rael / en commanoeert ober be Indianen, ende be Recbterg 3 ijn 19 ? 0 . boolten ban'thof:'theeft Gonincs lijeke Officieren/ een 2siftrbop/ en tCatbedzael Deken i en 'tCapit: tel: \&znoe dele 19zouintie bergaoert Gaer reffost en andere / en' to 1 anot is goet/en alles goet koop/en ticlf: de dat Merico ig/gelijck oock te bo. ren berbaelt is.

In Galifco ig een Conincklijck Ban Galie Hof / oc lelfoe die in Guatimala is/ ulet een 39zefident / en é capitepn Benerael bie commandeert / theeft kechterg / Die ooc 1920boolten uan thof 3ijn. Be 2Buficbop/enl Catbes Dzael / en al die bobe en de reft ban oe 19zobintien zijn als boozept ig. Be gouberuementé ban Lamai- Berbeel ba ca, Veragua, en Nicaragua, en andere pe andoere bebben een Bauberneur en $T$ apis tepn Benerael / een Conincklijcke lititen (b)ficicts/op de manier bã bet gouvernenient ban Popayan. theeft gimenten eenen dcele ban gout/Die de ozoinarite zijn/aen ans bere ban filber/en andere metalen/ als açgeroert ts. Bet nteu Đpacn= gien is too lanck en fo bebolcht als Piru, en bzeeder:'theeft ontallicke bele Ingebozene ébittené/ en an= oere ontoecktede / die op bet point siin ban geconquefteert te wozoen/ gbelijck als bet nieu Merico is / en anoere ontallichbepot van 1920 . vintien die gefien en ontoeckat juju/ 
en meet ban de belft jijnoer gefien en antbeclit. 3ck legge van oe lie= jublisiclte uan de Jungelioenen/yet Felfoe bat van Piru, ende die van de su.uenfebe/oock bet lelfoe : Cen in Piru,en Nueva Elpagnia, enin de res ite wert bet gheregeert/gegonber = nectt/ende anderfyoulden met leuen armen/ of 1eserclotiche tacken/Die een berbotgenlichaem maket/ons beulicly Catyoliic geloof bet looft jlinde/aie dele ziin: Dozloch/z]nto : inen /'t Goltuetnenient / Jultitie' Bereeringlyen/Stacien/en oe co: nincklickte $\eta^{2}$ atronagie.

Deconums In oe Coninckliicke Kaest ua!! bail Indien, be Indiens luerot int gljemeen glje= banoelt / en int butonder van alle Die materien bie oittinctelijck ghes beult jtin in oe anoere tiaden ban iin S9ajeltept / om cat die in die trijcken van een qualiteut 3 ijn/ $\mathrm{cn}$ inde 19 2ovintien ban Indien, wiens Stenniffe en derpacho ghelzocljt is tot die Conincklijcke Haet van oe Indiens. Ẽn oock die dingé die aen=

De ara:t ban Stact. gaen met reden de katet ban Staet, als jijn oe bebouoinge/ver felerin : ge/en bermeeroeringe/ of aentwag ban ae Indiens, nieutwe conquelten/ nizulue inffellingen ban liecljten) De gedutricbeut uan renten/en de commnnocringben ban oe Indiens, ilemende de manier / en faunen-lit = tingle met oe Commandeurs die die beblet / Die de Lantboutwerpe aengaet / $е \bar{e}$ particuliere pient van oe Indlanen, twelck cen is van oe àloertwaertfe oingé ban oit tînck.

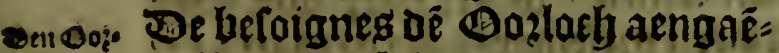
locy. de (bu De tocleke ecnige onoerloos jen van ae Raet wan Bozloge van tune sajeftcpt) am oe 3eplegets te verfié / ztmaden / Barnifoenen/ Genctalen/zadmiranten/9gaeltar del Campo, Capiteunen/ en anoere - Officiers/ fo bel te 7 ande/ als ter Zee/oock booz de Conquelten/als oock oe macht uan oe Indiens, en bare J rontieren ien voo z of beplic: bepat pan de repren van de atrma- en Blooten uan oe tocht op Indiens, en oun te ljandelen uan oc gheltadige ootlogen/die in ectige trijelien altoos getsandelt wetoen met oe Jugefetenen/als baer jijn int Lijets bat Chili, en bet Goubers nement uan Santa-cruz de la Sierra, intet de Indianen. Mojos en Chiris guanaes, en albet gene oat is over de andere fipe van de Cordillera, bat noch ig om t'ontoectien en te canquefteren: Fn de probintie van ae ECmerauden, en De p poluintie ba Cucumbios, in de provintie tian Quito, ende in de Indiens, genaemte Pixzos, uan bet gouluernement van Popayan, en die die genoemt werdê Cerribes ban 'tnientwe Rijclt tuan Granada, die upanden dic oe żee konnen infelteren /en yare nen/met haer altmaden en feljepê. De ifoffe uan bet incomen/wert sourthet its wel gbeweten hoe vachethaer ch oberuloedich bet veruoleb is / nae dien batter unt be lndiens getrot= lien werden alle' twaelf IBflioenen boo? fpne 29 aje Itcpt/en particulier in gontit/filber/ peerlen/ernerauden/bupden/fupe= het/corbenille/bout/çarça, gember/ ç anoere dingé bã groote baleur.

De leven in de weleke beltaet De selesen rente Uan fune Dajeftept Die bu in ban be ten Indien jeeft I ban oe welclie ljp boecken en reecteninghen lyecft in rijn Conincklijcke fonat-kifte / zijn thien op ofie forme. De viftoe ban triluer en gout/en oe groote git tin' ge/De impofitien of tollen ban tuee ten honoert / de renten uan be tri: bujten van be Indiers, oe bercochs. te Dffietien/ de Cruzada, oe tribupt pan bet inconten/ oe quicklillecen/bet gouts ofe amenden ban Camara, de negen= den ban de Ecclefiaftique tenten el:traozoinarig. In bet Ituck van tgolloernemit heeft oe Liaet groo. te becoumering be en arbepot/ ont oat bpnaeft alle die faken oie vooz: camen ziin ban bien ftoffe/ foo bel

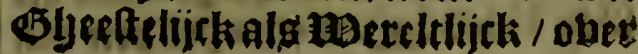

(3) 2 welcke 
Fol.28.

Eyghentljicke Befchryvinghe

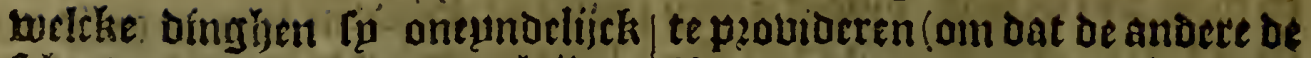
frber.ben/ena ace locrat berl tijots berdeten int fien tan loo beel bies = ben/en om ract te febaffen met foo een linguliere lazglye / met loo beel Crdullen/1Dzobifien/ en Ozdinan= tien/ Die/ om datter foo Licel waren beeft het tegen-[p?aeck gemaeckt/ oum ljet welcke de kaet beeft gepze= tenoeert te maken een Tiectiel, op De vort ats in Cartilien bã de Met. tê / cn allyoelwel dat N. uan Euzinas begint / fullendoer bite 25 arcken ges Deuck wozoen/met meerder confi= ocratie/werfant en blijt ban oe $\mathrm{Li}$. centiaet Zorilla, Ziect\}ter ปã Quiro.

Pait be Ju:

In Caken van Juftitie/ comen te rabe de Vifiten, en Refidentien, die De Bicerous tot yaer lait genomen hebbê/1Pzefioenten/Lechters/刃ie= naersen efficters ban oe loven! cn oe Eouberneturs / Capiteunen Generaels/Cozregidoozs/ cnoe al wat acluraeclit aen ' $\$$ Coninex in: comen/of Conincklijeke Offirier:s/ fiactoozs/Crelogiers/en lieeckenmetlters/en die latcken die voo? de tweede fupplicatie kamen/cnde op thegulter ban Duurent en viffbon= Dert en andere Blepten/en faké die aenga de gouluernententen/en toes oeplingê ban de Indiens, De welcke confozm ae Bot bâ Mechelen wer Den begannen in be Jouen/alwaer fu gheljoot serden bu partpen/en de hewufingen ontfangen twerden/ en de lake befloten zijnde / be par. turen geciteert jijnde $i$ werde de ogi= gimelen gerenitteert aen oe Raet' alwaer fu gedetermineett werden/ conform unet oe 3 ultitie.

vasueters So beel alterer gebanoelt is/gelt crrutgen of tof uerceringen of gratien/ oin Dat

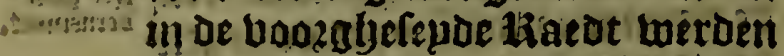
berfien Biceropg 1. Bzectioenten / liechters/ plobooften van 'thof/ Defficiers/ ellalle de Dienacrs! en Bfficiers uan de gouen uan de Indiens, Bouluerneurs/ Capitepinen/ Generaels/en de Coaregioooss die in oe Raed gljetwente beluben ou Bicerops acngaen) Delgelijex oock oe zlominiltratien / en andet offi: ciet Lan 'tlieclot/ en oe plume / en Coninckliteke Officiers / De cont: manderien en rentê van de Indicns,

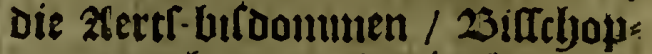
pen/Deccltens/en Capittelen/enoe 1) zebenoen uan alle oe Cathedzale Hercken/en andere 5 paeniflye be: neficien/en de Tngeboienen/als oe coltupme is in be 1iaet/andere ber: ecringlyen en befyllpingen ban on: kolten/ald daer jiljn de habitos van oe Dzie Bzozen/luan Santjago, Cala. trava, en Aleantara, confulterende neet fune 99 ajeltept van De perfoo= nen en verdientten/in de weicke bu gijedient beeft / en de tptulen ban Adelantado, en Maerfchalcken, enoe anoets.

De faken Dit be Contncklijcke Banbe ces 1)attonagie aengaen / werben ge = vaturkilichage yanoelt en gljedetermineert in of ghc Tepoe Zhaet/ dat jijn gajeltept Gatroon ly van oe indiens, ell oat bp aenneme of patronagie/als Conquetteerder ban die / en dat oe 25icluen en zipoltolijckre 23 ulleul en alle oe $25 e n i f i c i e n / e n$ onderwp. ringljen die gep?obidere werden in oe Indiens, pock in Gbeelteliyclie als Hereltlijcke/fullen maetê con= fozmi jijn de patronagie/en oe full= oeringhen van oe tercken / Cloo: (teren / parochien en Galtijuu[en! oat de 23 iff́choppen Eoicten fullen Atllen/en die haer daer tegljen lulIen ftellen / fullen baer met baer tween pzefenterê booz de platroon; diat oe vicerou fal zijn / of gacli $^{2}$ Dent / ofte Bouberneur ban eleke 13zobintie/op dat bu exnulete/enos noeme int eene amobile ad nutum, ende niet in titulo perpetuo, om Dat oat gbereferveert wozot boo? fune gsajeftept / ch de Riaedt / en die de abenoemoe eptel gegeluen berde. ILaet de 23utlen en 25tietuen in oe lined Ktomen/ en alle de do?pachos uan Roomen, en laft die niet mos gl)en 


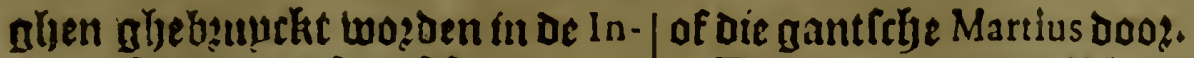

diens, fonder bat lu gefift 3ijn doo?

De twecde/ Dat oe Dlootell nae

Die bootende liaet/cul latet op eeth ander manier glyefuppliceert woz= oen/enoe lact lyet niet berbult wer: ben/ tot dat jijn bejlicbept gbetil= fozmecte lu.

Op aat wu dan befuuren dit

Dit hupg ball Contr sevills.

oe Indiens, tot ons \$uaengien/leg= gibe ick / Dat oun te lien bu nae de grootlyept van ac Indiens, en is nict meer noodich oan te fien bet buyg van be contractic uan oe Indiens in Sevilien, om Dat alljier fal glye: licn wozden bare groothepdet uan intomen/ om dat aloaer een 13 zefi= Dent is/cen Crefogier/een Tietkemeelter/een ffactoog/hechters/een fitcal/bie al lieclyters en officiers siin. Oe jpelioent beeft tot sijn Ialt de Deparcho uan de Galioenế blooten en ztrmaden $/$ en Sclyepen uan aouple ote van Indien gaen/ confozme de ozoze die daer ghege= ven wozot / en belet welende / Daer Dzacelyt een lozge vooz ban oe relt; confo?m bet genoeinde. Dacr $3 i$. Lîecken-meefters van oe zuerpe en andere Bienaer\$/ en Bfficiers. Catidenen. Eistk Jaer weroender gedefya. theert leve of acht Galioenen naer 'tualte $\mathbb{Z}$ anot/met eenige Patachen, ont te halé de febat tupt oe Indiens, ru betenghen cen Generatl / een zal= mirant / en Capitepnen uan uoctbolck/ en uan be Zee / en eetl rertio ban boet bolck/ Tonder 2000 tghe fallen / 'twelck conform is nae de vacht van elck Galioen / feet toel ghe wayent / en met glyefcljut ber roecht.

be tist ont te gaeit/cn teaunen. het biffe $\mathbb{Z}$ and / vooz befte tijot Jebbé / de Oofte twinden ill Jant: ario / of ten minlten een ga asnt te vozen eer be Balioenen af- baren op oat fp tljot hebben om baer Cargalapen te uertioopen/enoe te uergaderen.

De deroe/dac de bollagit ban oe Galioenenis/upt te loopen uan oc Barra, van San Lucar , of de baye van Caliz, enoe men loopt oatmen uerkent oe Eulanden van Canaria, en van haer baertmen tot bntmen verkent ae Eulanden uan Doninica, en Matalino, die verlaten jitin/ en Datr tenighe Indianen zijn die (B)olloch voeren / albiex nemen fu uerberffyinglje ban water, ende Daer werot berbolebt op bet ber bolch vanyet balte zland/tot bat= men lyauent in Carragena, dat bet cerfte bebolckte zeand is ban bet valte zandt / en Lan albicr werde uerboleljt de refe uan Porto belo; en tot de refterende Dcelen/glyclisk alteede berbaelt is/om dat ick ljet gerept bebbe/ alboewcl ulet groo: te periculen en arbentot/tu de weles fie een uJer ljem ftelt die $\mathfrak{r}$ cuft / en over 官e gaet: $\mathfrak{P a}$ acr ban alle die beeft mu de beer berloft / gbelijck ick bem altoos baot Demoedelijck Dooz de midoel uan jiin aloerlyen. Inclye Crupg/aen 'twelch ick ozoi. naerlijck devoot ben glsewect / mu berbitioende alle daechg met nien= we gunlt en gratien; in recompen fe ban dien/heefe bet mu gort glye. Docht/ niet ondanckbaer jtjnde op te offeren miin arbepot; en oock te frbepuen een 2boeck van zijn triumplyen. Albet weleke biode ick: oe beer dat bet fu tot 3 in beplige Dienit/en berbeffinglye vanzijn zl. ocrbenltsblte Daem. 
aet : +

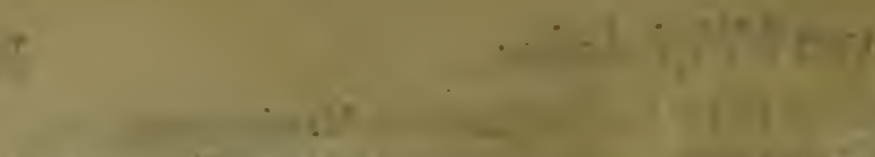

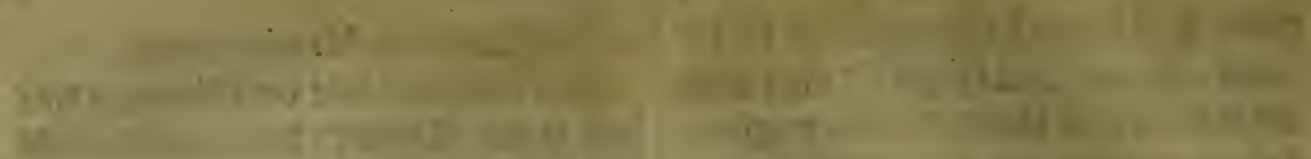

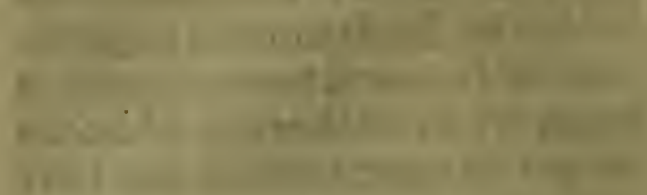

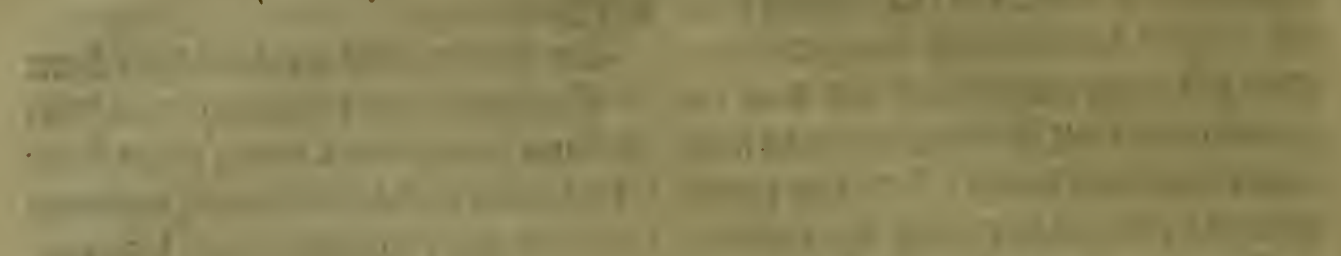

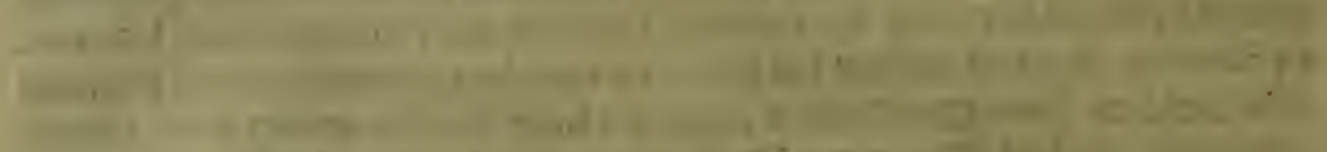

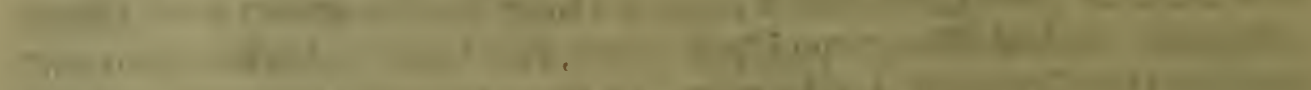

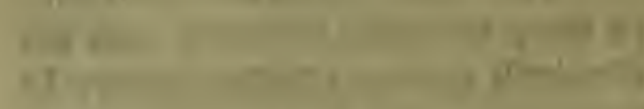

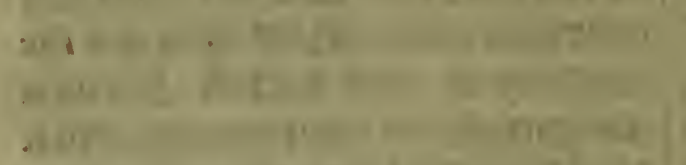

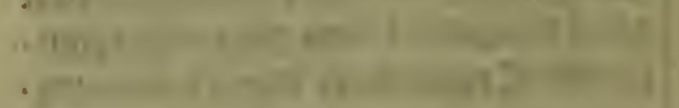

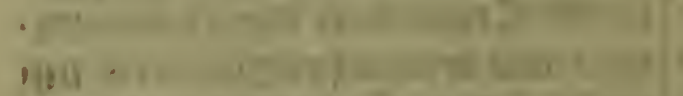
mis

- b2xif

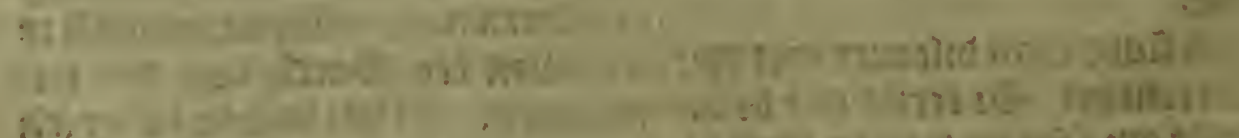

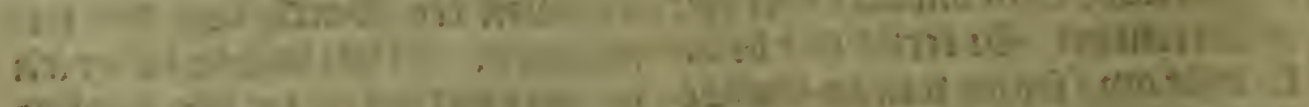

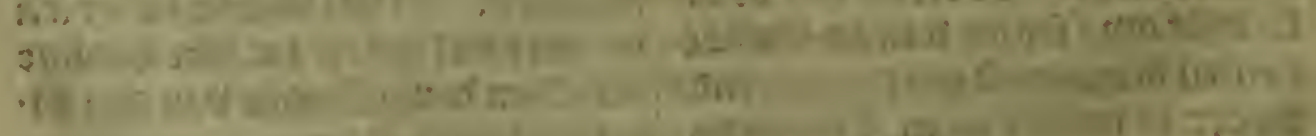

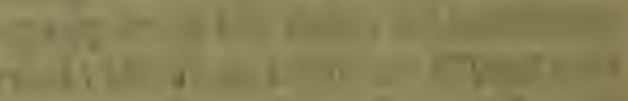

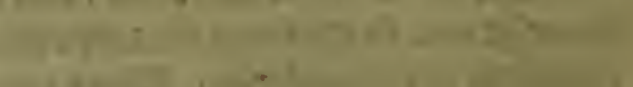

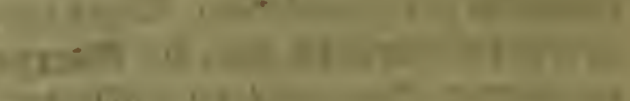

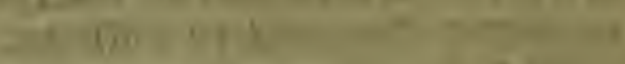

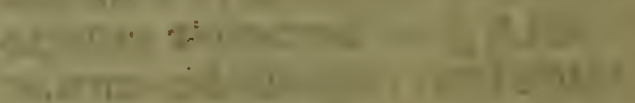

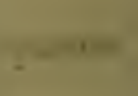

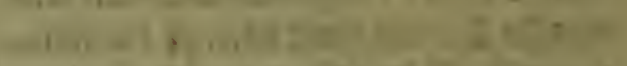

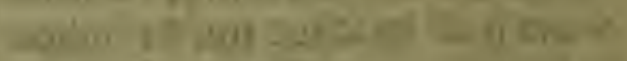

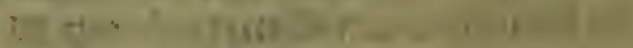

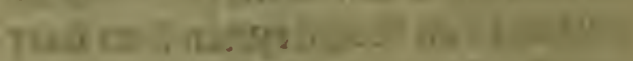

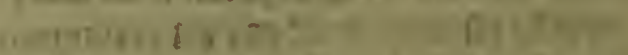
inoos is mint twate.

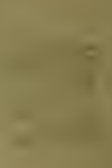

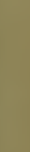

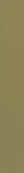
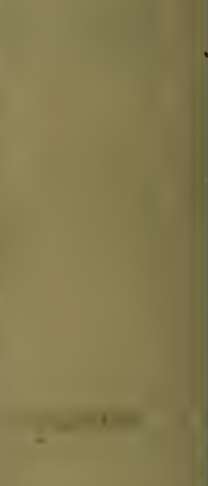
$+$
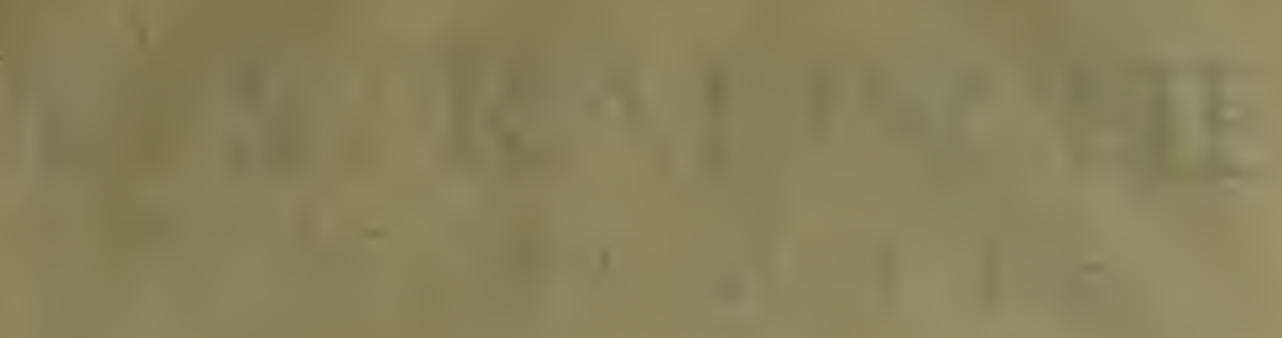

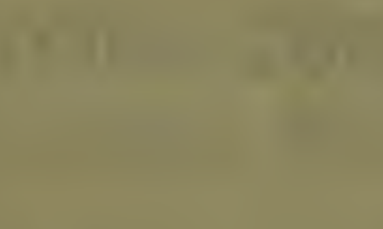

$-$

It she

.
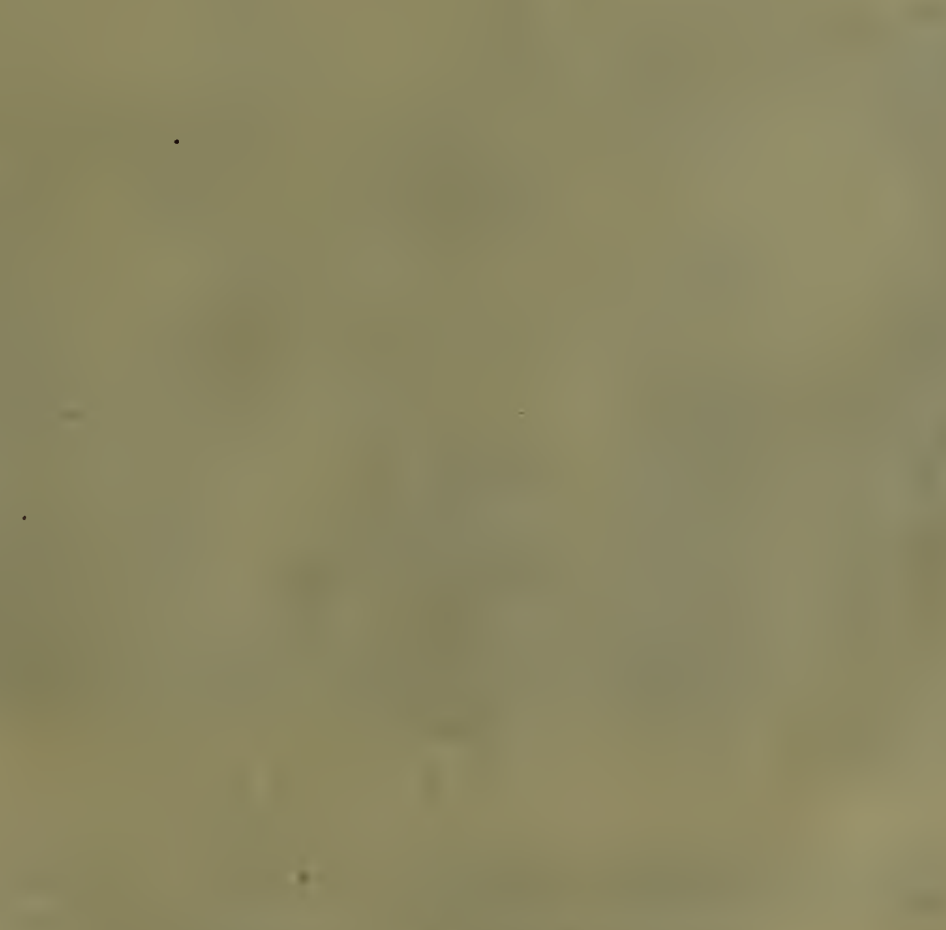

- 



\section{SPIEGHEL}

$D E R$

AVSTRALISCHE

N A V I G A T I E.

$$
\sigma_{\text {oro } s e y}
$$

aสaijt bermaerdenende cloeck = mardighan $\mathbb{Z}$ ce-lydlot/

I A C O B L E M A IRE,

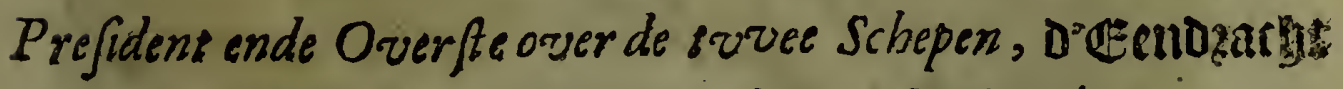

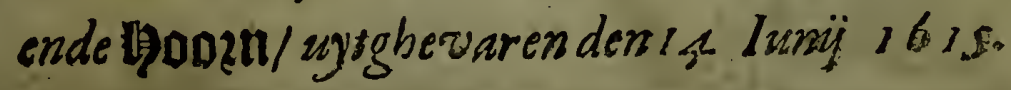

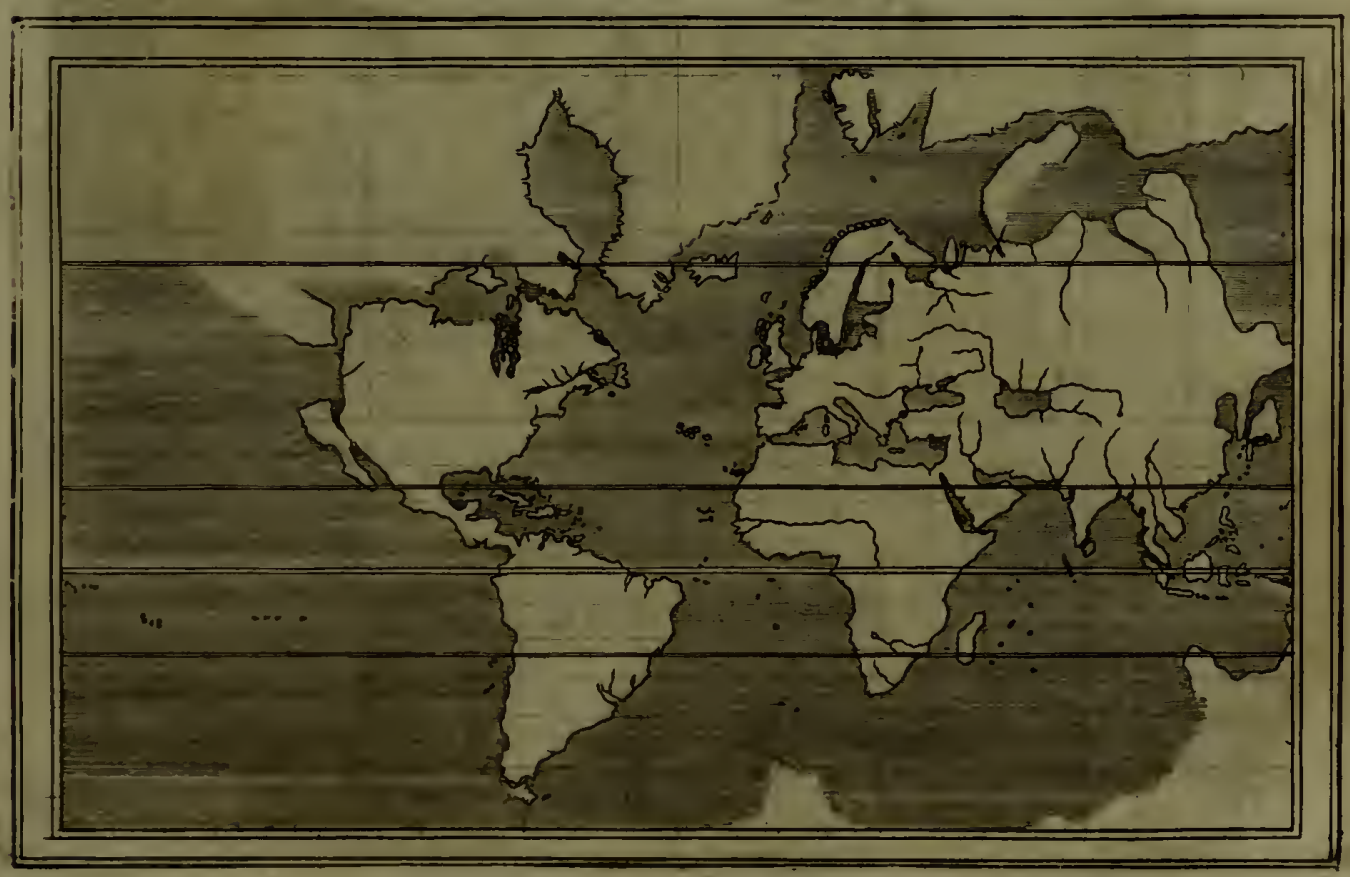

I'A MSTERDAM,

25p Michiel Colïn, 25oech-uetcoouer op't Amatte

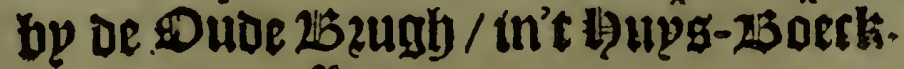




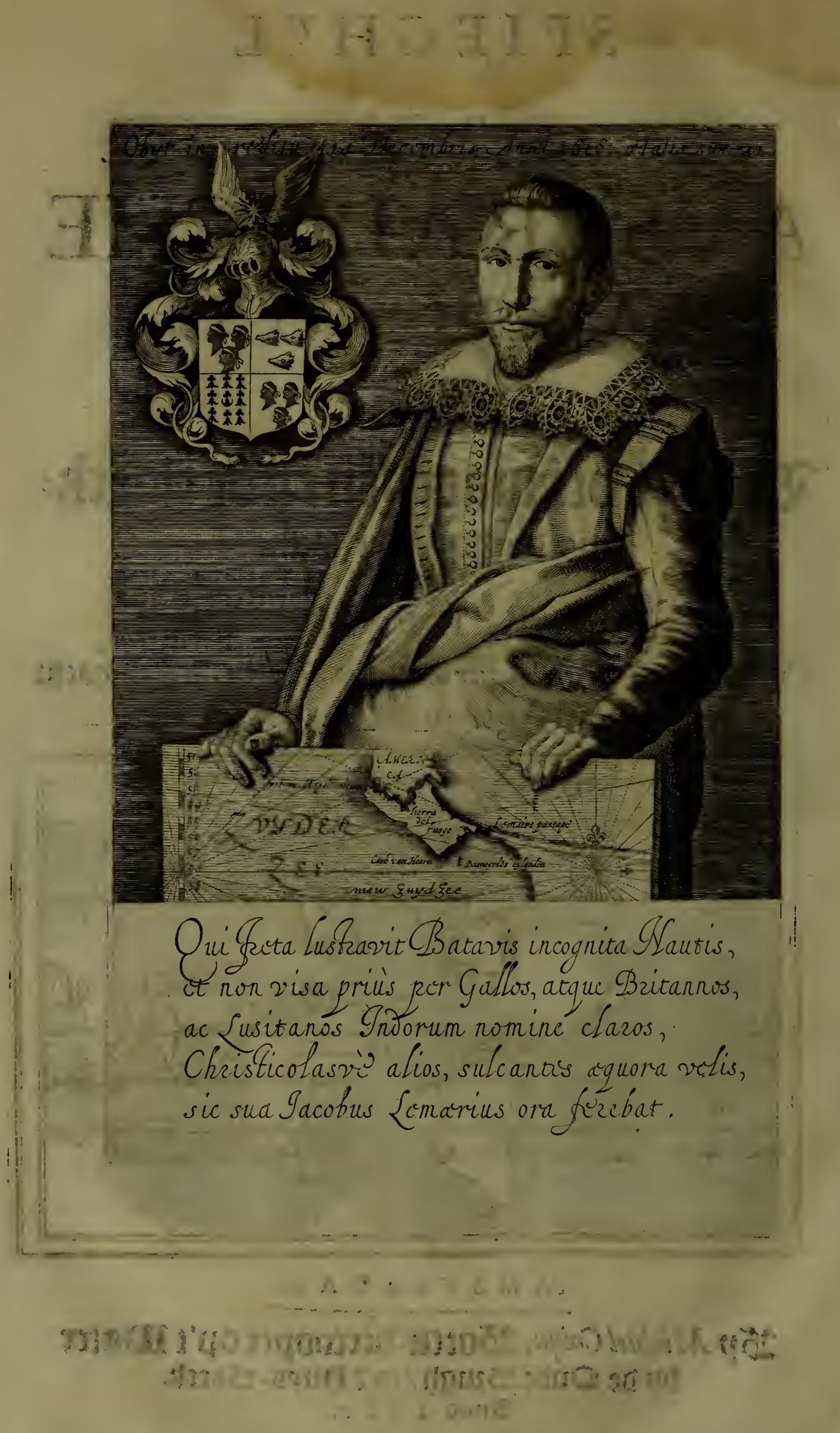




\section{AEN DE DOOR. L VCHTIGE GROOT. MOGHENDE ED. HEEREN STATEN GENERAEL,}

\section{Van de Vereenichde Nederlanden.}

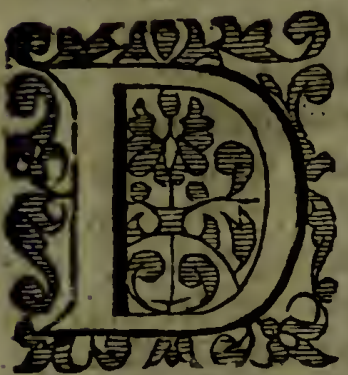

E Ambitie ende groot-moedicheyt van den Coninck Alexander de Groote, doen hy was in de fleur fijnder overwinninghen en Conqueften, en fouden hem niet connen verfaden, noch te vreden ftellen, waert by al dien dat hy gheweten hadde, datter meerder ghedeelten van de Werelt te conquefteren hadden ghewect, als die gene die hy focht met fijn Wapenen en Crijfch-knechende overwinnen, om te voldoen fijn groote begheerten die hy hadde over de gheheele Werelt te moghen donineren : Noch oock de cloecke Romeynen, die nae den Coninck Alexawder fijn ghecomen, en meerder machts en moghentheyts hebben weten by een te brenghen, als de voornoemde Coninck, om alles te dwinghen, en haèr tefubjugeren, dat eenichfins bekent was, en foude van ghelijcken hün groote begheerte en ambitie die zy hadden om te heerfchen en domineren niet hebben connen voldoen, indien fy-luyden gheweten hadden, ghelijck in defe onfe tijden gheopenbaert is, datter noch twee onbekende ghedeelten des VVerelts waren gheweeft, als namentlick het gheheele groote Landt van America, met fijn by-legghende Eylanden; dat weynich minder is, als de oude bekende VVerelt, ende oock het deel, oft nieuwe werelt van Auftralia, noch maer ten. deele bekent, en met de hulpe en fegheninghe des Heeren eer langhe jaren door de Navigaiie meer en meer tot voordeel en dienfte van defe Landen bekent fal werden.

Maer al fchoon ghenomen, dat de Coninck Alexander in fijnen tijdt, en de Romeynen nae hem, alle goede informatic en wetenfchap vah de twee nieuwe ghedeelten van de werelt hadden ghehadt, foo en was hith macht fulcx niet datfe fouden middel ghehadt hebben de felve te fubégeren; en t'onderbrengen, al hoe wel haer macht van Ruyteren en Knechten, als doen excedeerde de macht die men nu ter tijt foude weten' by een te brenghen, ende dat mits de voorfchreven twee nieuwe ghedeelten van de oude Werelt, door de Zee waren ghefepareert, en dat zy midts dien daer toe gheen bequaemheyt van macht en Schepen hadden, om fulcke conqueften te weghe te brenghen : oock foo heeft Godt de Heere de ontdeckinghe ende overwinninghe van de voorfchreven VVerelts-ghedeelten, foo het fchijit, ghereferveert voor andere Coninghen en Princen, als ghebleecken heeft, dat aen d'eene fijde de Coninck van Hipanien, door de bequaemheyt vah fij in Landt, ende Zeevaert, het meeftendeel van America heeft ö̈er wonnen, en onder $\left.f_{1}\right) \mathrm{n}$ fubjectie ghebracht, en dat aen d'ander fijde de Coninghen van Pertugael, maer eenighe conquerten hebben ghedaen; maer nier van fulcke

$$
(\therefore) \cdot 2 \text { us impor- }
$$




\section{Toe-eygheningh.}

importantie, als t'ghene door de Spaenjaerden te weghe ghebtacht is, mits de Conqueften van de Portugeefen meêt beftaet in d'ondeckinghe vàn plaetfen en Landen inde onder-werelt gheleghen, die te voorenzijn bekent gheweent, fulcx dat de felve meer voor ontdeckinghen, als Conqueíten moeten ghenomen worden, ende mits dien ghèen ghelijckeniffe en hebben by de overwinninghe die de Coninck van Hifpanien in umerica heefr ghe. daen, die in dat deel daer inne te verghelijcken is ter Zee, by Alexander de Groote te lande, hebbende hy (beneffens dat hy in Europa een feer groot Coninck is) volcomelick ecn nieuwe Werelt gheconquefteert, die hem feer groote rijckdom jaerlicx inbrenght tot maintienement van fijn ftaet, als yeder een bekent is, welcke rchatrijcke ghedeelte van de Werelt de Coninghen van Hifpanien met cleyne en gheringhe middelen fijn aenghecomen, als fulcx inde eerfte inftantie, foo de Hiftorien vermelden, verereghen hebbende met eens fefthien duyfent Ducaten te verfchieten, dat ghecoif heeft de eerfte equipage en tocruftinghe waer mede Christophorus Colimibus de Weft. Indien ontdeckt, ende ten deele ghewonnen heeft, welcke fefthien duyrent Ducaten by de Coninginne Ifabella van Cafilien oock befwaerlick in haer Riicke conden ghevonden worden.... Mct fulcke cleyne beginfelen, ende weynich middelen fijn de Weft-Indien ontdecke, en voorts met der tijdt-aen de Croone van Hifpanien ghevoecht, en langhe tijdt voor de Castilianen alleen ghehouden, want de Conin ck Ferdinandus van UArragon; ghetrouit heb. bende Dona Ifabella van Castilien, alfoo fy de penninghen tot defe entreprinfe uyt hare middelen alleene hadde verfchoten, cedeerde alle de Conqueiten ende profijten, dieder uyt fouden refulteren tot behoef ende voordeel vande Croone van Caftilien, excluderende daer uyt fijne vaffalen urragonezen, en andere Onderfaten, waer af contracten en conditien wierden ghemaeckt tot contemplatie van de Coninginne Ifabella, welcke conditien gheduyrt hebben en onderhouden fijn gheweeft dat gheen Aragonnoys, oft Vaffal van de Coninck Ferdirandus in $W e f t$-Indien heeft moghen handelen, tot foo langhe toe dat de Croonen van Castilien, Arragon, en andere Coninckrijcken van Spanien door houwelicken en erffeniffen te famen zijn ghevoecht, fulcx dat de Inwoonders van Spanien int ghemeen fonder onderfcheydt de beneficien trecken van defé vreemde Vaert. Iae felfs. de Genevoyfen, overmits die in de protectie van den Coninck van Hijpanien fijn, wefende alleene de Onderfaten van defe Landen, die mede Vaffalen, van de Coninck van Spaigrien doen ter tijt zijn gheweent, daer uyt ghenoten. Ende al hoe wel de lng:efetene van defe vereenichde Nederlanden in de Zee-vaert alle Natien van Europa, jae van de gantfche Werelt (fonder roem ghefproocken) ovcrtreffen en pafferen in ervarentheyt, induftrie, en practijcke, om de Zee op alle verde en vreemde Paffagien te bouwen, en te frequenteren, foo is nochtans voor defen by niemanden uyt defe Landen, eenighe nieuwe Paffagien, Havenen, of Landen die ghedenck-waerdich fijn ghevonden of ontdeckt, niet teghentaende t'felfde door verfcheyden perfoonen foo int Noorden als an. derweechs onderleyt is gheweeft, alle t'welcke de Bewint-hebbers van de Auftraelfche Compaignie, uwe Hooch-Moghende onderdanighe Dienaren, in rijpe deliberatie ghenomen hebbende, ende gheencouragecrt wefende, door den particulieren Octroye by uwe Hooghe Mog. Ed. fijn de felve beweecht gheweeft tot eere en reputatie van haer lieve Vaderlant, cen loflicke Entreprinfe voor te nemen : te weten, om een Paffagie int Suyden te foecken, daer door te varen; en voorts de Landen in de Suyt-Zce gheleghen, en voor defen onbekent, foo vecl doenlick foude fijn, aen te doen, en 


\section{Toe-eygheningh.}

frequenteren. En hebben dien volghende inden Iare fefthien hondert vijfthien binnen de Stede Hoorn toegheruft twee Schepen ghenaemt de Eendracbe ende Hoorn, daer op ftellende voor Prefident en Opper-Coopman den welervarenen, ende gheexperimenteerden laques le uMaire, die met de felfde Schepen fulcken Voyage heeft gedaen, en int Suyden foodanighen facile, bequame, en gantfch dienftighe Paffagie, die eenighe Mijlen van de Straet van CMagallanes is difterende, uytghevonden, ende Landen en Eylanden inde Suyt-Zee ontdeckt heefe, als in defe fijne Iournale vervaettet wort, aen welcke Landen alfoo nae alle apparentie t'Landt van Auftralia feer groot is, jae fulckx dat het recht wel voor cen derde-part van de Werelt mach ghereeckent worden, onghetwijfelt tot feer groot voordeel defer Landen, tréf felicke Coopmanfchap en negotic fal connen werden gheftabileert, ende Lal by experientie bevonden worden, dat aen de felve Landen foo wel als aent $t^{\prime} L$ andt van America goede profijten fullen wefen te doen, alfoo veel der felver Landen op een goet climaet fijn legghende, fulckx datals nii claerlick onder-vonden is, dat de oude Schrijvers die de Werelt ghedeelt hebben in drie deelen, als in Europa, Afa en Africa, feẹr grovelicken gefailleert hebben, en dat de felve drie deelen met recht niet meerder als voor een derdepart van de Werelt moghen gereeckent worden. Lmerica voor het tweede derdendeel, en Lafitralia voor het derde derdendeel, en naer alle apparentien, en conjecturen, foudemen met feér goet fondament connen argueren en ftaendehouden, dat het ghedeelte van sufralia het grootfte van de drie deelen is, gheconfidereert dat het eerfte en tweede ghedeelte, namelick de oude onbekende Werelt, ende 4 America, meeft beitaende fijn van de hoochte nederwaerts van Rio de Plata, en Cabo de bona E/perança, legghende beyde de voorfchreven plaetfen:ten naeften by ghereeckent op de hoochte van vijf-en dertich graden int Suyden, fonder dat van de voorfehreven hooch te op : pa: ralellicq eenighe Landen int Suyden bekent zijn; als alleen het Lant van de Pasagones, ftreckende van Rio de la Plata tot de Straet van Magallaines toe, dat feer weynich importeerr, en dat gheheel Europa en Afia meeft gheleghen fijin van 35 .graden Noortwaerts te nemen, naer de hoochte van de Straet ván Gibraltar af, daer uyt men voor feecker can befluyten en infereren dat int Suyden noch veel Lants is t'ontdecken, fijnde niet ghelooflick dat Godt de Heere, die volcomen en perfect is, in alle fijne wercken, de Länden van dé Werelt alleen foude ghefchapen hebben int Noorden, en dat int Suyden hiet dan Zee en Water foude wefen te vinden, nademael de Sonne fijn crachit; operatie, en virtuyt doet, en int werck ftelt, foo wel int Suyden als' int Noorden, ghevende elck ghedeelte fijn tijt en fayfoen om het Aertrijckite verwarmen, en te fruatifieren, ghelijck de Conincklicke Propheet David in fijn 89.Pfalm claerlick feyt, dat Godt de Heere, $t$ 'Noorden en t'Suyden' heeff ghefchapen, ghevende alfoo het Suyden foo veel voordeel en preeninentie als het Noorden, foo dat daer uyt vaftelick te prefumeren is ; dat het Súydérghedeelte van de Werelt; hoe wel tot nu toe onbekent, de felfde qualiteyten in fich felven moet hebben als .het Noorden, trwelck die vande Aufträlfche Compaignie, indient de almoghende Godt toelaet, en dat uwe Ho. Mó: $\mathrm{Ed}$. het Oatroy aen henluvden verleent fonder eenighe reftriate ofré limitatie volcomen effect laten forteren, in feer corte jaren door de nieuwe öntdeckte Strate Le Maire, tot cere en reputatie van de gheuniecrirde 'P rovincieñ, voorder en meerder verhopen uyt te vinderi, en ontdecken: Ende al ift dat feeckere invidieufe en afgun ttighe perfoonen dé, voorfchireven uytghevonden Strate, gheerne in clecn -achtinghe fouden brengen, en uwe Ho. Mo.Ed.

$$
(\cdot \circ) 3 \text { met }
$$




\section{Toe-eygheningh.}

met finiftre practijquen, foecken te imprefferen, dat de felve gantichelick gheen voordeel voor defe Landen foude connen gheven, foo vertrouwen nochtans de Bewinthebbers voornoemt, dat uwe Ho. Mog. Ed.na hare wijfe difcretic wel fullen connen oordeelen, dat door het uytvinden varn de felfde Paffagie, de Landen een uytnemende voordeel is ghefchiet, ten refpecte van de trafijcq die daer door al omme int Auftraelfche Lant, en ooock aen alle ander Länden aen de Suyt-Zee gheleghen fal connen werden gedreven, fijnde waerachtich dat de bequaemheyt ende faciliteyt van de felfde Strate fulcx is, dat gheene Paffagien die imınermeer int Noorden ontdeckt oft uytghevonden fouden moghen werden, ten regarde van het over-groote coude Climaet datmen foude moeten pafferen, noch oock de fpineufe ende moeyclicke Strate van Magallanes in gheene deelen daer by te verghielijcken fijn, tot welcken eynde de Bewinthebbers goet ghedacht heeft, alhier te declareren de moeyelicke befwareniffen, en ghevaerlickheden die bejeghent fija, ende die tijdt die gheconfumeert is by alle die ghene die oyt de Strate van Migallanes hebben ghepaffeert, en eerftelick foo veel aengaer Ferdinando Magellanes felve, foo brenghen de Hiftorien mede, dat hy' met een Vloöt van vijf Schepen uyt Spiignien ghefeyle fijnde, inden beginne vande Maent van April, inden jare 1520 . gheraeckt is in Porto S.tulizen, legghende op $49^{\prime}$. graden befuyden de Linie Æquinoctiael; en dat hy niet eerder door de Strate heeft connen pafferen, en in de Suyt-Zee comen, als op dẽ achthienfte December int tfelfde jaer, fulcx dat de Ielfde Magellanes, den tijt van on trent acht Maenden ende cen half befich is gheweeft om uyt Porto $S$. Iuliaen door de voorfchreven Strate te gheraecken, in de Suyt-Zee:-fijnde de voorfchreven Magellanes, en die ghene die met hem de voorfchreven Strate hadden gheyonden, by de Keyferlicke Majefteyt ten hooch ften ghehonoreert en gheremunereert, fulcx de Hiltorien ende de contracten van dien fijn medebrenghende, en fijn van de voorfchreven vijf Schepen'; vier op de Reyfe vergaen, De tweede die de voorfchreven Strate aenghedaen heeft is gheweeft Don lustieres Carvaial, Spaengiaert, die met een Vloote van vicr Schepen ghevaren is uyt S paignien, met confent en licentie van de Hoo. Mog. Keyfer Karle, om te pafferen de Strate van Magallanes, en ghecomen fijnde voor de Mont van de Strate, is daer met foodanich onweder en tormenten in de Strate overvallen, dat drie van fijn Schepen daer in ghebleven en vergaen zijn, met ontrent twee hondert vijftich Maninen daer op fijpde, en het vierde Schip hebbende twee en tfeftich daghen befich gheweelt, om de-voorrchreyen Straet te pafferen, is eyntlick met groot verlies van Anckers, en anders daer door gheraeckt, en ghenootfaeckrgheweelt te loopen' nae 'Calow de Limain Peru, fonder verder te connen feylen. , Capiteyn Don Alonfo LadrilleRo, Myt den naem en van weghen Don Garcia de Mendoza Gouverneur van Chili de derde, die de voorfchreven Strate heeft befochit, is lancx de Suyt. Zee, aen de voorfchreven Strate ghecomen mer twee Schepen, een van dewelcke door ftorm en onweder weder is ghekeert naer Chili, ende her ander met groote moeyte, en travaille is in de yoorfchreven Strate ghecomen, cn heeft foo verre ghefeylt dat hy de Noort-Zee heeft ghefien, en is daer naer wederomme ghekeert, hebbende befich gheweeit in de felfde Strate, ontrent drie Maenden. Don Fray Garcia de Loayfa vierde paffant van de voorfchreven Strate, is door laft van de hooch-ghedachte Keyfer, uyt Spaengien ghefeylt inden Iare duyfent vijf hondert vijfen twintich, en is op den ach. thienden Ianuarij I s 26 . ghearriveert inde Strate, a waer hen foodanighe fwaricheyt bejeghent is, dat hy door ftorm cens daer uyt ghedreven is, en 


\section{Toe-eygheningh.}

is den cerfen lunij dacr aen volghende ecrft daer door gheraeckt, achicrla. tende drie Schepen van fijn Vloote, dacr noyt tijdinghe van ghecomen is. Sir Francijco Draeck de vijfde, dic defe Stract heeft ghepaffect, is uyt Enghe. lant gheicylt met vijf Schepen den vijfthienden Norember $1577 . \mathrm{en}$ is den feften Scptember Anno acht en teventich gheracekt door de Stract in de Suyt-Zce, hebbende dentijt van ontrent fetthien daghen gheween in de voorfeyde Stract, en de felfde ghepaffeert op het geluckich fte van allen, doch daer is cen Schip van fijn Vlote weder in Enghclaint ghekecrt, fonder dattet t'felfde de Stract heeft connen pafieren. Capiteyn Pedro Sarmicnto de refte die de Strate heeft aenghicdaen, is door laft van Don Erancifco de Toledo, Viceroy van Peru, Anno I 579 . met twee Schepen ghevaren nac de voorfchreven Strate, on $S_{r}$ Francifro Draeck te gaen foccken, en is cen van de beyde Schepen ghenootfacckt gheweeft wederomme te keeren nac Lima, vervolghende de voorfchreven Sarmiento fijne Reyfe, en pafferende de voorfchreven Strate, ontrent in vijf en veertich daghen, is cyntlick gearrirecrt te $S_{t} . L_{z-}$ car. Sir Thomas Candifh. Enghelfinian, fijnde de fevende, is met twee Schepen, en een Barcque in Iulio 1 s 86. uyt Enghelant ghefsheyden, en den feften lanuarij I 8 8.inde Strate ghecomen, en den vicr en twintich ften Februarij dacr door gheracckt inde Suyt.Zec, hebtende befich gheweeft om de voorfehreven Straet te pafferen, den tijt van vijfiich daghen: De felve isandermael denacht en twintichften Augufti Anno 1 s 9 I. uyt Enghelant gefeylt, met drie grooote Schepen, en twce Barcquen, en is den twintich ften Martij I 92 . ghecomen aen de Stract, en de felve door ftorm en onweder nict connende paffeeren, is wederomme te rugghe ghckecrt inde Noort-Zce, fonder dat hy de reyfe conde volbrenghen, macr is ecn van fijne Schepen in Augufto dacr acn rolghende wederomme gheracckt in de Stract, en met groot perijckel en mocyte ghecomen fijnde in de Suyt-Zce, is door ftorm en onweder te rugghe ghedreven in de voorfchreven Strate, foo dat het felfde Schip ghenoorfäckt is gheweeft weder te kecren in de Noort-Zec, en is mitfdicn by gheen van de roerfchreven drie Schepen de reyfe volbracht. Capiteyn Thomas Haukÿns Enghel fmal\} de achtftc, is t'feyl ghegaen uyt Enghelant den twaclfden lunii I $593 . \mathrm{en}$ is yoor de Straet ghecomen den thienden Februarij is 94 . cnde gheracckt met groot perijckel, en miraculeufelick met rerlies van meeft alle fijn Anckers, den ncghen en twintichften Maert dacr aen volghende inde Suyt-Zce, fulcx dat hy den tijt van ontrent acht en reertich daghen in de voorfehreven Strate heeft ghefwermt. Iaques CMabicu de neghende, is uyt Rotterdam t'feyl ghegaen met rijf Schepen den feven en twintichften lunij, Anno I. 98 . cn den feften Aprilis ghecomen in de Strate, en de felve eerft ghepaffecrt den feften September dacraen volgende, behalven dat het Schip de Trowwe, dacr Capitcyn op was Sybold de Wecrz, ghenootfaecket is ghewecft wederomme te keeren, mits de groote fwaricheden hem bejeghent, ende zijn dic andere vier Schefen int pafieren van de voorfchreven Stratebefich gheweeft den tijt van ontrent vijf Maenden. Olivier. van Koort de thiende, is van Rotterdam t'feyl ghegaen den derthicnden September, Anno I 598. met vicr Schepen, en is den 24. October Anno I 599. inde roorfchreven Strate ghecomen, de welcke hy ghepaffert hecft met groote mocyte den 29. Februarij 1600. foo dat hy int pafferen van de roorfchreven Strate gheconfumecrt hecft den tijt van vicrMacnden en vijf dagen, De laetfte fijnde Capitcyn Toris Spilberghen, is met tes Schepen uyt defe Landen ghefeyliden acheften Augunti Anno I 6 I 4. en voor de voorfehreven Strate ghcarrircert den 2. Martij I 6 I s. de wclcke hy met groote mocyte ghcpaf- 


\section{Toe-eygheningh.}

ghepaffect hecft den 6. Meye daer àen volghende, alle welcke voorfchreval mocyelickheden en befwareniffen den ghenen bejeghent die oyt de Stratte van chagallanes hebbch ghefocht te pafferen, ghecom pareert fijnde, jeghents de facilitegt cridc bequaemheydt vande voorfchreven lpatieufe en com mo dieule Paflagic Le Mair, dicontrent acht Mijlen wijt, en feren Mijilen land is, en dooigaens vecrtich, vijftich, treftich Vademen diepte is, hebbende dacer door de voornoemde laques le Meir met het Schip' d'Eendracht in èen dach fonder cenighe befwaerlickheyt ghepaffeert, ende in de volle Suyt-Zee glieconinen is, blijckt clacrlick dat de felfde Paffagie in alle decien, de Palla gic van de Strate van Magallanis te prefereren is, cnde dat mitrdien de relve icn regarde van de goede gheleghentheden voordelicker en diennigher rôor defelanden is, als eenige paffagie, dic ter Werclt immermecr uytgeronden fal connen worden, te meer nae dien men de felve (foo voorfeyt is) fonder ed. highe ghevacrlickheyt can pafferen: en datmen daer door comende, terftont in een ghefont Climaet gheraeckt, fijnde waerachtich, dat door de ghenảdê Godts, niet ecn man van het voorfchreven fchip d' Eendracht op de uyt-rey gheftorven is, voor cn al cer datfe aen het Landt van lava gheconien fijir. Ende alfoo de Bewinthebbers voornoeñt valtelick vertrouwen dat de felîtec palfagie by uwe Hog. Mog. Ed. in fulcke achtinghe ghenomen fal werden, als de felve ten acnfien van de fituatic ende bequaemheyden is meritereinde: Soo heblhen de felve de vrijnioedicheyt ghenomen, uwe Ho. Mog. Ed, hare Soúveraine Orericheden dit louinael van haren Prefident Iaques le Maire te dëdiceren, en onder der fel ver proteetic int licht uyt te gheven, demoedelick verfocckende dat uwe Ho. Mog. Ed. den gheringhen arbeyt ghelieve voo acnghenacm ie ontfanghen, op dat wy daer door te nicerder verootraccke cn ghe-cucouragcert moghen worden, on voorts met de hulpe dës Heeren, de principaelfte Landen cnde Cunten vain Aufralia onfe lieve Vaderlant tot vorderniffe cn welftant ván dien bekent te maecken,énde nàct onfe gantrche democdighe en dienftighie ghebiedeniffe, fullen wy den Alimoghenden Godt bidden, uwe

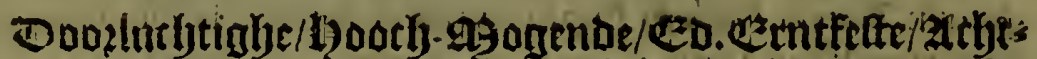

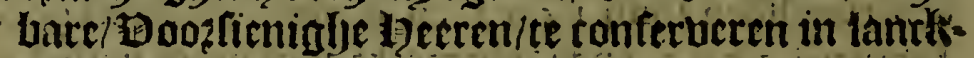

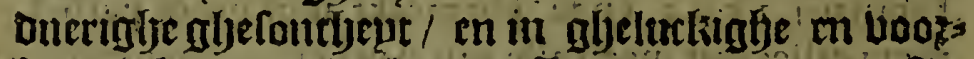

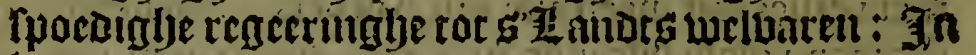
1) ongu Den 1 . A pilit I $62 \%$.

\section{Yvue Ho. Mog Ed.gantfch dienftruilli- ghe en onderdanighe Dienaren.}

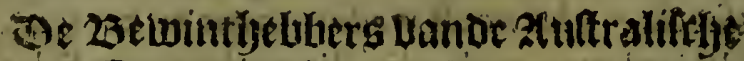 Eolnpnignis.}




\section{Voor-reden aen den goet- willighen Lefer.}

Z2ro de 2brtwint-hebteren bant

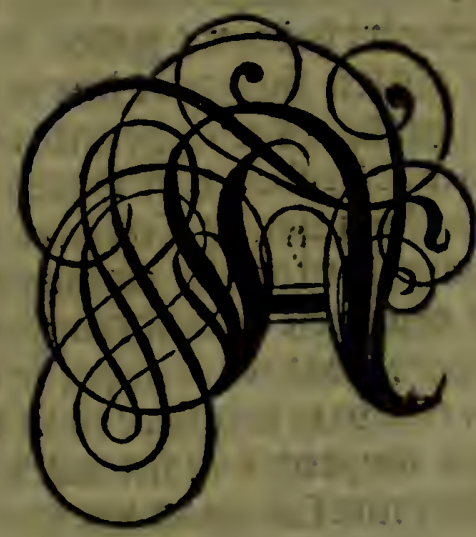

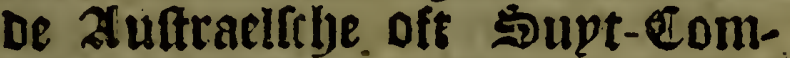
paitnic boorgljenomen bebben in datuk te laten untgaen let opterljt bigimat/ en: autentujeq $\mathbf{3}$ ournarl ban wijlen Saligber lacob le Maire, tre als Commanderm / op= per-13ooft / ell oochmede 25elwime-bebliex van of relue Coulpaignre olycuarm becft op de Sebepen thellarul d'Ecridracht end Hoorn, toeglierult en afghebaren fijude unn de stadt boozn / moe gsasnt ban linio; Anno i 6 i s. elloe dien uolghende ben

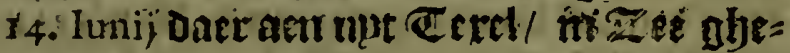

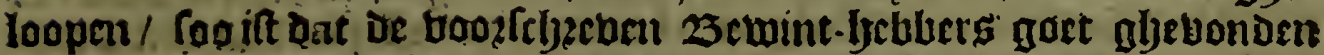

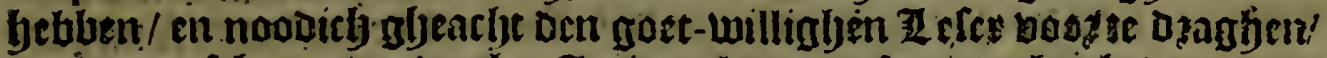

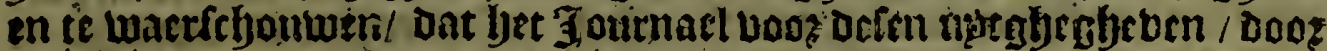

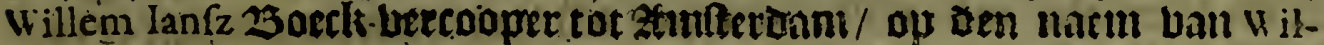
lem Cornelis Schouten. Seljippet / niet in is bet opzechte Jourmacl tant

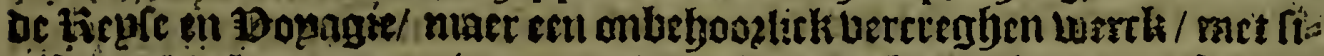
rifter modoeten/ en manieren bail boen bu cen gheboecht/ en berfahtelt/

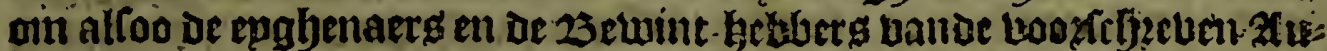
itraeliche Compaignie dic het torcome fuldr 3 oumat en werels trit te

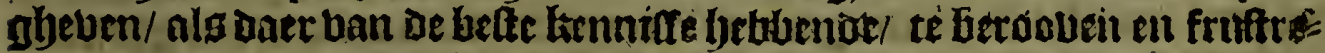

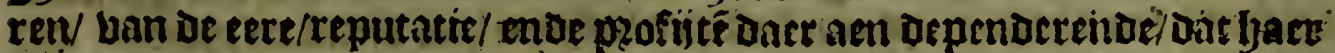

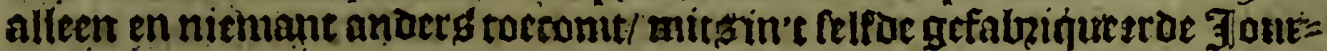

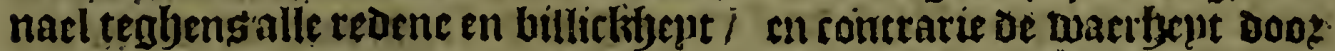
Willem lanfz de eere gtjegeven woat aen willem Cornelis Schout als bin=

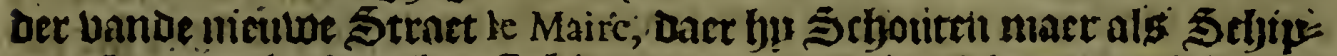
per gljebaren lyeeft op lyet Srlyip de Ecndraehr, Die noeljtans norly inuen=

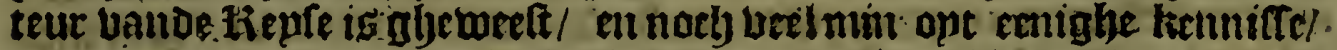

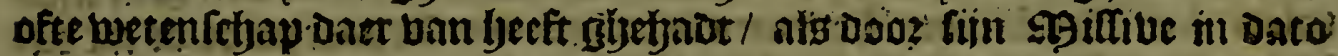
oen tweeden November, Anmo i 6 × 8. aen Ifackle Mair, vader batt de

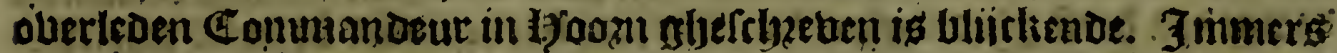

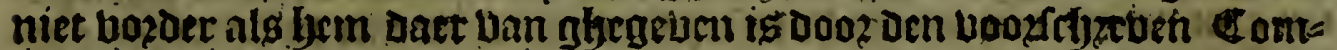

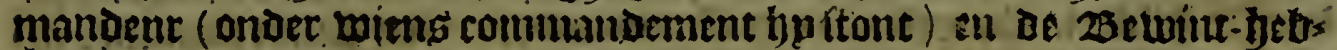

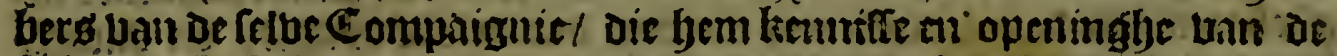

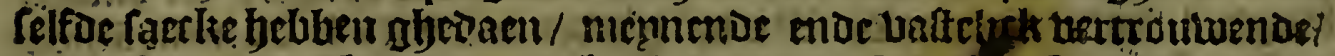
aen defen $D$ an ghebonden te heblen een petfoen vic C npribel en be=.

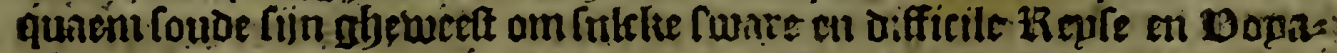

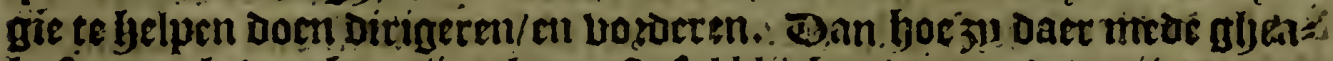
bureert en beogoghen sijn gineweet/ fatbhicken dooz de oeductic en ber:

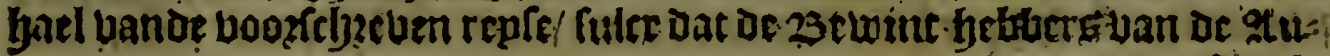

$$
(\because) \quad \text { atacls }
$$




\section{Voor-reden.}

ftraclictye Compaignie ten boochlyen fycbben te beclagljen ban wegyen

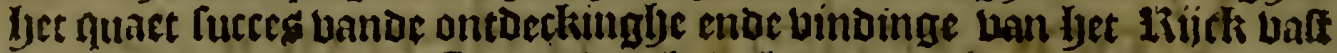
lant Jaut Auftralia inve 5 met-Zee gljelegletn / waer ban oe faute meet te umputeren is/ en te gljeben Den vooefrlyzeben Schipper / Die Doos [iin

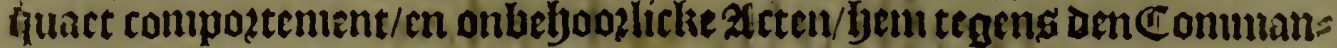
beur in alle oeclen beeft abeltelt / ende oe felue too qualick glyetrac. terert ell mirhandelt / en dat daer dooz belet is glyeweet / fijn berrlicken jerotcque beffenu cn vooenemen/tot fijn groot bart-leet en lect-Lelen te effecturen en volbenglyen : Dat Conder twijfel met be ljulpe ende feglies

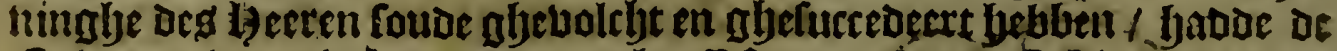

- Scbipper bem whelecondeert en nbeaftitteert/ alsen Schipper met ces ren toeftout/en yem gljegmeten en ghedaghen brooe ald penigbe nnoere

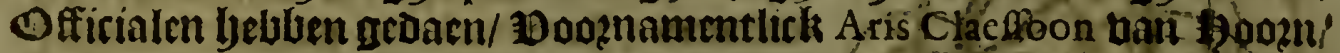
Conmuig op let Srljip Hoorn, die naeft den perfoon ban be Conuman. ocur de meefte eere ende lof toeconit/ als beblende fermin alle civilitept

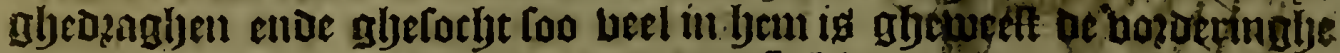

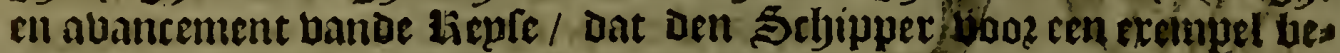

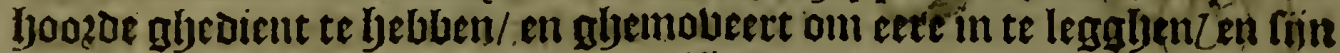
quade meneen en penctiquen int miffepden en mupten ban fet bolch/

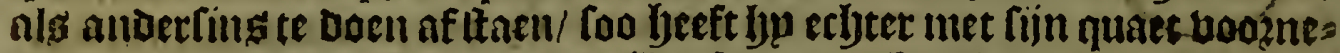
inen eventwel booztgegaen om de Lieufe te berachteré en onbuchthaer te

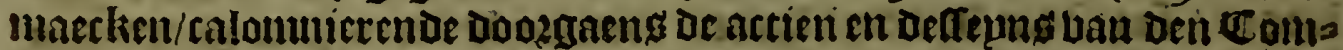

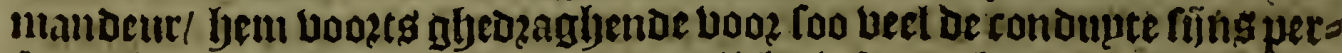

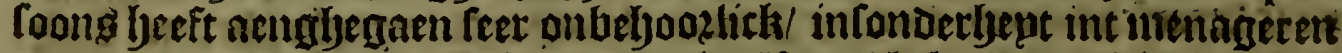
uande Dictualie/ ende Wijn/contratie alt wel bejoozde gefrbiet te tijm/ op fulcke laughe perijtkeleule tienlen : Dan onacngyelien ben noot die

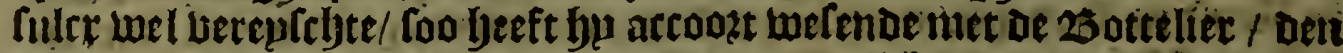
31jiu dte foo noodich uan Doen was/ onnuttetick uerniclt/en glyetonfus meert / De Baten buyten ozoinaris befocbt en. glyequelt / Datte epundelicfs Die ledicly glyemaeclit/ onnuttelick verdaen/ verniclt ende gljeconfinmeert

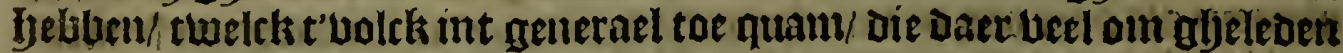

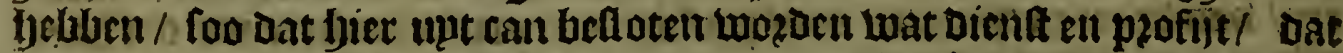
ocre willem Schouten, niet meriterende ae plaete th lietlecbeit bie by

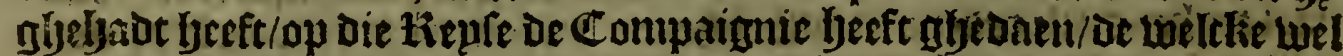
ingljelien en gheconlidereert veel ninder liin als batn oemputtgert van't

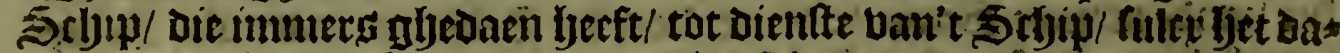

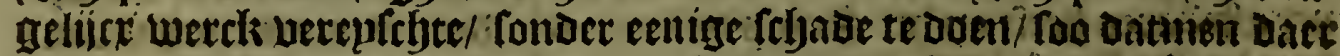
int can befupten met wat redene en fondament willen lanfer fjent inti: tuleet den Zee-1iidoer/ benemende en beroobende daer mebe de ect at

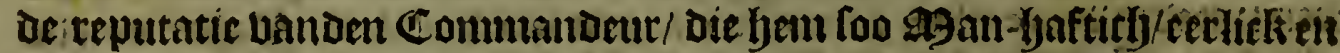

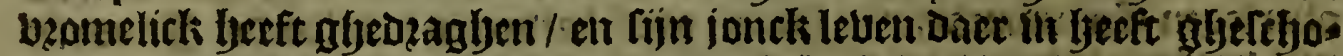

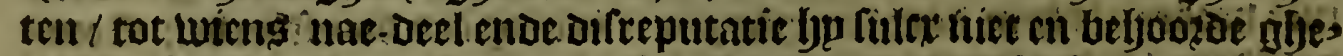

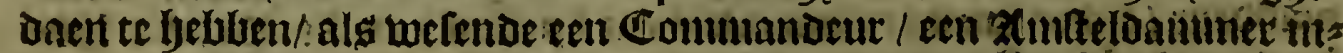

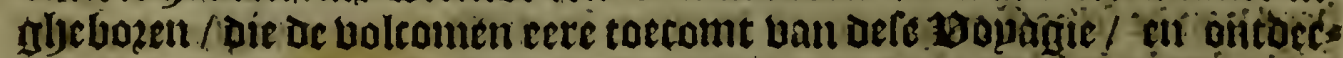

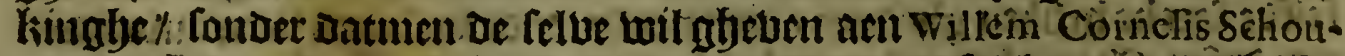

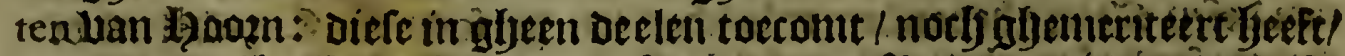

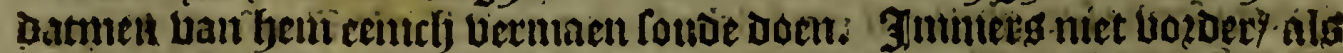

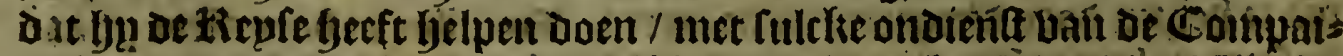

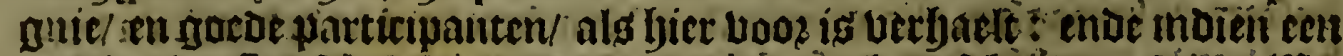

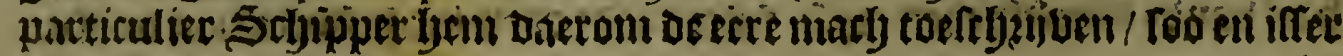




$$
\text { Voor-reden. }
$$

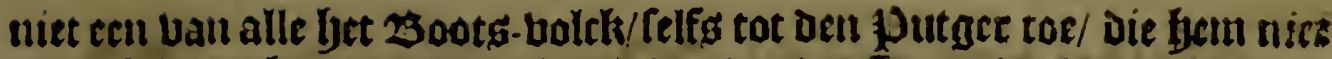
mede fal mogben roemen als vinder baube Seraet le Mayre, th gbe=

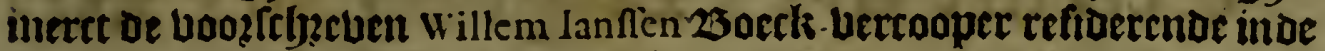

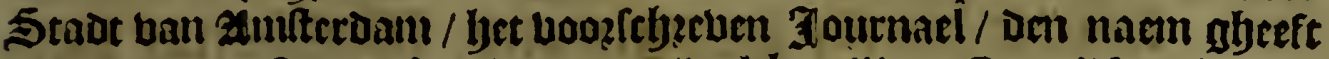
uan Willem Cornelifz Schouten : t'welcli Wiliem Cornclifz Schouten ontkent / en oefawonecte fijn wercli te rijin / als bp fiin milfibe blijcke.

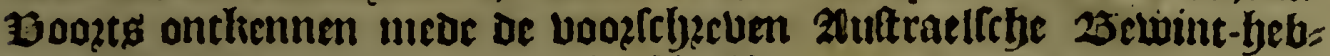
bers; fulcr Doos Willem Ianien in fijn Dooz-reden ach ocn goetwilli= byen zefer / wert gljefept / als te weten / Dat tulthen lfaac le Maire, en Willem Cornclis Schouten, wegfien defe 1ieple eenich oberlegh foude fijn glsedaen/ Joe men de felfoe bett conoe beginnen/ en achleggben/ et

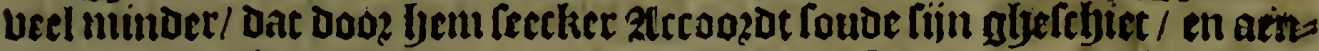
gljegaen wegleen bet furneren vande Benninglen/ alg anderfins / wes fente betert Willem Cornclis fintpelick boo? Sctjipper aengbenomen/ Conder dat boozoer pets met yem is gljeaccozoeert / maer wel waerachstidj/ Dat de boozfryetben Ifac le Maire, met Pieter en lan Clementfen Kies, פgit'gaders Ian Ianfoon Molen-werf $t^{\prime}$ famen zijt gljeaccozoeert/ weghen ben aenuancli ban orfe Dopagie/ bebbende Den Schipper dues beupckt tot bozoeringbe ban bet werck / die bent dace inne foo quatick beeft ghedzaglyen en gbequeten / Date fum Daer grootelicr ober ljebirm te beclagben: gbelijels in tijt en wijle fullex bzecoet vertlaect en ghededuceet fal wozocn : tnoe op dat oe obetwllighe ziler foube mogbet weten en oozbeten met wat fonbament / en in wat nianiete be Zus Araelfefje Compaignic begoft en aengbebanghen is / Calmen bis nate int langbe en bzet bertiact.

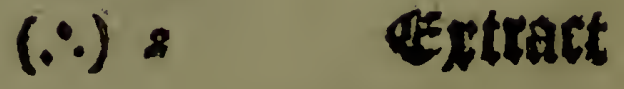




\section{Extract upt t Regifter Der $2 R \ell=$

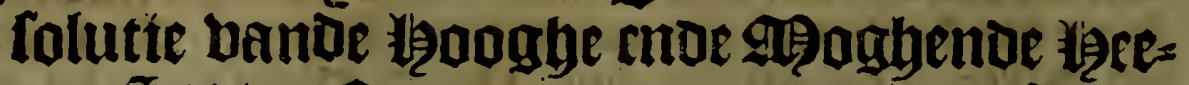

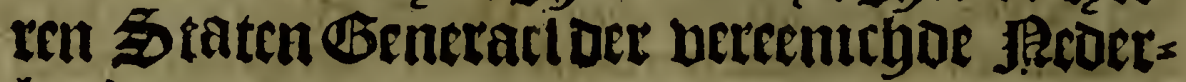 lanoen.}

- Iovis den xxvij. in Mérte 1614.

-

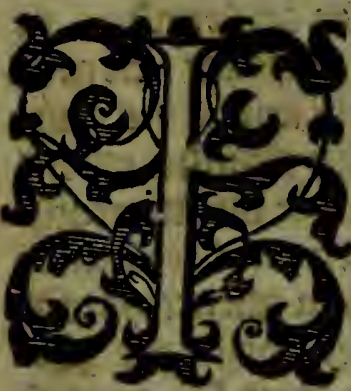

$S$ gbelefen de Requeste van ver fcheyden Coopluyden, Ingefetenen vande vereenichde Provsncien, verfoeckende vrÿdom dat zy de Pa[jagie, Landen ende Eylanden, die tot nu toe niet ontdeckt oft ghefrequenteert en zijn gheweeft, ende dic by baer door Godes $3^{\text {henaden }}$ ende bulpe onidecke fullen noghen werden; fullen vry mogben ghebruycken voor d'eerize fes reyfen, - fonder dat yemant anders als fy Suppliant en uye de fe vereeniclide Provincien die fullen moghen befeylen of fe freguenter en, directelsck noch indireczelick, voor ende al eer dat $\int y$ supplianten de voorScbreven Ses rey fen, int gbebeel volbracht ende ghedaen fullen bebben. Na deliberatie is verftaen ende gbere folveert datmen de Suppliantes bet verfoche Octroy, of fe conce Sie fal accorderen, gbelyck bun dat gbeaccordecr wert, mits de fen, voor vier rey $\int e n$, mides.

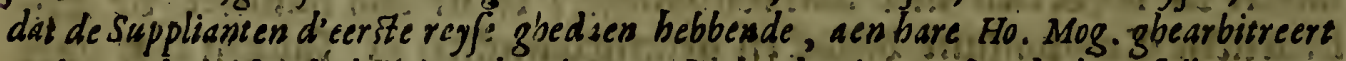

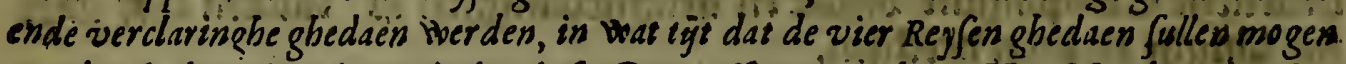
werden, behoudelick oock dat defe Conceffien van hare Ho. Moghende niet en fullen prejudiceren eenighe voorgaende Odroyen ende Conteffien van hare Ho. Mog. ende mits conditie, by foo verre al]fer twee of $t$ meer Compaignien in een Iare fulcke Landen ofte Pa/Jagien vinden, dat d' Selve t famen dit beneficie ende Pri. vilegie fullenghenieten, exde dat de verfchillen die defen aexgaende, of te anderfins val. len fullen, de felve ftaen tot decifie van bare Ho. Moghende.

GENE- 


\section{GENERAEL OCTROY}

Voor die ghene die eenighe nieuvve P affagien, Havenen, Landen of i Platetfen fullen ontdecker.

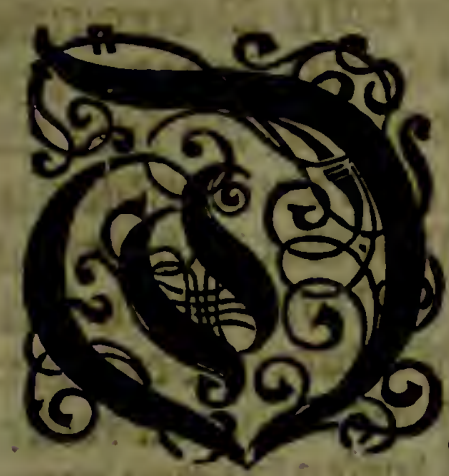

Ile Staten Bencrael banoe 1Ber= eenichoe \{edoerlanden / Zallen Den genen Die defe jegbenwoozoighe fullen lien of boozen lefen/ falupt: D O E T TE WET E N, alfoo wy verftaen/eerlick/Dienit = lick ende pzofiitelick boo defe zanden/ ende tot vozoeringhe vanden welftant vall dien / oock tot unoerbout ban bet Zee-bacende-volck te welen / Dat die goede Jngheletenen betweckt ende gheencourageert wozoen / omme ben te ens ploperen ende vercloucken int onderfoecken ende ontoeckell

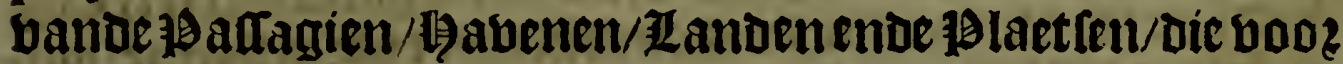
Defen niet ontDeckt ofte bevaren zijn greweet / eñ by eenigbe Cooplupden ons openinghe is ghedaen/ oat zylupden doo? Godes ghenadige hulpe/met Duligentie/moepten/periculen ende coften/ hen daer toe fulcx verbopen te emploperen/ Dat Daecban goede bauchten rouben ftaen te berwachten/indien onfe beliefte wate/hen te octroperen/pzivilegieten/eñ bege:

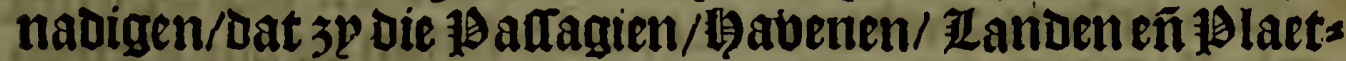
fen/bp henlupoen van nieus te vinoê ende ontoecken/alleen touden mogen bebaren/beleplen ende frequentetenvoo? les revfen in recompenfe bau bare coften/moepten ende pericus= len/ggit interdictie Dat niemant/ oirectelic ofte inditectelic/

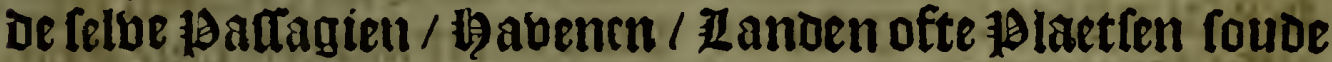
mogljen bebaren/belevlen ofte frequenteren/booz enoe aleet Die eerfte ontoeckers ofte bevinders ber felber / De boo2 $\sqrt{3}$ tes repenfonden beblen yolb?acbt. SO O I T, Dat we be voo2f 5 aecke rijpelick obergewagen hebbende/ ende bevin= Denoe als hier vozen beebaelt is/het vook [3 vooznemen booz Den welftant oer vereenich oe Zanden loffelíck / eerlick ende bientelick: Eende woillende bet befoucken booz alle ende een pegbelick bande Ingbetetenen oefer zanoen bze eñ gemeen maecken / hebben by delen allen eñe een pegljelick vanoen Jngbeletenen der vereeniebde facoerlanten tot het vooz onder toeck bp deren willen noodigen/ ende over fulcx geortropeett ende geconfenteert/octroperen ende confenteten bp Deten/Dat die gbene die eenigbe nieuwe 1\$allagie//abenen/

$$
(\because) 3 \quad \text { Zan }=
$$


Yalloen ofte felve alleenlick Lal bevaren ofte Doen bebaren voo bier reps fet1/ Conder Dat pentunt anders Direttelick ofte inoirertelick De felue nieule ontDeckte ende gevonden 39 a tagien/ /)ave. nen / Inanoen ofte 19laetren fal moghen uptte eaterenichoe Zlanden befeplen / bevaten of te frequenterell / booz dat den eerften bebinder ende onfdeclier de felve vter repien felfs fal Iyebben ghedaen / ofte doen Doen / Spp pene ban confiteatie vande Sebepen ende goederen daer mede bier jegljens tal wozoen glyeattenteert / enoe een multte bau bifftich oupfent

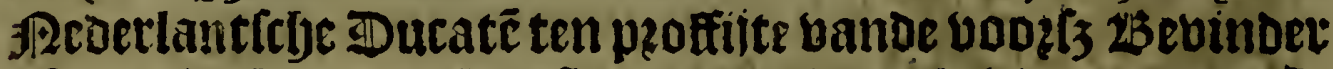
ofte ontoeclier : Hecluerffaende Dat Den zBebinder oue ecrite revte gibeaen bebbende/ ghebouden fat weten binnen beete thien Dagen nae fije intemifte bande letuerevie ons te doen pertinentrappost vanoe vook 3 ontockingen ende fijn toes Dervaten/omme t'felve ghebookt/bp ons ghearbitreect entoe berclaert te worden(na de gljelegentlyept ende diftantie) binnen wat trjot be voozf yier repren volbzacht fullen moeten wo 2ten/Lbeljondelick dat mp by de [en niet en bet ftaen eeniglye peeinditie ofte berminderinglje te doen aen onte booz= saende Detropen ende Conckien / Ende too betre in ofte onittêt een tijt / ofte in cen jaer/ een ofte meer Compaignien

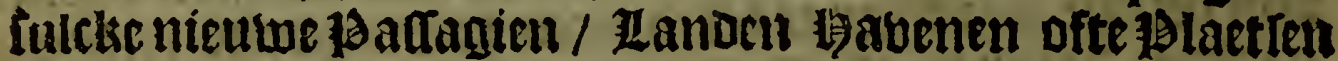
willden chde ont Decken/Dat de felve te famen dit ons Detroy ende 19tivilegie fullen gljenisten/ Eende inglievalle etniglye gljefrlyillen ofte differenten beien aengaende ofte anderfints

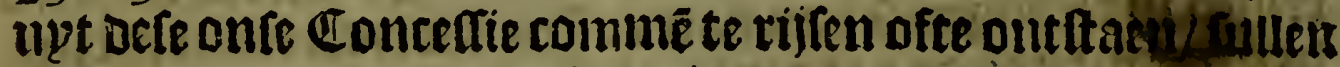
De felve by ong woz̧den gljedecideett/waee na bem een yege = liek fal ljeblon te reguleren. Eende ten epube defe onte Con= ecfie tot cen pegljelifex kenmiae macy commen / bebbetl we

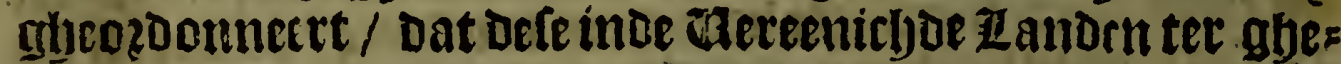
woonlitker platten glyepublicect enie aengheflaghen fal

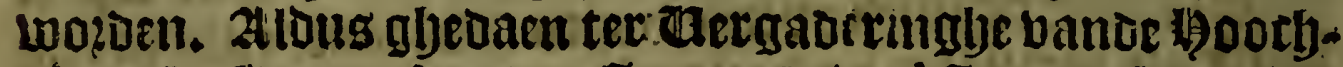

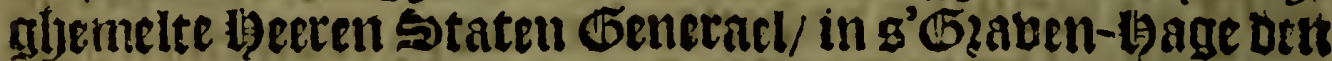
fewen-en-twinticl)(ten 29 artij feltbien bondert ende seer. thien. moas geparapljeert I.van Oldenbarnevelt vr. Onder ftont/

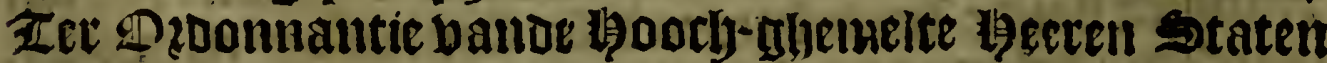
Generael. Gljeteetkent C.Aernen. Zijnoe daee op geburtit bet Contre-Zeglel der feiper 销a âclue. 


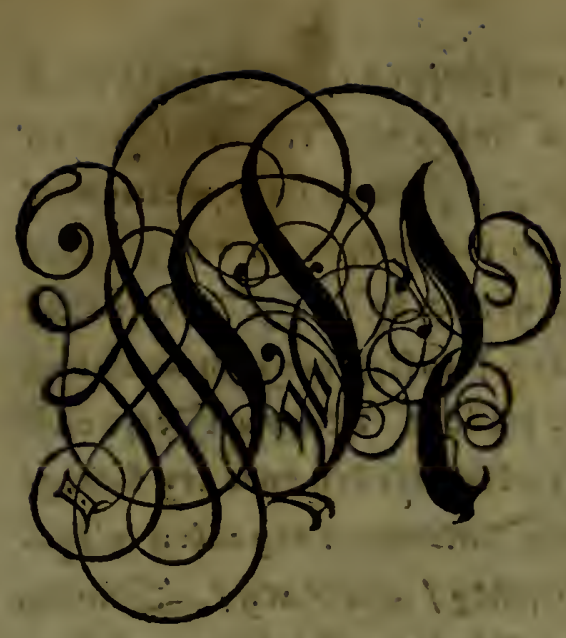

Aurits / 羽ince ban

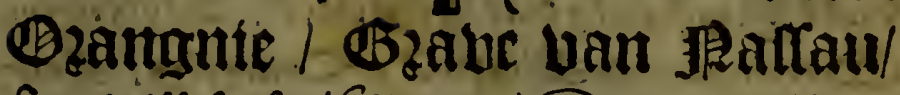
Catzenefféogffe / Soiandus / Sittz / ctc. Chat

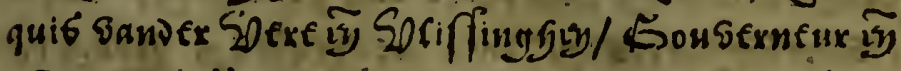

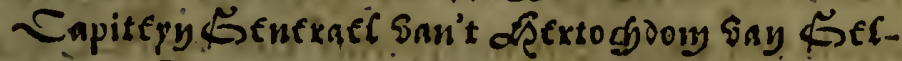

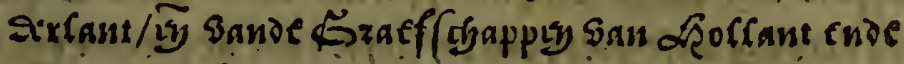

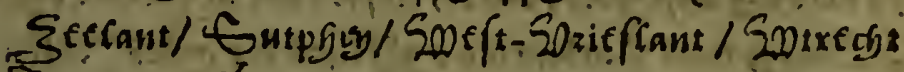

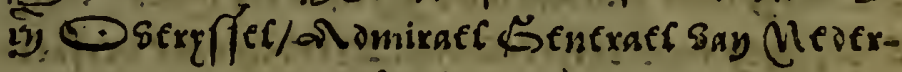

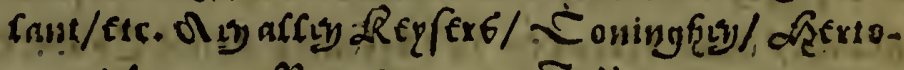

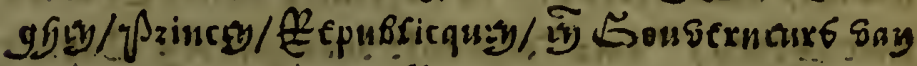

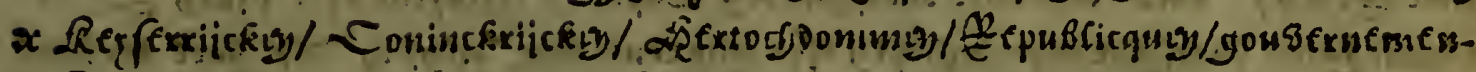

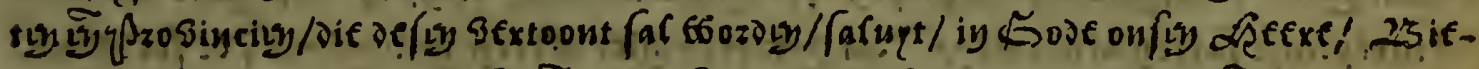

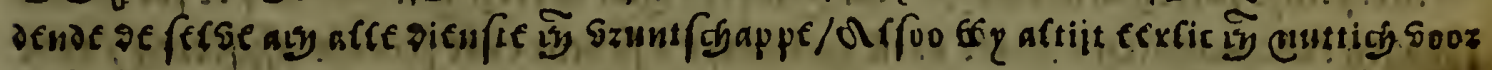

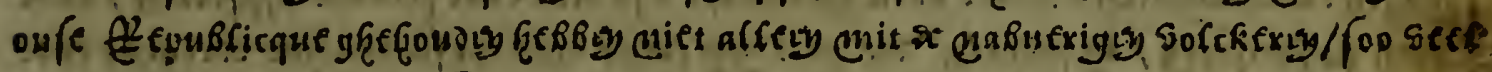

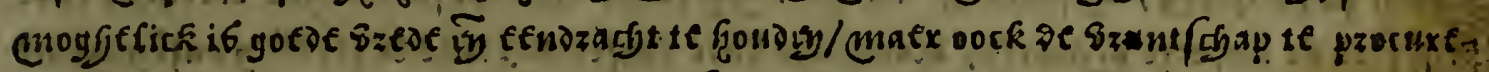

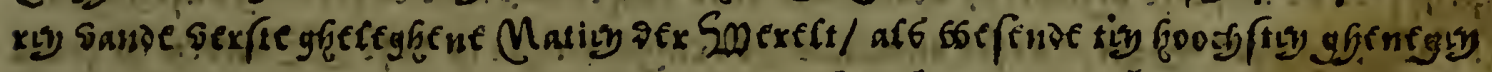

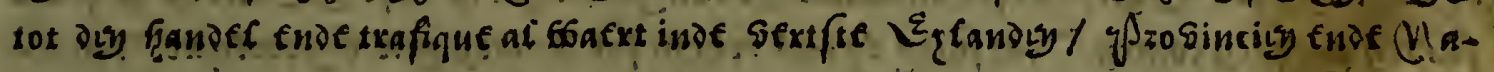

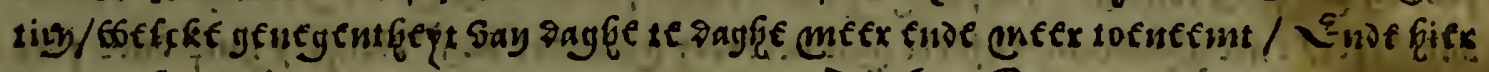

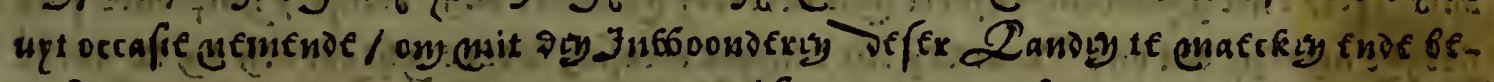

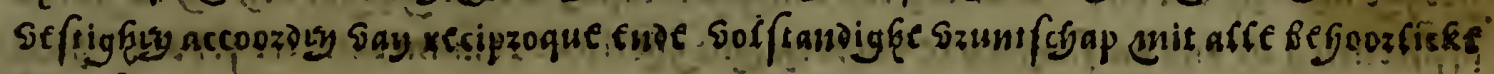

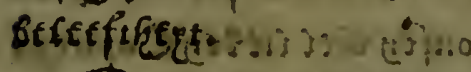

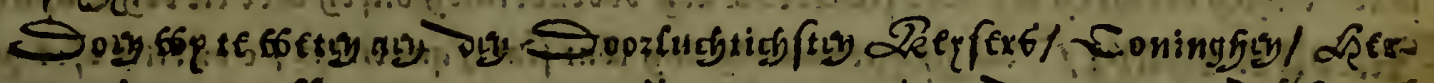

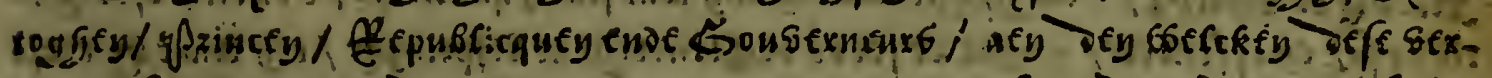

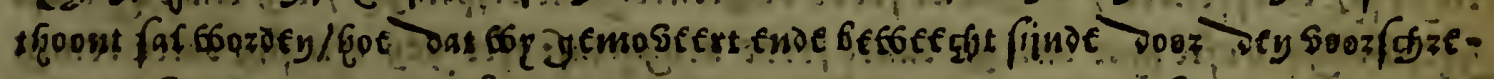

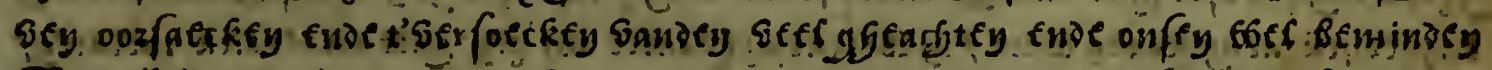

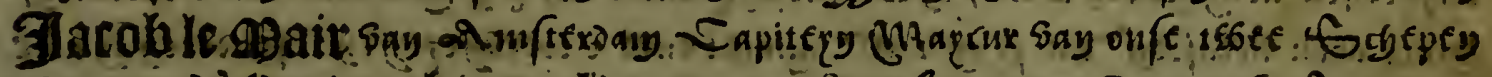

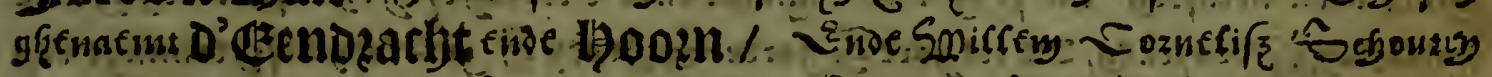

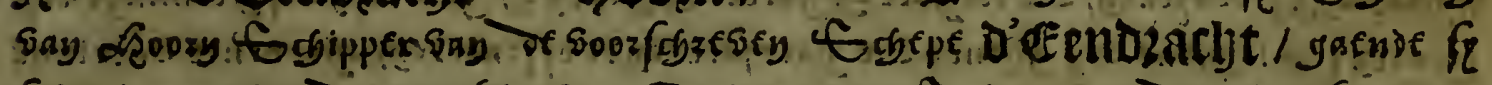

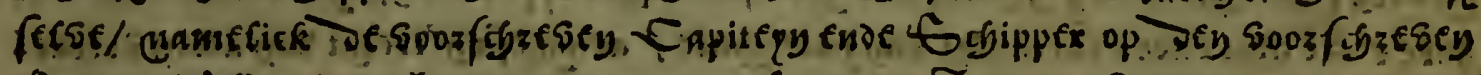

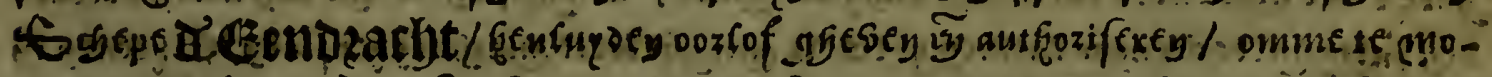

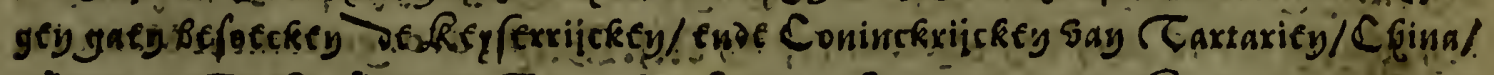

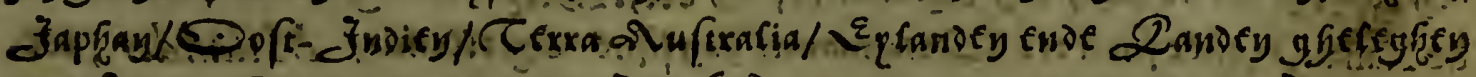

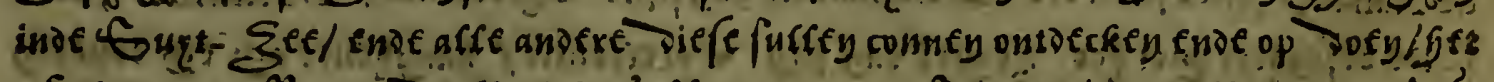

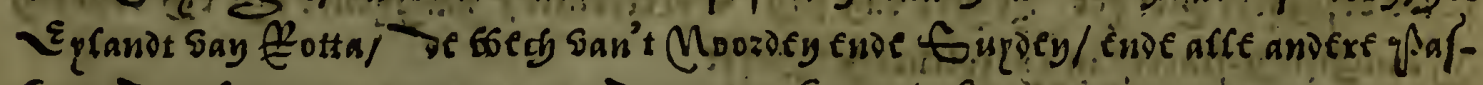

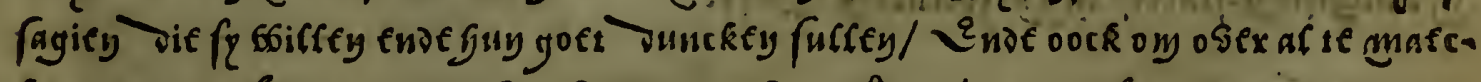
fey contract of te contxactey say gzede ende gzuntfojap/ ende txafiquexey ende gandeley mit Dey Jnf6oondexty/ende say Gafx roopende/sexcoopende ende teggent Gatx suqfenoe affexfande fooztfy say Coopmanfobappey / qfefterijey/ Gijoty/ Epece.

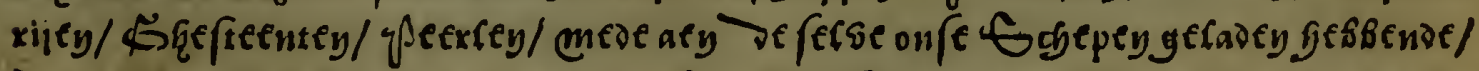

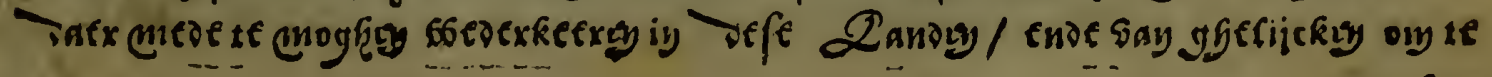




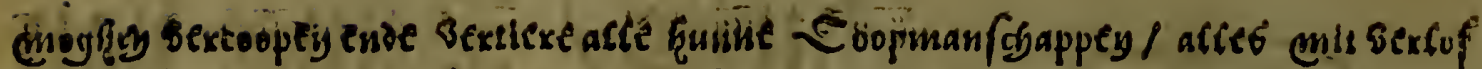

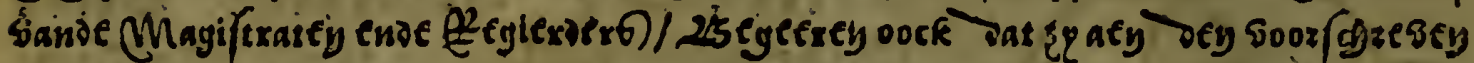

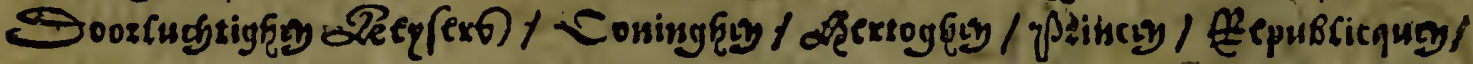

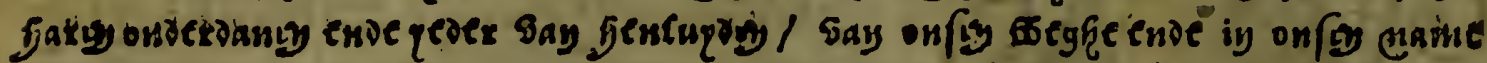

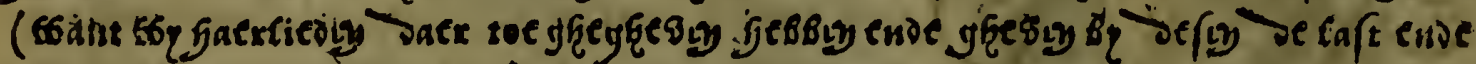

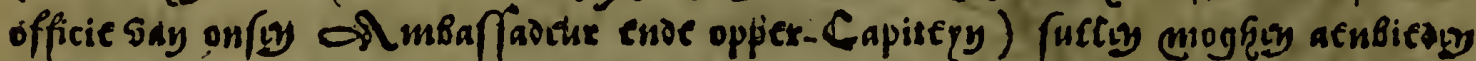

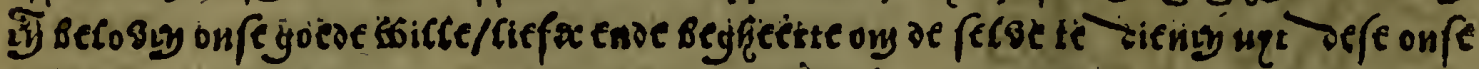

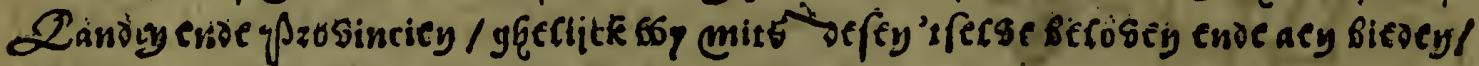

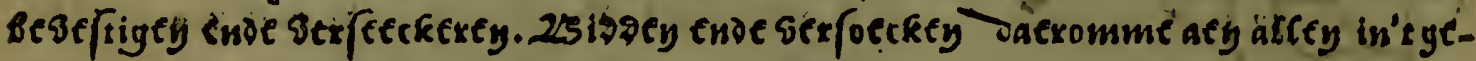

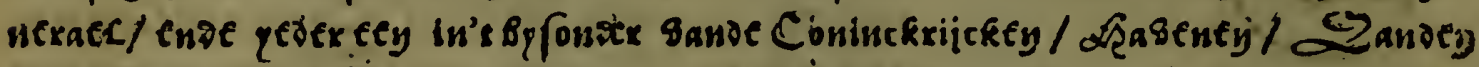

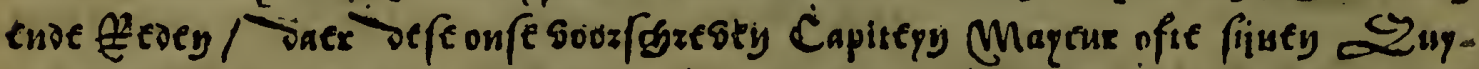

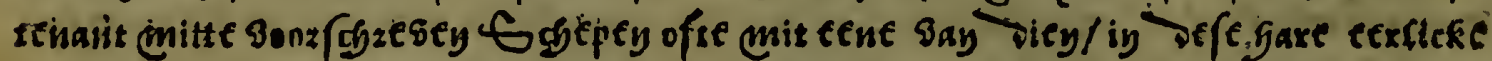

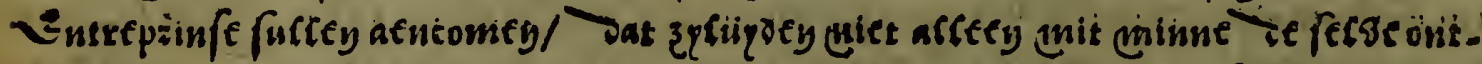

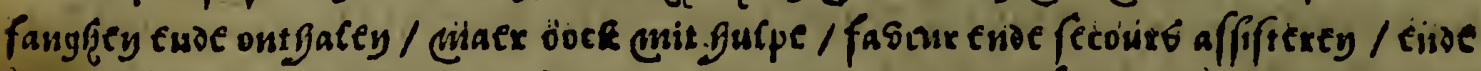
Joey fasozifexey / iy faeckey Jie iq Begfietrey/ ende tot foodanigfie textiicke cube pziiffericke impzefe begoozey/ Befosende ende serfetcketende a fefse iy onfey ciasue

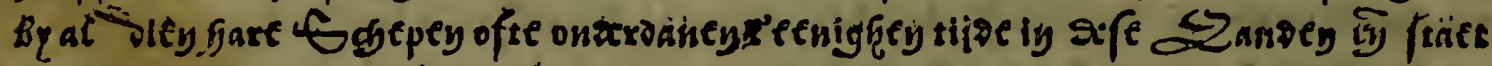

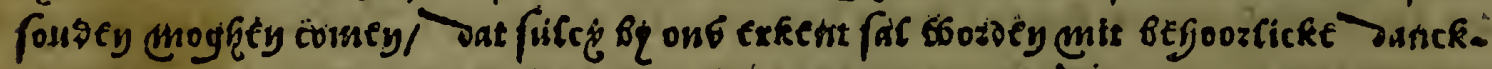

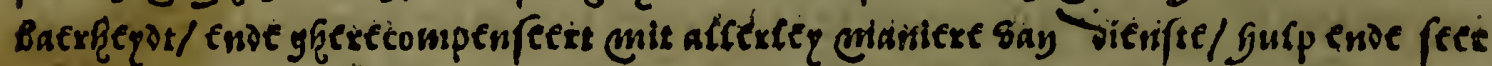

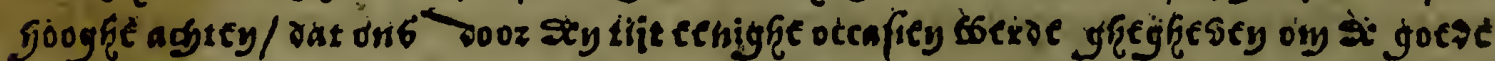

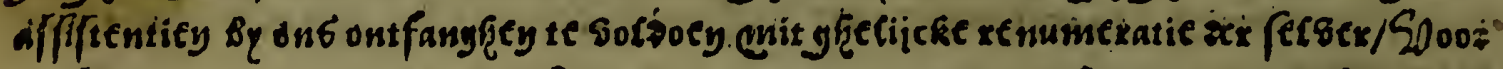

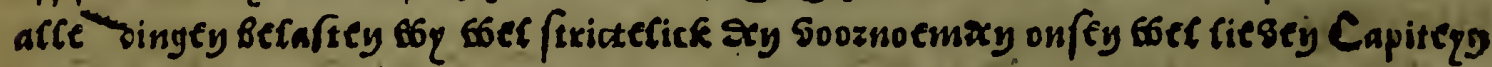

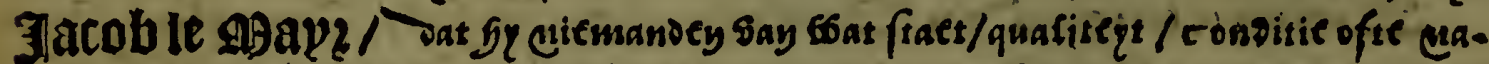

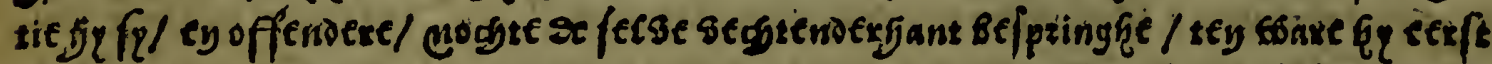

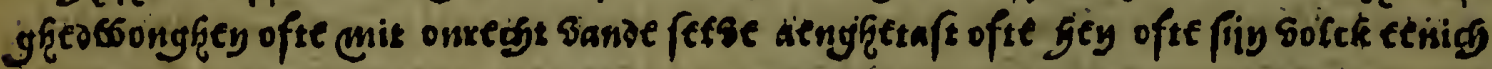

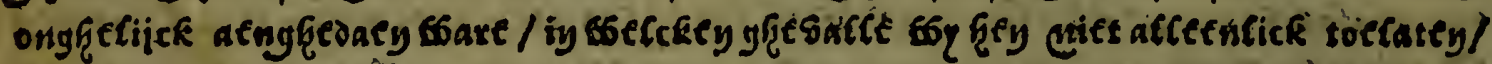

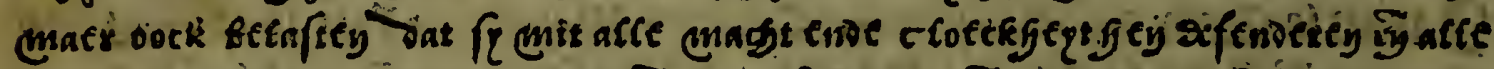

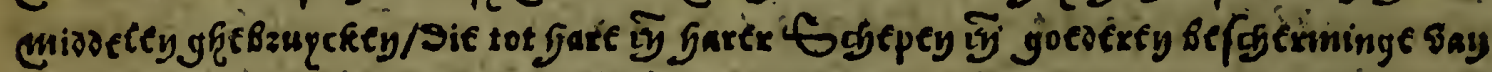

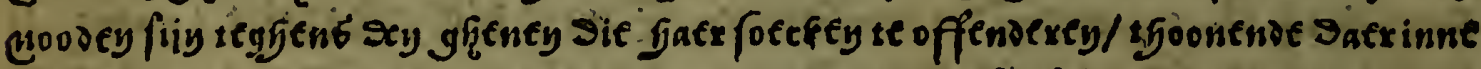

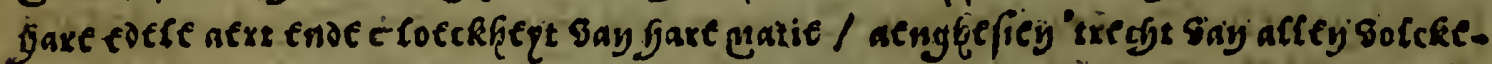

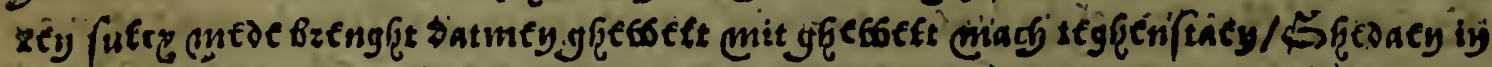

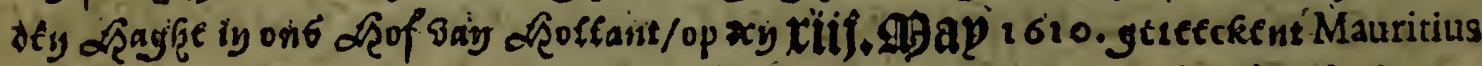

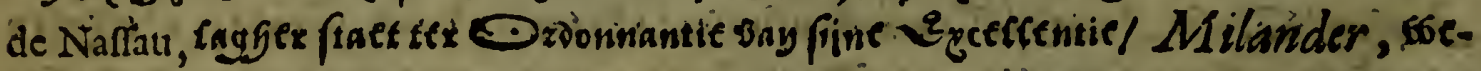

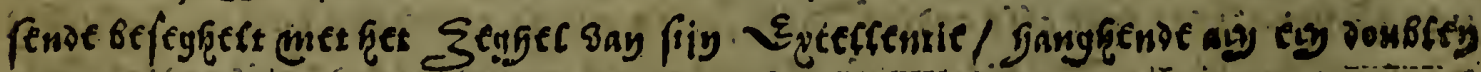

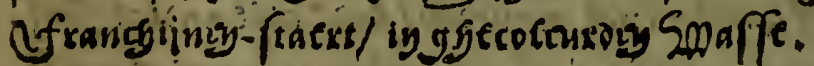




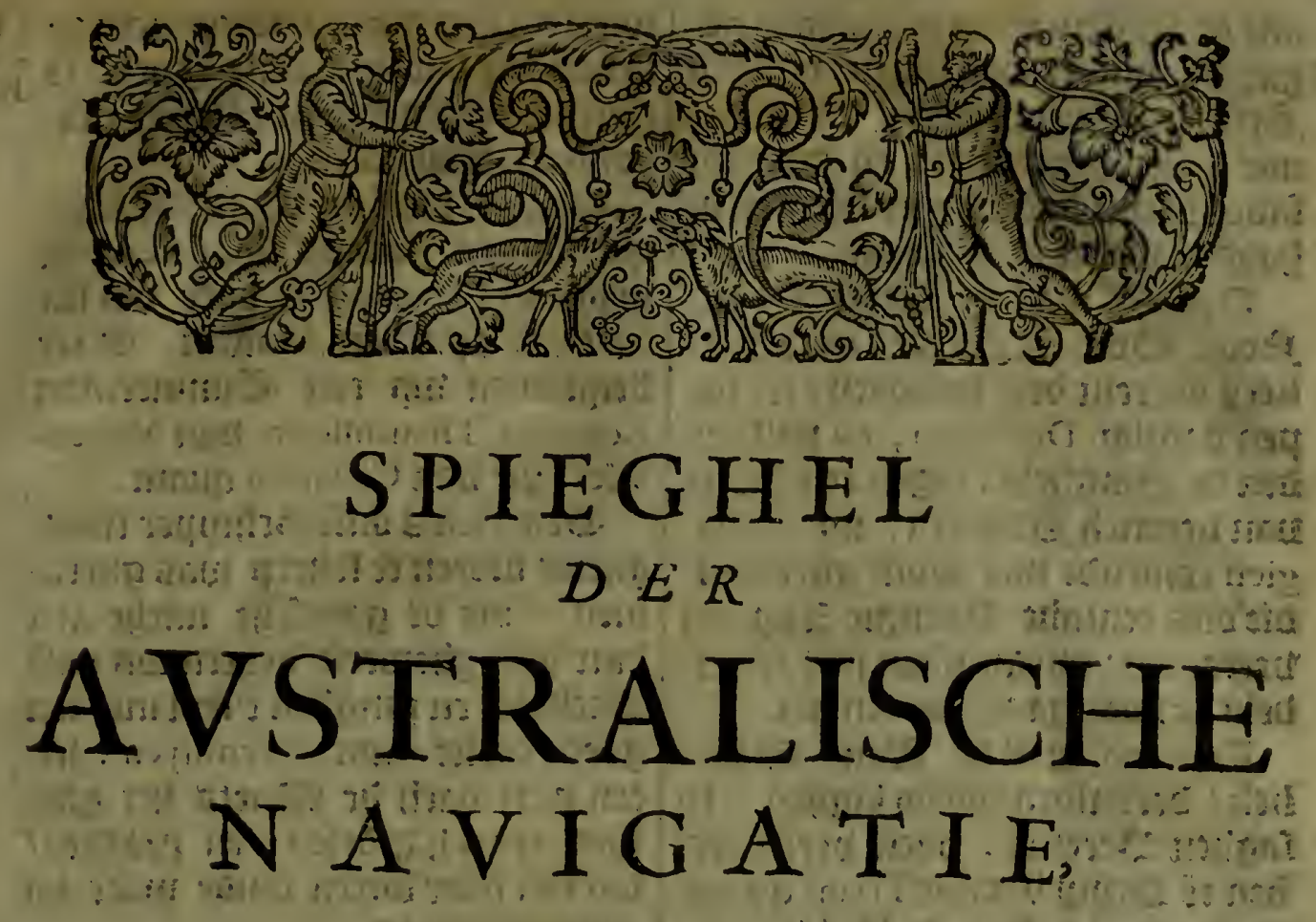

\section{Jaco den}

\section{Gaijt bermaerdenende cloeck= mocoughen zer-teldt/.}

\section{A COB LE M A I R E}

Prefident ende Overfte over de tvuee Scbepen, d'Eendracít ende Hoorn, uytghervaren den if. Iunÿ 1615 .

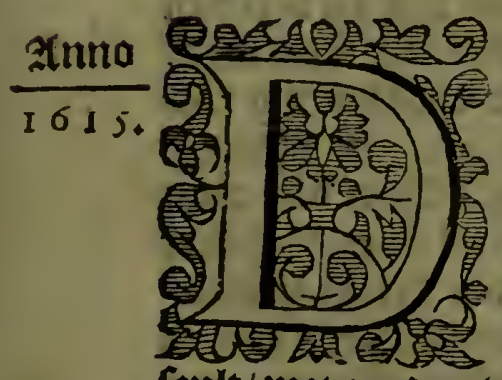

feplt/ met gocdent booztganck.

Den I s. wag De wint als vozet/ ginglien oulen cours nae de looof= Den / met goet weder. Een Daif= Ictjer ban Zierickzee uetipzaerken wp dien Dach / verttonden dat lyet Vecre gat Doit Sunt-Doft vau ons lach? ontrent Den Roene.

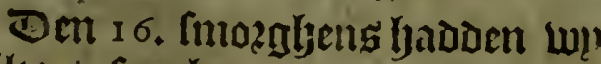
ftilte / Carghen Duynkercken, en
Calis : Dacuen net labuer coelte Iunij. Doo? De l)oaforn met de (Eblu: ber = Cuzarcken cen 99 an ban Hoorn, Die bain ons tilduglye bzocijt aen de liecuers: Smaclits bebuen wu bu glyelelut doo? at contratie bint.

Den 17 . Wag de Wint nocly at contratic / liepen derballen in Dupns/en fettent ander de Caltee = len. Zl lyer werde oufe Sclyipper, Willem Schout, aĩ Danicl le Majre acn Lant gyeret/ ountane Doeveren te garal / en ean Conltapel te hue= ren/ t'welcli glyefdyiede / en bulden oure lecglje Butcnmet Dater.

Dell I 8. contumectue de contera rie Luint:DutePrefidēt lacob leMaire, is met fiunen 25zocorr Daniel eñ 
1 6 I 5. alle oe affiffenten naer Zantwits ge=

Iunius. gaen / on te bernenten nae een Sefjecps-cimmertuan/ die aldaer niet te becomen was: en quamen fauonts nae dat 3 yaer berberfcht Gadoen weder aent booet.

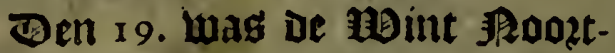
\$oolt-Bolt. Ilichteden onfe alne = fiers ontrent den midoacly/en lie= pen uooklup Doeveren, en patteer= Den de Singhels : fagen een blote van ueertich Schepen / uut Spaen gien comende met Sout glyeladen/ Die ons entighe Dzangic ztppelen vereerden : wu gingben met een re= Delicke boo:tganck dit ctmael.

Den 20. was de Hoint Helte= Iirk / Derbaluen wp bu laghen / en faghen 5 arefier: oock berfpzaec=

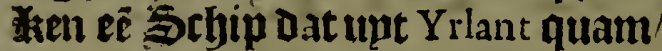
met een anaer ban $S^{t}$. Michiel, unt be Blaemiche Eplanden / Dien wp buitant beden ban eenigle roggen $252000 \mathrm{en} / \mathrm{en}$ andere $13200 i f i c /$ als Storkuis.

Den $2 x$, hadoen wp noct den 3u-Leggher / en liepen ober en we= จer/loo dat wp niet berbozderde.

Den 22, was het lelfoe weder/ oothmotadytich/en liepé in moictst: De Srljipper dede bliit onl een cimnterman te lyueren/ maer con= de gljeen becomen.

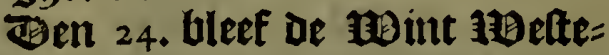
lick. Doent ginck oufe 13zefident met fijn affittenten / ende de Cam= mijg ban't $\mathbf{J}$ acht Aris Claeffoon, naer Nicuport ont een Timmer= man / maer 'tghebiel niet: Daer werden ban lyaer-lieden twee clep= ne Bercliens frbeep ghebzoclyt/ om uet te maecken / tot Schecpg-p2o= fiit.

Den 2 s.fmozgheng als be rioint goet was / lichteden wop onfe atne= lkers / maer bleef niet langlye alfoo: liepen over en weder tot dat wp in larmuyen quanten/ fetten let op feltijien Badem.

Det 26.als de Mint fmozgheng \$ookt-3oott-Boft was/gingben
Lup tfenl met goede bootganck: en 16 I 5 . beluonden dat wp onfe Jacht te $\frac{1 \text { so }}{\text { Iunius. }}$ cloec int feplen waren : liepen t' $\mathrm{ta}=$ uoutg uoozbu Portlant.

Den 27. bleef de Bint ald boaren / waren neffeng Gout ftert, en liepen in Pleymuyen, Daet wp bet fetteden op feben Badem. Baer bequamen wp een Cimmerman Cornelis Theunifoon ban Medenblick, die ban Canarien quam.

Den 28,als onfe Schipper fmozglyens bzoech te tebeep was ghecomen / die be gantiche nacht aen lant was gbeweet/ lichten we onfe Ztnckers/ en ginghen l'tepl met een goede coelte / upt Pleymuyen : lieten dien Dach oe maets bet glyeweer eeng handelen / en pzoeben/ Dat een poer weten coude waet bu opgheltelt was.

Den 29. waren wp op 38 . graben/de mint bariabel. Belen Dach bebben wa upt-ghedeelt / aen ss. Agan/twee hondert en vijf-en-t' $\mathrm{C}_{\mathrm{e}}=$ bentich Hafen / vooz de gantitye repre.

Ben 1.2. ende 3. Julij quelden Iulius. ons bariable winden/ oe locht was motachticly.

Den 4. yadoen wp de Haint Foozt-Bolt : Imiddaechg lyadoen wp de hoochte ban daie en veertich graden uiif en veertich $\mathfrak{s i n u t e n .}$ Dit wał de eerlte dach dat wu rãts foen untDeploen atn De gsaets/ te weten/ booz elck gran vict pont $232000 t$ / en een lyalf pend 250 ter 5 weecr/om op haer $23200 t$ te Inee= ren / ende een kanne 23iers Daechs van een gringhelen. Cot naerder beraet : t'fauants liep oe toint \#200zodijick.

Den 5. 6.ende 7. De mint varia= bel/'fmidoacchg de hoocljte ban vertich graden biiftliē grinuten.

Den 8. liep de wint 2002t-pBeft: Doen waren wp neffent de $25 a r$ : rels: op dien bach ouerleet op't Jacljt oe Bnder-rinumer-man Claes Pieterfoon ban Edam:en men 


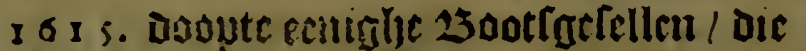
Ialius. nopic oe Barrels glyepnafert hnoden. De glisto bueire werde te vier uren naenosme ouer boost gbelet/ en cen 今̆turli log glyelclyoten.

Den 9. Whas De 10int Noozde= licli met ftube coelte / cu blecf al= roo twee daglien : foo dat wu den 11. fmozgens lagbeil Porte Sancto; cli Madcra

Den 12. limozglyeng ontrent òen viijen laglyen wu bet Eplant Salvages, diner ennigbe Bifteljers op= woonen / en lepllaent ontrent den

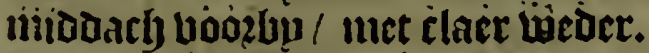
Pae de Élanden bain Canarien toe / Daer factheii wo een Sthip [culen / niet betchoe ban waer/ 'twas theit van ons.

Den I 3. De Hoint Roort-Dolt outg courg Supdelick. Des linoz= ghens laeglien wu Teneriffa, met namen oock de Pijck, lacli 19 eet ban ong: eenl wennich daer naer faglyen wh groot Canarien, latenioe télfoe aen bagitiooet legglyen/lepil= Den fulteljeit bejioeii Deur / welende oe [patie bait thien diunt the $29 i j=$ len/ Imet cen ljerde coelte.

Den 14.'miog gljeng creghen wp

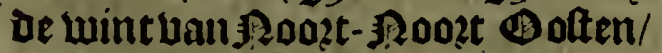
met gocde vooetganck: onle booch = te Lad furidoaechs viif en twintich graden/ beertictjginuten/ loo dat wu doen glycpalteert waren den Tropicum Cancri, die Moint conti= tuecede den gantíctyen dacly.

Den I s. was het een betoghen Toclit / waren lmiobaeclis op be Joocbte vain tuee en twintich gra Den veertich gimuten : loo dat ium gifteden viif en veetricl $\mathfrak{g}$ ijlen $\mathfrak{g} \mathfrak{e}=$ fenlt te bebluet in bier en twintich upzen. Dooj oe niidonely raeckten bu in een gibetentperde ljitte / 't'a = bouts loas onle cours I Supt ten Telten tot den daighetact toe.

Ben 16.finiodacclys Indoct wp

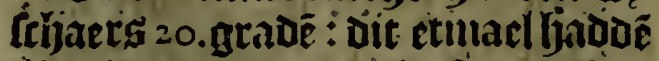

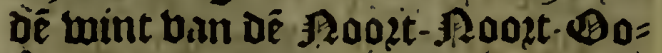
Gen foost-(Boften/ met (tijue coel= te/ch thander vooztganc: fettedé onfe

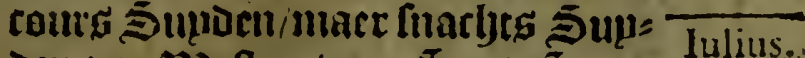
Dcu ten Weften / cll Supt-SultMorit / ont de BDal wat te muiden/. chl te [rljouluen of dzoochte ban Capo Blanco : lyet Muater was beel fwiart / waer unt gilten nuet verre wain lant te fiin/ ont wun nocljtang niet contbert fien / bet waepoe een

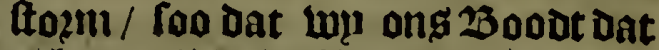
acljter aeii Aeeptén fmozgents als wo oe bzoedh-Coft aten/ uerloozen: en Daer wasi gijeen niodel oimte fal= vecen / alfoo bet de Eee bol tiba texs gluevult badoe / en dzecf ach = ter upt / eer wu het Srbip op de Z11 wicrpen. Al boe wel de Son= lie itt \% Enith badoe / waepae bet Daglieliicx een ftoznt/twelck fefijint Gier ofoinaris te fiin/ als of Sou= tie hooglye gaet. De sloctyt was Coo haricly datmen de sonute nau rien conde : 19p bernamen vooz midoactlys twee Schecpen aen ftuer-230ots ban ong/comende ban Capo Verde, oft Guinea. $\mathscr{A g}$ con= oe qualick de Soune peplen on

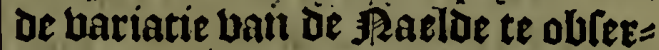
uceren/ niet te nin imen gilte of Gooctite vain twintich graden der= tich \&gintiten.

Den r 7. dito hadoé wp be hoochs te bau lebenthien graden / Dertich Sginuten/ dit etmael is de cours gbecontinueert Supden / De proint

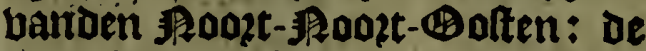
boozleden nacht beftont bet $\mathrm{BB}_{\mathrm{a}}=$ ter te Aecljten / net mop weder. woj faeglien bier dagbelijer beel vliegende siltchen/met lees watm ineser.

Den r. mozgheng ladoden wn calnite / Daijbende ging en weder: naer de midoach oe mint opeoes

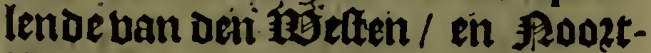
Moeften/cost ban't Ropzien/liepenSunoen / en Supoen ten Bolten aen / niet tamelicken booztganck: en leer warn weder. Het lieliefoe onté 1ozelident lacob le Maire de= ten dach of thate te betgaederen If 2 enAris 
fiol.4.

I 6 I s. en Aris Clacfioon, enl Ian Schout Iillius. Sibhipper Lan't Iacht aen bookt tontbieden/ omterefolberen naer Sierra Liona te loopen / on daer te berberiflien / t'welek oock alloo is bedoten/en bleluen bp ong fmidDaeclis op't Roen-inael / Iadoen Docn De ljoocljte Jan Tedfinen graet moe cen balf.

Eell I9. Ijadoen Intidoaectys de loorljte uan uiffthien graden / en Dit rtmacl een labber coeltje/ met eeniglie ftilte / gitten de langte ban Cabo Verde : twas leer heet weder: frughen ueel Steen-kroog Dzuben/ Dat een teecken was niet berde van't $\mathcal{I}_{\text {ant te }}$ lijit.

Den zo.înozgliens/als de Son op ginck / bẻbben wp bet ghelet onoer. Cabo Verde; alfoo wu het niet bouen conden [enlen/berbielen oes midoernaclits int tiveede quar =

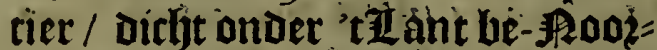
den de Caep; niet lonóner groot pe= rijekel/bulonder vain lject Jarht/on= trent de G9ont ban bé kebiere ban Zenega op $3 \frac{1}{2}$. baem tiolaters en het groot Schip op acht baem / gin= Glyen Heet ten Supoen / Lantx de abal.' Binghen lrier op de gront cenighe Bifry inet loocken : Oock warent wp aen lyet 7 achts-boozot/ om te fien boe jp gheffèlt warêt.

Den 21. was de mint Sunde= lick/ lichten onfe slurkerg / en gin= ghen t'repl / met wepnich coelte/ meer goet weder: maer ontrent Den abont trethen wp een Travado met Bonder en 25 litem fubiit unt bet lant/geduerende andéthalf ure.

Den 22. finozgljeng lichten wp ons Arncker / alloo de mint fterck was:-naer ontrent de $\mathfrak{g i b}$ daty/ betien wu gantech in calmute. De Capo Verde lach ontent ban ons Díte פsillen / Bolt aen / fettent op thee el dertich 19adeni/fant gront/ biefeer effen en ththoön was om te legghen/ wo faghen inneluaetets al leegh dumich Zant / groen en bồ 230 aftyagien.
Den 23. Dito omidoaeclyg gheen i 6 I s: hoochte conden wa becomen/ doo $\frac{6}{\text { Iulius. }}$ dien wo dit etmael unt bet ghefíche uan de Capo met variable Min= Den / en reghen 7 en betoghen locks wheplaeclit uerden: Sae de mid= Dáth huam de woint goet / en naer beél moejten / alloo ong de ftroom feer om de poost fettedo / te reede getomen/op $7 \frac{1}{2}$. Badem lant giront. Daer ons teritont veel Siwarten aen boogt quanten/alle bzientrelyap toe fegrgbelide / jae alle nootoruft' om ons te refraitberen / twótlck wn namaels contrarij bebonden.

Den 24. Jebben wp na ons 23 ier en Mater ghefien/bebonden groo= te leckagie / alloo de Baten ronder prere Lauben. waren: maerkten voozts gujereetrtyap om water te Jalen. Doen quamen ons énighe Swarten aen 250oit / (enoen 'abe= fonden te welen ban den Alcayro, die feer onbeleft marew/ epirbende

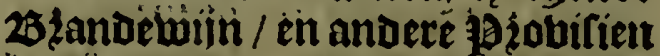
in loleftrben / Bingthen bien Dach Bifth booz bepde Srhepen.

Den is.quam de Alcayro; (dat ig Goubernetir) gbenaeme Gafpar Gonfalvo aen fiodet / een $\mathfrak{g a n} \mathfrak{n} \mathfrak{e}=$ ghenen beertich taren out/met een Almadia, met biet 9sannen/wuj DE= ben ben goede rietre / in ceten en Deincken / Daer hy matich in was actozdertien met bent booz acht Staben ürers / Too veel Baters te balen alg ong beliefoe : outh be bonglj Iju een fieftebe Sparns

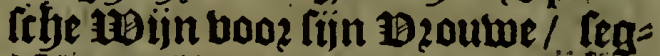
gibnde alloo de maniere te wetêt. - Onie lozéfioent lacob le Maire, (pzac aloerhande Coalen met hem

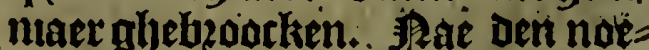
ne quam ons Bolch beoer aen

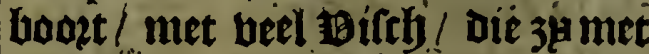
de Segben havoen gebaugen aent aoupen Eplant.

Den 26 . ninte bet Mater yeel fol unt ber Zze / oock de barde faint/ Reghen / . Donder/ ende 25lixem beletteden ong bet waater 
16 s s. halcu/ dooz or 23arringlje. Booz

lulius. de iniodach quam ex̃ bzecunt 5 chip bil outs op of litede / was een frimlina!.

Den 27 . if al quaet weder glye werit ! twelelt bier beroozfacclite oe [pimgly bande nienue פBante: filacbts wert lyet Atille/ en cregtjen toclte unt Den Noozoci van't lant: yaeloch Den Doty uner twee boots 1Bater. Dien Dacly cregljen wu weoer \$warten aen boozt/en ber= Torlyten 1. oft 2. Dlefteben maijng boo? lyacr Alcayro, oat met mo= geliick was te uepgeren. \$nachts ontrent twee $\mathfrak{A}$ gillen biti ons anc kerde ent כrlyup in Rcfrefco, liet cen pzinte blagglie blieghen/ be= vonoen Dat liet ectl thotterdams §clyip was / bain uectrich ZLaften/ oul op oc Cult te ljandelen / en Dan naer Brafilien te loopen.

Den 28. Ijebluen de 9 Satroofen met onfe twee 2500 ts / fes tochten FBaters glyelyaelt / cn binglyen fa= uonts de Saode / bp frlyoon ghe tempert weder.

Den 29. Ievldae onle jozefident. lacob le Maire, ende lijuen 25 zoeder Daniel le Maire', met De Juaclit / nae Refrefco, om ecnigitse verberfchin= glye te becomen vali Ozangien, tn gL imoenen / alloo aen oe Caep niet ell was: Imaer Guillam Block oe liotterdainmer / Cepoe niet ten be iten te wefen/ quan oerljaluen we $=$ orroin fonocr verberfebinglye / Dat ous feer bedactoe.

Oell 30 . Wuat é̃ ftille dach weers/ inet groote fitte: Bnte gazefioent die de meelte lozge voos ons dzocely/. lacob le Maire, is niet een fiefeh Wiins aen 3 ant ghetuaren/ent den Alcayer ghegraet/ oie ljem leer fees = fecrde. Hy was uan meeninglye ceniglise grobe 3 eelteli te coopen/ die Dace nivalicli te becomen zijn/ om datte te boozeli unt het zanot moeten ontboom boerden : Doch fyp coclje exil feljoone Gapt; on eent Stacf $\mu$ ters: en vercerde hen miet ecn clepu 3 unllelten : oat was al of 16 is. ueruericlytiglye die Iy bequnm. Iulius. pluckeden ten Deel \$octerlelie de Mer. Defen Alcayr ljadoe 4. Bzous Wuen / en res ltimberen / was een Cljiften/ feer armelick glyelogert. Én alfoo apparent wass niet meer uerberfeljinghe te connen beco= men / refoliberde lye om lyet landich aet boozot coment oer Swarten/ [mozafjens bzoecli tleul te gaen/ als foo wy uljereet waren / en al ons tobater in ljaboen.

Bent lelten fmozgheng/ wag oe Dint mt der $\mathbb{Z} e e:$ Doen quam bu ong het in atteroamirlye Srbip Dat ilt Refrefco gljelenljeil jaode / ghes naemt bet bliegljende Hert: De Coopnian Block bleef Imiodaechs onife Galt. Tit etmael wag bet icljoon weder / maer oes inachts Gadoen. luy een Travado, upt fet Zlant/ ntet Donder / 23lixem/ TRe? oljen/en ljarde Rout.

هen i ziugulti zijn wn met onfe Auguti twee Schepen: : en de Rotterdam:

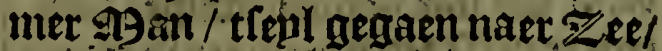
met ect Pooz-3oozt- Daltè nint: onfe cours was Sunt-meat : Imio Daecly nan oe HotteroammetgGan fiin affetbent / en tiep fijner wegtient

Den - de wint als boozen/ met redelicke toelte / Ctáecken ouer nae De Cuff ban Brafilien : Deg abonts wert bet lyeet ftil / en Dzebent Den gantechent viacht oorts in ltitte.

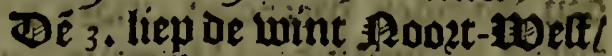
met redelicken booztganck / aten Dien Dath ong Blect Dat wp op Cabo Verde becoment fadaden : biele Den daer een tyeplingen dach âf batt

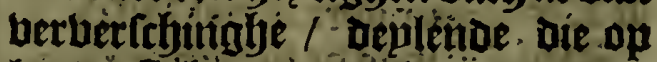
bevoe Sitjeper / oork oe loeterles lie de Mer. Smidoaechy ljadoent wo de fooclite bair $13 \frac{1}{4}$.graet. Dent gantechen nacht ginghen wo eent redelicken rours.

Den 4.crentien wa een ichoone coude ban dzie oft bier upeen bans Den Rookden: De cours was Sunt- 


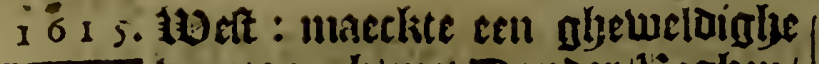
Angufti vooptganck/met Donder/lieghen/

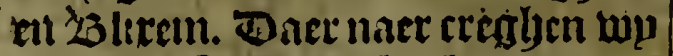

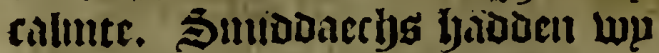
de lyoorfyte vall Derthien graden.

Den s. Imitboaecljs de booclite twaelf grnoeti. Dit etmael land=

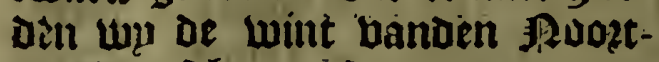
Feoclten/ felyoon bieaer/ met goede vooztganclt: fag̣ben aclst oagben lanck Leel Sefjili-1Padoen / oork' romtiits Albecoré, bit Bonniet.

Jell 6. Imixdacchg de Joochte ban I $0_{2}^{\prime}$. graden / dit etmael was oe wint als uoozen / feboon weder/ oe kimmen betoghen en Bijficly ocfe wint bzocht baij wat coude Ly/en Libas gbetemperder weder.

Den 7.8. ende 9. al betoogljen Zlocht / Dat men gheen yoocljte en conde peulen : Dicklwils oork kaec= ken en bläeghen met reglien / wp tagen Dien Dach twee toalbifteben/ en vingljêt oen eerften Bonniet.

Den ro. was de wint meet ba= riabel. Sagljent luachts een Caravelle, dat nae Brafilien wilde / loch = tent te berfpleecken/maer was ons fmozgheus ont teple boos tfilte/ Dat bet ons ontroept was.

Den 11 . Imidoaechs bevonden wp ons op de boochte bain negben graoen/45. Isinuten. Dit etulaêl neet ati: doen beltönt oe reglen op te youden / en claet bieder te wozaent.

ten 1 , en 2 4. haroen wint ban ben Supben dief ons / maer ber: tier dé weunich ooo de grobe ZeẼ die liet 5 fljip feer aingberden.

Q en i ó peploen wo de booctisté tchen graben. De wint als voozen/ oe Zee is fier outuer al vol Dilcly.

Den 1 7. was pe Gooctie 7 . gra ôn/ Dit etniáel ís oe wint toat gle= tuijiut. Oelen dack vinghen wo onile ecrite Do orado.

Bein i 8. en is. continatle wint uut den Supoen queloen ons too bât niet moghelirk was enighe joorfte te winnen / refolvecroe de
Poefioent/met den tiat nate Sier- 1615 . ra Liona te loopen / om dat wu ure $=\frac{}{\text { Augufti }}$ maede / Dat dic hoinc wel wat Imoclite ambouten : Dace-cul-tur= frben fonden wh olls cannen ber = verfeben! oun dat de Sifjentbunch begoft te Donimeren: cock becef= oen wo dat wu langlye onber de Linic Æquinodiael morlyten in (filte Daijuen. Op Den midanely badoeli wu de hoocljte ban leg gra= Den 52 . 2 ginuten.

Den 20.Alumut : yndoen fmio: Daechs de boochte ban feloen gra= Den. Bit ettnael continueerden wu onfe courd: Bolt tien Sumben / en Boft aen/ om Sierra Liona te be= Iepiten: De wint Gadoen wu Sups oen : en beranderingle ban water/ wipt oen groenen en twarten. Rae Den noen laghen wp groote rabe= linglie van froom / wierpen bet loot op les en bertich toaden fant= gront: feploen alfoo naer lant toel tot dat wp Dettich Badem wiet pen al ateffe gront : boozt loopen = de tot midoertiaclye/ op twinticfi Badem/ mendent tZeebaert om bet deooghen/maer Imotgens ant's Ferdell wp op achthien Bademen: en waren aen oe 1 Betet-cant bam of Bai xos ban S: Anna.

Den 21. met Sonnen opganclt Caghen wp 't Lant uan Sierra Lio.

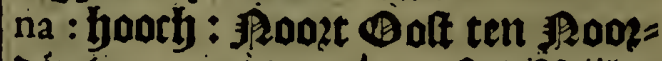
Dén ban ong ! ontient fes gigilen aent Sunt-Boften fagleen glyeen lant boozoer loopen / oan wel aent \$Roozden/ wefende leeglier 3 anat: ragen oork een Clippe/ oft Ênlan= Dekien van Mabrabomba, teghenss jooch lant aenlegghen in een keep of the de bain't gheberthte/ oerbals ben ong Alinelter. lithteben / en met cleene toclte baer op aen bieloety Dzongende beel fechtgeng / foo dat men oefe beele of uft bie feet vlack loopt mach looen in $z$ ee of bup= ten tgheficlite ban't lant wel veer = thien gijilen. Hop liepen al ober de Baixos ban S.Anna, al ceptende 
161 s. nact thimic toe: Het wepuniclj coelte/ $\frac{1}{\text { Auguti }}$ foo dat wu list miet bu daghe con= oen aen leplen/met de sonnen on= berganck ghelet op uiif Badem tebaers: Iet whetii oe gmck bier op en neer cen vadem : t'fabonts als wo Ropat-buact feploen bieptent/ maer Ooft-wacte bzoochtet : De Bloet loopt 5 upoen en Roozoen/ bet wag ftyoon weder / en beldere locht.

Jen 22. lmozglyens booz daghe begonnen wu wedet pesparatie te maecken / onfe Schipper boer aen 't Jarht / feuloe booz unt ong te waerfehouben / roeckenoe een be= quame reede:liepen maer cen 25ape bosz bp 't Ezlant / ell de punten en boecken uan't bafte lant / die mbet en Supt-10eft in Tee Itrecken: bebbent alfoo tegbens den abont gljelet neffeng t'ljoogbe lanot / op bier badem frfaets / lterck gront/ tis hier ouer al reer black , bebbent hier bu pzobulie ghetet/uertjopende dat dit de theede foude welen. Den joéfiomt voer noclj dien auont aen landt/ om naer bolek en ber $=$ berlelyinglye te bernemen / berna= men gheen ghewach of teethen uan volck/ dan ouer al 250 ofterhagie/dat mer niet $D 002$ en conoe. Bock foo bozlten $3 p$ baer niet diep int landt begheven / lijnde maer reebt uooz aen ltrant.

Ben 23 , voer onfe $2500 t$ on te diepen' oul dieper water te Coecken/ ende met ous Sthip daer te fet= ten. Den 1 perioent is vooz de mio= bach met onle 23oot en twaelf ghe $=$ wapende $\mathfrak{g a n n e n}$ naer lant glje $=$ baren om bolek te bernemen / ber= berferhinghe ende water te trijgen/ roepende naer een lant- 5 trant te: ghens t'hooghe glyeberchte / oaer buleer feljoon water bonden / co= menoe tuffehen de tteenen Dooz/ opt Stranot gheloopen / Doots mareerende de 5 trant 5 uut. Boft aen/of lancr; fagljen ober al alon= bantie ban water aen ftrant: Gin= glyen fomtiijotg wat lantwaets in/ vindende dan Bilt gbediert/ ban Berefteris / Blpphanten / Otren/ 25ocken/ggepz-Catten/en andere/ bol ban 250 fichagie: quamen ten laetften geroent en fagen een mont ball een Reviere ban beel berfeb water Daer $3 . \mathrm{en} 2 \frac{1}{\mathrm{i}} \cdot \mathrm{en} 2$, Badem was / met halff waftende water/ wel een glijle opwaertg roepende/ bonden die geel crom / en ban ghe= lijeke wiitte: het ghelogfyelte long feer lieffelick ; 'twag aen bepde fijden leegh 3 and dat de $2300 m e n$ int water hinghen / oaer wp eeni= ghe palmiten pluckten : quamen t'fabontg laet weoer aen boost.

Den 24. beeft de 19zerioent beu= de de 5oots elck aen een quartier ghelonoen om te bernemen nae de Tieviere / en een Eplandt Daer de Swarten moelten zijn / wel ghe= wapent ziinde. Setteden ons frbip op biif 1saden.

Den 2 s.tmozgens/quamen beps de de boots aen boozt/ tadoden noch Dozp/nocligenteljen ghevonden/ maer d'ectie $2500 t$ badoe een tie bier/ wel dafie oft bier 29 ijlen op gljeweelt meenende het Eulandt te binden/3p gingyen aen lant bir een dozren 23oom feel ljoordy / en bon= Den daer een 230 omgaert net $\mathbf{1} p=$ moenen/ Daer $\mathfrak{j} \mathrm{p}$ Lel leven lyonoert mede batljen. De jozefident / re= folberdue niae de kievier te leplen/ om te pzoeluen of wup daer in conde/ oul foo beel is pmoenen te crijgben allt mogbelick was/ baer comenoe/ bewont datter een deunuel of on= diepte vooz was : yebbent daer ge let / en alloo onle befident int I acht was ghetreden / en dat ong Schip voleboe/is fyp net oe febuyt/ en Aris Claefz Coopmaz ban't jacht in de Hevier gbeluaren / en ' $t$ Jadjt glyeozoonneert te leplen Sulnt= waert op / al waer wo cen leeglye Caep lagben/met twee eplanoen! mepunende dat dat de Supt eraep
Augufti

\section{.}


I 6 I 5. Luas dact Candifch af Irlyzifft: foo Auguiti lyet felfoe het land of plactere was Dat lyp daer [oude blijuen $/$ coo lyp glyeen Tievier/ met:lst Gulandat uonot /. Dat lju weder wooz ae $\mathbf{Z} \mathrm{L}=$ moen lievier fonse conien fetten/ Coo is 't Jacht oen wif en twinticy= Aten onder fenl ahegaen / ende den

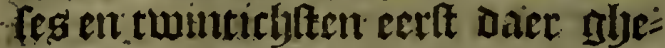
comen dowas itilte en contrarie wint. Sgidler tiit is de Prefident, met lyet Schuutgen in de kebier glyefeplt tot dzic plaetíen / Daer by : Duwfent Lier yondent. I pmoenê biant/ maer inet noepte:/ tot de midoel tae doo? thater en : $91902 a s$ l wemmenoe: tuec thacliten uut ghetweelt beb= Uende/cer 3 bet condế binden/ met grodeterglien an doucker.

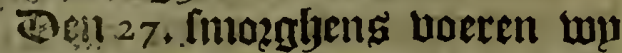
Lucoer hae een leun liebierken/ Daer wes meenden roorle glyefien te Jebben/ Docls vernamen niet/ maer Jonden Datrifín/Buevaers/Cro= codillen / in Schtlphoden: oocki Oefters die. aen de Clacken van 2500 men groepden/ dif mt water bingtien.

बen 28. Imozglyens als un een tieviere hadaen ghefren/eeil gijle oft mere beneden de Supt-ljaeck/ daet ons ban bere Doclyt dat een

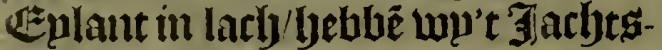
frljupt/ met Claes Ianfoon, ende den Schipper lan Schoute derwaerts ghéonden / hopende dat lyet de reclje platte coude fijn: Oock dat wu nljeeren bolck foude (pzeec= fien. Dock fonden wu anfe Bock, am te diepen thoe fien of wh met onfe Scljepen daer in conden / am 'twoater beneffens het 5 rij!p te felyeppen/en or $\Xi$ chjepen int beriely water frhoon fonde wozoen / maer beuanacn ondiepten. 500 is ll illem Cornelifz met onien $250 c k$ aen 'tualte laut aen oe 3 mut-boeck te= glyens over de liebier aen lant ghe baren / $t$ 'fuluonts weder camende badoe gheen vertely water beca= men/nach glyeen uolcti glyefien/ $\mathrm{fa}=$ ofen twee 23uffels : 'twas al black/ I $6.1 \mathrm{~s}$. datte voo? 't9logas nae de 5 chupt $\frac{}{\text { Augufti }}$ qualnetr/ foo dat wo Gevonden met te welen in de rechte Tieviere. Den 29. licljte wo oufe âneliers/ om loo bouen of be- Poozoen Den borck ban D'Eplanoen uan Mábrobomba te comen : De wint was.5unden/liepen lancx De Cuft/ quamen aen. een hoecls die hem Helt in Zee otreckte/ Daer wu t'la= Uonts acliter liepen / en ginglen Boft ten SupDen foo beel wu con= Den met dit ghetijoe aenbenghen/ alfoo bet ltil was / quamen ontrent een glil binnen de Capo, en fet= tent dicht be lant op bijftyien 10 a Denien.

Ben 30 . met de Bloot ginghen wu onder feul/ oe bint Supuen/ loopende een gsille berder/om nae De Leviere te feulen/ Dan alfoo ong de wint contrat ij was/en ber oogp faglen boven opt lanot/ hebluen wijt daer booz glyefet / fagljen bet berfey water upt de Clippen loo= pen / en 'tuolck riep ons toe / om aen lant te comten / Daer ober beeft onfe Prefident, A ris Claeffoón Coop= man ban't 7 acht $/$ en Claes Iañoon aen lant gljeronden/ Leedercomen= de bechten De Capiteun ban't Darp/ende den colck Emanuel, ende ent 5 wart aen liooat om met ons te accoederen on water te mo= ghen yalen: houdende Aris Claerfoon, met den booch-23ootfman aen lant in oltagie: Lun accozdect= den terftont mits gevende de ie $10 a=$ Demen ilinwaets en den Tolets een Badem loaz fijn fpeecken/ coa Dat wu terttont begonnen water te balen/en ruploen vooz Craelen en Sgetten / eeniglje sumoenen en Sananneng: Daer maren glyeen Hoenderen noch trijg.

Den 3 I. fmozgengs boo den da. glye / begonnen wp water te halen/ den ganteflyen dach/loo dat wp alle de baten uol creghen : midler tijt heluben boos Meraen en Goracticis 


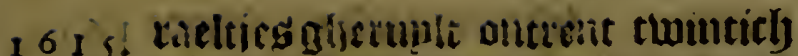
Augunti Dunfent \& munoenen/ en waer benoe

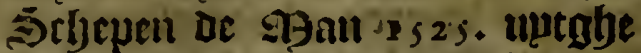
Declt : eregen ontrenc twee ljondert treltich oft twee ljenidert bijf en treventicly vooz cen Facka, of $\$ 9$ es/ en matenle met een grandelien. Defe Darly quantender twee Canoen balt tegljeng ober daer ech Coninck Faramborey woont / in D'cene was cen Coninct Swager/ en 2 ? inen warew/ oul ons te befichtigheni/ wat wu bzocljten / De Coninck [eu. De / ju yadoen luel Marfil en Gout/

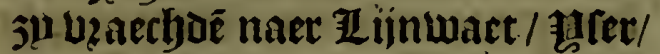
Cozactics / Sout /. Copere Brt. Keng/en Letels : Ieniden datter biu. nen bectlyien dacglen wel taijg Coude comen/ Die nocly op't Belt Itont / 5 u uerclaerden oock Datter

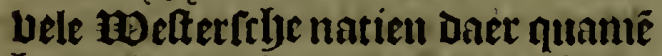
handelen/ Juronoer oe Eengelf́tyen Die ont root $\xi_{\text {out }}$ quamen bat oacr opt gheberibte watt. Een शgiile weechs van dele onfe water-plaet= te/ is een leer goede recde/ men leut op acht vadenten 5ant-gronot/ Dan Daer gaet een leet Aterck glye= tijde/ ban Gble en Doet/men lewt Daer bewaert booz alle Hoinderi/ uptghenomen voó welt \$ooztHeat : en Roort-tereft / Dan yet is nae lu eet befloten Gaven / Datter gheen yol water ean gaen/ oin de Dzoocljte die uooz de 23 aue Ieput/ Daer De zer op beeclit.

Septēb. Den certen $\Xi$ eptembeis als wu verberftht waren/ jijn wh fmid= Daechs met de ebue alloo lyet ltil was en inde wint/ uptwaertg aen= glyedecben/'tlauonts weder gljeret/ onttêt cen lyalf פgijle vande 马uฺt Caep / wefende een פgijle vozber als daer wute boozen laglien / $\mathrm{ctc}=$ tent boos een clepme sape / in= gaende als cen teveier / fijnde= fant-gront/ Dact upt bel ong $\mathbf{3}$, aclit lette en feljoon naeckte / met een daglyeliiex glyetijoe/laghen lyier op 7.en 8, Dadem Sant-gront / viclit onder de wal en be Glippen/ mtaet 'was buer met feer goet legglyen.

Den 2.isten partic ban't wolek aen lant.ghefet oul palmutes/ $12 \mu=$ moenen/ tî bananes te loecken/ of Wilt te fetjicten/of te biffrben/On=

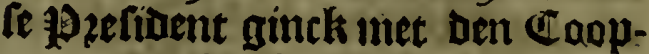
man bant 3 lacfjt A ris. Clacfoon ent= Defijnen $2520 e d e r$ Danicl atn laut/ alloo bumeende batter cen (Dozp was/ om de Capiteph te fpzectkeni/ mits de Negros ons ricpen als Loo= ren : maer voniden niet Dan Dzse

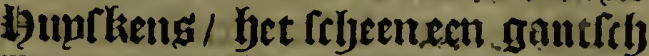
Dozp geweelt te hebluen maner ver= bzant / Dete punt wag onbekent/ milt / bol 25 offethanie/: en palmi= tes / oockeeniglye tivijs bonden wo Lu de L)upfliens / met nilie / maer beel I pmoenen int wilde: gheen gedierte dan poapegaepen en Ren"= gets: Initbaectys quam on s volek weder en bzocljten Steenbzafeniz/ met een Tioch / yelibende een lteert uan een 10abeni lanck/ en tauonts bzochten $3 \mu$ eetl 9 etug-Catte / en een A ritilop Die jonck was / in een Itrick ghebanglen/Die van de Mil= Den glje tet was: $t$ 'wagkals een clen= ne timbe/ en uan tfelfoe coleur/al= lecti t'booft en loozentieg uall een ander fatloen ; wu bonden hier ooc gocde dzumuê/ indiê die wel gebou= wet warẽ/Coude goede Maiin gevê.

Den 3. ginck weder een deel Wan ong bolrk aen landt / en bad= Den gljeweelt Daer oule Prefident gljeweelt ljabde daeclys te boozen/ cul hadoen beel IL unioenen glyelion = Den / Uan de welcke $3 p$ een goede partiic niede bzocliten/ Daer af elck bondert en vijftich deulde. / en hads. Den te voozen/ 125. vooz baer Deel. En alloo wo lier onfe Dingben ge= daen badoen/ an' $t$ Schip cen wey= nich frboon glyemnieclit/refolveerde (moeghents uzoecly t'tenl te gaeni.

Den 4.volglyenoe onfe refolutiel ginglyen met de ceble tlepl/ unt Sierra Liona, met wepnicly coeltel nae midonels is de wint gefeljerpt/ 
I 6 I s. foo ont wh ouer bevoe boegen geen Septēb. voozocel dedé : \$200zt-waerts Dooz dien de Cult 9:0ort- moeft ftrecke/ conoen wu niet bedziiben/en Suptwaert conden bolen de Baixos niet feplen/en wp molten 10 elt in Zeel om int bater-nater te conthen 60 . ovijlen ban't lant: lieten tlabonts täncker ballen op veetthien $1 \mathrm{~B} a=$ uem zant gront : alloo men bier op be Culte mach anckeren tot io.en Is.99ijlen/m meer ban't lant/rijn= oe feet vlack dere beele Culte / met fetht water/bebonden dat ong de ebbe Supt-wacts fettede / en de Oloet reclje naer't lant trock.

Den 6.7.en 8. bleben al onoer Sierra Liona, fonoer te connen daer af raecken:uernamen ltercke ftroo= men/met bele reghens/en Supde= Jicke winden/al-te-met calmte/al-te met harde winden/ loo dat wu dic $=$ wils anckerden / doog't onweder ban Donder en 25 lixem.

Ben 9.10.11. en I2. Jadoen lup variable winden / al-te-net onder fepl/ al-te-met Leeder aen ztncker/ op 32. 1月obem eñ 29. met wepnige nootganck/fmidoaechg badoen de joochte bant 9. gradê twinticly $\mathfrak{g} \mathrm{i}=$ nuten / den Prefident dede ljet gantiche fcheepg-bolek den zlrtiie = kel-252ief boopleten.

Den I 3.en 14.bleben bph leggen/ berwachtento andere wint.

- Den I s.fmozgeng booz dage zijn wu weder onder fepl ghegaen/eett met frherpe wint / Daer nae rupm= Defe : copt na oe midoach begon bet weder te maepen van den HeltSupt-meltê/foo dat wp oen gront berloozen / eñ bernamen wederom Ulieghende 1 is : twag de gantlebe nache beel caeckich weoer.

Den 16.fmozgeng met een caec= ke wert bet ftille / foo Dat wo bet Suut waerts oberwendeden:coets Daer naer quam daer weder een raeck/met groote regen/ foo dat wo bet 3 lacht niet conden fien/heblent Derbalven geret/ ambet Jacht in te wachten / feljoten oock een feyoot i 6 1 s. Inachts/en 'twaende een ftozm/ foo $\frac{1}{\text { Septéb. }}$ dat wu waeten ghenootfaeclat de iraes te terijcken/t welck nopt Defe reufe toas gljefebiet.

Oen 17. bleven wn aen zuncker leggiten/ om het quaet weder. Ten 1 8. bettont bet te befadigen/trocken onfe raes weder op/en gingé tiepl/ conden 'tancker qualick op crij= gljen/om't grobe water/[oo dat ofe zidriftentell molten belpen winden. Tiefolbeerden lyalf weder nae Sierra Liona te loopen / alloo oe thadige Supde winden ong plaechoen / die Daer getwaept badoen leven en arbt weecken : alloo de 3. winter 99 aen= Den aldaer $\mathbf{3}$ ulitis/ztugultus/5ep= tentber/ regen en quaet weder fuls= ject zijn.

Den 19. Pae de uzaech-Colt was de wint 1pettelick / Daer nae Supt melt/ roo dat inp refolveer= Den weder nae Sierra Liona te loo= pern/twelc onts wel Geozoefor $/$ nochs tang belt vooz ons was/ Dooz oen barden ftroom die wp gilteren ach = ter-midoach bernamen/dit ons om de Supt fettede / foo dat wp vzeefs den bu ralnite in de 25 ocht te ver $=$ ballen : letteden derbalben onfe cours na Sierra Liona : tegens uen abolit quam bet Jacht by ong/ fep= de / dat lyet een anteker berloozen Gndoe int lieljten/ en vijftbien $1 B a=$ oems Cous/[nachts ltadige regen/ met variable coelte.

Ben 20. (1mozgheng hadoen wo weder gront op Derticly vademen/ die wint Helt / De cours Boft aen/ Inet mop weder / en goede boozt= ganck: op de miodadh liep oe wint

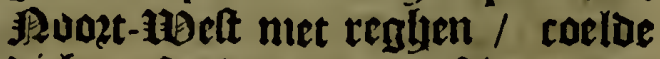
dicht / foo dat wu ter Zee-waett lepden/Sumben aen: hadden (nivo: Daeclys de hoochte van acht graden Dertich ginuten.

Cot lyet epnde van defe $99 a e n e$ toe: ginglyen wo valt vertelyepoene courfen/Dooz de berfchepdene win= oen : Ladoen oock vecl regend had= 


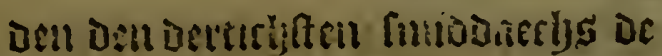
I 6 j ;. lyoordjte Uall buf givach.

Oatob. ए)en r. Ortober badorn wu ver= frijeudorne Luinden/ an courfen : foo Dat ljet 5 efjip weynicly uertierde/ Doos de đappiclyent orr windé. De= fen darb fagé wu een coetelounue/

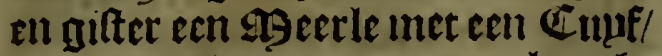
bout bau Deeren met cal langye beck/als een इuippe: oock dzie oft vicr Stwalumen/die ceniglye dagen butt Schip bielden: vermoedende Dat ju vant balte lant quamen.

Den 2. Dito: liepen de winden bariabel / Gappe coelten / De 更ocijt meet inclimerende nae bet $\mathrm{PRog}^{2}=$ Den : als oocliop den Detoen defer/ Inactyts na lyet Surden: beluben bet eerttequartier in ftilte glyedzeluen/ met mottige $\mathbb{Z}$ ocht/ fauonts claer = oet op / fmozghens crentyen wou een haeck ban den Roort-Heften / Die guerde 2.glaren / Daer naer wozde bet ftilimet betogen of oclyt/ loo dat Lou lyeel moepelick wozden.

Den 4. [middaechy yadoen de jooclyte uan 4.graden derticly $99 i=$ nuten / dit etmael d'eerlfe quartier Decuen we ilt ttilte / met betoghen I ocht : coets daer naer cregten wy cen coelte/ uan den Supt- - $u$ t-

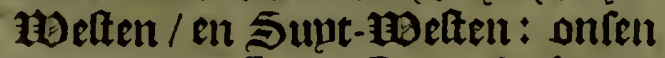
cours wats Sunt-5upt-Doft / cll Suut-Ooft : Daer maer feljerpte de wint/ coo dat wu wentaen 1 zaelt. waerts ober/met ithoone coclte/ bie wu in laugen niet badoen gebadt.

Den s.peplaé wu de ljoocijte ban

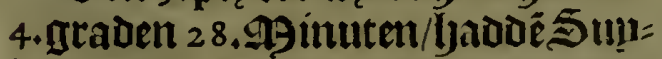
Den luint: De dimninglyen quameit oock uan Den Simoen beel grof/ ontrent dien tijot waffer cen groot gljedonmmal vooz het 5 chip/dat die onder waren meenden dat lyet op eenClippe ftiet/maer bebondé buy= ten toefiende/ dat de $\approx$ ee was glye $=$ uerwet / wilten niet wat Dencken: maet als wu in Porto Defire qua=

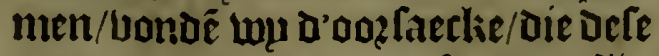
was / dat een $\mathbb{Z}$ eemonfter met fiin hoozne hadde tegeng bet 5 chip ge= fluommen 7. vostê oitoel lyet water i 6 I 5 .

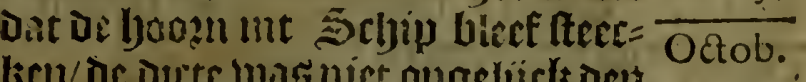
licti/ de Dute Luas niet ongelijeli dent genecue Oluplyants tant/ Las in= gljedzeuen int hout wel een lalue boet / en ech clęune ljallue boet daex. buuten/Datmet gelwélt cozt wass af: gebzoockê/ waer dooz bet Zeenton=. fter foo glyelueldoicy fjadoe glychloet.

Den 6.7.8.jiin de winden al ba= riabel gljeloopen/met grooteltilte/ IepDen dickwils ouer en weer ober/. met gljeftadiglie reglyen.

Den 9. Gelfont Den darly met flap= pe coelte uan Den Suut-Delten/

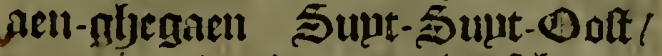
maer int tweede quartier trljerpte de wint / met ueel regliens/cul be= ftont bear te maepen / dat wu onfe Igarfeuls malten ltrijcken.

Den I c.fmidonaclyg luebondê de yoochte bait $3 \frac{1}{4} \cdot$ gradoen:de $\mathbb{Z}$ ee was heel tlecht met een eenparige wint/ fo dat het 5 eljip gocae filede lyielt: bingen dien dach acht Dorados, en giffer beel Bonietten : en twee ban de 93 atrolen werden in de boepen ghettelt $/$ an dat $\mathfrak{z}^{u}$ gheplockbauat innoden.

Cot Den I 4. Handoen bu hatit al cen beuer/miet aroot uerderiet/ ouer en lweder loopende : fagen beel ra= beling ban froom / tgintet loo ont boclis wel foo hart boot als te boo= ten / coo dat wul Lermoededen / al was ong betitecli ober be liondert Mijlen uan Sierra Liona, Dat wu Inaerder ve Guineefche Culte twa= ren als wu meenden/ Doot dien wu? foo ueel cleume Dorado laglyend Die yier acmeenlick leven : foo dat bu

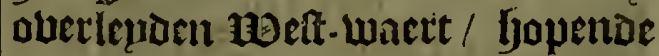
Daer beter winden te becoumen / als wu naerder Brafilien fouden jiin! alloo entige obletuatien treue ons te vertach gatuen/ Derlialben réol= ueerden roo langlye Heft ouer te loopen als wou foudoen connen / Coo Iniet Ooft-waett. Defe dacly uin= ghen wh weel biffy/ foo oat be Scheeps-coft gljelpaert wozde. 
fjol.12. Spieghel der Auftralifche Navizatie,

I 6 I s. Ten is. Pnididaeclys oe lyoochte Octob. twee graden en biif en berticly $\mathfrak{A g} i=$ nuten/de wiut Supt-19ert : Waren al befich unet de Diflelyerije/t'welck voozecl gaf / alloo onle rewle nocl lauck Dueren [nude/vonthen delen Dach unozt gantirlye Srbeepluolck roo beel ju in twee dangen conden eten / hier was de Zee foo vol bis Dat onfe $B$ oghen feyemeroen.

Teen 16 . funidaaeclys badoen wu De hoocljte van een graet / en een vierendeel : hadoen een eenparighe coelte / met frboon dzooch weder: fagen Deten Dach beel maluilfeben/ die d'andere verdzcuen / [oo dat maer een Bonit glyetchoten werde.

De tavolghende dagen wag het al til weder / met flappe coelte/ Alechte $\mathbb{Z}$ ee / Coo datter beel Bifrh ghevanghen werbe booz tijt-ber= Daiiff.

Den 20. Inachtg pafterdon wp de Ilinie Equinoctiael : alloo wp oen een en twintichlten badden de Iloochte van Derticly gginuten beSupden de I inie. De bint beltont al-te-met wat te rummen / dat onfe cours viel Sunt-zaett ten Supdé! ril Supt-20ett. Han daglye let = teden wp tweezluckers bande bou= glye int rụm.

Den 21 . hadoen wn de yoochte Lam een graet twaelf $\mathfrak{g}$ inuten beSupaen ae I inie: met goede coel= te/ De Zee beitont wat grober te gaen / en ons bocht dat wp thabe= ling ban ftroom laghen/ tot op den vier en twinticlytte als verande= ringe wan wint boozviel unt BoltSint- Ooft / met een doozgaende coeite: De 23linde raeckte aen ltuc= fen dooz de holle $\mathbb{Z} e$. Onte be= Iteck was be-Deften D'U Eplanden ban Don Fernando Loronha, Doch niet whefien / noch gheen uhebo= gelte vernomen/meenden even wel Dat wu veel Boltelicker waren.

Den 25. hadoen de boorlyte ban 5:. Iraden : Onfe courg en wint als boozen: twag caecliachticly we= Der/ met reghen betmengt: en cre $=16$ I s. gen dit etmael woch fryoene coelte: $\frac{}{\text { Octob. }}$ Alloo wp nu op biff graden waren/ hebben we onfe Stier-lieden/ar = filtenten / en Officieren berclaert dat onle meeningbe was te baren nae Terram Auftralem, en baerlie = den yet memoziael bai Quiros, Dooz Claes Ianfoon inde li)ut voozglyele = len / om te animeeren / foo date groote lult en couragie daer in cres glyen: De paftagie bebiel de Stierlieden oock redelick wel / die Daer feer in berblijt waren : oock alle let volek die den naem ban Terra Auftralia niet en conden ontlyou= den/ frlyetbent met crijt in de פgut= [en / andere op 25ardekens / eeni= ghe in haer Jafel-25oecken: has pende booz baer reeckeninghe bau foo een treffelicke rep[e/goet profiit te doen.

Jen 26. Imidoaechs hadoen wp de loochte ban leg graden derticly gsinuten / hebluen dit etmael de Luint glyebaot van den Supt-Dos Itetr gingben Supt aen / nu wier= oen lup ghe waer dat wp too $\mathrm{MB}_{\mathrm{e}}=$ Itelick niet en waren als ong lot = Iteck / en metten ltroom al Bolte = lick molten ghedreven jijn / en al= foo wu nu de Culten van Brafilien aen bookt creghen/ gilten Dat in nu oock inde ftroom quamen / Die oul de 5tut Dees tijot staerg loopt.

(Den 27. Desmid daechs wag De yooclyte Lau acht graben frlyaers/ dit etmael is de wint glerupmt/ en glyeweelt banden Dolten / comtiits Bolt-\$odat-Boit : Moegben onfe nientwe 9 garfepls en 23 linonn aen: Bnfe Prefident lacob le Maire, was met de Srhipper int Jacht / oul haer oock bekent te maecké werwaerts wp baren louDen / de Officieren in de Cajuut ontluicuende / en is hun alle gader wel bevallen.

Jnt laetlte van October al Stuudeliclie moinden/ladben geen lant/ norh 


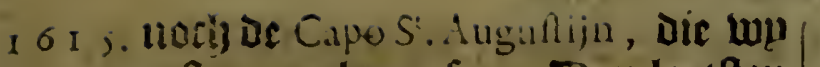

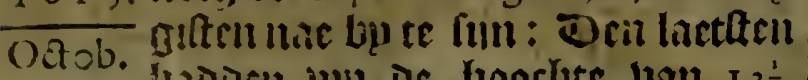
Gadocn wn or boorlyte ban $3^{\frac{1}{2}}$ griach / badoen dit etmiacl de Luint uut den Oolten / an Oolt-SupatOoft / met frboon claer weder en Doozgacntoe coclte. Sagtien twee

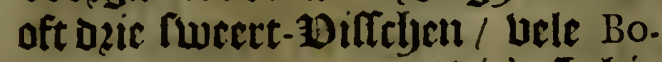
nictten, ent Albecoren Die't 5 rbiu atroich bolchjoen / wa lyadoen bier feer febjoon blau water / en fagben Dagheliict unt oun laut / en feplen/ maer te verglyecfs: uermoedende oock oul bat we glyech baet ban ftroom hadoen / Dat wu nocly cen goet ftuck Daer af waren : Get bleec oork unt de \$oozt-Dolteringhe Uan of Racloc uan acht graden/ twelck is ontrent trefticlj \$gijlen/ nae onfe calculatie: Sonnua wo bebonoen Dat wp het baii al wat om Den welten haoden laten gaé/ on rupme winoen te crijglyen / en ban De Cuft Laul Guinca te getaec= Ken : en tig leecker / Doen wu oule cours melt-waerts eerit oluerlette = Den/ Dat wu al be lengte van de Capo de drie puntas hadoen/als lwp oe witte mecultens taglyen / en ber = anderinglye deg waters. Doen werde gheraemt loo langlye te $\mathfrak{l}=$ ten 10 elt obertaen / als Den. wint foude rupmen / alloo ceniglye glye = felyziften ong fegghen. Exet ous befteck bande Stier-licoen/ wareli al arbter Dooz het lantot uan Brafi. filien gljefeplt / vooz oe mont ban Moranhou, neffents Fernando de Laronho.

Novēb. Den I, JRoluentuer : laadden Lụ de fjoochte batl i $s_{3}^{2}$. graden / en dit etmael den wint gljebad bamben \$oozt-Ooften / enọe \$oost-Ooft ten Oolten : ginglen aen Suyden? en 5upuen ten Dolten/ met f́lyoo= nen vootganck booz de wint cn claer weacr.

Den 2. Imidoaeclys de lyoochte ban $17 \frac{3}{4}$. graden/ oen wint hadoen noch ban den Roort-Ooften: De Eee ltadich ban acljter / Conder Dij=
Oen was oc booct

ghenthien graden. Cozts uac de midoacls fagen wu cen ban De $\mathbb{E}^{z} p=$ lanoen uan Martin Vaz; te loef= waert aen Gackboozt ban/ong / een clepn Ijackelich Eylant / meenton dat fyet Afcention was / niet feer booch / laglyen certa menichte ban fwarte en graulue Ian van Genten, oork entigfe rlepme agecumen die wit Laren. Beranderoen onfe cours / oin naer lyet ander Gzplant te loopen / en nae de luafte Culf te gieren / om wat naerder bu de wal in oe ftreeft uan de ftroom te co= men / die om oc Supt nu liep / foo wu'teplantot faglien febooten een reboot on' $t$ ' 3 actist t'abuileren : De= fen dacly pafterden wo d'A brol hos, en lagljen oock dit \&ejlant/ Dat wel met ong beltecli ouer een quam.

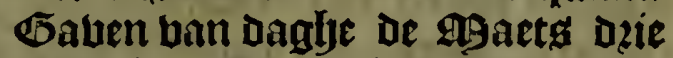
glutfkeng moing inde plaet fe ban I tot een teeclien uan blijélyap dat wa die periculetufe dzoochten glye paltert waren.

Den 4. [nidoaecljs de booclite uan twintich graden. Dit etmael oe wint gheljad unt Den \$Roort-

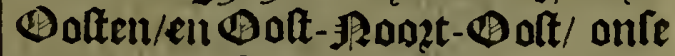
cours was \$unt Red : twas dup= factachticly weder / Datmen naulw ljoorlite conde nemen / 'twBater flecht / ent t'begolt coeloer te werdấ twelelt men al olver twee oft Dzie Dagljen conde boelen: wu fagljen oock glyen Bifrly uter.

Den s. De wiint Roort-Doft:

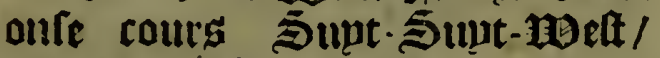
tweeroc duifteraclitich met betoo= ghen luclyt / Den boozleden narbt. hadoen wu regen / en oock ban da= ghe een dichte vlaghe. Dariatie elfgraden ban Roopt Oofteringe/ roo Dat ous Sunt-tiorit ten Sulv= Den/ was Sult- welt.

Den 6. finidoaeclys de lyooclyte uall 22 . griaden/ dit etmael was de

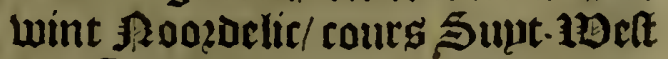
ten Sulnden/met gocoe coelte. 
fiol. 14

1615 .

Novēb.
Sen 7. De yeorlyte van 46. 99i= Iuten min als de Tropicus Capricorni, dit etmael calmi gheweeft/ ore wint \$upt-\$upt-10rat / met clep= ne vooztganth:

Den 8: fmidoacth oe boorbete van Deic en twinticly gradế $35.99 i=$ nuten / dit etmael de wunt ban den Ooften / met lieffelick weder / $\mathfrak{c h}$ flecht water / Gaghen noch $\mathfrak{g}$ hecn Seplen/yoe wel wus maer twintich oft biff en twintich'gilế yan S! Sebaftiaens Eplait waren.

Jell 9.10.1 1+12,13, 14, de wint al unt den Bolten / cn इunt-Bo= then / met tlaer wejer/al-te-met \$Roout-Boft.

Den I s. Intiddatetys de lyooctyte vall $32 \frac{1}{6}$ graden. De wint upt den

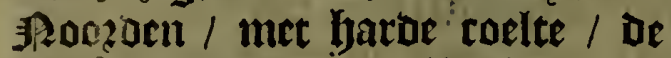
rourg Supden ten Horlten : wa cregthen yeden een rebzickelicken reghen / waren vertwondert Dooz oc fubpte ueranderinge des bints/ Die rontom liep: foa dat wh inde woint cresblyen.

Den 16,17 , I 8, Dzooch helaer weder / De wint unt oen Sunden/

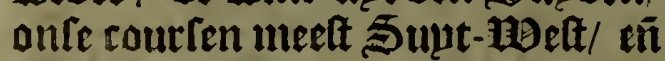

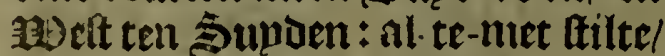
Ijadoen fntiodarchys de lyooct)te van Dier en Dertich graden tljen 99 ututch/ en der Soumen opganck Tess griaen be - Sunden t'o oat / ende onderganc be-Sunden thaet / wel bectrich graden/ is feuenthien gra=

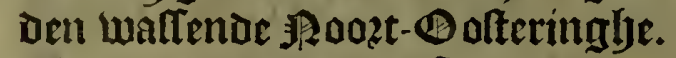
Ggethden Dat ons de ftroom groot vozisel ghedanch hadoe.

Den I 9. fintodatefys de lyoochte ban viif en Dertich graden feuen tn twinticly $\mathfrak{A g}$ inuten / Atll weder met een cleen labber coeltie / van daglye Docht ans het watter te beranderen/ te tweten batun en dicker allt glye= wetef mas / want faglient beel on= fuzuerhents en grupg int water alls clęue fquallekeng/ met twere joozentits Dere lectoen / en waren foe wit als Criftal / met een betum plackien aenit tyoft: : wu adjeteden

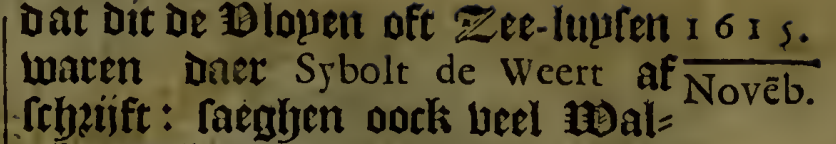
fchot Dziiben: H)et was feer abe= telupert weder : verthoonden eeni= glye Conituen.

Den 20.fmiddaectys de jooclyte ban Teuen en derticly graden/ unin thien gginuten / be bint ban den

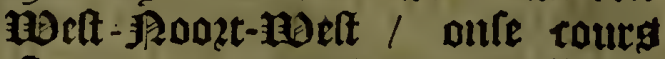
Suuben ten Meften / ta \$upt-

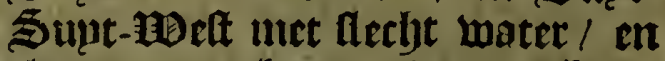
claer beder / hadoen uan dagtye de variatie van leftyien gradé nooztDofteringlye.

Den 2 I. yadoen wuy de fyoochte van 38 . graden/Dit etmael pe wint gfitjadt van moet \$Roozt-nert/ met flappe coette/ onfe courg Supt Sunt-neet / ginck wel wooat / met Ifljoon weder / tn aecljt waieter/ t'welck te boozell ouls Dorlit te ber= anderen / maer nu beder beupn wozde / foo eat wun lyet 3 oot wier $=$ pert/maer met Ijondert baem bots gheen gront. Heden ragben wo een tactken van een $2300 m$ met Gladeren Datr aen daijuet / melen= De moghelick Croos: ent twee wit=

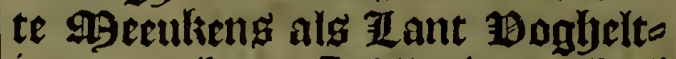
jens : oock ect Schilt pladode ljeel groot / Coo dat beneffens het was ter Dat beramuert en groen werdet. voozfectkere teeckenen faghen ban nist berre van ILandet te lijn / en hatet aront te tewerpen. Defe Cult is heel Uhack / roo dat-men gront Igecft ertmen 'tzant fiet/ foo Olivicr than Noort, odefk gheouserurelt beeft : wog waren berwondert uan onfe looclite / maer deftroom fyadoe ong om de Supt ghefet : en nerchtecoen Dat wp fewen ent twintich 98 iflen dit etmael gadden vertiert. bet bergon al bice al weder wat warmer te wozden.

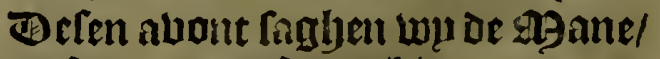
welende maer feventfien uren out/ alg Dr. Soltue cen bierendecl upts yadoe glyetueelf / om dat lyet fod belder weer was / an de kimment tlact. 
161 ; claer. De Dijuiligen bigomuen acty=

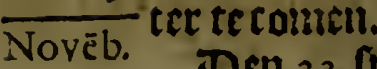

Den 22. mindonectys fadden wu

de hooclste bain neglsen en beetict graden 48. Aginuten : dit etmael oe wint gebaot uanden 1 Beft Roozt-

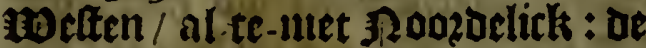
cours was 5upt-13)eft : nocly al ithoon weder: ontrent ocn Roene licple Roozden. Saglen beden beel Croos / en twitte agecukens; oock veraitoertigly van iwater: $\mathfrak{l} a=$ gben oock Conijnen / en een groo= ten Biffy inet een vinite op oe rug $=$ of́e / die water uut ' 'ppoch als een Walbifty. Onfe Prefident ontboot de Schipper en Coopman uan't Jaclyt aen boost/lyun glyebende bet journael ban Olivier, ont de cout= fen en t'Bant aen te toecken / dir= courerende/ en conferetende niet malcanderen.

Den 23. Mniodacthas de boothte van een en beertich graden s. 99 inuten $/$ dit etmael is de lwint glye= weeff upt de \$roozoelicker banat/ net pzopere coelte / met feljoon we= aer/ enide fiectit water: Leden had= Den wp beranteringhe ban luater

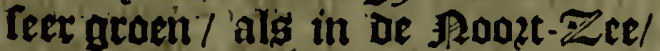
becl ravelingben ban ftroom: oock

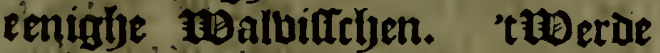
oock beden theltatueert/ dat elck ggatroos foude Daechs booztaen eet muttke Spaentrte mijn yed= ben / ende ter weeck een gloutl= Fen Blic/ om dat of 25 oter ende de firantrbe Wijn gbecoufumeet baren.

Ten 24 . baoden wep op oen mid= dach de hoochte ban een en weertich araden/acht en weertich grinuten: inde nache beviel ons cen ttozm fa= bijtelick/ foo dat men de 99 arffepls qualick tonoe in crijghen. Dooz dit onweder laghen wp beel groote en cleene 2 seeitwen op liet water. De \&9álefiten tickten int water glye tiick lan Huyghen teljeifft : ontrent den noene claerdet op/en lagen een groote Halbilth up bet Schip.
Den 25. all 26 . ginglyen wh, $t e=16$ is. delick wooat / t water wag groen/ Novēb. nae Turquois blau / meenden ber= baluen dat wus berder ban lant wa= cen als gifteren: alloo wo nu boo= gher of Supdelicker aengarnae/ t'lantyen oock Eeltelick ontualt/ met ten inborbt bel Supt-10ert ten Supden inde Caerte.

Den 27. ende 28. variabte win= den queloen ong / oock al te-met een errabade.

Den 29. Imidoaechad boochte ban bilf en beertich graden nin thien grinuten : wint en couts ba= riabel: Taglien beel Croog datjuen: oock eentge $\mathbb{Z}$ et - mooluen : terseng Den abont was fet Schip beel int/ alsoock de Septen op ueel platet= len: een teecken in ong Lant ban onweder/ oat ons oock baeclis daet naer aen quam upt ben JRobzt: Molten.

Den leften / faboen iun groote coelte enoe goeden udoztganck / te = gbeng den abont wag bet water fubijt uerandert/ wierpen bet I oot en vonden goeen gront oj fjondert so. Dadem : Ente onder-Stuer= man'reeckenoe baln dagbe te liin bijf en beertich geiflen bant lant/ ende ouer ue bondert ajijlen ban Porto Defire.

Den r. Oefentuer: Imidoaechis Decêb. de boochte lan reven en beertidy graden tiet. Dit etmael de mint gebad ban toeft-liclter hant/aen= gljegaen Suyt-Suit-10Beft: Dele naclit was doch coutten nacht die wu opt gljeljadt baobet.

Den 2. Imiodaeclys de joochte van acht en uectich graden / min thien grinuten/ die wint ibelte= lick / int laetfte quartier Nodzoe= fick/niet feboone coelte/chioe goede booztsanck: bou conden niet hoo = gber Teplen en behouden als 1 uert Sunt-12seft / t Welck bier lather tyec lant loopt/ en feer uepuirlyadert/ ligghende Porto Defre lave / of wel rod Roosolite wan on's, solin= 
fiol. 16. Spieghel der Auffralifcbe Navigatie,

I 6 I s. ma wu ent conton de wal met defe Decēb. [checpe winden niet aen bookt crij = glien / en raeckteden bencoen / Dat fect verdeieticly is /. Helubente oe Cuft wat te fect gljetchout : waren the oe onder-Stuninani befteck viif en trelticly astilà lain Porto Défire, en een ch Dertich Asijlen uan't lant / of be Capo die op deré joodjte leut.

Den $3 . \mathfrak{e n} 4$, 刃iverféle courfen: fagtyen beel ghebogelte / met bleeck water: 'tghejoelen was niet berre uan lant te jiin.

Den 4. Imidoaectys de booctjte uan 47 - graden: creglyen loo barde

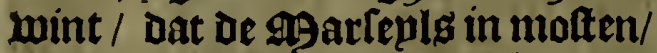
De Z ee ginck upter maten bol. Iacht 19ookn/wierp tfauonts grōt/ op biff en treventich padem: wp fagben bleeck water/ an beel gbe= boghelte.

Dê s. dit etmael de wint gefadt bariabel : wo gingben bu naet Moeftelick / om't lant aen boozt te rrijgyen: dit wag feer fefjoon we= Der / nochtang loo cout dat wp ong Bopeten in de kou niet conden ver = inarmen : wp ragben beel teecke= nen ban't lant/mBalbilfebe / Croog/ Doode pinguiing / Bleuglyels ban Doode Boglyelen : wierpen gront op 64. Bandemen / feer grau nwert jant/ thauonts wierp of 3 acht 54 . Bademen : laghen bleeck water: die coelte was van Den Foozden/

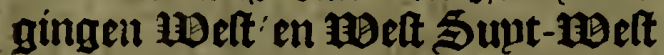
aen/frljoon claer weder : Defe mid= oach gilteden de Stierlieden/ noch rettlien भiilen wan lant te welen.

Den 6. fmidbaethy de hoochte vail 47 .graden 25.9 ginuten. Dit etmael ae wint ghejadt unt ben Roozden / ginghen Noet SuptHelt/ ten naelten lop [oo hoodj als wo feplen conden/ wierpen uan be mosgenltont gront op bijftich 1 a $=$ Dem: op oe midoach yadoen wn twee en beertich en baie en beertich Babem / wit en noart lant-gront/ net cleenelteentieg; bet was beosn

warner weder / foo dat wo maeck $=16$ I s.

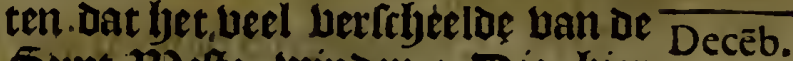
Stupt-1Delfe, winden : Die bier wacuen fijn meet tultetien $\mathrm{P}_{2002 t}$ en weelt / relden van Den Dolten. - Dack obrerbeerden wp lier / dat met den Somer alt nu lier is / allt lart begint te waepen / een ltijue coelte/ oft lto $2 \mathrm{~m} /$ dat het niet langh en Duret/ comende dickwils lubijt oberballen als een oradade/ macr Daie/ vijf oft les upzen Dace naer/ of een dacly ten lancketten/ ift al weder mop weder / en aecht water : allt bier coelt beeft men beelgseeumen/ en ghelogbelte groot en cleqn/ Daut alft reboon weder is niet veel. Olivier ban Noort lacij op acht en Der= ticl Bademen lant\$/en wu yadoen nul 42.en 43. Bademen : bju feplde daie Daghen merttelick naer bet lant / al gront bebluende / Coo dat dere Cuff leer black loopt / en buu= ten glueficht ban lant altijt gront/ uan $R$ io de la plata : tot Magallanes: Ban daglye fetteden wu onie tleene Sloupe in Stljip op / om in Porto Defire ljaeft ghedaen te hebben/ D'een 25oot om water / d'ander out ander verberftehinghe / oock om in be langlye daghen on de supt te raecken / en de Somer te geluzupe= ken. t'washeden een frboon wez oer / met cen claren Hemel. aln= Nota dere die ons na-conten moeten de benc. Cult foo niet frboutwen/maer'tlant ftout aentoeclien / Conber eenighe uzee [e/ gelijek Magellanes, Draeck, ende andere glyedaen beuben : en upt Porto Defire gaễe 'tlant lantr youden.

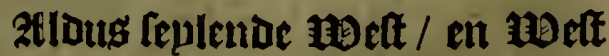
ten $\mathbb{Z}$ upden / met een moue coelte/ fagen ontrent Sunt-moefter Son= ne lyet balte lant ban America, le = \#opzon Porto defire, nae de wal feplende / wicrpen al-te-net twin= ticb/na thien Bademen/ eerit fant/ Dacr naer finglyel: [enulben tot on= trent cen en yalf steil uante wal/ en fettedent opthien Badenm lin= gljel-grant. 


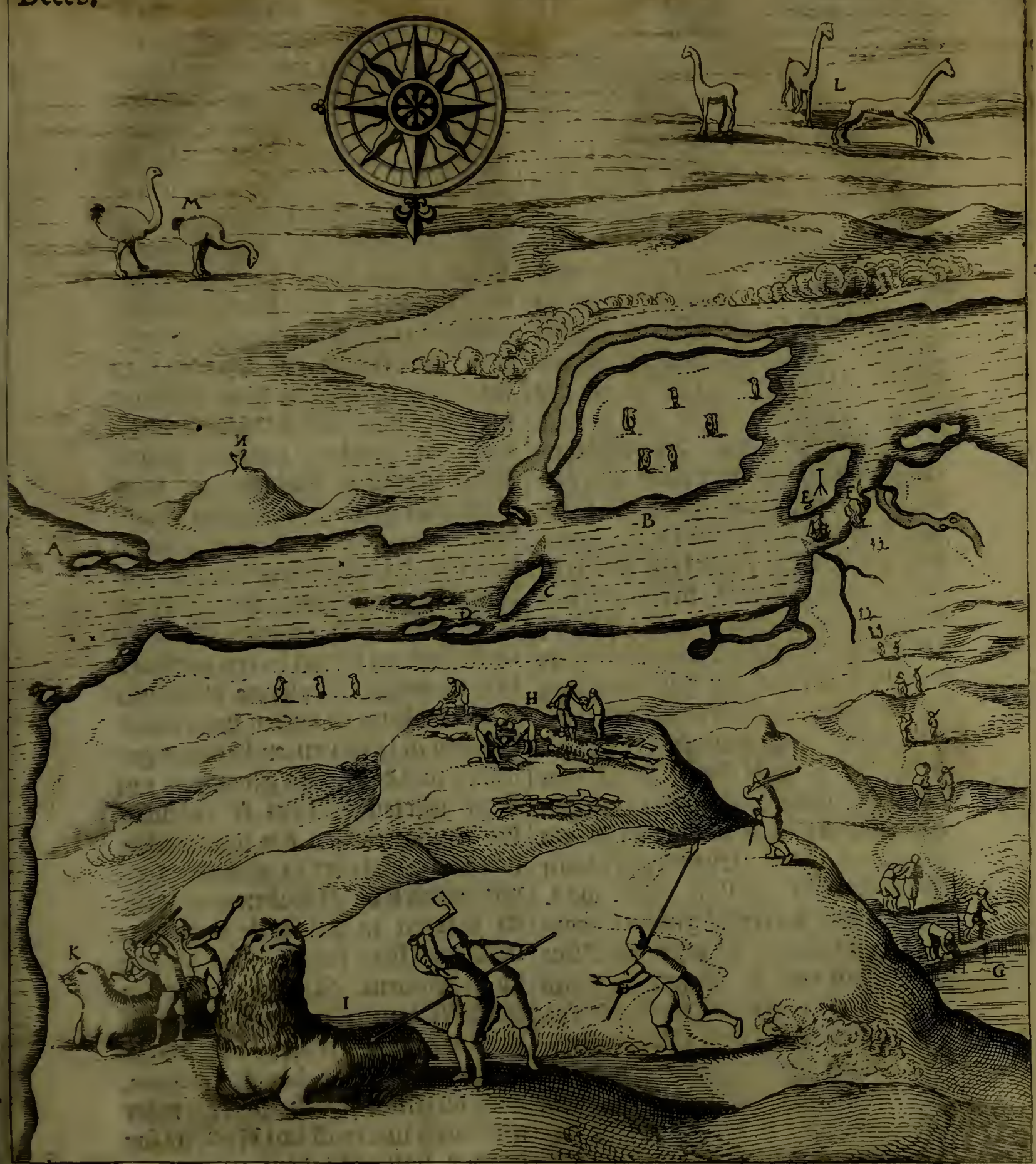

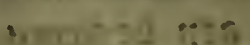

\section{Numero $i$.}

ล. T 5 ac รpiciungh พap.

B. 1 De plact in iner wo met oe stbepen in groot perijefiel aen de lual do̧even.

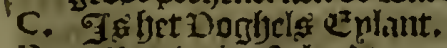

D. t li malumi-Eplanot.

E. Det cauinex-échlanot birce lup anfe Ecliepen frijoon maectien.

F. . xcrbzant oug Tacht.

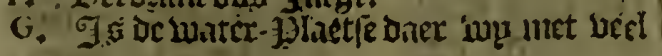

moepten water lyacloen.

$\mathrm{H}$, Eijn de graben unuto Patagones op looo abe. Clippelv/ daer maren beenen onder wall this cli elf bacten lang.

I. $K$, Dijn twee Eee-Il ecuburn.

1. Sijn (i)arten met feer langlfe fyalfai

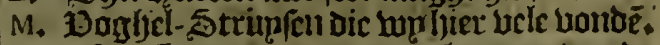

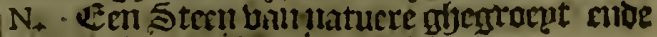
vertljogitt ficljalg cen inicli, 
I 6 I s. gijel grout. Dondont geraden befe DecēG. narfit bert te anclieren en nict naer Erete leggljen / om te beljouwen dat wh badoen/ alloo de ttroom buet fear om oe Sulut gaet / lae dat int fetten de betingly' roochte bant t 3 id dat foo fuel te clufe unt bloody/ dat wu ise lefristean / of liet foude Gibcbiant licbura : Daer gencli focly acject mit/ als of het met gacue coclie fenllo: actyende dat lyet de Coble is / Dis unt oe Roort comt.

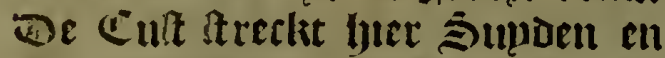
Poozden'en faglyen Ruozt Roozt-

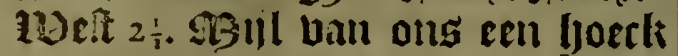

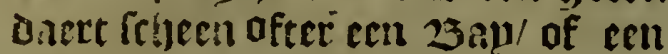
tnwircls tulfelyen 't!ant inliep/ tur= frlyea twe boecken / die ouer mal= canberen fucden/ net twee $\mathbf{3}$ ergen of groote Clippen. Defe Clippen uan lyier onder fepl gẫde/en $\mathbb{R}_{2002}=$ Den ten Weitê/ of wel foo Heftelic ban ong legglyende/ berloozen die: en fagen Doen cen groot fucls ban't limbe/ ban buuten eenen boeck/ de weleke filyeen of bet bem bant lant affonderde / tweick wp nadertyant beuonden / all 姃oozden ten aBe $=$ Iten / en Roort-SRoost-welt ban o: Is lacly een groote fpacie tultrlyen Gmoe fijnde / coo dat bet de plaets was Daer Olivier felyeiff glyemeent te bebluen dat bet Port Defire was. Ong Docht Datter ré groote ftroom upt moft vallen / al waer de Geble of Itroom unt be \$Roozt teglyengs crumite/ oft teghens aen Ineet / roo bat loet batmoe als oft Clippen wa = ren: maer wu bewonden / dat lyet De แntual wast wan den Ebbe / upt dien inbocht / Dat tegbeng bet an= Det water aen liep.

Den 7. (mozgljeng vzoecty licly= teden wu't Ztncker/ cn feuldê neer= waerts om Porto Defire de liaben te foecken/meteen achter-uloet/een cil en balf פstile oe Cult lanev/ was een Ietjoon 10elt-achticly Iecely lant/wit/ of lyet Criit en Sant wa= re: flucljts aen be Sterten yad=

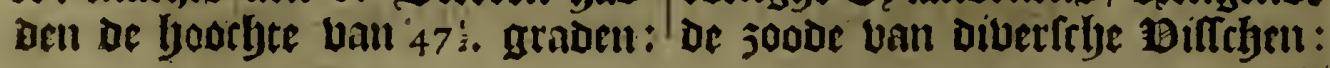

Cerftont quamen wp ect Ezlant 1615 . te fien / Dat een goet ttuck ban de Culte lacly / waer aen wu beniercl: Decêb. ten/ Dat lyer de l)aben moft Lefen: terftont werden wu Porto Defire kennende/ wetende leegly lanit/ Den noott boeck als wutte Clippan/ Daer wu cen eplandoken ban $\mathrm{f}=$ gljen ligalyeiv/ be lisauen / mfientos. De Roogt-lyoeck hooglyer filndel als de Sult-hoctk: hebluen dus al

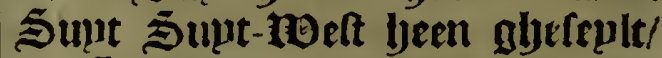
en Suparn / met feboon weder. Gadorn cozts een dzoorlyte berno= men daert fes oft feué Badrm diep was / en de ftroom daer tralyens rabeloe / Daer wu daagljende ljiel= Den / en creglyen tertiont Dirper water weerthyen Badem : dit was al funghel gront / laglyen be-Sunz Den Porto Defire, Dirp in $t$ lanot beel booch backelicly geberclyte / $8 \varepsilon=$ mely blau/ecnicly wit ofter fnecu op lach. ADu fevloen met oe Blort na De Haven $/ e n$ lecpen in een gat Dase veel dzoociten cn clepue clippiglye Eplandekeng en rutfen waren/feat nau om in te comen/en lyebbent ge: fet/ en met oe 2500 ts gljebaren om beter abeleglientlyeut te Coeckem/ die rappozteerdm dat wn 'treibte gat of Camael niet in glyerequt wan= ren/ Dwelck be- Joozoen ons larty en waeren in een lislle daer mm nieulwers been conde / wp laghen op $4 \frac{1}{2}$. Badent. 2Alg men vooz oit gat is/liet ment ljeel diep inloopen/ en wijt alseen tielsier / oft als oe Iiljiin outrent Schencken-Schans, oock dicp met boorly water/ maer met laech water if bol liut $/ \mathrm{en} /$ foo bat lyet balt 3. Tadem op en neer. Bop laglyen ljer al aen be gront en t'ioer ban't Sthip glyeknelt/ Dat yet Cortclicls buiectjoe / yadort quaet weder getuallen/ wo fouden't quaet genoerh geljad beliben van Den Oolt Bunt-Doltế. t'כanont quallen de 20ots weder ban of rotfighe \&enlanoeliens / baentende 


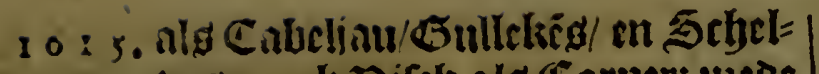
Decēb. Jis : oocli Difch als Carper: ulede upter maten geoote Splerinch lancli felthien oupmen/Daceom wol Defe intuitek noemoen des 5 pierinebalp: oocli uette 29 oftelen : fináchts quam oule ander Slocp aen boozt/ bzengthenoe lyonaete biffticl jon= glise pomaniing/ en 2, groote 叉reKobben / ban lyet Bingnijng- (E) = lisit / twelck ttiif twec 99 iil 'tzec. wacrts'Oolt Buut-Ooft tñ SuptBoft ten Doften ban Porto Defire leut / Dace abondantic uan Boglie= Ien is.

Deir s.fmozntyeils booz dage/ de

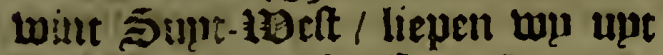
bet gat/out vooz bet Cannel te raec = tack: wu fugen ua Somuch opgane ech grooten roocli opgaen/ Docljeen bat Ijet bin oe Pangones was: oock raghen wu veel boute 23 zannfeljê thater Dooz baec hooft blafen/ haoden bwerfe fteerten / niet onge= Iijclide 25aun-Fiatlyen bie Sebalt de licet in fijn Caerte ltelt. Sitio = darerjis fagen wunoth eeng de rooc op gach bain de J numoonderg. IRae mivoacli liepen de bauen in ban Porto Defire bu naet tot een sgiil/ baec wo bet letteden / Dat was biex tulfeljen z.ftenige Clippen $\mathrm{mbal}=$ leti/oater t twintich en bif en twin = tich Bademen diep was/ al ltee= nen Clip-geondt / loo dat $t$ 'anc= fer niet willoe bouden / en 'twaep= De ttiff Lait Den \$ootot-melten/ foo out wop met ons warp en da= sigeliiex zuncker dooz ginghen / als

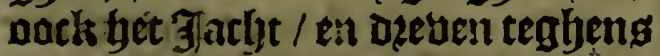
be lecgljer wal : tn bebielen met oe babe fyeel Dzooth/ nuts bet hier $2^{\div}-\mathrm{cn}$ Dxie toadem op en ne= oer ulazue / Dach t'bebiel op een dechtekcefelinghe iteen-gront/beel op fiin kimunen/ en lagen in groot parijekel atjter int rijfen/maer yet sortin frhoot met een hozt baer af/ en bialdact teglyens aen: badoe bet baet op blijuen ataen/:en foo bebal= len/ t'badoe ongewallen:bop? bleef bet beel blot/en alfod bet inet buels- $161 \mathrm{~s}$. boozt tegeng de wal en fteenê lacly/ Decteb. was bet aen ltier-boozt fes/ fetuen/ aclit Bademen diep/ foo dat bet te= gljens becl fteple Clippen lact): fod dat wa met ftil water/ en wint ons werp atnelser upt bzochten / elt wondent vall of wal/ en acn twee Anckers unt glselept: 't de Blort coels ulat raecliende/ wonocht pork uan de wal/ maer cen torm follde olls weder aen de toal syedeben betuen. 5a= vonts quam onfe Sloep aen bookt ende Indoe '\$Coninex-Eplandt ontoecht / bzocht de balve Scljupt bol eperen : oe 23oot ban't Facljt brocht ueel Bogbels / als thot. Eanlen : badoe oorlt veel tiobl= ben bernomen op be Clippen; baer was oock goede gljeleglyent= yept om met ben Seeghen te Bit= Íljen.

Den 9. walf claer Sonne-tetyijh

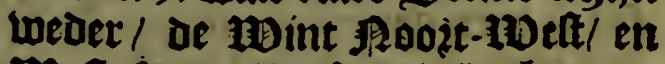

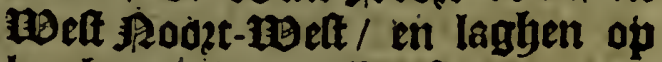
leegber wal/ en effen fteen-gtont/ boott watp-xintket / en een nwaet itneker / maer gingtjen booz / ten laetften bielt. De Gloet incomen= oe/ maeckten bu Sepl / in oe wint laberende / tot 'gConincx- Eplant/ maer niet baet achtet/oaer't zacht wel quam / Ong Prefident ginck aen tielfoe eplanot oilt te lien nae eeniglye 25tieben oft teeckens van Spilberghen, oft antere/ maer vont niet/ Dan een thae ban eet 2Befaen : twas een rupefi Eplanot/ van clepue 2500 mkens/Daer uptter maten veel greeuwen in neftelen/ en ontelbaer ezeren / bace top foo veel creghen als bo beglteerden/ ooch waren meet al buipl?

Den 10. Cont onfe Prefident oulte groote 250ot / atn be -3oost-fijoe Uan't bafte lant / om naer water te vernemen /: bant niet glétiotident als fout water in een ballcue?-gin= gben boozts op D'ejulanoen out onfe colt te foecken/ mede betrigen= C 2 be 
I 6 I s. Ue youltaert biif en tlebenticly water

Decēb. Dogijels / fwart vaul coleur / met Ejeren van differente rooztê groot ell clent : nach een Kieuger/ en cen pittoir / die daer beel waren: wa Londen op'c geoen Doglyel-Ceplant tectiens uan ollantrue Sche= pen / als fticken ban potten / en

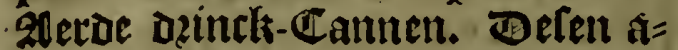
bont raeckte Canincx-Ceplant in Jzant / Dooz ongljeluck ban't Byer gbeltaockt oun te warmen / dat foo feer roacktede/ datmen beefoe de Patagones daer mede warclier te ma= Fen. Ong valck dat aen lant ghe=weeft hadoe / beenã niet dan lpooz ban gbedierte utet glyec louenc lau= wen/ t'obeherchte meelt ban witte fachtelteen.

Den II. aede de Prefident de $2500 t$ weoer manmen/en font neode Aris Claeffoon, ende Çlaes Ianfoon, met d'affiftenten ! om te gaen nae de 33 aeck aen Zee ltaende $/$ om al $=$ roo te becomen teeckené uan $\overline{5}$ che= pen die daer gheweet waren / oft 25zieven : maer twas al uergheefs: - oock hielden ie 3 lournalen dat aen be Supt-zijoe vant yalte lant foet water te becomen wag / maer bon= oen niet.

Dell 12.is de Prefident lelfg met Iijn23 zoeder Daniel le Maire met de Sloupe aen de Roozt-rijoe van't Eulanot gljeuaren / met twintich 9) ufquettierg/ en guncli ooos thant - 1.95iil/ Doos cet doze chanuai= sule [onder ghelioonte/ geen verf́ly water vernemende/ booz de ozooch = te /un fagluen grooten laock op= gaen acliter een Berch/: al waer wu bolcly uermaededen: oack ved 2ailts als Harten/ met feer langlye balren / reer- frbou uan ong. Bont? den eeniobe Gagum ban de Patagonen op hooge C lippen en Steen. Tioten/, dact alftenen op glyelept waren/Daer waren 25 cenderen on: ber yan thien en elf voeten lanck:

Jat r 3 ifject of Brefident weder de Sloupe Doen manten/ ende is met alle de zlctiftentê net de oe thevier meer als twee opwaerts oberoçut / gaentae decie maels te lande / twee mad aen oe

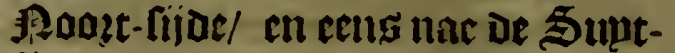
fijoe / cen groat ltuckifueectyg bu de Zec-cant lamer ouer $23 \mathrm{crch} \mathrm{cn}$ dal; fach luel twee frhoont diepe gracne Dalleuen/ Daer water it was ghe= weelt. Olivicr en Candifch ban fijn twee reputen feggljen datter berfef water moet wefen aen de Supt. (iijae/ maer wis uanoent niet.

Den 14.is ie Prefident, met Aris Claefoon ent twee Alfiftenten neets wacrts nae d: Sult-haeck abcua= ren/ daer Huydecoper begraben i\$/ en na oe Put daer Cãdif́ch af f́lyeijft nuer uonden niet. De Prefident meende cenl loart te fefjieten/maer raeckte niet: ginck in ie \$loepel cn boeren aen't groene soghelen Eulant/en bzocljt ontrent ljonoert Bogbelen : feboat oock oe fegben ell crercly $1^{\frac{1}{2}}$ Con Spierinck/ende anocre foozt ân ban Bif́ch/cn bzacht= Ie aell boost. Ra noen voet yp wes

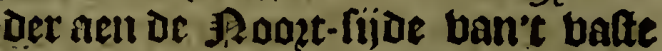
lant/teglyeng ober yet Eplant/ oaer wu! lagljen / cen wepnich ober bet gheberchte in cen groene Ballepe/ dede ly cen Cupl delben ban bije en twinticly uact int ronde / en bijfs thien voeten diep / maer alfe elf en twaelf yoet diep was / begon bet water booz doztoeren ban onder/ en van lijoen/ uut Den \$Ropzien en daeften te comen/beel claec/ maer bzack.

Den I s.izdesloepe wedernaer

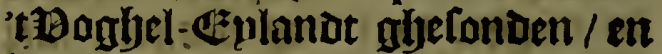
bzocht twee on Spierinck:en tfe. Gich verfelyepde toozten ban BogeIen! : Jadoen twee Zer-Zlecumen gbeflaghen / op een Clippe fijnoe uan Crêlyien voet lantk/thapz aen Den hals fijnde wel een singher tancli/ enoc op't lijf cost: $3 p$ waren foo groot als em jonck 18eert: men confe met cen bant-[pact nau boot taen/ in biiftich Slaeghen : feet $+0^{\circ}$ bzete- 
1615. bectelick chi mondacrbaner om ach te Decéb. Cietl / Dack ban ( Cop en l)als / voo? twee bzecoe h)andá en acliter twee/ warer mede $j^{u}$ inel counell dooz't water fwetument : ljet bel is fwart en de bupte lancli veertjien voeten/ cn onglyeliieli dicher als of befte Odtenlyupoen ban Indien, aft in 1)ollant. Het Diecich is goet / cri redelicli van fmaeck. 9 en dolf fier cen groef of Bookn nae de jut uan 'talyeluerclyte af / oun de regljen Daer in te Ienoen/ en foo bertely Later te trijglien.

Den I 6.beeft het.5rljeepr-uolek t'water upe de put gljeljaelt/maer t'was onbequacm oooz of bract = beut ounte dancken.

Den 17. is de Prefident meder met lyet Jachts 2300 nectwaerts gljebaren / oin berfefy water te be= comen. Ban Dagle bzochten wp onts felyip Eendracht aen de . Rooztfijoe aen oe wal/met joorb water/ beuielen beel bzooch / foo dat bet beel frfjoon ghemaeclst wozbe aen back-boost dien dach.

Den r 8.ig ong Seljip Eendracht beel frboon gljemaeckt: vallende met oe fpringly van de nieulwe Maen heel dzooch/ foo datmen Daer ont conde gaen/in Clep gront/met wat fingel/ ballende beethien boet waters. op lingel-gront/. maerfyeel ozooch/ twee $\mathfrak{M 0}$ fquet frbeuten ban ong Srljip: Be rupchte daer mede wop bandeden baeloen wa bant bafte lant / Daer oork bequame cteen is tot ballaft.

Den 19. nae miobach is de Prefident met de 5 chipper bie fiectsticlt

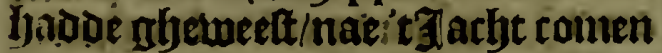
buandeten/omte fien yoe yjet frjoon gbenaeclet wozde/bertrocken copts Daer maet / en men beftont met ruuchte de :2sack-boozts lijoe te bzanden / maer De bzant vattede Coo ljefticly inde Cajupt en infjet. Foant / bat het bper niet te Llu[: [c]jen wasten wecbsande alfoo ghe? ycel iu ong gofycliclyt: finathts ginclis 6 I s. jet crupt eerlt aen bzant/ en de $\frac{6}{\text { Decōb. }}$ Stucket/ Daet poet booz oljebzelt yadoe.

Den 2o.izbe Prefident, met alle oe zefriftenten / aen bet berbzanoe Jact) gljebaren / cu lebluei oen banut gljelelt / fagljen datter niet beel ouerich Wuas : Dat ngrex / choe D'Heren fjoepent werden inde floep geladen ren nen't \$ctjip Eendracht gljebzoctid/dat nu al ban lant was/ oock het obefrlynt / en o'zincliers. ADp focljten feer nae bet nbelt ach)= tec op de kicl ban't $5 \mathrm{fljip} /$ oor bup= ten int water op de Strant/ dan coltelit niet binden/maer twas met te Aach vant Crupt int water olje: Imieten/ Daer upt wuj feet hocly von= oen / t'fauonts coeckende lauce de Strant / eit bonden met bet leecly= Ite Mater een clomp gtjefmolten Silver / Dat foo hwart was dat Frans Claeffoon ztuiltent Die bet ljaeloe niet en kende/en irk t felfoe Keninende/ alloo daer een beele ties ael ban acfiten in ltack $/$ en boozts roeckende net alle man vonden in alles viif en dertich pont Silbers. Den 2 . Imazgeng na de bzoech-

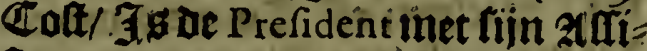
ftenten weder aen't lant ghebarenl maer thater was niet laech gye= noedy: macr t'auants onitrent ozic aren / ginck fou tweder aen lant en bont noch tiver pont silloers.

$\because$ Den.22, noej al aen lyet wark abefoopt. Dele berleden naclit beeft bet too glyeweloich acluaept/ en Ductoc defe gantfefye oach / oat de Sloupe die oln Big upt Laas/ niet conde atlit Stlip comen/ende De maets bleben friactits in de cou= de wint en regberi : roo oat ljet beel ergher was als cen' Travade: ofe 25olck:bañgliers módteir aet.

Den 23. quam oe "3oude aen boozt/met wis en bougels glyela Den vooz tacfitentich maninen boir

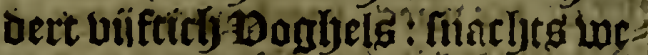
oer eenuliegriende ltosin.

$$
\mathbb{C}_{3}
$$

Den 24. 
$161 \mathrm{s.}$ Dell 24. 3iji wul weaer Doende

Decéb. Glyebeet aen.'t 3 acht te Aoopen/ Cocliten weder nae verteb. Aater/ mate bonden niet.

Den 2 ;. leeft de Prefident glye= remarqueert dat ecniglye lecten/ cnae vieruoetiglige aljedierten / tot az: diuerfebe refren ouer ljet ghe= be:cljec quamen/owersinevens ons Sclup/beclue bu ljent in Dat $3 \mu$ bace quamen oni 'tuerfelje mater/ ghelisck lju oock betumben badoe aien be Capo de bonne Efperance, fout ftrarx eenich wolck derwaetts! d: Direr vonden in de Balleuen tluee butell met water/ Itaet wa= re: 1 met diep gljenoety/ oerbalben mil die disptc! oni meer te criighen.

Bell 26. walt filjoon weber / foo Dat ac Slocp weder all de Coft unt gine: bzocht ontrent twee han = Dert Boglyels. Smozgheng is de Scljipper aen landt gljegaen met Aris Claeffoon, om oe water-plate fen te vifiteren/en bonden nu abon= Dantic: foo dat wus uan dagbe noch ach loozt bzacljten bier Baten wa= ters'twelcls wit en lysel dick wag.

Den 7. fmozgeng/ is ong bolek aen lant glitgaen oum water te ba= Ien/ bzochiten elf Connen water/ dat wel dick ban Clep was / maer fos foet als een Rote. Wadoen weber fulliken itozm dat bet frlyeen Dat oe 29 aft ober boost fouve gewaept Gebhen: coo dat het volck flauber= tich wisoe/ oooz tadighe onweder: t'ljadoe sock fijn (maricljepot om met ljet Schip unt de enge Reviere te tomen / Daer too quade alntkergront wag.

Den 28. oberviel ons foo groote topm als bin out hadoen glyehadt/ Coo dat bet Scthip op twee anckers logthente / Doo? ginck op facte Clen gront/ en teghen 'gConincr. Evland Decef/zijnoe al ateen en Cliw/, coo dat wp met bet acljterStljiu aen grent faten : maer als fiet Lo ter wies/ raeckteden Daer af Dooz Gouts bulpe. Maren ban das glye bęficly oull water te balen/twele
reer moepelicls wag Dooz oneffen weghen / met Balep vatekeng op be fibouderen: Dit. water was ooek wit en dick / Dooz ae tritkuozteclyen en andere ghedierte: moften oots wacht upt fetter / vooz den actuval ver Patagoncn. Het wacpoe bier alle dagljen fdjzickelick.

Den 29. Uerlief lyen weder de wint Imazgens. De Prefident giutls met o'zteniftenten aen lant / aen be water-plactle/ Daer de agatroolen bet berfdy water feljepteden / die dzoeglien foo blijticly dat bet bel wan lyaer feyouberen gintk / jae 75. Saleu-Uartiens en balf 9 ijle weeclys : enighe waren gheladen net $2 g$ ufquetten/ om niet oberbal= Ien te toozoell: Befe grocue bol waters ftrectede ben lancr of gant= fthe Ballepe.

Den 30. is de Prefident aen lant gljebaren/met onfe Tozposat/Aris Claeffoon, entoe Daniel le Maire en twee \$ootighefellen/ om bet zoot Dat feer onghefien en fwett was te utrimeltel / Dat upt be J Jacht ghe= bercht was: en bergoten dien dath res hondert ponts li oots / alles in baren / arbepoende in de hitte / ter wijl de andere befitl waren met water te yalen: Be Prcfident liet ban Dagbe booz n'cerfte teple fiem de Caerte ban Aúftralia, t'welck onte Opper-Stierman / en booch. 25oetiman wel beljacthoe: wu cte= ghem ban dagde thich sacen wa= terg aen boozt.

Den 3 I. Des fmozgheng wat fchoon weder : t'volck weder aen bet water balen; ende een partic

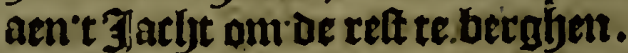

Jen eerten Januariug/ waff 1616 . feer feboon weder / foo dat de $50=\frac{1616 .}{\text { mer een fraepen aenbanck nam:de }}$
lanuarij gsaets dzoegen vaft de Biten vol. De Prefident boer aen de Suptfijde aen een Ëplant daer jpe meen. de eenith milt te fefjetten/ maer thag te felyou / Ctark bet aen bzant/ 
1616. chl bealloe dao: oc Dzooclye beel af. En alloo lyet ban Dagile nienue. 3ares-darty' quamen eenuglye ban't voleli / met Cromumelen / Crom: petten/ en Sulitrlje filupten vace De (Cajupt / lyct metrue Gace lpe= Iende / Dari voor ju bereet Lerden

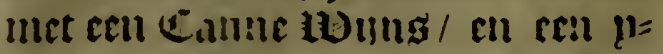
Der Uan de agacts nae or vaoerlyCo.t cen 29 Jurticn Wunt

Den 2. firgiseil wa twer liegenuoglisu / Daree cen ulagije reghens nier bolitjoe c Conden ong uoldi we= Der aen lant oll water. De Prefident beeft ban baglje de refte bant Luse en Cun gluefmolten / en aen bouzt glyedzacist' alles in baren ban biif pant / cot ontrent Duplent vier boituert pant Loot / en beerticly po it Uinne.

Den 3. De boozleden nathe was bet féjooil wider / chochet lyadoe sijedout: maer twee ulyen nae de werech. Colt/ berlyefte hem or wint

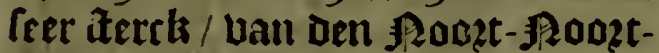
Oolten/ geduerende tot Den abont/ roo dat op goet weder bier gheen reckeninge is te maerken:bu lueb= bell ball Dage ong Schup ban buy. ten gheteert : De Prefident lyeft beden liin refolutie beginné te ttel= len/en ueritont ban Aris Clacffoon, aat oe §.ly:pper oe felfae nict wil= de teeckencil/, londer redin daer af te glycuen : rregen lyeden fruen $1 B a=$ ten watcis aen borzt.

Dell 4. gingljen oe s9ntrooren om onle ozomarie pzotific ban war= rer te balsu. Or Onder. Dtiermath a:ll de 50 soe ; die ee:l wonderlicke Difch uincls: hidode een lteert als ecn Rastie / Uier groote beede bin= nen / en ren finut aen Je mont met perfecte Canoen bojen' en allocr)

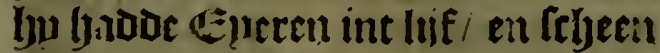
ecri looate te welen ban een lgaye/ conder frlyobluen / becl glimumende/ en vilden ljem.

Den s. ljeden werdender thien Daten waters aen boozt gheluzoctjt foo groat en cleen. alsi de Prefi- dent metriin volck atn Tafel fiac/ 1610. lect bem of Seljipuer ontuallew/ $\frac{1}{12 n u a r i j}$ dat byl wrlac nacr Oolt-Indien gaếl dat or pulcot en Claes lanfloon boozoen/en op minleanderen fagen! chlju lacljte up ljem felfs / ware mede bu foo veel te ueghe bzorby/ antmen lyet boleli ntet en becft ule bet booft connen waten / bian naer Oout-Indion te gaen: foo Dut lyet nict gialclinae de wille vall de Prefident. Dits volel faglyen iren de Water-plactle date Struug $1 \mathrm{Do}=$ gljels/ fijnde de erette die gbefien waren.

Bet 6. is de Prefident De Tituier opglycuaren / om fun ftreckinglye te fien/en wact datfe belangde/Dan Dace itack op groote Ito?m tot ocm abont toe / Dat ly weocr leerde. wop waren alle feer berflaghen/ Dooz dit continued onlwcocr $/ \mathrm{en}$ be $=$ Conder allos wp een perticuleug er: plotet vooz handen badoen. 1)t= dent liet de Prefident Claes lanfloon lefen fiin inltructie bãde Ratt booz de eertte repre: en berozonncerde een Bat gheluzandewiju ban de Coopmanfrtyap tot probifie ban't Schip/ om in Zee uut te beelen/ creghen heden twaelf con waters aen bookt.

Den 7: Ateloe de Prefident een pael op't \&zlant/met een $\mathbb{1}$ oot/en een 2 blicken burken/of Cokerken/ in t'welck was een sotief / verlja= Iende van ons arrivement aldatr/ en uat oule gantítle reple. En re= Colicerde ter gheleghener tijot af te izijuet/ om a aechg te leggben.

Den 8. Dreben wn op diep mee oe Ebbe/ buuten het Eplant/ had= den banoe mozacl een ltijue Don= ocr ulagbe/en oock regtien : wo fet= tcacilt terltont / Daer bolctjoe wint ch regen niet wepnich/twelek beel de courngie benam om t'supben te pafferen/bplonder als be Schip= per [epoc / Dat lyet noch dickwils Daer fuer unt foude lien: jae Ian Schouren die een ander yoozde t'a= nimeeren! 


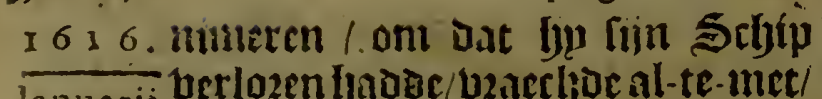
Lanuarij berlozen thadae/ozacelyoe al-te-met/ bancit wilde bechent : alle die p2o= pooiten waren werdaletirly om ljoo= ren. Befe Darly wert nict upt= aljereclit on t'oniweors.

Jen 9. Warpide het weder een balue ftom (lov Dat wu niet conden t'feul aaen: lieten de $300 t$ noch cens om t'lartfe louter baren mae De modacly/en cregljen dat aen borots.

Detı 10. jün wu net een ptoper coelte/en mou weder tfęl glyegaen/ maer op de nidodarly quam ong de wint teglyents unt oer $\mathbb{Z} \mathfrak{c e}:$ ong $2300 \mathrm{t}$ yoer aen t'Robben Eulandet/ eit de Slacp acn't boglyelslant/ vinglyen ueel bifrly/ ell Bo= glyelent.

Dein r2. werde onfe Sloeph ghe = ronden naer de Pinguijns Epland $\tilde{e}$ / om Pinguijns te halen ende die te Couten/ Dock om UEeren. De $2500 t$ voer'aen't Robben ezhlandt/ Daerfe ontrent twaelf $\mathbb{Z} e$ :-nolwen met \$o ufquetten soot feboten.

j: Den 13. quam onte Slocp met be Pinguijns weder aen boot / Die (natyts inde 5 pierinck-25au had = oe gheleghen / waren meet bedoz= beit / Dooz De bitte / maer Couteden cen Bcrbooft vol. Sagten oock Difie oft luict Zee-montters / ljebs= benoe urau ztruerwich lyapz / met langhe 3ecken / als Crocodillen. Ders na midDaectys teploen wu unt Porto Defre; ent Dooz ltille wint Cettedent / maer als dic cozts daer naer warliserde/. liepent wh't 芯e watert.

Den x s. Inozglyens hadoen unp Donder en 23 lixen / met ljerde da= oben / en ueel togljel carcken van

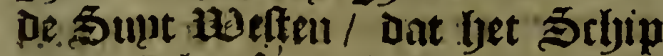
put beelplacten wir was : conclu= Dectoen megeneraet bat het Sup= benili equipollentie beel cauder ig/ algljet fopgoen : en wag met defe Subden wint becl couber als met ons de poopde moint into therift: 1616 .

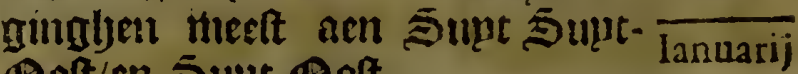
- Boft/cn supt-@olt.

Ben 16 . lieffelicli weder : yad = Den fnidoaeclys de yoochte van viifticly graden / thien פoinuten min : en maecliten giffinge dat ons Deftroom om de Supt fettede / oft Sunt- Moeft IIacr De Stract Ma: gellanis : wu faglyen nocly eetuiglye Pinguijns loo dat wun niet uecre Lan lant waren / cul twater beel bzupu: wu Ienoen ons mette ftroom t'gat om te Dziiuen be-5unden / om al= foode wint ons ban't lant wan be Stract Iout/ Die luy geene fonden verkent beblien. Dzeven fuaclyts in tílte.

Ðell 17. fmozgens lwalt ftil/liet= lick weder / maer fmidoneclys Doos de betoghen luebt gljeen boocljte âjepaèlt. Weden jijn onle zuti: itenten wat befryzolt om fyaer def= perate propoolten/Die ju gebzupck= ten ban wel te willen naet hups varen / met de welcke jpandere de couragie feer benamen.

Den i 8. was be wint val ben Supden : fagen vecl abalbiffrben: De Prefident arlyter upt De Galerije fiende / fach De Eulanden ban Zebold de Waert, die fyp Itelt inde $29 a=$ gellaenfelje Caert / en laghen on= trent Dofie griilen uan ons / SultOoft: cri legglyen ban De Straet

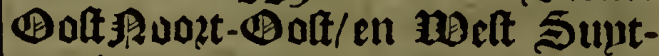
meft ontrent viifticlj gijlen : wh hadden rechts te voozen grewent/ anderg cotiden wp daer bu lance been gheloopen bebben / foo bau Daer un bouen of be-nortten lies pen : Imidoaechs booz betontjent locljt conden gueen boochte beco= men / De Son nocljtants lyem Daer naer openbarende ! bebolnden dat wo Couben geljabt bebben $51_{5}^{2}$. gra= Den: en Dat De ftroom ons on be Supt fetteve/ travonts begont te coelen ! namen be פartepls in: maet bet weber beterde/ en hietente ve ganticlje nacljt ftaen / Dooz dien bet 
16 I 6. Ijet tamelitio ricljt was: t'was biet Ianuarij Leter weder als in Porio Dedire.

Den 19 . fmotgheng was yet fret Mtọ Larmaclsticls weder / fierlje water / De wint Weltelicls / wu fa=

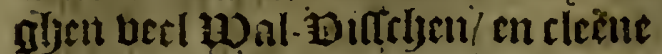

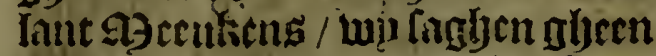
Croos Driluch / coo Dat int Sull= Den gliecu Eulanden Inoeten we= fen/ch Later rimaten groote dijnull=

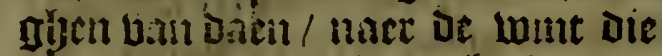
Dner warnde / fmiddaeclys hndorn Oe boocifte lian twee en uufticly gea = Den / t Thbonts mectiten giffunge oe lyoodijte te joblien bande 5 traet ban Magellanes, bup bebenden bat al De bis dic um in Porto Defire

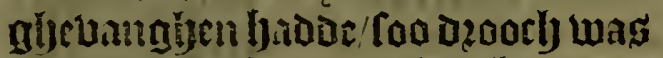

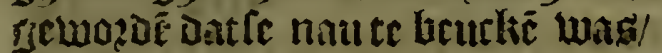
ist al baire fmacels berloozen lyado: trauonts began bet wat te coelen ban den ioeften / Coo dat bet te natidt ftozmae / [eplende met feljo= ber-Seula/ noclytans twater niet lol/ mits ljet een opperwal was/ maer t'sthip ainglierde ghelad= Dicly.

Dent 20. fmozghens began tyet quaet weder te ftillen / wacuense met buwen ban oe 10etteliiclier bint / met betorghen locht: faghen becl Croos Daijuen / en bortjels Die ander twater queceliten/ Cónder twijfel uan be striet uan Magalianes: handon fmidoactlys de hoocly= te van daic en bifftitl graden/t'we= Der te begon te claren : twater noch

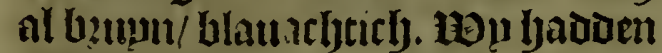
tredert wil tut Porto Defire liepen Lothtiglye locht / foo out het alle dinck ontliet/agatmelade/honirly/ het pareliement van ot Caerten in De Cajunt / wul waren in groote hoope dat ong oc ftroom om de Sulut fetteor/ an dooz t'Suuder/ of naet het Smboer gat trock/ t'a

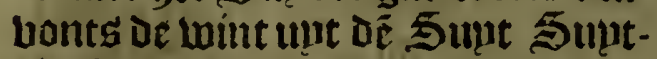
Terlten/lendent Weit- warrt ouer/ intet ftil weder/ Coo Dat wo wepnicl] bertietden / te mecr ont wul feer groote dijningyen treglyen upt oen

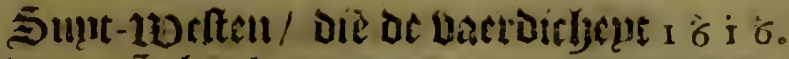

ban't 今

Doch 2 I . Was lyet fmozdjeng lzed

Tanuarij lieffelicli treorr / or nont sumtWelt/ gavien ban daglie cen barde reglinti-caseck / Dorb cout / maretilte/

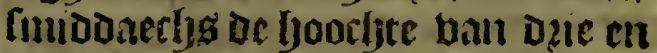

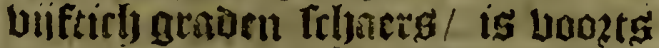
Den gljeljetem Dacly frfjom word gljelileuen. 10u fitopten uan Daghe cen leck boden oe tumpes / Dat hed groat was /. daxer mese tup ban't pompen verlaf maren: Snaclyts conisen wa in onfe Coler ons hiet vertwarmett.

Detl 22. was het fyeel tal wedery uertierden nict.

Den 23 . fmidonerly decten wit ill (tilte / cat urierpé tloot op vijfticli Oonach fianglyel-gront: inden ach ter-noen aengaende Sumoen ten Welten fanffen lou of lyet Eulan= oekens en begecken waren / fettes Den onle cotus aen Sunt-Boft/om lyet $\mathbb{Z}$ ant misen boven te loopen: Inaclyts Lierpen wo lyet I oot op beerthien Dadem / ante Prefident fiip diell naclyt niet/ mits ly in groot bedentcken was hoe de faecls foude afloopen / mits ly olluers wacht dit lant bernam.

Den 24. ftroztheng faghen wu vooz unt niet Dan lant/ Dare doo? Sunt-Boften têl Boften aen ginglien/ lijinde inet oe bariatic SintOolt / Coo als bet IL ant ftreckte / te Weten/ Sisnt-Ooft ten Ooften/ en Sunt-Ooft : waer ouer onfe Prefident feer berflaglien was/ meenen' de dat bet nae bet Caerticn ban Willem Ianffoon al balt lant was/ cn alfop lyem de Culte nict onl de Sult en ontuiel/ maer feet Ooftes lick ftrecktede / beectoe lyu dat lyet nocls meer Ooftelictier fonide ftree=

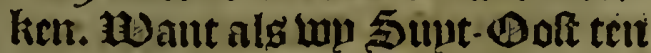
Supoen aenginghen/ Taglyen norb

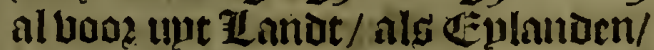
ljecl booch/dat ons boelit met fnecti bedecket te fiin/ maer gheleecla glyes bzootken Zlant meett. Cen laetfteit 


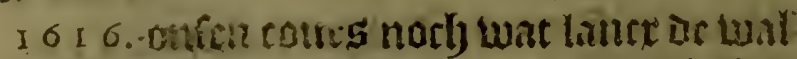

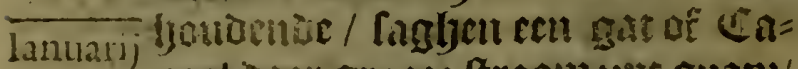
nacl aetr giooie ftrodul unt muany/

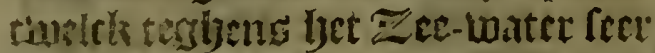
lirrt inet gat quam unt ballcu/ foo Dat liet fod rauclde / ch bzande te= gbens malcindocen / Dat ous lyet

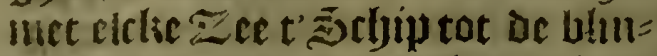

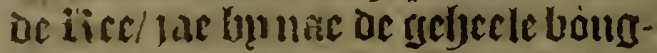

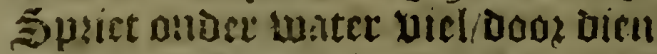
fiet whter too teglyeng milcander Ífjermbe: wa badacn hier ghecu

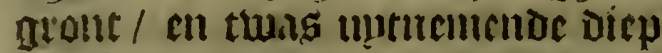
int Camarl: namen oe boorlyte dip 5;.ginden felyacrs. Nae den noe= ile licyen wun une lyet gat toe/ wer= wherts ous oorl de vlore track/ mage allog fyet ttiloe / en be wuint Dusg tegcu nusan/ lyobuent de nurfyt big giselyouren.

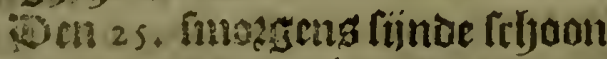
woture / Captoen al bet Canacl in/

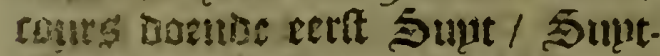

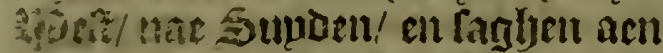
knepe flidem lanot dat 3 erely-acly= ticis cul bacteclicls was : wictpen in oc. Stract cen sgijle ban het lant bast asn bucli-boozt lacly / bet Woot

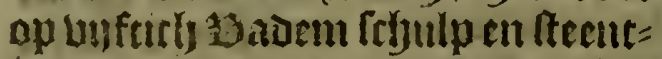
irme: alfon met aroote luacuclioe feptoen lus boos ae Royale paffagie : Gobt unmeltende dat lyu cent beclaberlecht lowded nae tglene war ton berlangine Inodoci. Waer nater wert uset gljencene refolutie acs tiacts/ yet meltelickite lant gibeninent Mauritius de Naffau : ent bet Odftelickifte Staten lant. Jilue

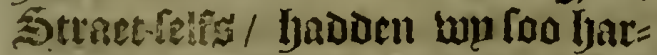

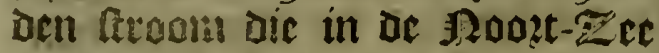
stel Dat ban bien nautuclicle conden boor Equlat met top-[euls coelte/

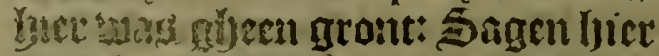

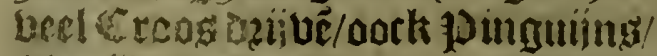

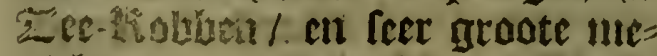
inclyte ban 10 al-biftlyen foo inde

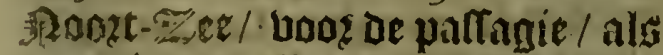
mece indos paftagie telfs enoc inde

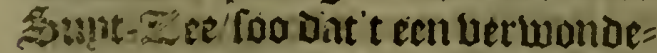
ringlie watg te ficn/ oocls dat het Solinp int replen dic moelte wije lim ende coms bermocren. OH C pprt- Stiermm lyeft looz De Lanzarij panthers sen Cabbelint getuangen. Onfe. Stictman was aen oe fren=

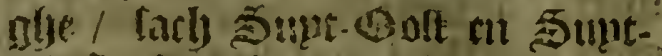
Delt ghern lanot. Op Manriius.

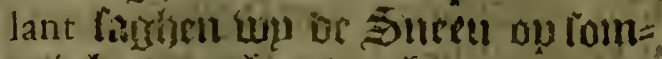
uniglye 23 erclien tegotsen/ mate op Staten lant oudlse ong cenige grocti" te te fun. bet felject banter aen lien= de fijurn goede lictoen whren / at b)atuang / met al be intuijoket! at

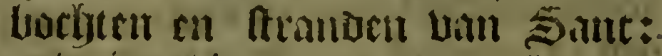
principacl in Staten lant, al waner Roozdent af cen Ėulant fryest te legolyen. Het sont Mauritius licp Roott- waerts af fect Ieca-nct tichy

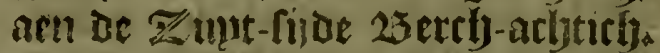

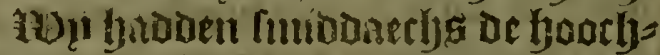
te ban viif en vijftrdy graoen fes en

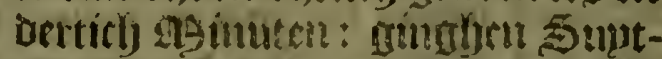

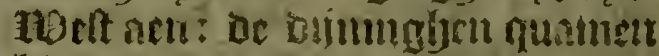
Gier ban bozen/cu thater dat malte:

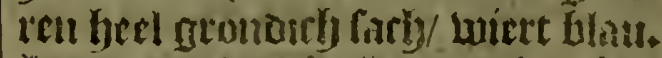
i)et Staten lant friglyen wurtectlest acn ox Supt-fijoe mett ooft/ cr Mauritius ftreclite binmen oolt ten

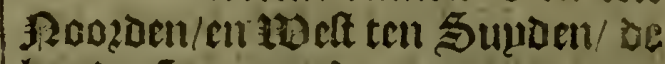
becoe Sunt en noozt ontrent Io. Agiilen: En ontoeckte (icl)'tlant Dort op foos verre als men fien morlis : en Staten lande foo berre Dolt Solnt- Ooft up allimen met rlaer weate bat fyoaglye lant oo $=$ aljen conde. Coets nae de nuidoarby wert fyet mottirlj weder / met naede coelte / be diininglyen ban boosen/

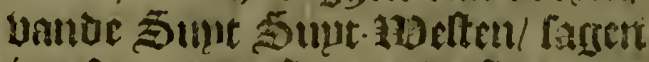

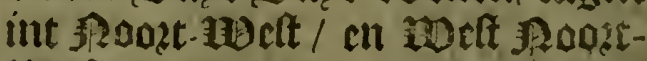
neften veel yoorfy lant/celijuentio gljebzooclten lant te 3iju/dat be= Stuben de Straet lept. Cegitens de nadje licp of luint tenfyeng/ met

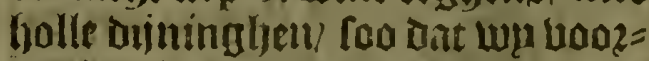
fectier etatuecroen tat lum nu de

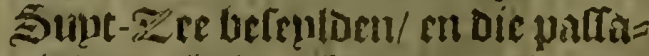

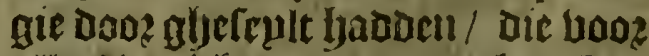
alle slsenfetyen tot nu verboanben Gndoe oljewert. bier was groote meniclite Dogfielen.

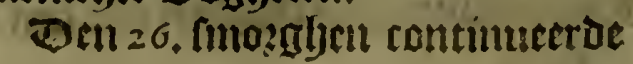





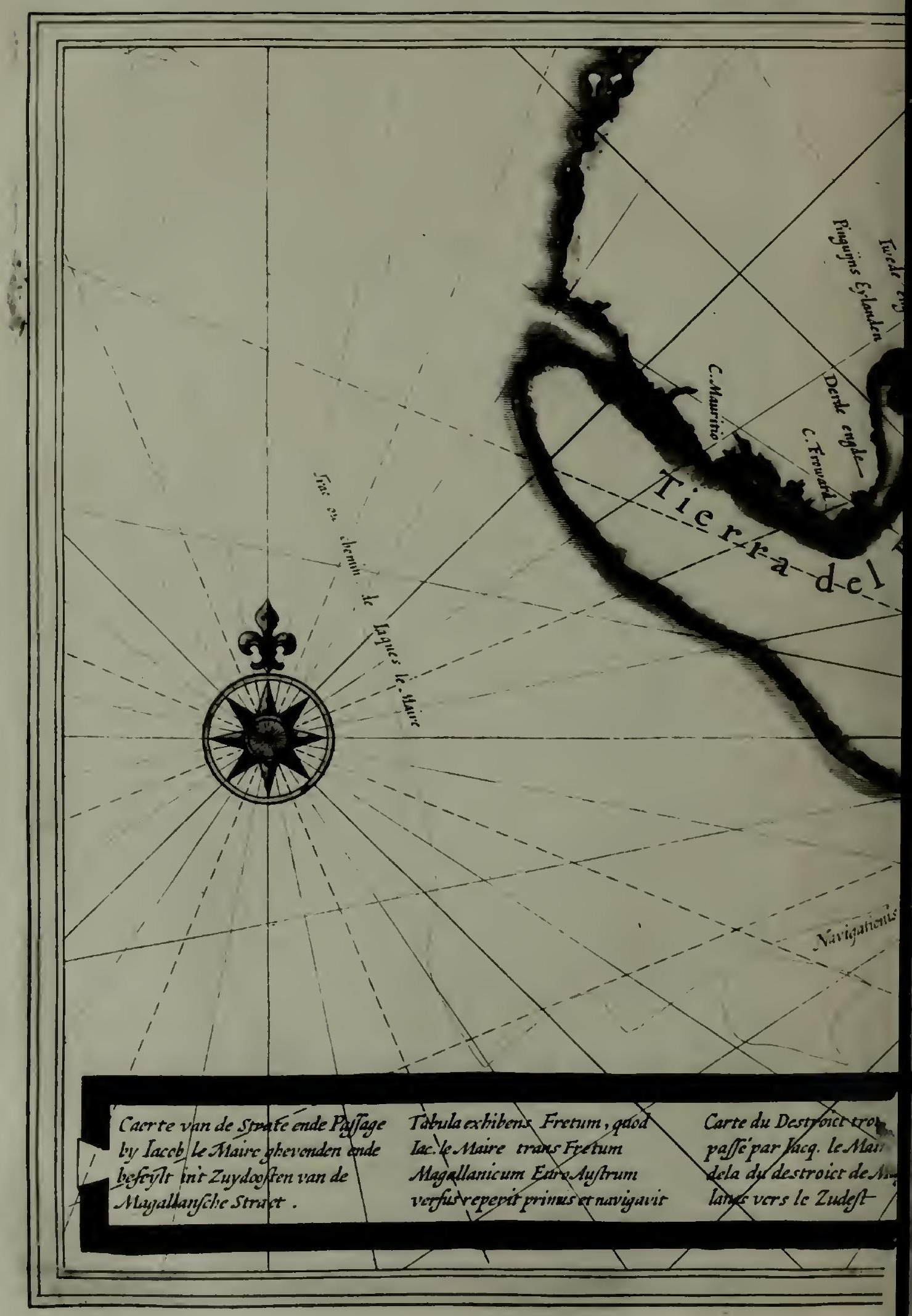



st 6. Je Wefte wine/ met regljen en $19 \mathfrak{a}=$

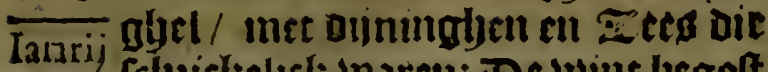
frlyzirkelteli wasten: Be wint begoft hem te verbeffen/ en in ttoun te Ueranderen i ont lyet water ouer't refip ftoztede. Simiodoneclys lyaden oc ljooclyte bail 57 . graden : en gin=

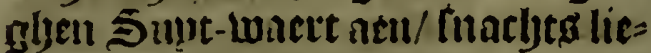
pen wu meeft Hocltwanert.

Den 27. Whis let weder wat be= bact. Gadoc fimidoneclys oe lyooch= te van ; $6_{3}^{\prime}$. gradew. Sagtjen biet Lecl Tau bail Gentell : yadoen die gropette conde ban dantje dic wy out alisljat bebuen : finaclits cen pzopere coelte/oner uace befticl) uan Deil Sint-ne(ten.

Dett 2s. Imozdlunts watt tret Ectjoan liefticls weder : yndoen oc yoori):e banl ; $6_{\%}^{\prime}$. arndoen / fettende

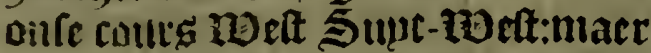
coets dace naer liep oe wint \$oo?oelic/en Deecf ons geweldicf boozt. De Prefident was Luel 3. of 4. macl

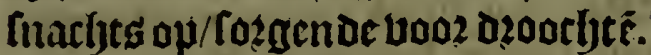

Den 29. Wuis bet betogê locht/ be wint Supt. Ooft / Tagen laut in 114 ban otts / Wett en Belt ten Sup= den:en wat op clarende fagben wu Dat lyet Ijeel perfecte Eplanden wa= ren/leguenoe ontent twee asiilent bain maleanderen/ of let glyebzooc= Fen lant bare: lycboen aenglyelocft om Daer bousent te teplen, be froom fettede oing lyier Danper oilt de

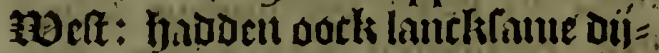
ningljen uut de $\$$ unt- Weiten/cen teeclien ban volle $\mathbb{Z} \mathrm{ce} /$ twater blij: ventor bir ciin coleur. 2t lous voos bu loet I-ant feplende gaf bet berfelyen= ocu gibedaenten/faglien geen Haal-

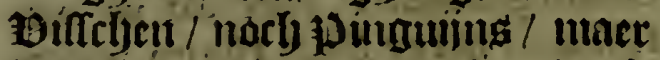
Dogeleti als in Porto Defire. Bete Eplandeftés leggende op de ljooely= te ball 57 . atroen / be-5tudem de zlinie Equinoctiael, noemoe onte Prefident Barnevelts Elulanderi/ ter ecren ball de li)eere tohan ran $\mathrm{Ol}$ denbarnevelt, licjen ten laetiten. Sunt-welt $/$ conacn bouen of bieSupton de Eulanden nict Teulen

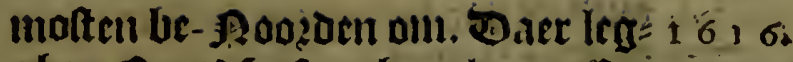

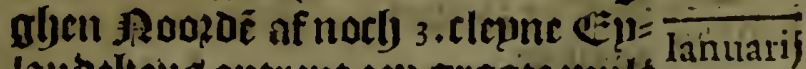
lanbelicitg ontrent cen groote mile van oe \$ookt ch Sumt.fiide/cul be= vontan meteltelicli bat ons of Atroum oul de 1 belt littede oc midoacls landien wu lant Roojt

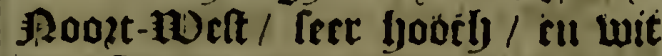
Lan Succu / en faglien twee boo= glje/ 23rrglyeh int wertten : nif̂ch = be Dat daet bet ljoogbe landot teit cunae licp' alloo luy de cundarn coll= oen fien/met rlaer weder : twelels onfe Prefident ter ecten bes stadts ban Hoorn llocnide Capo deHoorn: larb op oe yootjete ban iruen en

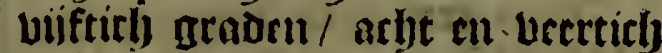
aginuten. Dill Defen l,oecli te uiijocn lepuaculjet $\$$ ųt-Laert a= ber och gantefljennadjt / mae nde: ateliels.

सen 30. Laalt of wint ibeltelickj ouer lanckfame diiningen/ het.water blau/ t'melek too groen beupti was bu De! Eulandeliens gifter/ Dacrom thaticetocu baftelic bat bu muin ae sunt: Ze al binteli. oen fniodoaceljs foochte van $37 \frac{1}{2}$. graden : Juetlingbende reer bau de Deftelicke Lininen/ en biininglyen

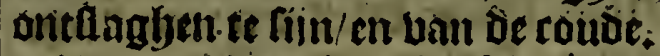

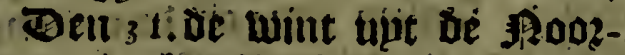
oen/ griughen Herf aen': wp fagen gbecu meer lant / tredert bup Capo de Hoorn hadaen berlaten/ bebonbell [ntidoneclys hoochte ban s8: graden nin thien פgintiten: tn bat wo nu in oen ácnuanck ban de Supt. Ze waten. Ra Den noene liep de wint ten merten.

(ben $x+$ fichzllarij : de - wint ald Februa woozen/ met ftosin : levoent ober. tegen den nachst Sult-waert! maet Jet bedaetoe finaclits.

Den 2. finosglisens was bet teni-! peeft ouer/de wilutheftelick/timioDaecljg be hoocljte ban s 8, graden: Caglien Croos/cn 2 ant-פg ceutwen: den ljeclé dach cu naclit hadoen wo cen bun-leggljer: niaer fabonts otll: De fyet: loet unater is bier tertant 
I 6 I, outfelt yoe het waept/terftont gaet

Eebrua. oe Zee lentel boach/en blijft lang ontitclit.

Decis. was of wint al Hoefte= lick / maer matigber: fugben oocli Dat de ftroom ong om de waelt let= teder

Deti 4. Walt tlaer wedoer//nido= bacelys de boorlyte ban s $6 \div$.graden/ cil alloo wo urranderinge van wa= ter fantyen/wenden Stupt-waerts/ trawonts (1B)elt-waerts : Inaclyto ginclit vooz de - wint / maer cruppt Jaeft weder foodt wert / alloo de Weltelicke winoé lyier meelt waep = cu/ en feloen Doft oft Supt-Boft. Den s. eertt walt irljoon weder/ nae noen ftozm / too dat whe al ong tupth moiten innenen / en orijuen op Sodts ghenade.

Den 7. meenden wp dat we be mont uan de Magellaenfche straet

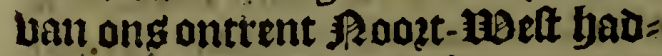
oen : miet reglyen/ naer ltilte.

Den 8 . en volghende daghen vele toinden upt Den Boelten/ met hagliel/4hegen bermengt/en groo: tecoude.

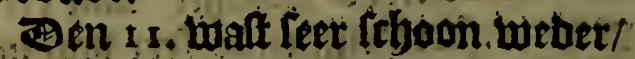
de toint Supt-abet t ninghen Poost \$oozt Mett aen : met fiecbe water: Caglen twee of Dzie rletne kobben/ maectsten giftinghe oat Die bainde Magellanes quamen!lyads ben fmidoatech s7.graden vifftith Sginuten : en fouden detticl 29 (ij= Ien glerenle beloben/ dat niet conoe weren : reertente oerbalven bat ails of ftroan! te baet gbecomen was. 't19s as Jett bacb beel warn.

Toen 1.2 deberptede de luint/ ban oe Roozolicker hamot / ghewent Meft-waet: : Ons verwonverbe bat ton finut be Boft de Straet/ nu baghelijar begonoen too goet weaer te srijghen / met foo aecht water $/$ baer bet ftheijuen van danoere couttarie is. Heden is alle fet $S$ tlyeeps-volck met Dubluet 1ianthoen bereert gbebeet/ tot een bentyst-tectien Dat wp nu in De \$tupt- Zee Waeren/ en De Strate Ma- I 61 ; gellanes al bouen / jae atjtet lyad $=\frac{}{\text { Febra }}$ aen. Back wert bu Generael ad= vijg vanden beeroen liaet geitemt/ bat imen de Straet, oft nieulue doo: $=$ tacljt tuffeljen de landen Màuritius en Staten Zlant / den name gheben loude de plaflage oft Siraet le Maire, Dooz wieng tloeckmoedicheprot ent tandigbe logghe dit treffelick / on= tterffelick warck is belent / en tivt: glyevaert : Derbiluen t'lijuder ec= ren beeft oe gantedyen thatet / Defe zlete ljiter naex bolghende/net haer epglien bant onderteectient.

$\mathrm{I}$ den name Godes. De welcké $I$ is het begin, maecker en Schepper van Hemel en Aerde, ende fon:der de welcke niet goets wert ghemaeckt, begonnen en gheconfervecrt: Alle goede beginfelen beftaende by en door Gode; en in fijnen naem alles. moet aenvanck nemen, tot fijnder eeren ende gloric vàn fijnen. heylighen Naem. En want het feer eerlick en vorderlidk is, voor alle Landen, Provincien, en Staten, byfonder die by de Zee. vaert beftaen, door middel:van de felve haer te doen valeten, en bethoonen de animofiteyt van him na. tie, tot welftant en proffijt van hare Landeh, en Inghefetenen: Sy notoir en kennelick aen allen den ghenien, die defen Inftrumente fullen fien, oft hooren lefen, faluyt. Dat wy onderghefchreven verclaten ende ghetuyghen, dat inde Maent van Ianuario, van het teghenwoordich. Iaer ferthien hondert en fefthien nae Chrifti gheboorte, naer dat wy uyt. Hollandt van de Stadt Hoorn waren gheleylt, met onfe twee 5 chepen

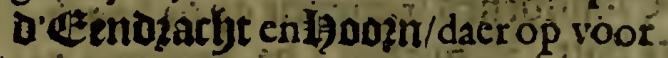
Prefident is lasob le Maire, ende Schipper Willem Cornelis Schouts onder de protectie, patenten, en banieren vande Edele, Hooch-Moghende Heeren Staten Gererael, van de geünieerdé Provincien, en fijn Prin licelicke Excellentis Mauritius van 
16 1 6. Naffau: Admiracl van de Belgifche urua Zee, \&c. Omme te ontdecken de Paffagien, Landen en Eylanden vant onbekende Suyder ghedeelte des Werelts, in conforniteyt van hun vergunde Oetroyen èn Patenten: Dat wy naer veel periculen en travaillen door Godts ghenade, gevonden, ontdeckt, en ghefeylt hebben, door ende langs cente nieuwe paftagic, ons leydende vain de groote Occan, in de Suyt Zee. Te weten by Suyden en boven omme de Strate van Magallanes, ende Eylanden daer annex, en dist tot oinder de latitude van 58 .gra. den, en neghen en vijftich Minuten. Al waer wy onderghefehreven ghetuyghen, en verfeeckeren ghevonden te hebben een heel Royale ruyme Zce; by Suyden de Landen, en Eylanden van America, daer door by ons ghenocmt de nieuwe SuytZee, hebbende int Suyden van de felfde gheen Landt, noch apparentie van dien yernomen; oordeclende bock vafteliek door fijne qualiteyten van fich feer wijt en breet te ftrecken, en te extenderen. Experimen. terende dat lanckx hier den voor:Ichreven Ocean, ende Noort-Zee embraffecrt, en ghemeynfchap heeft mer de fpaieufe Suyder-Zee, tor faciliteyt vande navigatiè. Teftificerende oock voor de waerheyt; dat wy niet en weten, of oyt ghehoort hebben dar eenighe Volckeren uyt Eu. ropa oft andere deelen des Wicrelts; defe paffagie befeylt en doorghevaren zijn, waer door wy ten opfien endc opmerckinghe van't felve, ont. deckendé drie Eylanden, legghende ter fijden af in Zee, ten Suyt-Ooften het Landt America ghenaemt hebben, Staten Landt, Mauritius Lant: en Barnecelis Eylandt ende de Paffagie die dacr by lancx valt lacoble Mairs Paffagic. Yan welck Landt, paflágic, ende Zce, uyt cracht en authorifatie voorfchreven apprehenderen de cerfte poffeffie en preferentie voor, ende in den nanie vande yoornoemde Edele, Hooch-Moghende
Heeren Statew, en fijn Princelicke i $\sigma$ I 6 . Excellentic, als eerfte ontdeckers, Februa: ende befeylders, volghiende d'oude ufantie. In teftimonie en tot verifi. catie van alle $t^{\prime}$ welcke voorfchreven is, hebben defen oijderieeckent. Actum int Schip Eentzäryt:

\section{laques le Maire.}

\section{VVillen Cornelifs.Scbouts.}

Iaš Dirckfs. van Hiorn. Opper Stierman op Hoorn.

Coen Dirckss. van Oofferblocker: Stierman op Hoorn.

Claes Piezerjz. van nofthiugen, opper-Piloos, op Eendracht.

Cornelis Kineryz. van RÿJdam; Onder-Stierman op Eendrachs.

Deil I $_{3}$ al bijfich tinottich we ber / jet water blau: fagben eeni:

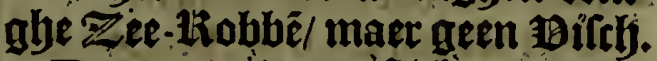

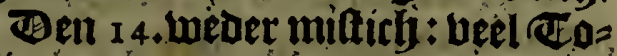
niinen en 2sunin-Bifferen bu't Schip/ de boochte fmidoaechs si. graden / faghen eenich Croos bąij: ben / t'welck met oe ttroom ban't ILant moet comen.

Den I s. continuétoen wo ons

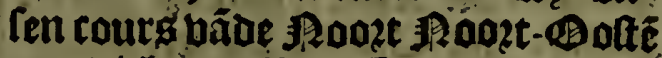
met dicke gsift : Snidoaectys de boochte ban uijftich gradęn/ bijf= ticly goinuten.

Den i 7 . faggen wp veranderin= ghe ban water/ in bleeck in groen/. bierpen bet I oot/op yondert 10 a dem gljegn gront.

Den 24. quam ons bq de genes rále Supue wint/ met goede boortganck / [miobanechs de boochte ban bier en ueertich graden/ les en oet: ticl ginuten / foo dat-wp oit et= mael derticly gijlen bertiert bad =: ben. Saglyen bier baglyelict veel Ilan bain Senten/. en gramue en witte gsecukeng; ef gsalefijten. Geden bzochten wa ons ghefrljut boben/ en fettent ter boost.

Den 2 s. Imitodarcljs de joochte

ए 3

batI 
I 6 I 6 . vall $42 \frac{1}{6}$ gradocn/ en. fetteden onle

Fcbrua. 25 zamienls op/alfoo wu nul waren iii reil gljerulte $\bar{z} r e$.

Den 27. continueerde noely de Irboone Supdelicke bint: fagtjen cen ionglye hoblue buit వeljip / een teeclien niet verte van lant te rijn:

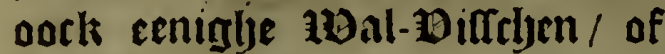
pots ljoofacn.

Den 28. [midoaetlyg de jooclyte Uan 38 .graden adyt פginutell. Oe= Ten dacl) Lueroe van oe Prefident in volle kiaet befloten / oll oe victua= lie te ftrecken / rnde te veruerf́ljen te lonpen acn de Eulnuben van lan Fernando : alfoo ong 23ootluolc met de 3 (l)enrbuucls genuelt wag/ anviet weumicly ban oc tơmcutect was.

Bell 29. contimuetde de Sultoe wint. / Imidonechs de ljoorbte yam 3 s.graden / viffticl] asmuten : na =

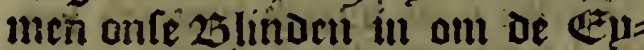
latioen niet bood lou te fepleti.

Martius Den erttcil 99 artii : fmozgheng met ocn baglietaet faclj or Dnoer = Atreculau ban oc fenglie t'landt: Smidoaechy : op de lyooctje bail Date en derticls gradé/ twee en vije= ticli gr iititech / waten wo een balf 29iile vant Sumbelitlitte "Eulant: wierpen bet Zl oot / jadooen gheen gront: Iot Ceplant Dat ten Supt11Beften Ient/ ghelijett bisel Dorte/ ftenl/ Clippidh/ lyee en Daer wat

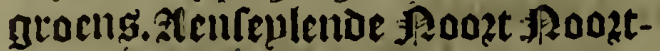

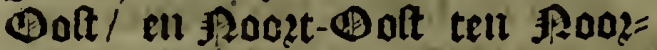
Den/ ghenaeckten t'lant loo lancr roo meer / al conder gront / fiinder feer fjooch / fteul/ hackelich lant/ berfenlorn den Sundelicken boeck/ baut Eulant $/$ Daer Lo Datr Bofte $=$ liclt Doo? in Z ee fagthen/ en tregen bet anoer groot Eulanoc op fijoe Dat boven groen en bol theboomte wiris: Jadoden vele variable win= Den en ftilte / foo dat luis nac onfen. wille acii lant nict colident romen: lieten be witte volagerlye uni de Conthaignie Lachen/miet wetende

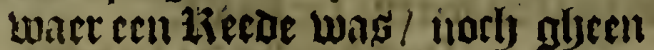

gront bernemende waren berfuft. 16 I 6 . Oe Prefident relatcerde de lienulte $\frac{16}{\text { Martius }}$ Dic lju upt ceniglye felyiften lyadoe/ maer werde baul of Scljipper niet glyeaclyt / t'weleli ong Daer naer fcljadclicli wag : en fictioe gheen inwiicken liet lye oe $2300 t$ unt fet ten / Cont Den Ondoerfuetulun met Den Onder-Coopman na orti wall bevondon cen rrocliclsen inbocht? en de Dicpte Derticly en vectticl) Da= beril / rwant fant/ bicljt onder oe wat : twee ban oe igacts liepen acn lant / culaglyen cen tiiole ban verfeh water/ unut ech wallewe cos mende in Eee ftozten / oock beel Liobla:n / 23ocken / Senten / ende andere viervortige gediertê: bzocty = ten de foode ban thien crostolna: Den/ en cell 25:alem aen boott/met boecken opgliebaelt / fagljen oock verberffhinglje ban frunt : mate wu condé olls Scljip niet op gront batngyen/om t'aticker te werpen/ fo Dooz ftilte/ en foljerpe wint. hiel= den fnachts bu.

Jen 2. faglien wur ont wo feec verre baur't Efllant ter Zee-waert glyedzeven waren booz ocn ftroom: wuy decuen den gantétyen Dach in teilte / conben glyeen gronde noch recoe crijglyen : en dic de recoe wil befeplen die ghelegen is int $\mathbf{R o 0 z}$ Den / in en groene ballepe / moet be-Doften t'Eplant ouloopen/ co= imende upt de Sunt. Smiboacths yndoé wu de hooclyte ban $33^{\frac{1}{2}}$ gra Den. Onfe boot was aen lant / eit bzoclyt wederkeerende wel dzie ljon= Dert Biffelyen al met de bangljel gljeluanglyen / Iegglyende aen oe decgglje onoer yet lant. $Z_{\mathbb{Z}} \mathrm{fa}=$ oen ootli veel 23 ocken/ en Gepter? ros ban coleur met 15 acroen/ et booknch/ oock jonglye Capzitieng/ dic win niet conden vanglyen / Doo? oc rupelite viluditenor. $\mathfrak{g}$ ent [acl] daer oocfifcljoone groote Creeften: Agen gljelooft bat hiet be spacng=

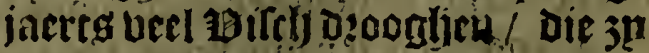
aen't baite lant ustuoeren. 
16 16. Ditl 3. hutyen wa dat wa dit Marius cturacl meer verloozen yadden dan othe toumen / en wel bier 29 il ban bet Enllamot waren: refoluectaen acrlmalven bet liser op te doutwen/ En gaen oule cours nae Terram AuPralem: berlieten allo o bet feboone Lant / alleenlick becomenoe wat witels/en Ditely/ Iegghende op of oxic an Dertich graden vectich $90 \mathrm{i}=$ nuten.

Den 4. tinglyen whante cours: Inet D'atide caclte/Landocin Sotuden/ slide Sunt-Doften / met Idjoone booztganck arbe en Derticy agill iu éti etm.rel.

- Oen 8. Whas onfe Prefident hit wel: ar Srlipper unas aen de loop: hict-te-min lettede de Prefident op bet luerte wolclijen t'welclt men lilet fien en tany dan aljoe Sonne ect wiiltie ts ander gheweet / en rach een ander wolctijen aen be Pool blect wit/ natuerlicts algeen litlye deijuende wolckielt.

Uen i 1 . Inadeen wo fmoeghens Eell goede roelte / fmidoaechy de Ijcorbte ban deie en twintith gea = ben twaelf $\mathfrak{A s}$ inuten: rojo Dat bou intu booz de tweede reufe den Tropicuni Cancri pafferden : bier cte= thlyen wu ben alglyemeenen Bolten cll Oalt Sult-Oolte wint/gingen Proort-nuret aen.

Den 17 . yadoen wa de lyoortyte ban negfientljien graden / hadoen Gijeen limot jernamen: Doen oe boochete gljenomen was / lyeeft of Prefident doen vergaderé de Stier= lieden en Den liaet/en refolbeerden aengheficn Luu nu daie etmacl woet aen tyadom ghegaen / onfe coutrs te Leranderen/om aen te feplen op't booclyfte $\mathbb{1}$ int ban Terra Aufra is, en gingljetl aen de voosgbeno= ment tours.

Den 19. contiutectaen wp Den boosfebretuch taurs/met fefjoon we= ter/fyrdoen (midonect)s be hoochte batt $17 \frac{1}{2}$. graden : foo dant way gifte = ben te fijit ozie fondert vijftich
Pliilen un de culte ban Peru. is 160 Den 20. anto: De wint Dofte = licls / Cagtjen ceniglis witte joijl= ferten/en berl uliegende Diffrien: (midonechs de joociste bain febaers rebentljien titionen:

Den 2 ibterf be roelte/met aroos te onningfien Uimben Sripden/als in or Spaenfrite zece : we fagtjer

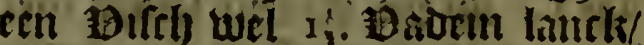
met ten Speet oft 5 wect toos

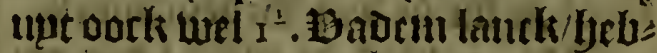
benoe fret troote binnen op , oe tingabe/oien reninge de naem ga= uen ban Hcorenvirch: het ginck alle daghen ltif voout.

Den 2.4. badoen fejoone toelte/ unt oen Sturt. Bolten/ caurg reclit

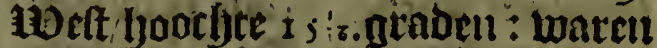
nu vicr ljonoert treltitygrsiilen van Peru, berlatmoen feer naer tlanty coo dat wo bejertoen datter gheen Terra Auftralia was : oock feude de Schipper in dien bp badoe gljewe: ten Datter gijeen Hollantich Indieh, oft Aultralis Terra, aen dees fiide Isan Salomons $\mathbb{u}^{z}$ ulanden ghetweet ware / hu fovide nopt een yoet ine Schip ghefet bebben: Een praet bie niet en floot.

- Den 3I. walt beel fthoon bes Dex / fagljen gljeen lant / ntaet beel Bonlyelen : wu waren bertwondert dat low liet ezplant S. Paulo niet ent faghen Dat op oefe foachte leggen moet : yadoen de ljoochte ban I s:graden/ yadoen norlj groote bij= nitulyen tuanden sumden.

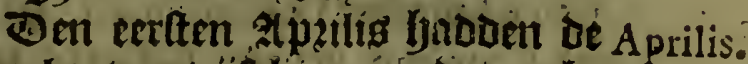
jourbte ban vijftlien graben acht Isinuten.

Den 3. welende paeteljoach: een $\mathfrak{A g a e n t}$ Dat wp ban bet Ep lant lan Fermando leplDen : wartit nu leven jontaert biif en twintich Buptrfye gsiilen uan Peru. De Scljeurbumeli beltont leer onder onste bounineren. 25 emerchtedent glyeen afurickinge/ maer of paels de trock Poozden en Simoen.

Den 9.hnaden noclj goede coette 


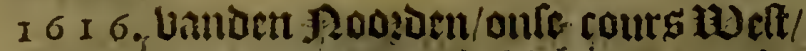
Aprilis waren ftif op uijftlien iraden : la gijen uain Dangle tuiec cleene 250 a nietten/ an Stere-99eruwen Met inenichte: daer was ope ued Ditch/ maer twe condenle niet yamgryen. Defen dach oucrleca lan Cornelifz Schouten : Scljipper op t Jaclje ge wect $/ 25$ oever van oule Sthipper/ nae oat by cen esaent hung niet wel Ijode gljeweef it liju boal niet beij (ijnor uan Scljenrbupeli.

Den 10. Luas de wint noch $r e=$ ivelick/ inet reglien en caeclien : wan fetteden beden het doode lichaem buuten boozt/ met ozocffenifte. bet

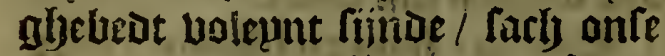
Sarbier lande : licpen oat track lance oui Ztncker-oront te biituen/ maer bonoen niet De slocpluer De gleniant/ell afglberonden / Dedoe ooch räpjoat / Datter silyed ápparentic was om te landen Doo oe Latninglye. Daer naer gincli de 1)ooch-2Sootfman nact land on quam gljenlackelick dact op / macr wederkeerende molte ben met cen Counae den $250 a t /$ uan bet lant int water beglieben/ en roo nwem= inen niet groot periickel. Bertelde Datter glyeen bertely, water op fet lant was / dan wel eenighe plaf= kens untnemende bitter / Die Daer inachten gyeregene ziin/ maer beel toifel onder bet lant / beel $\mathfrak{A e c u}=$ twen en glichogelte Dat op of $2500=$ men netteloe. Dit is het [epulant/ dat Magallanes Ijeft brefnlt / en bu jell gljenaemt Defueutturada, Daer

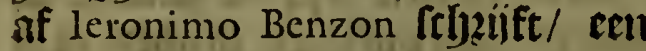

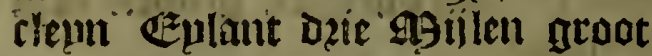
int ronde/fiinde feer leegly op foun= mighe plactien / foo dat met boocb ghetijoe t: $\mathbb{Z}$ ecwater tot int mid= Den vairt Gulant loopt. Dit Ey. lant ftact bol BDatertictite / Wais= lende acn clevule $2500 m$ lieng frer hitter ban fmacels/Doch leet goet

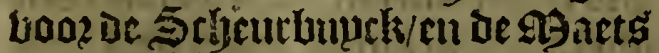
bzocliten cen goet ocel daer ban acu loozt : Jut lieet als Dzagon / bet macrlite afgancly cu verberityteide i 616

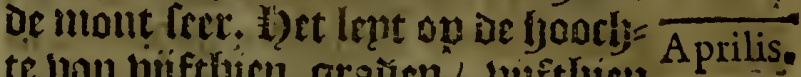
te uan bijfetbien graũen/ unfitlien Pintiten : an ban oe Gitute ban Peru negen fondert twintef 9911 2 len. EE om Dat $3 \mu$ gljefien hodDen

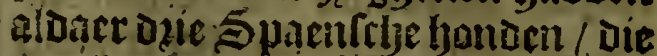
thatjer waren! noemoen wu bet Honden Evlant / tât beel unnei

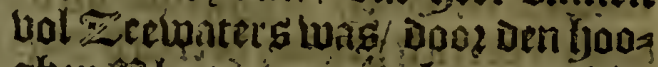
ghen bloct 7 wprginghen ban bier loet aen nae de Cpianden ban Salomon.

Den I . De Luint banden 3 soortDoften / rmidoaceljs lyadoen de loocijte net uan vilftlien graden: fnachts coeloe het Dapper aen / wi beecioen daer ergljens reniglye less ge Eulanum/als Tiberones mochs: ten legglyen / daer bu felgub ban Couden crijgijen / maer Godt lof PReril.

Den 12. yecft oc Prefident ben Opper en onoer-Stictnian lium Cacrteri ban Auftalia, en Nova Guinea gljeglyeben / on! Datie use couorn meglien fien/ en giffinglje maerken.

Den i. cen cenpariglje coelte vanden Ooften. theden was yet tbien gsaenden dat wy nut Cerel feuloen/en tou meenoen Dat wu in thien ggaenden/ Terram Aufralem Couden beteulen:naer mibnacly facly Baholomeus Pieters cen ₹ffaltent/

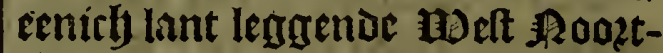
12Belt ban ons / otitrent twee 99 ij Ien/ Daet op ftachde centch glye= boomte/als De Tuberones, cen groot Eplant / maer Leel leeglye / foo Dat wu lyeel bethenefist waren / bulons oer De Cranclien / om de berbert= febingbe. Fetteden onfe cours oer= waerts wefteliclt acil / oun aent Noopt-epnoe ban't landet daer bos uen te loopen' ' ch ztudier-grout te coeclien.zilons feplcude fagl)en ecu Camoa/ met biet geele naette man= nen uner ong canen : ju bielden een ftick bant 5 chip/en riepen en cres

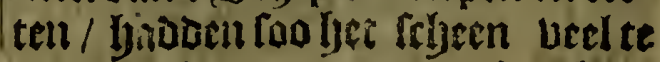


1616. fegthet / mact conden thace niet ueritaen / nocls 3ll ans / wat wu wenckteden / riepen / Crampetten blicten/ or witte Dlagge ult wiet =

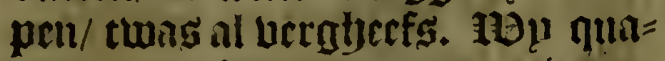
men ander bet lant / maer wierpen gliesn gront/ licpen t'Terwaet: Capts luer nase comt weder een andere Cinoe / riepen en tieroen

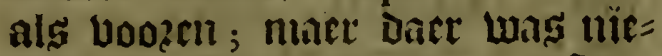
mant ban doufe dic baer uerftacn conde: :aset ern dinmene vicl fact Canoe oul/ maer ju terljtedan dien met cell rafieticubet: rn alloo tun niet conden anclicien/ refoluecrden

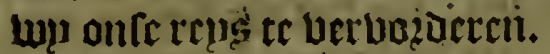

Dit Eulanc ig by githinge thum=

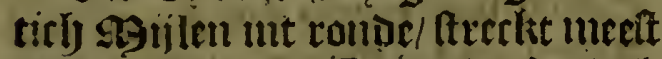

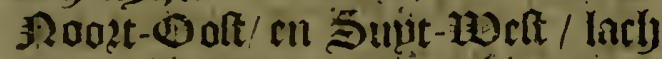
op oe boocljte ban uecttijien gra = ten viffen ocrticlj-29unten/fimoc fere leegly lant als or Turberones, en uol Cocos 250 amien : Dact was oock water midoen int (E) ulanot.

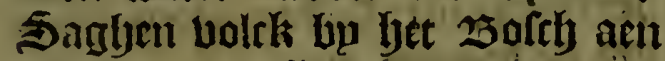
of water-cant fitten / $t$ ' was untter = matell geel! nac sen rooden tree = ltende/ Mér perk nwert lantk bauz/ inden pecli opglyeluomern. De Prefident farij cen SBan bie een wit Clectien on fim midodel lyadoe/ die in een Psantien feljeptedae.

Dell I s. Imozdyens / als wn een ftuck ter $\mathbb{Z}$ ee Luaren/ Ijicloen weder nar bet Eulandt toe/ en hadoen boape van atnclier-gront / om do Witte fant-Strant : macr vonden gitseen : bet volels ban't laint wenck= té ans met lyaer Clecticng'en groes= ne Carken dat wul arn lant cou= Den camen/ en lyet Silyip volulyen= De: Or Prefident fonde ous Slacp unt om te diepen/ Daer-eni tuffeljen quan een Canoe of met Dzie man= nen/die met lyaer te wöpt quamen/ maer condonle niet beritaen/en ga= benfe deie 9 effen ende wat $\mathbb{C o}=$ raeltics / dat lyact wel am ttont. Eu gauest ans volck terftant de nincker ()ant/en maeckiten bzient= folyn met bim/ leggende boogt ach boost: tenl leften weren bier à'onfer 616.

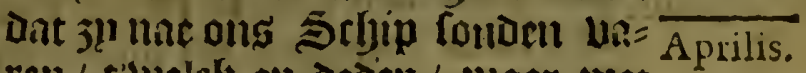
ren/ twelelt ju deden / maer met

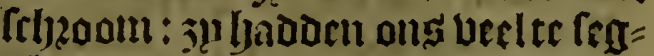
gljen en loegljen en uerwanaerden

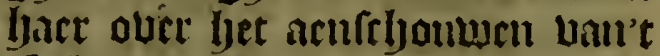
Scljip / dat ljet foo ljoodj was / cn foo booglye feulen / maer aberca= men Luilden ju net / bliibende met fiact Dzant acn boozt leggen: frtyouse = lsen bace te dzinclien / water paoff= Dent qualick / en mae laugli whirb)= ten bicloen oufe Seljacl: ju thoon= uen daté wel Merer begectoen/ Inad= ven ju de maclit gheljad / $3 n$ [oit= Den of $\mu$ rere 5 pijcliers en 23 anten unt bet Srbip glyetracken bebuen! procluenoe ouer al : hoo dat wo hace ture oft dzic इpiiclierg gaven/Daer ju blijt ant waren : wu gawen ljaer 25,00ten Lives/ t'welck ju neder lepocll. All Ererwerck was yaer gadingh/macr yaduen niet booz ons. Zu badden lluat bap? / Dat ernige op bonden bouen opt looft/ oft acliter in oe Recke / $3 \mu$ zin beel baunu gecl ban bel / de bingljers al gljepicquect met fwerte langlye ftrepen / en aock met tiaderliengl. en inuten uol werex : waren bet gelat/ in lferck / groot ban ftature? en cozpulent : plataditige seuren/ gaten in D'Oozé/ Daner jul de Spiic= fiers en Cozalen die 3 uan ons cregfyen in fteecken : bedectiten al= leen de frbamelyent met een laps Iten / oftern fijn Guinces 9 gatian oin baer midoel/ tulteljen oe 25er nen Dooagljelyalt. $\mathbb{Z}$ il bletuen al Lu de 5 locp / daer 3 g guecrue de Ozegalje untegeftolen badoen: qua= men oock wel in De Galerije/ macr daelden terftont weder neder. Tent laetlfen befloct de Prefident Datmen de Slocp anoerwerf foude upe fert= den am alle ghelegljentbept te lue= tê//Cont 6.9Dinfquettiers naer lant/ met Aris Clacffoon, en Claes Ianffoon, met want rupl goet/alfoo jp te ueritaen gaven dat bater Derchend en foénácen waren / cn lagfyen e wer 
I6 16. veel Cocos $\$ 0$ omen : aen lant $c 0=$ Aprilis. mente conden nitt te recljte raee = ken/mits ju terltont lyet volek aen= tafteden/moe facken foectiendenaer

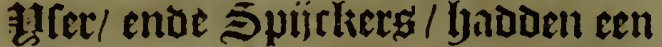
ontaer de boet / die ju ouer weldich= Den / en twee docoglien su teglyeng baet danck nae lyet 3 bordy / dat

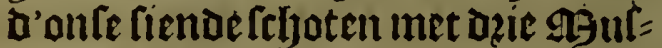
quetten inden boop / Daen felyen= Denfe. $\mathbb{E}$ alloo lyier gheen grout te crijuglyen was / gaben het Ej: lant de naem uan fonder gront, Dat rontfom met Palmites 250oune was belet / en bait binnen net fout wa: ter verbult: Gljeen apparentie van bzientfelyap fiende bielocin dzagen= ae inden acljter-noen / volghende

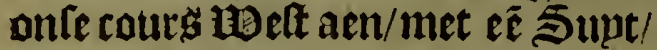
Sulut-Dolten wint / hadoen fa= bonts groote reglien. Wet Hater liep feer flecljt / Jetmoededen der= haluen dat lier noch eenich zand noof ontrent wefen ' ban D' $\mathbb{E}$ plant= Den uall Salomon, bft. Terræ AuAtralis : Dit Eulandt lacly op $14 \frac{1}{2}$. graden: wo conden niet mercken Dat bet opt van pemanot befepilt was:mits d'arinoede die Daer bas: be 13 zouwen yaduen Clectieg om't lijf van de 25 tupck tot beneden de knyen: frbenen baer $\mathfrak{a t a n s}$ te be= kiituen/ Dat $3^{\mu}$ foo rota met dounfe (jeljandelt hadoen / Daerom wp tooz gingben.

Jen I 6.een ure booz de Sonne/ fach oule Sthipper weder landt/ heel leegly/ fandich/clippich en ved gljeboomte/maer nocly Cocos, nocly Falnites / bocht ons onbetwoont. Sonden de Sloep ont te diepeil/ wierp veertich Bade waters/ maer niet bequarm om te leggen: D'onte ginghen aen lant / vonden Cuplen met vartefy water. . Bonden oocks Chuun-Cars / en Ooft-Indirche Salaet / Conder andets: Bulden bier Baten waters / conoen niet uneer crijglyen om de barninghe. 23zachten oock een fack Cars / en Salact dace wu 190 of af coocliten/
Ginglen onre cours macti Suptwert/met labluer weer ! encleunen booztgancls: begberenoe op oft bouen de bijftyien grauen te we= ftil / om de 25aue vall Quiros niet boozlou te feulen/alfoo wp vermoe= deden t'ualte lant lyier ten Supden diclyt up inolt wetell. De Prefident nocmbe dit lant/ I aterlant, om dat wo lont buaters gljecreglyen had= oen/ allier legglyende op urerthien graden les en weertich 29 inuten.

Den 17 . fagljen wu berfeljepoen Cookten uan 10oghelen met bacece uléugelen: Coo dat bu reecter waren datter lande int SHuden was.s. Baven van Dagbe fes g9utfkents water/ oni die 19aten die wu aljecreghen fyadoen : ang betuiel oork een goederegljen / die beel waters bu bizoclyt.

Jon 18 . mait nopi titi weder/ aecht water / laghen in oe il $\mu$ weder leegh lant : finiddaeclys fyadoen wu de lyooclite van i $s_{\frac{1}{3}}$.graden: lach ontrent twinticly $\mathfrak{A}$ ijlet luat Waterlant, Supt-1⿴囗十) ban ong. Sonden teritont de 2500 aen lant/ Uonden niet ban dat bzack luater was/ faghen een bilt glsenfey loopen net een 250 ghe in rijn thanot aen tarant of lyu pifch wilde febieten / groot ban ftature: en D'oufe gheen ghetweer hebbende vertracKen ltraci: als $3 \mu$ op de vaert waren / verthoonoen fjare fes $\mathfrak{A} 9$ annen op de Strant / die oockiftratr int 25 ofely wederfiecroen. $\$$ in dit Ezulandt was foo groote meniclyte uan Bliegben / Dat onfe $\mathfrak{g a c t}$ de $2500 t /$ de triemen/en alles ljadden befet: jae uloghen ons nae daie Dagben lanck / tot dat een dappere coelte/ fyaer berdzect : noemben deryalben defe plaetfe. Wet Vlicghen Epilant.

Oen 22. fjadoen Dzoef weder/met een \$oozoelicke wint/ weel Donbers en 25 lirents int Supden/gingljen 20 elt aen. 
16 16 . Den 27 . yndoen weगer diinin=

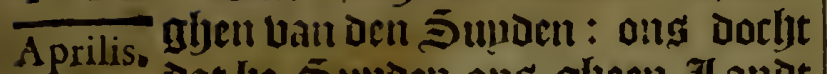
Dat be-5imben ons gheen zlandt conde wefen / of 'tmofte verre ban ong fiin / Yavoen fmiodaectys oe hoochte buifthien graben bifftidl g)inuten.

Den 24. De caelte unt ae Dolfe= licker lyandat / al-te-inet met ecn caeck do reglyet bu bzoche/ daer af lyet water werde glyegnett : faigletl beel glyetuagiselt.

Den 28 . funtodaectys ide yooctyte I $5_{6}^{\frac{1}{6}}$ gradoen / oc wut al continu= ceroe bamocli ouften / cours meet

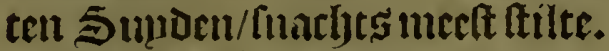

Majus. Den ecrlten $\mathfrak{B a n}$ roelde de Oofteliclie wint mop op: onfe cours mis welt ten Smusen : De diinin=

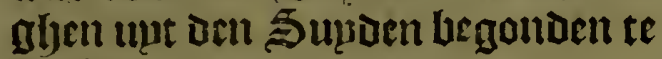
ntechten.

Oen 3. [miosaretys hasden de boocljte bain bijftljien gradé. Dien Dach was ons Woick meeft ghefont glyewozden / vande Clyuuncozs te eten/foo dat fetyeen dat de כefjeur= buuck unt lyet Scljip glyebannen was / Lun faglien d'eerite biif oft fes Dorados indé 5 muten groot / met eeniglie blieghende Diffeyen. Defemidoacly was onle reeckeninglye dat wu waren wan yet balte Zumot uan Peru Derthien jondert 29 iilen.

Den 4.5.6.7.meeft al eenê Luint/ en tours als boozen.

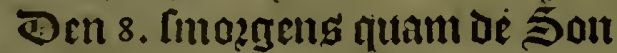
claec op : ual dagtye fagen wu teec= kenen uan lant / cleune Cackiens ban $2300 \mathrm{men} /$ of glyewas ronder loof : oock fagen ecn Qual / en beel 5onietten vooz oe $250 e c t$. Pae De midoach lach de Prefident in de

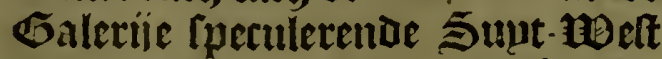
tupt / facty wat wits blinckende/ waer alser lyn de pliloot riep/ on't felfde oocli te fie/ naerder comende/ fagent boor cen 5 epl aen/ in forme ban een 23 efacil/ en quan techt op ons aen unt de 5 upt/en wilde nac cen alloer lanot/ bet onder leljoot

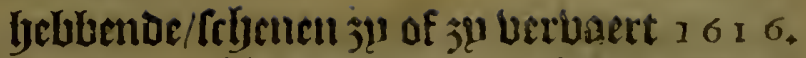
waren / en hicloen uan ons of : wp fetteden of Sloup unt / Dace earlyt פ9ultictters in glyefteit wozocn/su alloo d'onfe faglen dat al de mams bouen ftonden en al de sifngaicn

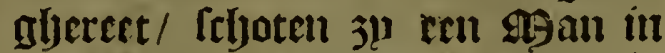
fiun titigglise cen feljami foljoot/ in clcke Frboudorblat cen gat / die

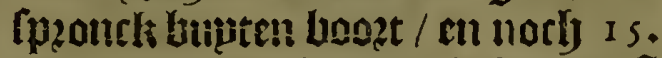
of i 6. met lyetu: Itum nenfielyten equt nwert maeckende met ziffere of $j \mu$ ter Doot ginglien / latende alle ive 19 2autwen en linilloren / met een oude $99 a n /$ dic een grijle 3 aert hadoc/en een $\mathbf{3}$ ongman int leljecp= lien / die int water uteenden yaer te fallueren net fwemmen / ter tiit toe dat wu weely fonden jiin: Een gadde een liint in fijn arm/ dack utede Inu in't water fpzoncli : donte enterden lyet Schecplien / en ituer= Dent volefs na ons toe/en t5elyeep= ien oork aen onls boogt / wierpen eerlt veel agaticns ober boozt / en Goenderen / en fteloen yaer niet te weer: aen boost comende fagyen Dat bet gede Indianen waren / al verftonden glyeen val onfe Calem/ wu Deden de 9 gang abercomen en Dedele goet tractement/ De ozoul= wen claecljoen met traenen / Dat lyaer 99ans uerdzoncken waren/ maer ju yebluente Daer naer alle weder glyevilelyt / alloo $\mathfrak{z}^{\mathrm{l} \text { leer goé- }}$ De fwenumers waren. Onte Sloep cregfien noch twee uan die glyene die bulten boozsot gljelpzonaben Luaren / teglien den alont fetteDen wy lyar in lyaer Sclyeepken/ bu de B zoumen / die baer loenden. beuonden bu haer eniglye CocosNoten, Obes-Wortelen, Matieng/ en Cleederen wan soaft / Daer ju ac relyaente mede Deckten. rcljenen Zee-water te daincken/gavent haer jongye tionderen/ die dzie in't ghetal waeren / oork ecnighe de ouder taren. De Prefident font Aris Clacffoon met Daniel le Maire, met ceniglje inuplteringbe om oe

$$
\mathfrak{E}_{2}
$$

30 ?०u- 
fol. 36

Spieghel der Auffraligche Navigutie,

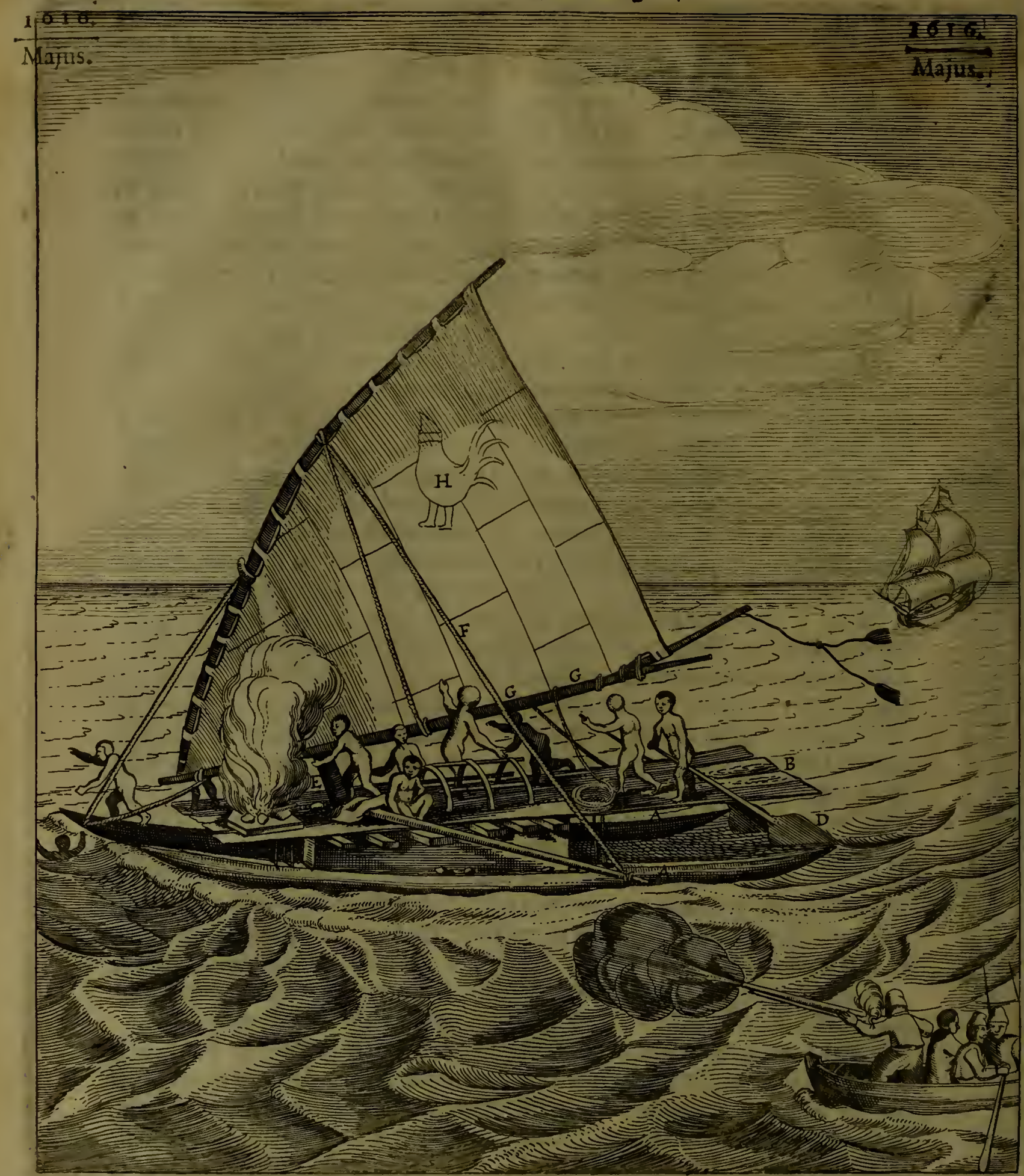

Numero 2" 
$\$ 616$. Dzantwente berceren/ diet terftont Majus. om aen l)als/ en op De $250 z$ t tyin= gljen / werenoe auer mede beel ber= bliit. Fne afgace figlyen, we dat een bzaliw feer wechioe onl yaer g9an / oue ju mectide verdzoncken te wefen. Zun gaven twee Cacos Boten en twre 99 aties Aris Claeffoon boo: fijn perfenten/ om dart wul laer niet nufoorn fouden: Eu yadoen rwert biny/ entiglye los/ eenighe wat gecrolt'maer met glye= crifpt. Oe Denthen was bet bay? tozt afgliefneden/ foo Datmenle nict en berkende/ Dam aen de $230 z$ ften. Zu hadien ect Dzollighe fatfoen ban een ferjecpjen / met ee! loloe= ring op twee befloten Bzosuliens/ Die aitoerfalf Dadm ban imalcan= oer quamen / owerts ober glyebon= aen met Balclien/ ra Stoclian / rul Dater 1) lanclien over glyelepte / daser op Gndoente een thutien of ent= jen uan 99 atten lancwerpich/ Dacr

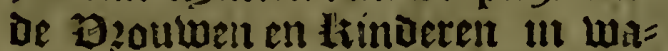
ren : ent ticroen met hiemen booz en achter/ Die wel dick en grof wa: = ren. We gsaft tont int voozfdjip/ op een owars ljout olyebeche / imet hooft-Canwen/ oock Gndoen een langhe $\Xi p$ ziet befacrig wile/ cn conden wel aen oe wint fenten : 5 u hadoen felyoon Cou uan diffetente Itoffe en fatcoen feer meetterlicts ge: mactit: lyet bocufel ban't barticel. was ban root 1)out/ als \$upckerFilten: [oo dat alles actoich quant hadaen ect Diffel ban ent ruerte Cact-5teen/aen een ftockglyebon= Den/ Die jul niet gheerne miften wil= Den : Inacr 5 cul was tuan 99 atten/ bet frbeen dat $;$ op de giffinghe boeren / bebbende gineen Compas bu bacr. Doen ju uan otts fepl= oen rettesen baer cours Suyt- Ooft aten.

Den 10 . fmozghents lyadren uup alelje water/. en een proper coclte uan Suut-Oolt: onfe conts wet ten Supaen / De Prefident ber= moedede gifteren al lanot/ maer nu fuclumen een fooglie sergit als 1 6 I 6. Ternate, oozdeelende tfelfoe vooz Majusi cen Eplande / en lacly Supt- Hert

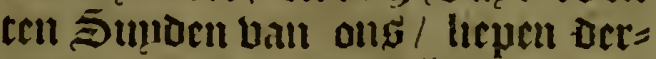
wacrts! lach feven asiile van ong/ gnoben groue dijninghen ban boo=

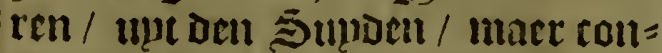
Den bet Ceplanot by Dadbe ktet be= [culcu. Rac midoar!yg clam de Prefident acn oe Itenglje/ en ontdeckte

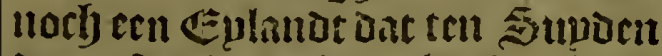
dact af lacty / werende lesth-achtich an backrlicy. Oefe tuee Eulant= Den / d'ene groot / ende cen clepit/

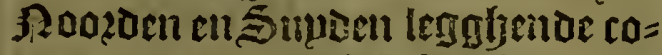
nien ouer ecil met bet fegghen ban Quiros, hopende oat wu De relt oock foonaer aduenant fouden binden/ ente Terram Auftralem baett lou= Den fien. Coats daee naer fagyen

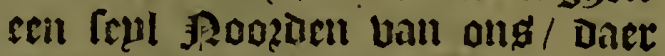
naer nocly ech feul tulfeyen bepde Eplanden / meenoen dat bet Bif= fchers twaren/alfoo ju fnachts bict = Den.

- Oen I I. fmozgyrns / Jieltoen wu Sulloclick ach boucn bet Eplant/ ae wint oftelick / wierpen ontoer 'clant op veertljicn Daocm gront! feljoon clęme feljulp als grujg met faut/ Daer oute fiinde / creglyen fec, thien / twainticly / 25. 26. 10ndem diepte / Con dat wen de gront weder uerlieten / en t'water fieclyter Laier= be/ t'welek teghetis lyet Rif acn= back / dat Roozocn af Lan't Ey= laut lept. Saghen twe Diffelycts/ Decte qunm dicht bu ons Schip/ judoe beel te ferglyen / maer conde bem niet veritaen/ noch aen boozt crijglyen of locken met Cozacltjes. Choonde ons Difcly/ die bu in fijn. back gadoe / Lun licten ecu gelenDattien af met een cou/ oun te griipen / maer Ijp bont daer twee Cocos \$oten inde trollter aen/ met een feer groote blieglyende wifty: en wu fyacloenfe nact ong / $3 \mu$ be= keecken onle bactien/en ginglyent dace neac dooz/ om lyet pirer oocly = tell wp. Ontrent den uniddacts c 3 quas 
Jrol.38? Spieghel der Auftralifche 2edvigatie;

I 6 I 6. quamen wu but tẹlant dact gront

Majus. Las/ Roozoen uan t' Eplant clepn fant en fetjulp-gronat / oock wat crael gront / taglyen aen de \$Ropat \$oopt- moeft tijoe / niet: uerre van een lyoeck twee Gotelinglyen fefjoot ban't il ant' op fes en twinticly $1 \mathrm{~B} a=$ Dem. Twee Diffeljers quamen uut= ter zee / loopende naer een ander Ëplandt Dat twee gsijlen uan lyet ander lach be-5upoen / niet feer boocly/ maer greot : Luiloen ong glyeerue Dertwaerts bebluen / maer als ju Cagen Dat wu bet naelte $\mathfrak{E} \nu=$ lande colen / quamen rontom ons Schip. Onle Sloep ginck te die = pe/ beuont buftljien / veertljien/ thacif yademen frijulp-gront: top liepent acint epune ban't Eulanot en fetteont op biffen twinticl $1 \mathrm{~B} a=$ uem fant-gront: De albiliden volcly= Den ons nae. Dosften eerit niet by comen / Daer naer clommen $3 \mu$ wat baijunocuigher op get boozt: toch Dooz beel noodes quamender twee inde Cajulet/ Dat D'andere liende/ quamen alle upt de Canoen bouen/ op de Contpaignie/Daer Dirck Boe-

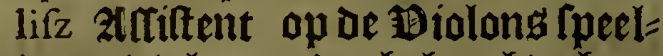
De/3p dit lyoozende/ heljaecljoe baer wel / feer onder malcanderen lac = yende/ en ons Oolck ghelijek $\mathfrak{g a} a$ troos leer dzollicly is beltont te Danfen / Dat Deoen $3 u^{u}$ oocls terltont mede: $\mathbb{z}$ fyadoen ouer al fin in/ Coo vertregen wo ljaec baientfrlyap: wp bediesen haer datle Cocos-Noten, Banannes, Oubas, Berckens/ I) oenueren / of anders wat fouden

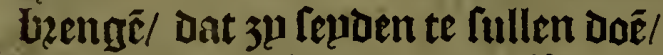
an liention al't felfoe / en wilten te legglyen datle gyenoech hadoen/en noely ueel meer dingen diefe noem= Den : wu pefenteerden baer $\$$ pije= liers en ander goet voo? frupt van 't2Lant: ju boeren en quamen we= Der met wel twee yondert CocosNoten : Darernaer quamender ong foo beel aen boost / Dat wu niet wi= Aten Lat Doen: $3 \mathrm{p} \mathrm{bonden} \mathrm{haer} \mathrm{Co-}$ cos oul de Jaifen/ en fuommien

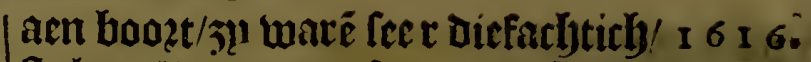
Italen alles wat $j \mu$ fagen/oe $5 p i j=\frac{}{\text { Majus }}$ liers upt trecliende met abewelt: namen de Cogels/ oock onle Cockjonglien een ases upt fijn handt Dat $3 \mu$ al riju Dingljerch Doos [ne= den / en lpzounck ouer boozt: $\mathfrak{E}$ en ander nam ean Coperen inclit- $\mathbb{C a}=$ kerken/unt ecn Cou:D'antar nam cen 23 ultfacli / Oozentlen / 23olck. uanglyer / an fprong in $\mathbb{Z}$ ee. De acrde witg in Conttapels Camer Doende/ too Datnien met yondere ooghen moft toe fien. Onfe Slocp uan lant afrocuende / werde ban thien / twaelf Canocs befet/cu na= nen 'toicp-iloot met gheluelt ban De Stierman / en decpeljoen d'onte nut Autegaien/ D'onte frboten twee mael met loos Crupt / Daer $\mathfrak{j}^{\mu}$ nict op een palteden: Doen feljootmen op de Capitepn in riin 250 et die neder-viel / Doen vlooden $5 \mu$ alle= gader van o'onfe af. Dit was meelt al pouer wolek / conder Coninclis of Buerljooft / waren rontom beelect met grobe bielen/ als MBildemand! Gaer cieraet was een dzantien oun Den jalg / met cen toot Slatienhupfien / daer aen ghebonden een Duuue / die $3 \mu$ ong wilden uercoo= pen : Commighe yadoen perlemoes Ders-fchulpen in plaets ban $\mathfrak{M a} a=$ dalien : faqfen oock ecn peecle= moeders Alrm-timguen on Det Alrm bebluen/ bouen Den Elleboge/ alyelifick de Tumoonders dam Capo

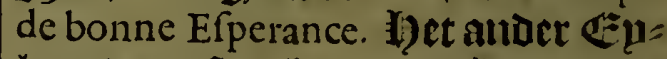
lant was feer betwoont fouls aen Gups/ t'was ouer al faut ftrant/te= gljeng de 25ottchagien/tegens den abont faglyen wo beel vieckeng/ denctiende dat een poer liin Colt coocktede. Defe $\mathbf{T}$ niwbonders wa= ren fraepe 9 gannen van lijue / ro= buft / conder clecoinglye / daul alleen oe fthaemte / bet hape cierden $\mathbf{j}^{\mu}$ uertefyepdelick / a'een badoe bet aen Ulectiten opgelonden/ d' ander ynd = de hat cierlick ghefrifeet / de derde

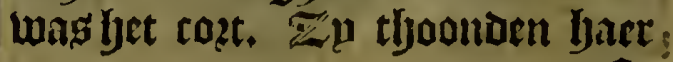
conte 


\section{Docr racoble vinive. Jie!. 39}

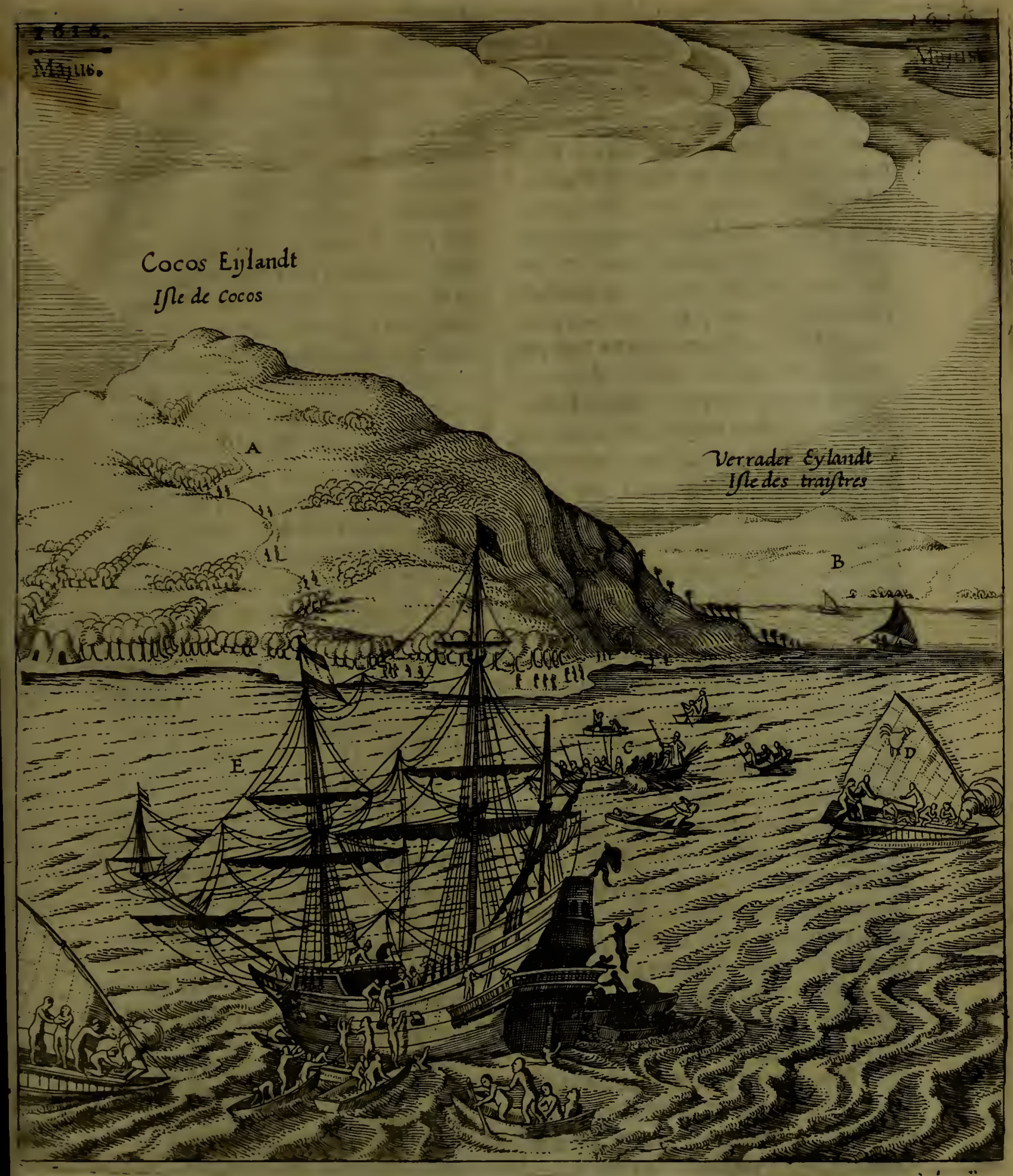

Nuritero 3. 
Fol.40.

16 I 6. conlte utt twenumen. Dit Cocos:

Majus. Enlant Icut op oe ljoocljte van rel= thien graden thien gyinuten.

Den I 2. fularghens walt dijfich wrder / Imet Lieglyen-vlagben / Coo lyaelt oe reglien glyecefteert was/ quamender uuf en Dertuch Canoes aen bookt/ al met Cocos, foo dat hup ban daglye wel feuen lyondert viif= tirlj Cocos-Noten rutulacn / en met Dic van gifterm wel thien lyoniert IItet ved Bananas, en Oubas-Wortelen: Dan Daglye maecliten wu cen merelst met ljaet / ban bijf Cocos. Noten vooz ech enckel midoel- $\Re a=$ gryel / ture enckele fnoctkens met. Cozalen uooz uiff Cocos: oock itel= De wu in dat ju ong ecrlt ljet goet fouden leveren / Dan betaelden wu haer/ oock dat men int epnde vande Galerije foube ruulen/ en nout bo: uen: Dat inen ooclt foo wel bu nacht als bu darse moft toefien/ foo dat nu oe Coop-lyandel ljeel mackielicli toe gincli/ als ju ledicl waren/en t'ba= re ljadoen / fpgonglyen ju ban bo= ven neder in $\mathbb{Z} e r$ / als wilde $\mathfrak{A g e n}=$ fthen/ en lwammen naer ljaer $\mathbb{C}_{\mathfrak{a}}=$ noes: als $j$ aen quamen boocken 3u onder ie Canoen Dooz/ om aent Srlyip te comen. Zu weicn ong Dat ie Ouerite of Coninck van't Zant aen boozt londe comen / die ong vereerde een Berthen / een )aen/cen linuclien/en diet bzoch= ten begliecrden glieen recompenfe. Gen ure Dacr naer faglyen wo ech Seul af comen / met ech Canoe/ Daer den : ocl in was/ net wel viif cn dorticly Canoen bergefelfcljapt/ cil fat int uluden / met cen groote fiine $99 a t t e /$ als en cente ouer fiil ()ooft. Alls ljp ontrent ons Srluip quam / dat feer folemuelicli gibeicliede/ riep lyu dacemael met groot gelupt/ mater de bierde repure/ riepen ył allegader yem nac. $\mathbb{E}^{z} \mathfrak{}$ Ije betures ons groote reberentic met liju banion booz't looft te naen / ent tf men in de Recls leg = ghende: fout ena aen boost/ die een
Overlte feheen te belen / met een I 6 I 6

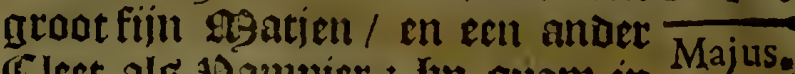
Clect als poampier : Ijp quam in oufe Galerii met fijn Gadlen / Die feer cozpulent en liat maren: top

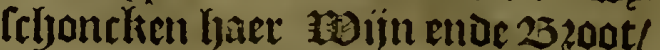
mace ten finaeckte lyaer niet: wo lieten ar Duerte fien een Dainte uan fiin Extel. Gtacf 23 antity/ met buetende of lyn't uerftant : oock ect Cam ens Spicghel daer fyu goet beljaglyen in hadod/ maer onle Prefident gaf bem die niet: maer als lyn ijem fiju peetent ghegeven land= de / gaf hu hem weder cen baem Tiinuaets / een 23ijl: twee 23of= iens Cozalen / Dat een ban allen aenbattede/ en [pzonglyen daer ule= ac over boazt/en bzacht lyet aen $\mathbb{C} a=$ ninck/die alleen een 9 atten Clect= ict am't liff hadoe/ ontfinclit in' danck/legghende lyet in lijn neck: thooft buuglyende/ oock op' tyooft ban biif oft les Codlieden: wri

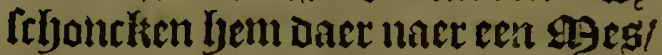

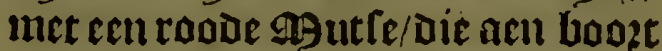
mag die oock commandererde d'an= Dere te vertrerken / alfoo ju te urel waren/fegtễ ar Fanou, en wech wij= fende utet de handen/ foo dede hu int aencomen de 10zaulwen achter af legaen: Gauen ong te uertarn Dat haet Coninck ban bet ginfte Evlant was / en dit liin Sone/ ju lepden ons beel dingljen/maer con= aen niet verltaen. Dit veritonden wa dat wo ons ztncker lomide lich = ten / ch comen bp faner / dace was alderlyande ververfeljinglye bait Derckeng/l)enแê/ / loęuen/firuut: wu thoonaen dat wu eret lyier mo= ften water lyalen/ Dan Couden wi comen/alloo bertrocken jil uict luwe roepen/ choe wap met crammelen/ en Crampetten / de 1) 2 ede-1Dach

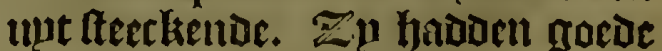
linne in de Prefidents tiem die ge= bozoutert was / en oock in al lyet ges tue dat Hefer ljadoc. Bace was cen

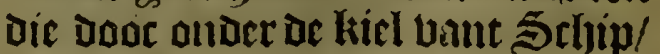
om ae Spijckers unt te balen/maer 


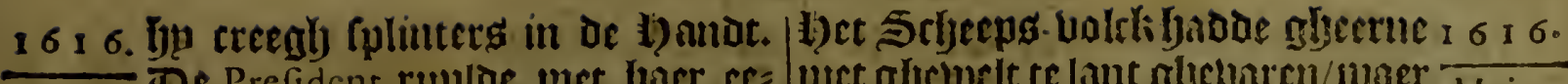
Majus. De Prefident rmulde met haer $\mathfrak{c e}=$ ofye Dichoecken / Dan ljet Difely= tuuely/ twilaen j! noode uercoapen. t'was [ecr ecrbicoicly volels/ vielen op yaer limuen en culteden onte poeten / feer uerwondert olver ons Scljip. En lyndon ac 23clleliens ban o' O ozc ougerulitit/ en loo lang getracken / Dat jutrefier op oe feljou= Deren lymgen/yadoen oock al blatl= we grautue placlicn op't lijf / glye= liicli ban 23 offectunt gebzant. Gacr gauz was beritijęiden / bouen de mont lyousen of kneucls af ghe frbjoen/ oe 23act onder de limine/ die ooclicacl wass.

Den 1 3. fmozgijens booz Son lagliender al twee Canoen aen boozt/ die d'oude boet lyielden in De Coopmantefjap / maer daer maer quainender uel bijf en bectich met Cocos, Bananes, Berckeng/ Hocn= Deren: Een Bercken voo enti viifDupm/ ruploen in twee uren ueel reven yondert Cocos, an andere Darch. Daer naer fagljen wu $\mathrm{ee}=$ nige Camoen ban acheeren comen/ en ons Dochte Dat de Coninclidien 3u Latou feeten / daer ill lwas: $: \mathfrak{n}$ quamen al om ons 5chip/ daer wu acjterdocht in creglyen: het pain= ripaefte 5 epl was ghelchildert met en figuere wan een Haen/ wan co= Ieur ros en grau / dat volchoen jp allegader / en Dat voer dooz de vloot/ als ozoze ttellende : als ju nuin farbozoze waren/. Coude wu ons Ancker licheren / glyeliche fyeb= bende / reulden / Doen quan ons aen boozt legeen een ban yaer $\mathbb{C} a=$ noen ; llet groot glyclupt/of ju ons wildê Uerbaren/en wierpen fel met Stcenen: en wu feljotē twee of dzie frboten/ foo dat eenige op oe plaets bleben leggljen / andere fwommen Deur/ met de Dzouben die bu haer waren. Mu befloten waftelick dat zu alle lyaer maclit bu een yadoen glyebaacht / mits batter wel thien gondert 9 gentcljen bp een waren. Majus. twerde bu de Prefidét chac ae kinet afgitiedaghen.

Oe Ouerfie vic Dacelys te uoosen acn't Schip quan/dic pecfentectde Dat Prefident cen vaturljt oft gewas Daer cen BDoztel aen Wns/ Ditu ly mict wiloe armenchen/en gaffe we= Der: Maet Daeclys Daef naev banclyt bu bet wederonv/ chae gaft ųe fint Canoa ace fijn Wolefi/ bie lyem dien bzochten/en betijoondent ontfe goet was oull te cten / felfs dic bittende en funglientar / maet fpogbent Le= ocr une / waer uut Iju fufpitie nam dat 3 bem wilden of bergheduen / of Lan fimen berooben: alloo men fulcke Dzartyten clocrs bint. Het Eplant dat cen asijl ban ons lacbl gauen wu den naem ban Verraders Eylandt, om Dat den meeften yoop uan't Zanot van die plactle was gljecounen.

Den I 4. Was lyet Duplter weder: alft op-claeroc fanten wo ten $\mathrm{WB} e=$ Iten cen ander Eplant/ reben $\mathfrak{g S i j =}$ len uan ong: Docly niet foo boocly als Cocos- Eulanot / lach oertich Bgijlen uan bet ander: L)et f(y)en twee agijlen lanck te welen 马upt en \$2oozt / op de yoochte uan Cocos-Ezlande feltyien graden ; triif/ als wo onder de wal quamen/zijn terftont Canotiens naer ons gye = comen / wel 27. foo dat wp qualick conden looden / waeren oock diefs actycicy/en wilden de a iin grijpen/

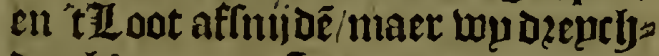
Den ljaer met \$teenen te werpen unt or Gaeloeriie/trocken aen de 25outen. Onfe Sloep ginck naer lant olu te diepen/Dien meenden $3 \mu$ t'ouerballen / maer als d'onfe twee fefoten glyedaen yadoen / belden jul der thee / die inde gront ftortes oen/cn quamen nict weder op:Doen bluchteden $3 p$ alle aen lant / en ries pen bou, bou, bou : gljeen kennifs le beblende ban't Icljieten. Jaer quam een Canoe uan't lant die nae if De Doo? 
fiol. 42 ? Spieghel der Auftralifche Ravigazie,

I 6 × 6. DE Dodden Conde fien. Majus. vont geell gront/ Dan met dé neers op of wal te legglyen / wernde weeck 召teen-uront / ban twinticly tot Dertich / veerticly / vijfticly $1 B a=$ Dem. Eap lagljen veel Cocos-Boo. men, en veel butten : taghen lyet bolck lanct bet $Z_{\text {and }}$ loopell / jae bouen op bet glyeberchte: De aerbe luis fwert aen te fien / joozoen de Doghelen uan't landt fingljen feer melodientelick. Tit was een bolek uan fatfoen / met bet voozgaenor: su en wilten niet ban berckeng oft i) oenderen / als be Prefiderit lyaer bie noentie in jaer clale Wacka en omo : foo dat lup fier niet crendjen ban die vier vlieghende Biftenen die wu eerit met haer vertuploen booz enighe bondellieng Cozalen/ boen wu nocly bitenden waren / en cunden oeck wel isienden abeble = uen beblien / hadoen 30 het op ter ract niet aenghelept: Bnfe Prefident hitcar gheerue alroo bu ong groot ghebzeck vam water was/ met bet 3 chip aen oen obuer gbe= legen/ ende niet grobestucken een wepnich athefpeelt / om een clace bane te maeclien / en oun de Sloep te mannen met 99 uf quetten/en too met ghe weit water te halen / maer jes twerde oberftemt. Git Eplandt noembe onfe Prefident de goede bo= pe / om Dat wo allegader gbeboopt jaboen daer ververfelyinge te crij= glyen/ oie wu wel gyebzeck badoen.

Den is. fmidoaechs gadoen de jooclite ban felthien graden twaelf Sginuten / De wint banden Supt. Dolten/onte cours belt : Daer wa = ten entigye die' tael gljewilt had =

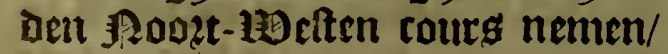
maer onle Prefident en bont niet gigeraden.

Den $x$. fmozgens waft leer lief= felicli weore: yadoen Imidonectys de boothe van i6. graden rupm. Defe dach Luerdenoer by oe Prefident ozore gijeltelt op't water bat feer minderde/ en dat een noer fou=

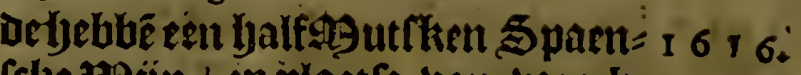
fclje 1 Bijn / in plaetfe ban bzoecij- Majus. zillicozet aeri cen Hecrlemocoer Hanglyel: werde beden befloten bu de Prefident ende de liarot toef́t Roozt-Hoelt aen te gaen.

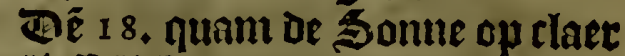
en lieffelicl: : yadoen oen alyebelen Dach tamelicke coelte van oen toes

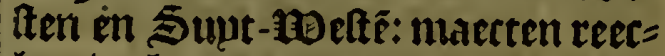
keniuglye nute belen dupfent bije londert viiftity mijlen van oe Cult van Peru : up badoen glyeen dijninglyen ult eeniglye quartieren/ oat ong Dede deneken ontrent lant te fijn / of ten minitten int Supoen ban ong: Uan't 12oozden yadoen oock gheen diininghen ghehat: De pilote comende in ie Galerije fepoe tegljen onfe Prefident, Dat

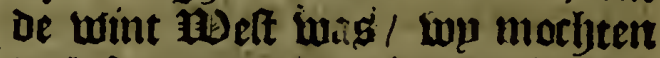
wel 积eczen aengaen : Eit gingben Supt-melt ten Supden aen: yet Leviel onfe Srhipper teer wel/ent wilde lyen perfuaderen t'lioer ter tont op te Doutuen en Poot aet te gaen/ Daer op yp fepde/ batment tot de midoarh toe coude laeten Doszltaen / hoctyte ghenomen heb = bende / fullen mairandeten wel fpreecken: yu hatde wel ghewilt Datmen al hoeft aenglyefpoozt yad = De/ om lyet goet gyeluoelen dat by hadoe / Dat wep noch lant aen Defe fijde van Nova Guinea rcuden bin= Den: Doch fyp werde met met batoe ghelaten/ ly molte refolberen : feg = gibende/Dat fyermeelloe dat wp bup= fent les bondert 9 ijlen fouden fept Ien eermen cours fonde verande: ren / oft Daer van lpzeecken / Dau bu fach wel twilde niet ballen: ftembe betlyalben alfoo of wint niet langle me eltick foudeblijuen/ghe tiick bp fabonts oock al weoex Supt waepio / dat wp dan JooztFelt fouben aengaen / recbt op'c epnt ban Nova Guinea aen! en foa fouben woet en ßroperoen bepoe aengaen/ en al met eenen wegtyel 


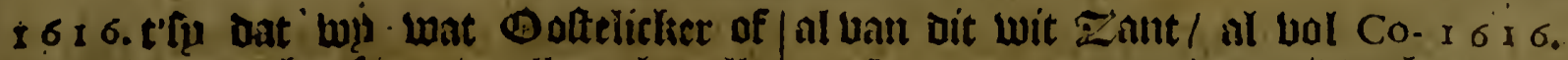
Majus. Weftictser fiun : in allen gyeballe

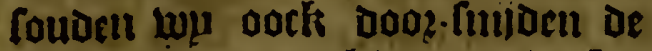
Eulantacn op ocrtlien graden be. Doozaen de 3aue/ en loo fouden twu Docn als wn feffuloicly waren. Daet op loo antwoozso de Bilote/ Dat wu Lel op't mioden ball Nova Guinea rouben uerballen / en loo eenige fyaturl plact te boosbu gacn: Dace toerde Doen in conformitept ban refolutie beflotel Angozorn aen te gaen tot thate en elf graden toe/ bet cerlte Ilant lyet befte oin Water. Gadoen fluaclits beel $r e=$ oljen / en bergadedoen ontent luif latten water vooz't Sthip.

Den 19. rmozalyeng was bet claer weber / mare ftille / nae noen coeloet unt den Supoen / cours ten \$oozden: Ijadoen de loochte ban triftbien graden fejatrs: te= glyens den abont fagben ive laint int Roozt-Ooft ten Bolten van ons / twee Eplanden / ontrent frg Seijlen ban ons / 'tfeljeen tame= fick yooch J anot/ Daer inné upp al te famen leer verblijt waeren: liepen berwaerts met fappe toels te $2002 t$ ooft aen : inachts yad =

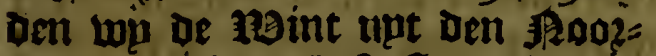
Den / gingyen al Dolt-wacrtgten naelten bu aen.

Den 20. Dito/ fmozgyengt faghein Jet Lanot / en waeren baij wat ghenadert / maet lach nocly luel Giif ozie झsiilen Rooztliick bin ons/ en laghen een grooten roock op't groottte Eplanot: en faghen Datter twec Eplanien waren / cẹn groot / en een Elepn: 'twas ben gautíthen darb fehoon beder/maer contrarie Wint / too Dat wu't Zlanat quaelick conden criighen/

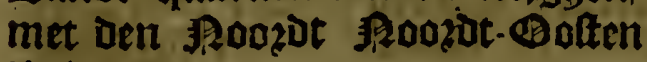
50int.

Ben 2x. waren wpit Ilant wel esn goet ftuck gbenabert / dan was ren wel twee 29 ijlen Daer noch af: faeglien bat ljet leet booct landt was/ en Golgheboomte/ be Strant cos-Boomen : ontrent den midparly Thajus. fagljen becl Canocs ban berfeben= oen plact ten/ uan benoc de $\mathbb{E}$ plan= Deri comen/ quamen acliter / en ten fioe van Jet Srlyip Lel vicr ch twinticl Canoes/in elck Dzic mnul cn machliten groot gljetiec/ t'welef cen teecken van bullecome is / bu ricpen Lucocrom fo lupDe als 3 y/ Crompettedoch / Dan feljecenden jui weder: 'twas meelt een glyedacute Uan Canaes; en volckials aen't ant= der lant ban goede hope / uan co= Icur / banz/ / pzopostic / tale / mater ons Doclit Dat ju wat gruliger wa= ren : ju bzachten niet te coop/ dan quamen alleen om bzienticlyap te marcken/gaben faer bat Coaaclts. iens / cil Spiickers / Datr ju lect blijoe mede waren / gaven bueder twee / dace clepne Bif́kens. Bit volcli was feer begeericlis an untne= ménoe diefactjtich / want clonimen bu defijoe op / quanten onder de fijoe van de Galerijel en namen De Prefidents ljemit dat upt bet wa: tex opglyebaelt welende / aen bet Coutien noch foo landye hing dat die bande Canoes dat mochten be repcken/ oe opper-Coopman ban't Fache witoe haer betweghen dat jp. bet lyemoe lowoen weder gljebeal. maer jú uepgerich jilinue wierpen hem inet lteenen nae bet $\$$ ooft/ ge: lijck gu in rebenge oock met cen Iterit nae lyact wictp/ waer op ue: niant van't feljip.bolel' fonder laft Dace toe te beuben/ eev grof Stucls gljeladen leggende op Den Canoes los ftboot / Daer wozoen cenigfent gljetaeckt / want die bau't Scbip. eenighe faeglien bloeden / enve $3 \mu$ met macht wech fcljeppende waier pen lyet bemoe in zere / loo tearly= ten is/ Dooz beele ban baren 0 veriten.

Bacr naer quamẽ wroer eenige Camoes ond bu/en wu lepden datte ons Cocos en alloere ffrupt uan't lant balen fotioen: En frijonelten

$$
\text { ff } 2 \text { ent }
$$


fiol. 44 . Spieghel der Auftralifche Ravigatic,

16 I 6. efl rift Colarlties aen haer (b) ver =

Najus. Ite / t'weleli wn niet weten of bp

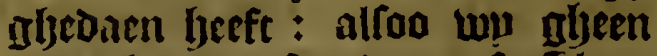
arout lyadoen/fetteoen onfe Sloep net de hooclj-23ootiman en opperStucrmam upt/met acijt $\mathfrak{\text { Salquet }}=$ tiets oul te diepen / maer londen gljecn gront oul te anckeren: als onfe Slocp uertrocli uan de wal/ wiloenfe de Sloep befetten / lieten De sitfagaien ouer 'tlyooft waepen/ cu quanten loo un dat $3 \mathrm{p}$ met knod= fen oug lolcli foitoen ghedaglyen bebluen: Doen gauen d'onte uper/ unet hun Steenltucken en 19 utkèt= ten / /rbotender dacie doot / en tre= glyen een Ganoa/ daer eeh Doot $\mathfrak{G}_{\mathfrak{i}}$ an unet fin been onder Den Doft valt was glyeftyoten / en lach met bet liff int water / dandere waren int water gbefpzonghen: Daer la= gljen ooclit twee Cinodren in / Doo? bet aenozinglen ban Den booch. 25 ootrman ijp beweectis belende met aduij: vanden onder-Stieŕ= man/Dno? oen noot บâ water/alloo wonnaer een wat water yaboen/ wiert diê nacht bet beflupt van Den Raedean Roopt op te gaen aen Zee/wearlyonden enaefijn voozitel abevolche/ van bu te houtoen dichte bp Ijet lant/ om op den oach met de Sloepe fin bet lant langs te baren/ ente abewapender yant water te roeckent.

Den 22.fmozglyens tregfyen wp een Aachbough/ Dat wp vęu boorh feploen/ met een reglyen-caeck / Dat wou reclit midoen van't eplant aen ftevenoen / tot onlen grooten ghe lutk. Deden De Sloep Diepen/bont so. vadem lant en frbulp-gront/ Direr over ban feer verblijt waren/ mits wo een tiebierken fagen ban eill groote Balleue booz of $2500=$ men Dooz comé Daett niet en barn = De/ en leer goet acilcomen was/ wp feploen woos die tiit de wal foo nael als goet was ; tot Dat we gront hadoen/ tot antrent bijf en beerticly Baden. Onfe Slocp die werde nu wat gemijt/ belonder alfoo daer y 616 . ecn \$19ulket log ginck anberboets/ Majus. die Iryzick maeckte. Ban dagbe beftonden wo te ruplen fes hondert of feven lyondert Cocos, water/ $\mathscr{C}_{\mathfrak{a}}=$ labaften/1Datattes/en anders meer: baer quamen aen boozt eeniglye die bzocliten een Eoztel Acona, op yaer l)ooft al bupglyende / en tup= pende in de $\$$ Recli legglyende/ een out man dede eertt een (D)atie/ daer D'andere int cjunde al op fetyzecudé, maer bu ontfinglyenfe niet / Doen wierpen ju dien in't water / wp fas gijen wel dat'e dien knauweden/ maer niet aten / daerom jp dien ritet weoer bzocjten : wp bedieden yaer datte Cocos, Bonanas, Bert= kens eilitoenders/louden bzengen/ Dat beoen 3 oock/ en upu gaven yace Cozaeltients/ en Spijckers. Zu bzochten be eerlte Berckeng gyeluzaden/maer galf ghenoech/ en feer grufich/ mitg ljet hooft bebloet was / badoen bet Jinghewant upt= ghetrocken/en gloequende beete lee= nen van binnen ingbefteecleen / en met groen Crupt gbebult. Defe Steenen waren daet in ghelept om uan binnen oock te bzaden. Map meenden datle dagelijex Derckengs molten eten / om dat wp daer beel aen lant laghen Joopen/ dorfi twij= felden of defe van den Duerten mocbten comen / al boe wel wu die betaeloen/net een gres/ een Itbeer= ken/ en enighe Cozacltieng. Ooch yet Itheen dat bet derde lebende Bercken quam als een lchentkagie uan den oberiten / welende een clepn tamelicts 3ercken / Dat met een Canoa alleen / met twee oude Mannen bail landt afglyetonden wetoe/ ban cen plaetle daer haer Belay, Dat is Deg Comincr titplacte was: Zultaeckent ban berre op/ en bonoent terltont aen een toutwe dat wu ban de Galerije af lieten/ foo wp alle lyet goet ban dage fad = ben ghedaen / ronder dat pemant binnen lefeeps-230opt quam: wp bandel= 
I 616 . bande lden oork leer vectich unet dit Majus. bolels uan daghe / en betrondeden ong ljun waren / nict poogljende wat te ftelen. Daer quain cen par = tic 10zoulwen af / met een l)zantwe/ cen teeclien uan beientfeljap/be=

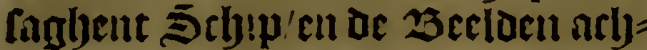
ter acn de saelocrie / met groote berwantocringe/ (taredten baer bin= therg ach het Ceer en \$ecli ban't Scljip/ els roockent / 'tbebucl fuut Lel: wai fpecloen op Crompetten cut bet Decltie / Dat bebaecljoe lyaer

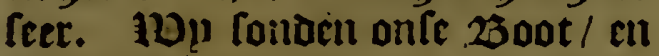
Ð) ten thoutglye Dattec echl (cljoone 23au was otll te legasben//eer Aecht en fil als in een laven/bu een tie = bicrken / Coo diclyt aen lanat / dat men uut het Scljip bet bolck [oude conmen beb?ij den oin water te Ijn= len/ en goede ZAnclier-gront. Wr Sctjipper \{epde/ dat lyu nu Terram Auftraliam badoe ghetuonden / foo wel alt ben beuiel/een tieluier ban uerfeb water/ ueel Berckens aen laut/ en anders/ Dat onle 9 gaets leer wel befjaecljoe. Be 5 chipper Itelde vooz bier vijf raeken te blii= ben legghen / en den Sloep op te tetten: Bap ruplden fes groote bilie= ghende Dilleljen/maer 3 hadoente feet lief/ en molten een Spijcker of rifken Cozalen booz een Bifty glyeuen. Creghen oock een Crocovade booz een groate 5 pijcker.

Detl 23. cotteden wu ons Seljip aen de wal in de boctyt / aen de wa= terplactle: t'volck van't lant fach= men met troupen / meett bp de Belay, Daer wel biif hondert $\mathfrak{a g e n}=$ frben vergaderden / om thaet te tjouden loo bet fcheen / met ttock= jens. De bzoulwen ente linderen bielen in plaukens en quaenten ons Schip befichtighen. Oe liw= Den ban ander Dozpliens quanten met Cackiens uan 230omen / Daer witte Baentieng ban af wacpion/ tot eell bzede-teecken/houdende die boozbaer lapoft: andere beden re=

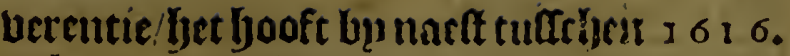
oe limen bunglyende / cregben ban Majus. daghe wel res ljondart Cocu. $t$. $\$$ a bouts quan ecu griis out maแ ban lant/bzocije bicr Comnterliangjon= abe Cocos conder bunt / die frfene= lende ban uneglen den overtel

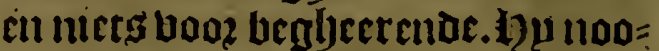
dicljae ons aen lant te comin/ maet whl bedicaen hem dant we? dat nict deden fonder Oftagiers. Die aen't

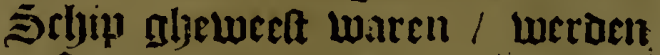
terftont ouncungelt/oun te diffourte

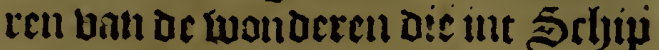
wareti.

Dell 24. (mozglyeng gutk Aris Clacfioon; Reynier Symonfoon, Den jonglye Caimut-wachter / alfoo in confoemutept uan't getsene un bum gilteren beaupaen aen lant: $3 \mu$ quanten met vier Bifagiers ooch af / wip dedenle inde Caiuut deine= lien en eten : lyet frbenen feer tref= feliclie perfoonen te 3ijn/ uan qua= liteut: hadoen twee Sonen ati boots / die incoe quamen/ oe feljoon= te Fonghelingtyen die nen uont/

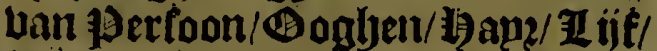
Leden / en loo goede contenantien Dat lyet wonder was. De onle gin= ghen aen lant/ met veel beulelin= gen tot pzelenten/als Cozarltienz/ bzant-Spieghels / een glafe liet= ting/ een roode 9 qute : 3u jaboen groote fin in lyet locut van Aris, dat foo wit was / foo dat hy cont ont cen 1 emt. De Prefident gaf een van fiin eughen bemben/cont dact bp twet $\$ 2$ ClTen / twee groote $25 \mathrm{el}=$ Ien : als A ris aen lant quam / wert In wel ontfaugben. De Coninck Dede jein groote reberentie/ en ne: dzicheut beuijende / lach bu rang. een balf ue met de banden tha= men / d'aenficht tegliens dofterde uuckende / en fittende op bare Indiaens, al perpler / blect coo langlye tijt líggen/Dat Aris Claeffoon oock die reberentie dede/ en molte fiem opzechten / Ijp cultede Aris Claerfoon Boeten / en Handen. 延

$$
f^{3}: \text { ander }
$$




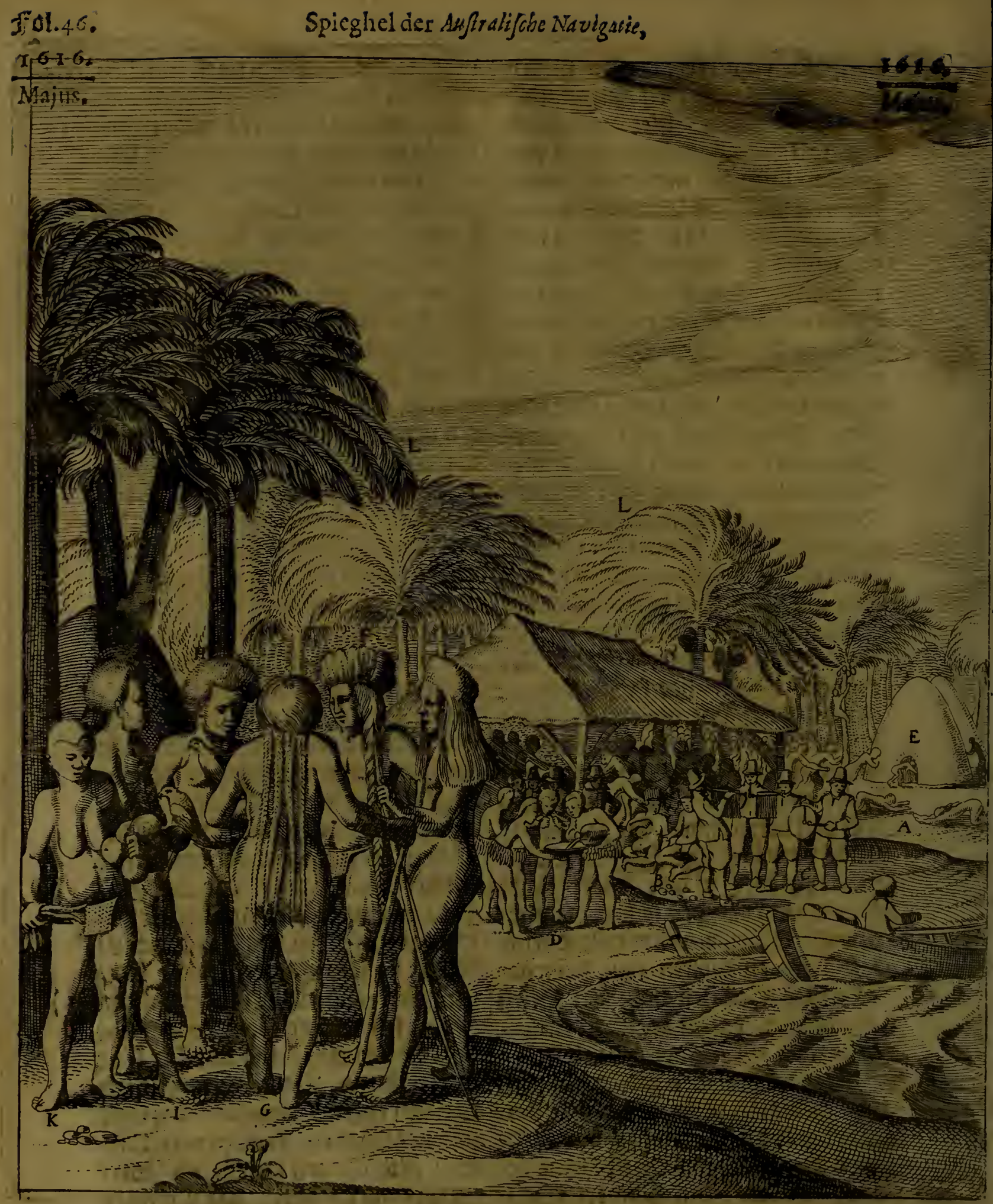

\section{Numero $4:$}

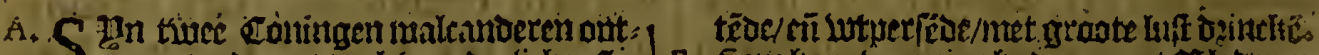

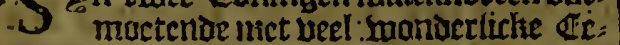
- ranonic ende jertuillecamen.

B. Bo felf octiuce Coninglsen fittente in byer pontificrisl op twee nesatjen: neffenti ulu: cintueren mutacr de Belay.

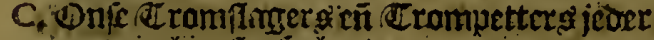
omt cierlijerite lpel clide tat groat conten: teirient bantoe Coninglicn.

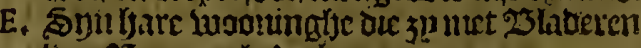
Dan 25 ad nued bedeclien.

F. Id Jen Goung/ende op luat manier yg glteciert io.

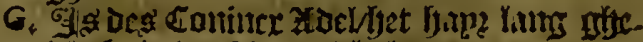
literbt in verfelyende blecljten.

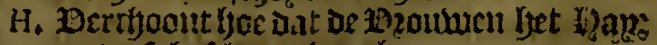
cost afuhticlyoren bustoch.

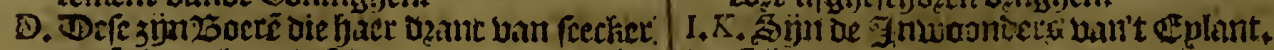

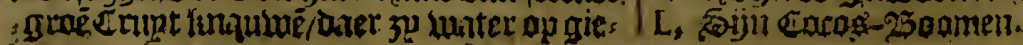


I6 16. ander pzincipale man dic bus beiu Majus. fat acoe bau gbeliielien/ dic buploc en creet als een litit//yndoe wonder te leggtien en te wiilen/ lju Iepue de Doeten Lall Aris in fiin Neck/ocde

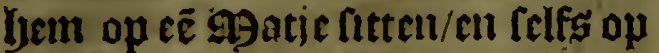
D'acrde: lien bernederende als een Toazm. l)p dede ljen de peefenten langljen/aie bem feer aemglyenaem twaren / frgglyentide Awoo, Awoo, Sont ach buott Drie oft bier ianglye Dereltetis. Doetr werde oock toe glyelaten water te lyaclen / too Dat biif 2000 teit verbult werden: als pemanat ong belecteoe/ Cont ziiń Dienaers: lju floecljecn tozoutue Dateq qualicli conde op ftren / foo furicus was Ijp. De Prefident fielt Dacr-en-tuffeljen of Ottagiers/ die gljecrne werb twilden / ntet lpelen op oe Cieter ende Diôlong / bock met danfen / febzeef yaer tale op. Daer-en-tultebet quamen beel $\mathbb{C}$ a noes aen boote / en ruploen alder = lyande toaet : nae ong giftinghe warenoer wel 40 . Too met 10 zott Loen ende kinderen gheladen / dat bet een plapfier was te fien: Daer clam een achter aen dectamer/ende nameen Sabel/ wp roepden bem nae/ntaer conden fem niet trijgen/ Defen Dogljel aen landt comerioel mert hen de Sabel ontnomeri / en booz ben Coninck aen boozt ahe = Conoen / en in de Caiunt gebsacht) Dat ons wel hebiel / $3 \mathrm{p}$ welen aen haer ljals / meenende foo oc Herico, Dat is de Coninck/ Den ztutbar geweten fnode/noch coude In bem 'ti'sooft afglyefmeden bebben. De Coninck lyadoe gheluoden oat mu eens fouden lebieten fonder fryade/ Dat glyefthiede / maer bp wag be= beecti / Dat by weely milde / maex Aris Claeffoon bielt bem / toonende bat In glyeen feljade foube hebben. 5 midoarelys fettede bu onfe ofta = giers aen lande / ende 3 de baete. Ban bagbe berde bet kantioen verbetert.

Den 25, waft een fchoone dach

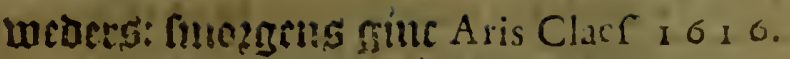
foon, Danicl le Maire dess Prefidents Maju. 23zorder/ en Clacs Laniz aen landr. De Prefident font aen de Latou tot een peefent cen Coperen 3 eerlien/ met en 93 as Cozaclen dic Lvit waren / cll wat 3act booz Kindig/ t'welek fien feet nenglyenacm wais. Ons Ieljuntgen ginck te Difrchety uingly cen clęme foods. Dic ban't lant quacuten met Cocos, maet D'onfe marente nti fat. Oaer qua= men ceniglje ftertite $\mathfrak{g a m}$ anen ong aen boozt / als Sergianten/ die groote autjoztejt ouer d'andere yadoen van 'gConinex weghen/ maer wu bielden lyact niet in ofta = gis/ coo dat bet nul al valte yecue was. Cen out פoan quan met. en leboon 25zactuercké ach boozt: en bereerde ons dat/met de ootmoe: dicifte rewerentie ter ilOerelt / Laj vercerden hem weder cen $9 \mathrm{ses} / \mathrm{ket}$ Spiicker/ en mat Cozalen / fü ginck daer mede doos bel gliecon= tentert. Atndere ban zldel tot Dzie/. of bier toe/ muamê [eet bzijmoedict aen boozt/niet taciken's daer bzedeBaentjes in hinghen in de hanoti en waten met groen il oof uan $\mathrm{Co}_{0}$ cos-Bladeren oum den ligals behan= gten : ju leppen lyaer zlengeficliten plat op't Sefjip nedor/ oe lyanden t'famen in de frboot vall de Prefident legghende/culteden fijne ten/een lantye wijle niet derluende op tien : lagen geeten d Dliphants Canden: Iju liet lyaer oe lozolo= gien/25ellen/Spienhels/psiftoolen fien/en gaf baex luat. Jaer be midoach gingen 3 b weder aen laut/ en bzochten Den Coninclieen pet $=$ rent: Een Cinné lepel/baer vooz de Coninck frlyonek/ een uet bers= ken : en cen Bogel op een tockien:!

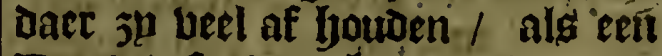
Dupue: Conden ben ueder een glaten tetting / een mas Cozalen/ en een baunerken.

Den 2 6.finozglieng íp onte Prefident, met Aris Claeffoon aen laut $\mathfrak{b} \mathfrak{b} \mathfrak{b} \mathfrak{a}=$ 
I 6 I 6. getuaren/medentmendacen/erom=

Majus. Wetter/ an een Wefent vooz Den Co= ninck / cen Cloot-\$pieglyel / wat Coralcm / wat Gout-Dzact en is $0=$ verties: aen lant comembe lacly de Conincli op de Sttant / op de ftee = neu/geel neder glyebogyen/De han= Den t'famen/Daenficht tegen d'ace=

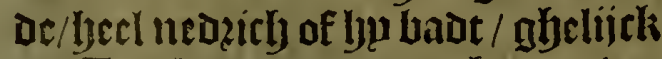
de Curclien : wu! deden yen relue= rentie mede/en Deden lyem opfacn/ gincli met ons onder de Belay, om Den reglyen / Dede een g-atte vooz fpecuen Daer fye ons dede op fitten: hip ginck relfs befijoen de 19 atte fitten / als of lyp't niet weredicly was. De Prefident Dede de Cront= petters blafen / Dactfe feer in uer= wondert waren : [egglyende A wo, awo. EEenighe waren bu naet uer = baert/ ou' tgeluut dat lyet maeclite. H) Dede ons een Canafter met $\mathfrak{e}=$ niglye Cocos-Noten bzenglyen / Die fettede cerlt in fijn lyals / en buy= ghende tot op der alerden/levpae die booz lijn looeten/om te eten/alloo by dede. Jaer naer quam de an= Der-Conincts bu ons / al Conder cpzectken/al met bet alenficlyt naer ons toe / eerit ten lijoen unt/ met De 1)anden in maltanoeren / vooz liin Doglyen / Dan latende banghen: daer naer liep achter unt / ecnighe boozoen ouerlunt / met groote au= thozitent/ lperecliễae/ lpzong hoory een fprong / en Liel willeng op fiin uaers neder/de boetê op \{ijn Snij= Ders onier bet lijf taende / ent dat op de Stecnen neder/Daer naer fpzack hueen ozatie/ met nroote ftatichept voo alle de Ounfitters/ niemant aenfiende / Dan 'tglyeficht naer D'aerde / of nae Den Hemel Maende/Dat glyedaen fijnde/beltont wat te eten / namentlick bail een Jumoen/ een grobe 13 zuclit Conder Imaeck/ met rimpels uan bunten

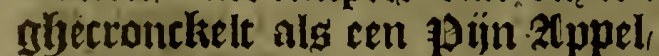
cll elc F reeedy foo ecn op een blinde= ken / elck Dooz Den Dienacr boosggedicnt fijnde! daner op dzantken
3! Uan Den Dzailcli der WOaztelen 1616 . Atona fyet Sap/ Dien ju ectit linau= $\frac{1616 .}{\text { Majus. }}$ wen/ en Dall op cen hoop Iegoben/ waftejen dan bner i)amaen/en gie= ten daser watter ou/dam nemtale ern yant vol Hacrer en clepndent daet Dooz/ Dacr ball ju elels wat gryeben/ in cen blad ban cen $2500 m /$ Dat 34 wetcn te beręuden / als cen peper= buus. Onfe Prefident feljoncli Det Coninck ach Cloot-Dpicglel fyent bedicdende dat liet of forme was

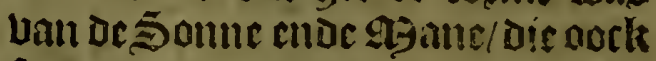
foo ront en blinckende waren: lyu werg ljaer dat ju dien aen de 23 alch Jan de Belay ronde bautghen/ als j" oock Deden. Onfe Sanets vingljen dien vach twee feltfame biffelyen Eeer dick ban liff/en dick van Cop/ fiinde glyeuleckt unan bouen op lyet Oel alseen $5 p e r w e r /$ met witte Doghen op't beupne Pel / Gadoen twee Blatugen of feer groote 1 in = nen/ roo langhe alg ecn lioetheBteert/ Daer tuffelyen cenfect Dun= ine Stect finck: was bu naelt bet fatfoen van een pleer-29ups.

Cer wiil de Prefident, en Aris Claeffoon bu de Coninglian faten/ Dede lyu baer alle cere ach : jae bet quan foo uerde tot de Prefident, en Aris Claeffoon oe Croonen uan or Coninglien op't daer werde onfe Prefident als ett Jongman die fulcke cere wel toe quan vooz fiju onuptrpzecticlicke moepte/ en rozglye / Die lyp int be= giil ende uptuoerẽ ban loo moenes licke reufe glyedaen badoe / glye= croont Conintk ban yet Eulant; $\mathfrak{e n}$ Aris Claeffoon onder-Coninck. Dele Croonen waren ghemaetkt ban witte langhe fmalle beders! dic bouen en ander aent epude ber = riert waren/ met clepule roode en grocne Bectiong ban Pcrequiten, en Dunuen / die bp lyarr in groot achtinghe ziin : mits alle deg $\mathbb{C a}=$ niner Tiaets-IL iceen / hebben ecn Bupf op en ftockjen fitterioe / bn baer / als ju ontent oen Coninck 
$1616.3 i j n:$ het Dupftien is bau bouen

Majus. wit tot oc Dleuglyels toe / unozts fwert/die 23unels is met roode Bee= renl glyefdit loert. Cer wiile 31 met de Coninglyen Doende waren/yael= oen wu al water'oun ous op te reple te uerfien.

Denl 27. vaeren ceniglye ban D'onfe Lueder aen lantot/ ntet de Crampetters aum de Conutek met blafen te bereeren / ende oun Dere liens / mede memende alderljande Coopunaufeljap/ maer conden nict rrijgljen te coop of te febenck: mits 3u weten dat jute telf gljebzecli badoen/ trocken lyaer 23 upck in/cn wiloen feggljen dat wou ljaer eten rouben upt lyet $\Xi$ thip bzenglyen. Ontrent den abont vinglyen D'onfe en loode / Daer af ju de Coniucl ceniglje uereerue / diefe al teritont rau op at' met poft cil Stecrt/ cil 3ingletwant / elt was lycm feer acnglyenaem. Ong bolck Danlten/ fonglien/ en fpeelaen unet De $\mathbf{T n}=$ woondersilldelt פ̧ane/3u [pecloen op yaer Crommel / en Daniten op baer unaniere / en D'ante Danlfeden op onfe maniere een ronde Dang/ Daer ju feer omi loeglyen. A ris Claeffoon ende Claes Ianfioon Ílyerm= Den teglyeng malcanderen met lyet

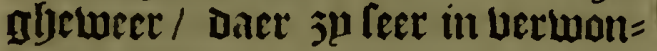
Dert waren.

Den 28, creglyen wu noch twee Beretictis uan de Coninck ende onder-Counitus / maer wlu malten Dien dierder betalen:De $\mathfrak{\text { Saets }}$ wa= ren walt beficly onit water te halen. 1)eoet quan de Latou bant ander Enlant lun Defen/ oul te befien wat wu uooz uolck waren: Defe febeen met groooter fupte / en autlyosi= tept/cn magnificentic te comế/mits Ijp ban Defe glyeweldicly gheeert wozde/ en twas maer ecn 5one unin D'ander Coniucli / foo dat Aris berbeert was/maer werde bant lyent gljeanimeert our te bliiben oul te eten. Rae noen giucli de Prefident relfs aen lant met liin piftool/ en cen Tioer/ en 't Bierfach/alfoo bet i 6 I 6 .

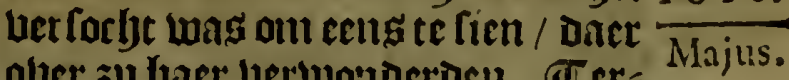
ober jul lyaer berwondoetden. Cer = font maeckteden 3!" gljerectldyap om te eten / Daev werde gljebzocljt fon groote meniclite bun Acona,

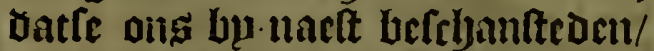
oocli gljebanden Oufij, en Cocos: lyet feljijut dit de manicte te weten Dat d'ene Comincli d'ander loo ontlyaclt : ju lizacelien al de Acona aen Itucken / en maeckteden een Drancli Daer af / bie $j^{\mu}$ al in't life Dzonclien : Daer naer vertrock de Conuck / Dare wa blijut om wa= ren/ om dat wu te bziicr waren.

Den 29. arbejpende d'ante tee= ducly int Later balen. De Prefident lict bent acl lant letten/ ont bet gljeberchte te fien/en de ander fijde Uam lyet lant/aen de $\mathbb{Z}$ ec-caut: bon= Den onder weglye des Coninclis 25 zocder alleen fittê/ of by ong ber= Lachtede/ en ginck met ons/lyadoe ons glyeene beneden gljeljouden/ maer waren baer niet mede abe= Dient / foo verre ong in't ILande te Leggeven / an Datle dicklwilg Boz= loggbe teglyeng malcanderen $\mathfrak{y e b}=$ ben: int opgaen quam de onderConinck ans gljevoldyt / Daer in wp [eer verblit Luaren dat $3^{p}$ ong conduifecrden / Dooz den reclyten wecly/ voozgaende/ ons wakrfelyou= wende : ginglyen uan D'eene 25 erch op d'ander/aen de Laterfpzongben onte Dzinclien sie zu witten : Daer naer lendếfe ans weder naer (ttant/ onder-weglye Dozit crijgljende/clam De onder-Conituck relfs/ met een Strick om liju boeten om laa= Itichept te gbeluen / clont op à Cocos-Boomen, met fulcken riffichept als een Cat: en plucktede de belte Cocos af / oie openende op een lon= Derlingye naniere: giuglyen fitten ruften in een feljoone plactle bau't 25 ufrly/ Daer ued gljebogljelte wasl en dzonckẽ Lande Cocos foo fmaec= liclick als opt tot twee toe: Die feere bol Laren: wu fagljen een ander. 


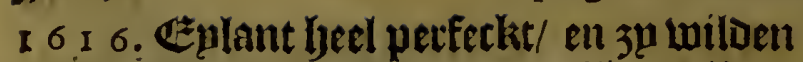

- Majus. Dat de Prefident net lijn joiftool Diner naer felyeten foude/ om dat 3 ! yacr fouden bzaveeren / mits ju - orloge lyadoen/en al-te-met ober = comen / en frbunlen lyaer in de bo $=$ Ien / Iteecliende facr geluoomte aen beant. Ba an't glyeberrijte comende/ quamen ons ine Dequmen te glye= moet / die ju vooz ons deden dan= fen. En alfoo yet uniodach was/ nanet wus de onder. Conints met ons aen boogt on te eten/aen Ca= fel fittende / fepuocı wu / dat wunu waters gyenoch badoen/macr dot wu noch thien Berckens/ Cocos, Vbes-Wortels, in wat gSaties ban Doen fyoden / als wa die badoen vercregen/Dat wu Dan binnen twee daglyen Conden uertrecken / bu Dat hoorende / areet oluer-lupdoe / ende Jep in ie Gaeloeriie/ tae-raepende oie gaede tijoinglye fijn volckien: mits su veeroen dat wu al hace fpiile fousen op eten / alloo ju felfs niet beel en hadoen : Defe onder Conincls wag leer nieug oierich/ belieect alles/onter en bouen/ende in De Mars climmende/fiende oock [eer naer bet 2 beelt-werck aen De Gaelderije.

区aacrentultehe comt lelfs $\mathrm{de} \mathbb{C} a=$ nindt net fiin atoel aet boost / me= de. bzengliende een Barclien/ ende een Canatter Cocos, dic hy pzefen= tertde/ legghende in Siin bals/ bue= licnde tot den ouetloop/Lettende het voo? de voeten uan de Prefident, en blecflos vooz yem legglyen of hy lem aen bast: Doen bp op ftont/ fettede bu de Coninck bu hem ne= Der op een Cuffen/ Daen dede de Coninc Dê Prefident, en Aris Claeffoon, wan liin \&loel tillen en bef= fen met groate Cermonie/ bouen baer hooft / en bieloente foo een wiile/ Doen lettedenle weder neder/ met cen treffelicke magnificentie/ alft de Coninck glyelioadt. (1)et fcljiint dat dit een teecken bu lyaer is van groote bzuntheyap en erre. De Prefident leude yem inde $\mathbb{C}_{a=1} 616$ : iupt/ en thoonde ben een Spiegel/ $\overline{\text { Majus. }}$ Daer lje niet in Doafte fien : fijoncli DeConincls fiin 23elle in lijn daep= plaetle / en twee $\mathfrak{\text { Iseften/ met noch }}$ andere beurelinglyen : ban daer werde bju ghelept inde Contbupg/ ell onder int כexip/ daer bu alle de Stucken oetticy in getale fach! dede daer voo oock liin reverentic: oock int rumu daer bu alle de va= ten met waters facty/ D'Ancliers/en 23allatt: Daer nacr in ConltapelsCanter / Daer j̆l alle ouer berwon= derden. Be Prefident leuze/ dat lys Daer uede Donder en 23 litem con= oe maeclietu/glyclijclidie inde porlyt ocmunert / liet bem do poompe cn Compas fien / Dat alles bem ect nientuiclyeut was te fien. Allles buel glyefien beblende/ ginck bu met onfe 500 / en oute Prefident naet lant/ met foo grooten vaefe / of fjg een Gout badde geweelt. 1)peñ de Conincli lwerde upt De boot nae de Belay glyedzanfen /, en onfe Gene= racl guncti met hem oaer onder fit= tế / tot dat wul wat aten en Dzonckế: Daer naer ginel bu met de onderConincli wandelen/om bet 25orch/ t'Lant an intwoonders woonin= glyen te befien / cul fach de 25 aeren

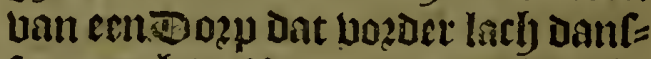

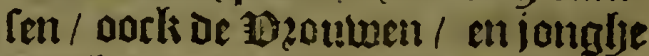
Dochters/Daer onfe Matrooren al te Loacu Lealicli andoer waren met Danfen en fpeelsar. Ceghen Den alont qunmente al!egader weder aen Loozt/ente Daniel le Maire inde manefelijn genck met een seet wan D'onfe Diftchen / met Aris Claeffoon, als j!" De focde glyeuanglyen hadoen gingtyen ju bu de Coninck/ die ju bonden gateaccompaigmeert van ecniglye jonglie Dorbters / die nacelit vooz lyeni dantteden/ op een [onderlingh Inttrument/ als een ponue/ ell op oat gelunt maeckten 5u yact fpzoutgen/ Dat een luft was on fien / bu foo een 23arbarifrbe Patie: opck D'onfe uergaten yaer 
\$.6 1 б. relben niet / om baer te vermaec= Majus. ken bu oen Coninck / die ntet baer in oe hant doeth / en lachtede / en was foo untmemende bzolick / oat wander was. Cer-wijle d'onte Danften en fpeelden bu den $\mathbb{C}_{0}=$ nincls/ badoen de 3 inwoonders leg oft Ceuch Diffefen glyenomen / Dat wp de Coninelit aen gaven / die leer onteltelt was/ en groote Jnquifitie Dede/ Maelloe twee uande Jnwoon= oers foo dapper / Dat bue bp naelt Doot-aoecly. $\mathbb{Z} p$ aten allegader de Bifcb heel rau op.

Den 30 . fmozgeng op oe bzoechCoft / quain een Canoe aen boozt/ ons Senecael beenghende een pze= fent ban twee 1 Berckeng/ en eenige Cocos, uan de Latou bereert/ wup gauenie wat Bifty te eten van onfe Cafel/oie jp met groote puer aten/ en leckeloaerden als of $\mathfrak{j} 25$ ancket aten / om dat $\mathfrak{z}^{p}$ dien rau eten / jae quallen: gavente elck een Spiic=

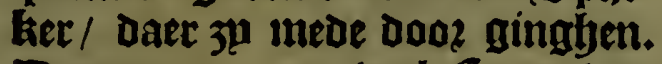
Daer naer quam beel ffrupt ban Den Coninck/ont dat by alde $250 e=$ ren gljecommanoeert badoe wat berfch op te bzenghen/tegbens ong bertreck. A ris Claeffoon ende $\mathrm{Da}$ niel le Maire, oe $23 z$ oeder uan onfe Eenerael/bzochten Imidoaechg oen onder-Coninck te galt/a la portion, dien wp treffelick tracteetden. Cort baer naer lagben wu Den Coninck uan bet ander Eplant / met al lijn Coelen / al met f́ljoone פgatiens/ wel ghecleet / en baer boulwen/ met extughe Canoes te water $/$ met $=$ be-bzengende bun etel ban ffrup= ten; bu detenC oninc comende/groe= tenoe maleanderen met dzollighe Ceremonien / laghen op d'Alerde met lyaer aengefichten / en banden/ op oe Steenen : dan gingljente leg= ghen teghen bet Schip/Dan teghen de keviere. Fen laetiten ginghen 3u be malcanderen fitten/ fingende baer Adova, en dit wis de groote Orankay, of oppertfe uan bepoe $\mathbb{C}^{z} \mathrm{p}$ landen. Onle Prefident, of Gene. rael, finck felfs aen lant oul dit 1616

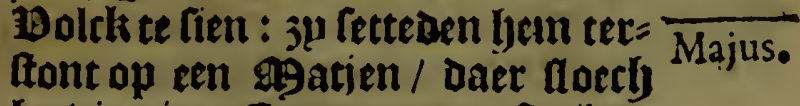
fnu vier int Crupt / en ontlack oe Zuont / en Claes lanffen feboot oe Biftool af/Daer $3 p$ over berwonder= Den : $\mathbb{Z}$ beglyeerben dat nen nae de Cocosichjeten louden/Dat werde ghedaen / Dat bet toater daer upt lirp. Die op't Stbip frboten een licbt Stuck af naer lanot op bet gheluerchte aen / Dat feer balderdel zp Larell foo ver baert / Dat $3 \mu$ be $=$ Gtonoen te bluefien: Daer naer $[a=$ gen lop dat $\mathfrak{\jmath} \mu$ Berckeng bzochten/ negen int getale/ ende werden ong ban be Arikis ghetrboncken / met baer oude reberentien / en lap de= Denle terltont aen boozt bzenglyen: wa gaven baer oock Cozalen / een 23ijle/ 2.Inetfen / Coper 25eckent en kingen : die $3 \mathrm{u}$ deelden aen biel die de Berckeng gelozactjt hadoen. Doen beltonden 3 te etelubzotbten eerte 19 ottelen ban Acona, en t'ges was : en fetteoent oe Coningen/ oe [edelen/en Bgoulwen vooz:oork een Deel gebzadẽ Berckens/met Crupt gljevult/[oo datter wel Spijfe wag vooz soo. Agentrbe die ontrent ong laten:wp cregben oock onfe poztie. Defpijfe weroe alloo beregt/ $3 \mathrm{p}$ bes Itonden allegader dit Crupt te caus wen/en alft clepn genoech was/na= men jp bet upt De Sganden/en berfameloent in een grooten bouten 23ack / verfamelt fiinde / goten ju Daer water op/mengoent en kneeoent onder een / en pzelenteerdēt oe Coningen om te dzincken/en oock ben xlael. Daer werden veel VbesWortelen gheglyeten : oock Ceatyien ghebzaden Bertlteng/ loo grupfich toegemaeckt/Dat prelick on fien is. Daer-en tuftenen vereerden haer onte Crompetters ende CronmeGagfer met bee lieffelicke gljelunt Gaerder $¥$ nftrumenten/ daer de negen yondert bergaderde 99 enlchen groote luft ín badoen.

Den 3r. Imozgheng waren . wy $\mathrm{B}_{2}$ boenoed 
. 6 1 6. Doende oum ong Znckers te redoen. / Fens die aen Strant ftonden/ glye $=1616$.

Majus. Dae De bzoech-Colt comen de ben= de Coningljen aen boozt/cn bzocly= teu les bereliens/tot een vereenin= obe / an De agaetg uerruplaen veel Oufy, Bananes, en Cocos, foo dat elck Maan feuen Cocos creech. De Latouw rieat den grooten Caniuck Dat lyp foube bet Schip befien / als 31 alle lyare Ceremonien en licden genblolueert hadoen / maer Iju doi= fte lyet niet beltaen : Doen nam onfe Prefident Den : Grooten Coninck tnu Dect ljant/Dat een feer frnp bequaem 99an-was / - feer Docil en bzitnde= lick / en lęloe hem inde Cajunt/ Dace lyu ben thoonde delozinte ban (iin Eercellentie/ fegghende/ oat dat onfe Ariki mas / heel in't Hapen: thoonde bem oock een इpiegliel/ en Dellen/ an De Caert / wers liem ons Lant/riinzant ten naelten bu/ en . Icheen Dat hy daer wan wat ap= preljendectoc $/$ bu frbanck clek een reljale Fbiing/ en Deocn berdyert: Ban Daer Ienoere inoe Gaelderiiel en foo boozts jooz't beele Scljip: baer werde weder bereert / elck een Loffen Cozalen/een Iges/é Caul/ en unat fpijckers/ mace elcke EDel: Inan een Spiicker. Daer was ecn van doaldel die Cuppers ziferē booz ghetaeckt badoe / ban onte $\mathbb{C o}=$ nunck van oe Bay, hp Dat fiende (Ioecly lyen in De Beck / wel Dap= per / foo dat Iju van't bouen Jet moft frljanuetr.

Cozts Daer naet gincle onfe $6 \mathfrak{e}=$ incracl met A ris Claeffoon aen lant/ on lyem nocly eens te waftelyen in De łiebiete/ Dat lju oock dede / Daer Hace gingljen $3 y$ nae de Coningen toe/ oill Gaer te groeten en zloieu te ceggen / 3u fpzenoen gratiens boos ong/ om op te fitten / en als $3 p$ ver = Itaen hadoen dat wu datelick t'fep!

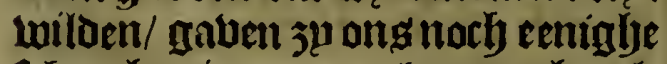
fefjenctagien/ en wu haer oock / al= coo ghelepoen ju ong aen Strant/ en frljenden ban bacx. Defe $\mathfrak{B g e n}=$ feljen leefoicn feer armulick/in lyupe bout ban 23 laderen ban $2500 m e n / \overline{M a j u s . ~}$ yadoen inden omring biff en twin= ticl poeten/en nau twaelf boeten ljooclj. De deure moeftnen al bucLende in-cruppen / yet louplraet was een beel bzooch Cruut/ om Daer op te rulten / hier en Daer in rell boeck tont eell bengel/ eloers een Cnobfe: Dat Huluraet fagluen wn in 'gConintkg zougie. $t$ ' $\mathbf{I g}$ cen fterck tolck / groot ban lijuel twelcliblecte Daer aen / als onfe Generacl ban het gelerelyte quam en mordé was / prefentecroe de onDer:Coninck bein op fiin inaeckte Srfjoutaeren te nemen / e ch coo af te Daggen/ Daer wute quact genoech Gadoen alleen dooz de quarde gangljen te gaen: Zul waren vati coleur Laun Ged/ hooleersich in baex 1) al! dat betcljepden was gberient / in't binoen / in friferen/ in't viechtan : Eeniglje: foljeenen 3osftelen op't looft te heluben//oo Itepliftontet. Het weou-bolck was leelick / met Lamghende 250 aten: lyet feljeen dat $j u$ ban d'eerbaerbent niet veel waetten yadoen / Cod gljemech was bet bu baer de $\mathbf{I p}$ miten ober te tresen. 23u baer was glicen Gcodts- vientt / lectDen als 25 eeften / hadoen gljeen kennilfe ban Cooplyandel. Het frhijnt Dat dit Dolck is val DeerAte Eecule/ om dat al hatr nootDatuft bincli aen De $2300 m e n$ en wies/ en de Dis die $3 p$ binghen aten sy rall. De Generacl noemde tefe Culanden ter exren van de Stadt Hoorn, Ot Hoornfche Eylanden, ell de Bay, De Ecndrachts Bay, ter cren van ong Schip.

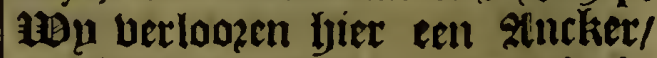
en ginglen $t$ 'teul/ met een oolt/ en Boif Roozt- Bolte caelte / anfe cours was Poozdon/ende \$RoozDen ten Melten. Onle plaetre Daer wu glyelegijen lyadoe/ lach op veertljien graden / Ies en vijeticly ginuten. 
fol. 44 1616. Iunius. o ons Supt Supt-Ooft:[nidDaechs de boochte van I 3 , graden veertich Aginaten.

Den 2. Imozgens quam de Son= ne claer op/ de Eint Lan Boftelic= ker hant: oute cours witg $\$$ Roozden ten pelten: Imidoaeclyg hadden de hoochte ban $12 \frac{1}{4}$ graden : wu $\mathfrak{c}=$ gljen noch glyeen lant :en de Gene = raels glyeuoelen was/dat D'Ezlant uan goede Hope, en de Hoornfche Eylanden, ar Ęulandenjijn/diemen Salomonis noemt ; altijt $3 \mu$ bebluen goede confozmitept met bet leggen Quiros: en t'ig reecker dat hier on= trent Auftralia Terra is : wp Taghen gheen gheuoghelt / dan een clepn ilant-פgecuken.

Den 3 , ginglyen al den outoen cours / met cenpariglye nint: $\mathfrak{l a}=$ ghen gheen lant/ onfe Stierman badoe giflinghe abemaeckt/dat wu al verce acliter Nova Guinea cou= oe welen : maer onfe Generael was altijt van contrarie opine: [mid= daechs hadoen be hoochte ban $12 \frac{1}{2}$. graden/ begomnen nu Albecoretten te lien.

Den relten/fmozglyens quamı de Sonne claer op/ faghen al noch gijeen Tant / onte rours was noct

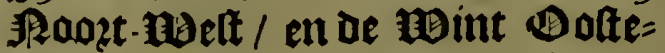
lick. Onle Prefident quan in dif = cours / en hadde groot achterdene= ken met de Srhipper en Stier= lieden ban veel pBeftelicker te Le = fen / en Nova Guinea al rupm op lijoe te hebuen / waer dooz wp met malcanderen réolbeerdé als bolcht Uan \$oosoen aen te gaen / om te fien waer wa waren / en foo lan= glye onfe cours te houden / dat ons Docht dat wp upt den dzoom waren : foo dat wp op de vzoech-Coft \$oozat aen ginglien : midoaechs Jadoen de joochte ban elf grae den.

Den 12. quam de Sonne on= Naer op: Defen abont werde refo= lutie ghenomen nuet de bolle thaet/ 1626 . en alle de Stierlieden/ yan onfe Iunius. courg Heft aen teltellen / om onfe reple te bozderen : Hadoen inid= Daechs de hoochee ban dzie graden les en veertich aginuten.

Den I s. Imozoens cours en wint als bozen / met f́boone coelte / en grof blau water/grobe dijningben uan den Suut-Dotten/Dat wu leet finglyerden/foo dat wu noch gheen bercljut hadoen ban de Supt-Doft Steert ban Nova Guinea, die wp beljoozoen te lyebben / als wp Daer be-neeften waren : Imidoaechs de Gooclyte ban $4 \frac{1}{i}$.graden.

Den I 8. De wint uan de Roozde= licker lyant / onle cours noeft : wn vinglyen een Boniet, fagen veel $190=$ glyelen / en Biftl : Imiddaechs we boochte van $4 \frac{3}{4}$.graden.

Den 20. quam de poiloot t'fabonts met groote blijottryap fegghen dat bu lant racy: eerlt Suptwaerts ban ong/ met groote 1)euuelen en $23 e r g e n$ : en lach al boben De s.gradeu : wn dzeben den gantfrlyen nache londer 5 epl/ om op'c lant niet te loopen.

Den 21. Imozgheng maeckten wp weder 5 eul / en faglen dat bet fecr leegly 21 aut was : daer naer fagljen noch neer Eulanden / groot en clepn tot les toe / reer leegly en clenn / met gljebaante. De Prefident giftede dat yet 1 geubelich lant dat wu gifteren faglyen/ ean beginrel was ban Nova Guinea : als wp nu beter toe faglyen / bevonden bat lyet al aen malcanderen valt wag/ booz een leeglye vlacke Strant / op eenige plaetfen wat yoochaclyticty rijnoe als leegle witte Duunen:lagen een lif afloopen ten \$oozoen

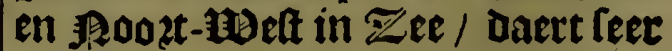
op barnde / een groot ftuck in ₹ee/ en lepldenaer be-\$oozden oni: wu faeghen ban't Eulanot naet ons toe-comen twee Canoes met Bolck/Daer in wu feer berblijt waren/bopende berkent te raecken/ 


\section{Door Incob le Maire.}

ficl.ss:

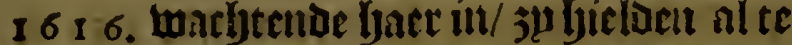
loef-wate ban ous/ an'tfelyeen dat zu beruaert waren / D'eertte gnode biet 9 San / en b'anbere fes/ waren ghewapent met 23 ogen en piilen: wu gavenle wat Cozacltiens / Daer $3 p$ (eer graecly naer wartn / en ga= bente liennen dat ju die yaer $\mathbf{O}=$ berftefouden bzenglyen aen Iant. Dit bolcli was al wat bzumnder en fwarter/ als aen 'tl)oozufthe Ẽu= lant: yet Hay? gelachtich/ Jaer lineucls waren oock afgliefneden: Onder hace Lageen out g9an met eeil geelen griifen 2 saert / Geer dick en uet / mede gljebrant en gljepte= queert oft gryelilaut was/ gljeliicli in oe vozighe Enlanden: 3u hadoen becl figueré op of Atrnien en 250 a $=$ aten / Jeckteden oocli baer frlyaente met 25laderen of $25 a f t e n /$ alieen Clections. De Canoes en t'rbep= pen als booz henen / maer gheen liicuen als D'andere on te vittrhế: - Oe Canoes oock niet foo poliit/het feljeen datfe Stocken hadden in plaet fe van tiieden : en yadoen een fpzaeclie met Die aen't Hoornfche

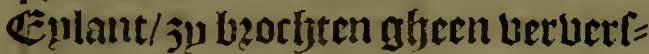
fchinge / al ift dat bet yant bol Cocos-Boomen ftant' en lienten Oufij, Licu, Fontij, Povacca: en feurdeu A jouta ne ay, Dat op bact laut geen en Lens/Dan wel beel aen bet ander lant / Dat ju ong weten welt aen te Iegurisen / Daer Den A riki unononde: wu bonden lyier ghern âncker. gront/fetteden oerlyaluê onfe routs (U)eft-waert. "'gפgidoarefys lyad= ben lun de yoorfyte luan vier graden 47. Asinten.

Den 22. De Mint continuertde/ Ooft en Ooft Bunt-Doit / met fchoone coelte / wu hadon imio= Daechy $4 \frac{3}{4}$. graden. Ontrent ien

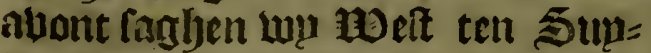
Den ban ons! ontrent $2 \frac{1}{2}$. श9iile/ een Iecgly-Culant/Daer wu bePoozoen olli hielen / en 'tqueloe ong dat oe IL anden foo leecly tua= relt : t'was van fyet voosgandie 32 .
Pijlen/ Doft en Welt atreliento/ I 6 I 6 . en dit Eulant ontrêt \$odat-120elt/ $\frac{1 \sigma r}{\text { Iunius. }}$

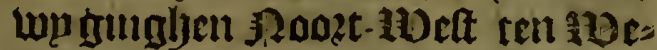

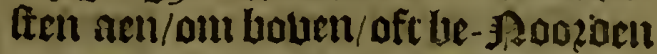
om 'teplant te faulew/ en te frhou= wê thee rlepue dzommelften\$2300= men / die girelijclie water ftonden/ rozghende Joo? Jzoorbte. Bit Eu= lant noentoe oufe Prefident Marcken, om de glyelijelietuife diet Daet mede yador.

Den 23, ninglyen wu ten ouden cours / en faglyen cenighe WaterSlanglyen / Dat ena tecelien is van lant gisemeenlict.

Den 24. fmidoarclys fracoen wn de froochte vant vier graden bijf ent thuinticly 9 gimuten. Coats nae de boochte gljenomen was/ fach onfe Crompetter lant. Oe Prefident ginck felfs nae hoven / chote facly hooch lant uoos unt: cn waré ture

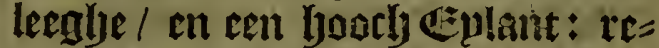
caluerden onfen cours te nemen Sont-10eft aen/ en faghen dat yet al boorfy lant was/tepl opgryelriclst en Clippicl) Daert feer terens barn= De. 23oben warenfe felyoon / en ef= fen int aenfien / feer plapfant met ueel gheloomte / en uptuentende urel Cocos-Boomen, intaer conden op een 99 iil aen lant geen Anckiterfrent ritighen. Onfe Prefident noemuefe de Groenc Eulanden: int boozt feulen deden fyaer noch clepne Eenlandeltenssop/ maer lietenfe al= foo oen abont aenquaul : bieloen Den gantfchen nacht bus/ met Suu= aclicke taut / tuffeljen luende $\mathbb{E p}=$ landen / Die biffthien פstílen van maltanderen lasthen.

Den 2 s. fmergy een looch Culant/ met ec Deel ben= belkens / hieloen Doer boben ten Stwoen out / en nacr toe : terttont daet naer datoeclite frem int wae= Iten ban oins / ljeel booch eftroon lant / dat wur gifter auont nictghe= fien en hibant / foo dat de Prefident refolbeerde oerfuatets te hou= oen : twilfetoen of lyet oe Crifte 
I 6 I 6. Lan Guinea foube ziin / Uerlatende Iunius. yet ander lyooch Equlant: dat van De Prefident genoemit werde lohannes (Eplant / en lacly op de yooclyte

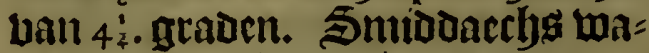
ren wu feer dirfyt bu de Culte / bat wu Ggentrben op de Strant / ende Guplen faglyen / maer conden geen atncker-gront crijgben : wp fagen beel rooct op't gljeluerclite/en 250r= frljagie/ oock Cocos-Boomen feer plapfant om tien : wu quamen by en boeck Daer wo boclyten alnc= ker-gront te welen/ en mandé onle Sloep gliewapent / oin te diepen: macr eer de Sloep af mas/ laglyen mu een Canoe afcomen nae de Sloepe/ die lefticly nae de 29 aets wierp ende fingljerde/ oock flit= fen gbebaupckte / maer loo baeft D'onte ễ feljoot gaf/ ginck die Doos. De Sloep wedergljecomen fijnde/ ginglyen onle cours / t'volck ban't lant liep en ricp ons naer / al gup= thende en wenckende met cacken ban 25oomen. Lier febeen een 25au te fiin / en cregljen gront dirfjt on = Der 'tlant / op vijfticls toadenv/ fant en cleun keefeliuglien / lettent op bijf en veertich toadem: Sawonts/ en bu de gGaen quamen twee $\mathbb{C}_{a}=$ nocs on't Srbip lyenglyelen/maer wo berftonoen jaer niet/ wu trom= melden / tromppetteden / en lpeel= oen op $25 e c k e n s$ daer $j !$ goet be= haghen in yadoen : maer waren feer bevzeeft : wp quamen loo uerde Dat wu baer Gozalen thoonden in

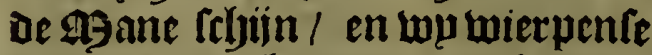
baer toe/ derbaluen ju lacijtcoen/ en badoen wonder te leggen onder malcanderen: men gaf tyaer oocl wat fpiickerg iaer $3 \Perp$ op baer ma= niex ons af danclteden.

Oen 26. quamen ong finozgeng bzoecly wel 7. 1Dzanlwen aen boozt/ en boeren balt rontom / maer bad= ben niet booz ons/ dan zllagapen/ Steenen/ Groden/ houte Sweer= oen / en Slingbers / nochtang ga = ben wu baer Cozalen / en al-Der= = Bercken/ Dat ap uerruplden : wa 1616 Dat zy ong Berckeng/ Cocos, Bana- Iunius. nes foudê halen: maer jp bettonden met Slingbers op ons Schip te werpen en pogben/ loo oat wu met een 25oeglyftuti daer onder fpeel= Den/ Doen vergonck baer de coura= gie / en fefyepteden allegader wech: donle fetteden ftracr de $2500 t$ unt/ en vervoleboeden gare Bpant / int water doot - daende / en tteckende/ al die $3 \mu$ conden crijgljen : loo dat ter wel thien bleben / en daie glyea vanglens creghen / die donte aen boozt bzacliten oin te rantloeneren vooz Bictualie / macr waren feer glyequetet : oock veroberoen 30 bier 1) zauluen / Daer ju eenige uan baer Wapenen in vontuen/ en verbzan= Defe. De gljequetften berbontmen. maer d'ecne terfftract.

De Prefident glyeboot na de mid= dach dat men de twee ghewangeng in en glyemande Sloep foude fet= ten / en met lyaer lancr de mbal ba= $\mathfrak{r e n} /$ on te tenteeren oft men ceni= ghe pzobific booz haer conoe beco= men boog't Hantfoen : men ber = nam eerlt slyeen bulck / de bzete wafter in : ten lactlten quamender dzie unt bet 25 ofely op een lingbelftrant / eu oufe gljevangens ricpen yacr toe: men roepde boost op een plaeté daer cen booch belt met glyefacpt graen ftont / lanck met Ztyzen/cn daer quantuiemant booz Den Dacly / Doe!: lieten we den ghe= quetten glyevaughen aen lant loo= pen/en nien gaf lyen nocb Cozaelt=

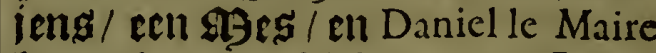
yadoe yen ecn ftịcli uan een Slaep laecken glyercljonclien / om yem te wiften / allog hu feet bloedede y en Dat opgoet glyeloof dat bu bercFens foude bzengen : nits wu dere twee op thien berckens gbeftelt yadoen: oic bn ons biecf wasten

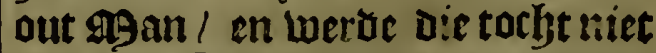
verloft. Sauonts quasuen ong bu twee Canoen / yebuende een cleun cregyen 
16 1 6. cregtjen oock cell txellent 2305 Iunius. Bananes.

Defe Patic hadoc acu bende (ii)= ocn acr Jeufeiv/gatcu gljeboott/in De welelie 3 ntiingen bnoden/vaoz= waer ecu feltfaem fpectaectkel.

Dell 27.alloo bet oetoepert uan olls water weder unt was $/$ - voldoen wn weacr anfe $2500 t e n$. Heden cres glien wop een bet Berclien om te Uerruplen/wu gaben dace booz ecn 23ecken / wat Cozacitieng / twee Spijckers.

Den 28. quam ons ecn groote 9) 2autwa aen boot/ Dace wel ecnen twinticly Egan in was / lijnoe acly= ter en booz heel boorly/cn fracp op= glichocnt / bit volck was ecrit wat felyou: Dace naer quamen nacrder/ uefaglyen oe 23 ecloen./ moe 1 eeu= wen ban de Gaelderije met bet= wonoeringfe. Dact nace quanı nocly cell Canoe acn boost / Dacr in een $\mathfrak{x}$ an yadoe een 230 s Pinange op fiin looft beenghenoe als een pzefent. at Ls onfe ghebanghen niet glyeloft werde / fettcaen wa bem aen lant : wy hoozoen daet naer op de Croumel fpelen / wo ber = moededen dat bet was dooz blijt= rchap.

Bere J, nwoonters gingen meet al nacckt / oeckten nu oc febaemte/ fijin niet of twepnich op't lichaem gijepicqueert / dan fadoen becl lit= tecckens / ootk warch $3 p$ niet ghe= bzant als oe voozgacnoe Eplan= Ders: ban midoelbare groote/ uct en bel gljeluoedet / beel azoegtien ecn Calabaltien Dacr Calck itigfe= flotell kass/Dactec Pinang met eten:

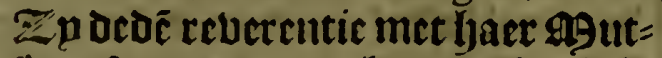
ien af te boen / cn bouen 'tljooft in De lyant te youden : Gace gletweer beftont in Atagaien / Slinghers/

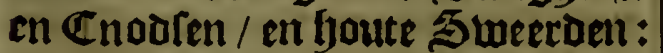
ju beten als gonoen : oe glyeuan= ghens beten in oe doften / jae alle be Coutwen aen ftucken : als won Conder gheweer waren / viclen $3 p$ ons aen't lijf te bijten: in fomma/ twas een quact vertidoicly Dolcli: 1636 .

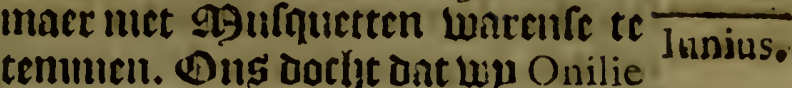

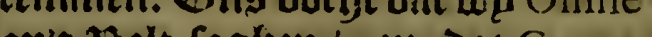
op't Belt fingljen / en dat Cocos-. Nooten yacr befte eten was: $10 \mu$ yoozocn poapegacuen rocpen / cth faglen gracne als in. $\mathrm{U}$ eft-Indien. 3n oe narfit lichteden wp ons alne= kier/ mict clęute coelte.

Den 29. Imozgheng waren wp neffens de Bookt-melter bouck vall oe Baye, beluente te nache miet ftilte maet ontrent $I \frac{1}{2}$. Mijle ver $=$ tiert, be wint uut oen Supden: Imidoaectys hadoen wu de boochte Uan $33^{3} \cdot$ grthoen / gacibe lancx de

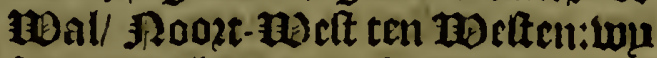
fagen dat lyet cen ualte Cufte was/ cli booz-freclier Nova Guinea: De Inwoonders ziin ghenaemt $\mathrm{Pa}$ poos, meett ruert / ontrent oen $a=$ vont faghen twa noch ozie hooglye Eplanden/ dic לan't groot Uplant laghen (es 99 ii Ien.

Den leften / funozgljents was dic EBoint bariabel: cn wọ waren ne vents de Cacp taer heni tlant ont= vicl oin de tWelt/ Daer naer $\$ 2002 t=$ lick febell een Baye te wefen. Hap rregben vecl: Canoen van't zant feer fraen opgheboept / en fcljoont= ieg met 25 eelt-werck uptsfiblous wen/en ghefclildert. $Z$ p bzorbten ons gant'ly gheen veruerfthinghe mede : beoen wel alle teectienen vall reberentie in die lyanden en grtutren op't hooft leggljende/oock yaer Spietfrn entigbe aen tue= len beethende / dat jp thoonden oat $\mathfrak{s}^{\mu}$ ons niet wiloen befdyadi= gljen/ contende feel beijmoedirh by ons inje Gaeloerije / cnde Cajupt. ₹u deckteden haer fchaemte met 25 ladeliens: wp lieten baer onfe Biftoolen affebicten : ju giughen feer manierlick wech d'een d'ander plaets maeckende : fonghen eeni= glje Ticoekeng ouer ag aeltije it tcecken uan Danckbaerbeudt/ Dat wo baer Cozacltieng gaven: foo overbzoclyten wuden dacly, feer pais 
fiol.5 8 .

1616 . fibelick / conber uet te telen. $\mathbb{Z} p$

lunius. toncken feer naer bet of fmeer / als Die aen Capo de bonne Efperance. Haer tale was anders als die gene Die aen't ualte lant / die oock baer upanden waren / $3 p$ badoen oock beel litteecleeng int liff $/$ beel oe = form int aenficlist/met canufe Rets $^{-}$ fen / groote Rippen / en Rgonden. Zu belteecken baer saerden met Calck / en baer hapz op't \$pooft/ waer mede $3 p$ [eer baer boovaerdije thoonden. Ban oit Bolck qumn niet / al ljoe wel wp baer bewelen dat wo wat ghebzect badoen.

Iulius.

Den eertten Julii/fmozgeng Dze= ben wp in Atilte / waren te narbt qualick twee giilen mett aen ber = tiert. Paer be bzoech. Colt quamen ueel Canoes af/ Die wp meenden Dat ous eenighe verberfethinghe back = ten / maer baboen wat anders in = De finne. Zू p quamé beel ltout aen! en baboen anders niet als 1 Bape nen / thoonenie ond die / of wap bat goet wel conoen : nu $3 p$ bingen aen en quetteden onfe 990 ples die opestncker ftont in lijn 2sillen/net een zulfegaie / noch een ander on= oer lijn armen: Daer op oock alle banoere begonnen aen te ballen/ met Slinglierg en Steenen: Doen waft onfe beurte / en loofden vier grobe Stuclien met een deel 9 gur= quetten/ loo datter een bofijn bleef. derftant berbolchoe baer onfe Slocp/ en treech een Canoe, dact een in boot lach / De andere twee bluebteden in't Hater / ban die welcke een werde ooot ghedlagen/ danoer werende een iongbelinck uan achtlien jaren/ namen zughe= banghen : en noemden bem grgop= feg/ nae de naem ban die glyene die ofequett was: : en oock het Eplant Daer onder befe erragedie wag ge= felyiet. De andere Canoen blucly= teden alte haers weechs. Smid=

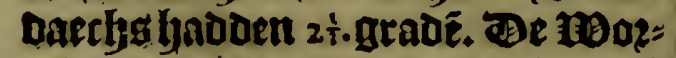
telen ban $2500 \mathrm{men}$ aten bit bolck in plactle ban $23200 t$.
Den 2 . Imozgliens in ip vooz nute I 6 I 6. weft van ons fagljen wy oock $\frac{}{\text { lalius. }}$ bet upterfte lant bial giftere about al uaft aen / finde tutachen bevom een beel frboone $23 a p e$ ofte inwile/ al leegh lant: too dat defe cult met bocljten uut en in loopt : Dat op De lijoe ban ong af lach / glyeleeck feet de Culte ban Guinea, wp bieloen

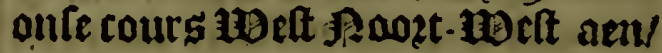
naer een Caep uan boodjactjtief lant/ Daer arliex fagljen weer leech Land allengfkens ontballmoe. Smidoaechg de boochte van $3 \div 0$ graden / wo liepen ontrent twee Agiilen bupten 'tlant / en fagljen een groote 3 erely.

Den 3. fmozgens met den daglye badoen wp ljet boonlye lant op oe zijde : en gingljen tiif boozt : en las gben ftract boozt gljeen lant wrotr. had men bet gljevoelen ban ten Prefident ghebouden en gljevoltyt/ men roude aen 25agboopt altoos 'tlant ghebolelyt hebtuen/[oo wel ln nacbt als bp oagbe pet int gyeficht bebbende. Bntrent den auont fa= ghen wp weder 10eft Supt-10eft ban ong boofb lant.

Den 4. [mozglyeng. Sat booth lant fagben wy vol glyeboomten groen ! en aock roock: Cer wijl weg alfoo doos de Eulanoen liepen! raghen wp aen Stuer-boozt bele. clepne Eplandekeng / op de lyooch: te ban twee graden / ocrticly $99 i=$

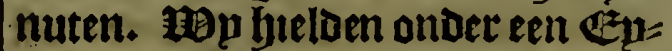
lant om't Xlneker te Luerpen / maer be nacht onderliep ons/ en ficlomt bu de gantedye nacht/Dat ons geen boozdefl dede / loo dat wo lyet lant niet aen ocoen / onfe Sclyipuer meende dat lyet Ceram was.

Den s.badoen wp becl kaecken ende Bonder/ en 23lixem: Baria= ble minde/ 5 mitodaeths de loorty= te ban Dzie gtaden / acht 9 sinuten: wo bebondé bier tamelicke froom om de roelt te gaell. Sauonts $\mathrm{ta}=$ I glyen wo weder ten roelten ban 
1636. ong in lii / twee leeglis Eulanøen! Iulius daerom feploen wu foo booch als uu canden/acnlocuende om bouen 'tlant te feulen / al इunt op tot de yoocljte baul banda, cn dull woeft aell.

Den 6. fondoen wy Den beelen Dacl) Uariabel weoer/ met regljen en loint. 5 midoaretys bevonden wu De boochte bau vier graden thiê

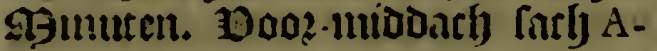
ris Clacfoon, lant / ecu $2 \mathfrak{c r c h}$ glye= liicls or Goudenapij in Banda : onfe ङfljipper affirmectue dat lyet Banda LuIs / Mtaer men beuont coets contrarij / mitg mel daer bu wel noch Daie oft bier fpitfe $23 \mathrm{erghen}$ racb die bealloen : ell derlyaluen Vulcani 25ergljen gljennemt wer=Den. SAen vont goet bet lant aen te doen/onerom oock de lyecle nacht Uus bieloell:

कell 7. Imlozglyeng/ waren wa etn goet ftucli wereljg vande Vulcaen 2 Bercly ( Daer wol naer toe feyls den / meenende daer boven te fen= len/tuftrben bet Culant en ljet ba= Ete lant dente/Docl] conden nict/ [epl oen beneoen out / en bieloen dicht bu. 'lant lantr / en quamen teritout in Zee/sijnoeture oft drie Canoes nace ong toe $/$ nuar witren banghe ban ons/ riepen ong toek maer cons? den bet uiet beritaen ?: noch oock onte obeunangene Moyfes, nae up thant comende quantn twacif $\mathrm{Ca}$

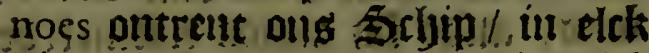

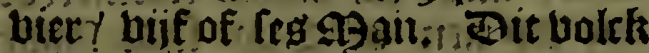
wag veel gecloer / alt boqzgaendes ooclt Jaer Ganoes waren bun an

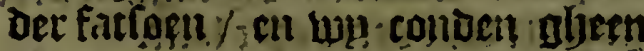
ancker gront eriiglien: an de $\mathrm{T} n=$ sljeletelien bzocliten ghecn berbere Ichinge/perbaluen refolucetoen top te tenlen, nace ect andere lecthe boeck vair lyet groot lant af ftef

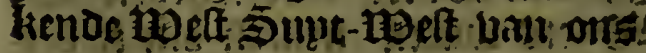
too verre wo fen contocn : alous Cenlende niet inop weder str rnumen biut faglica weaes twee of

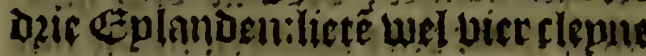

Enlanden/ footh aen be ainteker $=1616$. baut Dooet-waerts ban ons leg- Iulius. gljen/Dater unn olls een bocljte contimucliclis te rooclian : (midoaedys lacly oen Vulcacn ontent $x_{\mathrm{r}}$. Igilite baul ons oolt ten Poozdon. Wet water creecly bier berfchepoen ro= Icuren of wul bu een tieluier waren! gljecl/wit en groen/wp faghen bier Dejijuen beel bouts / $2300 \mathrm{men}$ met woztelen/ Caclien ban $2500 \mathrm{men}$. Defe Harljt dozften we niet feplen/ cu faglyen dzie Vulcanen, dzijuende olil oe Melt.

Ben 8. ginghen lwp Baettelick acn / en waren fitachtg glyedzeluen rectit bouen liet terglje lant / of wa gijefeult bavoen/ fagen weder voo? unt een hooch Eulant/en Noeft upt lach neer lant / reoclick boocly/al continuerenoe/ all gingben loo al voozt / Smidoarchg hadom wo de yoorljte van daie graden ache en uectich sinuten. \$abonts nae ben eten / banden we op tiebentich 1Baden gront / Daer quamen ong Çanoes aen loodet/ met twaelf man/ recbt acljter ac. Gaeloerite/ feer bes bzerf jiinde: wa gaven elck wat Craelticg/ maer hadoen gheen bers berftchinghe/ het fcheen oat $3 \mathrm{p}$ feet graecly waren naet linné Z aecken

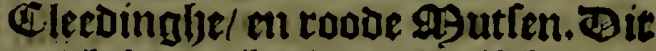
bolck feljeen beel 2Barbarifeb/ozoló lich uan fatroen: Moyres conde haer niet verftaen/ en was booz baer bebzed 1 Doutt bu faner nict aen lande gaen : beetende mogbelick dat jp bem Conder eten. : thapz was yaex beck twert; - Ifet waren rectste Paposos: reec nieurnierich als Alpen/omet scljip tabefien: 25e Uonden hier deljoochte ban 3 . gra= Det3 43. 93 inuten.

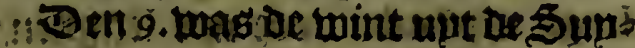
gelicker hant/ foo oat wo niet achs teke den hourh in to Baye bie wp connen fien/ colten comen. Won Conden oule Stoep om den boecti

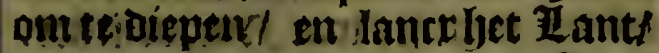
un buapmoe dien met twet fteen2 Stucktrm! 
fiol.60. Spieghel der Auftralifche Ruvigatie,

16 16.5tucken/ en thien $29 u\{q u e t t i e r s /$

Iulius. wont ouer al goedo gront : wut licly= ten ons atntlier/ en bonaen twee Dozpen/terttont quamen vecttbien Canócs uan lant / Daer ueel CocosBoomen ftonton: wul lietent atne= lice uallen op les en twumticy $0 a=$ Dem : tetltont quamenle met vosou= well chl tiuderen oun 't Sethip ront= fout te helien/: jae fluangere Do zou= wet / 'twats cetu teecken ban dzede: ecniglye tjaDoen attegnumen / flit= frn/ oldeen Slinglyers/ noch Stce $=$ nen / toch Cinoden / su bzocljten eniglje Cocos, die wo haer afrtwl= oen met moente booz Cozaeltiens. Snacljts badoen wu Donder/23li= rent en keghen.

Den fo. quamen weder ueel men= fcljen aen foost / nuace bzochten niet Dan beertich oft bijftich Cocos : Lwp fparecken ban Derckens/ die $3 u$ be loofoen te bzenghen/mact quamen nopt : Ijet waren recht-feljapé men= fcljen eters/ bebuende een leclické colcur / Ladoen felt lame cieraet om bun hats /: uan toozentieg/ ende Eet-Sctulpe/ an Derckeng-can= Den / Ilaer thevelg' waten afghe:

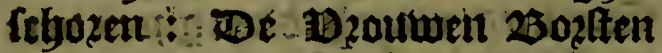
fivig ben tot oen Pabel toe! als ten Bonim: be 25 unck foo groot als een

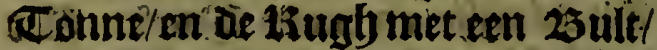
viner 3 p em 1 kint op retteden : mal

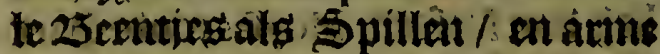
Itepranentidyen : enallon 3 ons niet en:bzorften/ reloluetroen'te biertenten:

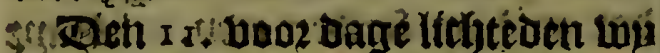

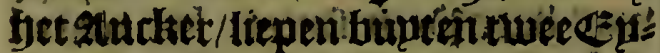

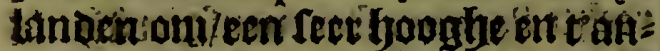

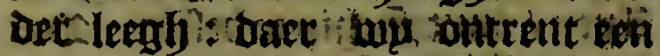

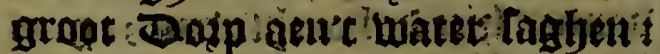
nae miodach fagben wu hover

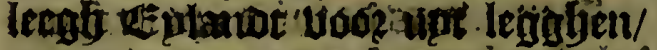

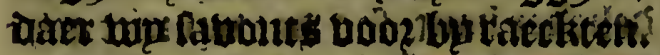

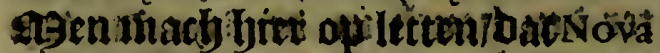

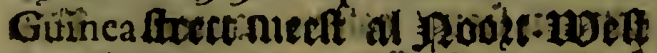

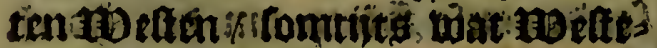

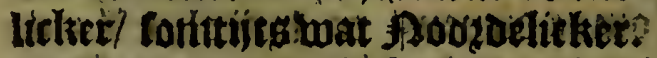

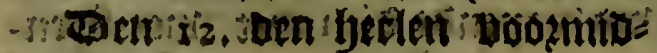
in:ilings: dacty hadoen wp al ftilte: bochten I 6 I 6. dat wp defe fpzingal beropoer we $=\frac{}{\text { lulius. }}$ Der foude obebadt fiebben / maer t'was al fryoon weder : foo dat wo al Coetics bu de Hal langs liepen

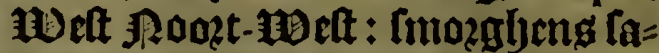
gljen wu lyet zanot wooz unt heel lanclt: fmidoacchg ae boochte ban Dzie graden : lagen veel youtg dzij= ven unt groote Revieren comendel Dace becl 9 goltels en Crabben aen biugen / gljeacconupaigueert met

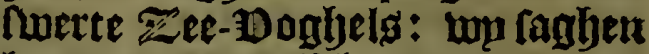
yeden ved Biâtyen van alders hande facly/ bebombē dat wu groot boozdeel ban de Stroom die ons om de melt lettede badoe / als defe. gantrdye Cult langs altoog is. 23 liremide leer alle nachten dooz de Daghelijextche ljitte.

Joen 13. Joch docht ong Dat bet een Doozgaende Culte was / met beel 25 ayen en bochten/en liabens: al-te-miet fooch ILant / romtijots leegh lant/ Inacljts badoen lop een Crabade.

Den ×4. Tequiton wpal lancx bet lant: ontrent biet of bif agijlen ban de Cult: Onfe Prefident had= be ghectte wat dicht aen ghewerf omilijn Caertien beter te maecken! maer onfe Schipper was feer ber= vacte boos "tlant. wu Leel Bifch/ en Jan ban Eens ten. Rubevont onfe Biloot wel

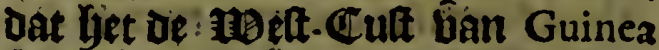
bad ll alroo by altoos fulfineerde bat we aen de Supt-fijoe foude verballen / fpzack derbalben bele oelpetate woozoen/ wo fagfien veel gtoente bañ verre op't lant. foen is. faghen wu leegbe fantStrant $/$ al bol gbebonte / tmido oated hadoent wo do boochte ban

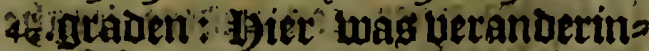
ghe bai water/ bleetk groen. Be gaets Laten graech om te bies pen/en wontion op uedrtich Badem gront 7 el 3 p faghen terfont twee oft dite Cãnoes, vie nae ong qua $\mathrm{men} / \mathrm{mact}$ baer bag cen in/Die nae ongt 
$1616.0 \mathrm{ng}$ wilder frjieten / foo feljoten wp lulius. certa met loos ( rüt / Daer mae un crult : op bet Seljip urecudente oocli dat ju onle Slocp overbiclen/ ell [chotch twee Gotelinglien af/ deene op Strant/ d'ander naer bet 230 fely / Darer 3!l met eraupen fton= Jen / en vlooden terftant weely: en alloo de becte un int Bolets wag/ werde gijetefolueert dat men op bet clentue Enllanst foude linden wel ghe wanent : foo dat or Brlyip= fer felfs mede boer / maet als or Tiln wontors laglyen dat ju! lan= ben uuloen / vocliten $j u$ loo furicus en munlick / dat jul met fjleten quettersen ueel ban o'onfe / jae wel fritlyen : donfe ftonden oack nict ttil. ch fuecloeber met Steen-ltue= lien chde $\mathfrak{R}$ surquetten onder dat l)et ltoof:als liet nu foo op een tam= pen ginch' wh is onle Selyipper leer berunctrt om aljequetlt te wozoen/ en croop onder oe Joft of 3 anicke baer ac lioeners op faten/al hoe wel lyu oozfaectie was dat donfe III ie Indianen eertt feboten / hacr bzaucrende met fijn Cootelag/aga troos die cadoicls is dit fiende/ noế De heni Daer naer Boft-trupper. De Prefident fiende ban't 5 elyip af oefe Bater-ftrij / enoe bat of Indianen ly:ftict) vortiteii/ (ctyoot fe=

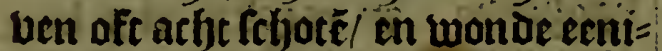
glye ban de Buation/ oerbaluen ju ltract oocti weeffethepten ae de Strant/ niet groot fuerticg.

Den 1 6. Imozgtiéng ljet anteker obeficht / en oicper titoe 3 ap obe= frult turterjen bepoe de eplanden! op 6/.toroin Cleu-ǵront/ we ber= nameri bien bact glueen Canoen, wain die thene bie ong baects te voosen bi waren glieweef. S9en refolbecroc oe sloej / ei boot upt te retten/alft oock glyerchiede/ gin= gljen toel gljenonteert been ! 30 Woecen âen eet clepn Enlant $/$ me of bienghende twee houdert tle titj Cocos-Notén. Ontrent Den avont quam een Indiaen als vesoe berfaecliende met fiin Canoc all $=1616$. trent ons / en wierp ons toe crn $\frac{}{\text { Iulius. }}$ f)oet dic dacefy te boozen verla= ren was int vectyten. Defe Zn woonoers 3 iju beel angyecleet.

Den 27 . Zijn wo wat beter in de 35 an ghecomen am ong Bolts te bebzijoen / die aen lande fouben gaen/ oft met lyaer jandelen. Baer werde gerefolvect ill dien $3 \mathrm{~g}$ uooz= midoach nict en quamen / dat men ocs nac midoneclys met ghetwelt de Cocos foutoen yalen. Bacr-en-tur= ftljen quam een ghemapent Sgan af / met een Canoe, en badoe eenis glje Cocos, die fy boben troam werpende/ bu ons liet dezijuen / ten laetften werde lju ftouter / quam onder de Gacloerije / en bont log of Cozacitiens die wp aent barn= bout balt gemaecke baoden. Doen quamen d'andare ooc bp/en bzocb: teit ons al dat wp wiloen / boos asellen / uerraelte Spijikers / en Cozaeltieng. Zp verruplden aen ons bare 25 ogtjen en pilen: $\mathbb{Z} \mu$ thoontoen oock groene Sember / en ceniǵbe geele HBozteltjeng / foo Dac ju treelmet ons wel te bzeden wa. ran.

Den 18. quamente fmozghens bzoectj al weoer by ong / wp babs Den den gantetyen dach glyenoech te doen om te bertuplen:loo dat wp creghen negen yonoert treftich Cocos : finiodaechs quam een 29 an met twee jo zoumen ons aen boot: en onfe 9 acts boeren om de foodel

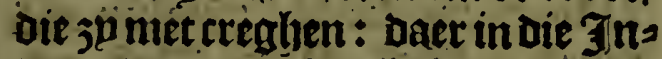
woonaers ons reer befulpicl wa. retr.

Den 19. Doog't berfoect van te geatroofen ou gaen te Bifteyen aen tgroote Ezplant/ gaf de Prefi. dentbertof/ en ont te fien of baer meer pirch was: t'melck ju oock deden met helieben der Inwoons deren/ die oen fegben hielpen trecs Iken. Hu faghen ban alle cantert bele 1) zautwen aenconten / oaerons wp onfe 9arets aen boozt riepenl 
fitot. $6=$

I 6 г 6. vermocoende quaet/mate ele quam Iulius. Coo veijmoediri\} aen boozt / dat wp ons verwonderden / mamglelende foo berl uu begerroen : $\mathbb{Z}$ ll noem=

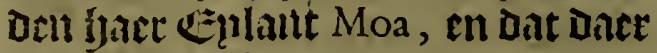
oluer lach / repuen sul wag Infou: omarr het fooglje Entlandt / nocul= Dell ou Artimoa, en waren loo bac $=$ Aticliomite lyandelen/Dat d'cen d'an= ber usidzongly/meelt roeckendeC $0=$ rillatl.

Dill 20 . (mo'sljens in De Daglye=

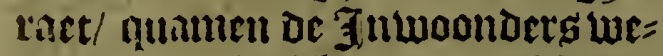
irer/ met twintith Canoen â boott: Darer onder oock groote [ell p) zau=

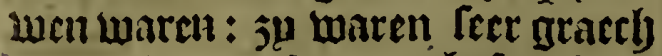
om rinlen / alfoo lup gibelcut lyad= ben Dat wul Luiloen van daglje werly [eplen/ ju becljten ang ued Cocos, foo dat cen uace wel viifticly poten rreech: pock foo uecl Bananas als wu begleerden : ju dzonglyen op De rijoe Uan't Schip malcauderen/ clef wilde de eerlte zijn/thoonende bacr toapenen/ljaer $5 p t i f e / a l$ om te ruwlen/ voos Spijiclers / cn ber= roeft goet. Dit volck was loo nieur= gieridy uiet on 5 eljepen te fien/ uerntocaen was oerbalben dat lyier wele Spaengiaerts glieweelt wa= xeny alloo wh oocli aen de Potteyfen merelsteden. Eit alloo de wint coet was / lichteden wo onszinc= Fir / als tup ban alles bertien ba= ien. Faer miodach feploen wn boozlou orie Eplanden die hooch warch/ waien Drie 9 g ijl groot/beel

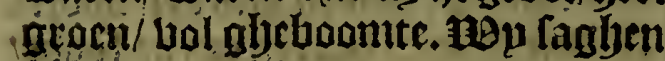
al boost upt lande / ginglen onfe comis al lBeft 12002t-110eft aen/ De bint Ooft tot Roozsen / varia = Wel/met goede coelte.

Den 2 r. finozglyens waft dunfter Weeser/: Dat wil lyet lant nauwelick tandeil fieli / too dat het met een bocht ong feljecti tontballen. Nae noen faglicn Ledoer lander / unacr al eplinour axeben dichtonder een clejn ejhanot en wierpen fmitfront t, en chelp op biffthien va=

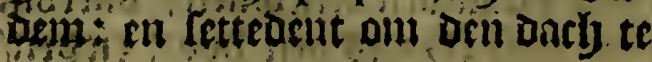
isy uerwachten / wu fagben al bieren 16 I 6 . op ofle plactlen ontrent onts: $\mathfrak{b}$ nd $=\frac{}{\text { Iulius. }}$ oen socli uecl reghen / 2 lixem/ an gljesonder.

Detl 22. ragen wp yeel perfect ot gront: Doen faghen wp dat yet i4. Eulanden waren / leggljende tas melicke lpatie uan een. 10p bads den Invidoaeclyg de joochte van eet graet befiupoen : Ong bolek bag upt onl Bifty / maer bingtyen niet oin de rudtighe Strant/ en hadoen beuonden de Eplandé enbewoont/ vol dicht 25ofrly: Itgghenoe arft en twinticly Mijl uan Moa. Họ refolvertaen bupten de Eplanden/ en be-\$oozaen om te loopen/ $\mathrm{en}$ dan de Cult te roecken / Die tyier

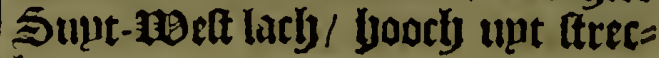
kende.

Den 23. Yichteden wp ong ancs Iter fonder frthade: wu feploen bo= uen de Eplanden / favonts laglen wu eell yoect: om daer bolen te cas men / dan t'bag feel clam/ en las glyen naer ons afcomen negen $\mathrm{C}_{2}$ noen, groot en cleyn/wat fooclj op oe Ternataenfche buife/de 3 zaumen redelick rupm/ datter een man in fitten conde: 3 quamen feer glyes wapent met fflit'en / waren eerft wat uerbaert / roepende Sano, oan wiinclende/dan de Cocos op't booft legghende / 3p roepden ong laux naer/met grooten puer / en wu des Den ogck teeckenen uan brede/ tot datte aen onle Salerije quamen! wu gavenle wat Cozaeltieng! $3 \mu$ itaccken terttont de yanden toe/ als grifpende naer een groote Sclyat/ oin dat $3 p$ die feer achten om den Gals te doen. 3 .1 cen ban defe Ca. noen was ect oberlte / die als fyet Jjem niet nae fijn fin ginck / ocbe alle de andere afteggljen / en be= gecrde datfenacr ong louden fflits fen / Datle niet begljeerden te Doen/ daer ober ljp oran wert / en floech Dapper fiin Slabe / die achter hema lat. Hp badoe een latting om fiin Gals/die onder fijn armbooz dincly טan 
I 6 I 6. van glyecolozeert balt / die acrdicly Iulius. glemsaeclit wis. Flis wu defe quilt waren/muall ball cell ander boecli cett anber foozte met langli glye $=$ crifpt bayz: gietende daet Hoater ober / tot een teechen ban bzede/ limglien Dooz of Reulgaten / die groen waren : cenugbe dooz ae $\mathrm{DO}=$ ren parlentoeder lingben om a zrumen : eenige baboen Cennenliinglyen doozt midoelfchot wan de Reus: Dele bzoclyten veel Cocos, twee hos Banannes, veel gljebzanden Bifty / Coeback / cen aach ban 3) zupmen nuet cmatte gladoe \$tec= nen/ oock Giseloench / die wp al boos Cozalen die jus Oaraija beeten/ en verbyande esgeiten cochten. Dit volck jadoe wejer een ander tale als oie van Moa, oock was bet een anoer Ratie/nici foo lwart/en lan= gher van lyap/ meer nae de Papoos treckende: en conom niet de Ooft. Indifche Cale/ norb Aru, Kay, Gilolo, Ternate, of Papoos : wu cre= ghen ban Gaer een Barmeetcop/ en era Schotel leer grof / met root en groen goferchilbert / 'twas 19o? = celepn/ oaer $3 p$ Teer licht affehepoe= on / booz twee riffens Cozalen/ foo dat onfe mepninghe was dat bier wel 5 paenfiche Schepen ghes fien waren/om dat $3 \mu$ loo meufgie = rich niet en waren nae ong Schip/ Jet bleeck oock aen geele Indiaen. fche Cozalm / als uan Amber, Die een bzoulwe hadoe/ die de Prefident verbanoelde ban hace / om cen rifken Cozalen. Bnachts bad oen wu ctilte / maer goede boozt= ganek Dooz den troom.

Den 24. Imidodecty helubende be booclyte van twintich ginuten/ feplaen we langs een feboon groot Eplanat/maer Dedent niet aen : twas een ban de Celanden die bz Nova Guinea laglyen / t'welck oe Prefident fuftineerde te boozen: all de Caep, de Caep van goede hope: om dat wp nu boosteecker wa= ren/ waer wo lectoen/ en goede bo=

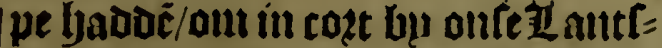
Stierboort nocl ecn cicpun Eulan= Deken Iegglyen/ Iepen tuIrljen Dat

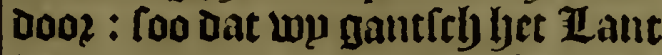
verloozen/ dat de Prefident feer ver: yeuclyoe / om dat alles nu nac liju calculatie toe gincly / wp atell feer bertelick uan ie Bifely / ende lyet firut dat ong glyelizaclit was. bet werde heden oock gereloluect beSupoen of Ilinie te blijoen/ ollt Coo bet moglyelick wag/ tulfeben Guinea, ende Gilolo Dooz te loopen: en onfe Srlyipper meende nocb Louua en Conbella te comen / als coo bu meende aclyter Gilolo te fiin: maer oe Prefident yadode de befte liennig / Datmé noch wat wat Cult fonde hebben / ghelijek wp daectys Daer naer bebonden.

Den 25. 26. faglyen wp aen bags boozt beel lant leeg en groot/ \$upt Supt-meft van ong: Daer nae we= oer wat booch : fauonts faglyen way Dat wo loo aengaende in een 3 ack gefeplt fouben lyebluen/Daerom gin,

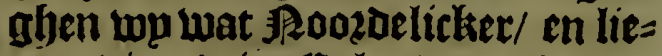
ten die Detie Eplanden die wh int \$oozoen laghen / aen Stierhooet legghen:noemende bet groot: ate lacobs eplant / oin dat bet op S. lacobrdach glyefien is ghetweet. De Cult liep noch al poozat.

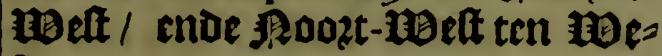
ten aen.

Den 27. Imozghens dede yem ide hooge Culte vall Nova Guinca bes Der op/ wh leploen Daer $1 \frac{1}{2}$. Alsijl ontrent: op eenighe plaetlen was het weder leegh: (miodaechs bad= den wh de hoochte uan neghen en twinticly ginuten be-5imben oe IL inie : wp couden gheerne eens aent booghe lanot ghelet bebluen/ maer het was te tille/ en Dzeben balt met de itroomlyeen.

Den 28. (mozgheng watt noch al Gil/en wo waren berre de Gult one trent viif $\mathscr{B g}$ ijlen : conden nocb al qualick bet epnde fien. 
1616. Den 29. Walt Diffich reglyenact)=

lulius. tich weder / en wy waren nu een

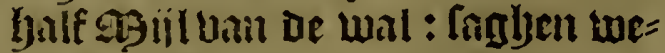
Dar beel Croos/ cll 23 laueren Daii= ven : outrent De midoach weroe wu al weder cleune Eulanden getware/ Die dirfyt onder ae Cult laglyen : lie= Jet: boozt lance lyet Lant / Dat bol of alliso ufyeen Canoes af quamen. (De

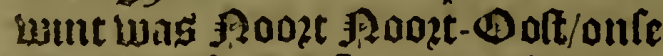
cours lUelt ten Suwden : (nact)ts [ijnoe entglje ghijlen Uan't lant/

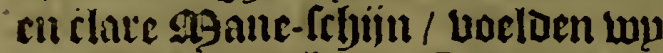
ecn 天્A ertbetinglye int Schip/ naer Duerde niet land: nochtans pertue= berde die loo de \$lacts dat ju uut De Copen fuzonghen/ entge meen= Den dat wel ftieten / men wierp lyet II oot/ men uont gljeen gront.

Oen 30 . Imozgheng faglyen wu yoo? une lant / daer nae feljeen lyet cen efelant te welen: naerder $C D=$ mende fagen wp loo wel aen back: bookt algaen ftier-boost lant: Dat wu glyebwongen waren bp te lyou= oen. Dien dacly hadoen up coo fcljzicltelictié bonderflach alg opt/ meenden dat $j u$ tebacde it ong - Scljiphadoe thedaen / foo beefoe Tet / en feheen beel dooz ae 25 lixem in bzant teltaen: Doen bolcboe te= slyen/die niet wepnicls wag.

Den lattiten : Uetuonden wa ons in een groote bocht/ foo dat wp routour belemmert waren met lant; ginglien \$20ozt aen/ nae de (Bofte= lickiften foeck wan't lant / om met - een te fien of unp eenige Canoes lou= Jen fien / Deftroom / en twater co. imende upt Revieren hielden ons vall De Culte: favonts wierpen wp 't Tloot / en wonden gront op tljien/ twinticly/derticly baden!: EOp Cettedent op twaelf 1 adem dicht onDer ljet lande : Dat ong Docht lyeel woelt en onluelwoont.

Auguf. Ten eerten zlugulti : Imorgens wag het gantrhy ftil/ foo dat wet oen gantf́clyen dach in ftilte dacluen: cul faghen boo? upt lant / beel verre om De Meft ftrecken/met ecn lyare/ I 6 I 6 . en een cleyn loocly Eulandat Daner $\frac{}{\text { Augurt. }}$ van legghen/ Jopende dat bet dner een epunt Coudo nemen / en decuen foo al met de ftroom lance de Cult: wu fagen oock vele frfyoone zapen en Jnwiicken/ Daer wel te legnden was / allmen pzofiit conde doen. bet lant was met $2500 m e n$ becleet/ als een mildernitfe : wu hadoen finidoaech ${ }^{\frac{1}{5}}$.part araets be-5upDen/ uac de Prefidents rectleninge: maer fagben wel $\mathbb{E}^{z}$ planden die beRoDzden be Il inie laghen / wp lieten fauonts bet atnctter vallen / op Craele/ cn fteenadjtiglye gront.

Joen 2. Iniogglyeng wacren wo glyenootfaeckt oun't Anckex te crifgben onder de Steenen ban daen: wu creghen yet cou onghercyent/ maer wp lieten be balbe bant onDer de Clippe. Beren dach feploen wu vooz de therede reple douz de Zinie/ fuactgts hadoen wu cen pzaper coelte / van de Supbelictier hatut / Deftroom felyeen ong om oe joopt te trectien.

Den 3. was once couts ten natIten bp Welt: en fagben ueel $\mathbb{E} p$ landen aen 25agboo?t / to2ts Daer naet quamen wy in groote calbelingbe ban water leplende/ban beel aecht water/in onltupmich / en faghen terltont oat bet een hif ett Dzoorbte luas / en lyadoen thitn/ twaelf/ vijfthien / achthien vadem fant-gront : maer alroo bet tegbent de nacljt gincls / wu bonden goet. bier te retten/en lietent ancker wallen op twaelf 1 adem. asen fach fes groote 20 aluiffenen: De Prefident arhtede dit De dzoochte te wefen / die in alle Caeten bentoen de Papoos legghen. Te Itroom \$Rael-

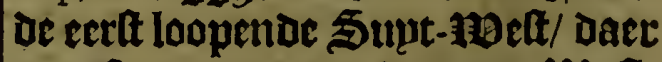

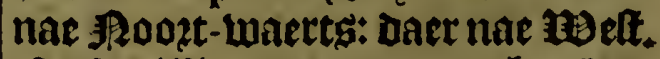
Onfe nilfinge was dat wp bet lant ban Nova Guinea gheepndicht had. Den/ als wp Daer lancx bu gbereult twee bondert tachtentich gs ijlen. Den 4 , Deren Dacly licpen wp in 



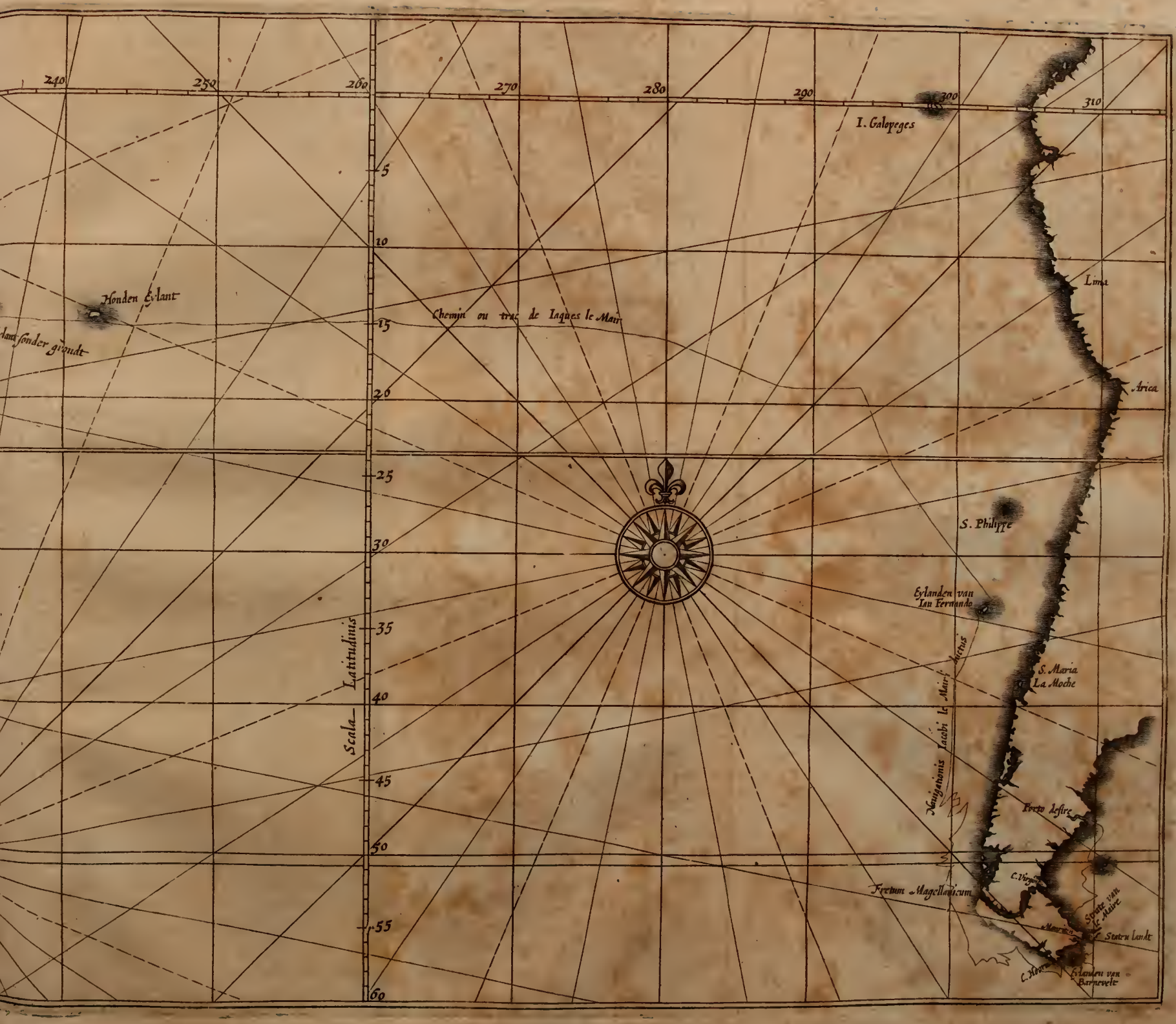


6. Ece/ faglisn gyject lant uneer: wu - Dedent olls befte am liet leeglje $\mathbb{C}^{z} p=$ lant tedefeplen / maer de Strooin urrlenae ong / lyier lyaden wu een feljeickeliclie wint : fitarijts lyieloen whi b!y' oll niet op't lant te feplen.

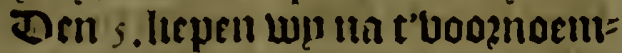
ae En!lanat / cul quantullt te betep= len : Te boorljte was finiodaetbs Derticl) asinuten be- Poo?doch. On= oer liet lant comende fagben ueel Cocos, mile Pa!mites. Boomen, corts Daer แner quau een Prau vol volet onder ljet lant/met ecti witte btedeDinen / Die niae ons tor quam / wp troumsetroen : matr $3 \mu$ vertrocken Dact ilact : terftont quaniender an= derc upt Die ons aen boozt quanten/ wu Ipearcheu inet lyner/Malays, Ter. nataens, ell Portuges. $\approx \mu$ weten ons dat wul be-sunden bet lant ont moften/eñ niet be- Roo?doct/ ch Dat wp III twec Daglleit wel in Bachian coitoen fint. Dele Ratie wagalg Ternatanen, Tydorefch,ecniglye als Boercn Uan Gilolo, altemael geel bail bunt/ met nwart 19aug/ gecleet ulet Indifche Cletienl/2 $320 \mathrm{eck}$ en/ joiitgeng/ tll Cullbanden/ lect cer= biedicly ons groetende/ goedé darb: Tabe Kuan:Laer PButKeng afooen= be op fijn Cyzilteng: wp faeghen oocli een met een nuarte Portugeefrche Bilt/ die oock Portugees fplat: 3) waren vain manieren als die in be Molucques waonen : 3u Cepben bat $3 \mu$ 990025 waren/ en van Dage Fitidaclj fijnde / Jjelden ju haer

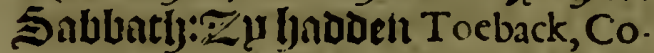
cos, Banannes, Rijit, Erweten, Boonen, Verckens, Hocnders, Vifch, en bzoryten bet ong : wu faglyen mede parndiig-Doglyels / daer wp baer ecn af ruplocil. Eetrigbe doegghen Cituch-ztrmiringljen / oock ban nuvoirs/ en gecle thinglen aen be Dingliers / weel uan onfe deebte IJacts / meenben Dat bet Gout was. Đou vertatuen naderfjant in Soppy dat defe plaets Jectede Maba, en Tic'orees [ijn : oock dozlten Gacr /uiet bouen begteben / Denckende 16 I 60 Dat wu bpanden ban of 5 pacn $=\frac{}{\text { Auguft. }}$ iaerc warcil/ 3 ! Laren leer beghee= ricljuac Manna, Manna, dat j̇in Cos ralen / 3u feuden ons nede bat lyet Jjer quaet legayen wag. Nor alle defe omitandicledecu fagen wp toel Dat wu acn ect Ijoetk ban Gilolo waren / en dat wu baef bp onfe vetenden fouben coinet / Daer wp goet tracktement vinden fouden/ inits ong dit frbecpg leben begon te verdzieten. Onfe gillingbe was Dat wo nu de betde reule onder de Zinie Æquinod tael waret.

Den 6. (moegbens watt tamelick goet weder / die ban bet lant qua inen weber thet comminge Prauwen, oul met ong te yandelen: t'welek $3 \mu$ oock ocden : imace de willt favozabel riinde/gingen Dooz/ oin ons reps te abrolueeren/maecle= ten onfeu couts/300zt aen/ om Den boekk ban Gilolo te winnen.

Den 7. als oe Dagheraet quam/ faglien wo glyeen lant / en be coelte continucerbe ban ben Supden:ons couts was \$Roozt aen: Imiboaecty baboen wp redben / tegbens ben abont claerdet op / en lagben joocti lant te loefluaert op ban ong : gif lende te wefen be fookt-cant ban Gilolo: oe boothte bebluende ban Dzie graden be-3roozoen: wp lie= pen Dan Leneden lantr/ Daer Mo.

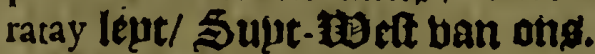

Den 8. fmozghens was bet oij: rich weder:ons touts wert 2 Roort melt / met bol water / Imidoaectss was de boochte 4. graden : Imachta was bet boos tereor / met aterek wint / en Duplter wedet / en beel regbeng/met 23litem en Donder. - Det 10. Imidoaechs de bootbte was / ban bzie graben bijf en beers ticly gs inuten / [nachts [cherptede oe wint/Dat men Supt \$ molten feplen : Hefolverden foa langlye ober befe 25 ough te repe Ien/als wa conden/ om boven Borneate feplen/en met de Supde wins 
I 6 I 6. Den un Celcbes lamex te loopen.

Augutt. Den I $1 . e n$ I 2 . finghen wul weder tamt / acm de Roore-lyoecli uan Gijolo, al-te-ntet dzeven wu Doo? den froom tuet bet abeficht.l al-te-met faghen wuy bet weocr / inace wu conoent neet befeulen / badocn bier beel Trarades.

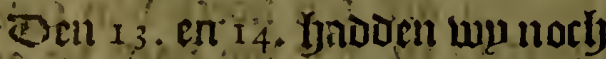
al oe bu-teggher met ueel regheng crítosu : of ftroom was ons hier De hatelte unarit.

- Ten r , yjudacn wu fuididaechs oc hoodjec uni, 3 . graden be-, $2002=$ Dew/ we en conden Doeng giben lant ficli.

Torn $x$ 7. Whas oc witht boozmid

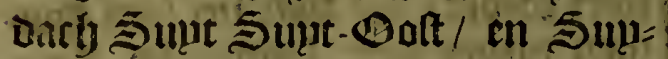
Den/Lu wendent Pocit-waerts ten ineften bu / oun't lant te beleplen/ welek why nawielick conden beco= men: Coeckende gronat / bonden gheen : en wp dectuen den ganticbe macje in ftilte.

ôn I 8. Laren wu vooz In den Roozt-Loecti van Gilolo, endozeven thet of Itoon in-wacrts. Jae nocn / quamen ơic 19zauberi wan lant naer ong toe/ met etr bzede-

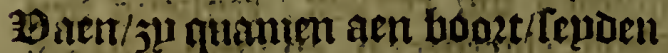
ons bait oe theleglienthept uan't lant: De wint liep Supt-apert / in un liepen nae de 25 ap booz Soppij, Dacr ons de Intwoonaers we = fen bat een wozp mas / en goede Ftncker-aront: Daer oock yet Jaclyt De Pau w gljeleglien bande/ ontrent twee \$aer gheleoen/ een goet ltuck uni't lamot was een lifif van lteengront / hadoe thien oft thaelf $10 a=$ oen diepte: Daer ober fijnde let= tcoen bur fyet op (es en twintich Ba abem/een fturlibimen de 23 anc. ₹p qunmen terifont ouer / mitg 31 Ternataens, en Hollandts olje fint jijn 1 st! conden wat Malays too ont un lyater beoupoen wie we waren / daer in wa en 31 feer wa= reñ terulitot: wul uertonoen oocli ban baer dat wy in twee daghen in" Tcrnate ronoen weren / met

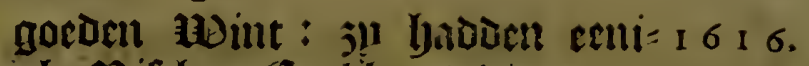
glye Bifty en Crabiben / Die ju ans A Auguft. vercacljten voos Cozalen: chl wa= ren baul be Sangage ghelonoen / onl te Lefien wat bolck wu waeten/ wu waren ljeer op ie boocljte bau thee graden leven en veertich 29

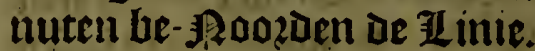

Dell I 9. wat fomerachtich we= aer: fmidoaedys oe woint van de Moozacliclier bant fiinde / liepen wu nae de 23 au / en letteden op twaelf Daden 5 tbulp gront. De Inglyelanocu quamen terftont met ueruerffebinglye acn boost/ vercoo= pende ong thils /, Scljilt-1sadoen. Sagu, Hoenders en vifth uoaz $\mathbb{C} \tilde{a}=$ men/ 23ellen / Sptickers/ Daer js wel mede gljedient waren.

Den 20. waren wu noch al lie= rich met berwiftelen / cregfjen ued Sagu : Oe thiis wiloen ju: alleen booz ylinwaet vertuplen. De= [en dach quam alljier ech Correcor om Kiijs ban Ternate, Die $\mathrm{ftu}=$ De datter welfeuentie groote Srbe: pen in Ternati lagben / te weten Gollanders die na Manille wilden. ABy trocken ljuer met onfe $\mathbb{E}$ egm Bis.

De nae-volgbende Daghen ver= fagtjen wu ans noch al van alder=

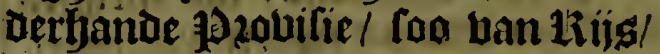
als mater : oock wan verflye bis! als Steen-23taremis : Banannes, Cocos-Noren treghen laz in abon= Dantie. Dit Bolck was goet ban natuer / Bunnoen ban de Spang= jaerts / Itaende onder lyet glyebiet bau de Coninck bau Ternati, die jp oock booz lyarr Souverepu hiel= oen. 't 3 b niet te legglien / of te fcheiiuen / yoe groote veranderillglye ende blijfrjap yet ong gljeweelt is / te sijn in een plactlé daer wp too biij-baren : en loo beel Bictualie conden. becommen als wa beglyecroen / helobende afgheleut foo een moenelickel periculoole repure t'feljecp bebluende biif $\mathrm{en}$ tachtentich cloeclie g9annen / fon- 


\section{Door Iacob le Maire.}

fol. $6 \%$ ?

16 1 6. Der bodtritet bu thaet / ch alle noot= Augutt. Dzuft ten cludoe : Coo Dat Lup Goac batmberticlyeut boo? ons.

Din 2 . altoo wi ber ohedaen hadocn / gimglsen wh 'trevt/, unt oe Liecde van Soppy: ban alled wel uerfien fimoe.

Cot lyet epnoc ban oefe $99 a c i i t$ liepen wun al ober en weder obier met bettheydorne winden gljequelt rijnot.

Septēb. Wen eerten September 7 tn oe tweede was De wint meeft Sunt= beit woy liepen dicht onder lyet

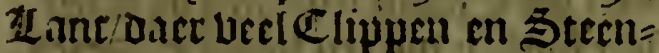

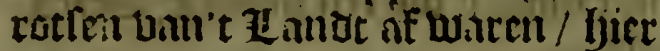
wata ons de Stroom fece teghens: twn licuen aclsec cen hoecli ban't Eulatiot Dat aíbewoont was/cli leteroent op vectich Batuen/ al fefjoone gront / onle פAarts waren aen lant / vinglien eal roode/ ch= oe bzochten bijfticly Cocos aen boost : jn! badocnder beel aen lant shegbeten.

Den 3. bleven wy alliggtjen on= ber bet lant / badocn calliely weder met reglien. De Prefident met fiin 23 foedcr Daniel, til de Stierman gingljen aen laubt/ cloumen op't obeberchte/ meunende daer boluen op te collien/ oui ouer te fien/ oe gheleabentljendt ban't lanot oace acheer/ofter eenige paffagie moefste rijn/ maér 3 conden niet bouen $\mathrm{Co}=$ men/alloo't fect wilt en woelt wns: int riédergaen liepen $3 \mu$ aroot $\mu \mathrm{e}=$ riicliel uan neber-ttozten / als ju een verkeerden weeb) namen / chiot ten lactifter beljotioen by ons qua= men: alloo wp befich waren neet bettely water te Deincken / Daer $j^{\mu}$ mede of ozoncken: atls $j \mathrm{p}$ treljeep ghecamen waren / voeret wu met onfe 500 t Dooz en lolle Clippe/ Die in't water ttont / als Dooe een Slunfe / wa fagben terttont we der een cleput Eplant, ont Den hoeck/ ntaet roenden nae boozt.

Den. 4, nae Dat we lyier $1 \frac{i}{2} \mathrm{et}=1$ meacl Gaddent glyeleglycui / ghequelt i 6 I $60^{\circ}$

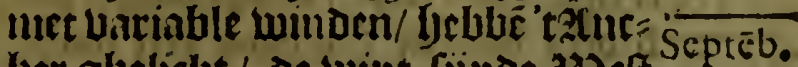
lier glyelicht/ oe wint fiinoe welt Rooge IBect / mecneribe dat lup oe arcom te lact yadoen / om tulfthen or Eulaniden/en lec balte lant doo? te comen: wh liepen bier ouer en weder ober met wejuich pzofiits; foo dat wu de Uijf eulanden per fert faglien dooz ntalcanderen / bet cerlte was glyenaemit Bihoa : bet tweede daer of Prefident op was Doij, ljet neroe Tuacaro, fiet vierde Poir, lict vijfor Salangarij, Dnt if geclachticly/ en Icecty/legabende na of Cult uan Gammacanorre. Dit jijn De viif Eplanden van Moro.

Derl s. Lleven woy Gier een gant= [cljen Dacly legglyell / Dooz of con= trarie wint : We Bifteljers voeren upt om Biffehen/ vingen wat Cro. covade, een leer frboone Big/terwijl ons bolcki aen lanot was / quamen bzic 9Sannen feet Ictyielick upt yet Jofch vallen / met Sthilt en \$a: bel frljermut lerende / niet wetende. oft ju Daer mede getkteden / oft int ernit lwas / $3 \mu$ dit liende rie pen : Orang Hollanda : Dit is : Wy zijn Hollanders, foo liepen $3 p$ ter ftont aet Strant $/$ en gooten 1 Ba $=$ ter op baer Hooft in teecken bati penel. en namen d'onle in Den arm/ en lachteden:voeren met donle aen boozt / bzochten een gebzaden obig: ju quaemen baer vall Gammacanorre oin Sagu int 25oleth te roess lien: wo Cetteden haer weder aen lant/ als wp haer vereert lyadoen / met wat Cozaeltits / belouende Berc keng en anoer goet te bentuen: Gammacanorre larb doen norb dzie Agijlties Lan ong/ [oo jp lepoen.

Den 6. liepen wa tegheng ober yet Eplanot Salangarij : wiecpen xincker tot feventyien sademen fant gront/ een Goottelinck Irboot van't lant.

Den 7. Deden wp weder nieule bliit / Conder pzofifi : Imiodaechs 32 cres 
fol. 68

I 6 I 6. clegluen wur ect Prau aen boozt/ die

Scptéb. hzorlye booz de Capinno een foode Dis: Lnt frijondicn tjem wedet Dzie

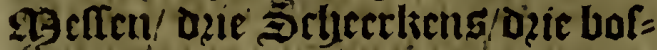
Feng E Eperbzant/ bat Corallen/ en Ditit Slive-glacfliens: foo Dat be bis onts dier ruante tane - Ong boldt ginch oner nace bittefjen/en ritrghed roo beel Dis als alle or ìietscanden ren:

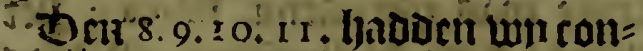

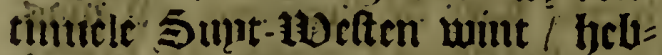
Eende alle Dete Datglien Doos cont= triric whit ende calmite./ niet meer Dan twee Sijlen gewounm. Dute Preff Acne lliet Aris Clacfión, Iman= Deli de Slocp/an noeren'tiact Gamniacaliore, matr ju quamen dace tilet / maer outeme cen gisit oft twer dare bu findoen gljeweet : foo Dat ons iniet mogrjelicli was Supt= Weft riente loopen naer Ternate.

ôn 12. De wint nortj als vozen: Oitre Stjipper willem Cornelif: Schout, gincls met een welgliemon= tectde Slocp nat Tcrnate, Dact fo wet bekent was/ in meende enti= glje uerbertrdifinglie boos ong te becanme ten feluen dagbe lictite= oftr wis weder ons zturlter: ntaer lietent ballen op bijfthien Badem rinit gront' Dat ons frer berbzoot.

Din $r_{3}$. boeten onfe gis acts on te Diffelien / bzorijten'ons foo beel Difrth als wun begliberdoen/als oock

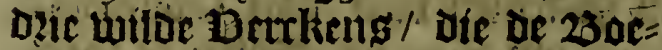
ren bhi' 'tlaut baer byachten/ Die

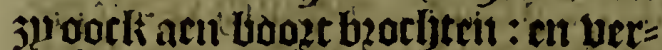
clarrueti an's dit ju die becomen Ifidoen mee de Jaclye Der Gonden:

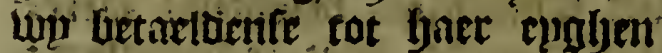
fegrelisen:

Oat r. viel or.wint met lyet lant/

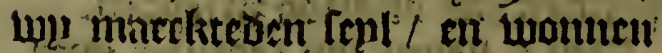

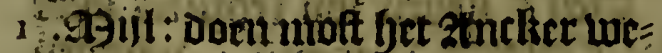
bet te gront.

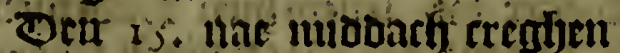
wit cet pecpet coelec/linper lante oe Mol / cll. ecn goet ftuck weetigs

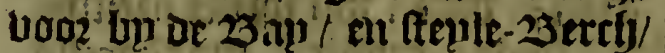
oirt inn int incamen unir Loloda filche Navigatie,

penfterden/ Lefende den boerli ban I 616 . de Eulanden Toggefongij fer glye- $\overline{\text { Seprēb. }}$

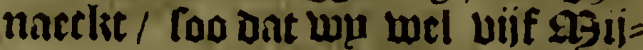
Ien Lertieraen / Defen Dacls bebbeil= de de ftroom te baet.

Den 16. Imozglyens waren tup

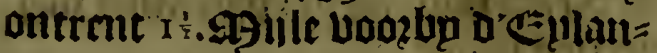
ocı Toggefongii, cu Loloda : unecn bocljt leggljende: t'was diffich we= Der / al-te-net regljende lyet : vooz oe inidonclj clactort op / et fagljen Dell 23 erclj uall Gammacanorre bo=

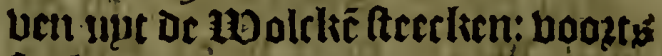
Caglyen wus Tcrnatc en Tidor, als oocli Iset Eulant Irij : aen de neclt-

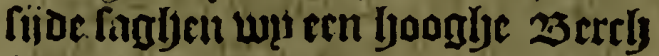
Sabuba.

Det1 17. wag de wint bariabel/ met tamelieke coelte/Deden ong luet om Ternatc aen boost te trijglien/ dan comende booz de borlit ban Scbou, quam daer een Scljip upt/ de Morghcintcrie van tiotterbam/ rijnde retl bail Spi'berghens Srlje= pen/ die doen al ober een balf jaer in De Molucces ghelweet wag: it Dit Schip was or ztomirael Verhaghen; ;il de Gouberneur lafper Ianf. foon : alg oock onfe Srljipper / Die roo langly by dit volck obetweet was / en wp quamen oefen bad' urpoe booz Ternate. Barende ben Commandeur net de Stljipper terftont aen lambt / alloo Dner cmi 25oot mam uande Gencracl oun bace te balen'/ t'weleli boen ter tijt Laurens Reael (alfoo de Generaet Gerrie Reynt, tot Iacatra ouerleden was) Dic ons wel onthatide / oock be gantle be that ban Indien, die Doễ ter tiit daer meeft luas. Dookts verffonaen IDy hoe oe reple ball Spilberghen was afgheloopen $/$ Dat tiin Thatht booz de g9ont um de Straet Magellanes ontuoert wagy bor bat al eer fju daer nuam in dét Ric= vicr Spirito Sancto beet bait fiju volck berloozen Inode. Tat In turee ga aenben tijes doozoticbarljt Jjeft in Dooztorlit uall de Straet. Doos ziinde de Cult ban Chili, en Pedu 
16 I 6. Peru liunci glictenlt liteft/censtad

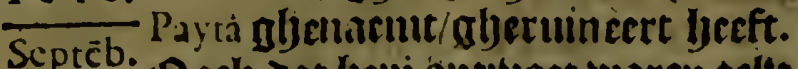
Oock Dut beni ontunoet waren arijt

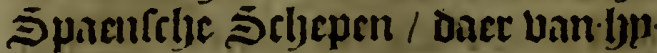
De zlamurael/ ende biec-atduniracl met noch een ondergebzoclje lyeeft/ met Lepuncly bunts / en uerlies uan fiiu Lolels. Bocli Dat lye Lima, en uecl 5 anden! en Julyanunen dooz: finffelt lyado/ met glieen pzofiit: Derljallen lantr oe cult ban Nova Hilpania, llike oe Manillas Luag oucralieftecelien / en foo Loozts une yet Baderlant glyercuit. Datter oocli thien teffelicke Schepen ge= reet wuren/om de B loote in oe $\mathrm{Ma}$ nillas leggentae/ dic op Ternate luas. untegherult te be[psinghen. Oock

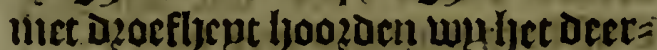
licli onglielucti ban oen zomirnel Pieter Bot : Jae lye- op Mauririus ge bleven Was / en nau De belft ban't bolef glyefalueert. 300 dat ons licf was te uertaen be ltaet en gbes legbentbeut ban onle ZL antflicoent: cu belonder ban de ltant ong $1 \mathrm{~B} a=$ Derlantes:

Gntrent den avont biterpen wa ons_Afinclict/vooz-Malege in:Ternare; Gebantó op ele Baden fant grout. Dnfe $\mathrm{f}$ refident glyearcoumpaigneert met Den Schippet lieten baer Itracx aen lait fetten / ont bu betr Sente= racl-Laurens Reacl, Dic in Geraerts Reynits plicte guecofent inag te co: unen/ en ben baec mocneliclie reufe te uerhaelen: hu tractcarde haet miaquifelick/ dact pectene warentide

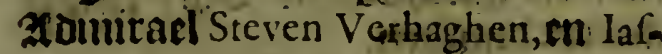
per lanfroon, (5aubertucuir ban Ambon, Efauauts quituente-Lueder aed1 hoost.

Den i s.alloa un beel plonde= ringlie ober badoen / van bet ver

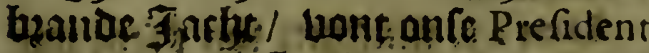
goet / met oe, Schipuec/. lyet alyente Dat wu te bet badoé/: te bercoopen: twelk jp oock deden: ghele mate lsende van aufe bejuer sloeven/.en vier Soottelingljen ban't Facht/ oork uan rm goed partic Tloots. oner b̨) Lwaren neglien stucliet's/

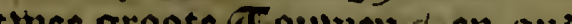
ounglien : t'belien in alles aunfent dzic yondere bifticly liealen wan arbten. \$oo dat bepar partuen wel gljecontentect Laarell.

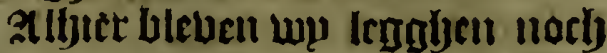
ache dagen/alfica wu niet clater wa= reil / all t'fepl te gaen : oacli lieten

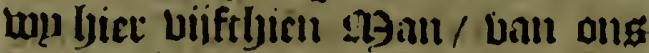
volck bliibẽ/ met lyaten bait Luille! alfoo jul lec cerlt berforljete/ch Dane: lner baer berbuerden aen de OoftIndifche Compangmie /. met gorde ueclueteringlje Uan gagit. Doozta bebleen whlyier onfe ledige baten aljesult net water : an oin oat oule coft op't $\$$ elyip meet op was/ corfy= ten wun ban den Generael/twee ląt

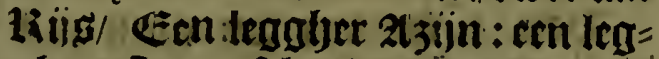
aber Epaentche wiili: als oorli die Daten Btefely: Letalende baer mace - jact contentement/gyelijef 3t! ollg ootk deden.

Den 26. \{moznheng waeren te Prcfident, en oufre Schipper bu deti Senteracl t ontbijen / die baer met Dentanticlye Hact/ ecn eerlick unt= gelep dede' ban acljeren bolgende?. ch: cainende op a te trant / net tent Dacndel : Doldacteni die in volle atafjozoze odie mael ijacregurfuct: ten affetioten tot een ztDieu. Alen boozt ro.uende/walt ttille/maer on= trent de midoarh ereglien wu ect coelte ban DE:Suutartliclter bant/clt ginghen ander fepl / met den Morghenfterre Uan Spilberghen, die wu becl te clock twaren int fequlen / Die nac Motir bilde: epp namen ober Monficur de:Breen, en Ian Philippe Tije-fchut ban amt Senernal/ am hace te Bantam te bzenglyen: De S. eeroe ons met wat pozcelepu / en Hecreu/i en sen pottile met 2boter. Ten 27:Deeben wu Den abeljecle Dach in ftilte/ on der be wal van Tidor : aicht booz be oe foztrefte det Spaenfeben/ foo dat $3 \mu$ bu naet foiven outs conneli gefrboten licls: 33 ben? 
16 × 6. ben / hidoden Lub wat naerocr gje $=$ Septeb. Weet : Den froom mede bebluen= Dc/ cn cen cocltien crijgljende/ qua men wu Delen dach vooz bu pot= tebackers Evilaut / gljenacnit Pulo cavalle, par Lun fauonts ueffeng Mo tir waren: fuachts feqloen wp op oc lanclite unu Machian.

Don 28: fmozglyeng faghen wp De Exilanden van Caijo en Gourities, en Desuen in ttilte. Pat onte giftinglye pafterden : wy vooz oe vietdereufe de il mie Equinoctiael?

Joll 29: fagen wu Bachiam voo? fteven cen feer groot wiit ltreckent Zlant / lesactytich / en feet flanclie = rende met beel hoecken en bocljten/ Looz de midaach warễ un neffenis Machiam, al in lailte dzijbenoe/ oock den gantltyen nacht.

Den: 3 o badoen wu noch Bac hiam aet .Stier-bookt / en Gilolo aen backbooozt/ en fagben booz upe ęniglye andere rlepne Ëplanden.

Oetob. Ben certten (Betoluer faghen wa aen 3 agboozt obebzoocken Lant/ met fommighe isembels / wefende/ tSupt-equae uan Gilolo. - Darer naer tagljen lup een groot Enlant/ IJjenaemt Obij.

Den 2. fagen wp het lant Zeran, en fettedent onfe rours Supt SuptFoelt / om Burro aen boost te crij= gljen.

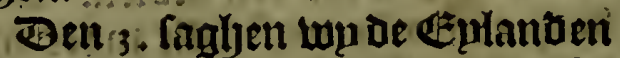
Manipa, Kilang, Burro. 5 midoaechs hadoen we Burro aen 25aglookt/ op 3.graoen be-5indoen de Plinie:

Den $4: 5 \cdot 6$. Wuag de bint meelt 5upt-Doft licpen voozlup burro, Cabeffa Calica, Den feuentten paf: feeroen wer Cambona : en waeren fmadyts niet verre bã Buqueronnes.

Dril 8: fmozghens be wint bai ie Ooftelicker ljant / fauonts par= feeroen wu twee éplanden die clech waten: en oocf 'tleeglie lant ban Celebes, ance op wp beel bieren lagfen.

Den 9. (midoaeclys waeren wp ontrent Macafzar: en liepen om Ma- dura aen boost te auglien / fuachis 1616. yadoen wul al ectupariglje coclte.

Den I . Whas or wint/ en cours als voozen / fabonts cocloe het op/ ban de ooftelicker lyandot / en lop. yadoen nae Intodacly op uijftlien Badem grant gljelauepen / bu cen Kif/ dat Inen noeme Pater Nofters, Dat wu Late te nace waire/ chl mocly=' tetl grocue plectien / barninglen/ ent dzoochte fien.

Oen 12. ell I 3 norty de lelfoe couts en wint: wn faglien oclen Dach/ bet Evplant van Madura aen backloogt van ons.

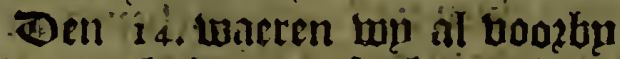
Madura gljefeult: en fautben of $\mathbb{C}$ ll $=$ Ite unin lava : en feulden dien bacly voozlu .Tuban : foo bat wp veel Giftrljerg vertuatnê met faer Prauwen tupt $\pi$ ee comtentat.

Den 6. fjadje lup de coelte upt fet lant: nae midoaechs quamen. un book Iapara, fettende bet op $s^{\frac{1}{2}}$. Baden Clep-grant / meffeng bet Schip Hollandia, uan A miterdam, dat hier gljec onten was om thijs/en in Ternate te boren. Befen dach voer oufe Plefident aen lant/ oum berberfelyinghe te coopen/-alloo de Jeeften lyier genoech en goet coop waten/als odck oe 19oenoetê/ Cepes ren / Canuarinoen/ \&lrack/ Bifrg: jae alle Iliftocht.

Den r7. en i s. was de wint inachts upt liet lant / en ober-dach unt ter Zee / wa ueruerferten ons bier treffelitk: ban kiis / $2300 n t=$ ies / en gheo?oorljoe 13 is/ cn alle andere dinglien.

Den 22 . yadoen we alle orife pzovifie in / wop berloozen trier een altucker/ alloo hier veel Travaden waepen/ die ong bet cou aen thus= ken bzack:

Ten 23 . gingtyen wu t'tenl nate lacatra, wu baoden doell in ons Sthip vijf levendige $19002 n-25 e e ?$ ten/om onderwegben te eten.

Den 28 . Des abonts lettedent wp neffeng den kiupggen-joects op Ders 
16 1 6. op ocrtbich Dadam (findar 3.99 ij $=$ Oitob. Ien bint lacaura.

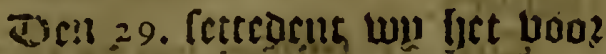
Iacatm, op vect'tyien Darachich ; wy

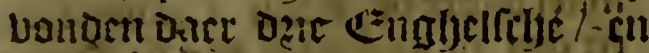
Uicr wiuntebe scbepen / alsoock sen lonckt, ate nia lianda wilac on te bunoelen/ alfor jutconrent lyobe

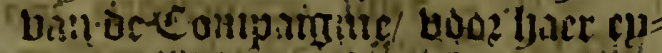
Thet p:offit. Tefenaclit oustecot

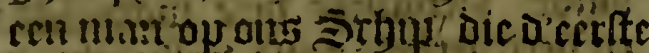
wis binn or repre inaet ap De Jacht

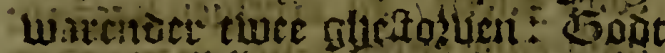

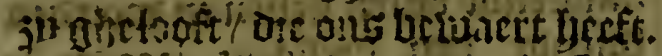
- vert 3 o. ttacatrir un ante $5 \mathrm{kp}=$ leir af: enduchs pucthat bacl teir wa alle oitr Sictien teulant: op orma-mandal; q:anm oocti boo? lacatra of Prefident Ian Picterfen Cocnen, met yet Sclyy Bantam glacmatiut.

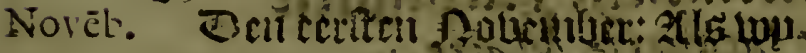

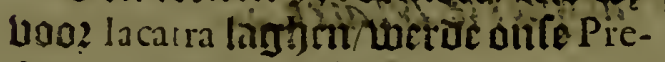
lidene, met oe priticyule wan ons uoleli ontbeden van oe Prefident, Die met filn uolle liaet was verga= Dete / en bin uerelacrde aldaer / Dat wis mofte!n afiłacu ball al 'taliene

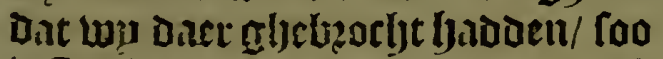
t Schip ell Goet / ell dat unt laî van de Ooiz-Indifche Compaignie/ twelels onfe Prefident niet bel en uelyaeilyoe / en badoe gljeerne defe actientet bele redenen te niet ghe $=$ Daren : Darer op hem gijeantwoout werde bat bu die int giseljeel fonde in b)ollaut vinden / Dace neen reclit Coude wiifen ban't gewelt dat ljetu acughedacn wozie/ [oo dat lyu glye= imong.jen was alles te verlaten:

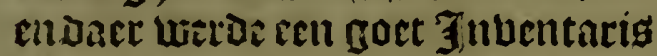
af gefjoudet ban't glyene dat ober $=$ friclevert wazde. Dit was recljt op Floerlyeplighan-Darh / primo No. vembric, na otite computatie/ en op Den tweeden dito maer de reeclie= ningeóntes natiel alfoo wu weat waert aen tut lyet baderlant glje= baren waten/su cens den gantfelye Gloot des Aertriicrmet de Sonire omglyefcult / roo lyadoen tau een maclye of Bounell ondergane nuin $=1,616$ der glicljad als doule : cll julie $=\frac{1}{\text { Novéb. }}$ ben ate vạn de 1Beit-tant/ teglueng ljet Doften Laren gljebaren / yad = ben ceil Dacly of Sommen onder: Gaucli mectoer gljelyad als wp/ twelch vier en twentidy. uten Diffe= rectio: waren lon oerljaluen glje $=$ suondjen onte reecheninge te ber= ainderen uai de tiit/ en. Derloozen ecu andy/malarende den Diinge Dacts f, tellende ban de 2 arndacly/ tot op Wuenfoarb/ foo dat ong oe Wecli was bau fes dangen.

Ats Wị alfoo ous Schip quift waten; thntender ceniglye van onfe goxets Die haer berbuerde alonex: inaer onte Prefident, met fijnen 23zoeder Daniel le Maire entoe de Scljipper unz Ecndracht mer nocly Thicu nuan Lacroen oljefet op het Sctuif Amferedam, dât nae bet pa: orrlant witoe. OSaer Aris Claeffoon,ende Claes Pieterfen Stierman, met andere tljien van oufe $\mathfrak{A g a t t}$ / werden beltelt op het Schip Zeelant : en ginglien t'fel uan Bantam, Den vijftljienden December. Decēb:

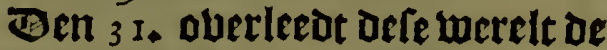
couragieule en beme Jongelinek/ (aljeweelt helubende onle Prefident) laques le Maire, wieng lult en dile= gentie treffelick beft gljebleecken de gantiche reple/om die met groo= te ecre uyt te boeren/ an te balbzen= aljen.

Den I. Januarij நjadoen top een Ianuar. Roots-1Beften woint/ met ltawn/ en dzeven fonder Sepl/maer fiende nocly lyet इrljip Zcclant.

Denl 2. Dito Uerlozé wu van ons Dooz den ltown lyet Schip Zcelant, maer bu vondét daet naer weder.

Den 23. laglyen wu bet Eplant Mauritius, en Den vier en twinticlyIten quame wu ap oe thecde aldaer/ en fjeblienong water op fet alder= fpocdiclifte imgenomen/ foo dat wu Den dertichlten claer raeckteden.

Den 7. A9eert : fenlden wu Loo= Martij. ven de Capo de bonnc Efperance, 
I 6 1 $\%$ dare wu feer ober verbliit waren/

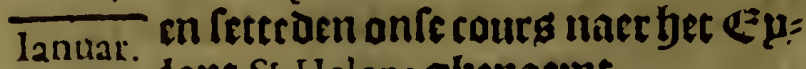
lant S'.Helena gljenaemt.

Aprilis. Den 1. Aprilis booz de nitodath/ fagljen lop bet Eplanot $S_{t}$.Helena, en daechg daer na/ lettedent wop bet onder lyet lant daee bop met bzcucht boiloen bet Scbip Zeelandt, Dat berowirelt laig ban ong / maer ec= uighe dagfen uooz ong. daex ghe= arribeet.

Jen 7. Dito. Saer bat wen ban alleg ons wel uerfien badoen / gin =

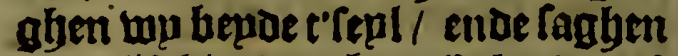
oen uifftbienden bat Geplanot Afcenfion.

Den 24. werben twee Schepen gefien vall oe פgaets/ op de fooch=/ baode. te uan ecin geact bi. Supden bi $\mathbb{L} i=1617$. nie / maer jp condenfe nict ver $=\overline{\text { Aprilis. }}$ kiennell.

Den 2 ;. Ceploen wp of bijfoe. mael ondor oe Linie Æquinoctiacl, ni den 29. berlebeen ons de RooztSterce/ die wp nu wel twintich 9aenden gfemiat badoen.

Dell 2. Tulii acriberden wh Iulij. met bet Sebip A mfterdam in ₹ee= lanot. Gebluende bele mocpelictie en verdaietigbe repre bolbzache/ in twee Taren / en arhthien dagben: Halatende onien lieven en weeraen bziendot oen Prefident lacques le Maire, bie glyecrne bet epnoe ban orfe labozenfe kente / ghtefien

\section{Wigrepnoicht de zultralitche Jeavtgatic van Den Prefident Lacquesle Maire}




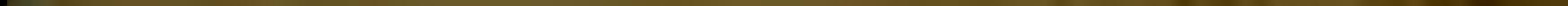




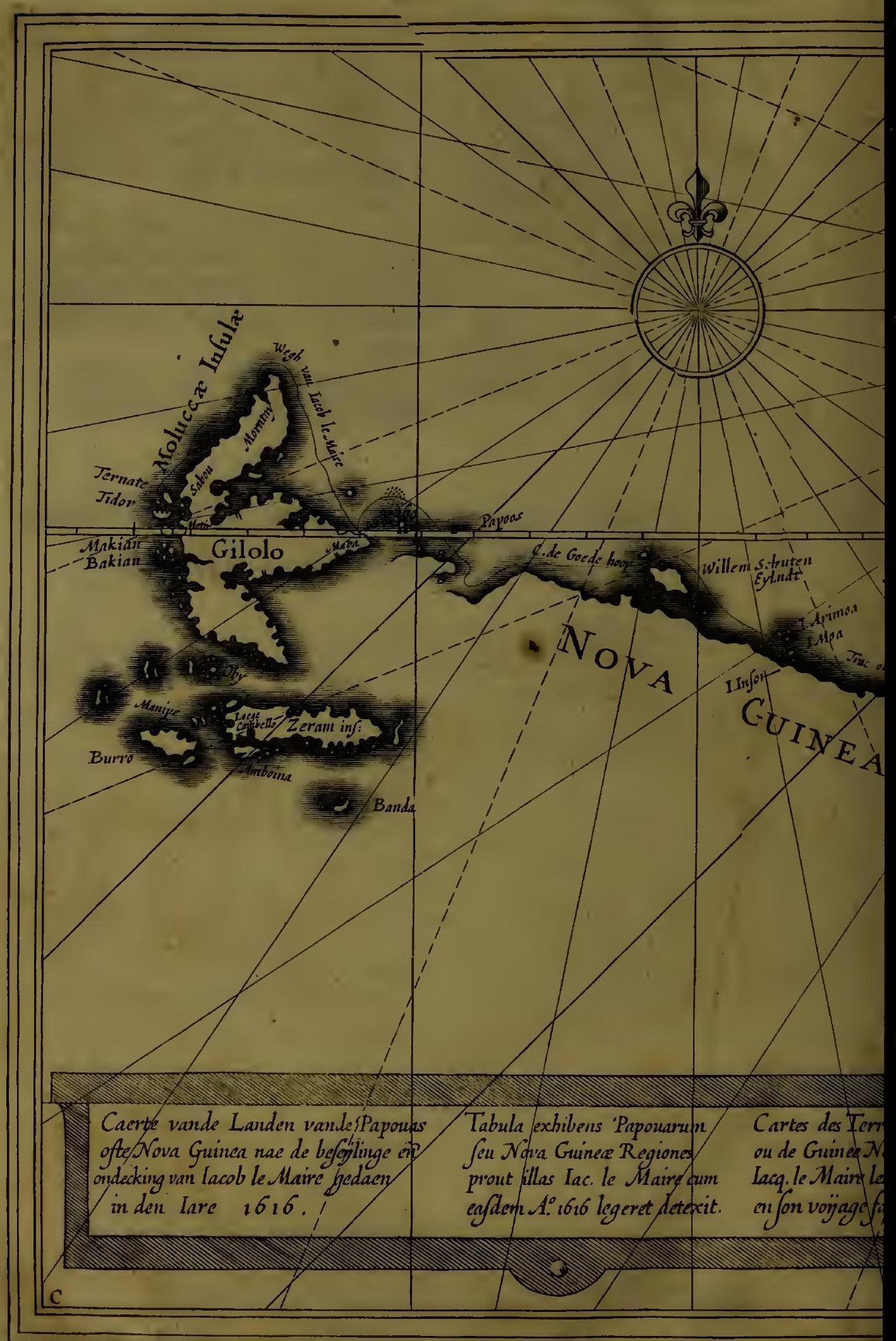




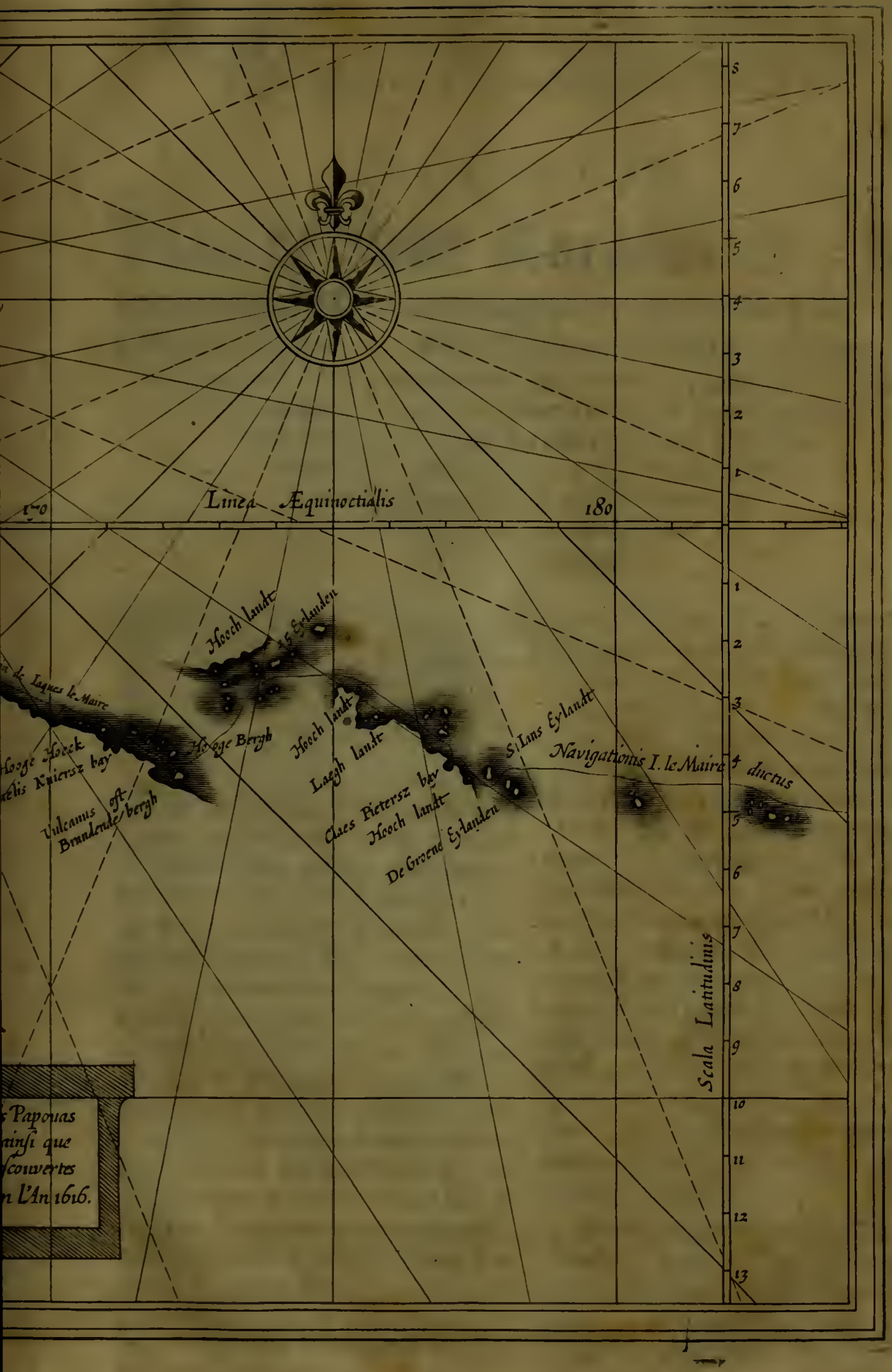




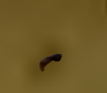




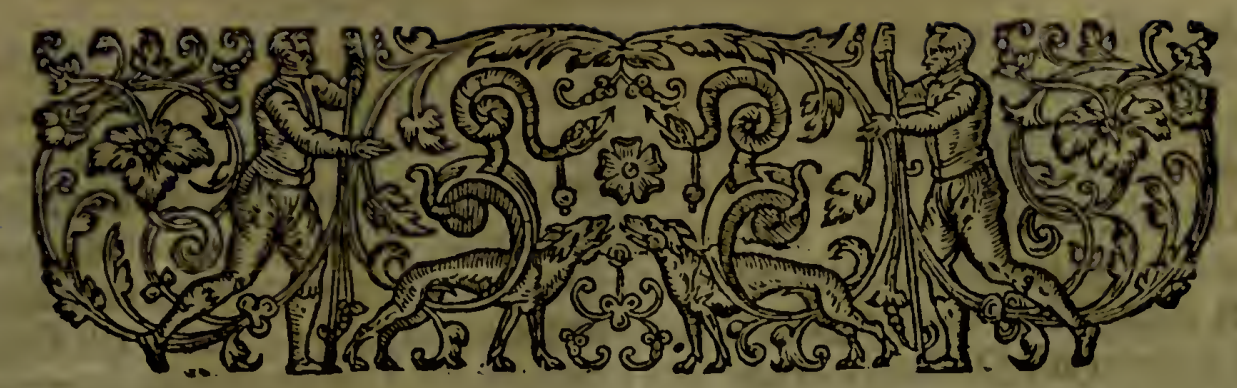

\section{R E L A T I E}

\section{Ghan oe tivee fratabellen / Die}

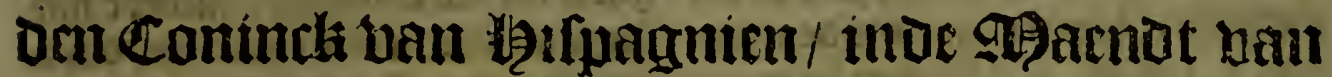

DCtober Znno 1618. ban Zlilbona beft upt-gefonderiom

te ontaecken/ ende beliclytigljen / het Frerum oft jaaftagie Le Maire

in't Zupoen gyelegtyen / onder conoutte uan Capitepn

Don Iuan de More, welclic twee Caravellen, weoce=

oint in Siville inde maent Auguftusi 6r 9. jiin

getteert/ende Dace ban ben Coniuck

rappozt ban ljull weder-ba=

ren yeduen gedaen.

1618.

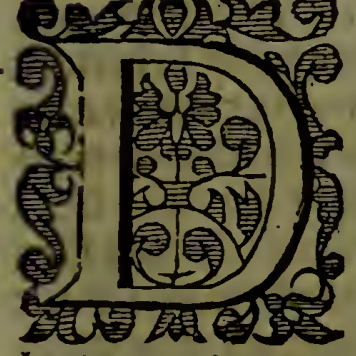

这Caninth uan Hilpanien beluenoe ber $=$ ltaen ie ontoec= Dingtye ban ljet Fretum le Maire, dooz die bã 190 l= lamor/ oul aat daer ban de aubijten il Spaengien waren gljeglyeben/ omi dace ban de bolcamene jerlece= kertjeyt en befcheet te lyebbê / heeft geozoonneert dat ban Lir bona fou. Den toe-gljemaeckit wozoen 2. Ca: ravellen/ele groot welende outrent ban bondert laft/ met 60 . פgannen op elcke Carabelle/ en Loozts wel berfien met glyefeljut/antonitic ban Oozloghe/ en Biuzes vooz ontrent twee Faren/ om liet lelfoe Fretum oft palfagie te laecken/cn te beficly= tiglien / tyebbende daer toe glyeltre = ghen unt dele zanden - cenige 19al= lantlelie 5 tier-lieden/en onder an= Deren een lan de Witte ban Amfterdam. Een dien volghjende loo ziin inde imaent uan Oetob. Anno I 6 I 8.
De boozetyzeuen Carauellen/ onder yet bewint en commanoement ban de boozfrtjetuen Capitano Don Iuan de More Lan Liffebona gljefepult / en

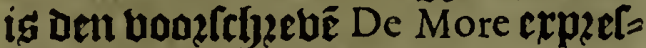
[elic belaft/ dat ljp op alles loel lou= de letten/ en goede opmerclinghe nenten/van de boozfch?even paffa = gien/en iriconoerbept oft Daer nitid= Del fonde jiin am die te fuptê/ met eenige fouten en Calteelen/ op het Tande te malien/ op welcke fonda= ment en dit te vernemen/ Die booz= [cljecben Carabellen alleen jiju upt gherecuet / alloo de Coninck leker= liick witte / en wel gbeinfozmeert was Dooz rappozt sam eenige lanotlebe ङtier-licoen / Dat aen of vindinglye uan de paffagie niet en was te twijffelen.

Dies niet tegenftaende den Co= ninck Daer mede niet sbeconten= teert juinde / ont alle particulari = teut en fekerhepot te weten/ op lyet beftupe van de vooefchzeben pafta= gie/ beeft euen-Lel de voosfetg?even (t) Cara= 
I 6 I 8 . Carnuellen oerwaerts ghelonoen/ De welclie voosfpoedichlicli jitin ge= arribeert ill Rio de Genero, aen oe liulte uan Brafilicn, al-waer fp jiin verberfelye / uan water / en fulcks meer alg fuluatl noode badoen/ende ban Daer zin fy boost gefeplt nae ljet Frerum oft paffagic / ende sijul Kouteil te beruallen / een wepuich Jeooften de Strate Uan Magellanes,al waer ontrent lyet Il andet komenoe ech groote 25 alu bebluen bevonden uptwaerts aen/ ili' $\mathcal{Z}$ andot loopen= oe/en om te befichtighen jijn fu oie umglyebaren/glyeuenoe die de naem bau S. Icorge. Eull hebben Dacr acn

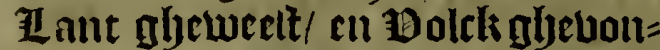

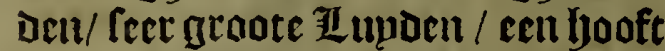
langer als de grootíte II unden/ Diemen biet te I ande bint/die baer al=

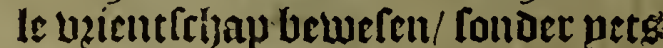
te mildoen. De Capitano Don Iuan de More, lynmbelde in ie boosfrly?e ben 25 ap / met de Indianen, en ber $=$

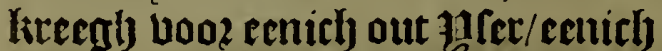
Gout/ ende onder andere cen ltuck gouts werfende anderbalf boet lane wan de $\$$ warten / Inaer ljoe wicly= tich bet was bebluen oe bollantiche \$tire- lieoen niet connen weten/al= fo bet de Capitano fecreet byelt:beb= ben oocls miet tonmen bernemen of jet felfoe Gout daer was geuallem' of bel eloers/ nuts de Capitano den ljandel alleen ozeef.

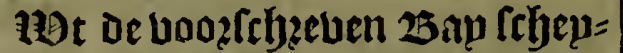
Dende/siin de Carauellen gevaren! ooftelicls aen lyet 12 andet verisolgen= de/ en 't telfae in't gheficht houden= de/ om alloo dic paffagie dies te be= ter te vinden/en t'ontdecken/enzijn alloo feulende vooz de paltagie ge= Fomen / bebluenoe bet lelfoe paŭa = $\mathfrak{g e} /$ of Fretum le Maire, foo bebon= oen/als be 25 ewint-lyebbers uande Auftraelfche Compaignie, haer caer = te dieg aentaende hebben upt-glye= ghelen: alleen different daer-inne/ Datle rapposteren Dat het Fretum niet loo beet en wiit en is / als het bier onder ig gljeteeckent / Dan in oe lengljoe uan feuen grijlen i 6 is ftact fu fulckis toe/ ente sil-juoe-wel Dat fu bet Fretum havaen alreuon= Den/ en terltont kontiden toos $\mathrm{fep}=$ Ien: Coo beeft de Capitano Don Iuan de More ; Lolgendeder lalt hem dooz Den Conumgl ghegthetren/het felfoe Fretum vooz-bu glyebaren / uerbol = gljenoe lyet zallot aell d'oolt-zijoe gljelegljen'en geleplt Ooft en ooft ten ₹upoen aen / tot ontrent de lenglite oft Z ongitude ban oettich Epaenfthe miilen/ an ce belichti: gljen/oft Daer noch een andoere par= fagie oft boos-torliten waren/oin in

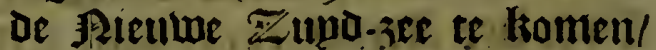
Imaer alieen glyeuonoen/ dal alleen baft gljefloten Iandt: Jubiceren= de ende dozoeelechde daer Doo? / Dat yet ualte $\mathbb{1}$ and ooftelick is loopen= De / en ftrecliende naer de Capo de bonne Efperance: Waar Doo? De twee Carnuclien weocromme gyelicert 3iiin / tocly met difficulteut alloo oe wint niet wel dienen en wilde nact bet Fretun, 'tfelfoe feer liclutelifels palferende/ in minter dan earen dach/ ende beni beuindende in ae

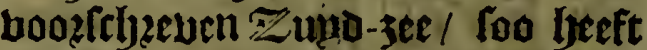
de Capitano volglyende oen latt hen gljeglyeuen/ bet voosfolyzeven lant legatjende beoulten bet boozftyze= ven Fretum, dat wp inde hollande fehe Caerte genoemt fyebluen Staten Landt, oock outrent De oertich mij= Ien beleult/ om te lien boe bet Zlant ttreet / en hebluen bebonden dat bet Z ant Daer beel beceoer was/algaen oe paftagie/fonoer eenige dooztocly= ten/ noch openinge te vintosn/maer wel boos-gneng/cen boocly/ttepl/en Klipacluticly Zlant / foo van buten als uan binnen / dat is aen.bepoe ziiden als \$oostweglien.

De boosfryetuen Carauellen kee= rende wederom Mefteliicls nae bet Fretum le Maire, jiin het felfoe booz bn. ghyefeult/ nacr Dat fu nochy in een $23 a p$ hadoen gheweet / alyeleghen op een hoeck uande felue palfagie/ aen oe $\mathbb{Z}$ upo-jec: Daer fn ulede vole heluben 
x 6 1 8. bebben glyetonoen / en oock als oe Scjepen Uando Auftraclfche Conpaignie betuen vernomen feer groo= te nentchte ball kBalvilfetyen/to in de Noest- 3 ee in oc padagie / als in De ₹upt-jec/ foo Dat lyet een farelie ban uerwonderingtye wag fulclien boop ball die gedicten te fien/ foul= ber dat fu de Srbepen uets mifae= Den. Sultoc vooets gereplt/en ge= uolefje ljet al ande (treckende aen de 3ijạe uantoe Magellanes ftract, Dooz ons glyenaentt Mauritius Eylandt, fp ontoreckeon meac ceniglie kileune Eulandetiens' die wh in onfe caer = te ghenocint Ijeliben harnerelts Ey. tandē,en oor fo uervolgende baren cours gljevonden bebuen bet Capo de Hoorn, op oierglyclijclise boochte uan graden / als die inde l)ollant = teje Caerte is gljeftelt/wefende de= ie Cacy bet Supdeliclifte II ant Dat out inve weerelt is beleplt:

Doozts uamar felfor Capo lyebluen oe twee Carauellen yaren kours vervalelye foo nae bet colferende als fu Konoen/ lisuluenoe altiit het felf= oe perfect int gryeficlite ghebouden/ en op ceniglise plaetien aen 2 Lanot slycueet / Daer fp ouer al Bolck/als te bozen verbaelt is Iycluben gevon= aen / Conoer dat fn bebben konten mereken ecnighe dooz-tochten / oft paffagien fonder upt de Magellaen. fche Atrate, nace oe zunat-3ee / als

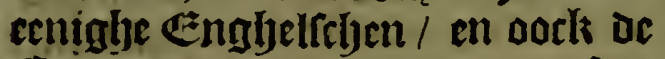
Comunandeur Speilbergen, met feer klepen fondament $\mathfrak{e n}$ wetenfriyap bebuen willen unt-gijecuen / en feg = gen : Dat van Capo Frouwacre leg; gljende ten naeften un midoc-werge ban de ftrate/ Daer een gat of Dooztoche Coude weren/oic na de ₹unosee conde loopen/twelek de 5 pan= niacrocn oock niet ghewonoen beb= ben. 99 acr in contraric alle bet Zut int of boozfthecuen Magellanes Straet oull-cingljelt / was dicht cn baft gijefloten tot ecu / fo dat liet epndelicken bliickt dat bet een $\mathbb{E} \mathrm{p}=$ tant is/'tsbene de $25 \mathrm{clwint}$-bebluers uande Auftracleche Compaignic $g e=1618$. naempt Jjeuben Mauritius Eylande, want foo dace eenigije doo?-tocisten waren / en Coude bet ghern Enlant wefen.

De twer Carabellen in de jurbzee bun bebumbende / fouden gljeer ne de culfe van Chili belen!t bebilué/ indien fu lael uan Diveg lyadje: uerfien geturelt/onn mits bec mant = quenuent ball bet le!foe/ en oat in plactre ban gljevictaliect te weicu booz twee Jaren / als de Conincls badoc geozoinecrt / qualick bives

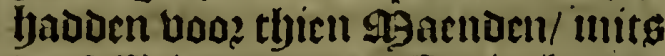
De Dfficiers banden Coninck / een acel uan de felfac ueteorlyt hadoen/ Dace mede bace pefiij onluchooslij: Coecliende/ foo zijn fu glyenoot faect geweelt / weoeronime (Fonuer meer te onder-foeclien) nae t)ups te feql= len / en alfoo baer de winot diende oll Dooz destlaet weseron te kee = reit / ende ae ftroom nea bebuen= De/siin doos de vookrchecuen 5tra= te feer vookfpocdelije uut de $\bar{z}$ upd= zee in de \$oozt-jee gljeractit: nae dat fu indestraet eenigfie plactlen badoen befichetet / en bn-ronoer ae plaetfe dace eertiits Philippus ftadt gebout uas/en dat doo? lait en be= Uel ban fijte פgajeftept טã \$pan= gien/ die de Commandeur dat bad= De belaú. Hebben oocls inde Straet eeniglie 25oomen gevonoen / waer uan de tebozfe was ban fonderlins abe gocüen renck / en foo fterclisen krachtich dat de joeper daer bu gễ comparatic en lyeft/ welclse [chyors? ren fu cen aeel mede nanten / $\mathrm{en}$ in Scville met groote begeert bercocht is felthien kiealen fyet pondot. ziin alfoo oe boo?frlyetuen Carabel= Ien bat de teraet weder in Spaets? gien / fonder cenielj ongheluclis we: Der-gljelieert/ ende ghearribeert tot booz de ftad ban Siville, bolgenide oen laft die fyun griegljeben was in Spangien taeder re keeren/ cun niet in Porrugacl, al- foe-Lucl de Capitano Don Iuan de More Uerlilarrael t. 2 Dat 
1618. Dat indian hu glyeweten yadoe / dat of Coninck te Liffebone badde ge= weelt / niet teglienitaende de booz= Icljecten latt / lju niet en loude gela= ten lyebluen in Portugael Leder te lieeten.

De tinee voozfelyzeduen CarnuelIen fiontende booz de ftede uan Seviile, op S. Laurents dach, welende de jatron uan ljet touyg van Borgondien, bebuen met reer groote triunwhe bact Glyerchut los glje= fcljoten / ende een פgan gaende op oe ltap doot-gefeljoten : en van ljet fucces ban de reule / beeft de Capitono aen liin 99 aieltept te Liffebone rappozt gedaen / en Daer ouer ljeeft de Coumek/met raet en aduiis ban fiinen liaet / ende die ball de Con= tratchtic in Seville olyeazonneert dat men in alle diligentie acht frbepen in Seville foude glyereet maken/ oin langlys de felfos jaffagie te fen, Den naer de Philippinas, met 5 olda. ten/en Zee-uarent uolck/en bootts IItet Gefrbut / ztmunonitie van - Bozloghe/Cou-werck/en aloerlep Schipg-beljoef/ on de Galioenen toe te ruffen die in de Manilles ziin/ en glyeliout werden / enmoeten die reet weren / en upt feplen tegiseng 3ouember i 6 I 9. Couder langber vertreck.

Hebben ooc die uan Spaengien gljerefolveert ouermids de goede gljeleghentlyept/'ende bequaenuept vande uooźcheven paffagie'ljaer= liicr langlyg dien wecly die inde Philippinas, ende Molucques te lecoure= ren. Aelro de Hollantílye Bilooten/ en onder andere éé Pieter Michielfz. ban Catdoelen, die mede booz $13 i=$ loot 9 a aio? / op een van de boo?l3. acbi Scbepen fal varen / den $\mathbb{C}_{0}=$ ninck ban 5 paengien valtelic toesijefept beeft/ongheluck gbeweert/ alle Taeren van Spaengien inde Philippinas, ende Molucques, te ba= xen / in arlyt of negben g9aenden/ nitats de loinden/en ftroomen inde Zupt zke/ loo fabozabel zijn/Dat fp

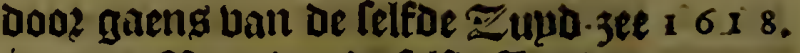
in twee shaenden de felfoe 1 anden nuet ktoeck en gljelondt Bolct tal konnen beleplen / conder fubject te welen eenige tijoen of faploen ban 'tjact / noclj dooz eenighe mouffonnes belet ziin oe Boyagie te boen. Een falie van leer groote aenifienj en confideratie/ yet 1 oofrk ghétont en frifth te bzengljen upt Europa, in of Philippinas, oft Molucques, in 8 . of 9. Elgaenden / Conder lubiect te welen eenige tiiden, windê of inouConnes, glyelijels.men anderwozpen is alfnie de felfoe wil belepleri lancr de Capo de bonne Efperance. "Tat ten regarde van de felfoe daet lante te befęulê gế contparatie norhy ges lijckenitte beeft als de erwarenthept ons nu foo langlye als de baent ges dupet becft uan oefe Bereeniclyoe 19zovintien/ op de Molucques claers licken aengetweten lyeeft/ en dat ten belten liamende de boozlchzebé rep= fen upt bollant naer de Molucques ghemepnlijek Dueren 1 4. bijfthien/ en Leltlien $\mathfrak{A g}$ aenden / en dickwils langher : en met fulcke 3 warichept ghedaen wozden / Dat genennlijek van bet Bolc en Soldaten die net die Schepen uaren/ een Derde-deel/ jae de belft/ en meer fterben eer dac fu in ofe ghediltineerde plaet $k \mathrm{k}=$ men : J ae het is gheliente dat eenigeSchepen beel meer berlozen bebben / als gbebeurt is bu differente DOlooten. "(S is waerachtich/en tan niet ontfient wozden / Dat de Sou berneut Gerrit Reynft, upt oele lan= Den met liine bloote nace BoffFndien : [efthien פ9aenden en deie daghen onder-Legljen isgtheweet/ eer bp tot Bantam is gljearriveert/ men laet ftaen inde Molucques, fyeb. bende berlozen meer als het bierdepart ban fijn bolelt. Intobelijer is de Conmandeur Adriaen Wouterfz. met lijn vier Scisepen van Wollandt tot Bantan, uegisentlien en een balf 9 aent onder-weghen gyebeeft:bebbende lyet Schip Vlif- 


\section{om te befichtighen, het Fretum le Miaire.}

fol.75.

16 1 8. Ginghen tan de felue blote hondert

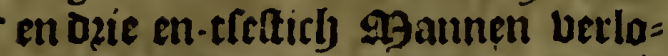
ren ban twee bonbert gsannen die bp op hadde/en De refterende feuen. en-Derticy meet liec en onbequain 3iinoe cenich feljecps were te doen. Ban gyelijelken de witten en fwar= ten 25euz / ziju oock op de vopagie

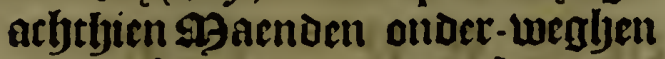
obeweet / en meer ; eer de felbe meDe tot Bantam ziin gearriveert: 1 )eb. bende defe Schepen verlozen fommighe cen quart! en een ocroendecl ban baer Bolck. De Scljepen Den Arendt Uan Zeelandt, De Eendracht ban Ámifteream, de Oraengie boom ban Hoorn, zijn onder- megyen geweet / twaslf / Dertljien ende meer Isaenden.

THaer Dooz blijckt boe jwaer de vacet be-doften de Cacp is / om de Molucques, Amboina, en andere plaetlen te bereplen / in relpect uan de nieube glyebonden paftagie/6oo parceren.

(5emiercht oat-men Dooz de booz= felyzeben paltagie/ upt dele Zanden varende met bequame faploen in ontrent biif gaenden acn de culte uan Chili en Peru, en in acht maenDen in oe Molucques, en $\mathbb{L}$ anden bier vooz uerbaelt conder verlies ban Bolck tan comen / als gbebleken beeft dooz bet Sctjip Eendrache van de Auftraelfche Compaignie, die daer ghearribert is conder Bolck te uerliefen : Daer ter con= trarie dickmils de Srbepen ban de Ooft - Indifche. Compaignie te Bantam arriberende/ bupten bet fap= foen nocljazie / bier / bijf ganenden berfliten eer fp bun repfe eon= nen bervozaeren / Conoer nody te reeckenen dien tijot / die lp moeten confumeeren oin op de Molucques; en andere Zanden te baren.

2 REIATIE

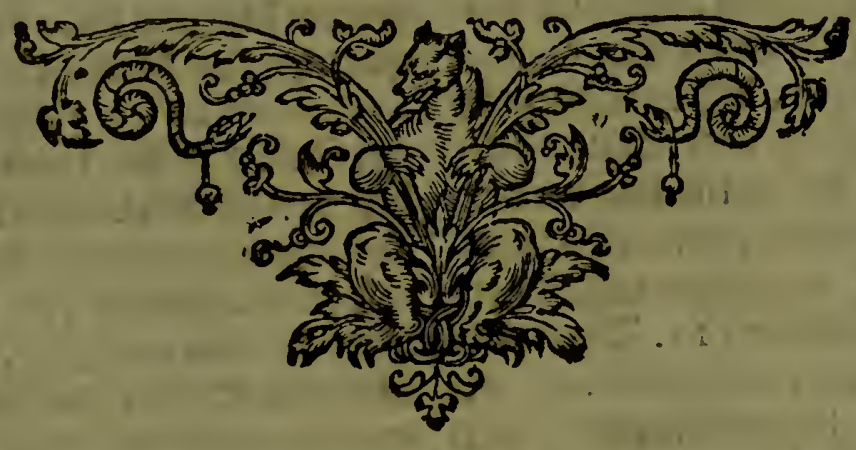




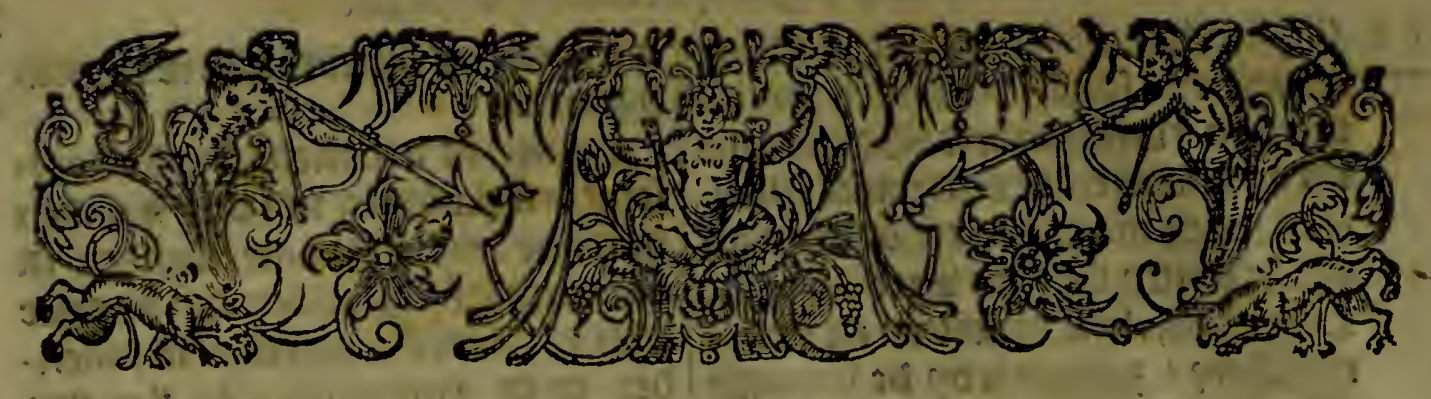

RE LA T IE in A B R E GE Gan alle oe Gopagiengbedaen nae de State ban Niagellanes.

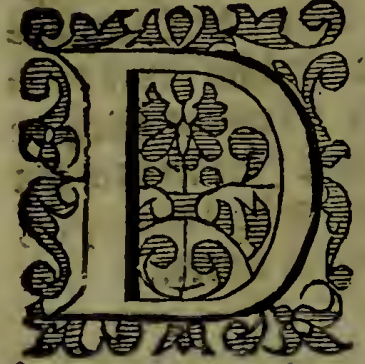

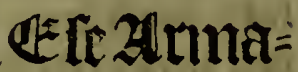
oe wat laet uer $=$ trocken gine $\mathrm{D} \ddot{\mathrm{e}}$ tienien ztugu= Iti I 5 I 9. ban Sevilla, quam in [eveangue Daghen bertoefoe / van Daer vertroclife den 3. Betoluer / nabigertbe vijfthien Dagen tot Dat= Ie quam aen oe kulte van Guinea, aen Capo Blanco, oft Daree ontrent/ Exegen hier Atilte ban twintich Da nlien lanck / ten epnoe van welcke/ hadoen een maent contrarien wint/ met tozment continuerende yacre Dopagie/ quam ben $\times 3$. Deremuer in Porto, of Rio genero in Brafil, al= waer fu lyace verberferjecion / Den

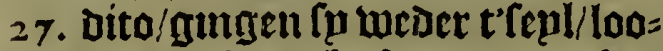
penoe lamglys de kulte. Den $7.7 a=$ nuarij i 20. ljadocn be lyoocljte van 34.graden/quamen in Rio de la Pla. ta : allwaer fu eeniglje batren verfery water in-namen / Den 6 . Jisbenarij/ verbolchoen lyaer reple/ coltrerende viif Daglyen een legua/ en finachts s. of 6 . leguas ban lant / op 40. ara oen uonoen een frboone 25 ape Sint Mattheus, alwaer fp wiloen in-Fromen/omte fien oft een \$ేtraet was/ macr glyeen gronot biniende om te anckeren/ liepen ban daer booktg in

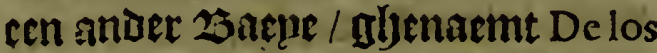
Patos, bonden aldaer ech lilepn Ep= 1520. lanot vol tioblen / $\mathrm{mr}$ lomguing/ veruolgell oe vootta fyaer repre/qua= necn ill een frjoone bape De los Trabayos, bun noozoen Port S. luhaen, de welclie fjum bequaem bocht .om te lyiterneren / om dat dacr te lande de zBinter begont tekamen/welen= :lpzil/en lyadố hicr groot toament/ ucrttoclien bau Daer de kultê lantr/ quamen den pBalm-fondacb in Porto S. Iulian op $49^{\prime}$. graden/Daer bun goet Docljt te ljiberneeren/ Cont uan ljier een Sefhip uoozocr aen/oul een Straet tontivetien / en vant twin= tich niilen verder een frboone thi= viere S. Cruz de Mayo, uonien ueel Biffechen en Zee-wolven / wan Daer willenoe vop?der feplen / quam een tozment dat lyet §chip teghen bet Jant gljebleven is/ nu werende ge= pafteert de s maenden van de win= tcr/ A pril, Majo, Iunio, Iulio, Augufto : vertrocken Den 24. Augufti, upt Porte S. Iulian, en quamen in RioS. Cruz, allwaer fp vertoefoen en ber= uerichten/ norly twoe inaenden Sep. tember en Oetober, feploen ban lyier ultimo Oetober, coltererende al zupte waert met beel moepten/ Dooz bet quaet weder / tot Dat'e quamen aen Capo de las Virginas, Den 6. November, feplion fp inde 5 traet/ den 27. 
Tweede cnde derde Reyfe door de Strate Magellanes.

I s 2 2. November, in twintich dagen qua imen fp inde $\Xi$ upd-jec/ ende namen haren cours nae iNoorden, met groot tozment / tot den achtifienden December, Datfe de hooctjte lyadoen ball $32 \div$. griden/ende gingljen uan Daer met goeden windot haren cours

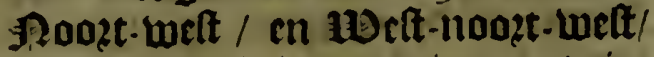
tot aen of 3 inie' Den vier-ent twin= ticlyeten December yndoen on de hooclyte ban fes-en-twainticligraden befupden de $\mathbb{Z}$ inic / yebbende meer alsoertich dagen genabigeert con= der Iantet te fien: In't lactfe out $=1 \leq 22$. occliten fur twee tilęne Eulantoe $=$ kens / Die onluelwoont waren/ Daer niet ten belten was / diefe noenden Defienturadas, Guido feglyt datfe Deqie naenden en twinticly daglyen fenloen fonder $\mathbb{Z}$ andot te fien. Anno I $;=2$. Den felten Scprember is lyet Sctjip Victoria in Sevilla gelionten/ of in Spaengien ghearribeert : ljeb= bende ap de rente gljeweelt 3.jarem/ [eg-en-twinticl] dagbetr.

\section{Japagie bande Gebepen bande 25imchou van jolatcuce.}

\section{DON IVIIERES}

CARVAIAL.
Etweede Dlo= te nae de Straet Lun Magellanes, is gbeweet toegherult vall de 25ifleljop van Pláence: jiinde in't gujetal van

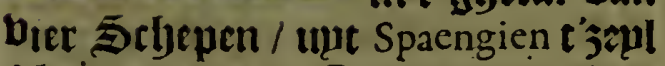
gyegaciv/ nac of Strinete/ oim die te paffecten/ om te gaen na de Molucques, allo oe bookftheeven 23 iftelyop Daer toe licentie baode glyekregben van de lieperer / met cen feer booz(poediglye windt ziin fu gibeltomen aen Dê Straet bã Magellanes, alwaer komende / ende wefende iil-glyeba = ren twintich miilen / lategdjen cen Itercken weiteliicken bint / Coo Dat Dzie Sclyepen teglyen bet Iant Dze= ven/aen oe Zund-jijoe / en geraec= ten gebeel in ftureen/ende het vier = De Schip Deefe wedeconme te rug= guje te $\widetilde{z} e$ / twelck als de ftroom gepaffeert was / lieerden wederont in De Strate / coeckende fijn Com= paigmie/en bont beel Boldkguande gebleten Schepen aen 3Lant/gaen= De langlys de thrande / maer de felje. pen waren tegen let Lant vergaen: bet Bolck Dat aen 1 anot was / riepen tot de glyene die in't 5 chip waren / maer oc Capitepu confidererende de klepnljept uan fiin इrbip/ enoe oe weunige Dictualie / vooz to beel Boler/wilde bu ljaer aen Xant niet gaen/maer berbożoerde lijn reple en pattertoe de Straet/en oul dat bn alleen was / relolverec iju niet nae de Molucques te leulen/Dant fetten fijnen cours naer de laulten uan Peru, en de ftadt Lima / aldaet aenkomende is lyet Srljip daer blijvenl leggen tot op deten dach/tot een mentozic : De $29 a f$ daer uan fettende booz het poalepg/'tbolck ban d andere dete frlyepen die inoe ftraet waren gisebleben tot het ghetal ban twee hondert en vijfticl $\mathfrak{A g e n}$ [rlyen/ waer af de Capitepn genaemt was Quiros, jinde nietners gheljoozt oft vernomen / tot op Den Darfy van gunden / wefende ontrent veertich Faren gheleden. 


\section{Je Derde Jopagiena de Straet Magellanes, Doch langtyd de Zupdt-sec.}

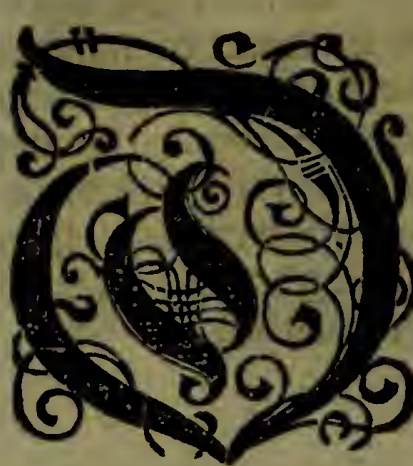

verteur uan Chili, Derwaerts len= Dende twee Schepen / met een Ca= pitcun Ladrillcro, welcke Capitenn Defelue Strate langlysdie jijoe gelagic is glye= weelt om die langlyes de Zuna-zijae Dooz B.Dze vã DonGar-
E Deroe lep=/ bonoen en gliepaffeet beeft / maee te ontoecliêl çia de Men. doza, 5ou= en Doeft hent niet berftouten / de Strate of de Engbte upt-waert\$ aen te loopen/maer is loo baelt als yp de \$oozdt-Zee berkent fyadoel wederom te rugghe gljekeert / booz bet berbolghen enl quaet beder/ om Dies-wille dat oe Bointer Daer al begonnen was / en waren naer lijn fegghen de baren ban de pookt-jee hell gemoetende/ Teer werljeven enoe ontifelt / en gantedy wit frbug= mende bau verbolghentlyept.

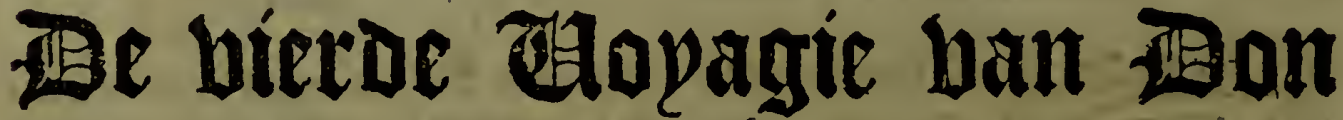 Fray Garcia, Lofre de Loayja. Anno is 2s. U1pt- sheronoen / met les Sebepen vall laepler Cacrle, om vozder t'ontderken.}

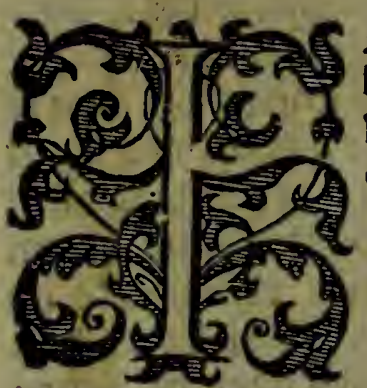

P Iulio gingen fp bau S. Lucar, verberferten int Eulanot vall $S$. Mattheus, $\mathfrak{l} \mathfrak{p}=$ lenoe van Daer Den 3. November, faghen fa Den vierden December, Brafil op 20 . Iraden: hadoen ban 14 , tot i 8.Ianuarij beel ltozm/ in't incomen uan oe 5 traet verlozen fueen \$rbip/bu Capo Virgine, en quamen oen i 8. dito in de Straet / meder raeckte= Den fu daer upt/ met berleglien lae= Der/en furkeloen in Rio S. Alephonfo, ende in Rio en Porto S. Cruz, Daer renige abupren en dílozden waren onoer't Bolck / gheractkten den 8 . A pril weder in ot Straet : Primo lunij quamenfe in de Zupt-zee/vin- bonden oock in Rio de Cruz, I'AfDende aen weder-zijdé van de mont piden ban beeloerlepacrt/en fteenen uan de Sotrate feer beel Eplanden op 48. grauen : yadoen s2: ungyen in oe Stratc booz-gelzoclyt/cu ber= lozen yaer jontaclje. Den bijfoen Darby Dat fu upt de Straet litpen/ Kreglyen fueen barde ttozm bart de Srbepen ban ect gfjerackkten / en berlozen de Capitana, die fp nopt meer faghen / duerde alloo bier oft viif baghen/datte glyeen lepl moch = ten boeren / Kreglen beel waters iu / berbolghoen haer renfe naer de Molucques, ent quamen aen in Mindanao: Sิu vonden aet de Straet Magellanes aen oe culten beel toalo vifteben op oe ftrandat/ die daer gberaeskten dooz ttozm en tempeets/ enoe ae berbolgljen Zee'op (muit/ twelc een teecken is ban beel quaet weder/ en hooch ulocpent Woater/ pie 
Reyfe van Sir. Francifco Draeck nae de Unagcllaenfcbe sitraet.

die blocot tempen / net ueel rari- | Doclj goet om t'eten : 5egghen meteyten/ bonden daer pock leer beel De/ Dat d'een en D'ander zijde ban Tejoone ende wel-rieckent Houbt/ oe Stract bewoont ís. en teer beel groene wilat Cancel:

\section{Gopagie ban Sir, Francifo susia Draeck nae De Magellaenfche straet. an in Jilisinit anno is 77 :}

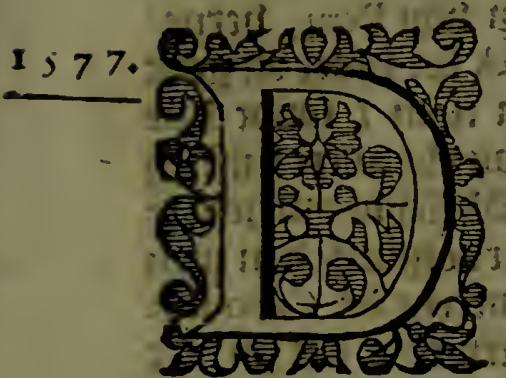

Cहn I s. Noven ber reulde iju upt Pleywouth, 0 oos contrárie wint quan we. Der tii. Denis. Décember revilde Iju looz de tweede mael unt/ nuet uiff Sclyepen groot'en klepn. Ben 2 s. quairien Ip bp Cabo Cantjen in Barbarien, ocu 27. quamen Ip aen't Eeplant Mogador, uaex lp hun pounalte op letteDew/ Ceploen booft Den 30. Decemb.

I s 7 . Ben 17. Ianuarij Anno I 578 . quaneen Ip aen Capo Blanco,iuren taben / en Celyeuben ban Daer Den 22 . Ianuarij, en uen 27. Uyilla de Majo: ben 29. quanen tuin Si lago, en nanien lyet Selyip uan Mino de Sylva, en namen lyeni mede/ om dat Iy cen goet jiloot was/gingljen ban bier naer bet equlandt Brava, en buloen enigfje Baten met betrly 1 bater/ namien baren cours. Uan bier naer Brafilien, hebluende antrent de, $\mathbf{i}$. nie dzie we Éten tilte/ quamen'tlant Uan Brafil te fient Jen eerlen April, op de lyoochte ban 33 . graden/ Con. Der eens 2 ant te fien/oft vertely water te nemen / baren cours berbolghende / tot Rio de la Plata, Daer Iy in repliden op 53.54 . undent/ende refepteaen beriely Hoater upt de Lii. biere neffens Scheeps boot / boost Ieploen ban bier/ende deoen faren cours uooztaen op tot op 39. gra-

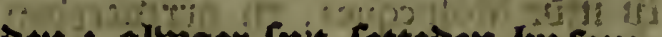
Den : almaer fp't fetteden up commialje clippen/ligghenoe vooz een 25ay/op de welcke ucel thobbe waren. en berlieten een Scljip of twee dat lp berbanden/alles Daer uutne mente / ende berbolghende ljaren coirs met Dander bier Srbepen/etr quamen Den 20. Iunij in Baya des las Inlas, aft Porto S.Iulian op 49. nraDen/- lagiben ljier 27. Dagben / berberichende haer: Ten I 7. Auguft, gingljen lp ban ljer t'repl/ liepen langbs de Kulte benen / tot ontrent $x_{\frac{1}{2}}$. miijle Leeclyg ban lyet $\mathbb{Z}$ andt af/ want bet ig daex al feboon en fupuer/ ban 20 . tot 25 . Badem dieptes en waren bier oft luij uaglien onder wegen tot den mont vande Strate/ de wint contrarie jijnde/ bertoefuen eeniglje dagben / eer [p uaer it liepen / en begolten in te gaen met een Boft \$0ozt Bolte wint ben 2 I. Augufti, Den 24. begonnen ly de traet innewaertg aen te loopen / en quamen dien dacly aen bet tiobuen $\mathbb{C E}_{\mathrm{p}}$. lant: binnen in be Straet laclende / liepen oaer booz nae baren will conoer eenich oberlalt ban weder of inint/ Jen 6. September, quamen fa

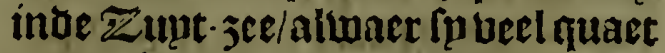
weder hadoê/ en malltamberen uerlosen/ten epnoe van melctie fu baer

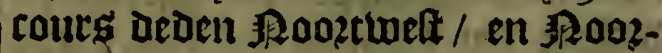
beu de kute langijs / beruericljende aen fommiglje Eplanden/ban Dacr baer cours nemende nex zupde wainDell / langly of wal / quamen nae ¿2 Chili, 
Reyfe van Sir. Francifco Draeck nae de cuagellaenfche Fr äet. Chili, ontrent S. lago. Den 17. Februarij i 579 thuamen fp bioó Aquapulco, in Nova-Efpagna, ban daiex t'feulgaende/ quamen op 43. gra= Den/alwaer fu oe locht to kout bon= den / dat fp fyaer nualijek konden verwarmen / van Daer quamen tp op 38. graoen / in ecr goeoe 25 ape/ oben atuit Nová, Álbion, in Ámerica âjeleglen. Den I 3. Oetober,qua= men fu aen cerighe Eeplanaen/ghe= leglyen aclyt graden be-noozden de I inie: Jen 14. November quamen ly inoe Molucques, $\mathfrak{e n}$ arribeerden in Engelant oen 3. November $1 ; 80$. en whiren wipt obluedt twee 3 aren/ Io isaenoen. Staet te noteren bat on in fevet ssaenden endo bier Dagijein feptoen ban Enghelandt in oc Straet: en oo of De stract in 16 , Danficti. Bock bat M. Iohn Win. ter ban de Compámie ban Draeck, wetentic inet banoet sthepen tie = feilt Dooz oe strate/ en welenae in ae wint/ niet nutret Leder/ is oet 8 . October van lijn Compitimie gbe= dimatic/loopende weoter te tugotie yooz de Straet/nemente fónt contrs naer loupg : en komt den ir. November weder upt de Straet/ in de Foozt-3ee/ palferentue beftratet witoer in fes en-twintich Dagben / en quam den tweedé Iunij Anno-1 5.79. weoê in Enghelande, bethithenbe tiloingtye/ yoe dat bie op ss. graben befupoen de Straet was gbeweet/ endealdaer ban ben gberaects.

De Bice-Lop uan Peru, ueme= inentoe Dat Dracek bet fillier en gout upt oe Styejen nam / Conbt boge Siblepen/met twee hondert bifftion פhanimen/ inet Ghefebut om betit te toecken/ en te terbdlghen/ băn bem niet bintoente ontent de Capo fint Franc, enoe betnentento bâ bu nitet wag ontrent Panama, mepnoen fp bat bu booz de straet we ber lwas gftefepit/ Daer ober is de blote bes orroin gbebarkn.

\section{Gapagie uan Pedro Sarmien- to, al-andevaren van Lima Anno is 79 . nate be Stratc uan Magellanes, om de felfoe ban oe Zupot- jijoe te gaen recogmófceren.}

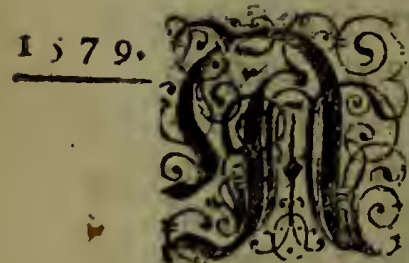

Pno 1 s79. To Draeck inde $\mathbb{Z}$ upd-zee langs de kulte vâ Peru bas iljemeet rooben/ko= mende Dooz de Stra= te ban Magellanes, tón yeft de Pice-tiop Don Francifco de Toledo, onimie daer inite te berfien/ en Iteinilfe vande faecke te betbien/ twee goede 5 chejen toe-gljerritt be welclie bain't Callou de Lima, t'sepl triigljen Den I 1 . October : Den eer= âell Novemb. pafterdé fp de zan= Den Defventuradas, op $25 \frac{1}{3}$. graben. bet eerte 2 allat dat [n berkenden was op $49 \frac{7}{2}$, graben/ alwaer lp qua= men met ien boosfpoedigbe repte/ui min als bertich daghen: loopende jee-waert in oin oe zzupde winuen te fchouben : alliter Dan bonden rpats in Archi-pelago veel ceplanoen /'en Sarmiento arguiceroe dat oe Straet fier moife welen/ twelch tp ondertorbten/loopende in uerrethepben 2 saruen/Tnwijctien/en CanaIten / Kliniviende op be hoogbe obes betrften/en ontoecten berfebenoen groote Zngangher / Ribieren/ en 10 oten/ bonden niet als glieboten en gyefedyento Xanden / tellenoe tuateben 
I ; 7 9. tufredyen dit en uifftirb graaen/meer alfr 8y. Eulanden/ groot cn klepu/ liepen ban bier weder zee-waert in/ alfoo fin gheen Straet lionden vilt= Den/en lireglyen grooten ttozm ende ollweder / foo Dat oe Scljepen mal= kattoeren berlozen' en niet weder te liet quamen : t'fanderdacelys glye= ouctoe nochy bet ghe welt bail wumbe dic blwars of te wal was/cenfiende Dc opentrine ce bet cannel int $\mathbf{x}$ ant/ Cagbell dat bet meer en meer inneLacets aen wag itreckentio / fchoon ell Diep : waer Dooz Ijaer berfeeclie. rende Dat aldaer de Straet wag/. bebonderi aen de boorlite oes Solis si griben: twelclis oe rechte boorlj= te is uan be Straet aen de Roortziibe: in De Zupdt-zec ondecliende

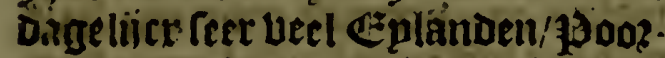
ten en libicren / Daer uan yp pos= rellie llah booz Den Coninck ban Ditpanieñ ? alle de plaetfen noems. De bujuet berfebeuden nanten : cn vonot op feecker plaetfe oljenaemt Porto Vermejo Befters vain be zet onet-inne waren teer fcljoone peerleii. Doende bain fier voozts fiin bopagie inde Strnte / vernam In bain de Intwoonoers Dat Draeck aldaet gljepalteert wag/ en verfpieDenoe een gelesentljent aen be eng = te vari be Straet/ om die te dupten/ en met foitereften te betwaren/par= reerden boozoer/en febben ueel cammunicatien/met o 3 Trimoonoers en = at Gigantes abeljad t ban De Straet: bolgljens dat-men in Argenfola le ren marb: ziinde alloo met goet we: Der en fonioer eeniglje zwaricjeut de Strate beel dooz geloopen/Komen = de aen of Roozot-zee ujit / en nam ban Daer fiin cour\$ na Capo Verde, tII ban Daer met liju 5 chip na Hifpanien: alwace bu replae nae bet iqof/ Daer bem fiju agajeftept groo= telijcks begacfoe / en dede op liin berfoec em groote Bloot toerwiten/ onder Diego Flores de Baldez om de Strnet te fortificeten.

Dukecrende tot De Bite-2abnii= rael/ die doogit onweder uectecelien x 79. was: Deden baer nuecrfteluefte $\mathbb{z}$ cewaret in te loopen / maer onl dat de 0 wint Dtwars op de wal / en feer giseweldicls was waepende / mepnoen flu vooz gljewis Dat fp vergaen lou= Den bebluen/en op de wal gberaeckt (alloo bet ontweder dzie dagen lanck Duerde) maer'tghebiel gantich cor. tracie / want bebonoen dat bet lane meer en meer inwacts/ enoc (Boltwaerder aen was wijckente / bet onweder gljedarn zijnde/bebonden baer op s6. grnoen / cm waren noch berre ban $31 a n t$ / feer bertonondert jijnoe. Hackuyt feljziift datfe in oefe navigatie oock waren op s8. graDen gbefeplt / bindende be-3updert De Straet becl Eplanden/ als oock een ander Straet/ beweleke Acolta oock affirmect / reggbenoe van een ander go ont / of Tintamite gelegen op meerder boochte/ Dooz oozfaechs van een reker groot Eplant gelegen aen oe Zupt-mont ban de Stract am de Zupt-3ijde / Gent la Campana. Ban bier reploe be Bice-sido= mirael oe fitute langhs nae Chili, m. valt daer nae Lima in Peru.

Anno 1;81. is booz be Conincts ban Hirpanien ( Too op't berfoect en perfuafie ban Sarmiento, als doos dien den roep was/Datter in Enghe. lant wederóm Stbepen nae Magellanes berden' toe-glicmaeckt) na de Strate vijefonden Don Diego Flores de Baldez, met 23 . Tepien / enoe 2500 . 99annen / Daer bur een nieu= wett Gotusernetur nae Chili met bijf bondert Soldaten unt pederlanot: inve Sefepen labende beel mate: rialen ont een fortrelle te maken it De engte uande fract/bolgende der raedt / ende bet aduijg / ban Sarmiento die met dete Srfyepen oock ginck/ met-nemende lent beel atmmonitie met beel bolekg/ agannen en Fzottoeu / om de Straet te papuleeren / ende een Colonie te maecken / Daer ober dat defe Sar- 
Eerfte Reyfe van Thomas Candisch, naer de Straet Magellanes.

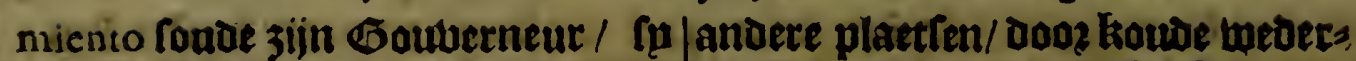
waret Faer eit Dacly eer oat [p in om keecende / en eenigbe Sctjepen be Straet quamen/ hiberenoe twee berbzoncken met beel Bolckf.

ofte Deie reph in Rio de Genero, etl

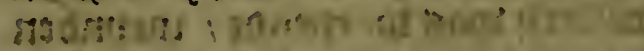

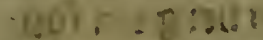

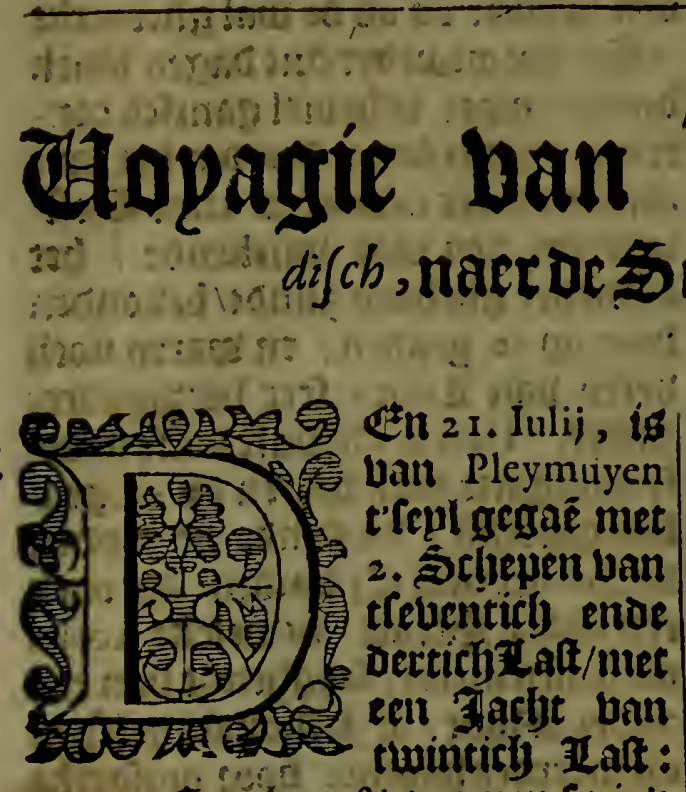

Den eerteli Augufti quamen op in't ghefichte uait Forte Ventura. Den neglyenden Augufti quamen ip in Capo Blanco. Den 26. dito qua = mentu in Sierra Liona, albaer berberlteden l.en tardecroen to is. da= glyen: Den 10. September boeren fp wan Ine Verde, of Madragumba, nae Brafil. Prímó November badoden fy Capo Frio in Brafil, (p verberlteden in't Eulanat S. Sebaftiaen twintich baglyen : voroer gingten fu den 23. November 't'repl/ Den 17 . December nuamen lp in Porto defire. Anno is 87 . Den 3. Ianuarii quamen lp aen Capo de Virgines. Dett letten Ianuarij quamen lp in de Straet/ en quas men bu oe 5 paengiaerts in Porto Famine, enl lagljen een 99 aent ttil. Inde Stracte waren $\underline{p}$ belet boos

ftozn en onweder tot dê 24. Februsa. rij, Doen quamen fp inde zupt-3ee: Zlinde in feuen meecken Dooz de Straet ghetomen / en krcgben op negben-en - beerticly ende een balt graden / bijf en ueettich Leguas ban t'Lant een 3 waren ftozm / upt den Roozoen / betlielende cen Schip wut bet gefictje dat in groos periics kel bas: : Den viifthienden Meere quamen fu onoer bet Eplandt fine Maria. Detn vierden November quanten lp aen bet Jant ban Califormias op 23 . graden. Den beers thienden lanuarij 1588 . quamen (p inde Philippines, Den eerteen Martii, quamen in in een thatuen bes 3upden lava Major. Den biifden September quamet of weder in En. ghelandt, op de rejgg geweet zijn de bifen twintich 9 aenoen biifthien Bagben : En waren thien Ggaenden achthien Jagben bano oe Straet tot inde Manillas : ende waren ban Pleymouth tot in oe Stratt ban Magellanes bijf 918aen= den lefthien saggen.

i.:

마
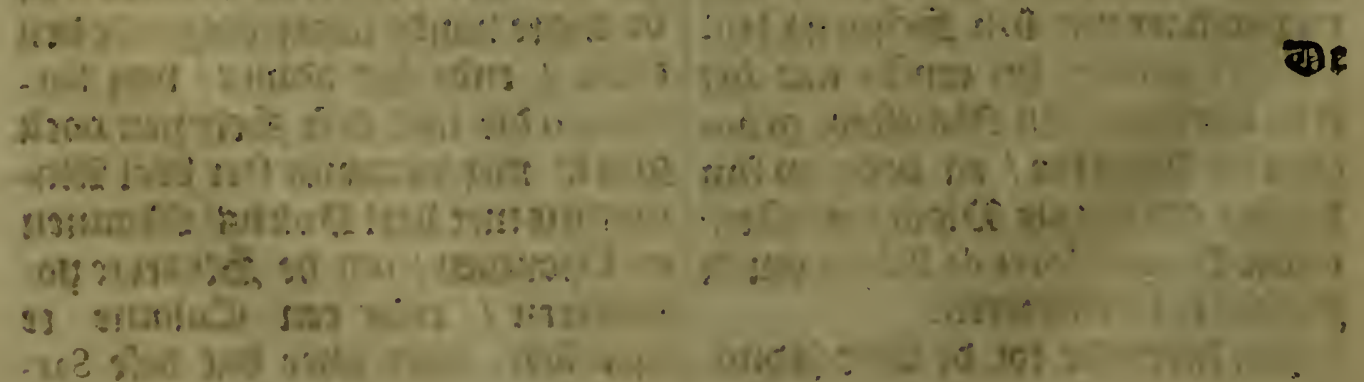
fiol.7s:

Tae tweede ende laetffe Kregre

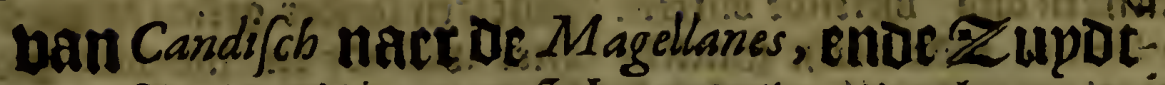

Zere thet doze groote Srbepen en twee:25artken.

1591.

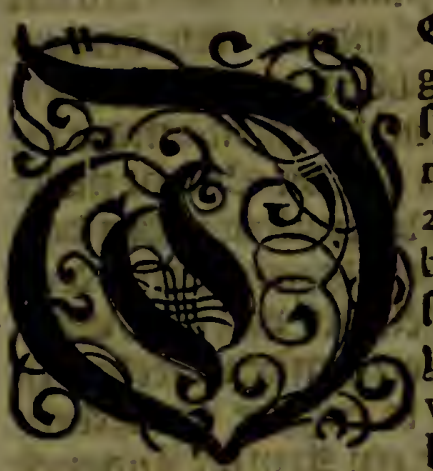

$\mathbb{E n}=6: \mathrm{Au}-$ gurti fenloen (1) uan Pley-. muy $\vec{c}:$ गु 29. November quamen Cu bu de ba= ue vant Sal. vador, op be liut vâ Brafil, twaelf leguas aen oefe zijof Capo Frio, Daer fp ban ftilte belopé wierDen / glieduerende tot Den tweeden December, Bp oien tijt namen ta een hlepne 23arcke/komende upt oe thibiere de la Plata jut 5 uncker en Bearog. De Sebipper bant 28 are $=$ ien b2ocht ljaec aen ieen Eplant $P$ ha. zentia, Dertich leguas Boeft uan $C$. po Frjo, Daer arciberden fu den s. December, fo 000 - fochten en plon = berben fes oft felo fuplen bemagnt uan Portugefen; : Taen ri. Dito [epl: Den (p ban Daet: en Jen 1 4.quamen [u aen S. Sebaftiaen, ban waer (p bertrocken on in te nemen bet fte= Deken uan de Santos: Ben r 4 ! 'tta: bonts ancfierden fy aen de 2 sarte ban Santos, en Lp quamen g'anderDaechs furozghenditot oe plactie: namen bet Stedelsen int en kregen baer beet berberffbingde met gbe= welt / nemende de Jinwoonoecs ge. banglyent Auptentoe die inoe kerek. OE $2 z_{3}$ Ianuarij Anno is 52 . vettrac: ken fu uan oe plaetfe: en bzanteden S. Vineent tot de grone af : Den 24. vito ainghen fu t'fepl en fetteden baren cours naer oe 5 traet. Den 7. Februarij hadoen fu cen groote Gozm. Den 8. dito was de boloote ghelepareert/Dooz de furie ban tem= peef. De Capitepn refolbeeroe te gaen na Porto deGire, op 48. graden.

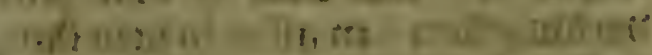

Den 16. Martij quam bi baer of $1 ; 92$. gounafe / Joopteden dat de Sene= rael daec komien foude/ om dat bu. fijn erfte reple daer goede betuer. rchinglie ghenomen badoe. : Ten: 6. Dito quamen fu met twee Sefbepen in Porto defire. Den r 6. Dito quam bu de boop de jDunate. Den 18. quamen Tp alle-gader bpen/ maer een barcke was weder te rug: ghe guekeert naer Englielandt. Jeet 20. dito bertrocken rp ban daer naer de Straet. Oen 8. A piil quamen fu aen of Straet/yebbende onder we= obeu gbeleben beet gróte ftozmien. Den is: paffectuen ifp dooz de ere:-

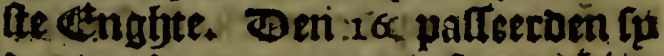
De tweede nawnte / welente thiet leguas ban oe eette. : . Tean 18: Dob:blecroen qu Capo Frouward: leggenoe op brie-en-biftidy en een half

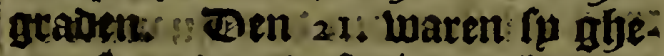
nootfaet boos de furie ban get we: oer te toopen in een fmal gat 4 . le: guas vanue Capo aen oe zunt-zijoel daer fu bleven tot Den bijfthienter May; in welcke tijt fp leden extreme koude / en ltoun / met glyedueriglye Ineew/en hongher/leefoen bu 250 Celen/water en lirupt ban de Zzed Daer dooz beel ban bact ftieruen. Capitepir Candrich wel wetende dat ve konocen fineeu uiet langhe oue: ren fonden/ badoe geerne booit geweeft dan meeft de Compaignie/en 't Bolek wiloen dat men een anoer reps foube aenuanglyen / of weder mae Brafil-Loopen / maer tijn refolutie wast te gaen nae Capo de bonne Efperance, dooz dien datter ban alles gbebieck was/ ende veel ellente waren fupposterende : lop wert ter conttacie gheperfuadect te gaen naer Brafil: foo dat fu den is. May II 3 meder 
Twecde Reyfe van Thomas Candifcb nae de Strate Magellanes.

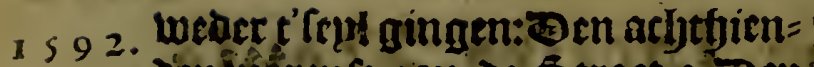

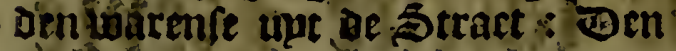
twinticfaten dito / berende ontrent Porto Defire, inde nache ginck be Gencrael ech anbere houts too tp menuden / darer doo? lp van bem verowaeloen / en alloo [u ben call= Decoacclys nìct en faglyen/ waten ip uan usectumglyc dat lju gegaen twas naer:Porto Defire, vindende aen de Iundet-sijoe van ve Porto een jout met berfcl] Bater. Den feften Augufti baer Oenerael niet beeriemen= De/ Ceploen naer't Pinguijns-Eýlant, Ieggende beneden Porto Défire, uan Daer letteden fu ljact kours weder nae de 5triet. - Ben negljenden dito baoden le groote to $2 \mathrm{~m} /$ roo dat fp haer feplen molten in nemié. Den bectthienben waren fq onoer ecri= uige Eulanden te voozen nopt ont= Deckt: I Iegghende i s. leguas ban't 1:ant/Doft aen be Roozoer Ip ban be Straet/ op de welcle fp conden ghedreuen bebben / en bergaen/too be ftozm niet en hadoe gheceffert/ Dant De Linot Boltelijet loopenoe/ fette ố fp baer kours nia de: Straet: Toen achthientoen dito quamen Ip In de Capo, net een teer bicke milt/ Den feluen nacht anckerden fu thien leguas ban de. Capo. Den neghen = thienden pafteerdé fp de ecrlte eng= te. - De tweede endbte den $21 . \mathrm{Dob}=$ Uleerden of Capo Frouward. Den 22. dito anckerden $f \mathrm{p}$ in cen biepe Ganael/ of ztem/ Daer fp beel wil= oen fagljen : nict tegfentaende be extreme koude / liepen defe Xupoen naeckt / chl leben in 25ofechen ende Eiloerniaten / als Satpzs / fu jijn upt-nemende fterck / ende bierpen uae baer met Ateenen / ban bier of biff pont / een onglyeloobeliiclse di= Amtic. Den vier-en-twntitg(ten feylden In ban daer $/$ en quamen

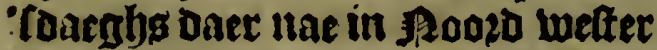
tinetk/oft Canael na oe ZupDzee. Den 2 s. anckerben $\{p$ ueerthien le= guas uan be Zupb-zee / willente in bie plaetfe bare Benerael berwach= ten / alfoo yet daer maer dacicleguas i s $9:$. bece en was $\%$ m lin niet en lianoe pafteren Conber gefien te wogzon. Hier welende tn lijoende groate koude / en of oictualie op gaendel uonoen fy goet te gaé na de ₹unajee / aen't éplanot S. Maria , in een gyétempert Climact / om berber= feljinglje te bebben/ende aldaer ben Eencract te berwachten/ont dat ly nootwiendicly aet dit eplant nicte kamen:- too bertrocken fp den bers thietioen Septémber, $\mathfrak{k}$ quame vien

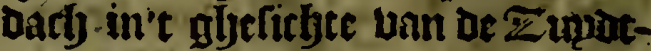
3et: Ben beetljienden wrraen of weacromme te rugghe abeazclem! en quariten in een 2 trm oft 3 ngand orie mijlen inde ltract / Ip fettratat ban thier weder boogts / m wetente acht ofte thim leguas Uan't Innut quam de wint feer futrieufelioth unat

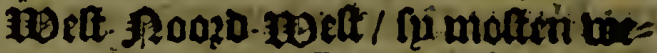
Derom thaer oe Stract te rusgity baer fu weider op de relfie plactse mickerden. Don tweeden Oatober, gheraecleten Ip weder in be Euput3 eet bzp uan alle x anden. De bols gbenoe natht begonft be wiviot we= ber feer te wapen ban noeft-noosmeef 1 . roo langhs foo nieer toz-hes mende/ foo datsp niet wilferi bat kours fu nemen fouben / Doof aien

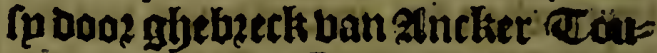
wen Gaet in de Straet' niet en bot= ten meder. begbeben. Den biat. den ende vijfoen Oetober, enve fos boozts/nam be ltozm al aen/foo bat fp inbe unterfte ellenbe waten:ente kregben ueel Haters in / maer bere elfoen October, tagljen tu de Capo Defirado in In van baer twee mï= len : wetende feer verleghen on thie te boubleeten / b00z dien Ip anders: aen't Iant melten brijben/ getax: liende te met bet naer bet $Y$ ant toel op een Galfmiil ban frant gaffyar Sobt dat Ip de Capo Doubieeroea/ Daer bp langlg vooz ober feplenved tot cen Setjecpg lengte of wepnich meer : Daer ban liepen fp in- batert be Strate met fulcken furie bau bint 
Rejfe nae de frraet van Magellames, van laguïs Mabu ende Symön de Cordes. Jiol. 80. wint / troom/ en barm/ Dat [u [cul=|gheweeft 3ijnoe cen-en-twinticl) en oetl buf-en-twintich leguas in tes $u=$ cen balf פacnden

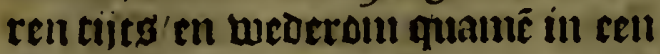
Slop ten ancker! en loo Lecoer aen Pinguijns Eylandt : Lerende uen 25 . Oetober. Den revé-en-twinticljeten dito / waren fp weder in de $\mathbf{R o s} 2 \mathrm{-}$ jee unt ban de Stract Detl ber= ticheten Odober warm lu belleden Porro Defire, aen de Pinguijns Eyfanden, loopende in't Porto om te 1593. verberfifyen : Anno I 593 . Den der = tirljeten lanuarij, quamen [u aell llá Plazen! ia aen de kulte van Brafil: en Den clfDetr Iunij itt Enghelandt, upt.

- Cis oock Laeraclyticfy dat Anno i s 9 s. x 595 . unt Enghelandt gljebaren ig Capitcpl Haukens, die gyekounen is inet bet Stljip dooz de Strate uan Magellanes, Daer zijnoe / beeft cen [ecr groote fto?m gljebadt/ $\mathrm{cn}$ is met kracty uan wind jupjwaerts aen glyeloopen tot op s6. graden/: Conder cenidy uat iland ghebon: oen te yebben / maer wer eenighe Eplanden/ onder de buelcke ljp bel 45. Dagljen navigeerde.

\section{Jopagie nae de Stract ban Magellanes, van viff $\$$ rbeunn van laques $M a s$ bu, ende Symon de Cordes, Anno is 98 . uan Rot- terdam aljefeplt.}

1598.

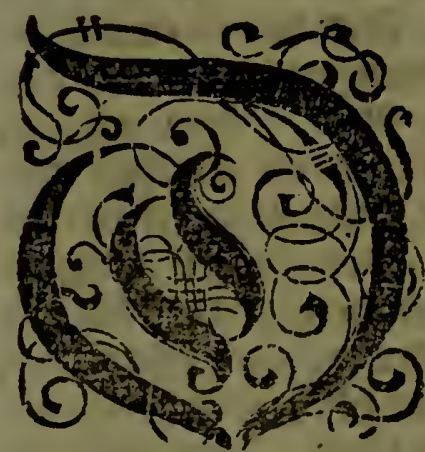
En 27. Iu-/ liin sut berballen op de Culte ban nij unt Goereefche gat tiin ta t'fepl gegaen met viif Sthes pen (โp qua $=$ mein laet eer fu aen de li= nie quamê/ oun die Conder contrarie wint te paf= Ceeren : welende outtent den leften Oetober, eer dat $\left\{\boldsymbol{p}\right.$ op $1 \frac{1}{3}$.grnden be $=$ filloen de Tinie waren: wefende Atif uier $\mathfrak{A g}$ aenden/ welcken tijdt $\mathfrak{f} p$ meeft met onnoodiglye exploitten oooz-bzaclytet / als bolglyen fal) nu met een Roost. Oofte PDint / upt Goeree t'feul glyegaen lijinoe choe onder Ėugelant comtende ziin/ Doroz contrarie wint in Dupns ghefet/ tot aen uiifthienden Iulij, Lederom t'epl gaende badoen eerft den tient= ben Augufti de booclite uan Capo S.Vincent, den neglentlyienden dito
Barbarijen, verre bupten haer gillin = glye/ ban lier bebben $3 u$ Gaer courg gljelet nae bet Eulanot uan Capo Verde, ende cregen den laetlten Augufti lyet Eulant S.Iago int gefichtel Daerle gbeerne eenigbe 25 arque of verber'́rginge betomen yadden/Dan areghen niet. Primo Septembris quas inetr $3 \mu$ onder Ina de Majo, albter gbeen water noch verbertrhingbe becomende / gingtyen $3 \mu$ ranuer= Daeclys t' 'Tepl nae S. Iago, al waer $3 \mu$ een oft twee Sthepen aengbeljaelt bebben/van baer berfoeckende booz gyelt/ met bzunttlyap eenighe bers verteljingtye / maer niet ban ozep= ghementen tot antwoozt crijgiendel yebhen $\boldsymbol{p} \boldsymbol{\mu}$ met ghewelt een plaet\$ àen tant fugbenoinen / Daer naer weder met bzuntrelyap bertoeckenide verberfelfinithe / wader niet ten be ten als Crupt en Xoot / foo dat 3 p met sbewelt berwaterden / oock doo? de onglyelonde Locht / werde alอar 
Reyli nae de fraet van Magellanes, van Laquis Mabuende Symon de Cordes.

I 9 8. aloaer lyet wolck fieck. Ten fetten Septembris ginglien ju weDer onoer [eul. Den thienden dito verlieten jul lyet Eulant/ Den elfften fiin jl! in Hila brana glyecomen / en daer bets= benle niet aroote mocute eenich berfely Hater lecomen / al waer $j !$ pock niet als vpantrclyap en bon= Ḍen: jiin deii uijfrhienuen Septembris Lecoer t'feul ghegaen /. met een \$ookt-Doffen itoint! courg fetten= De Sunt-Bolt: Den vierentwin= ticlyeten dito $\%$ is oe Generael alje $=$ ctoruen : op dato; betuonden ju haer fiecl diclyt bp lant $/$ ap regent twin= ticly Badenten waters: [oo dat $\mathbf{j} \mu$ den viif en twinticlyeten dito ozoze Itelden op lyumme cours / van bier af begon bet Srycurbuncli Dapper it be bloot te regmeeren. . Den ne= niben en twanticliften dito bebben $3 p$ yaer teps neerticly ueruolelyt / ya= ren cours lettende Sumt-10elt ten

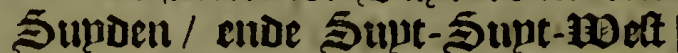
tot Den vierden October, Doen lie =

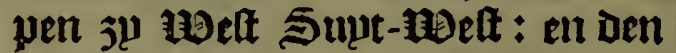
feften dito wederam (Boft. SuntBoft aen. Den acht en twinticly= Iten bebben 30 een tyenteene $25 i 0=$ bach glyelyouiden / welende op de boochte bail $I$. graden/ befupden De Linie. Den tweeden Novembris boos de lieckite van't bolck werde glyeraetfaent gevonden eenich lant aen te foecken / aun te berberfchen/ foo dat $j \mathfrak{p}$ haer courg naer Anno. bon beblien gelet \$Roort Bolt aen/. en wetoen teritont het Zlant glje $=$ waer / veel bupten giffinghe : ju ineenden bondert/en bondert twiil= tich en meer 19 ijlen ban de wBalle te welen/quamen op de foochte uan deie graden be-fupden / aen De $\mathbb{C}_{\mathfrak{u}}=$ te van Manicongo, en $j \notin$ liepen van bier te lande nae de Caep lopo Gonfalves, tnoe de Cult ban Guinea, al om verberftrhinglye te becomen/ Die niet veel en was / norljtang met het wepniglye quamen be fieclten meet wederom op oe been / maer Doos de quade ell ongheronde Iloctyt die dact is / weder invallende / Yjeluben i s 98. raetfacm glyebonden den neghen: aten. Decembris uan Daer t'fepl te gaen nae. Annobon: en den feftyien= den nae inidoacly bebluen ju't onder bet Ezplant ghefet/al daer bet Bozp inmentende / en met glicwelt vers uerfichende/ Doch foo is doos de on= glyefonde Iocht weder beel neet bette Cootten/ en andere Sieckten bebaugen : Yjet Scljeurunuck was ghenefen / maer t'ghetal der fiecken wasiniet ninder: Ben twecoen la. nuarij Anno I s 99. met cen Bupt- I s99. Sunt-Dolten wint lijn 3 unat Annabon t'teplghegaen/nae ac Straet Maghellanes, yaer cours fettende Siut-1bert. Den twee en twins ticliten op $8 \div$. graden / faghen $\mathfrak{j}^{\mu}$ Afcencion, Den laetften cregben $3 \mu$ de Sonne bovent thooft / ell voozt uarende / waren jl op $20_{3}^{\prime}$. graden/ Dé. Abrolhos pafferende / met een Foort-Boften Hount / lyaer courg Supt-1)eltten Suuden Ben ne= gyenifen Maert Jonden $j^{p}$ ljaer op $4 I^{\frac{1}{2}}$ graden. Den Defie en twintich $=$ Iten bito hadaen $j u$ twee oft Daie Das gljen groote 9 sift. Den acbt en twinticlyten dito de boorbte van so: graden / wierpen gront op trer= ticly Baben. Ben neglyen en twins ticlfften dito de fyooclyte van vijftich graden/yndoden taclytenticlj Baorm gront / en een gljelweloiglyen Itorm Zaelt-Poost-1Deft. Den Derticly= tenen eenendertichjlten dito / met

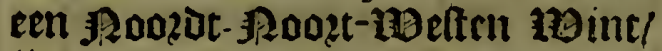

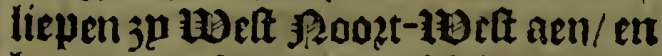
hadoen op reuen en telticly/entachs tentich Dademen al goede Sant= aronc. Den eer(ten April , faglyen $3 \mu$ 'tlant \$2ooztgyen/ yooch lant/ent wierpen gront op les en Derticly Dadem. Denle le iten A pril quamen ju in of Straetl met een Sumben point / wefende nuneglyen פgaenoen en negen das ghen / dat $3 p$ upt lyet Baderlinat ojefeplt waren/en ltif dzic Ała๕n= Den dat $j^{p}$ unt Annabon gheleult 
Reyfe nae de fraet van Magellanes, van Iaques Matios ende Symon de Cor àts. Fiol.8.1.

I s 9 s. Waren. But alfoo defe tijt be Win=। ter feer bart aen / quant badoen 3p berfchepoen foztupnen en tegthen= Cpact / Doozt t'quact weder / twelets Dooz oe coude / reglen en bonglyer bet bolifli feer berfwackite. ₹p jad = ben in Dele tiit int voozlte van April biif oft fes Dagljen gacbe bolt en Roost Bolte woinden / in welcke tiidet $3 \mathrm{p}$ de Straet Couben connen patcerel / maer dooz de verberf: frfjinghe bant t'volck / Fater en Gout baelen / als oock topletten yall een 5locpe / verfupmoen 34 ben wint bolconelisek te gbebzupe= ken/ghelick ju beboozoen/bebben= oevan oen feuenlten tot Den twin= tichlten Aprilis, redeliclie goede wint om te pađerely den woint Daer naer veranoerende van ben Suщs oen / en Supt-Weften / en als 3p wilden paueeren conden niet/ alfoo De DSap-פaent daer in of thinter is / met lece beel quact weder gbe= nootlaeckt sijnde / in be straet te berwinteren tot oell opie en twin= ticheten Auguft, $3 \mathrm{~g}$ lagben in oe groene 23 ape met groote armoedel op dito ginghen $3 p$ wedet t'cepl/ alte-met weder eeng anckerende / tot batle den tweeden Septembris, met een Boft Sunt-Bofte mint $t$ ' ginghen/ ende den derden lnachts oe Stract uptgelcplt / comende niet mop weder in oe Supt-Zee / beb= bendenu ontrent frs פgaenden in = De Straet gijelweelt. Ben bijfoen en reften ginglyen $3 u$ noch al met

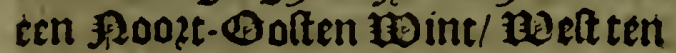
joozoen aen. De les Setjepen/ waer onoer de Sloepe was / noch albp oen anderen wetende / tot op ben fevenden September, begon bet ten Top- [epl te wapen / Doch noch glyeen ftozm / foo dat de Zee die Daer feer baeft ontitele ende berbef= te: fulcr dat de twee Sthepen / die Liefde ende de Trouwe, Daer of Weert, ende de Cordes waren/op oe Zije molten werpen/om baer boots in te retten : Ondertufteben de
Weert volcht den alomirael/die voof 1 s 98 . upt was / ter wijlen ober quam yet Jacht genaemt de blijde Boorfchap, Die loefwaert vant $t$ 'Geloove wag/ ecil onglyelucts/t'welckecen beginfel vanoe berftropinalje uande B loote mas/ Dat lijn 230 ug-\{phict/en booz= atengye ober boozt biel in perijekel mede vall oe focke-matt te berlie= (en booz bet glyemeldicl) (tampen bande Eee/ waer oooz Ijp alle of Seplen inmemende/een leboot beeft glyefchoten om bulpe. Capitepn de $\mathrm{V}$ cert liende beeftet oock op de 1 ije gbewozpen / en fiin Seulen inghenomen oil bp lyem te latell desuben: als oock dede oé Trouwc : Bndertulteben lyadoet Den stomirael laten vopzts Caen/ Doch oe Liefde bp oe anderen co= mende / nammede fijn sopeplen in: Too dat bier de bier Schepen/ van oe bijf bp een waren : Cozts baer naer ifter een dicken asift obebal. len / loo Dat $3 p$ malcandecen nite lien en conden: Docly loo bleben 34 ben betlen Dacis loo bp den andes ren Dzijbende / ondertulichen was oe Jacht befich om fiin graften te repareren: alloo dat oe ozite Sches. pen Des anderdaechg ben acheltent September bet Jlache met de Sloes pe / De 1 boftillon mitten / want $3 p$ met bum 3. Scbepen Dooz de groo= te en lwaerte meer nederwaerty ges: Dzeben waren : jp Dzeben dien dach nocl) bu ez/tot g'anderdaechs fmoz= ghens dat be Bite-2lonirael be Liefde(want yet niet gljeraden was langber alloo oxijuende ben gaes Den wint te berlupmen) een teet: ken dede van fepl te maecken / bo: penoe de twee Jachten booz upt te vinoen: gbelijek $3 p$ oock twee oft bzie uren baer naer t'fepl gaenve te loefwaert ban bun int gheficlyte creglien / die 3p met blistiffap in ghewacht bebben : hopende ben zomiral mede te vinden. Cordes en de wecrt leenden elck baer reime merman aen't 3acht/ om wederom. 
Reyfe nae de Stratt van Migellanes van Taques Mabu, en Simon de Cordes.

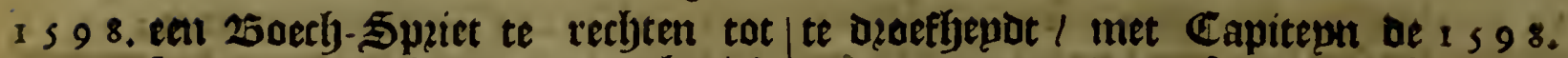
baren groote fryade en ongljerief/ want $3 \mathrm{p}$ die tredert niet weder en fagljen: obermits ju ganderdaerlys Den thienden Septembris de munt eregben upt Den \$oozdt-metten/ waet Dooz ju het wenden / en Sult-moeft aetilicpen : alloo de Zee Doo: or mint bace Dapper weder begon te verbeffen / yeeft de Taebt tegleng den avont liju 5 ep= len weserom inglyenomen/als oock oc Dice-2tantiracl bede / vooz unt fijnde / twelck t'Gheloove en Trouwe fiende / lietent mede dzijuen/ Doch alfoot nu Donclier nacht was/ Gaoden de Tacht ende de BiceLlomirael feul gljetuactht / conder eenicls teecken te doen / immers foo beel defe twee nacomende Sche= pen fien ofte fyoozen mochten: al= too bleef de Trouwe bp bet Gheloof, Jopende dat de Liefde ghe= waer wozdende / bat 311 niet ghe bolebt Leroe/ mede ope oxijuen ftel Ien foude / hier-en-tulferyen hiel de Stozm even Dapperiân : licht ghe = inozaen fijnde en facghen sheen fepl: t' welck vooz defe twee achter gljeblebeu Capitcunen een bitter beroziet was : maecke oerbaluen Capiteun oe . Weert een clepn feult = ien/ende nae Capitepn Cordes loo= pende / am met beni te befunten/ bat $j^{\prime}$ op malcanderen wat lou= ben palfen/ en fonder weder tece kenen aljecr Sepl te mimaeren/ noch meerderen : heliben su daer meoe wederont tepl gljemacelit/ $b_{0}=$ pende haier ghereltehap te acljter= balen: / of ter bettemaer plaetle te binden. Dien Dach twelck was oen refthienden Septembris, en de moint ten naeften bu wertido / en daecbs daer naer met ect Supden/ en Sipderi ten tBeften wint/ noely al den feluen caurg houbende / ver = ballen op s4\%. graden be-fupden ben Mont ban De Stract. Cen felfoen daglje ttictf de Scljipuer wall of Weert, ent loer alloo in groo= Cordes boozt : Oen felfoen bach liep de mint Supt Bolt ten $B 0=$ ften / loo dat $3 \mu$ un Roozoen ten metten aenboeren- en Des ande= ren daetyg oen mint Supt-5upt-

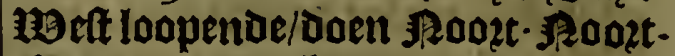
Boft aen. Boch Den neglienthiens den weder Roozden ten MBelten wacuende/ yieloent ten naelten bp. Cen felben dagye begon lyet met

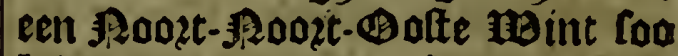
bart te maepen / en foo breecelick te Itozmen / dat 34 meeft fonder Seplen Daijuen moelten / in groo= ten noot ban omghelwozpen / of de fijoe inghedaghen te wozoen / crij= gbende veel tiBaterg in / in lulcker voeglyen voeren Defe twee Stje= pen bier en twintich daglyen / in de Supt-Zze / meeli daijuende can= oer / oft met wepnicls Sepls/ over= mits de gheweloighe en ghedue righe Stozmen : malcanderen foms tiits des mozgheng mifendid boch altijots meder vindende/ mits ju op malcanderen wat beter ghelet hebben: Zperegljen den vije in twinticfyeten Septembris weder eet barde ftozm upt den Poozoen / roo dat $p^{\prime}$ 't weder lieten Quijuen / boch daeclys daer naer clepu fepl maet= liende / liepen met een wefter Wint foout-mett acn / en ber = viclen die nacljt be-poozoen de Straet op it slanot: Conder bat 30 het lelfoe dooz de onnekerljent olye= fien lyadoct : oock gheen giffinglye baer op glyetnaeckt belubnde / mitg 3t lyun saer uoch wel 20. miil len af giften / si waren beel berbaedi bact op de lecgher nal bevin= Denoe/ met foo veel moints / Dat ju de פgars-5eulen tiet conbett boeren / onder-tulfeljen weraen $3 \mu$ het Iant keimende / want $3 p$ Ijaet Dzie Mijlen boven de Straet be= bonden/ en dooz den melten wint gheen midoel fijnde on van I ant af te comten / befloten tramen be Straet mederom te hiclen / oun een gocde 
Reyic nae de Straet san Migellanes van I.tques Mabu, en Simon de Cordes.

Fol.83.

1 598 . goede lirede te Junoen / bequactiI weder en wint bertwachtende: om De Bloot te volgen aie twee 9 gaet= oen in lyet Enlanot S. Maria fottoe wachten: siin alloo booz de wint weder naer de Straet toeglyeloo= pen / met de focke lancks bet lant feulende / mits of woind unt oen Poozot- \$oozdt-20eften feer fel $=$ leliicli wacpoc : liepen 5upt- Ooft ten Simoen aen / ch quanen te=

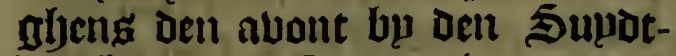
boceli ban de Stlaet / innewaets loopende / oul een lieede of 23ape te Coeclien : $3 \mu$ beuonden dat $3 \mu$ aen cen leeclier 2 ape teglyens de nacht willende Luljonden dooz den ftroom in een nache wel trs oft feuen $98 \mathrm{ij}=$ len te Strate-waert in ghedzeben waren / cil quamen in een 25 aue/. verwacljtende bequacmljepot om ball bier wederoull inde $\mathbb{Z} e t$ te loopen : ju hadoen bier cenigbe da = gljem tamelick weder $/$ yoe wel de wint meltelick waepende was tot Den laetlten September. Jen eer = Iten October hadoen ju een groote Atazm/ upt Den Poort-noeften ten ßoozoen / Dat $3 \mathrm{u}$ booz Dzie ztnc= kers leggtien moften / bet weder Daer naer twat Itillende ! hoopten altijt bat oe Somer beter weder foude inbenghen / macr werden feer gljeabufecert choe badoen noch iil twee $\mathfrak{g}$ aenden Daer Haer naule enten dacly / om baer Sevlen te dzooghen/Den beertlyienden bad= Den 3u wederoin geweldigen Itosul

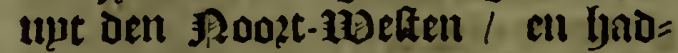
Den Daghelijelts oen enen ftozm op oen alloeren / met glyelueloiglye 23 upen ouer het gljeliereljte ballen= De/ Dat hate gljeen Couwen mach = ten bouben. Somun ju verfteten Ijier neglyen $\mathfrak{g}$ anenden tijts / inde Straet / Conder opt t'Sepl van de lieede te bebluen / oun lyare tijt/ en alle abantagie wace te nemen / de

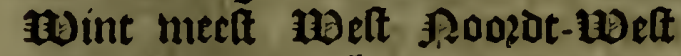
waeuende/als oocli den derden Norember met groote §to?m. achetten December "oberviel haer i 5.98 . fulcken ftopu als 3 tu out gyehoozt bebuen/-t'water bem tor of Iten=0 glye tor verlyefendoe / ecng cen lutte! tiits de wint banl den Bolten ch Roozt-Ooft bebluetioe / waren $3 \mu$ onder [ent/ en gljeraecken oe Wect en Cordes eleli op een berfetjentan plactle ten ancker / Dooz de contra = rie Wint/ foo dat jus nate den elfoen December malranderen niet meer en laegljen / maer de ll ccrt ghe?

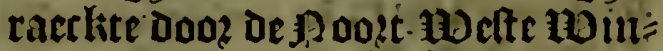
Den en ltozmen/ noclsureer Strâtwaets in/ tot int bje. Olivier ont= moete den Teltlyienden December; int midoen bau of Straet / ben twintieffiten dito / met cen SuytBofte wint met den anderen t'fenl gaenoe/ quiam teritont oc wint we= Derom roelt Roozt-nelt. Som= ma allo oe Weerts 5 bjip lyed buul/ en in twee Jaren niet frljoon gljes maetlten was / conde niet leplen/ nocly Olivier boldglyen / Dooz duen bu loo niet lauecren ch conde / is ber = halben ban Olivier uerlaten: De Weert ljadoe woots in oc 5 triet vele fostupuen / en fuaricheden/, Uerballende ban d'en ineonvenient in yet ander! tot dat ly bumten Ijope jijnde / wan bu Cordes te co= men/ of bu oc Dloote / niet teglyen= ataende of groote diligentie die bji dede / beeft dooz noots halben en per faulte van ceniglje .2002t $O D=$

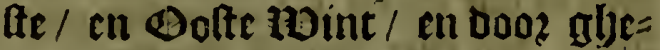
beecli ban alles/ de' Stract moe: ten uerlaten/ en nae buщ kecten: upt de 5traet feulende den ren en twointichften Fcbruarij : nae ont $\mathbf{3 \mu}$ Daer in negljen agaenden badoen gljefwerne / oen bier en twinticly= ften Martij quam Ije op de Culf ball Guinca,en midoden I Inio quam lu int Goereefe gat: nacr dat lyu unt glyetweelt lyadoe ontrent bije elt twinticly פgaenden.

Cordes met lyet Seljip oe Trouwe, gtjeraeclite wederom in de

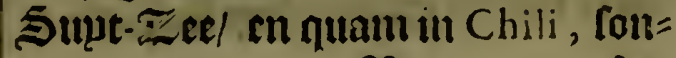


Rey fe nae de ftraet van Mazellares, van Iaques Mabue en Symon de Cordes.

I 59 8. Der fijn Compaignie te binden/ ro $=$ menoe aldaer om berbertrebinglye/ nameen Stedeken oft 5 fozt in wan De Spaemiaerden/ en plunderde het

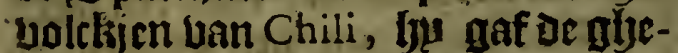

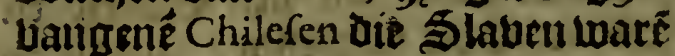
b2ii / twaerom oe ?ntwoonders hem tot Coninck wilden op-berpen. Bant bier gheloopen lantr oe Cuat/ uan Peru, willende fitin gheecelfrilap volglyen / nam verfthepoen Sclye. pen op de Cufte van Peru, en uan Daer quam ga in De Molucques, $\mathfrak{3} \mathfrak{n}$ Tidore Daer of pottugeelen met werratet yem lyet Sochip aflyensicty maeckten/ en den Srthipper Swarte Theun naer Malacca glyebanglyen ghevoert.

Be Jlacht uan Dirck Gerrits Die=

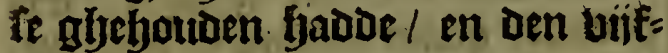
thientoen September met oe Liefde, ban die twee Sthepen te weten de VVeeris, entibe Cordes waren afgheowaelt : $\{0034$ s sanderdatchs ghemaer mierden / Dat bet in oe wint was / en de VVeert bon feventhienden op 54 ; be - Supben de Graet berviel: en den negthentyienden re-

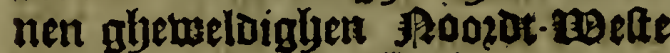
twint waetendoe/Datte vier ent twin. tich dagtyen lanck meelt conder fepl

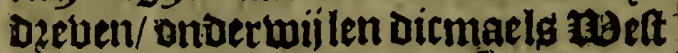

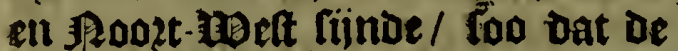
VVeert or straet ghenootfaeckt wass wederom in te loopen/ 0002 alle defe rontratie EBinden if ap. parent Dat Dirck Gerritfz Die glyebzeck aen liin 25oecly-Spziet / en forkematt haode / foo berte Supt. waerts is ghedecesen / namelick op bier en trétich graden ur-fupben of Stratt op die tyoothre weetenot/ fact int Supoen leggen lyeel yrooch 2 Berchachtich lando / wol Sneeus / als flet Il anot van Roozweghen!. beel Lit Gebeckt / en ftreclktede liem ails of het wae or explanoen van Salomon twilto loopen / van Irier is gh nae Chili abeloopen / eñae tyet Eulant van S'. Maria mittende / al

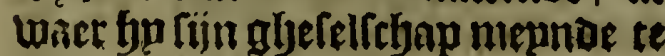

vinden / is inde taben ban S. Iago, 139 ,: re Val Parayfo anghbecomen / en gyeen vaientetlyap bindente/ inath. reloos zimbet ts in be banden vaan riin upandent moeten vallen.

De Dice-2lomirael de Liefde, eaer Stripper op wasa Iacob Quack, m Conumid Melechior Uâ Santvoort,

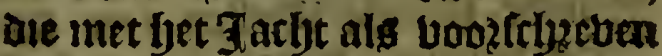
fepl niactlite / uan o'ander 2.Setjopent t'Gheloove en Trouwe berftateSen / is apparent mede zupt arn geozeven gljelwertt / oaer naer noth. tan is ha mede in Clisli ghesaectat" aencomende int Ëplanot $S$. Maria om fijn geteliclyap dat Daet beltent was te warbten/fijnoe nae alle apparentie van $t$ 'Tacht afglyeraectit/ t'belck als voozfelyzeven is nae $S_{\text {. }}$ Maria oock ginck: Teplenbe clck in een uertelyepoen placts: foo tat oe Liefde wos aen het punt uan Lamapie, aen bet balte lant om ecrighe uecberfichinghe te crijghen / maet alloo bem aldaer 23 . 295an boo? oe Indianen ooot glfefmeten wozonen/i ban Daer gheteplt nae Iapan met bat

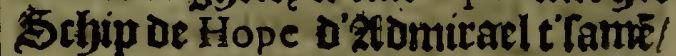
wefende aêt etplant Maria in lapan wel ontfangen/sijn daer gbebleben.

- Hoope a'sunirael unetentor onoz de Straet in be Supt. Z ece th alleen boos upt feplende / wert ban

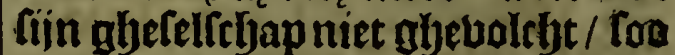
booz lyet quaret weoer/als bet on. ghelnck ban $t^{\text {? }}$ Iacht/ en anoer atci. oenten/ Coo dat Ip alleen ghebleben is : Wuefende vock onderwozpen oen generalen forden ftoen ban foopt Poozt-20ert/ende Woelt/ghedzeden werdende ten 5 upden in 54 . Dagm/ fijnde boen ter tije leer cout/ op t'lete redeliche wint wederom trijgenDe/ berboleboe fiju reple na oc Cuit ban Peru, berliefende dan D'cen dan banber / quam op de Cultevan Chili op tes en - veerticly graden/ Daer fint beftenut was malcande=

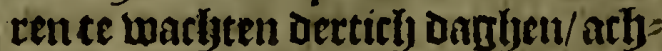
tervolghenoe twetcke foo bleef top fyier lexorlyen acljt en twinticlj oa: 
gljeu / verbetfebende / bindende / te: en foo ftelden $3 \mu$ yaer coursnae bet bolck uan't lant goet ban taa= 'tezplandt $S^{2}$. Maria, Daer aen't ba=

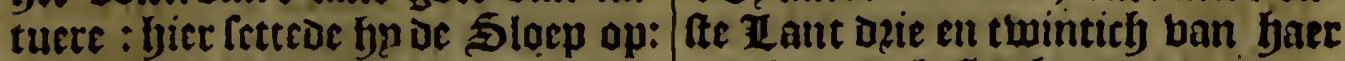

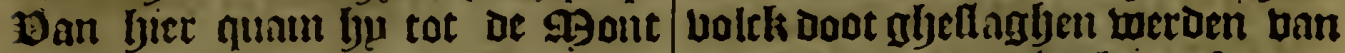
uan Baldivia, Doos fterclie Woint / en De Indianen : en gingben t'faemen liep Dace niet in/ Imacr nae la Mo. hun cours Lan $S^{2}$. Maria naer Ia.

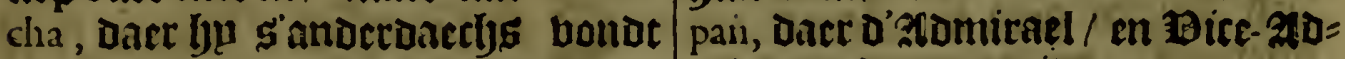
Jet Styip de Liefde, wan de Dloo=/miracl t' lamen arriberden.

\section{Japagie / ban Olivier ban

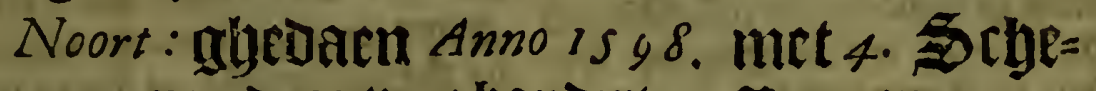 pen Doo? twee lonoert 48. 99 annen.}

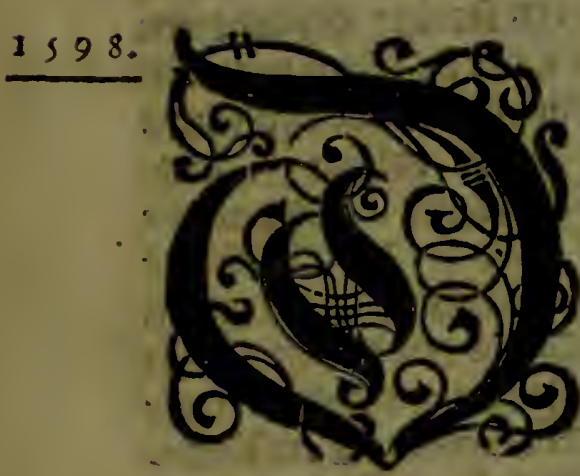

it periickel op't lant te teplen. Den 18. Dito $/$ op elf graden quam of Woint contraric / tut oen SuptWeitetl/Detl Derden November, wag oc 1)int noch 5 upoelick / cn had= den uan oen elfeten October allze eft tn Supde foinden/dace ntede glye= lavecre tot dat 30 berbielen opt zlant ban Guinea op dzic graden vier פrinuten. Den elfiten Decem. ber, quamen jp onder Ine of Prin1 s 99. cipe: Anno is 99 . Den cerften Ianuarij faghen $3 \mathfrak{p ~ A n n a b o n , ~ o p ~ D a t o ~}$ bebluen $3 p$ baer cours ghetet nae Rio Ianero, Den tlwaelfoen dito be= gonnen $3 \mu$ beel lieck polck in De Bloote te crijgyen : Den Derden Februarij faghen $3 p$ Brafil, op twins tich graden. Ben neglyenten dito quamen $j \mathrm{p}$ in Rio Ianero om Uer = berfichinghe! Dan werden met ghe= welt ban lanot gijeljouden. Ben Celthiendell quaemen $j \mu$ aen twee onbekende $\mathfrak{E}^{z}$ plambetiens / Daer $j p$
Ued \$) alnites en g9ofelen von $=1599$. oen : Ben twee ent twinticljeten di= to : quanten aent Eplant Sint Scbaftiaen, Dacr berbericht fyebbendel sijn ghereplt nat t'eplandot S.He. lena, Dan bet is haer gyemilt: Den Dertichften dito faegtjen $3 p$ weder Brafilien op twintich graden. Ben twecoen lunij fagben $3 p$ t' Ezplandt S. Clara, daer jp nootwendich mos Iten landen/ (bebbende maer acbt of thien ghe (onde 9 annen) om te verberffeben van be palmiten en

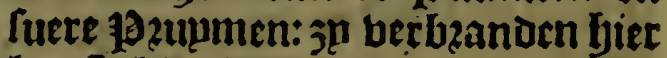
yet §chjip d'Eendracht : Den Derticly= Iten quamen $j \mu$ weder aen tot S.Se. baftiaen: Ben twinticfiften Septem. ber quamen $j u$ in Porto defire : ber: vertichende nuet Boglielen / Rob. ben, Pinguijns, en Eperen/ Daer mede $j_{p}$. leex ghelterckt / en meet op de been comende/ fjeblen $j p$ de Stljepen frljoon gljemaeclit : 3ijn op den Derticlyeten Oetober be: Der in ₹er gberaeclit / ben biet= Den November quamen $3 p$ aen de Capo Virgines, en bonden baer yet water bel leben Badem op en ne= Der blocpen. Den bijfiten November feplaen jn in Straet / nae dat 3p ucertljien 29 acnden / tot bier toe ladoen t'foeck gbemaeckt / en wel bondert $\mathfrak{B a n}$ ber loozen : Den vier entwiutichlten bito quamen fp lyed 
Reyre van Olivicr van Noort.

I 598 . ill of 马tratt / nae dat $j^{\mathrm{n}}$ Dit tot vijf Mlaels ingyelepilt yadoen / en ele = ke rcule Doof contrarie moint in atoom untaljedecten waerm/ Doo? De theft-jijoe bail oc Straet bon= Den $3 \mu$ Sebalt orll eert, die wel twin= ticl S9illen Doos oe Straet was gljeweet i cul weacrom moft kee= ren : Dewect wasteer berwon= Dert ouer ae uetticleepat / en glind= biclyent van Oliviers Galten / Die feer diffooft waren / Dooz dien j! ouer al berberfetyen / en oberbloet van alleg gefyad yadoen/begleacts uan oe. Weerdt ter contrarien feet magher en đapjijnde. Olivier is ntet de Weert fauonts onber lepl glecgaen , dati booz de oulueleplt= hepot / en onglyemaniettheut ban oe VVeerts 5rbip/ niet counende De Capo Mauritio bouen leplen / of laueren / moft te rugabe blijuen. Olivier boott aen pafterende / vont feer variable Stroom/ en Ebbe/ elt B̀loet. Den néglyen en twin= ticljten Februarij, quam hy in be Supt $\mathbb{Z} e e /$ llae bat lup in oe Straet wel hondert-mael fenl ghenaeckt GaDde/ en was ontent vier agaen= ben in oe Straet/ Date hy feer qua= lick/sn met Leel moente dooz qualu. I) u uonat veel tlepune Ejulanden ju Capo Defirado, lantks be Cult Uan Chili, Ieggljende Den twatlf=

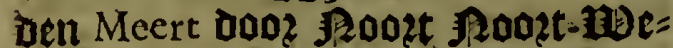
fte ftozm op [es en bectich graocn/ berloos lyu fijn Bite-ztomirael upt jet abeficlyte. Ben een en twin: tichifen dito quam Iju aen la Mocha, daer tamelick berverfejende. Den viifthienden September, quan fju in of Straet Manille, wefrente 6!.99aenden/ Dat lyp upt de 5traet Magellancs Las gljefeplt / Anno I 601 . 1601. Den neglentaen Februarij leul= be lin nut oe Straet Balaboam. Den viff cu twintichiften A prilis vernam Ijy t'Z int uall Capo de bona Efperauza , Ocu viff en twintichten Äu- gufti arriveerde bp in de $29 a k g /$ met 180 k een Schip/twaelf dnghen min alg Dzie \$aer uptgheweet bebbende.

S Ir Franfoys Draeck, $\mathfrak{l}$ epl $=$ Nora. De Den felten Septembet upt De Strnet / in be Stupt= Zee / Den fruenden dito een gract longitude wernac ban de ggont Uan oe Stract / creech fultké ftozhy Dat lyu wel twee hondert leguas Supt waerts ban be asorit ban= De Straet Decef / al waer jp quan op bijftlyien graden en neer: in be Gauen ban een leetker cejlant ten ancker/van waer de Bint uan ber Supden comende/fettede fijn rour: yeel poozt-waert tot op $5>\frac{1}{5}$. gra den/aen Commiglje onbeloonos Eplanoen / Daer hp verich matec en rommighe trachtigbe Crupoen bant/en loopende een wepnich bot: Der / quam bu aen een 23 ape / Daet vont lyp naecktegrgannen en 1 zous wen/batende in baer Canoen/ bau D'een Ezplant aen Dander om boet tel/ en jp handeloen met yen fultx als ju badoen / vinoende aldaiet beel ghewogbelte : Jebont mede dat Daer/ ende oock aen D'uptterlte \&u= landen de Sonne opt minte acht araden aen Tropicus Capricorni (ijnde / Den 趹ht maer feljatrs twee uren lantk was / merckenDe Daer hu als of sanue in $\mathrm{Ca}_{2}$ pricorno colnte 7 Dat bate ganterly ofeen natyt is 7 Draesk wederom hebbende becomen de foochte van de Strate / nam liin cours ßoontwaettg'en quam Den 29. Novenber in la Mocha op acljt en Dertich gra= Den : als lye glycloopen badoe van t'5upden van de Straet arbter= ony / tot de ljooclyte van be joopt. zijor ban de Stract / Conder landi tefien:

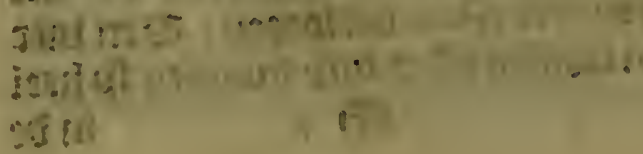




\section{YOCABVLAER: \\ Dft Cale ban deplanden \\ $\therefore=$ Salomonis.}

F. En. Tacij, oft Taci.

E. Cuce. Loua, of loa.

खie.

Bite.

Buif.

stg.

Cinien.

Comt $a \bar{e}$ boot.

Bact wech.

Becljen/ latn. - Backela.

Een Doulue. Herri.

Een Berrken. VVacka.

I oen of kiecke : Omo.

Be wint.

Augin. s.un

Bitch.

Ica:

Cen Bifthriet. Eca.

Cocog.noten. Alieuw.

25anannas.

(1) bos of oulues- Oufij, Oubi. sBoztel.

Gecft mp muja Toma may oufi. obos:

Sieck.

Mapii:

Jongbe Cocog. D'mauta.

cozalen.

Een S5ijeker.

ziser.

Bilfouck.

Obertte.

sten lant.

Soet pler.

3aa.

Lickafoa, of acachoa

Hakoubea.

Hequij.

Matau,

Iatou.

A jouita, of ajouda.

Moạij:

$D_{a}$ of jito.

Alick.wi.

Eé MBoztel too Acoua. genaemt/Ziet:

achticly' gemer =

beit op die batet.

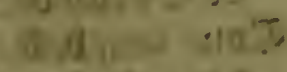

Sterten. Fitrous.

Dogben. ... Matta.:

Bozen. "Talinga.

Congye. Alello.it?

Xippen. Lamatori.

Bangen. Calâfou.

Strat of keel. Oya. li

23 azten. Chou. inner

Sherte. Fatra: is

भlant. Coloij...

Pacus: Efou. .tilc

23actr. Talaffa.

Canoen. Nyfoof lyfo.

cham - uan't Ourousg, ou. Hooft.

2Beenen. VVa2ij.

Ganot in Fatinga.lima, vingers.

Ragelen. Maij ninia.

20upch. Tinay.

.ve Rugghe. "Toua.

Icbonoers. Touauma,

De billen. Mouris ".

Eé jongê. Tama.

Oocliter. ... Toubou.

Bzoulue. Farri.

Slaapen. Mooij. iny

Banten. Pipi.

een loug of Fare.

Hutte.

Steen. Fattou.

$2500 m$. Talie, of taliei.

Hout.

急位.

Cralen.

Lachaai.

Hackoumiea.

Een Scljip. Wach.

Bercken. Pouacca:

eentisaen. Moa.

nater. VVaij.

Aeckinne. Oufa.

Zegfen. Oua.

Een 2 bill of Tocki gelrij.

Gamer. 
Tale van't Cocos-Eylandt.

Acrbe.

Kille.

tioper.

Tatto,

Ëit-Ctoel. Noffoa.

Gen 13 annekeh. elpentien. Şuppen.

Chienga:

Tatta.

Steecketr met een Tuamo. gigeg.

Eetilitcrat. ."Câfou.

Een gratien. D'fau.

Cocos-olie.

D'loló.

Gen zerglj. :Maoucha.

Heth-fepletr. Foulai.

Etet/ oft/cet-op; Tacki, necki:

Gaal-op. $\quad$ Foudij:

23anannag. Fouti:-

Obeg-wogtelen. Oufi.

Bater: VVaij:

Glie. it Eolo.

Itees. Poulaca:

Ren Stlject. Epouri:

Een bingex-rinets. Mamina -

Cien Trommel. Naffa:

een fort.

- $11 . .5$ neluij.

Een. Nécdel. Waijfogi.

Een IDEs.

- Fañi.

Lotant-spiegeltien Leffi iloa.

Cocos-bladers. Aes, Cifaror

Cotos-water.

Suptker.

Wacki:

Supther. Tolo.

23 alt van Corog. Pourou.

Een felier bacucht. Falafola:

Bactlemoeder.

Ėen 25 elle.

Cancel.

Een Couwetit. :

Y)et Buer.

Eenl

duet

Dziel

Bierl

Biif!

Tiffa, of Teffa.

Taula.

Kaij.

St\$l

SEtuenI

V.Vaffauw.

Oumou;

altht/

Regben

chietr.

Taci,

Loua,

Tolou.

Fa;

Lima;

Houno.

Fitou.

VValou,

Ywou.

Sp kandet nit

Ongefoula.

நoogfjer als thieut

tellen / wlu leerden

Gaet tot bot =

Dert tellen / met

baet tellingbe.

Ceif/

retonalf.

Ongcfouila taci.

Ongefoula lour.

Dit/of/ligier. Equi,

Tonct. - Màta.

Iaet betrin fié. Matta may.

Baer is niet. Neay, of Eay.

Reen. : : Eai.

3ae.,$\frac{1}{3}=$ Yio, of $\mathrm{X}$ jouw.

Dupbe: . Loupe:

Dact ig̀nict Eenw. micer.

Sanck.

Een teetken bã Lolle: willekom en adiets.

op't lijf.

Bzillen in't Mon. Danten.

Cen 25 etf met Nifo. booznetit.

Supcker. Lolo.

Llenn Bbos. Talo:

San Pampitr Kealiva.

gyefthilderde

Hiletjend.

Uen of met yooztens.

VVagga, wagga

Een wotelel die Acava : Atova; Ip hauluen/ Cava.

en Jaet Dzane af mlacthen.

Eet Sifipip. VVachi.

De namentian Tamay:en Fora,

twei docta

giers/beten=

De EDelliété.

cou/ uan balt/ Caffa Daer ip haet

klectieg mede

Jinuen om't

iiif.

zstm-ringlyen. : Tauwa pou:

Bingber.

Een groê ftuipt Lolou.

rappict.

คิที 
Talc oft fpraecke van de Cufte van Guinea nova.

Dins 23 zoot. Mafi.

Yever. Addc.

Berckeg leuer. Adde puacca.

Be-Eoninrk. Arikl.

Latoy, of latau. Oucxlte:

\section{ITale oft cplarke van De} Culte ban Guinea nova.

De Coniutr. Latieu. $w_{d}$

Cocos-3aten. Lamas.

Een binne.

Coocq.

Een 1Bercken. Tembor.

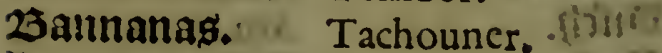

Goenders ezpes Palima Coo.

ren.

Bater. ....tic

Biftch.

Crabbekeng.

Dan oft Daan:

Hifföi.

Contecor.

25 etel of Spyij=

blaben.

pinangf.

Lkalck.

Dlic.

Cralen.

gicer.

Een भisk.

Kattang

c'boofi.

SReult.

Bazen.

Tanden.

1000z-booft.

lyape.

igant.

joeten.

2303 ften.

Alrman.

crangte.

Zippen.

Sctjouders.

23 upek.

kugghe.

singber.

23 illex.

Slapen.

25 aert

Eten.

Bzincken.

Hakê of IDảgê. Paring.

Pongliman, $11,1 \mathrm{C}$

Hermangh.

Tabaing vouling.

Haliyug.

Balang.

Baheing. . N!: :

Cateling liman.

Poutong.

Heim.

Incam beffer.

Nam Nam,

Anda.
Strot of litele.

Con Con Hăgh.

Hiet.

Een Storl.

Daan.

Stecnen.

Dutw2.

Zant: aen lant. Behoul. stare

Bif bourk.

laoul.

poaerlemaraer.

Sonne.

as ane.

Sterien.

CorronTāborin

Naas.

Calangh: :-

Maemetia.

Hnoafe van tjout. Hereris.

Ciffer of tartaruge Foun.

- De kingen die $3 \mu$ - láoull. Doaz: De neute dzagê/uan been.

Oliphants tant. Tembron bis. coundetieg / of Calcaloun. Iijnties.

De Zze. Taas.

Goute Stweerden. Scél. aterde / of roode Taar.

Alector

Sant.

Coon

Legber.

Ous.

Een aingher. Gimmio Halla:

Een Spiets ban Mareet. bout.

Een 2llagaiy. Houvan.

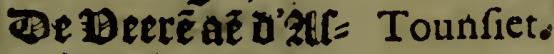

legaii.

gsentchen-bloet. Daar aug.

Berckendebloet. Daar de rembos.

glotten $/$ die jn Nandikea. Dzagljen.

Een Cansa. Takoup.

Zaepen of trheppẽ Gemoe Haloes.

c'gbeberthte. Faffer.

Cen ifet niet. Capte andefin. 
Tale ende Spraecke van defe twee Eylanden.

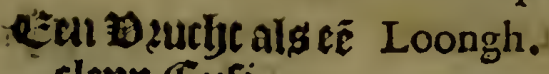
clepn Cufi.

Maachten.

Attingani.

Een naent ban $\mathfrak{e} \tilde{e}$ Tarhar lieuw. gevangen inan.

Jchen kent niet. Kin kabbeling lotgtee.

\section{Splaecke vant Eplant} Moyfes.

\section{Jaă. Onberepe bout. 23root. Sibnouders. 250elten. Intien. (1)oghe. Yaet fiet. Strot of vitele. Tonobe. 25aett. Reule. 23ananas. \\ Thtal bier comen teritont. \\ Eerckelt. \\ Cacog. \\ Mop. \\ Alep.} Ilu.

x: etew

2. Tiwe

3. Diel

4. Bier!

5. Tijel

6. 5et!

7. Seluell

3. 2telyt

9. Regluen!

10. Thien. LEen finente.

Sagu.

Pouhonnori.

Carracerreram. Sou foll.

Pou hanking. Mattanga. Matta may. Comieconnon. Caramme. Parre wourou. Wanfrugo: Hiwoundi, Taboun:

Kirrekir.

Camboulir

Lamas:

Tata.

Facht of tees Atont.

1. $\mathrm{KaOH}_{2}$

2. $\mathrm{Roa}$,

3. Tolou.

4. Wati,

5. Rima,

6. Eno.

7. Wijtrou.

s. Ejalou;

9. Siwa.

1o. Sangaponlo. Mitoa.
盟

Innode. Micoura. Mafirim.

Spzarcke gan't épland Moo, op twee graden: daer de fflitzen waven.

Cocos. 2bannannag: Bercken. Bater. Bember. Bifch. ROE. Hont Cralen. Cami ban 25eell. Marmauw. Spiicker of Bler. Bee. $23200 \mathrm{t}$.

2320ot in Coecken Soome: gbebacken. Cleedieng ban de Maije. Bzautmen. ZArmbanden. Hant-bogbe. . Partina. Biilen. $\quad$ : Bare. Slapen. Moune Bif́b febietett. I Tineanij: Berckens-tant sona. Een vier-boetich Pari-wou, Ghediert.

Sonne. 解任. Ardươ. Geel witte ogel. Mavi:kackerous, Een geele Moztel: Aou. als Gurtuma. Mitte Cozalen. Saffera poute. Riet/ niet/ ßeen/ Taop, Trop. neen.

Mecty uan bier. Hoijda, Een leectiet $e_{n}=$ Arti. landt.

$$
\text { FINIS. }
$$






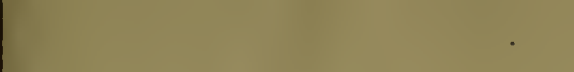



Collection of
W. L. SHE LDEN 


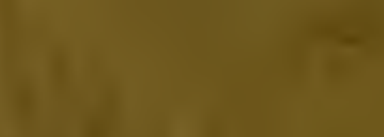

I

+

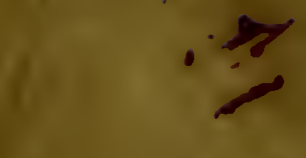

1 $101+10$

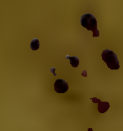

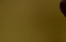

1

5

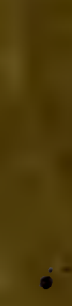

(1)

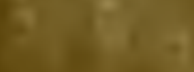

